Федеральное государственное бюджетное учреждение науки

ЦЕНТРАЛЬНЫЙ ЭКОНОМИКО-МАТЕМАТИЧЕСКИЙ ИНСТИТУТ РАН CENTRAL ECONOMICS AND MATHEMATICS INSTITUTE RAS

РОССИЙСКАЯ

RUSSIAN

АКАДЕМИЯ НАУК

ACADEMY OF SCIENCES

Л.Я. Клеппер

МРВ МОДЕЛИ ДЛЯ ПЛАНИРОВАНИЯ ЛТ

ЗЛОКАЧЕСТВЕННЫХ ОПУХОЛЕЙ

(справочное пособие

для лучевых терапевтов

и медицинских физиков) 


\section{ББК 28.071:В6}

K48

Клеппер Л.Я. МРВ модели для планирования ЛТ злокачественных опухолей (справочное К48 пособие для лучевых терапевтов и медицинских физиков) [Текст]: монография. - М.: ЦЭМИ РАН, 2020. - 195 с. (Рус.)

Работа посвящена проблеме математического моделирования и оптимизации планов лучевой терапии (ЛТ) злокачественных опухолей при помощи созданной математической модели, модифицированного распределения Вейбулла, (модели МРВ).

В работе приводятся результаты расчета оптимальных значений параметров модели МРВ для различных органов и тканей, полученные на основе систематизированных клинических данных, приведенных в работах Emami et al. и Kehwar et al. Они позволяют успешно использовать модель МРВ для планирования ЛТ злокачественных опухолей.

На основе модели МРВ разработаны математические модели для редукции неоднородных дозовых распределений в эквивалентные по ВЛО адекватные дозы (АД) однородного облучения органов и тканей. Показано, что эти модели могут быть также использованы для проверки согласованности исходных клинических данных.

Ключевые слова: моделирование, оптимизация, дозиметрия, радиобиология, лучевая терапия, неоднородные распределения дозы.

Klepper L.Ia. MDV model for planning RT malignant tumors (reference allowance for radiotherapist and medical physicist). - Moscow, CEMI Russian Academy of Sciences, 2020. - 195 p. (Rus.)

Work is dedicated to problem of mathematical modeling and optimization plan RT of the malignancies with the help of created mathematical model, modified distribution Veybull, (the model MDV). The results of the calculation of the best values parameter happen to in work to models MDV for different organ and tissues, received on base systematized clinical data, provided in work Emami et al and Kehwar et al. They allow successfully using the model MDV for planning RT malignancies. Mathematical models is designed on base of the models MDV for reduction non-uniform dose distribution in equivalent on NTCP the uniform irradiation organs and tissues. It Is Shown that these models can be are also used for check of consensus source clinical data. tribution.

Keywords: modeling, optimization, dosimetry, radiobiology, radiotherapy, non-uniform dose dis-

Рецензенты: Каткова И.П., д.м.н., гл.н.с. ИСЭПН РАН;

Царфин Л.В., к.э.н., с.н.с. ЦЭМИ РАН.

УДК $51-76: 616-006$

ББК 28.071:в6

ISBN 978-5-8211-0789-3

Текст. Клеппер Л.Я., 2020 г.

() ФГБУН Центральный экономико-математический институт РАН, 2020 г 


\section{ОГЛАВЛЕНИЕ}

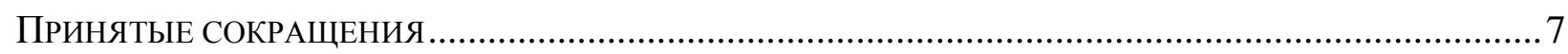

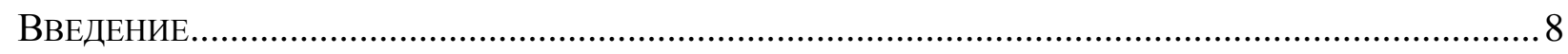

ГЛАВА 1. МАТЕМАТИЧЕСКИЕ МОДЕЛИ ДЛЯ РАСЧЕТА ТД В ОРГАНАХ И ТКАНЯХ

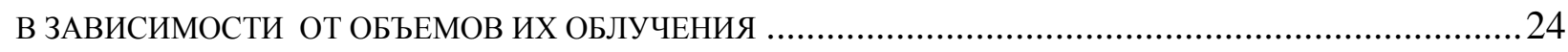

1.1. ТД для различных органов и тканей организма ........................................................2 24

1.2. Зависимость ТД от объема облученного органа или ткани ......................................25

1.3. Систематизированные клинические данные о вероятностях возникновения лучевых повреждениях органов и тканей в зависимости от дозы и объема облучения. Значение разовой дозы предполагается фиксированной

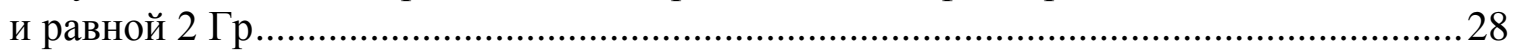

1.4. Математические модели для расчета ТД в зависимости от условий облучения ...... 32

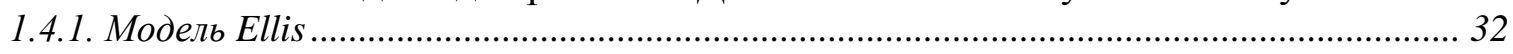

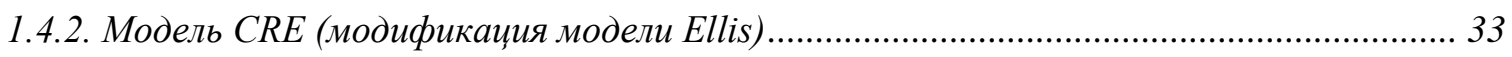

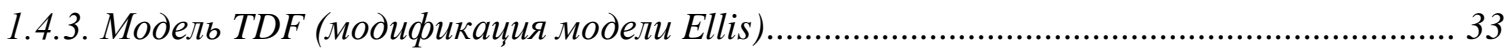

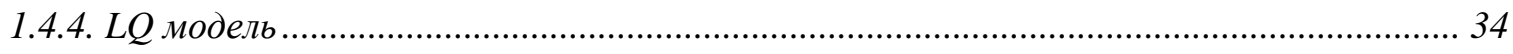

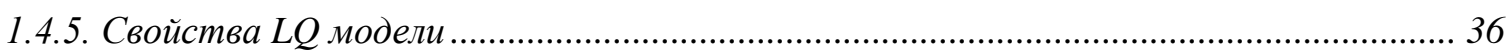

1.5. Анализ математических моделей фракционирования дозы для планирования ЛТ опухолевых заболеваний ….................................................. 38

ГЛАВА 2. МРВ МОДЕЛЬ ДЛЯ ОПИСАНИЯ ВЛО В ОРГАНАХ И ТКАНЯХ КАК ФУНКЦИИ

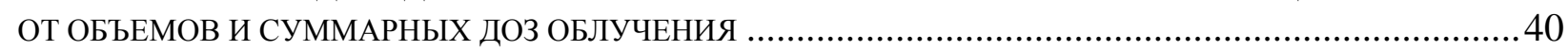

2.1. Модель МРВ (модифицированное распределения Вейбулла) .................................... 40

2.2. Экспресс-метод расчета ВЛО в органах и тканях при помощи модели МРВ ..........41

2.3. Модель НРВ (нормальное распределение вероятностей) для расчета ВЛО

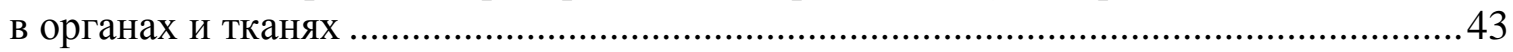

2.4. Анализ результатов расчета ВЛО в органах и тканях организма при помощи

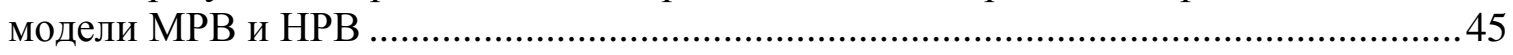

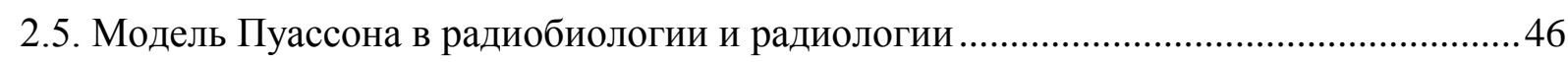

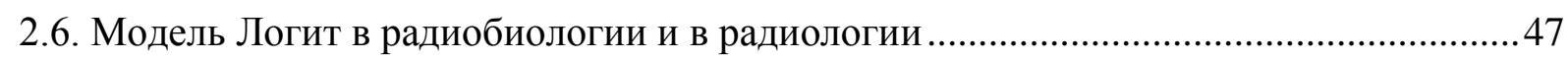

ГЛАВА 3. СИНТЕЗИРОВАННЫЕ МАТЕМАТИЧЕСКИЕ МОДЕЛИ (СМ МОДЕЛИ), СВЯЗЫВАЮЩИЕ МЕЖДУ СОБОЙ ЗНАЧЕНИЯ ВЛО, СУММАРНЫЕ И РАЗОВЫЕ ДОЗЫ, И ЗНАЧЕНИЯ ОБЪЕМОВ

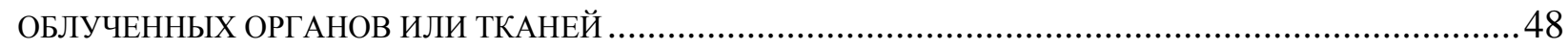

3.1. Синтезированные математические модели (СМ модели) ….......................................4 48

3.2. CM1 модель (модель Ellis + модель МРВ) ..............................................................50

3.3. СМ2 модель (модель Ellis + модель НРВ) …........................................................57

3.4. LQ модель. Связь ТД с разовой дозой и объемом облученной ткани.......................6 60

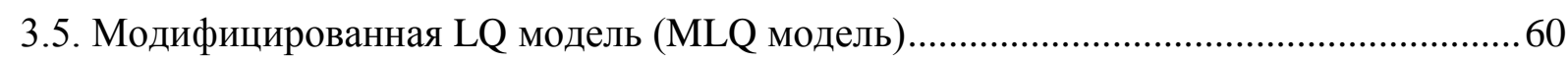

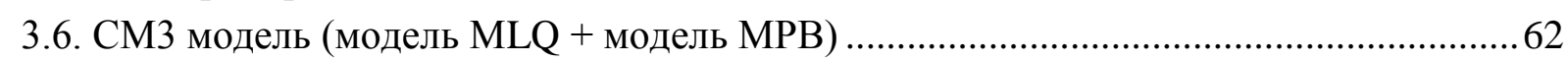

3.7. Использование CM3 модели и модели MPB для планирования облучения сердца. 
3.8. Упрощенные методы расчета зависимости между суммарными и разовыми

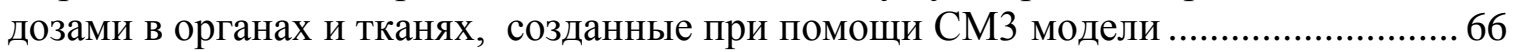

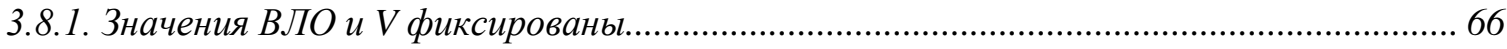

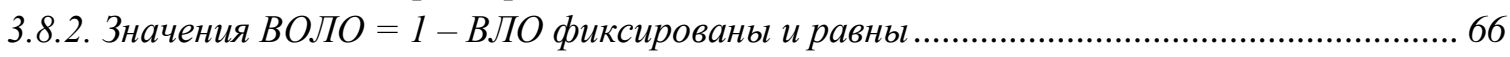

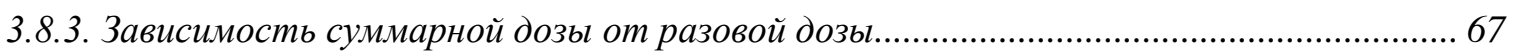

3.9. Описание ВЛИ ранней стадии РМЖ при помощи СМЗ модели

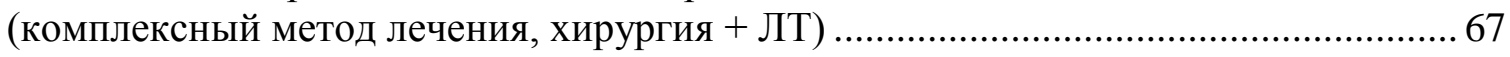

3.10. Параметры СМ3 модели, рассчитанные для некоторых органов и тканей ............ 69

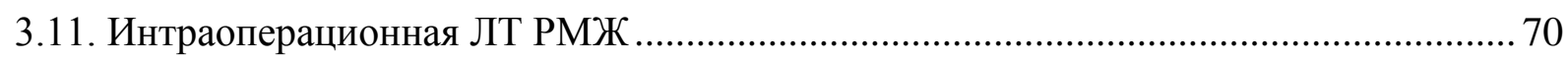

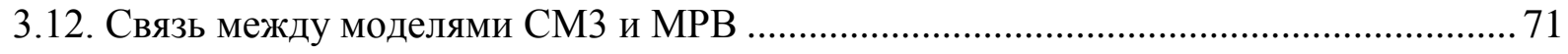

3.13. Синтезированная CM4 модель (LQ модел+модель MPB)......................................... 72

3.13.1. Расчет ВЛИ РМЖ при комплексном методе лечения РМЖ

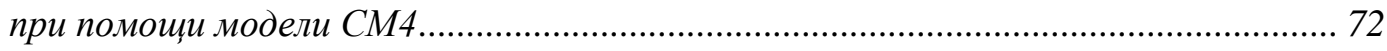

3.14. Синтезированная модель CM5 (MLQ модель + MPВ модель) …............................ 75

ГЛАВА 4. РАСЧЕТ ОПТИМАЛЬНЫХ ЗНАЧЕНИЙ ПАРАМЕТРОВ НАСТРОЙКИ МОДЕЛИ МРВ ДЛЯ ОРГАНОВ И ТКАНЕЙ НА ОСНОВЕ СИСТЕМАТИЗИРОВАННЫХ КЛИНИЧЕСКИХ ДАННЫХ

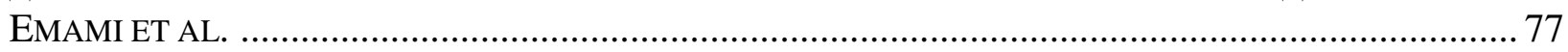

4.1. Мочевой пузырь. Осложнение: симптомы контрактуры и уменьшение объема ..... 80

4.2. Плечевое сплетение. Клинические повреждения нервов.......................................... 82

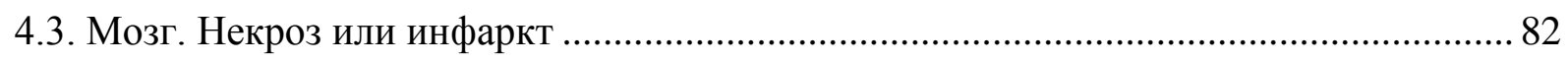

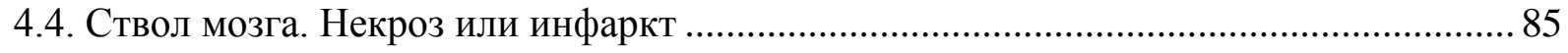

4.5. Конский хвост. Осложнение: клиническое проявление повреждения нервов......... 87

4.6. Кишка. Осложнение: обструкция, перфорация, изъязвление, фистула..................... 87

4.7. Среднее ухо 1. Осложнение: Острый серозный отит ……....................................... 89

4.8. Среднее ухо 2. Осложнение: хронический отит...................................................... 90

4.9. Пищевод. Осложнение: стриктура, перфорация ...................................................... 90

4.10. Головка и шейка бедра. Осложнение: Некроз ........................................................... 93

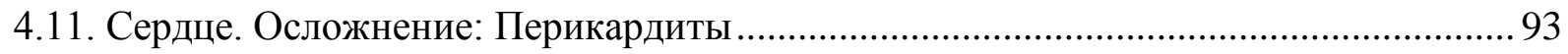

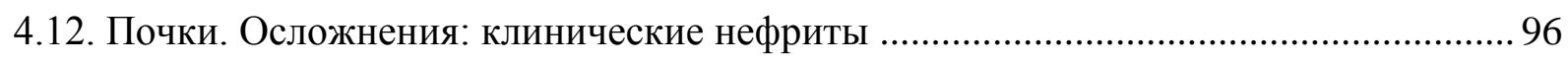

4.13. Гортань 1. Осложнение: Некроз хряща...................................................................... 98

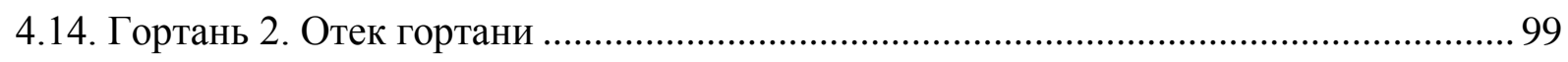

4.15. Хрусталик. Осложнение: катаракта, требующая вмешательства ........................... 100

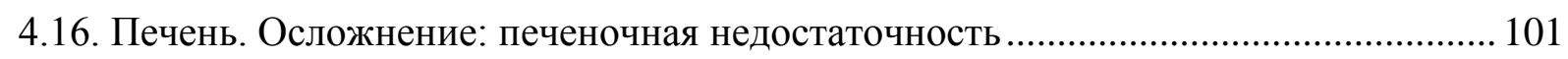

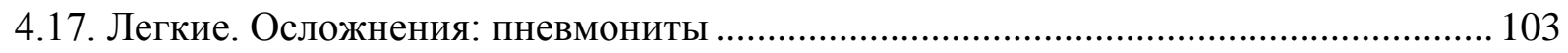

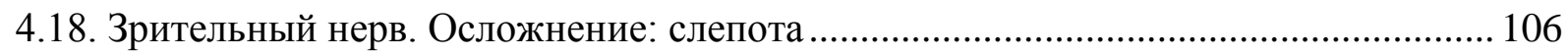

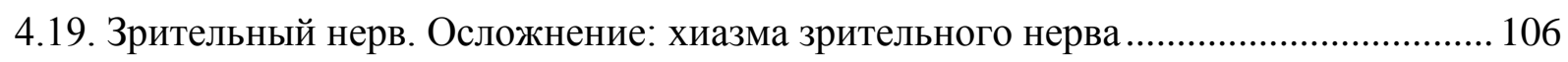

4.20. Околоушная слюнная железа. Осложнения: ксеростомия ……................................ 106

4.21. Прямая кишка. Осложнения: тяжелый практит, некроз, стеноз, свищ.................. 107

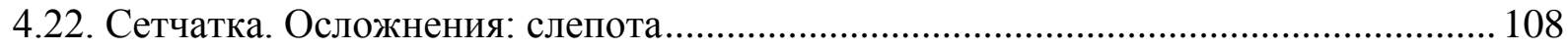

4.23. Ребро грудной клетки. Осложнение: патологический перелом ….......................... 108

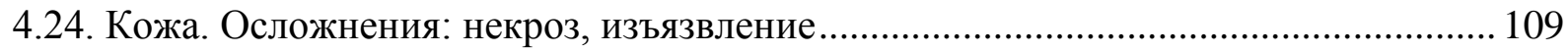

4.25. Тонкий кишечник. Осложнения: обструкция, перфорация ……….......................... 111 
4.26. Спинной мозг. Осложнения: миелиты, некрозы ................................................... 113

4.27. Желудок. Осложнения: изъязвление, перфорация ................................................... 114

4.28. Щитовидная железа. Осложнения: тиреоидит .................................................... 116

4.29. Кость, сустав нижней челюсти (КСНЧ). Осложнение: выраженное

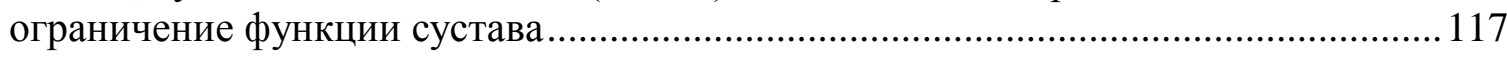

4.30. Кость, головка бедренной кости. Осложнение: некроз .......................................... 118

ГЛАВА 5. РАСЧЕТ ОПТИМАЛЬНЫХ ЗНАЧЕНИЙ ПАРАМЕТРОВ МОДЕЛИ МРВ ДЛЯ ОРГАНОВ И ТКАНЕЙ НА ОСНОВЕ СИСТЕМАТИЗИРОВАННОЙ КЛИНИЧЕСКОЙ ИНФОРМАЦИИ

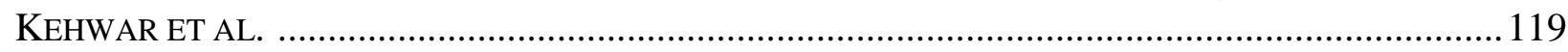

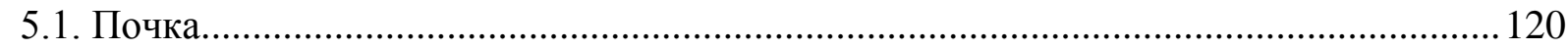

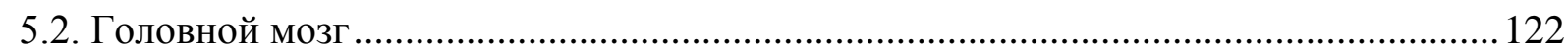

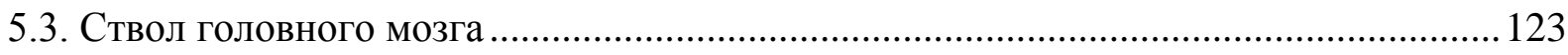

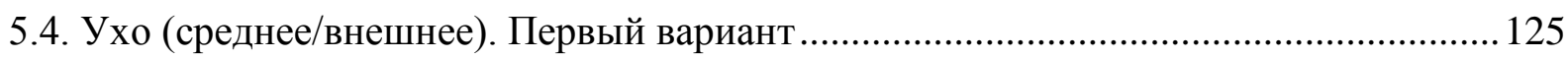

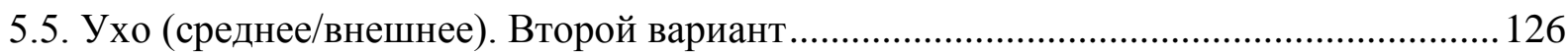

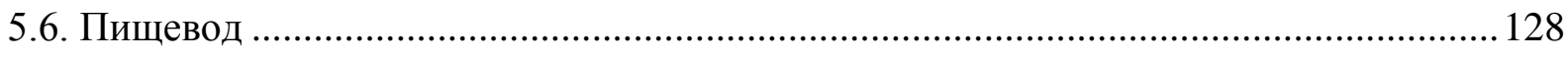

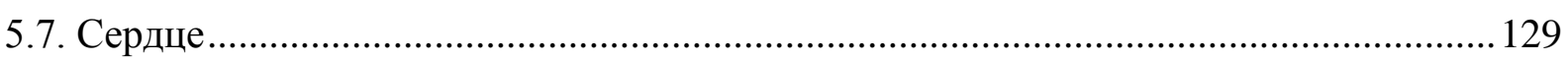

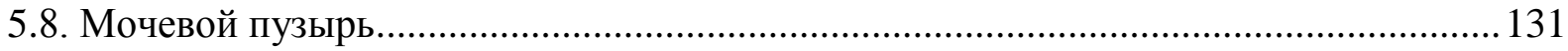

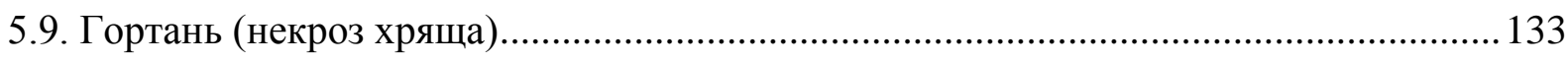

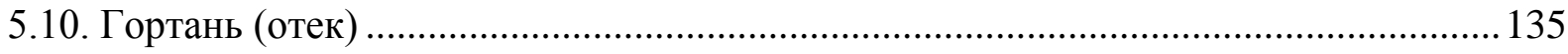

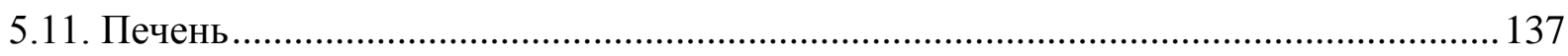

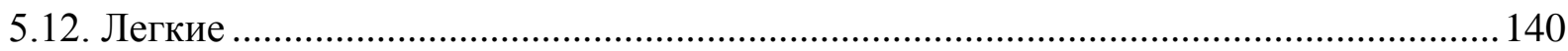

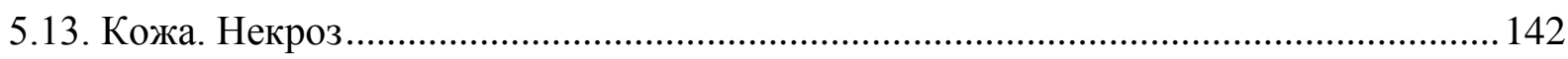

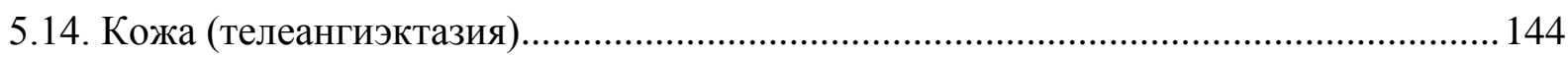

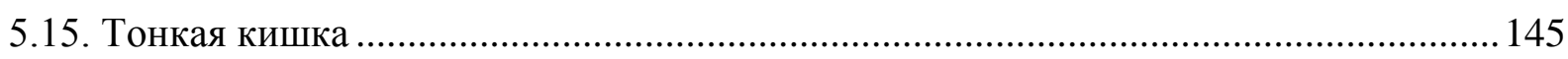

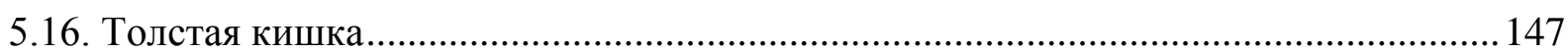

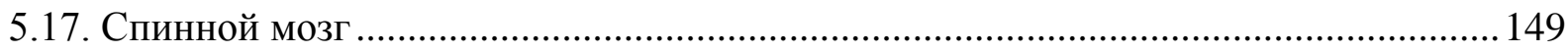

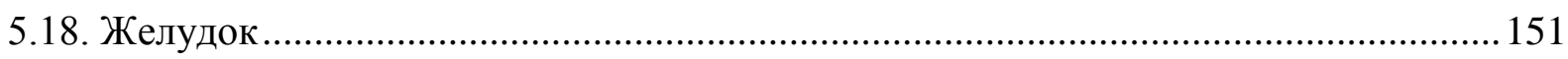

5.19. Височно-нижнечелюстной сустав и нижняя челюсть (ВНС и НЧ) ....................... 153

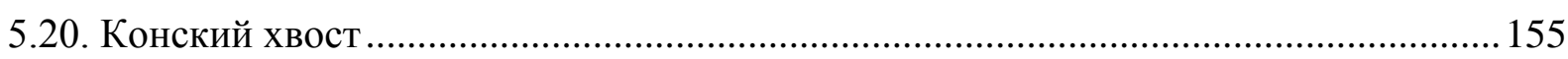

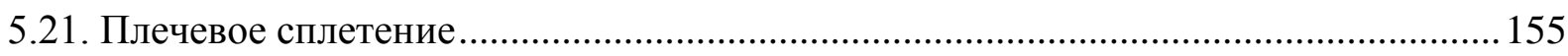

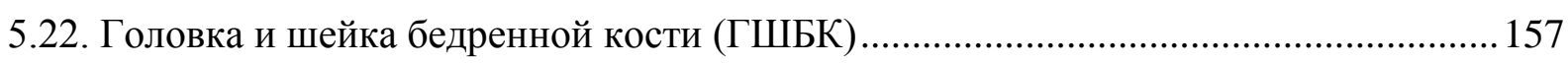

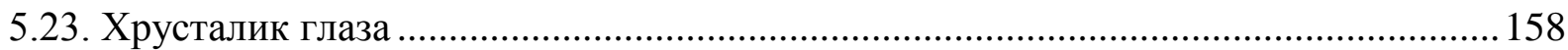

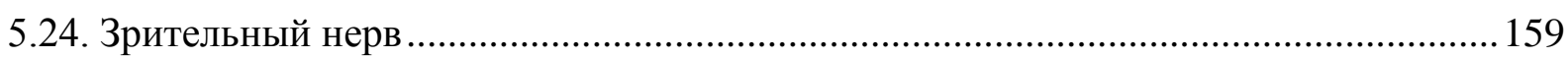

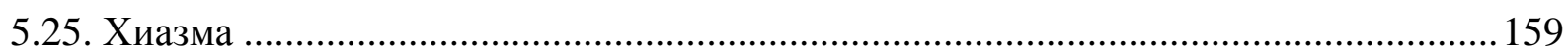

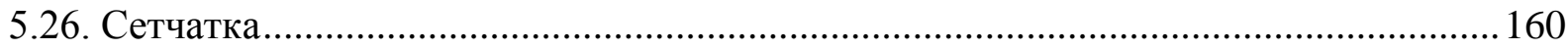

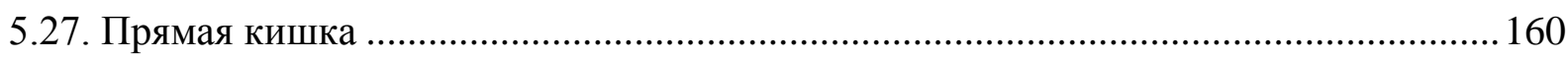

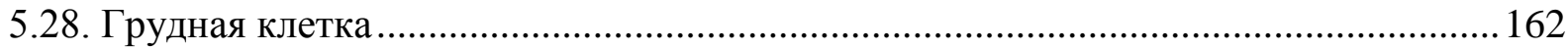

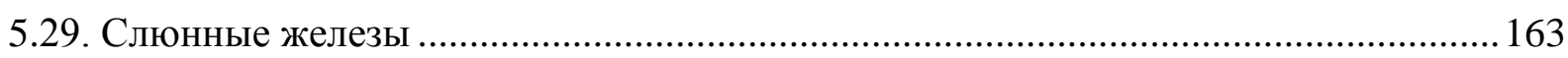

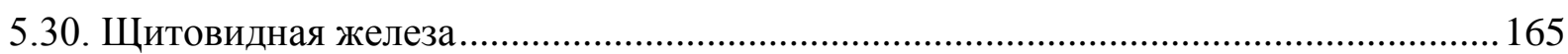

ГЛАВА 6. ОПТИМАЛЬНЫЕ ЗНАЧЕНИЯ ПАРАМЕТРОВ МОДЕЛИ МРВ ДЛЯ НЕКОТОРЫХ

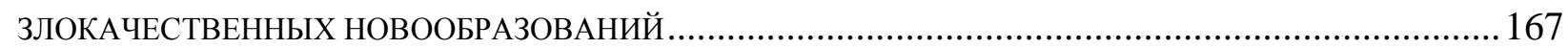

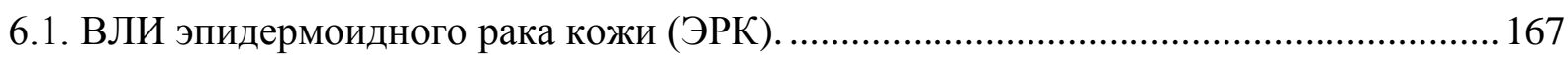




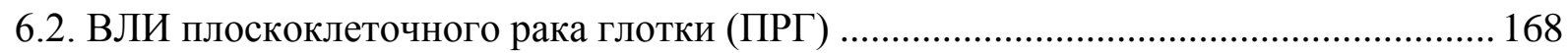

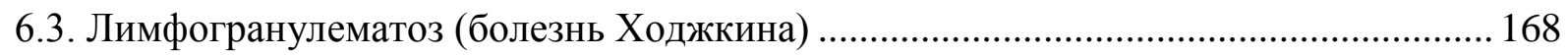

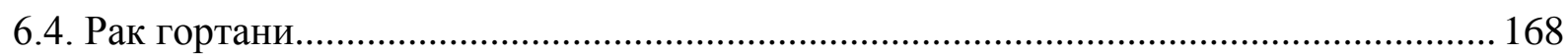

ГЛАВА 7. РЕДУКЦИЯ НЕОДНОРОДНЫХ ДОЗОВЫХ РАСПРЕДЕЛЕНИЙ В ОРГАНАХ И ТКАНЯХ В ОДНОРОДНЫЕ РАСПРЕДЕЛЕНИЯ ДОЗЫ (В АД). РАСЧЕТ СООТВЕТСТВУЮЩИХ РЕДУЦИРОВАННЫХ ЗНАЧЕНИЙ ВОЛОР (ВЛОР)

7.1. Проблема оценки неоднородного распределения дозы и оценка соответствующего значения ВЛО в органе или ткани при планировании ЛТ злокачественных опухолей.

7.2. Математические модели для расчета редуцированного значения дозы однородного облучения органа или ткани, и для расчета соответствующего ей редуцированного значения ВОЛОр (ВЛОр).

7.3. Свойства математических моделей (7.7) и (7.11).

7.4. Проверка согласованности систематизированной клинической информации для некоторых органов и тканей, основанная на расчете редуцированных значений ВОЛОр и АД при помощи моделей (7.7) и (7.11) ….............................. 176

7.5. Редукция неоднородного распределения ВОЛО при помощи модели (7.7) .......... 178

7.6. Редукция неоднородного распределения ТД в АД при помощи модели (7.11).... 179

ГЛАВА 8. ПОВЫШЕНИЕ ТОЧНОСТИ РЕДУКЦИИ НЕОДНОРОДНОГО ДОЗОВОГО РАСПРЕДЕЛЕНИЯ В РЕЗУЛЬТАТЕ «ПРИВЕДЕНИЯ НЕОДНОРОДНОГО ДОЗОВОГО РАСПРЕДЕЛЕНИЯ К ТОТАЛЬНЫМ ОБЪЕМАМ ОБЛУЧЕНИЯ» ПРИ ПОМОЩИ МОДЕЛИ МРВ.

ЗАКЛЮЧЕНИЕ

СПИСОК ЛИТЕРАТУРЫ 


\section{ПРИНЯТЫЕ СОКРАЩЕНИЯ}

ЛТ - лучевая терапия опухолевого заболевания.

ММ - математическая модель.

МЖ - молочная железа.

РМЖ - рак молочной железы.

ПРГ - плоскоклеточный рак гортани.

ЭРК - эпидермоидный рак кожи.

ТД - толерантная доза для здорового органа или ткани организма.

СОД - суммарная опухолевая доза облучения.

РОД - разовая опухолевая доза.

Гр - единица измерения дозы.

ВЛИ - вероятность локального излечения опухолевого заболевания.

ВЛО, ВОЛО - вероятность лучевого осложнения и вероятнсть отсутствия лучевого осложнения в здоровом органе или ткани.

ВЛОр, ВОЛОр - редуцированные значения ВЛО и ВОЛО.

МРВ - ММ, модифицированное распределение Вейбулла для расчета ВЛО (или ВЛИ) в зависимости от дозы и объема облучения.

НРВ - нормальное распределение вероятностей для расчета ВЛО (или ВЛИ).

ФД - фракционирование дозы.

LQ модель - линейно-квадратичная модель, предназначенная для планирования фракционированных схем (методов) ЛТ.

ДГДО, ИГДО - дифференциальная и интегральная гистограмма доза-объем.

CM1, ..., СМ5 - синтезированные математические модели, образованные в результате синтеза (объединения) двух моделей: модели, которая описывает эквивалентные по ВЛО, или ВЛИ, схемы ФД, и модели, которая описывает зависимость ВЛО (или ВЛИ) от дозы при фиксированной схеме ФД.

Критерий ТД - относительная оценка дозового распределения в виде отклонения построенного дозового распределения от заданного клинического распределения дозы в органе или ткани.

Критерий ВЛО - оценка вероятности возникновения лучевого осложнения в виде относительного отклонения теоретических (модельных) значений ВЛО от заданых клинических значений ВЛО в органе или ткани.

Критерий ВЛО + ТД - оценка дозового распределения по двум объединенным критериям, ВЛО и ТД. 


\section{ВВЕДЕНИЕ}

Настоящая работа является итогом многолетних исследований автора в рамках научного направления «Математическое моделирование и оптимизация планов лучевой терапии злокачественных опухолей», выполненных по планам НИР ЦЭМИ РАН. Это направление было организовано в институте на основе личной договоренности академика Н.П. Федоренко и президента АН СССР академика М.В. Келдыша.

Исследования выполнялись при активном участии сотрудников ЦОЛИУВ, НИИ рентгенологии и радиологии и других медицинских учреждений России.

Успех лучевого лечения опухолевого заболевания зависит от ряда факторов: локализации опухоли, типа опухолевого заболевания, радиочувствительности здоровых органов и тканей, окружающих патологический очаг заболевания, общего состояния пациента и эффективности выбранного плана облучения [1-11].

Опыт применения лучевой терапии (ЛТ) свидетельствует о том, что она может быть чрезвычайно эффективной как самостоятельный метод лечения, так и в сочетании с хирургией и химиотерапией. Успеху лучевого лечения способствует применение различных модификаторов, радиосенсибилизаторов и радиопротекторов.

В настоящее время задача планирования ЛТ осуществляется на основе толерантных доз (ТД) в здоровых органах и тканях и на терапевтических дозах в очаге опухолевого заболевания (мишени), выделенных коллективно лучевыми терапевтами, радиобиологами и медицинскими физиками. Эффективный план ЛТ должен создавать такое дозовое поле в очаге опухолевого заболевания (мишени), которое приведет к необратимому разрушению опухолевого процесса и сведет к минимуму лучевые повреждения здоровых органов и тканей организма. Выбор плана ЛТ осуществляется лучевым терапевтом и медицинским физиком. Для выбранного плана облучения, вручную, или при помощи ЭВМ, рассчитывается суммарное дозовое поле, которое анализируется лучевым терапевтом и медицинским физиком. В случае необходимости задаются альтернативные планы облучения, рассчитываются соответствующие им суммарные дозовые поля, и процесс анализа и сопоставления альтернативных дозовых распределений продолжается. Такая же процедура реализуется и в том случае, когда по заданным ограничениям на дозовое поле, в результате решения специальной экстремальной задачи, определяются оптимальные планы ЛТ.

Какими недостатками обладает приведенная схема определения плана ЛТ и что необходимо сделать для того, чтобы повысить эффективность планирования ЛТ злокачественных опухолей? 
1. ТД, которые являются обобщением коллективного опыта лучевых терапевтов и выступают как ограничения на дозовые поля в здоровых органах и тканях, в большинстве случаев рассматриваются как постоянные величины или как величины, лежащие в определенных границах. В настоящее время можно считать твердо установленным, что ТД зависят от объемов облученных органов и тканей и от распределения доз. Для формирования эффективных терапевтических дозовых полей необходимо учитывать как объемы, так и распределения доз в облученных органах и тканях.

2. Предполагается, что ТД приводят к ВЛО в органах и тканях, которые не превышают 3-5\% лучевых осложнений, но в процессе лучевого лечения нередки случаи, когда ВЛО в здоровых органах и тканях превышают толерантные уровни облучения. Для корректного планирования ЛТ необходимо создавать математические модели, которые описывают ВЛО в здоровых органах и тканях как функции от условий их облучения, т.е. обобщить понятие ТД и учитывать это в планировании ЛТ.

3. Для корректного планирования ЛТ необходимо создавать и использовать математические модели, описывающие ВЛО как функции от распределения доз и объемов облученных органов и тканей. Необходимо разрабатывать методы редукции неоднородных дозовых распределений в эквивалентные по ВЛО однородные дозовые распределения. Это чрезвычайно важно, так как в основном все разработанные в настоящее время математические модели, которые используются при планировании ЛТ, предполагают, что распределения доз в органах и тканях однородны.

4. ТД для здоровых органов и тканей зависят от схемы фракционирования дозы (ФД) во времени. От нее зависят также и ВЛО в органах и тканях. Установление зависимости ТД и ВЛО от ФД существенно упрощает проблему оценки альтернативных планов ЛТ и определение оптимальных планов облучения.

5. Одним из самых сложных вопросов планирования ЛТ злокачественных опухолей является вопрос о критериях оптимальности ЛТ. При планировании ЛТ на основе ТД для здоровых органов и тканей этот вопрос в известной степени нивелируется. Действительно, достаточно выбрать такой план облучения, при котором дозы в здоровых органах и тканях не превосходят толерантные значения ТД, как одну из главных задач планирования ЛТ можно считать решенной. В действительности все обстоит намного сложнее. Дело заключается в том, что в подавляющем большинстве случаев значения дозы в здоровых органах и тканях, особенно вблизи опухоли, превышают толерантные уровни. (Заметим, что так было и во всех тех случаях, совокупность которых образует клинический опыт ЛТ). Возникает другой важный вопрос, какие дозы в условиях ее неоднородного распределения в органах и тканях следует считать толерантной дозой? 
Для корректного планирования ЛТ злокачественных опухолей и оценки альтернативных планов облучения необходимо разрабатывать математические модели для перехода от неоднородных распределений дозы в органах и тканях к адекватным дозам (АД) эквивалентного по ВЛО их однородного облучения. С помощью «АД [12] или равноценной дозы», которая впервые была введена КейримМаркусом и его учениками [13] (мы предложили назвать ее адекватной дозой (АД) [12]), может быть осмыслен опыт ЛТ и сохранена традиционная для ЛТ терминология, «доза в органе или доза в ткани».

6. Возможность определять ВЛО в здоровых органах и тканях и использовать эту информацию при планировании ЛТ сразу же обнажает многокритериальную природу задачи планирования ЛТ, так как ВЛО в органах и тканях, а также ВЛИ опухолевого образования, выступают в качестве критериев оптимальности, которые имеют конкретный и хорошо понятный врачам-радиологам смысл. Они являются прогностическими оценками лучевых воздействий на опухолевые и здоровые органы и ткани организма. С помощью таких оценок, которые имеют конкретные числовые выражения и число которых относительно невелико, врачирадиологи и медицинские физики могут соразмерять риск онкозаболевания с риском возникновения необратимых лучевых осложнений в здоровых органах и тканях в результате ЛТ.

7. Основная цель ЛТ - добиться необратимой регрессии опухолевого процесса без серьезных лучевых осложнений в стромальных тканях (ложе опухоли), а также и в других здоровых органах и тканях организма. В настоящее время выбор опухолевой дозы и режима ФД в основном осуществляется лучевым терапевтом и медицинским физиком на основе накопленного коллективного опыта лучевой терапии.

8. Для повышения эффективности ЛТ необходимо совершенствовать математические модели, описывающие кинетику лучевых повреждений и восстановлений опухолевых и здоровых органов и тканей организма, и на их основе осуществлять объективный выбор оптимальных планов ЛТ.

В настоящей работе сделана попытка наметить пути решения некоторых поставленных вопросов. В ряде случаев недостаток клинических наблюдений затруднил проверку гипотез, при помощи которых может быть построена система непротиворечивых радиологических и радиобиологических моделей. Эффективная проверка этих гипотез возможна в результате целенаправленного сбора и обработки клинической и экспериментальной информации о лучевых реакциях в органах и тканях при различных методах их облучения и в тесном взаимодействии с лучевыми терапевтами и радиобиологами. 
Работа состоит из восьми глав. В первой главе рассматриваются математические модели, которые описывают ТД для здоровых органов и тканей организма в зависимости от условий их облучения. Среди множества существующих в настоящее время математических моделей мы использовали: модель Ellis и две еe модификации, CRE и TDF [14-16], а также, наиболее распространенную в настоящее время, LQ модель [17]. Эти модели были использованы для создания Синтезированных (объединенных) Математических моделей (СМ моделей), описание которых приводятся в главе $3[6,7,18-22]$.

Во второй главе приводится описание предложенного нами модифицированного распределения Вейбулла (МРВ модель [6, 7]). Она связывает между собой значение ВЛО, суммарную дозу $D$ и объем $V$ облученного органа или ткани. Разовая доза $d$ считается фиксиованной и мы, в случае необходимости, считали ее равной наиболее распространенной разовой дозе 2 Гр. Она не входит в число параметров модели МРВ. Опыт использования модели МРВ в планировании ЛТ опухолевых образований показал, что она может быть успешно использована в работе лучевых терапевтов как для описания лучевого воздействия на здоровые органы и ткани организма $[6,7]$, так и для описания лучевого воздействия на опухолевые ткани (см. главу 6).

Помимо МРВ модели $[6,7]$ для расчета ВЛО и ВЛИ в работе было использовано Нормальное Распределение Вероятностей, (НРВ) (модель, предложенная Lyman [23, 24]). Приводится сравнение клинических значений ТД с теоретическими (модельными) значениями ТД, рассчитанными при помощи модели МРВ и модели НРВ. Рассмотрены особенности применения модели Пуассона [25] в описании лучевых повреждений органов и тканей. В работе [26, 27] мы показали, что модель Пуассона может быть эффективно использована в проблеме определения радиобиологических параметров опухолевого образования на основе феноменологической описания зависимости ВЛИ опухолевого образования от дозы (метод «Три графика» [30]).

В третьей главе приводятся впервые разработанные нами синтезированные математические модели (СМ модели) [18-22], которые описывают связь между значением ВЛО, объемом облученного органа (или ткани), суммарной дозой, и, что очень важно, разовой дозой облучениия. Это следующие модели:

СМ1 модель - синтез модели МРВ и модели Ellis [28].

СМ2 модель - синтез модели НРВ (нормальное распределение вероятностей) и модели Ellis [23, 24].

СM3 модель - синтез модели MPB и модифицированной нами LQ модели $[17,19]$ (MLQ модели). 
В работе приводятся также СМ4 модель и СМ5 модель, которые получены в результате синтеза математической модели МРВ с некоторыми модификациями LQ и MLQ модели.

При помощи СМ3 модели (в частности, благодаря тому обстоятельству, что в ней в качестве параметров модели, наряду с ВЛО, объемом облучения $V$ и однородной суммарной дозой облучения $D$, используется еще и разовая доза $d$ ), нам удалось впервые построить математическую модель для выбора оптимальных условий облучения ранних стадий рака молочной железы (РМЖ) в комплексном методе лечения Системы «опухоль + ложе опухоли» $[10,29,30])$, и, в частности показать, как оптимальный план ЛТ связан с ГиперФ или с ГипоФ схемами фракционированного облучения (они определяются используемой разовой дозой облучения).

В четвертой главе приводятся результаты расчета оптимальных значений параметров модели МРВ, полученные на основе систематизированной клинической информации для ВЛО $=0,05$ и ВЛО $=0,50$, значений ТД, соответствующих трем относительным объемам, (или площадям), облучения, $(1,3 ; 2 / 3 ; 1,0)$, подготовленной Emami et al. [31].

Оптимальные значения параметров модели МРВ определялись в результате решения специальной экстремальной задачи, в которой минимизировались отклонения теоретических (модельных) значений ВЛО от заданных клинических значений, равных 0,05 и 0,50, (критерий ВЛО), или минимизировались отклонения теоретических (модельных) значений ТД от заданных клинических значений ТД (критерий ТД), либо одновременно использовались оба критерия (сумма приведенных относительных значений двух критериев).

Анализ результатов, полученных в главе 4, показал, что модель МРВ удовлетворительно описывает клинические значения ТД и хуже описывает клинические значения ВЛО. Это понятно, так как в настоящее время планирование ЛТ злокачественных опухолей в основном происходит по заданным значениям толерантных доз (ТД) в здоровых органах и тканях, и по заданной опухолевой дозе однородного облучения очага опухолевого заболевания. Значения ВЛО в органах и тканях и значения ВЛИ опухоли расчитываются редко, и точность их расчетов в большинстве случае осложняется неоднородностью распределения дозы в опухолевых и здоровых органах и тканях организма.

Таким образом, исходная систематизированная клиническая информация, которая необходима для настройки модели МРВ, состоит из значений дозы однородного облучения органа или ткани (значений ТД), значений ВЛО, которые возникают при однородном облучении заданного относительного объема (или площади) облученного органа или ткани. В этом легко убедиться, рассмотрев си- 
стематизированные клинические данные, приведенные в главе 1: Emami et al. [31] табл. 1.1, и Kehwar et al. [32, 33] - табл. 1.2.

Распределение доз в органе или в ткани могут быть неоднородными и тогда не адекватными могут оказаться значения параметров модели МРВ. Выйти из этого положения можно так, как это сделали Kehwar et al. [32, 33]. Систематизированные клинические данные о значениях ТД однородного облучения органов и тканей они приводят в виде значений доз с 95\% доверительными интервалами. Эта информация чрезвычайно важна и, как показали расчеты, точность настройки модели МРВ и СМ моделей, а также точность модельного воспроизведения клинических значений ТД оказывается выше, чем это наблюдается с клиническими данными Emami et al. [30]. Но при этом, в большинстве случаев, в стороне остается вопрос о точности расчета значений ВЛО в здоровых органах и тканях, и точности расчета ВЛИ опухолевого заболевания. Как известно, эти величины являются важными прогностическими оценками, которые характеризуют эффективность плана ЛТ. При помощи ВЛИ и ВЛО лучевой терапевт может для каждого альтернативного плана ЛТ соразмерить тяжесть онкозаболевания с риском вызвать тяжелые необратимые лучевые повреждения в здоровых органах и тканях организма и, тем самым, получить критериальные оценки планов ЛТ.

Можно ли улучшить ситуацию с расчетом значений ВЛИ и ВЛО? Мы полагаем, что можно, но для этого необходимо воспользоваться моделями редукции неоднородных дозовых распределений, использование которых приведет к расчету редуцированного однородного дозового распределения и соответствующего ему редуцированного значения ВОЛОр (ВЛОр). Таким образом, можно редуцировать неоднородность дозового распределения в ткани и рассчитать дозу ее однородного облучения, эквивалентного значению рассчитанного ВОЛОр (ВЛОр). Различные подходы к решению проблемы неоднородности распределения дозы рассмотрены нами в главе 7.

В пятой главе приводятся результаты расчета оптимальных значений параметров МРВ модели, основанные на клинических данных, систематизированных Kehwar et al. [32, 33] и представленных в табл. 1.2 (глава 1). Так же, как и в главе 4, определение оптимальных значений параметров модели МРВ осуществлялось с тремя различными критериями оптимальности. Первый критерий - ВЛО. Он приводит к наиболее близким значениям клинических и теоретических (модельных) значений ВЛО. Второй критерий - ТД, приводит к наиболее близким значениям клинических и теоретических (модельных) значений ТД. Поиск оптимальных значений параметров модели МРВ по третьему критерию заключался в определении наиболее близких теоретических (модельных) значений ВЛО и ТД (совместно) к их клиническим значениям. 
1. Анализ результатов моделирования клинической информации при помощи модели МРВ показал, что наиболее близкие рассчитанные (модельные) значения ТД к заданным клиническим значениям ТД получаются в том случае, когда используется критерий ТД. Это является следствием того, что в настоящее время планирование ЛТ осуществляется в основном по заданным значениям ТД.

2. Необходимо отметить, что значения ТД, которые приводятся в табл. 1.1 и 1.2, и на основе которых определяются оптимальные значения параметров модели МРВ, являются приближенными. Между тем считается, что клинические значения ТД соответствуют однородным распределениям доз в органах или тканях, в то время как реальные распределения доз в них большей частью являются неоднородными! Поэтому, в табл. 1.2, предложенной Kehwar et al., [32, 33], приводятся дозы в органах и тканях с 95\% доверительными интервалами.

3. Для достаточно точного расчета однородного дозового распределения в органе или в ткани необходимо осуществить редукцию неоднородного дозового распределения, которое может быть описано в виде ДГДО (или ИГДО).

В настоящей работе мы описали вывод «традиционной» математической модели для редукции неоднородного распределения дозы [9], и разработали новую, более «точную» математическую модель, основную на «Приведении неоднородного дозового распределения к тотальному облучению органа или ткани», которая может быть использвана для решения этой проблемы (см. главу 8).

4. Мы показали, что в том случае, когда модель редукции неоднородного дозового распределения выделена при помощи модели МРВ, тогда единственный параметр $A_{2}$, входящий в число параметров модели МРВ, (см. главу 7), используется и в математической модели, предназначенной для редукции неоднородных дозовых распределений. Для его точного расчета необходимо, чтобы в исходном систематизированном клиническом материале, который используется для расчета оптимальных значений параметров модели МРВ, ТД соответствовали бы однородным дозовым распределениям.

В настоящей работе мы не привели оптимальные значения параметров СМ модели из-за недостатка места и постараемся сделать это в нашей следующей работе. СМ модели, в отличие от существующих математических моделей, впервые позволят учитывать в планировании ЛТ разовую дозу и, мы полагаем, что это благтворно скажется на эффективности планирования ЛТ злокачественных опухолей. Ведь только благодаря СМ3 модели и возможности варьировать разовые дозы облучения Системы «опухоль + ложе опухоли», нам удалось разработать метод планирования ЛТ для комплексного лечения ранней стадии рака молочной железы. 
В шестой главе описываются параметры настройки модели МРВ для некоторых злокачественных новообразований, для которых нам удалось найти в литературе систематизированные клинические данные [34-37].

В седьмой главе описываются полученные нами результаты, посвященные выводу «традиционной» математической модели, [9], для редукции неоднородных дозовых распределений, выполненных на основе использования модели МРВ, а также для вывода редуцированного значения ВЛОр, которая соответствует редуцированному однородному распределению дозы [30]. (Вывод традиционной модели для редукции неоднородных распределений дозы в работе [9] не приводится).

Исследованы свойства созданных моделей. Установлена зависимость адекватной редуцированной дозы (АД) от параметра $A_{2}$, который входит в число параметров модели МРВ. Анализ неоднородных дозовых распределений и проблемы их свертки (редукции) в однородные дозовые распределения, позволил выявить интересную особенность, связанную с неоднородным облучением Системы «опухоль+ложе опухоли». Очевидно, что при облучении Системы однородными дозовыми полями, значения дозы в опухоли и в ложе опухоли идентичны. Но если Cистема «опухоль + ложе опухоли» облучается неоднородными дозовыми полями, тогда рассчитанные редуцированные дозы (АД), эквивалентного по ВЛОр однородного облучения опухоли и ложса опухоли, окажстся разными. Это призойдет в том случае, если параметры модели МРВ $A_{2}$ для опухоли и ложа опухоли будут отличаться друг от друга. Величина АД является возрастающей функцией от параметра $A_{2}$. Если $A_{2}$ для опухоли больше значения $A_{2}$ для ложа опухоли, тогда при облучении Системы неоднородным распределением дозы, редуцированная доза облучения в опухоли будет больше редуцированной дозы облучения в ложе опухоли. К сожалению, результаты настройки модели МРВ для опухоли и ложа опухоли свидетельствуют о том, что обычно, в рассмотренных нами случаях, параметр $A_{2}$ для опухоли оказывался меньше, чем соответствующее значение $A_{2}$ для ложа опухоли (пока это, правда, следует рассматривать как предположение!). В этом случае следует признать, что однородные дозовые распределения в Системе «опухоль + ложе опухоли» являются более эффективными, чем неоднородные дозовые распределения, так как при неоднородном распределении дозы значение АД в ложе опухоли оказывается больше, чем доза в самой опухоли. Если это действительно так, то следует признать, что лучевые терапевты оказались правы, когда стараются облучать Систему «опухоль + ложе опухоли» однородными дозовыми полями. Но, открытый нами «эффект» требует дальнейшего тщательного и всестороннего исследования.

В главе 7 мы показали, что математические модели, предназначенные для расчета редуцированных дозовых распределений, позволяют также оценить со- 
гласованность исходной систематизированной клинической информации. Дело заключается в том, что для ВЛО $=0,05$, (и для ВЛО =0,50), облучение 1/3 и 2/3 объема ткани соответствует ее тотальному неоднородному облучению. Поэтому, для нее можно рассчитать редуцированную дозу АД однородного облучения ткани. Но клиническая доза тотального однородного облучения ткани входит в исходную клиническую информацию (для ВЛО $=0,05$ - это $D_{3}$, для ВЛО $=0,50$ - это $D_{6}$, см. главу 4 и 5). Значения АД и $D_{3}$, (или соответствующие значения АД и $D_{6}$ ), должны быть равны, если клиническая информация является согласованной (непротиворечивой). В противном случае можно считать, что их различие характеризует степень рассогласованности исходной клинической информации. Полученный результат важен, так как в нашем распоряжении в настоящее время находится только прямой метод оценки, сравнения рассчитанных теоретических (модельных) значений ТД с их клиническими аналогами.

В восьмой главе описывается разработанный нами новый метод редукции неоднородных дозовых распределений, который позволяет повысить точность редукции неоднородных дозовых распределений. Мы предлагаем назвать его методом «Приведения неоднородного дозового распределения к тотальным объемам облучения». Дело заключается в том, что построение «Традиционной модели» для редукции неоднородных дозовых распределений базируется на предположениях 7.1 и 7.2 (глава 7), согласно которым существует приближенная модель для расчета ВЛО в элементарном объеме облученной ткани с учетом объема всей облученной ткани. Она и использовалась для расчета редуцированного значения ВЛО (ВЛОр) и АД. Разработанный нами новый метод расчета редуцированного неоднородного распределения доз, метод «Приведения неоднородных дозовых распределений к тотальным объемам облучения», оказался более точным. Он включает в себя следующие операции.

1. Пересчет неоднородных распределений доз с разными объемами облучения к эквивалентным по ВЛО тотальным облучениям ткани путем пересчета неоднородного распределения доз при помощи модели МРВ. Заметим, что в результате осуществления этой операции редуцированное неоднородное распределение ВЛОр в ткани остается неизменным.

2. Расчет редуцированного значения АД для неоднородного распределения пересчитанных доз. В табл. 7.4, (глава 7), показано, что редуцированное значение АД, рассчитанные при помощи разработанного «нового метода» позволяют получать более точные значения АД, более близкие к клиническим значениям, чем рассчитанные традиционным методом. 
В табл. 7.4 первые четыре строки (1-4), относятся к коже (Kehwar et al.), строки (5-8) и (9-12) - к головному мозгу (Emami et al. и Kehwar et al.). Для каждого рассмотренного органа или ткани, первые две строки из четырех, относятся к расчету редуцированных доз традиционным методом. Последующие две строки относятся к расчету редуцированных значений доз методом приведения неоднородных дозовых распределений к тотальным объемам облучения. В девятом и десятом столбцах табл. 7.4 приводятся значения АД, полученные в результате редукции неоднородных распределений доз, и отклонения клинических значений $D_{\text {тот }}$ от рассчитанных значений АД в виде $\varepsilon, \%=100\left(\right.$ АД $\left.-D_{\text {Тот }}\right) / D_{\text {ТОт }} \cdot\left(D_{\text {Тот }}-\right.$ это клинические толерантные дозы тотального облучения органа или ткани при ВЛО = 0,05 и при ВЛО = 0,50).

Анализ полученных результатов по облучению кожи, и головного мозга, приведенные в табл. 7.4 свидетельствуют о том, что:

1) во всех рассмотренных случаях, АД тотального облучения органа или ткани превышает соответствующее клиническое значение $D_{\text {тот}}$;

2) разработанный новый метод, «приведение неоднородного дозового распределения к тотальным объемам облучения» для редукции неоднородных дозовых распределений, оказался точнее традиционного метода редукции неоднородных дозовых распределений. В этом легко убедиться, сравнив значения отклонений, приведенных в последнем правом столбце табл. 7.4.

Например, для кожи, традиционный метод редукции неоднородных дозовых распределений приводит для ВЛО =0,05 к абсолютным значениям отклонений $D_{\text {тот }}$ от АД $-9,22 \%$, а для ВЛО $=0,50-$ к 6,82\%. Использование нового метода приводит к следующим отклонениям: для ВЛО $=0,05-$ к 8,60\% < 9,22\%, для ВЛО $=0,50-\kappa 6,24 \%<6,82 \%$.

Для головного мозга, данные Emami et al., новый метод редукции неоднородных дозовых распределений приводит к меньшим абсолютным значениям отклонений, чем традиционный метод. Для ВЛО $=0,05,9,47 \%<22,91 \%$, для ВЛО $=0,50,4,65 \%<16,48 \%$.

Аналогичный результат наблюдается для головного мозга и данных Kehwar et al. Для ВЛО =0,05 - 12,38 < 15,39\%, для ВЛО =0,50, 7,60\% < 10,29\%. Для головного мозга и клинических данных Kehwar et al. расчеты приводят к меньшим отклонениям $D_{\text {тот }}$ от АД, чем для клинических данных Emami et al. Это, скорее всего, связано с тем, что Kehwar et al. более тщательно провели статистическую обработку клинической информации.

Хочется надеяться, что, разаботанные в настоящей работе методы редукции неоднородных дозовых распределений и развитие этой области знаний в виде 
направленного формирования однородных и неоднородных дозовых распределений, позволит решить вопрос о повышении эффективности ЛТ злокачественных опухолей однородными и неоднородными дозовыми полями. Они также позволят разрешить старый вопрос, на который до настоящего времени нет ответа, в каких случаях неоднородное облучение опухоли через решетчатые диафрагмы может быть эффективней, чем ее облучение однородными дозовыми распределениями, (открытыми полями)?

Кроме того, необходимо отметить, и это очень важно, что проблема оценки однородных и неоднородных дозовых распределений, их изучение и направленное формирование, является актуальной проблемой не только в радиологии и радиобиологии для лечения онкологических заболеваний. Она актуальна везде, где человеку приходится иметь дело с радиоактивным излучением, и на земле, и в космическом пространстве.

Цель настоящей работы заключалась в анализе сложившейся ситуации, связанной с математическим моделированием и оптимальным планированием ЛТ злокачественных опухолей, в выделении сопутствующих актуальных проблем. Их анализ приводит нас к заключению, что дальнейший прогресс ЛТ опухолевых заболеваний может быть связан с разработкой методов оценок и направленного формирования как однородных, так и неоднородных терапевтических дозовых распределений. Они могут существенно расширить возможности ЛТ, но для их эффективного использования необходимо всесторонне исследовать свойства неоднородных дозовых распределений, разработать методы их моделирования для формирования оптимальных неоднородных (или однородных) терапевтических дозовых полей.

Необходимо особо отметить, что проблему планирования ЛТ опухолевых заболеваний следует рассматривать как комплексную проблему, прогресс в которой требует совместных усилий специалистов из различных областей знаний. (Анализ современного состояния медицинской физики и некоторые проблемы ее развития были рассмотрены В.А. Костылевым в работе [11].)

Отметим наиболее важные полученные нами результаты и вопросы, связанные с проблемами математического моделирования оптимальных однородных и неоднородных дозовых распределений, которые следует разрешить для повышения эффективности планирования ЛТ опухолевых заболеваний.

1. В отечественной и зарубежной литературе, посвященной ЛТ опухолевых заболеваний неоднородными дозовыми распределениями, речь в основном идет о поиске редуцированных значениях дозы, АД. Между тем, наряду с неоднородным распределением дозы, возникает неоднородное распределение ВЛО (ВОЛО) в элементарных объемах органов и тканей, характеризующих неоднородные дозовые 
распределения. Вопрос об определении редуцированного значения ВОЛОр (или ВЛОр) столь же важен и актуален, как и определение редуцированного значения дозы, АД. Более того, мы говорим о редуцированной дозе неоднородного облучения ткани АД, которая приводит к такому же значению ВОЛО, как и рассматриваемое неоднородное распределение дозы. Но таким значением ВОЛО как раз и должно быть редуцированное значение ВОЛОр. В настоящей работе мы показали, что ВОЛОр, АД и модель МРВ тесно связаны друг с другом.

2. Не следует забывать, что оценка неоднородных дозовых распределений приводит к установлению значений АД и ВОЛОр, но только ВОЛОр в здоровых органах и тканях и ВЛИр опухолевого заболевания могут служить глобальными многокритериальными оценками неоднородных дозовых распределений. Именно они позволяют соразмерять тяжесть онкозаболевания с риском вызвать необратимые лучевые повреждения в ложе опухоли и в здоровых органах и тканях организма.

3. Мы показали, каким образом можно получить традиционную математическую модель для редукции неоднородных дозовых распределений в АД и математическую модель для редукции ВЛО (ВОЛО) в ВЛОр (ВОЛОр), основанные на использовании математической модели МРВ.

4. В работах [10, 28-30] мы показали, что использование неоднородных дозовых распределений при облучении Системы «опухоль+ложе опухли» могут привести к тому, что дозы в опухоли и в ложе опухоли будут отличаться друг от друга. Этого нет при однородном облучении Системы.

5. В работе [30] мы показали, что определить параметры $\gamma$ для опухолевых тканей можно в результате обработки феноменологической зависимости ВЛИ опухоли от дозы облучения при помощи разработанного нами метода «Три графика» (ТГ). Метод ТГ был использован нами для проверки гипотезы (см. работу [9]): образуют ли опухоли системные единицы (системно связанные агломерации опухолевых клеток)? В результате проведенной работы мы пришли к заключению, что образуют. Удалось построить гипотетическую картину эволюции агломерации опухолевых клеток под действием ионизирующего излучения [30]. При облучении агломераций систем опухолевых клеток происходит их распад и к тому моменту, когда суммарная опухолевая доза достигает терапевтических значений, агломерации разрушаются и опухоль оказывается состоящей в основном из изолированных клоногенных опухолевых клеток, а также из погибших и поврежденных излучением опухолевых клеток. Эта работа была основана на использовании распределения вероятностей гибели-выживания опухолевых клеток моделью Пуассона, которая обычно «плохо» описывает эволюцию состояния популяции опухолевых клеток в процессе всего облучения [30], но удовлетворительно может описывать «мгновен- 
ные снимки» состояния популяции опухолевых клеток. Поскольку лучевого терапевта в основном волнует проблема уничтожения популяции опухолевых клеток, он в большинстве случаев имеет дело с конечной стадией облучения опухолевых клеток в больших дозах. Радиобиологические свойства таких опухолевых клеток могут значительно отличаться от состояния популяции опухолевых клеток на начальном этапе облучения.

6. Мировой опыт отечественных и зарубежных организаций здравоохранения по применению неоднородных дозовых распределений для ЛТ опухолевых заболеваний, образованных при помощи свинцовых решетчатых диафрагм (РД), свидетельствует о том, что в некоторых случаях она может быть чрезвычайно эффективной для лечения запущенных и тяжелых форм опухолевых заболеваний [77-80].

Во всех случаях рассматривались РД, в которых открытые участки РД образовывали правильную сетку. Со временем интерес к лучевой терапии неоднородными дозовыми распределениями пошел на убыль и прекратился совсем. Почему это произошло? На наш взгляд, это могло быть вызвано отсутствием надлежащей теоретической основы для анализа и развития такого подхода, отсутствием методов математического моделирования процессов неоднородного облучения и оптимизации планов ЛТ при помощи специальных методов решения сложных экстремальных задач. Также отсутствовали клиническая и специальная экспериментальная информация, столь необходимые для построения математических моделей, описывающих воздействие неоднородных дозовых распределений на опухолевые и здоровые органы и ткани организма. Хочется надеяться, что в настоящее время ситуация начнет изменяться к лучшему.

7. Следует отметить, что в настоящее время существуют методы ЛТ с направленным формированием неоднородных терапевтических дозовых распределений, которые образуются при помощи линейных источников радиоактивного излучения (иголок), радиоактивных гранул, и т.д. [4, 5]. Они образуют внутритканевые, внутриполостные и аппликационные методы облучения (брахитерапию). Именно с брахитерапии и началась вся лучевая терапия, которая используется и «здравствует» в настоящее время. Затем к ней добавилась дистанционная ЛТ, основанная на использовании различных пучков ионизирующего излучения, которые генерировались различными радиационными установками (аппаратами). Не исключено, что изучение неоднородных дозовых распределений, образованных одним или несколькими источниками излучения при внутритканевой и внутриполостной лучевой терапии, станут «тропинками» к изучению проблемы направленного формирования эффективных терапевтических неоднородных дозовых распределений. 
8. Созданные нами математические модели для редукции неоднородных дозовых распределений в эквивалентные по ВЛО однородные распределения дозы, позволяют выявить новые интересные аспекты проблемы планирования ЛТ злокачественных опухолей. Приведем некоторые из них (см. главу 7 и 8, а также работы [10, 26-30]).

8.1. ВЛИ опухолевого заболевания и ВЛО в здоровом органе или в ткани зависит от интегральной поглощенной дозы и распределения дозы. В работе [27, $28,30]$ мы показали, что при фиксированной интегральной дозе в органе или ткани минимальное значение ВЛО соответствует их однородному облучению средней дозой, которая равна интегральной дозе, деленной на объем облученного органа или ткани. При фиксированной интегральной дозе любое неоднородное облучение органа или ткани приводит к увеличению значения ВЛО!

Таким образом, для уменьшения лучевой нагрузки на здоровые органы и ткани организма необходимо стремиться к минимизации интегральной дозы и к равномерному распределении дозы в ткани. В случае равных интегральных доз предпочтение следует отдавать однородным дозовым распределениям. Они приводят к минимальным значениям ВЛО в ложе опухоли и в других здоровых органах и тканях.

8.2. Рассмотрим теперь облучение Системы «опухоль + ложе опухоли». При однородном облучении Системы, дозы в опухоли и в ложе опухоли будут равны. Но, при одном и том же неоднородном распределении доз редуцированные значения дозы, от которых зависят значения ВЛИ опухоли и ВЛО в ложе опухоли могут оказаться неравными, если неравными окажутся параметры $A_{2}$ в моделях редуцирования неоднородных доз. Причем, редуцированная доза будет тем больше, чем больше будет значение параметра $A_{2}$. Следовательно, если для ложа опухоли параметр $A_{2}$ будет больше аналогичного параметра для опухолевой ткани, выгодней облучать Систему «опухоль + ложе опухоли» однородными дозовыми распределениями. Если параметр $A_{2}$ для опухоли больше аналогичного параметра для ложа опухоли, тогда выгоднее облучать Систему неоднородным распределением дозы. Она приведет к большему значению редуцированной дозы в опухоли по сравнению с дозой в ложе опухоли. Правда, в этом случае возникает проблема выбора эффективного неоднородного распределения дозы, которое не приведет к рецидиву опухолевого заболевания. Возникает вопрос, на который у нас в настоящее время еще нет ответа: каким ограничениям должно удовлетворять неоднородное дозовое поле, чтобы эффективно уничтожить очаг опухолевого заболевания и не привести к рецидиву опухолевого заболевания. Очевидно, что при облучении Системы однородными дозовыми полями задача формирования терапевтического дозового поля выглядит намного проще. В этом случае не надо заботиться об участках опухоли с 
минимальными значениями дозы, которые могут привести к рецидиву опухолевого заболевания.

8.3. В современной радиологии требования к однородности дозового поля в объеме мишени (опухоли) достаточно высоки. Согласно рекомендациям ICRU [48] однородность дозового поля в опухоли необходимо поддерживать в пределах $[-5 \%,+7 \%]$. Возможно, что в этих пределах можно и нужно формировать неоднородные дозовые распределения в Системе «опухоль + ложе опухоли»?

8.4. В настоящее время существует брахитерапия, которая характерна чрезвычайно неоднородными дозовыми распределениями в опухоли, нарушающим рекомендации ICRU. Но, она является одним из наиболее эффективных методов ЛТ. А это означает, что медико-биологические критерии терапевтического дозового поля в опухоли не разработаны в должной мере. Опыт применения контактных методов ЛТ свидетельствует о том, что неоднородное терапевтическое дозовое поле в опухоли может быть достаточно эффективным [4, 5]. Проблема заключается в том, чтобы установить, каким критериям оно должно удовлетворять.

Если опухоль можно рассматривать как ткань со всеми присущими ткани свойствами, то оптимальным дозовым полем в опухоли при фиксированной интегральной дозе будет не однородное дозовое поле, которое предлагается формировать в настоящее время, а неоднородное дозовое поле! Оно приведет к максимальному значению редуцированной дозы и, следовательно, к максимальному значению ВЛИ. Заметим, что эта проблема до настоящего времени радиобиологами и лучевыми терапевтами понастоящему не рассматривалась.

8.5. Следует подчеркнуть актуальность затронутых проблем применения неоднородных дозовых распределений не только для лучевого лечения злокачественных новообразований. Они актуальны везде, где человек сталкивается с радиоактивным излучением, как на нашей планете, так и в космическом пространстве.

8.6. Проблема направленного формирования однородных и неоднородных дозовых распределений для лучевого лечения злокачественных опухолей является проблемой междисциплинарной и достаточно сложной. Для своего разрешения она требует тесного взаимодействия лучевых терапевтов, радиобиологов, медицинских физиков, математиков, программистов, а также других специалистов, которые могут быть полезными в деле формирования эффективных планов ЛТ злокачественных опухолей.

Автор надеется, что разработанные математические модели, методы оптимизации планов лучевой терапии и затронутые в работе актуальные проблемы поиска новых технологий для совершенствования методов ЛТ, помогут лучевым терапевтам, радиобиологам, медицинским физикам и другим специалистам, занятых 
лечением опухолевых заболеваний, в их благородной работе по сохранению здоровья и жизни людей.

В заключение хочу выразить глубокую благодарность руководителям Центрального экономико-математического института РАН (ранее АН СССР) нескольких поколений за понимание важности исследований в области планирования лучевой терапии злокачественных опухолей и постоянное внимание к развитию этого научного направления. В первую очередь это касается первого директора ЦЭМИ РАН академика Н.П. Федоренко и его заместителя по научной работе к.э.н. Ю.А. Олейника, которые способствовали зарождению и развитию в институте этого нового научного направления исследований «Математическое моделирование и оптимизация планов лучевой терапии злокачественных опухолей».

В настоящее время, я выражаю глубокую благодарность директору Центрального экономико-математического института РАН, члену-корреспонденту РАН А.Р. Бахтизину, научному руководителю института академику РАН В.Л. Макарову, ученому секретарю к.э.н. А.И. Ставчикову за понимание важности исследований в области лучевой терапии опухолевых образований и постоянное внимание к развитию этого научного направления. Считаю своим долгом выразить также искреннюю признательность всему коллективу научного Отделения теоретической экономики и математических исследований ЦЭМИ РАН, в котором я тружусь с 1963 г., за дружескую поддержку.

Мою признательность за внимание и помощь в работе прошу принять заведующих лабораториями ЦЭМИ РАН д.ф.-м.н. Л.А. Бекларяна, д.э.н. Р.М. Качалова и старшего научного сотрудника ЦЭМИ РАН, к.ф.-м.н. Л.В. Царфина - за помощь в подготовке текста рукописи и ряда полезных советов и замечаний.

Я бесконечно благодарен за сотрудничество врачам-онкологам и медицинским физикам, с которыми мне довелось вести совместные исследования:

ЦОЛИУВ: академику АМН А.С. Павлову, д.м.н. О.А. Замятину, к.м.н. Л.Д. Стиоп, к.т.н. М.А. Фадеевой и к.м.н. Л.И. Виноградову;

НИИ рентгенологии и радиологии: профессору д.ф.-м.н. А.Н. Кронгаузу, к.т.н. Р.С. Мильштейну;

РОНЦ им. Н.Н. Блохина: к.м.н. Т.В. Юрьевой, д.м.н. И.А. Гладилиной к.т.н. Т.Г. Ратнер;

Институт биофизики МЗ: д.ф.-м.н. И.Б. Кейрим-Маркусу, д.ф.-м.н. Д.П. Осанову, а также многим другим замечательным людям, без которых не удалось бы добиться успеха, и которых я вспоминаю с теплотой и признательностью. 


\section{Глава 1.}

\section{МАТЕМАТИЧЕСКИЕ МОДЕЛИ ДЛЯ РАСЧЕТА ТД \\ В ОРГАНАХ И ТКАНЯХ В ЗАВИСИМОСТИ ОТ ОБЪЕМОВ ИХ ОБЛУЧЕНИЯ}

\section{1. ТД для различных органов и тканей организма}

Предполагается, что равномерный режим фракционирования дозы (ФД) в ЛТ задан, если заданы: число сеансов облучения $N$, равные интервалы времени между сеансами облучения $t$, или общая длительность ЛТ, $T=(N-1) t$, и доза за фракцию $d$. Сокращенно это может быть записано в виде $(N, t, d)$ или $(N, T, D)$, где $D=N d$ - суммарная доза облучения. Обычно общая длительность лучевого лечения определяется интервалом времени между первым и последним сеансами облучения. Разовая доза при равномерном курсе ФД остается постоянной. Равномерный курс ЛТ, по самым разным причинам может быть разорван на два или более курсов (частей), интервалы времени между которыми существенно превышают интервал между двумя сеансами облучения на равномерном участке облучения. Такой курс ЛТ носит название сплит-курс и может быть описан следующим образом:

$$
\left(N_{1}, t_{1}, d_{1} ; T_{1}^{0}: N_{2}, t_{2}, d_{2} ; T_{2}^{0}: \ldots: N_{i}, t_{i}, d_{i} ; T_{i}^{0}: \ldots\right),
$$

где $\left(N_{i}, t_{i}, d_{i} ; T_{i}^{0}\right)$ - параметры $i$-го равномерного курса облучения, $T_{i}^{0}-$ интервал времени между $i$-м и $(i+1)$-м равномерным курсом ФД.

В ЛТ злокачественных опухолей одним из наиболее распространенных курсов ФД был и остается курс облучения, который состоит из 5 сеансов облучения в неделю, следующих друг за другом, начиная с понедельника так, что интервал времени между 5 сеансами на неделе равен 1 сут, а между неделями - 3 сут. Этот ритм ЛТ повторял ритм рабочей недели, был очень практичен, оказался эффективным с терапевтической точки зрения. Для $N=30$ при разовой дозе $d=2$ Гр в опухоли он получил название стандартного курса облучения. Понятно, что при дозе 2 Гр на опухоль (мишень) разовая доза в здоровых органах и тканях, окружсающих опухоль, может быть самой различной и даже превыщать 2 Гр. Поэтому было бы правильно, говоря о стандартном курсе облучения опухоли, считать разовую дозу в опухоли равной 2 Гр, а при стандартном облучении здоровых органов и тканей подразумевать только ритм ФД, всякий раз уточняя разовую дозу. Иногда стандартный курс облучения рассматривается как квазиравномерный режим ФД. При этом полагают $t=T /(N-1)$, где $T-$ общая длительность ЛТ, $T=7(k-1)+5$, где $k$ число недель. 
Будем называть схему ФД стандартизованной, если при 5 следующих друг за другом сеансами облучения в неделю с интервалом в 1 сут, и 3 сут между каждыми 5 сеансами облучения, число сеансов отличается от стандартного, т.е. $N>30$ или $N<30$.

Опыт ЛТ злокачественных опухолей нашел свое отражение в установлении суммарного значения канцерицидной дозы, которую следует подвести к опухоли в зависимости от ее типа и объема, при том или ином ритме ФД, в установлении ТД для большинства здоровых органов и тканей организма, которые могут оказаться в зоне интенсивного лучевого воздействия.

Определение. Под ТД в ЛТ подразумевают такую дозу ионизирующего излучения, превышение которой, при фиксированном режиме ФД и фиксированном объеме облученного органа или ткани, приводит к резкому увеличению вероятности возникновения лучевого осложнения (ВЛО) определенного клинически идентифицируемого лучевым терапевтом типа.

В настоящее время принято считать, что ТД однородного облучения приводит к ВЛО в органе или ткани, которая не превышает 3-5\% случаев лучевых осложнений за 5 лет наблюдения. Иногда для большей определенности ее полагают равной 5\%. Для большинства здоровых органов и тканей ТД были установлены эмпирически и являются обобщением коллективного опыта лучевых терапевтов [31-33]. Иногда ТД приводятся в виде некоторых интервалов, в которых с наибольшей вероятностью лежит ТД [32, 33]. Статистический разброс значений ТД определяется вариацией дозовых распределений, объемов облученных органов и тканей, а также биологической вариабельностью радиочувствительности пациента.

Выделение ТД, которые стали выступать как ограничения на дозы в здоровых органах и тканях, позволило сделать важный шаг на пути совершенствования методов ЛТ, поставить задачу выбора рациональных планов облучения, которая включает в себя выбор числа направлений облучения, размеров облучаемых полей, углов облучения, точек центрации пучков излучения и длительностей их экспозиции с тем, чтобы подвести к опухоли (мишени) необходимую канцерицидную дозу, не превысив ТД в здоровых органах и тканях [1, 2, 5-7, 49-61].

\section{2. Зависимость ТД от объема облученного органа или ткани}

Сначала для кожи [62], а затем и для других органов и тканей организма $[63,64]$ было установлено, что ТД зависят от объемов (или площадей) облученных органов и тканей. Исследование функциональной зависимости ТД от объемов (или площадей) облученных органов и тканей имеет большое значение для совершен- 
ствования методов ЛТ, правильной оценки эффективности планов облучения и определения оптимальных планов ЛТ злокачественных опухолей. В настоящее время одна из наиболее распространенных математических моделей зависимости ТД от объема облученной ткани носит степенной характер [64-66]:

$$
\frac{D\left(V_{1}\right)}{D\left(V_{2}\right)}=\left(\frac{V_{1}}{V_{2}}\right)^{-b},
$$

где $D\left(V_{1}\right), D\left(V_{2}\right)$ - ТД для отнсительных облученных объемов $V_{1}$ и $V_{2}$ одной и той же ткани организма, $b$ - параметр модели. Из (1.1) следует:

$$
D\left(V_{1}\right) V_{1}^{b}=D\left(V_{2}\right) V_{2}^{b}=D(1) \cdot 1^{b}=D(1)=\text { const, }
$$

т.е. произведение ТД на объем облученного органа или ткани в степени $b$ есть величина постоянная для рассматриваемой ткани, равная ТД единичного относительного объема (или площади) облученной ткани. Параметр $b$ зависит от типа ткани. Мы полагаем, что его значение лежит в интервале $0 \leq b \leq 1$, и не зависит от дозы и объема облученного органа или ткани.

Выражение (1.2) можно рассматривать как одну из первых «констант» в радиологии. Установление подобных констант, которые являются отражением закономерностей лучевого повреждения здоровых органов и тканей организма (тканевой уровень лучевого повреждения), может послужить основой для появления нового научного направления, количественной радиологии.

Мы уже отмечали, что в настоящее время принято считать ТД такую дозу однородного облучения органа или ткани, которая приводит к ВЛО порядка 3-5\% при фиксированном режиме ФД и фиксированном объеме облученного органа или ткани.

Полагаю, что было бы целесообразно выйти за рамки этого определения. Предлагаю считать ТД такую дозу, которая приводит не к ВЛО = 5\%, а к любому заданному лучевым терапевтом значению ВЛО = $P \%$. Такое определение ТД уровня ВЛО является естественным, если учесть большой интерес лучевых терапевтов к созданию и использованию математических моделей, которые описывают ВЛО в здоровых органах и тканях, и ВЛИ опухолевых заболеваний, и ту роль, которую они играют в совершенствовании методов планирования ЛТ.

Возникает чрезвычайно важный вопрос, сохранится ли зависимость «обобщенной» ТД уровня ВЛО от объема облученной ткани (1.1), останется ли неизменным параметр $b$, не будет ли он зависеть от ВЛО и объема облучения $V$. К сожалению, в настоящее время мы не можем однозначно ответить на этот важный вопрос. Поэтому мы выделим его в виде предположения, чтобы привлечь к нему внимание лучевых терапевтов, занимающихся сбором и обработкой радиологической инфор- 
мации. В настоящей работе мы будем предполагать, что модель (1.1) является адекватной и может быть использована в планировании ЛТ злокачественных опухолей.

Предположение 1.1. Для любого фиксированного режима ФД и при любом определении ТД уровня ВЛО зависимость ТД от объема облученного органа или ткани имеет следующий вид,

$$
\frac{D\left(P, V_{1}\right)}{D\left(P, V_{2}\right)}=\left(\frac{V_{1}}{V_{2}}\right)^{-b} \text {. }
$$

Таким образом, мы будем предполагать, что структура формулы (1.1.) сохраняется и для ТД уровня $P \%$. Из (1.3) следует, что при любом значении ВЛО $=P \%$, будет справедливо соотношение,

$$
D\left(P, V_{i}\right) V_{i}^{b}=D(P, 1), i=1, \ldots
$$

В двойном логарифмическом масштабе зависимость ТД от объема облученного органа или ткани для фиксированного значения $P$ описывается прямой линией. Следует подчеркнуть, что предположение 1.1 нуждается в экспериментальном и клиническом обосновании. Важность соотношения (1.3) и (1.4) для корректного планирования ЛТ, как это станет ясно в дальнейшем, трудно переоценить. Оно позволяет уточнять и существенно упрощать структуры математических моделей, описывающих ВЛО в тканях организма, облегчают анализ «надежности и достоверности» клинической информации при определении параметров моделей, описывающих ВЛО. В дальнейшем при создании математических моделей, описывающих ВЛО в здоровых органах и тканях организма, мы постоянно будем обращаться к предположению 1.1, проверять его и пытаться проследить адекватность его структуры реальным процессам лучевых повреждений различных органов и тканей организма при различных схемах ФД. Следует еще раз напомнить читателю, что предположение 1.1 следует рассматривать именно как предположение, которое нуждается в проверке и в экспериментальном и клиническом обосновании.

Система непротиворечивых предположений, при помощи которых в настоящей работе будут конструироваться радиологические модели, позволит в известной степени избежать возникновения эффекта «ползучего» эмпиризма, когда в рассмотрение вводятся новые модели или происходит расширение области применения хорошо известных моделей без должного логического, математического и экспериментального обоснования. Необходимость сосредоточить усилия на сборе радиологической информации и на ее качественной обработке становится ясной при анализе некоторых клинических данных, которые оказались в нашем распоряже- 
нии [6, 7], и при помощи которых мы провели анализ зависимостей (1.1)-(1.4). Основная цель анализа заключалась в попытке ответить на следующие вопросы:

1) справедливо ли предположение о том, что зависимость между дозами и объемами (площадями) облученных органов и тканей для фиксированного значения ВЛО описывается формулой (1.1) и (1.4);

2) зависит ли параметр $b$ от режима ФД.

Анализ полученных результатов расчета параметра $b$, которые вместе с доверительными интервалами приводятся в [32, 33], свидетельствуют о том, что в настоящее время мы еще не в состоянии однозначно ответить на поставленные вопросы. Для получения обоснованного ответа на эти вопросы необходимо иметь более обширную и хорошо систематизированную клиническую информацию. Такая информация появляется и она чрезвычайно полезна.

\section{3. Систематизированные клинические данные}

о вероятностях возникновения лучевых поврежсениях органов

и тканей в зависимости от дозы и объема облучения.

\section{Значение разовой дозы предполагается фиксированной и равной 2 Гр}

В настоящее время систематизированные клинические данные о зависимостях ВЛО от дозы и объема облучения органов и тканей наиболее полно представлены в работах Emami et al. [31, табл. 1.1], и Kehwar et al. [32, 33, табл. 1.2]. Заметим, что при использовании клинической информации из этих таблиц для настройки математических моделей для расчета ВЛО в органах и тканях, мы полагали разовую дозу фиксированной и равной 2 Гр.

В табл. 1.2 приводятся уточненные данные о зависимостях ВЛО от ТД для относительных объемов облученных органов и тканей $1 / 3,2 / 3$ и 1 с 95\% доверительными интервалами (ДИ). Эта таблица была построена в работе [32, 33] путем использования новых данных, дополняющих табл. 1.1 [31].

Таблицы 1.1 и 1.2 представляют собой значения ТД, которые приводят к ВЛО $=5 \%$ и ВЛО $=50 \%$ для фиксированных относительных объемов облученных органов и тканей, $(1 / 3,2 / 3,1)$, при стандартных схемах ФД (пять облучений в неделю с разовой дозой 2 Гр). Этими таблицами можно пользоваться как справочным пособием по ТД уровня ВЛО, равных 5 и 50\%, а также использовать ее для определения параметра $b$ с тем, чтобы вычислять ТД для произвольных значений объемов облучения (модели (1.1)-(1.4)), а также для определения ВЛО в органах и тканях, превышающих толерантные уровни облучения. 
Таблица 1.1

Толерантные дозы в органах и тканях уровня ВЛО 5 и 50\%

\section{и соответствующие им типы лучевых осложннений}

\begin{tabular}{|c|c|c|c|c|c|c|c|c|}
\hline \multirow{2}{*}{ № } & \multirow{2}{*}{ Органы и ткани } & \multicolumn{3}{|c|}{$D_{5 / 5}$, объемы } & \multicolumn{3}{|c|}{$D_{50 / 5}$, объемы } & \multirow{2}{*}{ Типы лучевых осложнений } \\
\hline & & $1 / 3$ & $2 / 3$ & 1 & $1 / 3$ & $2 / 3$ & 1 & \\
\hline 1 & Мочевой пузырь & & 80 & 65 & & 85 & 80 & $\begin{array}{l}\text { Симптомы контрактуры и уменьше- } \\
\text { ние объема }\end{array}$ \\
\hline 2 & Плечевое сплетение & & & 60 & & & 75 & Клинические повреждения нервов \\
\hline 3 & Мозг & 60 & 50 & 45 & 75 & 65 & 60 & Некроз или инфаркт \\
\hline 4 & Ствол мозга & 60 & 53 & 50 & & & 65 & Некроз или инфаркт \\
\hline 5 & Конский хвост & & & 60 & & & 75 & $\begin{array}{l}\text { Клиническое проявления } \\
\text { повреждения нервов }\end{array}$ \\
\hline 6 & Кишка & 55 & & 45 & 65 & & 55 & $\begin{array}{l}\text { Обструкция, перфорация, изъязвле- } \\
\text { ние фистула }\end{array}$ \\
\hline 7 & Среднее ухо & 30 & 30 & 30 & 40 & 40 & 40 & Острый серозный отит \\
\hline 8 & Среднее ухо & 55 & 55 & 55 & 65 & 65 & 65 & Хронический отит \\
\hline 9 & Пищевод & 60 & 58 & 55 & 72 & 70 & 68 & Стриктура, перфорация \\
\hline 10 & |Головка и шейка бедра & & & 52 & & & 65 & Некроз \\
\hline 11 & Сердце & 60 & 45 & 40 & 70 & 55 & 50 & Перикардиты \\
\hline 12 & Почки & 50 & 30 & 23 & & 40 & 28 & Клинические нефриты \\
\hline 13 & |Гортань 1 & 79 & 70 & 70 & 90 & 80 & 80 & Некроз хряща \\
\hline 14 & |Гортань 2 & 55 & 55 & 50 & 70 & 70 & 70 & Отек гортани \\
\hline 15 & Хрусталик & & & 10 & & & 18 & $\begin{array}{l}\text { Катаракта, требующая } \\
\text { вмешательства }\end{array}$ \\
\hline 16 & Печень & 50 & 35 & 30 & 55 & 45 & 40 & Печеночная недостаточность \\
\hline 17 & Легкие & 45 & 30 & 17,5 & 65 & 40 & 24,5 & Пневмониты \\
\hline 18 & Зрительный нерв & & & 50 & & & 65 & Слепота \\
\hline 19 & Хиазма зритель. Нерва & & & 50 & & & 65 & Слепота \\
\hline 20 & $\begin{array}{l}\text { Околоушная слюнная } \\
\text { железа }\end{array}$ & & 32 & 32 & & 46 & 46 & Ксеростомия \\
\hline 21 & Прямая кишка & & & 60 & & & 80 & $\begin{array}{l}\text { Тяжелый практит, некроз, стеноз, } \\
\text { свищ }\end{array}$ \\
\hline 22 & Сетчатка & & & 45 & & & 65 & Слепота \\
\hline 23 & Ребро грудной клетки & 50 & & & 65 & & & Патологический перелом \\
\hline 24 & Кожа & 70 & 60 & 55 & & & 70 & Некроз, изъязвление \\
\hline 25 & Тонкий кишечник & 50 & & 40 & 60 & & 55 & Обструкция, перфорация \\
\hline 26 & Спинной мозг & 50 & 50 & 47 & 70 & 70 & & Миелиты, некрозы \\
\hline 27 & Желудок & 60 & 55 & 50 & 70 & 67 & 65 & Изъязвление, перфорация \\
\hline 28 & Щитовидная железа & & & 45 & & & 80 & Тиреоидит \\
\hline 29 & $\begin{array}{l}\text { Кость, сустав нижней } \\
\text { челюсти }\end{array}$ & 65 & 60 & 60 & 77 & 72 & 72 & $\begin{array}{l}\text { Выраженное ограничение функции } \\
\text { сустава }\end{array}$ \\
\hline 30 & $\begin{array}{l}\text { Кость, головка бедренной } \\
\text { кости }\end{array}$ & & & 52 & & & 65 & Некроз \\
\hline
\end{tabular}

Примечание.

$D_{5 / 5}, D_{50 / 5}$ - суммарные дозы, которые приводят к ВЛО 5 и $50 \%$ за 5 -летний срок наблюдения.

Относительные объемы облучения тканей - 1/3, 2/3, 1 .

Разовую дозу мы считаем фиксированной и равной 2 Гр.

Толерантные дозы в органах и тканях уровня ВЛО 5 и $50 \%$ систематизированны в работах Emami et al. [31]. 
Таблица 1.2

\section{Толерантные дозы для органов и тканей уровня ВЛО, равной 5 и 50\%,}

\section{с 95\% доверительными интервалами}

\begin{tabular}{|c|c|c|c|c|c|c|c|}
\hline № & $\mathrm{Op}$ & $\begin{array}{c}T D_{5 / 5}(1 / 3) \\
+/-95 \% \text { ДИ } \\
\end{array}$ & $\begin{array}{c}T D_{5 / 5}(2 / 3) \\
+/-95 \% \text { ДИ }\end{array}$ & $\begin{array}{c}\mathrm{TD}_{5 / 5}(3 / 3) \\
+/-95 \% \text { ДИ } \\
\end{array}$ & $\begin{array}{l}T D_{50 / 5}(1 / 3) \\
+/-95 \% \text { ДИ } \\
\end{array}$ & $\begin{array}{l}T D_{50 / 5}(2 / 3) \\
+/-95 \% \text { ДИ } \\
\end{array}$ & $\begin{array}{l}T D_{50 / 5}(3 / 3) \\
+/-95 \% \text { ДИ } \\
\end{array}$ \\
\hline 1 & Почка & $\begin{array}{c}43,92 \\
41,31-46,54 \\
\end{array}$ & $\begin{array}{c}27,02 \\
24,40-29,63\end{array}$ & $\begin{array}{c}17,12 \\
14,51-19,74 \\
\end{array}$ & $\begin{array}{c}59,14 \\
56,53-61,76 \\
\end{array}$ & $\begin{array}{c}42,23 \\
39,62-44,85\end{array}$ & $\begin{array}{c}32,34 \\
29,73-34,95\end{array}$ \\
\hline 2 & Головной & $\begin{array}{c}58,56 \\
55,34-61,78 \\
\end{array}$ & $\begin{array}{c}51,42 \\
48,21-54,64\end{array}$ & $\begin{array}{c}47,25 \\
44,03-50,46\end{array}$ & $\begin{array}{c}80 \\
76,78-83,21 \\
\end{array}$ & $\begin{array}{c}72,86 \\
69,64-76,07 \\
\end{array}$ & $\begin{array}{c}68,68 \\
65,47-71,90\end{array}$ \\
\hline 3 & Ств & $\begin{array}{c}59,20 \\
56,10-52,31 \\
\end{array}$ & $\begin{array}{c}55,15 \\
52,05-58,26\end{array}$ & $\begin{array}{c}52,78 \\
49,67-55,89 \\
\end{array}$ & $\begin{array}{c}72,99 \\
69,88-76,09 \\
\end{array}$ & $\begin{array}{c}68,93 \\
65,83-72,04 \\
\end{array}$ & $\begin{array}{c}66,56 \\
63,46-69,67 \\
\end{array}$ \\
\hline 4 & Yxo & $\begin{array}{c}29,99 \\
29,99-30 \\
\end{array}$ & $\begin{array}{c}29,99 \\
29,99-30 \\
\end{array}$ & $\begin{array}{c}29,99 \\
29,99-30 \\
\end{array}$ & $\begin{array}{c}39,99 \\
39,99-40 \\
\end{array}$ & $\begin{array}{c}39,99 \\
39,99-40\end{array}$ & $\begin{array}{c}39,99 \\
39,99-40\end{array}$ \\
\hline 5 & $\mathrm{yxo}$ & $\begin{array}{c}57,30 \\
54,74-59,86\end{array}$ & $\begin{array}{c}56,41 \\
53,85-58,98\end{array}$ & $\begin{array}{c}55,9 \\
53,33-58,46\end{array}$ & $\begin{array}{c}68,66 \\
66,06-71,22\end{array}$ & $\begin{array}{c}67,77 \\
65,21-70,33 \\
\end{array}$ & $\begin{array}{c}67,25 \\
64,69-69,81\end{array}$ \\
\hline 6 & Пиш & $\begin{array}{c}59,10 \\
57,34-60,87 \\
\end{array}$ & $\begin{array}{c}57,82 \\
56,05-59,58\end{array}$ & $\begin{array}{c}57,07 \\
55,3-58,83 \\
\end{array}$ & $\begin{array}{c}74,1 \\
72,34-75,87 \\
\end{array}$ & $\begin{array}{c}72,82 \\
71,05-74,58 \\
\end{array}$ & $\begin{array}{c}72,07 \\
70,30-73,83 \\
\end{array}$ \\
\hline 7 & Cep & $\begin{array}{c}59,91 \\
58,25-61,56 \\
\end{array}$ & $\begin{array}{c}44,53 \\
42,88-46,19\end{array}$ & $\begin{array}{c}35,54 \\
33,88-37,20 \\
\end{array}$ & $\begin{array}{c}72,54 \\
70,89-74,20 \\
\end{array}$ & $\begin{array}{c}57,17 \\
55,51-58,83\end{array}$ & $\begin{array}{c}48,18 \\
46,52-49,84\end{array}$ \\
\hline 8 & Mor & $\begin{array}{c}59,40 \\
54,71-64,09 \\
\end{array}$ & $\begin{array}{c}57,1 \\
52,41-61,79\end{array}$ & $\begin{array}{c}55,75 \\
51,06-60,44 \\
\end{array}$ & $\begin{array}{c}90,14 \\
85,45-94,83 \\
\end{array}$ & $\begin{array}{c}87,84 \\
83,15-92,53 \\
\end{array}$ & $\begin{array}{c}86,49 \\
81,80-91,18\end{array}$ \\
\hline 9 & $\begin{array}{l}\text { Гортань (некроз } \\
\text { ща) }\end{array}$ & $\begin{array}{c}77,90 \\
76,52-79,26 \\
\end{array}$ & $\begin{array}{c}71,57 \\
70,19-72,96 \\
\end{array}$ & $\begin{array}{c}67,88 \\
66,49-69,26 \\
\end{array}$ & $\begin{array}{c}89,24 \\
87,85-90,62 \\
\end{array}$ & $\begin{array}{c}82,91 \\
81,53-84,29 \\
\end{array}$ & $\begin{array}{c}79,21 \\
77,83-80,60 \\
\end{array}$ \\
\hline 10 & Гоптань (отек) & $\begin{array}{c}41,05 \\
37,14-44,96 \\
\end{array}$ & $\begin{array}{c}55,69 \\
51,78-59,60 \\
\end{array}$ & $\begin{array}{c}64,25 \\
60,34-68,17 \\
\end{array}$ & $\begin{array}{c}64,92 \\
61,01-68,84\end{array}$ & $\begin{array}{c}79,56 \\
75,65-83,48 \\
\end{array}$ & $\begin{array}{c}88,13 \\
84,21-92,04\end{array}$ \\
\hline 11 & Пег & $\begin{array}{c}44,73 \\
42,51-46,94 \\
\end{array}$ & $\begin{array}{c}34,15 \\
31,94-36,37 \\
\end{array}$ & $\begin{array}{c}27,96 \\
25,75-30,18 \\
\end{array}$ & $\begin{array}{c}58,66 \\
56,45-60,88 \\
\end{array}$ & $\begin{array}{c}48,09 \\
45,87-50,30 \\
\end{array}$ & $\begin{array}{c}41,9 \\
39,69-44,12\end{array}$ \\
\hline 12 & Л & $\begin{array}{c}29,93 \\
21,51-38,34 \\
\end{array}$ & $\begin{array}{c}14,69 \\
6,269-23,10 \\
\end{array}$ & $\begin{array}{c}5,771 \\
-2,65-14,19 \\
\end{array}$ & $\begin{array}{c}61,18 \\
52,76-69,60 \\
\end{array}$ & $\begin{array}{c}45,94 \\
37,52-54,35 \\
\end{array}$ & $\begin{array}{c}37,02 \\
28,6-45,44\end{array}$ \\
\hline 13 & ox & $\begin{array}{c}60,84 \\
58,48-63,20 \\
\end{array}$ & $\begin{array}{c}55,97 \\
53,61-58,33 \\
\end{array}$ & $\begin{array}{c}53,12 \\
50,76-55,48 \\
\end{array}$ & $\begin{array}{c}77,92 \\
75,57-80,28 \\
\end{array}$ & $\begin{array}{c}73,06 \\
70,70-75,42\end{array}$ & $\begin{array}{c}70,21 \\
67,85-72,57\end{array}$ \\
\hline 14 & Кож & $\begin{array}{c}48,54 \\
47,51-49,58 \\
\end{array}$ & $\begin{array}{c}48,54 \\
47,51-49,58\end{array}$ & $\begin{array}{c}48,54 \\
47,51-49,58 \\
\end{array}$ & $\begin{array}{c}65,09 \\
64,06-66,13 \\
\end{array}$ & $\begin{array}{c}65,09 \\
64,06-66,13 \\
\end{array}$ & $\begin{array}{c}65,09 \\
64,06-66,13\end{array}$ \\
\hline 15 & Тон & $\begin{array}{c}48,17 \\
45,77-50,56 \\
\end{array}$ & $\begin{array}{c}45,75 \\
43,36-48,14 \\
\end{array}$ & $\begin{array}{c}44,33 \\
41,94-46,73 \\
\end{array}$ & $\begin{array}{c}61,83 \\
59,44-64,23 \\
\end{array}$ & $\begin{array}{c}59,41 \\
57,02-61,81 \\
\end{array}$ & $\begin{array}{c}58 \\
55,61-60,39 \\
\end{array}$ \\
\hline 16 & ה U U & $\begin{array}{c}55,00 \\
(0,0-0,0) \\
\end{array}$ & $\begin{array}{c}48,69 \\
(0,0-0,0) \\
\end{array}$ & $\begin{array}{c}45 \\
(0,0-0,0) \\
\end{array}$ & $\begin{array}{c}65 \\
(0,0-0,0) \\
\end{array}$ & $\begin{array}{c}58,69 \\
(0,0-0,0) \\
\end{array}$ & $\begin{array}{c}55 \\
(0,0-0,0) \\
\end{array}$ \\
\hline 17 & Спи & $\begin{array}{c}46,89 \\
43,58-50,19 \\
\end{array}$ & $\begin{array}{c}47,44 \\
44,13-50,75 \\
\end{array}$ & $\begin{array}{c}47,76 \\
44,45-51,07 \\
\end{array}$ & $\begin{array}{c}70,74 \\
67,44-74,05 \\
\end{array}$ & $\begin{array}{c}71,30 \\
67,99-74,61 \\
\end{array}$ & $\begin{array}{c}71,62 \\
68,31-74,93 \\
\end{array}$ \\
\hline 18 & Жел & $\begin{array}{c}58,33 \\
56,10-60,55 \\
\end{array}$ & $\begin{array}{c}51,04 \\
48,81-53,26 \\
\end{array}$ & $\begin{array}{c}46,77 \\
44,55-49,00 \\
\end{array}$ & $\begin{array}{c}73,45 \\
71,22-75,67 \\
\end{array}$ & $\begin{array}{c}66,16 \\
63,93-68,39 \\
\end{array}$ & $\begin{array}{c}61,9 \\
59,67-64,12 \\
\end{array}$ \\
\hline 19 & $\begin{array}{l}\text { Височно- } \\
\text { нижнечелюстной су- } \\
\text { став и нижняя челюсть }\end{array}$ & $\begin{array}{c}60,51 \\
57,75-63,27\end{array}$ & $\begin{array}{c}60,32 \\
57,56-63,08\end{array}$ & $\begin{array}{c}60,2 \\
57,44-62,96\end{array}$ & $\begin{array}{c}78,90 \\
76,14-81,66\end{array}$ & $\begin{array}{c}78,7 \\
75,94-81,46\end{array}$ & $\begin{array}{c}78,58 \\
75,82-81,35\end{array}$ \\
\hline 20 & Конский хвост & $\begin{array}{c}58,65 \\
47,06-70,25 \\
\end{array}$ & $\begin{array}{c}58,65 \\
47,06-70,25\end{array}$ & $\begin{array}{c}58,65 \\
47,06-70,25 \\
\end{array}$ & $\begin{array}{c}75,19 \\
63,60-86,79 \\
\end{array}$ & $\begin{array}{c}75,19 \\
63,60-86,79 \\
\end{array}$ & $\begin{array}{c}75,19 \\
63,60-86,79 \\
\end{array}$ \\
\hline 21 & Плеч & $\begin{array}{c}61,09 \\
59,70-62,48 \\
\end{array}$ & $\begin{array}{c}58,67 \\
57,27-60,06 \\
\end{array}$ & $\begin{array}{c}57,25 \\
55,86-58,64 \\
\end{array}$ & $\begin{array}{c}78,67 \\
77,28-80,06 \\
\end{array}$ & $\begin{array}{c}76,25 \\
4,86-77,64 \\
\end{array}$ & $\begin{array}{c}74,83 \\
3,44-76,23 \\
\end{array}$ \\
\hline 22 & $\begin{array}{l}\text { Головка и шейка бед- } \\
\text { ренной кости }\end{array}$ & $\begin{array}{c}51,61 \\
41,56-61,66 \\
\end{array}$ & $\begin{array}{c}51,61 \\
41,56-61,66 \\
\end{array}$ & $\begin{array}{c}51,61 \\
41,56-61,66 \\
\end{array}$ & $\begin{array}{c}63,70 \\
53,65-73,74 \\
\end{array}$ & $\begin{array}{c}63,7 \\
53,65-73,74 \\
\end{array}$ & $\begin{array}{c}63,7 \\
53,65-73,74\end{array}$ \\
\hline 23 & Хрусталики глаза & $\begin{array}{c}6,762 \\
4,29-9,23 \\
\end{array}$ & $\begin{array}{c}6,762 \\
4,294-9,229 \\
\end{array}$ & $\begin{array}{c}6,762 \\
4,294-9,229 \\
\end{array}$ & $\begin{array}{c}16,86 \\
14,39-19,32 \\
\end{array}$ & \begin{tabular}{c|}
16,86 \\
$14,39-19,32$ \\
\end{tabular} & $\begin{array}{c}16,86 \\
14,39-19,32 \\
\end{array}$ \\
\hline
\end{tabular}


Окончание табл. 1.2

\begin{tabular}{|c|c|c|c|c|c|c|c|}
\hline № & Орган & \begin{tabular}{|c|}
$T D_{5 / 5}(1 / 3)$ \\
$+/-95 \%$ ДИ \\
\end{tabular} & $\begin{array}{c}T D_{5 / 5}(2 / 3) \\
+/-95 \% \text { ДИ } \\
\end{array}$ & $\begin{array}{c}\mathrm{TD}_{5 / 5}(3 / 3) \\
+/-95 \% \text { ДИ } \\
\end{array}$ & \begin{tabular}{|l|}
$T D_{50 / 5}(1 / 3)$ \\
$+/-95 \%$ ДИ \\
\end{tabular} & $\begin{array}{l}T D_{50 / 5}(2 / 3) \\
+/-95 \% \text { ДИ } \\
\end{array}$ & $\begin{array}{l}T D_{50 / 5}(3 / 3) \\
+/-95 \% \text { ДИ } \\
\end{array}$ \\
\hline 24 & Зрительны & \begin{tabular}{c|}
49,34 \\
$46,06-52,62$
\end{tabular} & $\begin{array}{c}49,34 \\
46,06-52,62\end{array}$ & $\begin{array}{c}49,34 \\
46,06-52,62\end{array}$ & $\begin{array}{c}67,02 \\
63,74-70,31\end{array}$ & $\begin{array}{c}67,02 \\
63,74-70,31\end{array}$ & $\begin{array}{c}67,02 \\
63,74-70,31\end{array}$ \\
\hline 25 & Хиаз & \begin{tabular}{|c|}
49,54 \\
$37,54-61,54$ \\
\end{tabular} & $\begin{array}{c}49,54 \\
37,54-61,54 \\
\end{array}$ & $\begin{array}{c}49,54 \\
37,54-61,54 \\
\end{array}$ & \begin{tabular}{c|}
84,57 \\
$72,57-96,57$ \\
\end{tabular} & $\begin{array}{c}84,57 \\
72,57-96,57 \\
\end{array}$ & $\begin{array}{c}84,57 \\
72,57-96,57 \\
\end{array}$ \\
\hline 26 & Сетг & \begin{tabular}{c|}
44,67 \\
$43,04-46,29$ \\
\end{tabular} & $\begin{array}{c}44,67 \\
43,04-46,29 \\
\end{array}$ & $\begin{array}{c}44,67 \\
43,04-46,29\end{array}$ & \begin{tabular}{c|}
61,58 \\
$59,95-63,20$
\end{tabular} & $\begin{array}{c}61,58 \\
59,95-63,20\end{array}$ & $\begin{array}{c}61,58 \\
59,95-63,20\end{array}$ \\
\hline 27 & Прямая кишка & \begin{tabular}{|c|}
58,56 \\
$55,15-61,97$
\end{tabular} & $\begin{array}{c}55,73 \\
52,32-59,14\end{array}$ & $\begin{array}{c}54,08 \\
50,66-57,49\end{array}$ & \begin{tabular}{|c|}
88,42 \\
$85,00-91,83$ \\
\end{tabular} & $\begin{array}{c}85,59 \\
82,17-89,00\end{array}$ & $\begin{array}{c}83,93 \\
80,52-87,35\end{array}$ \\
\hline 28 & Груд & \begin{tabular}{c|}
52,23 \\
$49,78-54,69$ \\
\end{tabular} & $\begin{array}{c}52,23 \\
49,78-54,69 \\
\end{array}$ & $\begin{array}{c}52,23 \\
49,78-54,69 \\
\end{array}$ & \begin{tabular}{c|}
67,74 \\
$65,29-70,19$ \\
\end{tabular} & $\begin{array}{c}67,74 \\
65,29-70,19 \\
\end{array}$ & $\begin{array}{c}67,74 \\
65,29-70,19 \\
\end{array}$ \\
\hline 29 & Слюнные железы & $\begin{array}{c}26,38 \\
9,74-43,02\end{array}$ & $\begin{array}{c}26,14 \\
9,501-42,78\end{array}$ & $\begin{array}{c}26 \\
9,364-42,65\end{array}$ & \begin{tabular}{c|}
52,09 \\
$35,45-68,73$
\end{tabular} & $\begin{array}{c}51,86 \\
35,22-68,50\end{array}$ & $\begin{array}{c}51,72 \\
35,08-68,36\end{array}$ \\
\hline 30 & Щитовидная железа & \begin{tabular}{|c|}
27,50 \\
$-2,21-57,20$
\end{tabular} & $\begin{array}{c}27,5 \\
-2,2-57,2\end{array}$ & $\begin{array}{c}27,5 \\
-2,2-57,20\end{array}$ & \begin{tabular}{|c|}
132,5 \\
$102,8-162,2$
\end{tabular} & $\begin{array}{c}132,5 \\
102,8-162,2\end{array}$ & $\begin{array}{c}132,5 \\
102,8-162,2\end{array}$ \\
\hline
\end{tabular}

Примечание. Толерантные дозы для органов и тканей уровня ВЛО равной 5 и $50 \%$, с $95 \%$ доверительными интервалами, полученны Kehwar et al. [32,33] в результате уточнения данных, приведенных в работе Emami et al. [31], и использования результатов, полученных другими исследователями.

Пример 1.1. Из табл. 1.1 следует, что для сердца параметр $b$ принимает следующие значения:

- при ВЛО $=5 \%: b=\left|\frac{\ln (46 / 70)}{\ln (60 \% / 25 \%)}\right|=0,505$;

- при ВЛО $=50 \%: b=\left|\frac{\ln (55 / 80)}{\ln (60 \% / 25 \%)}\right|=0,428$.

Значения $b$ отличаются друг от друга на 18\% (довольно значительное отличие).

Для головного мозга:

- при ВЛО $=5 \%: b=\left|\frac{\ln (60 / 70)}{\ln (100 \% / 25 \%)}\right|=0,111$;

- при ВЛО $=50 \%: \quad b=\left|\frac{\ln (70 / 80)}{\ln (100 \% / 25 \%)}\right|=0,096$.

Различие составляет $15,6 \%$, и т.д.

Мы не будем останавливаться на детальном анализе табл. 1.1 и 1.2, так как в дальнейшем будем часто обращаться к ним, и вопрос о выполнении соотношения (1.1)-(1.4) будет иметь решающее значение при уточнении структуры моделей и вычислении параметров их настройки для описания ВЛО в здоровых органах и тканях организма.

Заметим, что опыт, накопленный нами при создании математических моделей, предназначенных для описания ТД и ВЛО в органах и тканях, показал, что гораздо полезней оценивать эффективность различных «частных» (вспомогательных) 
моделей, описывающих реакции тканей на облучения, не фрагментарно, а в рамках более сложной по структуре модели, куда они входят как составные части. Например, как это имеет место в предложенной нами модели МРВ или в синтезированных математических моделях (СМ моделях, см. главу 3).

\section{4. Математические модели для расчета ТД в зависимости от условий облучения}

В настоящее время существуют различные математические модели для расчета ТД. Описание большинства из них и анализ возможностей их применения для планирования ЛТ злокачественных опухолей, приводятся в работах $[6,7,9]$.

В настоящей работе для построения математических моделей, которые описывают ВЛО в здоровых органах и тканях, (тканевой уровень облучения), в зависимости от объема облучения, разовой и суммарной дозы, нам понадобится модель Ellis [14] и две ее модификации, CRE и TDF [15, 16], а также LQ модель [17]. Рассмотрим эти модели.

\subsection{1. Модель Ellis}

Феноменологическая модель Ellis была предложена в 1969 г. [14] и долгое время она была одной из наиболее распространенных моделей для расчета ТД при различных схемах ФД в ЛТ

$$
D(N, T)=N S D \cdot N^{k_{1}} T^{k_{2}},
$$

где $D(N, T)$ - суммарная ТД для $N$ равномерных сеансов облучения, реализованных за $T$ сут; NSD - коэффициент пропорциональности; $k_{1} \geq 0$ и $k_{2} \geq 0$ - параметры модели. Анализ показал, что модель Ellis описывает значения ТД тем точнее, чем ближе режимы облучения к стандартизованным режимам ФД, на основе которых она создавалась. Было установлено, что модель (1.5) дает приемлемые значения ТД для $N \geq 4$ и $4 \leq T \leq 100$ сут.

Заметим также, что модель (1.5), использованная Ellis для описания ТД при различных режимах ФД, хорошо известна в экономике как «производственная функция» и широко используется в эконометрических исследованиях.

Чтобы облегчить практическое использование модели Ellis и ввести в pacсмотрение такие важные параметры фракционированного облучения как разовая доза $d$, количество реализованных сеансов облучения $n$ и временной интервал между сеансами облучения $t$, были предложены две ее модификации, $C R E$ и TDF. 


\subsection{2. Модель CRE (модификация модели Ellis)}

В 1971 г. Kirk, Grey, Watson [15] предложили модель для расчета кумулятивного радиационного эффекта (CRE). Она имеет следующий вид

$$
C R E=\frac{D}{N^{k_{1}} T^{k_{2}}}=D \cdot N^{-k_{1}} T^{-k_{2}} .
$$

Полагая $d=D / N, t=T / N$, можно определить значение $C R E$, соответствующее толерантному уровню облучения

$$
C R E=d \cdot N^{1-k_{1}-k_{2}} t^{-k_{2}} .
$$

Заменяя в (1.7) $N$ на $n$ и считая параметры $n, d, t$ свободными переменными, получаем окончательный вид модели $C R E$

$$
\operatorname{CRE}(n, d, t)=d \cdot n^{1-k_{1}-k_{2}} t^{-k_{2}},
$$

которую можно использовать для оценки различных режимов облучения. Здесь $n-$ число реализованных сеансов облучения, $d$ - разовая доза, $t$ - интервал времени между сеансами облучения. В СССР эта модель была подготовлена для практического применения в клинике К.И. Жолкивером и др. [67].

\subsection{3. Модель TDF (модификация модели Ellis)}

Вместе с моделью $C R E$ широкое распространение получила также модель TDF (Orton, Ellis [16]), которая была основанна на предположении о существовании парциальной толерантности, которая оценивается в частях $N S D$

$$
P T(n, N)=N S D \frac{n}{N} .
$$

Здесь $N$ - толерантное число сеансов облучения, $n$ - число реализованных сеансов облучения. В работе $[8,16]$ было высказано предположение о том, что если две схемы облучения образуют ряды парциальных толерантностей $P T\left(n_{1}, N_{1}\right), \ldots$, $P T\left(n_{k}, N_{k}\right)$ и $P T\left(n_{1}^{*}, N_{1}^{*}\right), \ldots, P T\left(n_{r}^{*}, N_{r}^{*}\right)$, и приводят к одному и тому же радиобиологическому эффекту, то справедливо равенство

$$
\sum_{i=1}^{k} P T\left(n_{i}, N_{i}\right)=\sum_{i=1}^{r} P T\left(n_{i}^{*}, N_{i}^{*}\right) .
$$

Определим $N$ из уравнения Ellis (1.5), подставим его в (1.9) и, полагая $d=D / N, t=T / N$, получим:

$$
\begin{aligned}
& \operatorname{TDF}(n, d, t)=N S D^{1 /\left(1-k_{1}-k_{2}\right)} n d^{1 /\left(1-k_{1}-k_{2}\right)} t^{-k_{2} /\left(1-k_{1}-k_{2}\right)}, \\
& \sum_{i=1}^{k} P T\left(n_{i}, N_{i}\right)=\sum_{i=1}^{k} N S D^{1 /\left(1-k_{1}-k_{1}\right)} n_{i} d_{i}^{1 /\left(1-k_{1}-k_{1}\right)} t_{i}^{-k_{2} /\left(1-k_{1}-k_{2}\right)} .
\end{aligned}
$$


В обеих моделях степень исчерпания толерантности определяется в долях $N S D$. Изменяемая часть модели не зависит от $N S D$. Связь между CRE и TDF имеет следующий вид:

$$
\begin{aligned}
& \operatorname{CRE}(n, d, t)=\left[N S D^{1 /\left(1-k_{1}-k_{2}\right)} \operatorname{TDF}(n, d, t)\right]^{1-k_{1}-k_{2}}, \\
& \operatorname{TDF}(n, d, t)=[N S D \cdot \operatorname{CRE}(n, d, t)]^{1 /\left(1-k_{1}-k_{2}\right)} .
\end{aligned}
$$

Поскольку мы допускаем, что для фиксированного режима облучения справедливо предположение 1.1, естественно предположить, что формула Ellis c учетом объема облученной ткани и для фиксированного значения ВЛО может быть записана следующим образом

$$
D(P, V)=N S D(P, 1) N^{k_{1}} T^{k_{2}} V^{-b} .
$$

Предположение 1.2. В модели Ellis от ВЛО $=P($ или ВОЛО $=Q=1-P$ ) и объема облученного органа или ткани $V$ зависит только $N S D$.

$$
N S D(Q, V)=N S D(Q, 1) \cdot V^{b},
$$

где $\operatorname{NSD}(Q, 1)$ - коэффициент пропорциональности в модели Ellis, ее также определяют как номинальную стандартную дозу для ВОЛО $=Q$ и $V=1$. Таким образом, для фиксированного значения $Q$ получаем

$$
\frac{N S D\left(Q, V_{1}\right)}{N S D\left(Q, V_{2}\right)}=\frac{D\left(Q, V_{1}\right)}{D\left(Q, V_{2}\right)}=\left(\frac{V_{1}}{V_{2}}\right)^{-b} .
$$

Соотношение (1.17) понятно, так как $N S D(Q, V)$ по сути дела должна быть равна ТД однократного облучения уровня $Q$ (правда, формула Ellis справедлива только для $(4 \leq N \leq 40)$.

В работе Ellis [14] было установлено, что для кожи и соединительной ткани параметры модели принимают значения: $k_{1}=0,24, k_{2}=0,11$. В СССР методические рекомендации по применению модели CRE в клинической практике были подготовлены К.И. Жолкивером, И.Ф. Зевриевой, А.Х. Досхановым в 1983 г. [67], а по применению модели ТДФ - М.А. Фадеевой, К.Н. Костроминой, В.С. Даценко и др. в 1987 г. [68].

\subsubsection{LQ модель}

Для описания фракции выживших после облучения клеток широкое распространение получила LQ модель, предложенная в работах Chadwick, Leenhouts [69] и Kellerer, Rossi [70]

$$
C(d)=\exp \left(-\alpha d-\beta d^{2}\right),
$$

где $C(d)$ - фракция выживших клеток после облучения дозой $d ; \alpha$ и $\beta$ - параметры модели (1.18), характеризующие радиочувствительность клеток. Предполагается, 
что параметр $\alpha$ характеризует начальный наклон кривой и соответствует одномишенной и одноударной гибели клеток. Параметр $\beta$ характеризует сублетальные повреждения клеток. Предполагается также [71, 72], что параметры $\alpha$ и $\beta$ могут быть приближенно связаны с параметрами $I$ и $K$ в модели Videroe [73]

$$
q(d)=\exp (-I d)\left\{1-[1-\exp (-K d)]^{n}\right\},
$$

следующим образом $\alpha=I, \beta=\sqrt{K}$. Модель (1.18) можно рассматривать как часть более общей модели, которая описывает выжившую фракцию популяции клеток [9]

$$
C(d)=\exp \left(-\alpha d-\beta d^{2}-\gamma d^{3}-\ldots\right) \text {. }
$$

При неравномерном по разовой дозе фракционированном облучении клеток их выживаемость может быть описана следующим образом:

$$
C(d, n)=\prod_{i=1}^{n} \exp \left(-\alpha d_{i}-\beta d_{i}^{2}\right)=\exp \left(-\sum_{i=1}^{n} \alpha d_{i}-\beta d_{i}^{2}\right),
$$

где $n$ - число сеансов облучения.

Опыт показал, что LQ модель (1.21), предназначенная для описания фракции выживших в результате облучения клеток, может быть с успехом использована для расчета ТД для различных органов и тканей организма. Предполагалось, что при этом должны выполняться следующие условия [74]:

1) временной интервал между сеансами облучения выбирается таким, что к моменту нового облучения происходит полное восстановление клеток ткани от сублетальных радиационных повреждений;

2) пролиферативной активностью клеток ткани между сеансами облучения можно пренебречь;

$3)$ и главное, параметры модели, $\alpha$ и $\beta$, которые используются для описания лучевых осложнений в ткани, скорее всего отличаются от значений параметров $\alpha$ и $\beta$, которые используются для описания фракции выживших клеток. Модель одна и та же, но параметры двух моделей могут серьезно отличаться друг от друга.

LQ модель для описания толерантных уровней облучения органов и тканей была предложена Barendsen [17]. Для неравномерной схемы ФД она может быть представлена следующим образом:

$$
E=\sum_{i=1}^{n} \alpha d_{i}+\beta d_{i}^{2}=\alpha D+\beta \sum_{i=1}^{n} d_{i}^{2},
$$

где $d=\left(d_{1}, \ldots, d_{n}\right)$ - разовые дозы облучения; $D=\sum_{i=1}^{n} d_{i}-$ суммарная доза; $E-$ параметр, задающий уровень толерантности ткани; $(\alpha, \beta)$ - радиобиологические параметры ткани, которые, скорее всего не совпадают с радиобиологическими параметрами, образующих ее клеток. 
Важно отчетливо понимать, что LQ модель в нашей работе предназначена для описания ТД для органов и тканей, радиобиологические параметры которых, $\alpha$ и $\beta$, скорее всего, отличаются от характеристик составляющих их клеток.

Более подробно этот вопрос был рассмотрен нами в работе [30] для опухолевых тканей в связи с исследованием проблемы существования опухолевых единиц (тканеподобно связанных опухолевых клеток).

LQ модель может быть использована для описания эквивалентных уровней облучения опухолевых тканей, приводящих к одним и тем же значениям ВЛИ опухолевого заболевания. Не исключено, что радиобиологические характеристики опухолевых тканей могут отличаться от радиобиологических характеристик составляющих их клеток, но, как было показано нами в [30], по мере увеличения суммарной дозы, по мере приближения суммарной дозы к терапевтическим значениям, радиобиологические параметры опухолевой ткани будут приближсаться к радиобиологическим параметрам независимых опухолевых клеток. В этом может сказываться некоторая тканевая организация опухолевых клеток.

\subsection{5. Свойства LQ модели}

Рассмотрим равномерную схему ФД. Пусть $d$ - разовая доза (доза за сеанс), $n$ - число сеансов облучения, $D=n d$ - суммарная доза. Тогда из (1.22) получаем:

$$
E=\alpha D+\frac{\beta D^{2}}{n}=\alpha D+\beta D d .
$$

Для фиксированных значений $\alpha$ и $\beta$ из (1.23) можно получить зависимость $D$ от $n$ и $E$

$$
D=\left[\sqrt{\left(\frac{\alpha}{\beta}\right)^{2}+\frac{4 E}{\beta \cdot n}}-\frac{\alpha}{\beta}\right] \frac{n}{2} .
$$

Если предположить, что от ВЛО зависит только параметр $E$, который задает уровень толерантности ткани, мы можем получать различные синтезированные математические модели (СМ модели, см. главу 3 ), по разному задавая зависимости $E$ от ВЛО.

В модели (1.23) независимыми переменными являются $D$ и $n$ или $D$ и $d$. Формула (1.23) упрощается, если $d=$ const, а варьируются $n$ и $D$. В этом случае

$$
E=D(\alpha+\beta d)=\tau D=\tau \cdot n \cdot d,
$$

где $\tau=\alpha+\beta d$. При $n=$ const, получаем

$$
E=\alpha D+(\beta / n) D^{2}=\alpha D+\beta^{\prime} D^{2},
$$

где $\beta^{\prime}=\beta / n$. Из структуры LQ модели следует: 
1) уровень толерантности ткани $E$ является аддитивной величиной

$$
E=\sum_{i=1}^{n} \alpha d_{i}+\beta d_{i}^{2}=\sum_{i=1}^{n} E_{i}
$$

Действительно, слагаемые $E_{i}$ могут трактоваться как парциальные уровни толерантности;

2) можно усложнить LQ модель путем ввода в нее параметра, связанного с длительностью курса лучевого лечения $T$. Сделать это можно по-разному. Фактор времени можно ввести в форме экспоненциальной функции роста числа клеток. В этом случае уравнение (1.25) принимает следующий вид

$$
E=\alpha D+\beta D d-\lambda T,
$$

где $\lambda$ - параметр модели (1.28).

Влияние длительности курса лучевого лечения может быть учтено также в форме, предложенной Barendsen [17]

$$
D_{\Pi}(T)=\left(T-T_{p}\right)_{+} d^{*},
$$

где $D_{\Pi}(T)$ - поправка к толерантной дозе за весь курс лучевого лечения $T ; T_{p}-$ интервал времени, в течение которого восстановительный процесс в ткани можно не учитывать; $d^{*}$ - приращение дозы на увеличение времени лучевого лечения на 1 сут

$$
\left(T-T_{p}\right)_{+}=\left\{\begin{array}{l}
T-T_{p}, \text { если } T-T_{p}>0 ; \\
0, \text { если } T-T_{p}<0 .
\end{array}\right.
$$

Значение $d^{*}$ варьируют от 0,1 до 1 Гр/сут. Barendsen полагал, что значение $d^{*}=0,5$ Гр/сут является удовлетворительной для кожи.

В табл. 1.3 приводятся отношения $\gamma=\alpha / \beta$ для различных здоровых органов и тканей организма, для ранних и поздних проявлений лучевых реакций, систематизированных в работе [74].

Вопросы, связанные с учетом объема облученных органов и тканей при расчете радиобиологических параметров модифицированной LQ модели, будут рассмотрены нами в следующих разделах. 
Параметры LQмодели $\gamma=\alpha / \beta$, систематизированные в [74]

\begin{tabular}{|c|c|c|c|}
\hline Ткань & Тип осложнения & $\gamma=\alpha / \beta$ & Ссылки \\
\hline \multicolumn{4}{|c|}{ Ранние реакции } \\
\hline Кожа & Эритема & 9 & Tuesson, Notter, 1984 \\
\hline \multicolumn{4}{|c|}{ Поздние реакции } \\
\hline Позвоночник & Миелопатия & 2,5 & Cohen, Creditor, 1981 \\
\hline Мозг & Некроз & 4,2 & Cohen, Creditor, 1983 \\
\hline Гортань & Некроз, оттек & 3,8 & Maciejewsky, e.a., 1986 \\
\hline Кость & Остеорадионекроз & 2,5 & Overgard, 1985 \\
\hline Почки & Нефрограмма & 3,4 & Cohen, Creditor, 1983 \\
\hline \multirow[t]{3}{*}{ Легкие } & Пневмониты & $4,4-6,3$ & Fowler, 1984 \\
\hline & Пневмониты & 6,9 & Overgard, 1985 \\
\hline & Фиброзы & 3,6 & \\
\hline Кожа & Фиброзы & $3-5$ & Tuesson, Notter, 1984 \\
\hline Кишечник & Изъязвление & 7,9 & Cohen, Creditor, 1983 \\
\hline Ректум & Осложнения & 2,5 & Visser et al., 1985 \\
\hline
\end{tabular}

\section{5. Анализ математических моделей фракционирования дозы для планирования ЛТ опухолевых заболеваний}

Обоснованный вывод о ценности той или иной математической модели ФД можно сделать лишь на основе хорошо систематизированной клинической и экспериментальной информации, однако, до настоящего времени мы такой информацией не располагаем, и нам трудно сделать однозначный вывод о границах применимости математических моделей, описывающих изоэффективные режимы ФД. Клиническая и экспериментальная информация, которая необходима для тестирования математических моделей ФД, должна содержать данные о толерантных уровнях облучения различных органов и тканей с указанием их статистического разброса, и включать в себя:

- ТД при равномерном облучении органов и тканей (равные дозы-равные временные интервалы между сеансами облучения);

- ТД, соответствующие стандартным и стандартизованным схемам ФД;

- ТД, соответствующие частично или полностью неравномерным схемам ФД (равные дозы - неравные интервалы времени между сеансами облучения, неравные дозы - равные интервалы времени между сеансами облучения, неравные дозы - неравные интервалы времени между сеансами облучения);

- всю перечисленную информацию для других определений толерантных уровней облучения, для фиксированных значений ВЛО, отличных от 5\%.

Все рассмотренные модели построены на одном и том же постулате, согласно которому процесс лучевого воздействия на ткань, (или популяцию клеток, 
образующих ткань), и восстановительные процессы, протекающие в них, можно рассматривать как два независимых процесса. Этот постулат, который можно назвать принципом «отделимости», существенно облегчает процесс построения математических моделей ФД. Свойство отделимости прослеживается в модели Ellis, в которой число сеансов облучения и общее время лучевого лечения рассматривается как два независимых параметра, а также в LQ модели.

Одно из основных медико-биологических требований при планировании ЛТ заключается в образовании в опухоли-мишени однородного дозового поля заданной конфигурации и уровня. Поэтому лучевая нагрузка на ложе опухолевой ткани может с достаточной точностью характеризоваться значением дозы в мишени и такой актуальный вопрос, как влияние распределения доз на лучевые осложнения в ложе опухоли почти не возникает.

Иначе обстоит дело со здоровыми органами и тканями, которые находятся в зоне интенсивного лучевого воздействия, и в которых распределение доз может быть неоднородным. Можно ли в этом случае использовать уже рассмотренные математические модели ФД? Этот вопрос тем более важен, что в настоящее время модель Ellis и ее модификации $T D F, C R E$, а также LQ модель, уже используются в практической ЛТ.

Чрезвычайно актуальной и сложной проблемой в ЛТ является проблема создания математических моделей для перехода от неоднородных распределений дозы в здоровых органах и тканях, к эквивалентным по ВЛО однородным распределениям дозы. Такая же сложная проблема возникает при переходе от неоднородных дозовых распределений в опухолевых тканях к эквивалентным по ВЛИ однородным дозовым распределениям. Особенно остро эта проблема стоит в брахитерапии, (внутритканевой и внутриполостной ЛТ опухолевых заболеваний), где образовать однородное дозовое поле в принципе невозможно. 


\section{Глава 2.}

\section{МРВ МОДЕЛЬ ДЛЯ ОПИСАНИЯ ВЛО \\ В ОРГАНАХ И ТКАНЯХ КАК ФУНКЦИИ ОТ ОБЪЕМОВ И СУММАРНЫХ ДОЗ ОБЛУЧЕНИЯ}

\section{1. Модель МРВ (модифицированное распределения Вейбулла)}

При облучении фиксированного объема органа или ткани дозой $D$, (разовая доза также фиксирована), для описания ВЛО в органах и тканях мы предложили использовать модифицированное распределение Вейбулла (МРВ) $[6,7,75]$

$$
P(D)=1-Q(D)=1-\exp \left[-\left(D / A_{1}\right)^{A_{2}}\right], Q(D)=\exp \left[-\left(D / A_{1}\right)^{A_{2}}\right],
$$

где $A_{1}, A_{2}$ - параметры модели; $Q(D)=1-P(D)$ - ВОЛО в органе или ткани. Модель МРВ удобна тем, что позволяет в явном виде выразить дозу, которая приводит к заданному значению ВЛО

$$
D=A_{1}|\ln (Q)|^{1 / A_{2}} .
$$

Модели (2.1) и (2.2) описывают ВЛО = $P$ в зависимости от дозы облучения и для фиксированного объема облученного органа или ткани. Полагая, что для любого значения ВЛО = $P$ выполняется предположение 1.1-1.4, получаем следующую модификацию модели (2.1) с учетом объема $V$ облученной ткани $[6,7]$ :

$$
P(D, V)=1-Q(D, V)=1-\exp \left[-\left(D V^{b} / A_{1}\right)^{A_{2}}\right],
$$

где $b$ параметр модели, необходимый для учета объема облученной ткани. Из (2.3) легко получить различные полезные соотношения, которые связывают между собой три радиологических параметра, $Q, D$ и $V$ :

$$
\begin{aligned}
& D(Q, V)=A_{1}|\ln (Q)|^{1 / A_{2}} V^{-b}, \frac{D\left(Q, V_{1}\right)}{D\left(Q, V_{2}\right)}=\left(\frac{V_{1}}{V_{2}}\right)^{-b}, \\
& D(Q, 1)=A_{1}|\ln (Q)|^{1 / A_{2}}, V(Q, D)=\left[\frac{A_{1}|\ln (Q)|^{1 / A_{2}}}{D(Q, V)}\right]^{1 / b}, \\
& V(Q, D)=\left[\frac{A_{1}|\ln (Q)|^{1 / A_{2}}}{D(Q, V)}\right]^{1 / b}, b=\ln \left[\frac{A_{1}|\ln (Q)|^{1 / A_{2}}}{D(Q, V)}\right] / \ln [V] .
\end{aligned}
$$

Опыт применения модели (2.1) показал, что она с успехом может быть использовано и для описания ВЛИ опухолевых заболеваний в зависимости от условий облучения $[6,7]$. 


\section{2. Экспресс-метод расчета ВЛО}

\section{в органах и тканях при помощци модели МРВ}

Из (2.4) можно получить формулу для оперативного расчета ВОЛО $=Q$ в ткани при изменении объема облучения. Пусть задана функция $Q\left(D, V_{0}\right)$, которая описывает ВОЛО при однородном облучении ткани для фиксированного объема $V_{0}$ как функцию от дозы $D$. Докажем справедливость следующего утверждения:

Утверждения 2.1. Рассчитать ВОЛО для произвольного объема $V_{1}$ ткани при облучении ее дозой $D$ можно при помощи модели:

$$
Q\left(D, V_{1}\right)=Q\left(D, V_{0}\right)^{\left(V_{1} / V_{0}\right)^{b \cdot A_{2}}}=Q\left(D, V_{0}\right)^{\left(V_{1} / V_{0}\right)^{\Psi}},
$$

где $Q\left(D, V_{0}\right)$ - ВОЛО при облучении объема $V_{0}$ ткани дозой $D ; \Psi=b \cdot A_{2}-$ параметр, равный произведению двух параметров модели МРВ.

Доказательство. Действительно, ВОЛО для объемов $V_{0}$ и $V_{1}$ ткани при облучении ее дозой $D$ будут равны:

$$
Q\left(D, V_{0}\right)=\exp \left[-\left(D V_{0}^{b} / A_{1}\right)^{A_{2}}\right], Q\left(D, V_{1}\right)=\exp \left[-\left(D V_{1}^{b} / A_{1}\right)^{A_{2}}\right] \text {. }
$$

Отношение логарифмов значений $Q$ из (2.8) будет

$$
\frac{\ln \left(Q\left(D, V_{1}\right)\right)}{\ln \left(Q\left(D, V_{0}\right)\right)}=\left(\frac{V_{1}}{V_{0}}\right)^{b A_{2}}=\left(\frac{V_{1}}{V_{0}}\right)^{\Psi} \text {. }
$$

Из (2.8) и (2.9) получаем

$$
\begin{aligned}
& \ln \left(Q\left(D, V_{1}\right)\right)=\left(V_{1} / V_{0}\right)^{\Psi} \cdot \ln \left(Q\left(D, V_{0}\right)\right), \\
& Q\left(D, V_{1}\right)=Q\left(D, V_{0}\right)^{\left(V_{1} / V_{0}\right)^{\Psi}} .
\end{aligned}
$$

\section{Утверждение доказано.}

Таким образом, если построена зависимость $Q\left(D, V_{0}\right)$ от $D$ для фиксированного объема $V_{0}$ и известен параметр $\Psi=b \cdot A_{2}$, рассчитать ВОЛО для любого другого объема облучения этой же ткани можно по формуле (2.11). Параметр $\Psi$ можно рассчитать, если известны значения ВОЛО для двух режимов облучения с одинаковой дозой и разными объемами при помощи формулы (2.10)

$$
\Psi=\ln \left[\frac{\ln \left(Q\left(D, V_{1}\right)\right)}{\ln \left(Q\left(D, V_{0}\right)\right)}\right] / \ln \left(\frac{V_{1}}{V_{0}}\right) .
$$

Пример 2.1. В качестве примера рассмотрим расчет ВОЛО как функцию от объема облученного сердца. В работе [7] мы показали, что

$$
Q(D, V)=\exp \left[-\left(\frac{D V^{0,47}}{357,3}\right)^{14,5}\right]
$$


$\Psi=b \cdot A_{2}=0,47 \cdot 14,5=6,815$. При $V=30 \%$ облученного сердца дозой 60 Гр ВОЛО $=Q(60,30)=93,45 \%$, ВЛО $=P=6,55 \%$. Предположим, что объем облученного сердца увеличен на $10 \%$, т.е. $V_{1}=40 \%$, а доза осталась неизменной. Воспользовавшись формулой (2.12), получаем

$$
Q(60,40)=Q(60,30)^{(40 / 30)^{6,815}}=0,9345^{7,103}=0,618(61,8 \%) .
$$

Таким образом, при увеличении объема облучения на 10\% значение ВЛО увеличилось с 6,55 до 30,82\%. Непосредственное вычисление $Q(60,40)$ по формуле (2.13), как и следует ожидать, дает также 0,618. Ясно, что предлагаемый формализм необходимо использовать в тех случаях, когда функция $Q(D, V)$ задана в виде математической формулы, (или построена графически) и только для одного фиксированного значения $V$.

Утверждения 2.2. Пусть функция $Q\left(D_{0}, V\right)$ описывает ВОЛО при однородном облучении ткани для фиксированной дозы $D_{0}$ как функцию от объема облучения $V$. Тогда рассчитать ВОЛО для произвольной дозы $D_{1}$ при облучении объема $V$ ткани можно по формуле:

$$
\begin{aligned}
& Q\left(D_{1}, V\right)=Q\left(D_{0}, V\right)^{\left(D_{1} / D_{0}\right)^{\Psi}}, \\
& \Psi=b \cdot A_{2} .
\end{aligned}
$$

Доказательство. ВОЛО при облучении ткани дозой $D_{0}$ и $D_{1}$ равны:

$$
Q\left(D, V_{0}\right)=\exp \left[-\left(\frac{D_{0} V^{b}}{A_{1}}\right)^{A_{2}}\right], Q\left(D, V_{1}\right)=\exp \left[-\left(\frac{D_{1} V^{b}}{A_{1}}\right)^{A_{2}}\right] .
$$

Взяв отношение логарифмов (2.17), получаем

$$
\frac{\ln \left(Q\left(D_{1}, V\right)\right)}{\ln \left(Q\left(D_{0}, V\right)\right)}=\left(\frac{D_{1}}{D_{0}}\right)^{b \times A_{2}}=\left(\frac{D_{1}}{D_{0}}\right)^{\Psi} .
$$

Из (2.18) следует:

$$
Q\left(D_{1}, V\right)=Q\left(D_{0}, V\right)^{\left(D_{1} / D_{0}\right)^{\Psi}} .
$$

\section{Утверждение доказано.}

Пример 2.2. В качестве примера рассмотрим расчет ВОЛО как функцию от дозы облученного сердца. Как и в примере 2.1, ВОЛО в сердце как функция от объема и дозы описывается формулой (2.13). Значение $\Psi=b \cdot A_{2}=6,815$. При $V=30 \%$ облученного сердца дозой 60 Гр ВОЛО $=Q(60,30)=93,45 \%$. ВЛО $=P=6,55 \%$. Если дозу облученного сердца увеличить на 10 Гр, т.е. $D_{1}=70$ Гр, а объем облучения оставить неизменным, значение ВОЛО, рассчитанное при помощи формулы (2.19), будет:

$$
Q(70,30)=Q(60,30)^{(70 / 60)^{6,815}}=0,9345^{2,859}=0,824 .
$$


При увеличении дозы на 10 Гр значение ВОЛО уменьшилось с 0,9345 $(93,45 \%)$ до 0,824 (82,4\%), а ВЛО возросло с 6,55 до 17,6\%. Непосредственное вычисление $Q(70,40)$ по формуле (2.13) дает то же самое значение 0,824 . Предлагаемый метод удобно использовать в тех случаях, когда функция $Q(D, V)$ задана в виде формулы (или построена графически) только для одного фиксированного значения $D$.

В главе 7 показано, что параметр $\Psi=b \cdot A_{2}$ может служить мерой связанности клеток в тканевую систему.

\section{3. Модель НРВ (нормальное распределение вероятностей) для расчета ВЛО в органах и тканях}

Для фиксированного режима ФД и объема ткани $V$ для расчета ВЛО органах и тканях Lyman $[23,24,38]$ предложил использовать функцию HРB

$$
P\left(D^{\prime}\right)=\frac{1}{\sqrt{2 \pi \sigma}} \int_{-\infty}^{D^{\prime}} \exp \left[\frac{(D-D(0,5))^{2}}{2 \sigma^{2}}\right] d D,
$$

где $D(0,5)>0$ - доза, которая приводит к ВЛО $=0,5, \sigma>0$ - дисперсия случайной величины. Свойства НРВ хорошо изучены $[6,7,23,24,38]$. Сделав замену переменной $D$ на $y=(D-D(0,5)) / \sigma$, получим приведенное к нулю распределение НРВ:

$$
\begin{aligned}
& P(D)=\frac{1}{\sqrt{2 \pi \sigma}} \int_{-\infty}^{F} \exp \left[\frac{y^{2}}{2}\right] d y, \\
& F=\frac{D-D(0,5)}{\sigma} .
\end{aligned}
$$

Нетрудно видеть, что формула (2.23) полностью определяет значение ВЛО, которое можно получить, используя (2.22), так как она описывает единственный изменяемый параметр в (2.22). Поэтому, специалист может рассматривать модель (2.23) вместо модели (2.22).

Использование НРВ для описания ВЛО при фиксированном режиме ФД и фиксированном объеме $V$ облученной ткани не вызывает затруднений, хотя необходимость обращения к специальным подпрограммам (процедурам) или таблицам, невозможность рассчитывать ВЛО напрямую, (как это можно сделать используя модель МРВ), создает известные неудобства. Она усложняет задачу создания модели, описывающей ВЛО при любой схеме ФД, и может затруднить расчеты при определении оптимальных планов облучения с учетом ВЛО в здоровых органах и тканях. 
При фиксированном режиме ФД и фиксированном объеме облученной ткани для настройки модели НРВ на клиническом или экспериментальном материале необходимо определить всего два параметра, $D_{0,5}$ и $\sigma$. Задача становится намного сложнее, если необходимо учесть объем облученной ткани. Для учета объема облученной ткани в модели НРВ нам понадобится сделать два предположения.

Предположение 2.1. В модели (2.23) от объема зависят параметры $D(0,5)$ и $\sigma$. Параметр $F$ не зависит от объема облученной ткани, т.е.

$$
\begin{aligned}
& F(P)=\frac{D(V)-D(0,5 ; V)}{\sigma(V)}, \\
& D(P, V)=D(0,5 ; V)+F(P) \sigma(V) .
\end{aligned}
$$

Из предположения 2.1. и структуры модели (2.25) следует, что $D(V)$, $D(0,5 ; V)$ и $\sigma(V)$ должны одинаковым образом зависеть от $V$, так как $F(P)$ не зависит от $V$.

Предположение 2.2. В модели (2.25) зависимость параметра $\sigma$ от $V$ имеет следующий вид:

$$
\sigma(V)=\sigma(1) \cdot V^{-b}
$$

Действительно, в силу предположения 1.1, имеем:

$$
D(V)=D(1) \cdot V^{-b}
$$

Следовательно,

$$
D(0,5 ; V)=D(0,5 ; 1) \cdot V^{-b} .
$$

Необходимо, чтобы выполнялось соотношение

$$
\frac{D\left(P, V_{1}\right)}{D\left(P, V_{2}\right)}=\frac{D\left(0,5 ; V_{1}\right)+F(P) \sigma\left(V_{1}\right)}{D\left(0,5 ; V_{2}\right)+F(P) \sigma\left(V_{2}\right)}=\left(\frac{V_{1}}{V_{2}}\right)^{-b} \text {, }
$$

откуда следует, что $\sigma(V)=\sigma(1) \cdot V^{-b}$.

Анализ (2.26) и (2.28) свидетельствует о том, что с ростом $V$ происходит уменьшение $D(0,5 ; V)$ и $\sigma(v)$. С ростом $V$ график зависимости ВЛО от $V$ сдвигается влево и становится более крутым. Приведенная к единичному объему модель (2.25) будет иметь следующий вид:

$$
\begin{aligned}
& D(P, 1)=D(0,5 ; 1)+F(P) \sigma(1), \\
& D(P, V)=D(P, 1) \cdot V^{-b}=[D(0,5 ; 1)+F(P) \sigma(1)] \cdot V^{-b} .
\end{aligned}
$$




\section{4. Анализ результатов расчета ВЛО в органах и тканях организма при помощи модели МРВ и НРВ}

Для того чтобы выяснить, как влияет на результаты расчетов ВЛО выбор модели МРВ и НРВ, мы воспользовались результатами систематизации клинических данных о ТД уровня ВЛО 5 и 50\% для здоровых органов и тканей организма в зависимости от суммарной дозы и объема облучения, приведенных в табл. 1.1 (Emami et al. [31]) и табл. 1.2 (Kehwar et al. [32, 33]).

Таблица 2.1

\section{Толерантная доза}

\begin{tabular}{|c|c|c|c|c|c|c|c|}
\hline № & Орган или ткань & $\begin{array}{l}D_{5 / 5} \Gamma \mathrm{p} \\
V=1 / 3\end{array}$ & $\begin{array}{l}D_{5 / 5} \Gamma \mathrm{p} \\
V=2 / 3\end{array}$ & $\begin{array}{c}D_{5 / 5} \Gamma \mathrm{p} \\
V=1\end{array}$ & $\begin{array}{l}D_{50 / 5} \Gamma \mathrm{p} \\
V=1 / 3\end{array}$ & $\begin{array}{l}D_{50 / 5} \Gamma \mathrm{p} \\
V=2 / 3\end{array}$ & $\begin{array}{c}D_{50 / 5} \Gamma \mathrm{p} \\
V=1\end{array}$ \\
\hline$\overline{1}$ & Мочевой пузырь & & $77(80)\{80\}$ & $64(65)\{65\}$ & & $93(98)\{98\}$ & $78(80)\{80\}$ \\
\hline 2 & Плечевое сплетение & & & $60(60)\{60\}$ & & & $75(75)\{75\}$ \\
\hline 3 & Мозг & $59(60)\{60\}$ & $50(50)\{50\}$ & $45(45)\{45\}$ & $77(75)\{79\}$ & $65(65)\{67\}$ & $59(60)\{60\}$ \\
\hline 4 & Ствол мозга & $60(60)\{60\}$ & $53(53)\{53\}$ & $50(50)\{50\}$ & & & $65(65)\{65\}$ \\
\hline 5 & Конский хвост & & & $60(60)\{60\}$ & & & $75(75)\{75\}$ \\
\hline 6 & Кишка & $55(54)\{54\}$ & & $45(45)\{45\}$ & $66(66)\{66\}$ & & $54(55)\{55\}$ \\
\hline 7 & Среднее ухо 1 & $30(30)\{30\}$ & $30(30)\{30\}$ & $30(30)\{30\}$ & $40(40)\{40\}$ & $40(40)\{40\}$ & $40(40)\{40\}$ \\
\hline 8 & Среднее ухо 2 & $55(55)\{55\}$ & $55(55)\{55\}$ & $55(55)\{55\}$ & $65(66)\{66\}$ & $65(66)\{66\}$ & $65(66)\{66\}$ \\
\hline 9 & Пищевод & $60(60)\{60\}$ & $57(58)\{57\}$ & $56(55)\{56\}$ & $73(73)\{73\}$ & $70(70)\{70\}$ & $68(68)\{68\}$ \\
\hline 10 & Головка и шейка бедра & & & $52(52)\{52\}$ & & & $65(65)\{65\}$ \\
\hline 11 & Сердце & $59(60)\{59\}$ & $46(45)\{46\}$ & $40(40)\{40\}$ & $72(70)\{70\}$ & $56(55)\{55\}$ & $49(50)\{48\}$ \\
\hline 12 & Почки & $50(50)\{50\}$ & $30(30)\{31\}$ & $23(23)\{23\}$ & & $39(40)\{37\}$ & $29(28)\{28\}$ \\
\hline 13 & Гортань 1 & $77(79)\{79\}$ & $72(70)\{73\}$ & $68(70)\{70\}$ & $90(90)\{90\}$ & $83(80)\{84\}$ & $79(80)\{80\}$ \\
\hline 14 & Гортань 2 & $54(55)\{55\}$ & $53(52)\{52\}$ & $52(50)\{50\}$ & $72(70)\{76\}$ & $70(70)\{72\}$ & $69(70)\{70\}$ \\
\hline 15 & Хрусталик & & & $10(10)\{10\}$ & & & $18(18)\{18\}$ \\
\hline 16 & Печень & $48(50)\{43\}$ & $35(35)\{34\}$ & $29(30)\{30\}$ & $62(55)\{57\}$ & $45(45)\{46\}$ & $38(40)\{40\}$ \\
\hline 17 & Легкие & $44(45)\{45\}$ & $26(30)\{25\}$ & $\begin{array}{c}19(17,5) \\
\{17\}\end{array}$ & $66(65)\{64\}$ & $39(40)\{35\}$ & $\begin{array}{c}28(24,5) \\
\{25\}\end{array}$ \\
\hline 18 & Зрительный нерв & & & $50(50)\{50\}$ & & & $65(65)\{65\}$ \\
\hline 19 & $\begin{array}{l}\text { Хиазма зрительного } \\
\text { нерва }\end{array}$ & & & $50(50)\{50\}$ & & & $65(65)\{65\}$ \\
\hline 20 & $\begin{array}{l}\text { Околоушная слюнная } \\
\text { железа }\end{array}$ & & $32(32)\{32\}$ & $32(32)\{32\}$ & & $46(46)\{46\}$ & $46(46)\{46\}$ \\
\hline 21 & Прямая кишка & & & $60(60)\{60\}$ & & & $80(80)\{80\}$ \\
\hline 22 & Сетчатка & & & $45(45)\{45\}$ & & & $65(65)\{65\}$ \\
\hline 23 & Ребро грудной клетки & $50(50)\{50\}$ & & & $65(65)\{65\}$ & & \\
\hline 24 & Кожа & $69(70)\{71\}$ & $61(60)\{63\}$ & $54(55)\{56\}$ & & & $70(70)\{70\}$ \\
\hline 25 & Тонкий кишечник & $49(50)\{48\}$ & & $40(40)\{40\}$ & $64(60)\{65\}$ & & $53(55)\{55\}$ \\
\hline 26 & Спинной мозг & $50(50)\{51\}$ & $49(50)\{49\}$ & $48(47)\{47\}$ & $71(70)\{71\}$ & $69(70)\{69\}$ & \\
\hline 27 & Желудок & $59(60)\{59\}$ & $54(55)\{53\}$ & $51(50)\{50\}$ & $73(70)\{77\}$ & $67(67)\{69\}$ & $63(65)\{65\}$ \\
\hline 28 & Щитовидн & & & $45(45)\{46\}$ & & & $80(80)\{80\}$ \\
\hline 29 & $\begin{array}{l}\text { Кость, сустав нижней } \\
\text { челюсти }\end{array}$ & $64(65)\{65\}$ & $61(60)\{62\}$ & $59(60)\{60\}$ & $59(60)\{60\}$ & $73(72)\{74\}$ & $71(72)\{72\}$ \\
\hline 30 & $\begin{array}{l}\text { Кость, головка } \\
\text { бедренной кости }\end{array}$ & & & $52(52)\{52\}$ & & & $65(65)\{65\}$ \\
\hline
\end{tabular}

Примечание. ТД, систематизированные в работе Kehwar et al. [32, 33] приводятся в круглых скобках, их воспроизведения при помощи модели НРВ (2.21) - без скобок, а при помощи модели МРВ - в фигурных скобках.

Суммарные дозы $D_{5 / 5}$ и $D_{50 / 5}$ приводят к 5 и $50 \%$ лучевых осложнений за 5 -летний срок наблюдения. $V$ - относительный объем облученного органа или ткани. 
В табл. 2.1. приводятся результаты воспроизведения значений толерантных доз, приведенных в табл. 1.1 при помощи моделей МРВ и НРВ, которые для относительных объемов облученных органов и тканей $(1 / 3,2 / 3,1)$ приводят к ВЛО 5 и $50 \%$.

Анализ табл. 2.1 показал, что модели МРВ и НРВ не всегда воспроизводят ТД с одинаковой точностью. Этого следовало ожидать, поскольку значения суммарных доз и объемы облученных органов и тканей, приведенные в таблице, в отдельных случаях определялись интерактивно, приближенно. Также не учитывалась неоднородность дозовых распределений. Но появление систематизированных клинических значений ТД в виде табл. 1.1 и 1.2 следует признать явлением положительным и достаточно важным для радиологии. Они позволили лучевым терапевтам ориентироваться при оценке лучевых нагрузок на здоровые органы и ткани при планировании ЛТ опухолевых новообразований, явились стимулом для математического моделирования результатов лучевого воздействия на здоровые органы и ткани организма.

\section{5. Модель Пуассона в радиобиологии и радиологии}

Модель Пуассона играет значительную роль в радиобиологии и в радиологии. Дело заключается в том, что структура модели Пуассона [6,7,25-27,38] находится в полном соответствии с современными представлениями и требованиями радиобиологов к формированию дозовых полей в ЛТ опухолевых заболеваний. Согласно этим представлениям ВЛИ опухолевого заболевания следует рассматривать как функцию от начального числа клоногенных опухолевых клеток $N$, их радиочувствительности $\tau=\alpha+\beta d$, (где $d$ разовая доза), суммарной дозы облучения $D$ и числа выживших после облучения опухолевых клеток [9]. ВЛИ опухолевого заболевания рассматривается как вероятность того, что в живых не останется ни одной опухолевой клетки. Описание ВЛИ опухолевого заболевания при помощи модели Пуассона имеет следующий вид:

$$
\begin{aligned}
& P(D, N, d)=\exp (-M(D, d))=\exp [-N \cdot C(D, d)]= \\
& =\exp \{-N \exp [-D(\alpha+\beta d)]\}=\exp \{-N \exp [-\tau D]\}= \\
& =\exp \{-\rho V \exp [-\tau D]\},
\end{aligned}
$$

где $\tau=\alpha+\beta d ; V$ - объем опухолевой ткани; $\rho$ - плотность опухолевых клеток; $M(D, d)$ - число выживших опухолевых клеток. В модели Пуассона предполагается, что клоногенные опухолевые клетки независимы и ВЛИ опухолевого заболевания есть вероятность того, что все клоногенные опухолевые клетки будут инактивированы. Свойства модели Пуассона рассмотрены нами в работах [6, 7, 78-80]. 
График зависимости ВЛИ опухолевого заболевания от СОД, построенный при помощи модели Пуассона, представляет собой асимметричную выпукло-вогнутую кривую. Некоторые особенности, связанные с применения модели Пуассона для описания ВЛИ опухолевого заболевания в зависимости от СОД, рассмотрены нами в работе [27].

В табл. 2.2 приводятся радиобиологические параметры для некоторых типов опухолей, полученные in vitro, которые могут быть использованы в модели Пуассона [78-80].

Таблица 2.2

Параметры LQ функции для различных типов опухолевых клеток $(\tau=\alpha+2 \beta$, дя разовой дозы 2 Гр)

\begin{tabular}{c|l|c|c|c|c}
\hline № & \multicolumn{1}{|c|}{ Тип опухоли } & $\alpha, \Gamma \mathrm{p}^{-1}$ & $\beta, \Gamma \mathrm{p}^{-2}$ & $\alpha / \beta, \Gamma \mathrm{p}$ & $\tau, \Gamma \mathrm{p}^{-1}$ \\
\hline 1 & Глиобластома & 0,241 & 0,029 & 8,31 & 0,299 \\
2 & Меланома & 0,255 & 0,053 & 4,81 & 0,361 \\
3 & Плоскоклеточный рак & 0,273 & 0,045 & 6,07 & 0,363 \\
4 & Аденокарцинома & 0,311 & 0,055 & 5,65 & 0,421 \\
5 & Лимфома & 0,451 & 0,051 & 8,48 & 0,553 \\
6 & Мелкоклеточный рак & 0,650 & 0,081 & 8,02 & 0,812 \\
\hline
\end{tabular}

Примечание. Параметры LQ функции для различных типов опухолевых клеток, взяты из работы [78-80].

\section{6. Модель Логит в радиобиологии и в радиологии}

В работе [9] сообщается, что для описания зависимости ВЛИ $=P(D, d)$ от суммарной $D$ и разовой дозы $d$, чаще модели Пуассона используется модель Логит [9]:

$$
P(D, d)=\frac{\exp \left(a_{0}+a_{1} D+a_{2} D d+\ldots\right)}{1+\exp \left(a_{0}+a_{1} D+a_{2} D d+\ldots\right)},
$$

где $D, d$ - суммарная и разовая доза. В работе [9] сообщается, что феноменологическая модель (2.33) не поддается простой биологической интерпретации. Но, тем не менее, как указывается в работе [9], она «представляет удобный и гибкий инструмент для прогнозирования ВЛИ опухолевого заболевания на различные воздействия и широко используется в различных областях медицины, непосредственно с радиобиологией не связанных». Параметры $a_{1}$ и $a_{2}$ играют ту же роль, что и коэффициенты $\alpha$ и $\beta$ в LQ модели. Считается, что эти параметры точно не соответствуют $\alpha$ и $\beta$, но сохраняется только тождественность отношений $a_{1} / a_{2}$ и $\alpha / \beta$.

Надо полагать, что эта модель заинтересует специалистов, которые рассмотрят возможность ее использования в планировании ЛТ злокачественных опухолей. 


\section{Глава 3.}

\section{СИНТЕЗИРОВАННЫЕ МАТЕМАТИЧЕСКИЕ МОДЕЛИ (СМ МОДЕЛИ), СВЯЗЫВАЮЩИЕ МЕЖДУ СОБОЙ ЗНАЧЕНИЯ ВЛО, СУММАРНЫЕ И РАЗОВЫЕ ДОЗЫ, И ЗНАЧЕНИЯ ОБЬЕМОВ ОБЛУЧЕННЫХ ОРГАНОВ ИЛИ ТКАНЕЙ}

\section{1. Синтезированные математические модели (СМ модели)}

Актуальной проблемой современной ЛТ является создание математических моделей, которые позволяют рассчитывать эффективные планы облучения различных опухолевых заболеваний, наилучшим образом решающие основную задачу ЛТ: добиться излечения опухолевого заболевания без серьезных необратимых лучевых осложнений в здоровых органах и тканях организма. Эти модели описывают воздействие излучения на органы и ткани организма на тканевом уровне, но не на клеточном уровне. При этом уровень лучевого воздействия на опухолевые и здоровые органы и ткани организма описываются в виде ВЛИ и ВЛО как функции от объемов облучения, суммарной и разовой дозы.

Математическая интерпретация задачи эффективного планирования ЛТ опухолевых образований приводит к многокритериальной экстремальной задаче оптимизации. Возникает она в ЛТ из-за вполне естественного стремления врачарадиолога подвести к опухоли (мишени) большую дозу, чтобы избежать рецидива опухолевого заболевания, и, вместе с тем, уменьшить лучевые нагрузки на здоровые органы и ткани организма, которые образуют ложе опухоли и окружают очаг опухолевого заболевания. В подавляющем большинстве случаев эта операция приводит к несовместности (противоречивости) системы ограничений на дозовое поле, порождает пустое допустимое множество планов облучения. Выйти из этого положения можно только в результате разумного компромисса, завышения ограничений на дозы в некоторых, наименее «ценных» органах и тканях, (с точки зрения лучевого терапевта). Для его осуществления необходимо разрабатывать математические модели для оценки лучевых повреждений здоровых органов и тканей организма, многокритериального оценивания планов облучения и, следовательно, многокритериального планирования ЛТ. Оно включает в себя выделение Парето оптимальных планов облучения [39, 40], их интерактивный экспертный анализ, и, на его основе, выделение компромиссного (эффективного) плана ЛТ.

Опыт ЛТ свидетельствует о том, что в качестве критериев ЛТ разумно использовать ВЛИ опухолевого заболевания и ВЛО в здоровых органах и тканях ор- 
ганизма. Эти показатели естественны и хорошо понятны лучевым терапевтам. Они возникают в результате статистической обработки результатов ЛТ и позволяют соразмерять тяжесть онкозаболевания с риском вызвать необратимые лучевые осложнения в здоровых органах и тканях организма.

ВЛИ опухолевого заболевания и ВЛО в здоровых органах и тканях организма, наряду с ТД в здоровых органах и тканях, можно рассматривать как критериальные оценки плана ЛТ. Существующие в настоящее время математические модели позволяют в основном рассчитывать значения ТД, и реже корректно рассчитывать ВЛО в органах и тканях в зависимости от условий облучения. К сожалению, большинство из них не могут учитывать разовые дозы. Их значения обычно считаются фиксированными (равными 2 Гр), но, в действительности, это, в основном, относится к дозе в мишени (опухоли) и в ложе опухоли.

Актуальной проблемой современной ЛТ является создание математических моделей, которые позволят рассчитывать ВЛО и ТД для произвольных схем ФД, которые включают в число параметров модели объемы облученных тканей, суммарные и разовые дозы. Мы будем называть такие модели синтезированными математическими моделями (СМ моделями), так как они получаются в результате объединения (синтеза) математических моделей, которые позволяют рассчитывать значения ВЛО и значения ТД при заданных схемах ФД.

Настоящая глава посвящена проблемам создания СМ моделей, которые могут быть эффективно использованы для планирования ЛТ злокачественных опухолей. Они позволяют рассчитывать суммарную опухолевую дозу, (СОД), и дозы в здоровых органах и тканях организма, а также значений ВЛИ опухолевого заболевания и ВЛО в здоровых органах и тканях организма в зависимости от условий облучения. Эти параметры следует рассматривать как основные критериальные оценки планов ЛТ, на основе которых следует выбирать компромиссные эффективные планы ЛТ.

Проблема синтеза математических моделей для планирования ЛТ опухолевых образований была впервые рассмотрена нами еще 1986 г. [6]. Исследование этой проблемы было продолжено в последующие годы. Ряд полученных нами новых результатов был опубликован в нашей монографии в 1993 г [7]. Работа над созданием и совершенствованием СМ моделей продолжается нами и в настоящее время [10, 28-30].

Мы показали, что синтез математических моделей, (создание СМ моделей), которые связывают между собой значения ВЛО в здоровых органах и тканях организма, суммарные и разовые дозы, а также объемы облученных органов и тканей, оказывается возможным при некоторых предположениях, которые выделены в 
настоящей работе. Эти предположения нуждаются в проверке. Мы полагаем, что в этом направлении следует работать, так как СМ модели позволяют сделать серьезный шаг на пути совершенствования методов планирования ЛТ и прогнозирования ее результатов.

Создание СМ моделей основано на анализе структур синтезируемых математических моделей, учете размерностей их параметров, и естественным образом приводит к появлению радиологических инвариантов (констант). Эти константы могут стать основой для построения нового научного направления - количественной радиологии на тканевом уровне.

Настоятельная необходимость создания СМ моделей очевидна. Планирование ЛТ на их основе позволяют определять эффективные планы ЛТ, которые включают в себя: физико-технические условия облучения, схемы ФД и эффективные критериальные оценки планов облучения, ВЛИ опухолевых образований, ВЛО в ложе опухоли и в здоровых органах и тканях. Рассчитанные критериальные оценки одновременно являются и прогностическими оценкам ЛТ, (ВЛИ опухолевого заболевания и ВЛО в ложе опухоли и в здоровых органах и тканях организма). Некоторые подходы к созданию таких моделей были впервые рассмотрены нами в работах $[6,7]$.

Вместе с тем мы показали, что СМ модели позволяют полнее использовать клиническую информацию и, как это не покажется парадоксальным, для них легче определять значения параметров настройки моделей. Так как, чем разнообразнее клиническая информация, (чем больше отличаются друг от друга режимы ФД и соответствующие им значения ВЛО), тем точнее будут определены значения параметров СМ модели. (Напомним, что для настройки параметров моделей, предназначенных только для расчета традиционных ТД в зависимости от схемы ФД, было необходимо из клинической информации выделять только ту ее часть, которая соответствует толерантным уровням облучения (ВЛО $\approx 3 \%-5 \%)$.

Описание и использование созданных нами СМ моделей можно найти в работах [27-30] и в настоящей работе.

\subsection{CM1 модель (модель Ellis + модель МРВ)}

Рассмотрим синтез математической модели Ellis и модели MPB, создание СМ1 модели.

\section{Модель Ellis}

Первые усилия по математическому моделированию результатов ЛТ опухолевых заболеваний на тканевом уровне были предприняты лучевыми терапевта- 
ми, радиобиологами и медицинскими физиками. Они были направлены на описание толерантных (предельных) доз (ТД) для органов и тканей организма, которые не приводят к тяжелым, необратимым лучевым осложнениям. В настоящее время принято считать, что ТД, это такие сочетания суммарных и разовых доз облучения, использование которых приводит к ВЛО в органах и тканях определенного, клинически идентифицируемого типа лучевого осложнения, не превышающего 3\%-5\% случаев. Это модель Strandqvist 1944 [62], модель Ellis 1969 [14] и ее модификации, CRE (Kirk, Gray, Watson 1971 [15]), TDF (Orton, Ellis 1973 [16]) и др. Модель Ellis имеет следующий вид:

$$
D=N S D \cdot N^{k_{1}} \cdot T^{k_{2}}
$$

где $D$ - толерантная доза (ТД); NSD - коэффициент пропорциональности (номинальная стандартная доза); $N$ - число сеансов облучения; $T$ - длительность курса ЛТ (сут); (NSD, $\left.k_{1}, k_{2}\right)$ - параметры модели Ellis.

Список ТД для различных органов и тканей при разовой дозе 2 Гр была подготовлена К.И. Жолкивером (табл. 3.1) и опубликована в его диссертации на соискание ученой степени д.м.н. в 1969 г. Позднее, в 1976 г. она была приведена и в его работе [76].

Интересно, что структура модели Ellis полностью совпадает со структурой производственной функции, которая широко используется в экономометрических исследованиях. Таким образом, 1969 г. можно было бы считать началом использования экономических моделей в задачах моделирования результатов ЛТ опухолевых заболеваний. Шутка! Но, если говорить всерьез, то следует отметить, что, по своей сути, модели оптимального планирования ЛТ злокачественных опухолей имеют сходные черты с моделями, которые возникают при исследовании экономических процессов.

Пусть модель Ellis и модель МРВ описывают ТД уровня ВОЛО $=Q$ для одной и той же ткани, фиксированного объема $V$ и фиксированной схемы ФД = $(N, T)$. Модель Ellis имеет следующий вид [14]:

$$
D(Q, N, T, V)=N S D(Q, 1) \times N^{k_{1}} T^{k_{2}} V^{-b},
$$

где $(D, Q, N, T, V)$ - параметры модели Ellis: $D$ - суммарная доза; $Q-$ ВОЛО в ткани; $N$ - число сеансов облучения; $T$ - длительность курса ЛТ в сут.; $V$ - объем облученной ткани; NSD - номинальная стандартная доза, (коэффициент пропорциональности). Обычно предполагалось, что речь идет только о ТД и, следовательно, $Q=0,95$ (ВЛО =0,05). Модель Ellis приводит к адекватным значениям ТД только в том случае, когда число фракций превышает 4 сеанса облучения. Покажем, что СМ1 модель имеет следующий вид: 


$$
Q(D, N, T, V)=1-P(D, N, T, V)=\exp \left[-\left(\frac{D V^{b}}{A_{1}(N, T)}\right)^{A_{2}}\right]
$$

Таблица 3.1

\section{Значения параметра NSD для различных органов и тканей в модели Ellis}

\begin{tabular}{|c|c|c|}
\hline Орган, ткань & NSD (ret) & ТД Гр \\
\hline Кожа & $15,60-18,65$ & $50-65$ \\
\hline Волосистая часть головы & 18,9 & 66,0 \\
\hline Слизистые оболочки & 11,2 & 30,0 \\
\hline Глаз (хрусталик) & 15,0 & - \\
\hline \multicolumn{3}{|l|}{ Головной мозг } \\
\hline малый облученный объем (до 100 см³). & 18,8 & 66,5 \\
\hline большой облученный объем (1000 cм³) & 13,0 & 38,0 \\
\hline Основание мозга, продолговатый мозг & 10,2 & 26,0 \\
\hline \multicolumn{3}{|l|}{ Спинной мозг } \\
\hline до $20 \mathrm{~cm}$ & 16,95 & 57,0 \\
\hline более $20 \mathrm{~cm}$ & 11,2 & 30,0 \\
\hline Растущая кость & $9,0-11,3$ & $22,0-30,0$ \\
\hline Кость & 21,45 & 81,0 \\
\hline Зубы (у детей) & 15,2 & 49,0 \\
\hline Хрящ & 17,0 & 59,0 \\
\hline \multicolumn{3}{|l|}{ Мышцы } \\
\hline дети & 17,0 & 57,0 \\
\hline взрослые & 29,2 & 130,0 \\
\hline Сердце, аорта & 14,2 & 43,0 \\
\hline \multicolumn{3}{|l|}{ Легкие } \\
\hline одно & 11,2 & 30,0 \\
\hline оба & 7,55 & 16.5 \\
\hline Пищевод & 17,6 & 60,0 \\
\hline Желудок & 12,3 & 35,0 \\
\hline Тонкий кишечник & 12,3 & 35,0 \\
\hline Толстый кишечник, прямая кишка & 16,0 & 52,0 \\
\hline \multicolumn{3}{|l|}{ Печень } \\
\hline малый объем (до 200 см³) & 15,8 & 50,0 \\
\hline большой объем (1000 cм³) & 11,2 & 30,0 \\
\hline Почки & 6,3 & 13,0 \\
\hline Мочевой пузырь & 17,6 & 60,0 \\
\hline Кроветворная ткань & 5,0 & 9,0 \\
\hline Селезенка & 3,75 & 5.5 \\
\hline Лимфатические узлы & 15,0 & 4,80 \\
\hline Яички (без учета генетических последствий) & 2,5 & 3,0 \\
\hline Яичники (без учета генетических последствий) & 1,0 & - \\
\hline Вульва & 10,2 & 26,0 \\
\hline Влагалище & 21,25 & 80,0 \\
\hline
\end{tabular}

Примечание. Значения параметра NSD для различных органов и тканей в модели Ellis, подготовленны К.И. Жолкивером и приведенны в работе [76].

Для создания СM1 модели предположим, что выполняется следующее положение.

Предположение 3.1. В модели Ellis (3.1) от ВОЛО $=Q$ зависит только NSD, а в модели МРВ от режима ФД = $(N, T)$ зависит только параметр $A_{1}$. 
Поскольку мы считаем, что модель Ellis и модель МРВ описывают одно и то же значение ТД уровня $Q$, при одной и той же схеме ФД и объеме облучения, приравняем их:

$$
\begin{aligned}
& D(Q, N, T, V)=A_{1}(N, T)|\ln (Q)|^{1 / A_{2}} V^{-b}=N S D(Q, 1) N^{k_{1}} T^{k_{2}} V^{-b}, \\
& A_{1}(N, T)|\ln (Q)|^{1 / A_{2}}=N S D(Q, 1) N^{k_{1}} T^{k_{2}} .
\end{aligned}
$$

Перепишем (3.4) следующим образом

$$
A_{1}(N, T)=\frac{N S D(Q, 1)}{|\ln (Q)|^{1 / A_{2}}} N^{k_{1}} T^{k_{2}} .
$$

Поскольку параметр $A_{1}$ не зависит от $Q$ (это следует из построения модели MPB), необходимо допустить, что выполняются следующие условия:

Предположение 3.2. Синтез модели Ellis и модели MPB возможен в том случае, если:

1) параметры $A_{1}$ и $A_{2}$ не зависят от $Q$ (это условие очевидно);

2) параметр $A_{1}$ является функцией от схемы ФД, $A_{1}=A_{1}(N, T)$, а параметр $A_{2}-$ не является;

3) выполняется синтезирующее условие, которое следует из предположения 3.1. Синтезирующий модели параметр $K^{1}$ можно определить следующим образом:

$$
K^{1}=\frac{A_{1}}{N^{k_{1}} T^{k_{2}}}=\frac{N S D(Q, 1)}{|\ln (Q)|^{1 / A_{2}}}=\text { const. }
$$

Мы считаем, что $K^{1}$ является синтезирующим параметром модели (постоянным числом, радиологической константой (инвариантом)) для рассматриваемой ткани. Он не зависит от $Q$ и схемы ФД и имеет размерность такую же, как и NSD. Из (3.7) получаем линейную зависимость, приведенного к относительному единичному объему параметра $N S D(Q, 1)$ в модели Ellis, от значения $|\ln (Q)|^{1 / A_{2}}$

$$
N S D(Q, 1)=K^{1}|\ln (Q)|^{1 / A_{2}} \text {. }
$$

Он может быть использован для проверки Предположения 3.2 на клиническом материале. Кроме того, из (3.7) следует зависимость $A_{1}$ от схемы ФД $(N, T)$

$$
A_{1}(N, T)=K^{1} N^{k_{1}} T^{k_{2}} \text {. }
$$

Если бы модель Ellis работала, начиная с $N=1$ и $T=1$, мы могли бы написать $A_{1}(1,1)=K^{1}$, т.е. синтезирующий параметр $K^{1}$ численно был бы равен параметру $A_{1}$ в модели МРВ.

СM1 модель, которая работает для произвольного значения $Q$, с учетом (3.9) может быть записана следующим образом:

$$
D(Q, N, T, V)=K^{1}|\ln (Q)|^{1 / A_{2}} N^{k_{1}} T^{k_{2}} V^{-b} .
$$


Из (3.8) получаем формулу для расчета $N S D$ в зависимости от $Q$ и $V$

$$
N S D(Q, V)=K^{1}|\ln (Q)|^{1 / A_{2}} V^{-b} \text {. }
$$

Уравнения (3.7) и (3.11) могут быть использованы для определения параметров $K^{1}, b, A_{2}$. СМ1 модель, которая описывает ВОЛО в ткани для произвольного режима ФД, принимает следующий вид:

$$
\begin{aligned}
& Q(D, N, T, V)=\exp \left[-\left(\frac{D V^{b}}{A_{1}}\right)^{A_{2}}\right]=\exp \left[-\left(\frac{D V^{b}}{K^{1} N^{k_{1}} T^{k_{2}}}\right)^{A_{2}}\right]= \\
& =\exp \left[-\left|\frac{N S D \cdot C R E(1)}{K^{1}}\right|^{A_{2}}\right]=\exp \left[-\left|\frac{N S D \cdot T D F(1)^{k_{4}}}{K^{1}}\right|^{A_{2}}\right],
\end{aligned}
$$

где $k_{4}=1-k_{1}-k_{2}>0$. Параметр модели $A_{1}$ зависит от режима облучения (режима фракционирования дозы). Параметры $A_{2}$ и $b$ - не зависят. Они могут быть определены для произвольного фиксированного режима облучения, при котором определяется и $A_{1}$. Затем можно определить $K^{1}, k_{1}, k_{2}$. Когда параметры модели Ellis $\left(k_{1}, k_{2}\right)$ известны, можно привести все клинические наблюдения к одному и тому же стандартному режиму ФД, на множестве которых затем можно определить параметры CM1 модели, и которые позволят рассчитать ВЛО в ткани [6, 7]. Возможны также случаи, когда известными являются параметры модели МРВ $A_{1}$ и $A_{2}$, для некоторого фиксированного режима ФД, и параметры модели Ellis для фиксированного значения $Q$. В этом случае построение СМ1 модели сводится к определению значения синтезирующего параметра $K^{1}$ из уравнения:

$$
A_{1}(N, T)=K^{1} N^{k_{1}} T^{k_{2}} \text {. }
$$

Пример 3.1. Пусть для некоторого органа или ткани известны параметры модели Ellis $\left(k_{1}, k_{2}, b\right)$, задан режим ФД $=(N, T)$, и задан план облучения $(D, V)$. Необходимо при помощи СM1 модели определить ВОЛО (ВЛО) в ткани. Решение будет состоять из следующих действий (шагов):

1) из (3.12) следует определить $\operatorname{NSD}(Q, V)$ для любого режима облучения $(N, T)$;

2) определить предельную (толерантную) дозу $D$ уровня $Q$ и по формулам, которые приводятся ниже, рассчитать ВОЛО $=Q$ :

$$
\begin{aligned}
& Q(D, N, T, V)=\exp \left[-\left(\frac{D V^{b}}{A_{1}}\right)^{A_{2}}\right]=\exp \left[-\left(\frac{D V^{b}}{K^{1} N^{k_{1}} T^{k_{2}}}\right)^{A_{2}}\right]= \\
& =\exp \left[-\left|\frac{N S D(Q, 1) N^{k_{1}} T^{k_{2}}}{K^{1} N^{k_{1}} T^{k_{2}}}\right|^{A_{2}}\right]=\exp \left[-\left|\frac{N S D(Q, 1)}{K^{1}}\right|^{A_{2}}\right] .
\end{aligned}
$$

Следующие утверждения характеризуют свойства полученной СM1 модели. 
Утверждение 3.1. Для СМ1 модели (3.12) с ростом $N$ или $T$, или и $N$ и $T$, график, описывающий зависимость $Q$ (или $P$ ) от $D$, смещается вправо вдоль оси абсцисс.

Доказательство. Из (3.13) следует, что с ростом $N$ или $T$, или и $N$ и $T$, значение параметра $A_{1}$ увеличивается. Из уравнения

$$
D(Q, V)=A_{1}|\ln (Q)|^{1 / A_{2}} V^{-b}
$$

следует, что для фиксированных значений $Q$ и $V$ с ростом $A_{1}$ значение $D(Q, V)$ увеличивается. А это значит, что график $Q(D, V)$ будет смещаться вправо. Утверждение доказано.

Утверждение 3.2. С ростом параметра $A_{1}$ график зависимости ВЛО (или ВОЛО), от дозы становится более пологим.

Доказательство. Для доказательства рассмотрим два дозовых интервала, которые определяются значениями ВОЛО, равными $Q_{1}$ и $Q_{2}$, и зависят от значения параметра $A_{1}$. Зададим значения $A_{1}^{*}$ и $A_{2}^{*}$, причем $A_{1}^{*}>A_{2}^{*}$. Покажем, что дозовый интервал

$$
\left|D_{11}\left(Q_{1}, A_{1}^{*}\right)-D_{12}\left(Q_{2}, A_{1}^{*}\right)\right|>\left|D_{21}\left(Q_{1}, A_{2}^{*}\right)-D_{22}\left(Q_{2}, A_{2}^{*}\right)\right| .
$$

Действительно, суммарные дозы, которые получаются в результате использования параметров $A_{1}$ и $Q$, будут:

$$
\begin{aligned}
& D_{11}=A_{1}^{*}\left|\ln \left(Q_{1}\right)\right|^{1 / A_{2}} V^{-b}, D_{12}=A_{1}^{*}\left|\ln \left(Q_{2}\right)\right|^{1 / A_{2}} V^{-b}, \\
& D_{21}=A_{2}^{*}\left|\ln \left(Q_{1}\right)\right|^{1 / A_{2}} V^{-b}, D_{22}=A_{2}^{*}\left|\ln \left(Q_{2}\right)\right|^{1 / A_{2}} V^{-b} .
\end{aligned}
$$

Рассмотрим отношение дозовых интервалов (обозначим это отношение $\Lambda$ )

$$
\Lambda=\frac{\left|D_{11}-D_{12}\right|}{\left|D_{21}-D_{22}\right|}=\frac{\left.\left|A_{1}^{*}\right| \ln \left(Q_{1}\right)\right|^{1 / A_{2}} V^{-b}-A_{1}^{*}\left|\ln \left(Q_{2}\right)\right|^{1 / A_{2}} V^{-b} \mid}{\left.\left|A_{2}^{*}\right| \ln \left(Q_{1}\right)\right|^{1 / A_{2}} V^{-b}-A_{2}^{*}\left|\ln \left(Q_{2}\right)\right|^{1 / A_{2}} V^{-b} \mid}=\frac{A_{1}^{*}}{A_{2}^{*}}>1 .
$$

Отсюда следует, что выполняется неравенство $\left|D_{11}-D_{12}\right|>\left|D_{21}-D_{22}\right|$, и график зависимости $D$ от $Q$ (или $Q$ от $D$ ), для $A_{1}^{*}$ будет более пологим. Утверждение доказано.

Утверждение 3.3. Для СМ1 модели (3.12) с ростом $V$ график, описывающий зависимость $Q$ (или $P$ ) от $D$ смещается к оси ординат и кривая становится менее пологой.

Доказательство. Из (3.13) следует, что для фиксированного значения $Q$ с ростом $V$ значение $D(Q, V)$ уменьшается. Следовательно, с ростом $V$ график зависимости $Q$ от $D$ смещается влево, к оси ординат. Покажем теперь, что график кривой становится более крутым. Для этого достаточно показать, что для двух 
фиксированных значений ВОЛО $Q_{1}$ и $Q_{2}$, с ростом $V$ дозовый интервал $\left|D\left(Q_{1}, V\right)-D\left(Q_{2}, V\right)\right|$ уменьшается. Пусть $V_{1}>V_{2}$. Используя схему построения отношения (3.18), получаем:

$$
\begin{aligned}
& D_{11}\left(Q_{1}, V_{1}\right)=A_{1}\left|\ln \left(Q_{1}\right)\right|^{1 / A_{2}} V_{1}^{-b}, \\
& D_{12}\left(Q_{2}, V_{1}\right)=A_{1}\left|\ln \left(Q_{2}\right)\right|^{1 / A_{2}} V_{1}^{-b}, \\
& D_{21}\left(Q_{1}, V_{2}\right)=A_{1}\left|\ln \left(Q_{1}\right)\right|^{1 / A_{2}} V_{2}^{-b}, \\
& D_{22}\left(Q_{2}, V_{2}\right)=A_{1}\left|\ln \left(Q_{2}\right)\right|^{1 / A_{2}} V_{2}^{-b} .
\end{aligned}
$$

Рассмотрим отношение

$$
\begin{aligned}
& \Omega=\frac{\left|D_{11}\left(Q_{1}, V_{1}\right)-D_{12}\left(Q_{2}, V_{1}\right)\right|}{\left|D_{21}\left(Q_{1}, V_{2}\right)-D_{22}\left(Q_{2}, V_{2}\right)\right|}=\frac{\left.V_{1}^{-b}\left|A_{1}\right| \ln \left(Q_{1}\right)\right|^{1 / A_{2}}-A_{1}\left|\ln \left(Q_{2}\right)\right|^{1 / A_{2}} \mid}{\left.V_{2}^{-b}\left|A_{1}\right| \ln \left(Q_{1}\right)\right|^{1 / A_{2}}-A_{1}\left|\ln \left(Q_{2}\right)\right|^{1 / A_{2}} \mid}= \\
& =\left(\frac{V_{1}}{V_{2}}\right)^{-b}<1 .
\end{aligned}
$$

Из (3.24) следует, что с ростом $V_{1}$ дозовый интервал сокращается. А это означает, что график кривой $Q(D)$ станет круче. Утверждение доказано.

Параметры CM1 модели на множестве систематизированных клинических наблюдений $\left(Q_{i}, D_{i}, N_{i}, T_{i}, V_{i}\right), i=1, \ldots, n$, можно определить в результате решения одной из следующих нелинейных экстремальных задач,

$$
\begin{aligned}
& F_{1}=\sum_{i=1}^{n}\left\{\exp \left|-\left(\frac{D_{i} V_{i}^{b}}{K^{1} N_{i}^{k_{1}} T_{i}^{k_{2}}}\right)^{A_{2}}\right|-Q_{i}\right\}^{2} \rightarrow \min _{A_{2}, b, K^{1}, k_{1}, k_{2}}, \\
& F_{2}=\sum_{i=1}^{n}\left[K^{1} N_{i}^{k_{1}} T_{i}^{k_{2}}\left|\ln \left(Q_{i}\right)\right|^{1 / A_{2}} V_{i}^{-b}-D_{i}\right]^{2} \rightarrow \min _{A_{2}, b, K^{1}, k_{1}, k_{2}} .
\end{aligned}
$$

В табл. 3.2 приводятся параметры СМ1 моделей $A_{1}, A_{2}, b$ для стандартных режимов ФД. Они были определены либо в результате обработки клинических данных, либо, (как в случае сердца, печени и кишечника), были взяты такими же, как и для кожи (соединительной ткани) в работе Ellis. Работы, в которых приводятся значения $k_{1}$ и $k_{2}$, взяты из нашей работы [26].

Легко убедиться, что в случае неравномерной схемы ФД синтез модели Ellis и МРВ, которая описывает эквивалентные схемы ФД, осуществляется точно так же, как и в случае равномерного фракционирования дозы, так как синтезирующий параметр образуется в результате преобразования параметра $\mathrm{A}_{1}$, который не участвует в формировании адекватной по ВЛО дозы [7]. Проблема построения СМ1 модели в случаях сплит-курса и других схем ФД рассмотрена нами в работе [7]. 
Параметры СМ1 модели (модель Ellis + модель МРВ)

для различных здоровых органов и тканей организма

\begin{tabular}{l|c|c|c|c|c|c}
\hline \multirow{2}{*}{ Орган или ткань } & \multicolumn{7}{|c}{ Значения параметров } \\
\cline { 2 - 6 } & $A_{1}$ & $b$ & $A_{2}$ & $k_{1}$ & $k_{2}$ & $K^{1}$ \\
\hline Кожа & 162,1 & 0,175 & 10,80 & 0,24 & 0,11 & 47,8 \\
Легкие & 216,2 & 0,464 & 6,08 & 0,377 & 0,058 & 160,7 \\
& 848,9 & 0,718 & 3,39 & 0.377 & 0,058 & 120,1 \\
Сердце & 392,6 & 0,476 & 14,44 & 0,24 & 0,11 & 115,7 \\
Головной мозг & 111,4 & 0,104 & 18,00 & 0,56 & 0,03 & 14,8 \\
Печень & 260,8 & 0,412 & 9,61 & 0,24 & 0,11 & 79,9 \\
Кишечник & 91,0 & 0,098 & 11,05 & 0,29 & 0,08 & 25,3 \\
Почки & 131,8 & 0,324 & 7,81 & 0,25 & 0,19 & 27,9 \\
Желудок & 409,5 & 0,431 & 13,56 & 0,24 & 0,11 & 120,6 \\
Спинной мозг & 74,8 & 0,076 & 12,98 & 0,24 & 0,11 & 22,0 \\
Эпидермоидный рак & 9,63 & 0,076 & 1,00 & 0,25 & 0,04 & $0,104^{-1}$ \\
\hline
\end{tabular}

\section{3. СМ2 модель (модель Ellis + модель НРВ)}

Синтезированная модель СM2 связывает модель Ellis и нормальное распределение вероятностей (НРВ). Будем считать, что для создания СМ2 модели должны выполняться следующие предположения:

Предположение 3.3. В модели Ellis от ВЛО $=P$ зависит только $N S D(P, 1)$

$$
D(P, N, T, V)=N S D(P, 1) N^{k_{1}} T^{k_{2}} V^{-b},
$$

а в модели НРВ от режима ФД = $(N, T)$ зависят параметры модели $D(0,5 ; N, T, 1)$ и $\sigma(N, T, 1)$

$$
D(P, N, T, V)=D(0,5 ; N, T, V)+F(P) \sigma(N, T, V) .
$$

Пусть обе модели описывают одно и то же значение дозы $D(P, N, T, V)$, и им соответствуют одинаковые условия облучения.

Предположение 3.4. Создание синтезированной СМ2 модели оказывается возможным, если при любых значениях параметров синтезируемых моделей $(P, D, N, T, V)$ выполняются условия:

$$
\begin{aligned}
& D(P, N, T, V)=N S D(P, 1) N^{k_{1}} T^{k_{2}} V^{-b}= \\
& =[D(0,5 ; N, T, 1)+F(P) \sigma(N, T, 1)] V^{-b}, \\
& N S D(P, 1) N^{k_{1}} T^{k_{2}}=D(0,5 ; N, T, 1)+F(P) \sigma(N, T, 1) .
\end{aligned}
$$

Предположение 3.5. Синтез модели Ellis и НРB возможен, если выполняются следующие условия:

$$
\begin{aligned}
& D(0,5 ; N, T, 1)=N S D(0,5 ; 1) N^{k_{1}} T^{k_{2}}, \\
& \sigma(N, T, 1)=K^{2} N^{k_{1}} T^{k_{2}},
\end{aligned}
$$




$$
N S D(P, 1)=N S D(0,5 ; 1)+F(P) K^{2},
$$

где $K^{2}$ - синтезирующий параметр, инвариант (константа), которая имеет размерность такую же, как и $N S D$, и зависит только от вида облучаемой ткани.

Действительно, приравняв (3.27) и (3.28), получаем

$$
D(0,5 ; N, T, 1)+F(P) \sigma(N, T, 1)=N S D(P, 1) N^{k_{1}} T^{k_{2}} .
$$

Учитывая структуры моделей, входящие в (3.34), можем написать

$$
N S D(P, 1)=N S D(0,5 ; 1)+N S D(P, 1)-N S D(0,5 ; 1) .
$$

Учитывая (3.34) и (3.35), получаем

$$
\begin{aligned}
& D(0,5 ; N, T, 1)+F(P) \sigma(N, T, 1)= \\
& N S D(0,5 ; 1) N^{k_{1}} T^{k_{2}}+[N S D(P, 1)-N S D(0,5 ; 1)] N^{k_{1}} T^{k_{2}} .
\end{aligned}
$$

По определению

$$
D(0,5 ; N, T, 1)=N S D(0,5 ; 1) N^{k_{1}} T^{k_{2}} .
$$

Сгруппировав члены, которые содержат ВЛО $=P$, в соответствие с предположением 3.3 , получаем

$$
\frac{N S D(P, 1)-N S D(0,5 ; 1)}{F(P)}=\frac{\sigma(N, T, 1)}{N^{k_{1}} T^{k_{2}}}=K^{2}=\text { const. }
$$

Из (3.38) следует, что $N S D(P, 1)$ есть линейная функция от $F(P)$

$$
\begin{aligned}
& N S D(P, 1)=N S D(0,5 ; 1)+F(P) K^{2}, \\
& \sigma(N, T, 1)=K^{2} N^{k_{1}} T^{k_{2}} .
\end{aligned}
$$

Напомним, что $\sigma(N, T, 1)$, как и $D(0,5 ; N, T, 1)$, должна иметь размерность дозы. Следовательно, параметр $K^{2}$ должен иметь размерность такую же, как и NSD. СM2 модель может быть представлена следующим образом:

$$
D(P, N, T, V)=\left[N S D(0,5 ; 1)+K^{2} F(P)\right] N^{k_{1}} T^{k_{2}} V^{-b} .
$$

Здесь первое слагаемое описывает толерантный уровень облучения ткани объема $V$, когда под «толерантностью» подразумевается ВЛО $=0,5$, а второе слагаемое - поправку на отклонение $P$ от 0,5 . Значение $F(P)$ отрицательно при $P<0,5$ и положительно при $P>0,5$. При $P=0,5, F(P)=0$. Формулу (3.40) можно разрешить относительно $F(P)$

$$
F(P)=\left[\frac{D(V) V^{b}}{N^{k_{1}} T^{k_{2}}}-N S D(0,5 ; 1)\right] / K^{2} .
$$

При фиксированном значении Р и фиксированном режиме ФД $=(N, T)$, для разных объемов $V_{1}$ и $V_{2}$ облученной ткани, выполняется основное соотношение между толерантными дозами и объемами облучения тканей 


$$
\begin{aligned}
& D_{1}(0,83 ; 1)=42,86 \frac{3,41}{} \\
& \frac{D\left(P, N, T, V_{1}\right)}{D\left(P, N, T, V_{2}\right)}=\left(\frac{V_{1}}{V_{2}}\right)^{-b} .
\end{aligned}
$$

Следующие утверждения характеризуют свойства полученной СМ2 модели.

Утверждение 3.4. Для модели СМ2 с ростом $N$ или $T$, или и $N$ и $T$, график, описывающий зависимость $P$ (или $Q$ ) от $D$, смещается вправо и становится более пологим.

Доказательство. Оно следует из (3.41), так как с увеличением $N$ или $T$, или $N$ и $T$, происходит увеличение $D(0,5 ; N, T, V)$, график смещается вправо и при увеличении $\sigma(N, T, V)$ график зависимости ВЛО от $D$ становится более пологим.

\section{Утверждение доказано.}

Утверждение 5.5. Для СМ2 модели и фиксированных значениий $(N, T)$ с ростом $V$ график функции, описывающий зависимость $P$ (или $Q$ ) от $D$, смещается влево и становится менее пологим.

Доказательство. Из (3.41) видно, что с ростом $V$ параметры моделей, $D(0,5 ; N, T, V)$ и $\sigma(N, T, V)$, уменьшаются. А это означает, что с ростом $V$ график будет смещаться влево, в сторону малых доз и становится менее пологим (более крутым). Утверждение доказано.

Определить параметры СМ2 модели (3.41) или (3.42) на основе систематизированных клинических данных можно в результате решения следующих экстремальных задач

$$
\begin{aligned}
\Phi_{1} & =\sum_{i=1}^{M}\left\{\left[N S D(0,5 ; 1)+F\left(P_{i}\right) K^{2}\right] N_{i}^{k_{1}} T_{i}^{k_{2}} V_{i}^{-b}-D\left(P_{i}, N_{i}, T_{i}, V_{i}\right)\right\}^{2} \rightarrow \\
\rightarrow \min _{K^{2}, b, k_{1}, k_{2}}, & \\
\text { или } \quad \Phi_{2} & =\sum_{i=1}^{M}\left\{\left[\frac{D\left(V_{i}\right) V_{i}^{b}}{N_{i}^{k_{1}} T_{i}^{k_{2}}}-N S D(0,5 ; 1)\right] / K^{2}-F\left(P_{i}\right)\right\}^{2} \rightarrow \min _{K^{2}, b, k_{1}, k_{2}},
\end{aligned}
$$

где $M$ - число систематизированных клинических наблюдений. В зависимости от того, какие параметры моделей уже известны, могут быть рассмотрены различные модификации задачи определения параметров СМ2 модели. 


\subsection{LQ модель. Связь ТД с разовой дозой и объемом облученной ткани}

Среди математических моделей, которые в настоящее время используются в планировании ЛT, широкое распространение получила LQ модель, созданная на основе LQ функции, предложенной в работах Kellerer, Rossi 1969 [70] и Chadwick, Leenhauts 1973 [69] для описания фракции выживших клеток в результате их однократного или фракционированного облучения. Окончательный вид, для расчета ТД на тканевом уровне, LQ модель приобрела в работе Barendsen 1982 [17]

$$
E=D(\alpha+\beta d),
$$

где $E$ - параметр, задающий уровень толерантности ткани; $(D, d)$ - план облучения; $(\alpha, \beta)$ - параметры модели. В настоящее время LQ модель является одной из наиболее востребованных математических моделей, которая используется для описания ТД для различных схем фракционированного облучения нормальных или опухолевых тканей [76]. Для $n$ сеансов облучения с постоянным значением разовой дозы $d$, LQ модель может быть описана следующим образом:

$$
E=\alpha D+\frac{\beta D^{2}}{n}=\alpha D+\beta D d=D(\alpha+\beta d),
$$

где $D=n d$ - суммарная доза; $n$ - число сеансов облучения; $d$ - разовая доза; $E$ - параметр, задающий уровень толерантности ткани, обычно он соответствует толерантному уровню облучения органа или ткани; $(\alpha, \beta)$ - параметры модели [24]. Обычно параметр $E$ в LQ модели задает уровень ТД, т.е. дозы, которая приводит к ВЛО $=0,05$.

\section{5. Модифицированная LQ модель (MLQ модель)}

Для создания синтезированной математической модели СМ3, которая объединяет модель MPB и LQ модель, нам понадобится модифицированная LQ модель (MLQ модель). Для описания толерантных уровней облучения органа или ткани LQ модель имеет следующий вид:

$$
E=D(\alpha+\beta d)
$$

где $(D, d)$ - режим ФД; $(\alpha, \beta)$ - радиобиологические параметры модели; параметр $E$ задает уровень толерантности ткани.

Опыт применения LQ модели для планирования ЛТ показал, что, в отличие от модели Ellis, LQ модель может быть использована для расчета ТД, начиная с одного сеанса облучения, $n=1,2, \ldots$ Обзначим ТД однократного облучения как $D_{R}$. Мы можем модифицировать LQ модель (создать MLQ модель), введя в нее одно- 
кратную толерантную дозу $D_{R}$ в качестве параметра модели. По определению, поскольку LQ модель работает и для однократного облучения, имеет место равенство

$$
\begin{aligned}
& E=D(\alpha+\beta d D)=\alpha D_{R}+\beta D_{R}^{2}, \\
& D=\frac{\alpha D_{R}+\beta D_{R}^{2}}{\alpha+\beta d}=D_{R} \frac{\alpha+\beta D_{R}}{\alpha+\beta d}=D_{R} \frac{(\alpha / \beta)+D_{R}}{(\alpha / \beta)+d}=D_{R} \frac{\gamma+D_{R}}{\gamma+d}=D_{R} f(d),
\end{aligned}
$$

где $\gamma=\alpha / \beta$ - радиобиологический параметр для облучаемого органа или ткани. Из соотношения (3.51) следует, что толерантная доза равномерного курса фракционированного облучения ткани в $f(d)$ раз больше однократной толерантной дозы $f(d) \geq 1$.

MLQ модель имеет всего два параметра $\gamma$ и $D_{R}$. Эти параметры можно определить, если известны, по меньшей мере, два эквивалентных (по толерантности) режима облучения. Если их больше, значения параметров модели MLQ могут быть определены в результате решения соответствующей экстремальной задачи.

Проследим, как можно описать зависимость параметров MLQ модели от объема облученной ткани и ВЛО в ткани.

Предположение 3.6. В MLQ модели от Q (или от Р) зависит только однократная толерантная доза $D_{R}(Q)$. Таким образом, из (3.50) и (3.51) следует:

$$
\begin{aligned}
& E(Q)=\alpha D_{R}(Q)+\beta D_{R}^{2}(Q), \\
& D(Q)=\frac{\gamma D_{R}(Q)+D_{R}^{2}(Q)}{\gamma+d} .
\end{aligned}
$$

Предположение 3.7. В MLQ модели зависимость параметров $\left(D, d, D_{R}, \alpha\right.$, $\beta, \gamma)$ от объема $V$ облученной ткани имеет следующий вид:

$$
\begin{aligned}
& D(V)=D(1) V^{-b}, d(V)=d(1) V^{-b}, D_{R}(Q, V)=D_{R}(Q, 1) V^{-b}, \\
& \alpha(V)=\alpha(1) V^{b}, \beta(V)=\beta(1) V^{2 b}, \gamma(V)=\gamma(1) V^{-b},
\end{aligned}
$$

где $b$ - параметр модели, зависящий только от вида ткани.

Покажем, что из предположений 3.6 и 3.7 следует, что параметр $E$ не зависит от $V$. Действительно, при любом значении $V$ справедливо равенство

$$
E(Q)=\alpha(V) D_{R}(Q, V)+\beta(V) D_{R}^{2}(Q, V)=\alpha(1) D_{R}(Q, 1)+\beta(1) D_{R}^{2}(Q, 1) .
$$

Предположения 3.6 и 3.7 не противоречат сделанным нами ранее предположениям. 


\section{6. СМЗ модель (модель $M L Q+$ модель МРВ)}

Из соотношения (3.53) следует, что

$$
D(Q, V)=\frac{\alpha(V) D_{R}(Q, V)+\beta(V) D_{R}^{2}(Q, V)}{\alpha(V)+\beta(V) d(V)}=\frac{\gamma(1) D_{R}(Q, 1)+D_{R}^{2}(Q, 1)}{\gamma(1)+d(1)} V^{-b} .
$$

и мы получаем $\boldsymbol{C M 3}$ модель, которая имеет следующий вид:

$$
D(Q, V)=\frac{\gamma(1) D_{R}(Q, 1)+D_{R}^{2}(Q, 1)}{\gamma(1)+d(1)} V^{-b},
$$

где $\quad \gamma(1)=\frac{\alpha(1)}{\beta(1)} \Gamma \mathrm{p}$.

При тотальном облучении ткани из (3.57) получаем

$$
D=\frac{\gamma D_{R}+D_{R}^{2}}{\gamma+d}=D_{R}\left[1+\frac{D_{R}-d}{\gamma+d}\right] \text {. }
$$

Следующее утверждение характеризует свойство модели СМ3.

Утверждение 3.6. Для фиксированных значений $Q$ и $V$ с ростом разовой дозы $d$ график зависимости $D(Q, d, V)$ от $Q$ смещается влево и становится более «крутым».

Доказательство. При фиксированных условиях облучения с ростом $d$ величина $D$ в модели CM3 (3.58), уменьшается и происходит смещение графика зависимости $D(d)$ влево, к оси ординат. С ростом $V$ значение $D$ уменьшается и происходит уменьшение величины $Q(V)$ и интервал изменения величины $Q$ сжимается и смещается к оси ординат. Пусть $V_{1}>V_{2}$. Возьмем два произвольных значений $Q_{1}$ и $Q_{2}$, остальные параметры CM3 модели будем считать фиксированными. Рассмотрим отношение

$$
\frac{\left|D\left(Q_{1}, V_{1}\right)-D\left(Q_{2}, V_{1}\right)\right|}{\left|D\left(Q_{1}, V_{2}\right)-D\left(Q_{2}, V_{2}\right)\right|}=\left(\frac{V_{1}}{V_{2}}\right)^{-b}<1 .
$$

Из него следует, что чем больше объем облученной ткани, тем меньше дозовый интервал, в который заключен график. Утверждение доказано.

В качестве примера расчитаем параметры СМ3 модель для вычисления ВЛО в сердце как функцию от условий облучения. Для этого воспользуемся клиническими данными, подготовленными Kehwar et al. (табл. 1.2). Они приводятся в табл. 3.3 вместе с рассчитанными параметрами СМ3 модели $\left(\gamma(1), b, D_{R}(P, 1)\right)$, раздельно, для ВЛО = 0,05 и ВЛО =0,50. 
Параметры СМЗ модели $\left(\gamma(1), b, D_{R}(P, 1)\right)$ для сердца

\begin{tabular}{|c|c|c|c|c|c|c|c|}
\hline$P, \%$ & $V$, отн. ед. & $D_{\text {Кл }}, \Gamma \mathrm{p}$ & $D_{\mathrm{TEOP}}, \Gamma \mathrm{p}$ & Отклонение, $\varepsilon, \%$ & $b$ & $\gamma(1), \Gamma \mathrm{p}$ & $D_{R}(P, 1), \Gamma \mathrm{p}$ \\
\hline 5 & $1 / 3$ & 59,91 & 60,33 & $-0,71$ & \multirow{3}{*}{0,35} & \multirow{3}{*}{3,32} & \multirow{3}{*}{12,27} \\
\hline 5 & $2 / 3$ & 44,53 & 43,69 & 1,90 & & & \\
\hline 5 & 1 & 35,54 & 35,94 & $-1,13$ & & & \\
\hline 50 & $1 / 3$ & 72,54 & 72,85 & $-0,43$ & \multirow{3}{*}{0,29} & \multirow{3}{*}{3,47} & \multirow{3}{*}{14,65} \\
\hline 50 & $2 / 3$ & 57,17 & 56,51 & 1,16 & & & \\
\hline 50 & 1 & 48,18 & 48,52 & $-0,70$ & & & \\
\hline
\end{tabular}

Примечание. Параметры СМ3 модели определенны на основе клинических данных Kehwar et al. [32, 33] в результате решения экстремальных задач. Параметры модели определялись отдельно для

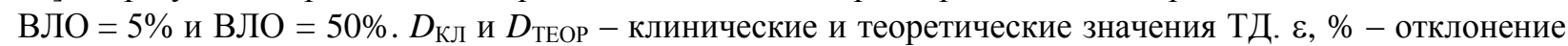
теоретических значений дозы от клинических значений.

Раздельное решение задачи определения параметров модели СМ3 для ВЛО $=0,05$ и ВЛО = 0,50 было осуществлено для того, чтобы убедиться, что вычисленные раздельно параметры модели близки друг к другу (согласованы). В известной степени это позволяет судить об адекватности СМ3 модели к исходной систематизированной клинической информации. Анализ табл. 3.3 свидетельствует о том, что СМ3 модель удовлетворительно описывает разделённые клинические данные о ТД в сердце, для уровней ВЛО = 5\% и ВЛО = 50\%. Максимальное отклонение клинической дозы от рассчитанной (модельной) дозы не превышает 1,9\%! Параметры модели $b$ и $\gamma(1)$ для разных значений ВЛО близки друг к другу, что также свидетельствует об адекватности СМ3 модели к исходной клинической информации. В табл. 3.4 приводятся параметры СМ3 модели, полученные в результате обработки всей клинической информации, для ВЛО $=0,05$ и ВЛО $=0,50$. Анализ табл. 3.4 свидетельствует о том, что СМ3 модель можно с успехом использовать для оценки планов облучения сердца. Максимальное отклонение клинического значения дозы от теоретического (модельного) значения не превышает 3,9\%!

Таблица 3.4

Параметры СМЗ модели (b, $\left.\gamma(1), A_{1}, A_{2}\right)$ для сердца (для ВЛО = 5\% и ВЛО = 50\%)

\begin{tabular}{|c|c|c|c|c|c|c|c|c|}
\hline$P, \%$ & $V$, отн. ед. & $D_{\text {Кл}}, \Gamma \mathrm{p}$ & $D_{\mathrm{TEOP}}, \Gamma \mathrm{p}$ & $\begin{array}{c}\text { Отклонение, } \\
\varepsilon, \%\end{array}$ & $b$ & $\gamma(1), \Gamma \mathrm{p}$ & $A_{1}, \Gamma \mathrm{p}$ & $A_{2}$ \\
\hline 5 & $1 / 3$ & 59,91 & 58,58 & 2,23 & \multirow{6}{*}{0,32} & \multirow{6}{*}{3,41} & \multirow{6}{*}{12,53} & \multirow{6}{*}{14,39} \\
\hline 5 & $2 / 3$ & 44,53 & 43,90 & 1,41 & & & & \\
\hline 5 & 1 & 35,54 & 36,91 & $-3,85$ & & & & \\
\hline 50 & $1 / 3$ & 72,54 & 75,09 & $-3,52$ & & & & \\
\hline 50 & $2 / 3$ & 57,17 & 56,28 & 1,55 & & & & \\
\hline 50 & 1 & 48,18 & 47,31 & 1,80 & & & & \\
\hline
\end{tabular}

Примечание. Параметры определенны на основе клинических данных Kehwar et al. [32, 33] в результате обработки всей клинической информации. 
Клинические данные Kehwar et al. [32, 33], использованные для определения параметров СМ3 модели, оказались более точными (эффективными), чем данные Emami et al. [31]. В этом можно убедиться, сравнив полученные результаты, с результатами расчетов, приведенных в табл. 3.5 и 3.6, и полученных на основе данных Emami et al. В обоих случаях параметр $\gamma(1) \approx 15$ Гр, т.е. принимает предельно большое разрешенное нами значение в экстремальной задаче определения параметров модели (3.58). Мы полагаем, что это может быть вызвано тем, что в исходной клинической информации разовая доза принимала не только одно фиксированное значение $d=2$ Гр. Вместе с тем следует отметить, что клинические и модельные значения суммарных доз близки друг к другу.

В табл. 3.5 приводятся результаты определения параметров СМ3 модели отдельно для $\mathrm{B} Л О=0,05$ и ВЛО $=0,50$.

Таблица 3.5

Параметры CMЗ модели $\left(b, \gamma(1), D_{R}(P, 1)\right)$ для сердца

\begin{tabular}{|c|c|c|c|c|c|c|c|}
\hline$P, \%$ & $V$, отн. ед. & $D_{\text {Кл, }}$, p & $D_{\mathrm{TEOP}}, \Gamma \mathrm{p}$ & Отклонение, $\varepsilon, \%$ & $b$ & $\gamma(1), \Gamma p$ & $D_{R}(P, 1), \Gamma \mathrm{p}$ \\
\hline 5 & $1 / 3$ & 60 & 59,49 & 0,85 & \multirow{3}{*}{0,34} & \multirow{3}{*}{$\underline{14,99}$} & \multirow{3}{*}{19,46} \\
\hline 5 & $2 / 3$ & 45 & 45,97 & $-2,16$ & & & \\
\hline 5 & 1 & 40 & 39,45 & 1,38 & & & \\
\hline 50 & $1 / 3$ & 70 & 69,49 & 0,73 & \multirow{3}{*}{0,28} & \multirow{3}{*}{$\underline{14,99}$} & \multirow{3}{*}{22,42} \\
\hline 50 & $2 / 3$ & 55 & 56,07 & $-1,05$ & & & \\
\hline 50 & 1 & 50 & 49,36 & 1,28 & & & \\
\hline
\end{tabular}

Примечание. Параметры СМ3 модели определенны на основе клинических данных Emami et al. [31] в результате решения экстремальной задачи. Параметры определялись отдельно для ВЛО $=5 \%$ и ВЛО $=50 \%$.

Таблица 3.6

Параметры CMЗ модели $\left(b, \gamma(1), D_{R}(P, 1)\right)$ для сердца на основе всей клинической информации

\begin{tabular}{|c|c|c|c|c|c|c|c|c|}
\hline$P, \%$ & $V$, отн. ед. & $D_{\text {Кл}}, \Gamma \mathrm{p}$ & $D_{\mathrm{TEOP}}, \Gamma \mathrm{p}$ & $\begin{array}{c}\text { Отклонение, } \\
\varepsilon, \%\end{array}$ & $b$ & $\gamma(1), \Gamma \mathrm{p}$ & $A_{1}$ & $A_{2}$ \\
\hline 5 & $1 / 3$ & 60 & 58,42 & 2,63 & \multirow{6}{*}{0,31} & \multirow{6}{*}{$\underline{14,99}$} & \multirow{6}{*}{22,60} & \multirow{6}{*}{21,38} \\
\hline 5 & $2 / 3$ & 45 & 46,13 & $-2,52$ & & & & \\
\hline 5 & 1 & 40 & 40,10 & $-0,25$ & & & & \\
\hline 50 & $1 / 3$ & 70 & 70,83 & $-1,19$ & & & & \\
\hline 50 & $2 / 3$ & 55 & 55,93 & $-1,69$ & & & & \\
\hline 50 & 1 & 50 & 48,62 & 2,76 & & & & \\
\hline
\end{tabular}

Примечание. Параметры СМ3 модели определенны на основе клинических данных Emami et al. [31] в результате решения экстремальной задачи на основе всей клинической информации.

Анализ показывает, что значения параметров CM3 модели, определенные при разделенной клинической информации, для ВЛО $=0,05$ и ВЛО $=0,50$, и совокупной клинической информации, ВЛО $=0,05$ и ВЛО $=0,50$, близки друг к другу. 


\section{7. Использование СМЗ модели и модели МРВ \\ для планирования облучения сердца}

Поскольку в использованной клинической информации в разделе 3.6 по облучению сердца разовая доза была фиксирована и ровна $d=2$ Гр, ВЛО зависит только от суммарной дозы $D$ и от относительного значения облученного объема $V$. В этом случае, помимо СМ3 модели, может быть успешно использована модель МРВ. Оптимальные значения ее параметров для двух вариантов рассматриваемой клинической информации, Kehwar et al. и Emami et al., приводятся в табл. 3.7 и 3.8 .

Таблица 3.7

Параметры модели МРВ (b, $\left.A_{1}, A_{2}\right)$ для сердца, определенные на основе клинических данных Kehwar et al. (табл. 1.2)

\begin{tabular}{|c|c|c|c|c|c|c|c|}
\hline$P, \%$ & $V$, отн. ед. & $D_{\text {КЛ }}, \Gamma \mathrm{p}$ & $D_{\text {TEOP }}, \Gamma \mathrm{p}$ & Отклонение, \% & $b$ & $A_{1}$ & $A_{2}$ \\
\hline 5 & $1 / 3$ & 59,91 & 56,93 & $-4,98$ & \multirow{6}{*}{0,37} & \multirow{6}{*}{50,28} & \multirow{6}{*}{10,52} \\
\hline 5 & $2 / 3$ & 44,53 & 44,05 & $-1,08$ & & & \\
\hline 5 & 1 & 35,54 & 37,91 & $-6,38$ & & & \\
\hline 50 & $1 / 3$ & 72,54 & 72,92 & 0,52 & & & \\
\hline 50 & $2 / 3$ & 57,17 & 56,42 & $-1,31$ & & & \\
\hline 50 & 1 & 48,18 & 48,56 & 0,79 & & & \\
\hline
\end{tabular}

Таблица 3.8

Параметры модели МРВ $\left(b, A_{1}, A_{2}\right)$ для сердца, определенные на основе клинических данных Emami et al. (табл. 1.1)

\begin{tabular}{|c|c|c|c|c|c|c|c|}
\hline$P, \%$ & $V$, отн. ед. & $D_{\text {КЛ}}, \Gamma \mathrm{p}$ & $D_{\mathrm{TEOP}}, \Gamma \mathrm{p}$ & Отклонение, \% & $b$ & $A_{1}$ & $A_{2}$ \\
\hline 5 & $1 / 3$ & 60 & 57,31 & $-4,49$ & \multirow{6}{*}{0,31} & \multirow{6}{*}{50,79} & \multirow{6}{*}{13,51} \\
\hline 5 & $2 / 3$ & 45 & 46,23 & 2,73 & & & \\
\hline 5 & 1 & 40 & 40,77 & 1,92 & & & \\
\hline 50 & $1 / 3$ & 70 & 69,48 & $-0,74$ & & & \\
\hline 50 & $2 / 3$ & 55 & 56,05 & 1,91 & & & \\
\hline 50 & 1 & 50 & 49,43 & $-1,14$ & & & \\
\hline
\end{tabular}

Анализ показывает, что они близки друг к другу. Несколько худшее согласие наблюдается между значениями параметра $b$ и $A_{2}$. 


\section{8. Упрощенные методы расчета зависимости}

\section{между суммарными и разовыми дозами в органах и тканях, созданные при помощи СМЗ модели}

Из (3.58) можно получить различные соотношения, которые могут оказаться полезными при анализе исходной клинической информации и при планировании ЛТ. Мы рассмотрим два из них. Остальные предоставляем читателю.

\subsection{1. Значения ВЛО и $V$ фиксированы}

Пусть для двух планов облучения, № 1 и № 2 имеем: $Q_{1}=Q_{2}=Q, V_{1}=V_{2}=V$, $d_{1} \neq d_{2}, D_{1} \neq D_{2}$. Тогда при помощи модели (3.58) получаем уравнения, связывающие суммарную дозу с разовой дозой при любых значениях объема облученной ткани и для любого фиксированного значения ВОЛО $=1-\mathrm{BЛО}=Q$. Имеем:

$$
D_{1}(Q, 1)=\frac{\gamma(1) D_{R}(Q, 1)+D_{R}^{2}(Q, 1)}{\gamma(1)+d_{1}(1)}, D_{2}(Q, 1)=\frac{\gamma(1) D_{R}(Q, 1)+D_{R}^{2}(Q, 1)}{\gamma(1)+d_{2}(1)} .
$$

Взяв отношение $D_{1}(Q, 1)$ к $D_{2}(Q, 1)$, для произвольного значения $V$, получаем:

$$
\begin{aligned}
& \frac{D_{1}(Q, 1)}{D_{2}(Q, 1)}=\frac{\gamma(1)+d_{2}(1)}{\gamma(1)+d_{1}(1)}, \\
& D_{1}(Q, V)=D_{2}(Q, V) \frac{\gamma(V)+d_{2}(V)}{\gamma(V)+d_{1}(V)}=D_{2}(Q, V) \cdot Z\left(d_{1}(V)\right),
\end{aligned}
$$

где $\quad Z\left(d_{1}(V), d_{2}(V)\right)=\frac{\gamma(V)+d_{2}(V)}{\gamma(V)+d_{1}(V)}=\frac{\gamma(1)+d_{2}(1)}{\gamma(1)+d_{1}(1)}$.

Заметим, что сходные соотношения можно получить из традиционной LQ модели, но только при условии, что параметр $\gamma$ не зависит от объема. Но, в этом случае точность расчетов может оказаться хуже, чем при помощи модели (3.64).

\subsection{2. Значения ВОЛО $=1-$ ВЛО фиксированы и равны}

Рассмотрим более сложный случай, когда $Q_{1}=Q_{2}=Q, V_{1} \neq V_{2}, d_{1} \neq d_{2}$, $D_{1} \neq D_{2}$. Используя (3.58), получаем:

$$
\begin{aligned}
& D_{1}\left(Q, V_{1}\right)=\frac{\gamma\left(V_{1}\right) D_{R}\left(Q, V_{1}\right)+D_{R}^{2}\left(Q, V_{1}\right)}{\gamma\left(V_{1}\right)+d_{1}\left(V_{1}\right)}=\frac{\gamma(1) D_{R}(Q, 1)+D_{R}^{2}(Q, 1)}{\gamma(1)+d_{1}(1)} V_{1}^{-b}, \\
& D_{2}\left(Q, V_{2}\right)=\frac{\gamma\left(V_{2}\right) D_{R}\left(Q, V_{2}\right)+D_{R}^{2}\left(Q, V_{2}\right)}{\gamma\left(V_{2}\right)+d_{1}\left(V_{2}\right)}=\frac{\gamma(1) D_{R}(Q, 1)+D_{R}^{2}(Q, 1)}{\gamma(1)+d_{2}(1)} V_{2}^{-b} .
\end{aligned}
$$


Взяв отношение (3.66) к (3.67), получаем уравнение, связывающее суммарную дозу с разовой дозой и объемом облученной ткани при любых фиксированных значениях ВОЛО $=1-$ ВЛО $=Q$

$$
\begin{aligned}
& \frac{D_{1}\left(Q, V_{1}\right)}{D_{2}\left(Q, V_{2}\right)}=\frac{\gamma(1)+d_{1}(1)}{\gamma(1)+d_{2}(1)}\left(\frac{V_{1}}{V_{2}}\right)^{-b}=\Theta\left(d_{1}(V), d_{2}(V)\right)\left(\frac{V_{1}}{V_{2}}\right)^{-b}, \\
& D_{1}\left(Q, V_{1}\right)=D_{2}\left(Q, V_{2}\right) \cdot \Theta\left(d_{1}(V), d_{2}(V)\right)\left(\frac{V_{1}}{V_{2}}\right)^{-b},
\end{aligned}
$$

где $\quad \Theta\left(d_{1}(V), d_{2}(V)\right)=\frac{\gamma(1)+d_{1}(1)}{\gamma(1)+d_{2}(1)}$.

\subsection{3. Зависимость суммарной дозы от разовой дозы}

Определим изменение суммарной дозы в зависимости от разовой дозы для фиксированного значения ВЛО при тотальном облучении сердца $V=1$. Воспользуемся значениями параметров СМ3 модели, приведенными в табл. 3.3 и рассчитаем значение дозы $D_{2}$, которая при тотальном облучении сердца при $V=1$ и при разовой дозе $d_{2}=2$ Гр приводит к ВЛО $=0,17($ ВОЛО $=0,83)$

$$
D_{2}=A_{1}|\ln (0,83)|^{1 / A_{2}} V^{-b}=50,28|\ln (0,83)|^{1 / 10,52} 1^{-0,37}=42,86 \text {. }
$$

Найдем дозу $D_{1}$, которая при тотальном облучении сердца с разовой дозой $d=2,8$ Гр приводит к ВОЛО $=0,83$. Из табл. 3.4 имеем: $\gamma(1)=3,41, b=0,32$. Используя формулу (3.62), находим

$$
D_{1}(0,83 ; 1)=42,86 \frac{3,41+2 \cdot 1^{0,32}}{3,41+2,8 \cdot 1^{0,32}}=37,34 \Gamma \mathrm{p} .
$$

Таким образом, при увеличении разовой дозы с 2 до 2,8 Гр суммарная толерантная доза для сердца уменьшается на 5,52 Гр.

Опыт показал, что СМ3 модели могут быть успешно исползованы для расчета ВЛИ опухолевых заболеваний.

\section{9. Описание ВЛИ ранней стадии РМЖ при помощи СМЗ модели (комплексный метод лечения, хирургия + ЛТ)}

Рассмотрим применение СМ3 модели для описания ВЛИ ранней стадии рака молочной железы (РМЖ) в комплексном методе лечения, который включает в себя секторальную резекцию опухоли и последующую тотальную ЛТ всей молочной железы. Тотальное $(V=1)$, послеперационное облучение молочной железы необходимо для инактивации опухолевых клеток, которые могли уцелеть в опера- 
ционном поле МЖ, или выйти за пределы операционного поля. Поскольку $V=1$, СМ3 модель будет иметь следующий вид:

$$
D(Q, d)=\frac{T_{1} \gamma|\ln (Q)|^{1 / T_{2}}+T_{1}^{2}|\ln (Q)|^{2 / T_{2}}}{\gamma+d},
$$

где $(D, d)$ - план ЛТ; $\left(\gamma, T_{1}, T_{2}\right)$ - параметры CM3 модели. Для идентификации параметров СМ3 модели мы воспользовались систематизированными клиническими данными, приведенными в работе [43]. Результаты вычисления параметров СМ3 модели для разных планов ЛТ приводятся в табл. 3.9.

Таблица 3.9

\section{Параметры СМЗ модели для описания ВЛИ ранних стадий}

\section{тотального облучения РМЖ}

\begin{tabular}{|c|c|c|c|c|c|c|c|c|c|c|c|}
\hline № & $P_{\text {Кл }}$ & $P_{\text {TEOP }}$ & $f \%$ & $d$ & $n$ & $D_{\text {КЛ }}$ & $D_{\text {TEOP }}$ & $g \%$ & $\gamma$ & $T_{1}$ & $T_{2}$ \\
\hline 1 & 0,968 & 0,969 & 0,07 & 2 & 25 & 50,0 & 49,46 & $-1,07$ & \multirow{7}{*}{0,94} & \multirow{7}{*}{4,07} & \multirow{7}{*}{1,18} \\
\hline 2 & 0,972 & 0,971 & $-0,06$ & 2,66 & 16 & 42,50 & 42,99 & 0,96 & & & \\
\hline 3 & 0,965 & 0,964 & $-0,09$ & 2,5 & 16 & 40,0 & 40,50 & 1,24 & & & \\
\hline 4 & 0,968 & 0,968 & $-0,04$ & 2,26 & 20 & 45,2 & 45,45 & 0,56 & & & \\
\hline 5 & 0,968 & 0,969 & 0,08 & 3,34 & 10 & 34,4 & 33,98 & $-1,21$ & & & \\
\hline 6 & 0,968 & 0,968 & $-0,04$ & 4,93 & 5 & 24,65 & 24,78 & 0,59 & & & \\
\hline 7 & 0,968 & 0,969 & 0,06 & 3,39 & 10 & 33,90 & 33,59 & $-0,91$ & & & \\
\hline
\end{tabular}

Примечание. Параметры СМЗ модели для описания ВЛИ ранних стадий тотального облучения РМЖ, рассчитанны путем решения экстремальной задачи на основе 7 планов облучения, приведенных в рабо-

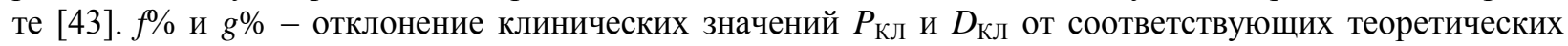
(модельных) значений $P_{\text {ТЕОР и }} D_{\text {ТЕОР. }}$

Анализ табл. 3.9 показывает, что наблюдается удовлетворительное согласие между теоретическими (модельными) и клиническими значениями СОД, а также между клиническими и теоретическими значениями ВЛИ РМЖ. Максимальное отклонение в значениях СОД не превышают 1,3\%, а отклонения в значениях ВЛИ РМЖ $=P$ не превышают 0,09\%! Следует также отметить, что в наших расчетах получилось значение параметра $\gamma=0,94$ Гр, которое меньше значений $\gamma$, приведенных в работе [43]. В ней указывается, что по данным разных авторов значение этого параметра лежит между 1,44 и 4,39 Гр.

На рис. 3.1 приводятся графики зависимостей ВИ РМЖ от СОД для разовых доз 1,8; 2,6 и 3,6 Гр, рассчитанные при помощи СМ3 модели. 


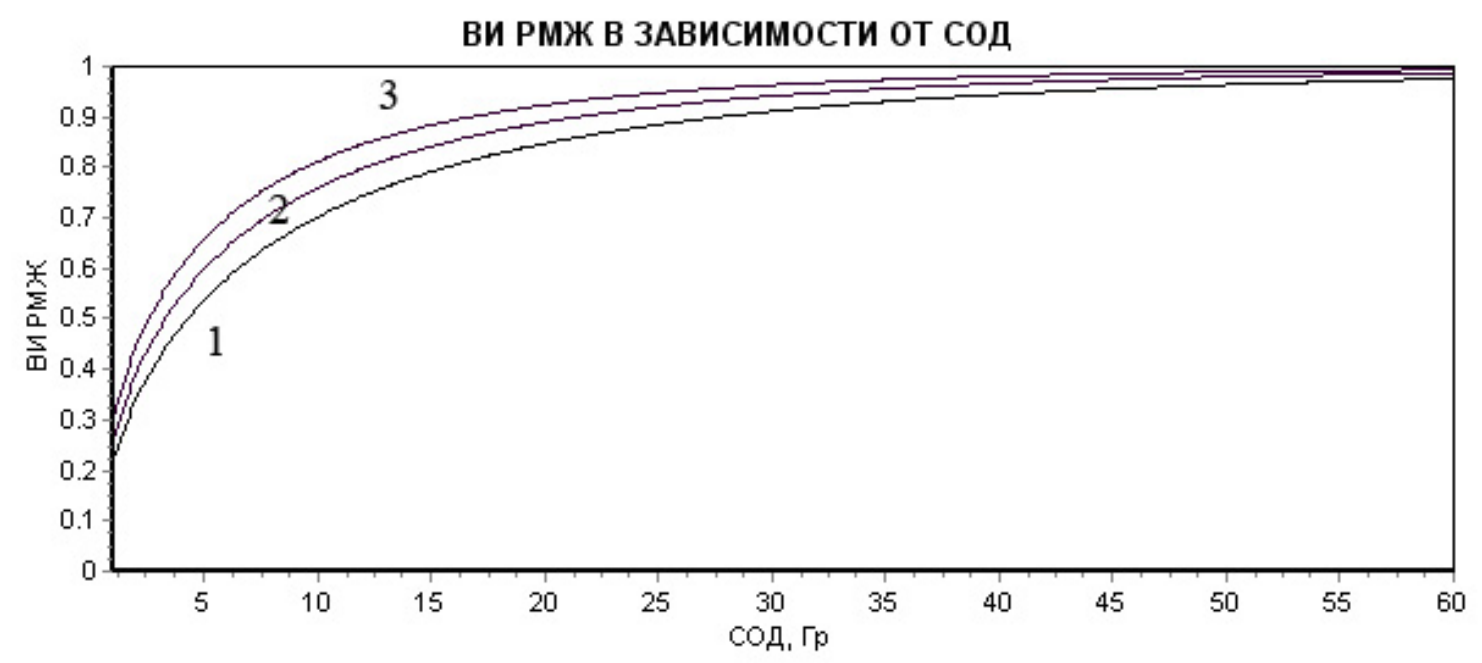

Рис. 3.1. Зависимости ВЛИ РМЖ от СОД для РОД

Примечание. График 1 - РОД = 1,8 Гр; график 2 - РОД = 2,6 Гр; график 3 - РОД = 3,6 Гр.

Расчеты велись при помощи СМ3 модели.

\subsection{0. Параметры СМЗ модели, рассчитанные для некоторых органов и тканей}

В качестве примера рассчитаем параметры СМ3 модели для некоторых органов и тканей, приведенных в табл. 1.1 (Емами et al.).

Таблица 3.10

Параметры СМЗ модели для некоторых органов и тканей

\begin{tabular}{l|c|c|c|c|c}
\hline \multicolumn{1}{c|}{ Орган или ткань } & $b$ & $\gamma, \Gamma \mathrm{p}$ & $A_{1}$ & $A_{2}$ & $\varepsilon, \%$ \\
\hline Мочевой пузырь & 0,185 & 0,52 & 13,83 & 37,78 & 4,0 \\
Головной мозг & 0,135 & 0,71 & 12,53 & 19,60 & 1,8 \\
Кишка & 0,110 & 1,72 & 13,60 & 26,63 & 0,9 \\
Пищевод & 0,035 & 0,45 & 12,85 & 26,30 & 1,5 \\
Сердце & 0,320 & $\mathbf{7 8 , 4 2}$ & 12,53 & 14,39 & 2,6 \\
Почки & 0,560 & 3,41 & 11,10 & 18,63 & 2,8 \\
Печень & 0,210 & 0,45 & 9,63 & 23,73 & 6,2 \\
Легкие & 0,635 & 2,55 & 9,98 & 13,38 & 6,8 \\
Кожа & 0,110 & 0,006 & 12,05 & 21,53 & 0,2 \\
Тонкий кишечник & 0,085 & 0,98 & 12,35 & 19,83 & 3,8 \\
Желудок & 0,085 & 3,59 & 17,43 & 23,03 & 3,6 \\
\hline Iримечане
\end{tabular}

Примечание. Параметры СМ3 модели взяты из табл. 1.1 (Emami et al.). Максимальное отклонение клинической дозы от рассчитанной теоретической приводится в виде $\varepsilon, \%$.

Анализ табл. 3.10 свидетельсвует о том, что СМ3 модель удовлетворительно описывает клинические данные. Максимальное отклонение клинической дозы от теоретической (модельной) дозы, рассчитанной при помощи СМЗ модели, для всех приведенных органов и тканей не превышает $6,8 \%$ (легкие). Для сердца параметр $\gamma$ принимает неестественно большое значение. Мы полагаем, что это может 
быть вызвано неточностью исходной клинической информации или отсутствием в исходном клиническом материале разнообразия в значениях разовых доз. Они все были взяты равными 2 Гр.

\subsection{1. Интраоперационная ЛТ РМЖ}

С 1998 г. во многих странах стала активно изучаться проблема интраоперационной ЛТ РМЖ после органосохраняющего оперативного вмешательства. В работе [10], например, сообщалось, что к ложу удаленного РМЖ подводилась доза однократного облучения в 17-21 Гр, и что эта доза эквивалентна 56-60 Гр при стандартном методе фракционирования дозы. В работе описываются показания для отбора пациентов для интраоперационной ЛТ. Опыт показал, что интраоперационной метод ЛТ клинически себя полностью оправдал. Пациентам такой метод лечения обходится намного дешевле (он ненамного превышает стоимость одного сеанса облучения).

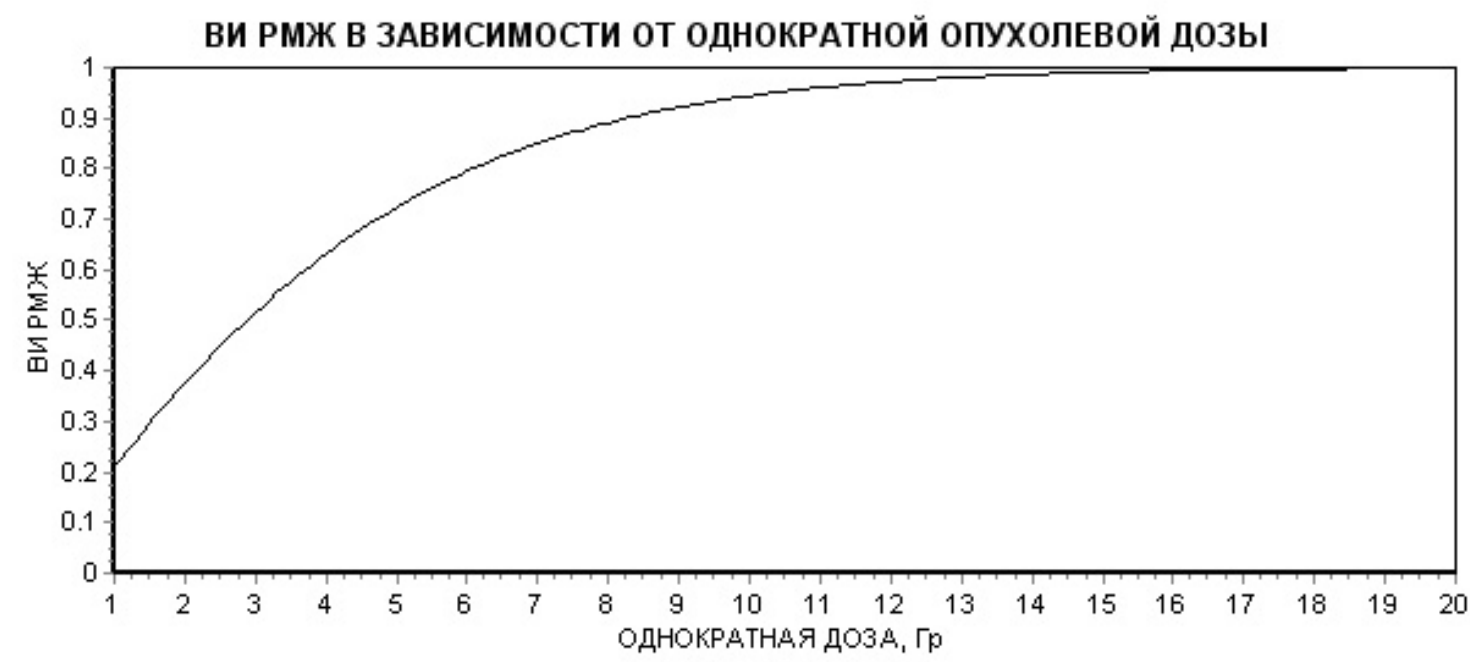

\section{Рис. 3.2. График зависимости ВЛИ РМЖ от однократной дозы, р ассчитанный при помощци СМЗ модели}

На рис. 3.2 приводится график зависимости ВИ РМЖ от однократной дозы облучения, когда для излечения РМЖ используется только основной, одноплановый метод облучения (см. [10]). График построен при помощи СМ3 модели. Параметры модели приведены в табл. 3.10.

В табл. 3.11 приведены значения ВЛИ РМЖ для ряда значений однократной дозы. Анализ рис. 3.2 и табл. 3.10 свидетельствует о том, что в рассматриваемом случае однократная доза в 14-15 Гр приводит к ВИ РМЖ, которая больше или равна 0,99 . 
В табл. 3.11 приводятся значения ВЛИ РМЖ в зависимости от изменения однократной дозы облучения, от 1 до 15 Гр с шагом 1 Гр.

\section{ВЛИ РМЖ = P, \% для значений однократной дозы $D_{\text {од }}$} от 1 до 15 Гр с шагом 1 Гр

\begin{tabular}{|c|c|c|c|c|c|c|c|c|c|c|c|c|c|c|c|}
\hline$D_{\text {од }}, \Gamma \mathrm{p}$ & 1 & 2 & 3 & 4 & 5 & 6 & 7 & 8 & 9 & 10 & 11 & 12 & 13 & 14 & 15 \\
\hline$P, \%$ & 21 & 38 & 52 & 63 & 72 & 80 & 85 & 89 & 92 & 94 & 96 & 97 & 98 & 99 & $>99$ \\
\hline
\end{tabular}

Аналогичные расчеты могут быть проведены для определения однократной дозы интраоперационного облучения молочной железы при би-плановой ЛТ, состоящей из основного и дополнительного (boost) облучения (см. гл. 7 в работе [10]).

\subsection{2. Связь между моделями СМЗ и МРВ}

Установим связь между моделями СМ3 и МРВ. Будем считать, что $\gamma(1)$ и $d(1)$ фиксированы и $\tau(1)=\gamma(1)+d(1)$. Приведенную к единичному объему СМ3 модель запишем в виде уравнения (3.74) следующим образом:

$$
A_{1}^{2}|\ln (Q)|^{2 / A_{2}}+\gamma(1) A_{1}|\ln (Q)|^{1 / A_{2}}-D(1)[\gamma(1)+d(1)]=0 .
$$

Значение ВОЛО $=Q$ из уравнения (3.74) будет:

$$
\begin{aligned}
& |\ln (Q)|^{1 / A_{2}}=\frac{-\gamma(1)+\sqrt{\gamma^{2}(1)+4 D(1)[\gamma(1)+d(1)]}}{2 A_{1}}, \\
& Q(D(1))=\exp \left[\left(-\frac{\sqrt{\gamma^{2}(1)+4 D(1)[\gamma(1)+d(1)]}-\gamma(1)}{2 A_{1}}\right)^{A_{2}}\right]= \\
& =\exp \left[\left(-\frac{D(1)}{A_{1}^{*}}\right)^{A_{2}}\right],
\end{aligned}
$$

где $\quad A_{1}^{*}=-\frac{2 A_{1}}{D(1)\left\{\sqrt{\gamma^{2}(1)+4 D(1)[\gamma(1)+d(1)]}-\gamma(1)\right\}}$.

Из (3.74)-(3.77) видно, каким образом можно осуществить переход от СМ3 модели к модели МРВ. Для этого необходимо знать $\gamma(1)$ рассчитать параметр $A_{1}^{*}$, параметр $A_{2}$ остается неизменным.

В табл. 3.12 приводятся результаты моделирования клинических данных по облучению сердца при помощи СМ3 модели и модели МРВ. Систематизированные клинические данные взяты из табл. 1.2 (Kehwar et al.). Сравнительный анализ показывает, что СМЗ модель описывает клинические данные точнее, чем модель МРВ. 
Кроме того, СМ3 модель может быть использована для произвольного значения разовой дозы, а МРВ модель нет.

Таблица 3.12

Результаты моделирования клинических данных о ВЛО в сердце в зависимости от условий облучения при помощци СМЗ модели и модели МРВ

\begin{tabular}{|c|c|c|c|c|c|c|}
\hline \multicolumn{3}{|c|}{ Исходные данные } & \multicolumn{2}{|c|}{ СМ3 модель } & \multicolumn{2}{|c|}{ МРВ модель } \\
\hline$P, \%$ & $V$, отн. ед. & $D_{\text {кл}}, \Gamma \mathrm{p}$ & $D_{\mathrm{TEOP}}, \Gamma \mathrm{p}$ & $\begin{array}{c}\text { Отклонение, } \\
\%\end{array}$ & $D_{\mathrm{TEOP}}, \Gamma \mathrm{p}$ & $\begin{array}{c}\text { Отклонение, } \\
\%\end{array}$ \\
\hline 5 & $1 / 3$ & 59,91 & 58,58 & 2,23 & 56,93 & 4,98 \\
\hline 5 & $2 / 3$ & 44,53 & 43,90 & 1,41 & 44,05 & 1,08 \\
\hline 5 & 1 & 35,54 & 36,91 & $-3,85$ & 37,91 & $-6,67$ \\
\hline 50 & $1 / 3$ & 72,54 & 75,09 & $-3,52$ & 72,92 & $-0,52$ \\
\hline 50 & $2 / 3$ & 57,17 & 56,28 & 1,55 & 56,42 & 1,31 \\
\hline 50 & 1 & 48,18 & 47,31 & 1,80 & 48,56 & $-0,79$ \\
\hline
\end{tabular}

Примечание. Исходные клинические данные взяты из работы Kehwar et al. и представлены в табл. 1.2.

\subsection{3. Синтезированная CM4 модель (LQмодел+модель МPB)}

CM4 модель можно получить в результате синтеза LQ модели и модели МРВ на основе следующего.

Предположение 3.8. B LQ модели

$$
E=D(\alpha+\beta d) \text {, }
$$

уровень толерантности органа или ткани может быть задан дозой его однократного облучения, соответствующего уровню $E$, который может быть описан при помощи модели МРВ

$$
E=A_{1}|\ln (Q)|^{1 / A_{2}} \text {. }
$$

В результате предположения 3.8 CM4 модель может быть представлена следующим образом:

$$
D(Q, d)=\frac{A_{1}|\ln (Q)|^{1 / A_{2}}}{\alpha+\beta d},
$$

где $(D, d)$ - план тотального облучения ткани, который приводит к ВОЛО = $Q$; $(\alpha, \beta)$ - радиобиологические параметры облученной ткани. Покажем, что модель СМ4 можно использовать для описания ВЛИ РМЖ.

\subsection{1. Расчет ВЛИ РМЖ при комплексном методе лечения РМЖ при} помощи модели СМ4

Относительный объем РМЖ был принят нами равным единице, так как в клинической информации, которой мы располагаем, он не указывается [10, 28-30]. Для настройки CM4 модели мы воспользовались клиническими данными, приве- 
денными в работе Qi et al. [43]. Информация, извлеченная из этой работы для ранних стадий РМЖ, приводится в табл. 3.13. В ней $n$ - число сеансов облучения; $\left(D_{\text {Кл}}, D_{\text {ТЕОР }}\right)$ и $\left(Q_{\text {кл}}, Q_{\text {ТЕоР }}\right)$ - клинические и теоретические значения дозы и вероятности отсутствия рецидивов опухолевых заболеваний. Длительность ЛТ - 35 сут; $g \%$ и $f \%$ - отклонения теоретических значений параметров $Q$ и $D$ от клинических в \%. Число сеансов облучения варьировалось от 5 до 25, суммарная доза от 24,65 до 50 Гр. Первый план ЛТ в табл. 3.13 является стандартным. В остальных шести планах облучения использовались ГипоФ методы ЛТ со значениями РОД > 2 Гр.

Параметры СМ4 модели были получены нами в результате решения специальной экстремальной задачи, в которой минимизировалась сумма квадратов отклонений клинических значений СОД от соответствующих теоретических (модельных) значений. Оптимальные значения параметров модели СM4 (3.80), приводятся в табл. 3.13.

Таблица 3.13

Параметры СМ4 модели для ранних стадий РМЖ, полученные в результате решения экстремальной задачи на основе семи основных планов ЛТ, взятых из работы [10].

\begin{tabular}{|c|c|c|c|c|c|c|c|c|c|c|c|c|}
\hline № & $Q_{\text {КЛ }}$ & $Q_{\text {TEOP }}$ & $g \%$ & $d$ & $N$ & $D_{\text {Кл }}$ & $D_{\mathrm{TEOP}}$ & $f \%$ & $\alpha$ & $\beta$ & $T_{1}$ & $T_{2}$ \\
\hline 1 & 0,032 & 0,0313 & $-2,15$ & 2 & 25 & 50,0 & 49,49 & $-1,01$ & \multirow{7}{*}{0,871} & \multirow{7}{*}{0,933} & \multirow{7}{*}{18,450} & \multirow{7}{*}{0,620} \\
\hline 2 & 0,028 & 0,0286 & 2,00 & 2,66 & 16 & 42,58 & 42,96 & 0,90 & & & & \\
\hline 3 & 0,035 & 0,0360 & 2,70 & 2,5 & 16 & 40,0 & 40,53 & 1,31 & & & & \\
\hline 4 & 0,032 & 0,0324 & 1,25 & 2,26 & 20 & 45,2 & 45,47 & 0,59 & & & & \\
\hline 5 & 0,032 & 0,0312 & $-2,63$ & 3,34 & 10 & 34,4 & 33,98 & $-1,23$ & & & & \\
\hline 6 & 0,032 & 0,0323 & 0,97 & 4,93 & 5 & 24,65 & 24,76 & 0,46 & & & & \\
\hline 7 & 0,032 & 0,0314 & $-1,99$ & 3,39 & 10 & 33,90 & 33,58 & $-0,94$ & & & & \\
\hline
\end{tabular}

Анализ табл. 3.13 показывает, что наблюдается удовлетворительное согласие между $D_{\text {Кл и }} D_{\text {ТЕор}}$, а также между $Q_{\text {Кл }}$ и $Q_{\text {ТЕор}}$, хотя в экстремальной задаче искалось только наилучшее согласие между клиническими и теоретическими зна-

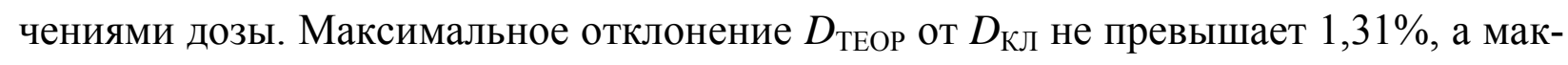

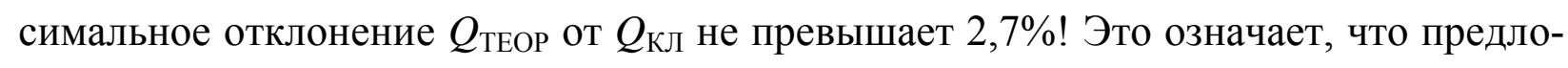
женная нами CM4 модель, может быть успешно использована для планирования стандартных и ГипоФ методов ЛТ ранних стадий РМЖ [10]. Более полно о возможности использования СМ4 модели в ЛТ покажут дальнейшие исследования.

Оптимальные значения параметров СМ4 модели $\left(\alpha, \beta, T_{1}, T_{2}\right)$, нуждаются в уточнении. (Нетрудно видеть, что величины $D, d$ и $Q$ не изменятся, если параметры модели $\left(T_{1}, \alpha, \beta\right)$ умножить на одно и то же число.) Значение параметра $\gamma=\alpha / \beta=0,871 / 0,933=0,934$. Интересно, что в результате использования СМ3 модели (табл. 3.9), мы получили значение параметра $\gamma=0,94$. 
Длительности курсов ЛТ для шести рассмотренных планов облучения изменяются от 1 до 5 недель. При расчете параметров СМ4 модели длительности курсов ЛТ не учитывалась, и это не сказалось на близости клинических и теоретических (модельных) значений СОД. Этот факт может указывать на то, что для рассмотренных условий облучения (табл. 3.12 и 3.13) восстановлением опухолевых клеток и их пролиферацией (для рассматриваемых планов ЛТ, стандартного и ГипоФ планов облучения) в первом приближении можно пренебречь.

Таким образом, СМ4 модель может быть успешно использована для планирования ЛТ ранних стадий РМЖ. Варьируя условия облучения РМЖ (РОД и СОД) и получая при помощи СМ4 модели оценку плана облучения в виде ВИ РМЖ $=P=1-Q$, лучевой терапевт может выбрать рациональный план ЛТ. Задав ВИ РМЖ, он может найти зависимость СОД от РОД (или РОД от СОД), применение которых приводит к заданному значению ВЛИ РМЖ.

Разумеется, для эффективного планирования лечения РМЖ необходимо уметь вычислять и другие критериальные оценки ЛТ: ВЛО в сердце, ВЛО в легких, ВЛО на коже, ВЛО в подкожной клетчатке, и т.д. (см. работу [76]). Необходимо из множества альтернативных планов ЛТ выбрать допустимые планы облучения, и на этом множестве при помощи лучевых терапевтов, радиобиологов, медицинских физиков, математиков и других экспертов выделить эффективный (компромиссный) план облучения РМЖ.

Представляет интерес точность, с которой рассчитывает клинические данные модель СМ3 и модель СМ4. Для СМ3 модели максимальное отклонение клинических значений ВЛО от теоретических (модельных) составило $0,09 \%$, а для суммарной дозы $-1,24 \%$. В результате использования СМ4 модели мы получили, (табл. 3.13): максимальное отклонение клинических значений ВЛО от теоретических (модельных) составило 2,7\%, а для суммарной дозы - 1,31\%. Заметим, что CM3 и CM4 модели приводят практически к одному и тому же значению параметра $\gamma$, они лежат в интервале $\gamma \in(0,93 ; 0,94)$ Гр.

Таким образом, отклонения ВЛО и СОД для СМ3 и СМ4 модели мало отличаются друг от друга, обе модели могут быть успешно использованы для планирования тотального облучения ранних стадий РМЖ после иссечения опухолевого образования.

Графики зависимости ВИ РМЖ от СОД, построенные с помощью СМЗ и CM4 модели приводятся на рис. 3.3. Они практически совпадают. 


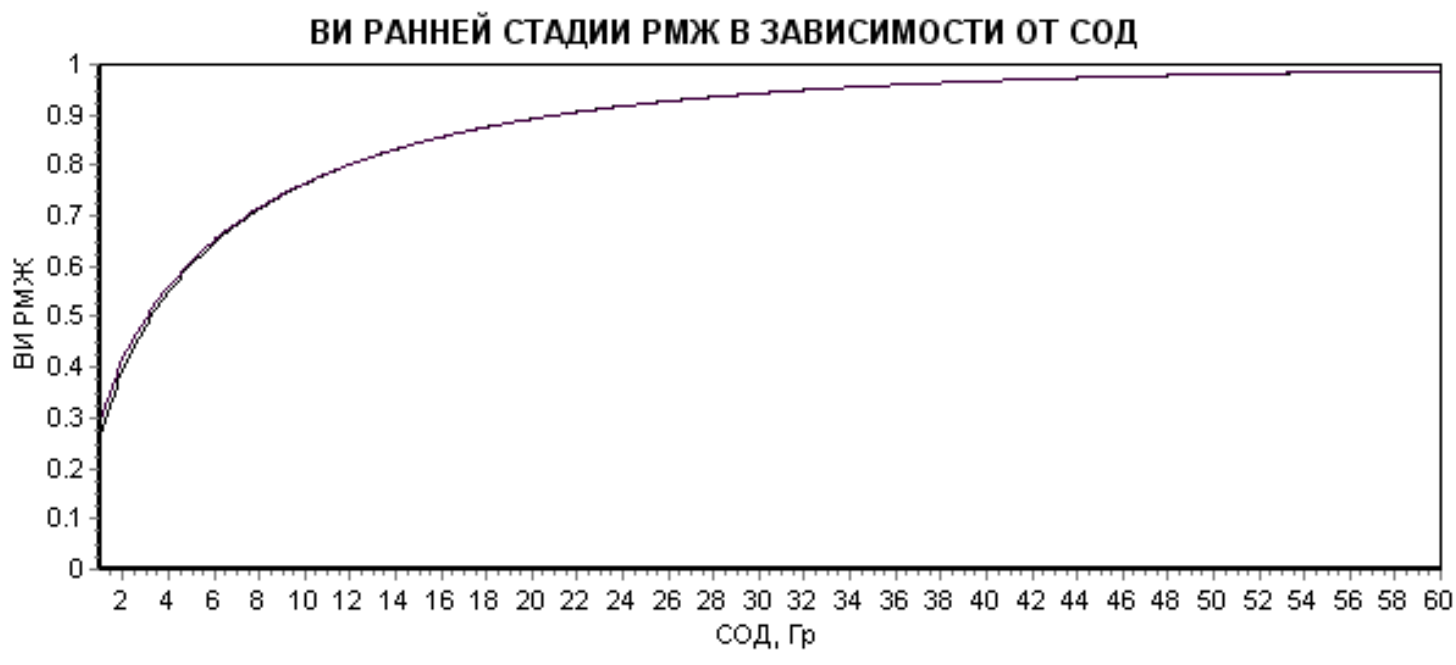

Рис. 3.3. Графики зависимости ВЛИ РМЖ от СОД, построенные при помощи СМЗ и СМ4 моделей

Примечание. Графики практически совпадают.

На рис. 3.4 приводятся графики зависимости ВЛИ РМЖ от СОД для РОД 1,$8 ; 2,6$ и 3,6 Гр, рассчитанные при помощи СМ3 модели.

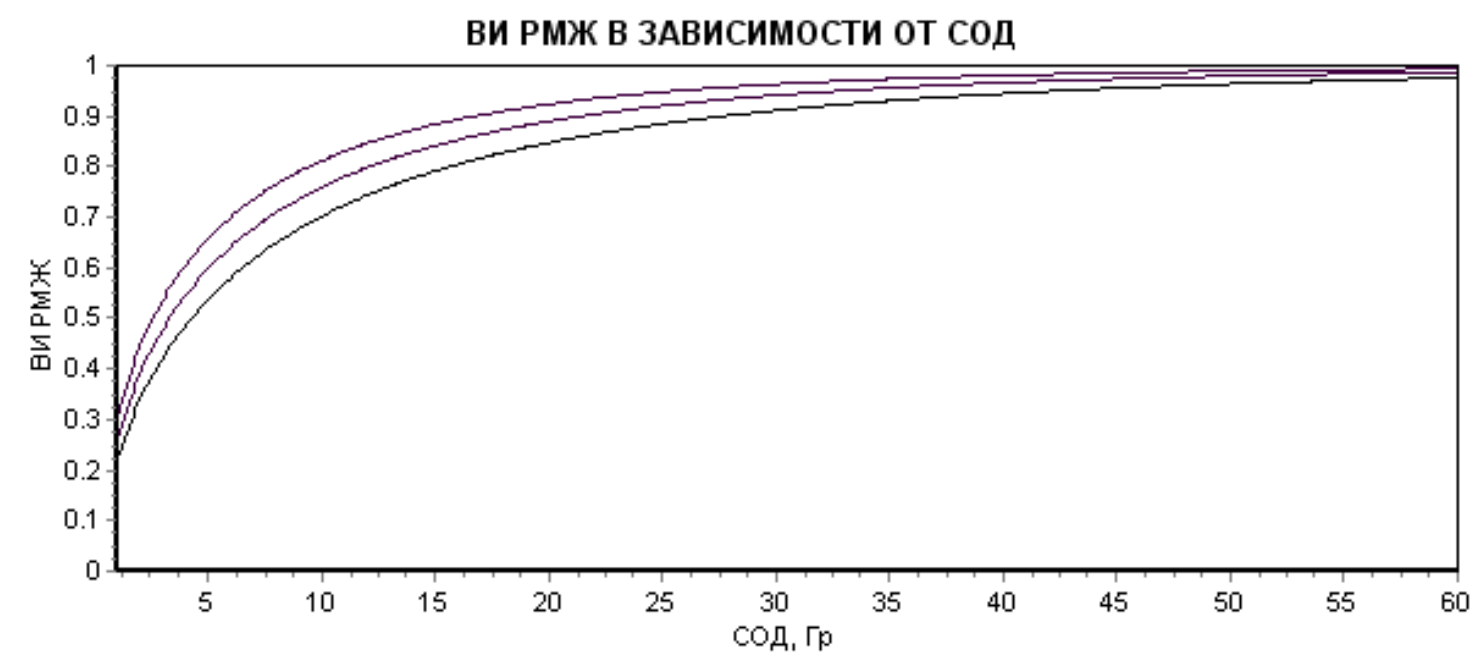

Рис. 3.4. Графки зависимости ВЛИ РМЖ от СОД для РОД

Примечание. Нижний график - РОД = 1,8 Гр, средний график - РОД = 2,6 Гр, верхний график - РОД = 3,6 Гр. Графики построенны при помощи СМ3 модели.

\subsection{4. Синтезированная модель CM5 (MLQмодель + МРВ модель)}

Создание модели СM5, также, как и модели СM4, основано на использовании LQ модели и модели MPB, а также на том, что эта модель может быть использована для любого числа сеансов облучения, включая и однократное облучение. 
Таким образом, для тотального облучения ткани мы можем представить LQ модель следующим образом:

$$
E=D(\alpha+\beta d)=D_{R}\left(\alpha+\beta D_{R}\right),
$$

где $D, d$ - суммарная и разовая доза фракционированного облучения ткани; $D=n d-$ ТД фракционированного облучения; $n$ - число сеансов облучения; $D_{R}(Q)-$ однократная толерантная доза, задающая уровень толерантности ткани ВОЛО $=Q$; $(\alpha, \beta)$ - радиобиологические параметры облучаемой ткани. Из (3.81) можно получить СМ5 модель:

$$
D=D_{R}(Q) \frac{\gamma+D_{R}(Q)}{\gamma+d},
$$

где $\gamma=\alpha / \beta$ - радиобиологический параметр ткани. Нетрудно видеть, что эта математическая модель хоть и похожа на модель СM4, но все же отлична от нее. Для проверки модели СМ5 и сравнения ее с СМ3 и СМ4 моделями, можно рассмотреть точность, с которой она описывает клиническую информацию, представленную в табл. 3.13. Эту работу мы предоставляем читателю.

Разумеется, для эффективного планирования РМЖ необходимо уметь вычислять и другие критериальные оценки ЛТ: ВЛО в сердце, ВЛО в легких, ВЛО на коже, ВЛО в подкожной клетчатке, и т.д. (см. работу [76]). Необходимо рассмотреть множество альтернативных планов ЛТ, выбрать допустимые планы облучения, и выделить Парето оптимальные планы облучения (несравнимые формально планы облучения) [39, 40]. На этом множестве при помощи специалистов-медиков, медицинских физиков, и др. экспертов следует интерактивно выделить компромиссный и эффективный план облучения. 


\section{Глава 4. \\ РАСЧЕТ ОПТИМАЛЬНЫХ ЗНАЧЕНИЙ ПАРАМЕТРОВ НАСТРОЙКИ МОДЕЛИ МРВ ДЛЯ ОРГАНОВ И ТКАНЕЙ НА ОСНОВЕ СИСТЕМАТИЗИРОВАННЫХ КЛИНИЧЕСКИХ ДАННЫХ ЕМАМІ et al.}

В главах 4 и 5 приводятся результаты расчета оптимальных значений параметров модели МРВ, которая связывает значения ВЛО с объемом облученного органа или ткани, и с суммарной дозой их облучения. Разовая доза считалась постоянной величиной. По умолчанию, мы считали ее равной 2 Гр, так как она наиболее часто используется в ЛТ.

Систематизированные клинические данные, на основе которых были расчитаны оптимальные значения параметров модели МРВ, приводятся в табл. 1.1, (см. главу 1), и были подготовлены Emami et al. [31]. Органы и ткани, для которых определяются оптимальные значения параметров модели МРВ, связаны с номером рассматриваемой главы и с их нумерацией в табл. 1.1.

Расчет оптимальных значений параметров модели МРВ осуществлялся с тремя различными критериями оптимальности. Первый критерий - ВЛО. Он приводит к наиболее близким значениям клинических и теоретических (модельных) значений ВЛО. Второй критерий толерантные дозы - ТД, приводит к наиболее близким значениям клинических и теоретических (модельных) значений ТД. Поиск оптимальных значений параметров модели МРВ по третьему критерию заключался в определении наиболее близких теоретических (модельных) значений ВЛО + ТД к их клиническим значениям. Полученные результаты расчетов позволяют придти к следующему заключению.

1. Анализ результатов моделирования клинической информации при помощи модели МРВ показал, что наиболее близкие рассчитанные (модельные) значения ТД к заданным клиническим значениям ТД получаются в том случае, когда используется критерий ТД. Это понятно, так как в настоящее время планирование ЛТ осуществляется в основном по значениям ТД.

2. Следует отметить, что оценка планов ЛТ по значениям ВЛИ в опухоли, и по значениям ВЛО в ложе опухоли, и в других здоровых органах и тканях организма, если они рассчитаны с достаточной точностью, очень важны. Они позволяют врачу радиологу сопоставить успех ЛТ опухолевого заболевания с риском вызвать необратимые лучевые повреждения в здоровых органах и тканях организма. 
3. Необходимо отметить, что клинические значения ТД, которые приводятся в табл. 1.1, и, на основе которых определяются оптимальные значения параметров модели $\mathrm{MPB}$, являются приближенными. Это связано с тем, что клинические значения ТД, которые должны соответствовать однородным распределениям доз в органах и тканях, часто являются неоднородными. Поэтому в табл. 1.2 (данные Кеhwar et al.), приводятся значения ТД как однородные дозы в органах и тканях с 95\% доверительными интервалами.

4. На графиках, которые описывают зависимости ВЛО от дозы, рассчитанные при помощи модели МРВ, относительные объемы облученных органов и тканей изменяются от 0,1 до 1,0 с шагом 0,1 .

5. Мы полагаем, что для расчета ВЛО однородного дозового распределения в органе или в ткани, необходимо осуществлять редукцию неоднородного дозового распределения, которое может быть представлено в виде ДГДО (или ИГДО). В работе $[26,27]$ мы описали вывод традиционной математической модели, известной давно [9], и разработали новую математическую модель, которая может быть использвана для решения этой проблемы (главы 3, 7 и 8).

6. Нам впервые удалось показать, что если математическая модель редукции неоднородного дозового распределения выделена при помощи модели МРВ, тогда единственный параметр $A_{2}$, входящий в число параметров модели МРВ, следует использовать в математической модели, предназначенной для редукции неоднородных дозовых распределений, для расчета эквивалентной по ВЛО редуцированной адекватной дозы (АД) однородного облучения ткани.

7. Используя СМ3 модель (глава 3) для однородных дозовых распределений в Системе «опухоль + ложе опухоли» мы разработали метод расчета оптимальных условий облучения Системы и установили, в каких случаях следует использовать ГиперФ, а в каких ГипоФ схему облучения рака молочной железы (РМЖ) [10, 27, $28,30]$. Разработанная методика была использована для планирования комплексного лечения ранней стадии рака молочной железы.

В четвертой главе мы приводим рассчитанные оптимальные значения параметров модели МРВ для тридцати различных органов и тканей организма, которые были подготовлены Emami et al. и приводятся в табл. 1.1. В каждой таблице приводятся клинические и теоретические (модельные) значения ВЛО и ТД, рассчитанные при помощи модели МРВ, а также нормированные отклонения исходных клинических данных ВЛО и ТД от их теоретических (модельных) значений в процентах.

В каждой таблице, предназначенной для размещения оптимальных значений параметров модели МРВ, в столбце 1 приводятся клинические значения ВЛО, 
в столбце 2 - теоретические значения ВЛО, в столбце 3 - отклонения клинических значений ВЛО от теоретических значений ВЛО в \%. Аналогично приводятся и клинические значения ТД. Рассчитываются оптимальные теоретические значения ТД и отклонения клинических значений ТД от теоретических в \% (столбцы 5-7). В столбце 4 приводятся относительные объемы облученных тканей. В столбцах 8-10 приводятся рассчитанные оптимальные значения параметров модели МРВ $\left(b, A_{1}, A_{2}\right)$.

Структуры таблиц, которые приводятся в главах 4 и 5, отличаются друг от друга. Это было сделано для того, чтобы было удобно различать клинические данные Emami et al. от клинических данных Kehwar et al.

Необходимо отметить, что в табл. 1.1 и 1.2 приводятся органы и ткани, для которых полная клиническая информация содержит шесть планов облучения. Но приводится также органы и ткани с урезанной клинической информацией, которая содержит от двух планов облучения до пяти планов облучения. Параметры модели МРВ были рассчитаны для всех указанных случаев.

В том случае, например, когда клиническая информация содержит всего два плана облучения, необходимо рассмотреть всего два уравнения:

$$
D_{1}=A_{1} \mid\left.\ln \left(\text { ВОЛО }_{1}\right)\right|^{A_{2}} V_{1}^{-b} \text { и } D_{2}=A_{1} \mid\left.\ln (\text { ВОЛО } 2)\right|^{A_{2}} V_{1}^{-b} \text {. }
$$

В этом случае, для того, чтобы настроить вырожденную модель МРВ необходимо, чтобы клиническая информация различалась двумя клиническими параметрами. Например, для клинической информации, которая состоит всего из двух планов облучения $\left(\right.$ ВОЛО $\left.{ }_{1}, D_{1}, V\right)$ и $\left(\mathrm{BOЛО}_{2}, D_{2}, V\right)$, система уравнений, которую следует рассмотреть, будет:

$$
D_{1}=A_{1} \mid\left.\ln (\text { ВОЛО } 1)\right|^{A_{2}}, D_{2}=A_{1} \mid\left.\ln \left(\text { ВОЛО }_{2}\right)\right|^{A_{2}}, V_{1}=V_{2}=V .
$$

Из (4.2) получаем математическую модель:

$$
\frac{D_{1}}{D_{2}}=\left|\ln \left(\frac{\mathrm{BОЛО}_{1}}{\text { ВОЛО }_{2}}\right)\right|^{A_{2}} \text {. }
$$

В том случае, когда ВОЛО ${ }_{1}=$ ВОЛО $_{2}=$ ВОЛО, $D_{1} \neq D_{2}$ и $V_{1} \neq V_{2}$, получаем:

$$
\frac{D_{1}}{D_{2}}=\left(\frac{V_{1}}{V_{2}}\right)^{-b}
$$

и т.д. Подобным образом можно строить другие зависимости между параметрами математической модели МРВ.

Ниже приводятся результаты определения параметров модели МРВ на основе систематизированных клинических данных, приведенных Emami et al. [31]. Для каждого набора птимальных значений параметров модели МРВ приводятся 
графики зависимости значений ВЛО от дозы для относительных объемов (или площадей) облученных органов и тканей, которые изменяются от 0,1 до $1,0 \%$ с шагом $0,1 \%$. Крайнему слева графику соответствует относительный объем облучения $0,1 \%$, крайнему справа - объем $1.0 \%$. Прочерки в таблицах указывают на отсутствие соответствующей клинической информации.

\section{1. Мочевой пузырь.}

\section{Осложнение: симптомы контрактуры и уменьщение объема}

Таблица 4.1.1

Мочевой пузырь. Оптимизация по ВЛО

\begin{tabular}{|c|c|c|c|c|c|c|c|c|c|}
\hline$P_{\text {КЛ }}$ & $P_{\text {TEOP }}$ & $\varepsilon, \%$ & $V$ & $D_{\text {КЛ }}$ & $D_{\text {TEOP }}$ & $\varepsilon, \%$ & $A_{1}$ & $A_{2}$ & $b$ \\
\hline- & - & - & - & - & - & - & \multirow{6}{*}{104,35} & \multirow{6}{*}{6,50} & \multirow{6}{*}{0,430} \\
\hline 0,05 & 0,056 & 11,11 & $2 / 3$ & 80 & 78,68 & $-1,65$ & & & \\
\hline 0,05 & 0,045 & $-10,03$ & 1 & 65 & 66,09 & 1,68 & & & \\
\hline- & - & - & - & - & - & - & & & \\
\hline 0,50 & 0,933 & 86,63 & $2 / 3$ & 85 & 68,94 & $-18,89$ & & & \\
\hline 0,50 & 0,163 & 67,45 & 1 & 80 & 98,63 & 23,29 & & & \\
\hline
\end{tabular}

ВЛО В ОРГАНЕ ИЛИ В ТКАНИ В ЗАВИСИМОСТИ ОТ ОБЪЕМА ОБЛУЧЕНИЯ и СУММАРНОЙ ДОЗЫ

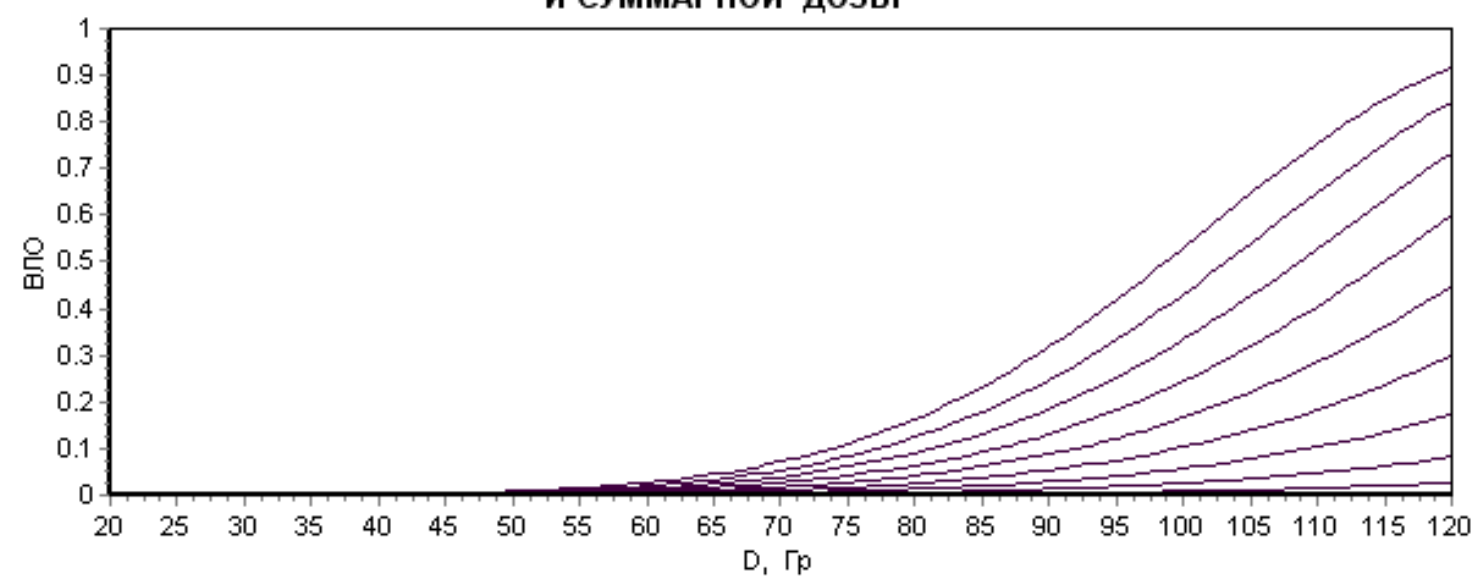

Рис. 4.1.1. Мочевой пузырь. Оптимизация по ВЛО

Таблица 4.1.2

Мочевой пузырь. Оптимизация по ТД

\begin{tabular}{|c|c|c|c|c|c|c|c|c|c|}
\hline$P_{\text {Кл }}$ & $P_{\mathrm{TEOP}}$ & $\varepsilon, \%$ & $V$ & $D_{\text {КЛ }}$ & $D_{\text {TEOP }}$ & $\varepsilon, \%$ & $A_{1}$ & $A_{2}$ & $b$ \\
\hline- & - & - & - & - & - & - & \multirow{6}{*}{85,744} & \multirow{6}{*}{16,385} & \multirow{6}{*}{0,040} \\
\hline 0,05 & 0.218 & 336,05 & $2 / 3$ & 80 & 72,70 & $-9,13$ & & & \\
\hline 0,05 & 0,010 & $-78,73$ & 1 & 65 & 71,53 & 10,04 & & & \\
\hline- & - & $\overline{-}$ & - & - & $\overline{-}$ & - & & & \\
\hline 0,50 & 0,776 & 55,21 & $2 / 3$ & 85 & 81,10 & 0,78 & & & \\
\hline 0,50 & 0,275 & $-45,08$ & 1 & 80 & 83,85 & 4,81 & & & \\
\hline
\end{tabular}


ВЛО В ОРГАНЕ ИЛИ В ТКАНИ В ЗАВИСИМОСТИ ОТ ОБЪЕМА ОБЛУЧЕНИЯ И СУММАРНОЙ ДОЗЫ

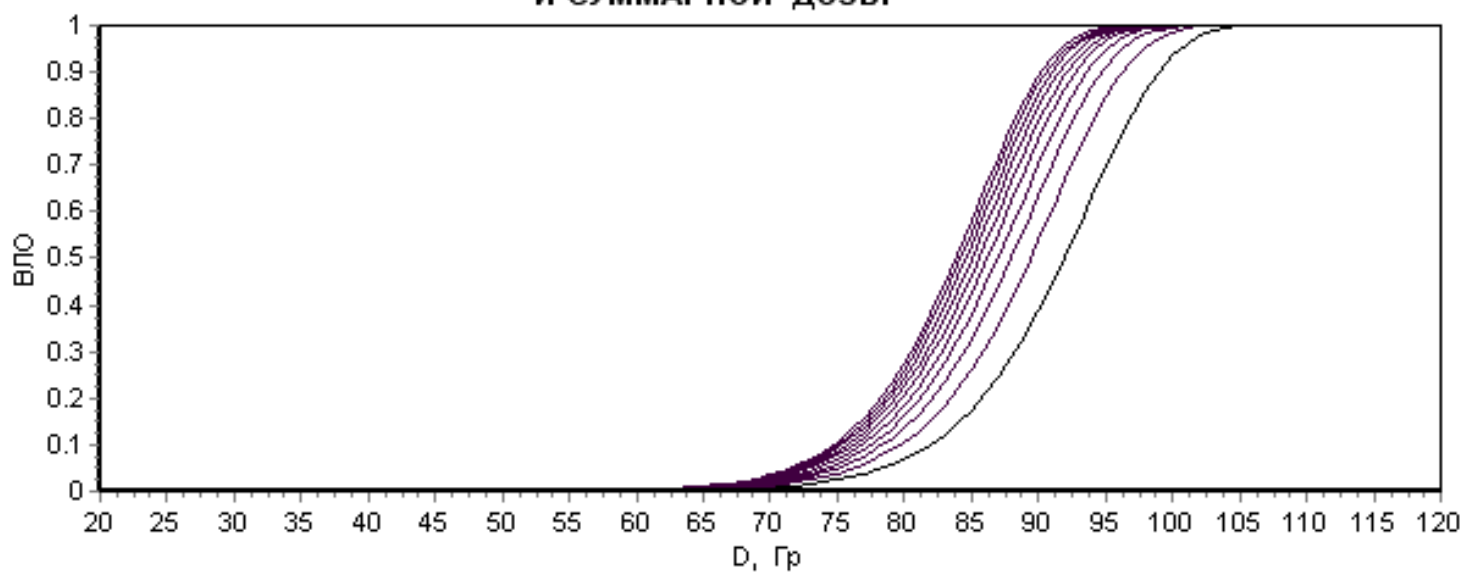

Рис. 4.1.2. Мочевой пузырь. Оптимизация по ТД

Таблица 4.3.3

Мочевой пузырь. Оптимизация по ВЛО + ТД

\begin{tabular}{|c|c|c|c|c|c|c|c|c|c|}
\hline$P_{\text {Кл }}$ & $P_{\mathrm{TEOP}}$ & $\varepsilon, \%$ & $V$ & $D_{\text {Кл }}$ & $D_{\mathrm{TEOP}}$ & $\varepsilon, \%$ & $A_{1}$ & $A_{2}$ & $b$ \\
\hline- & - & - & - & - & - & - & \multirow{6}{*}{102,63} & \multirow{6}{*}{6,853} & \multirow{6}{*}{0,397} \\
\hline 0,05 & 0,058 & 16,82 & $2 / 3$ & 80 & 78,15 & $-2,31$ & & & \\
\hline 0,05 & 0,043 & $-14,46$ & 1 & 65 & 66,53 & 2,36 & & & \\
\hline- & - & - & - & - & - & - & & & \\
\hline 0,50 & 0,929 & 85,86 & $2 / 3$ & 85 & 69,90 & $-17,77$ & & & \\
\hline 0,50 & 0,166 & $-66,83$ & 1 & 80 & 97,29 & 21,61 & & & \\
\hline
\end{tabular}

ВЛО В ОРГАНЕ ИЛИ В ТКАНИ В ЗАВИСИМОСТИ ОТ ОБЪЕМА ОБЛУЧЕНИЯ И СУММАРНОЙ ДОЗЫ

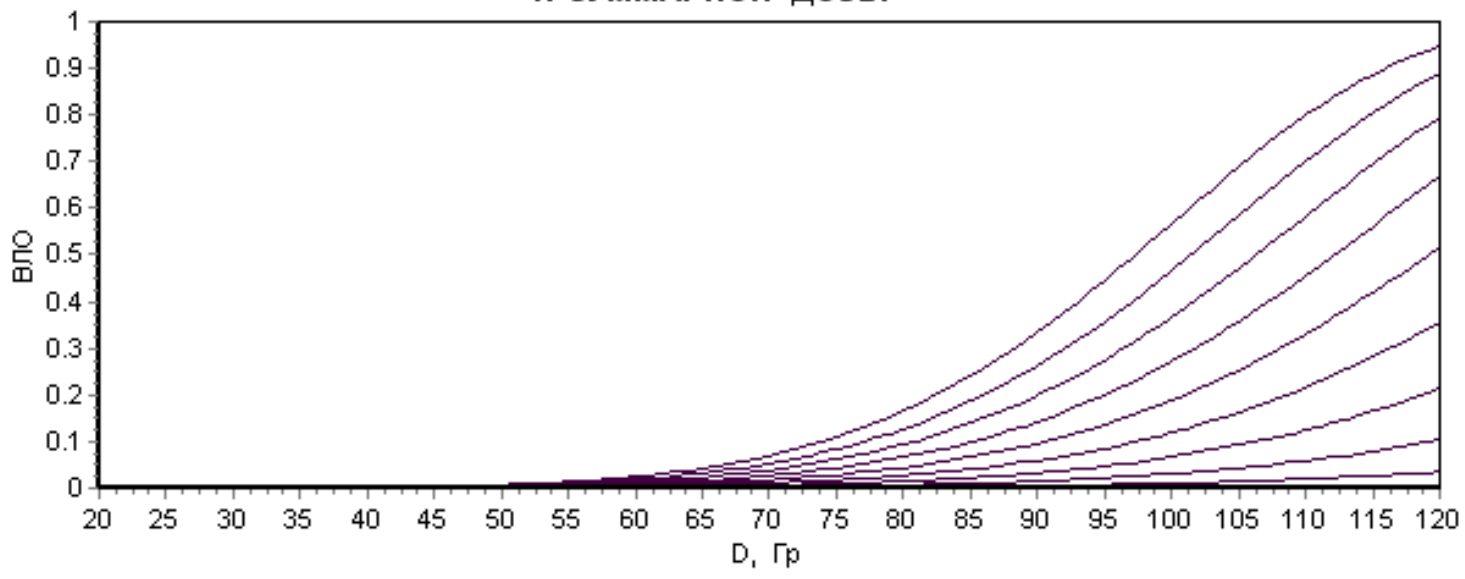

Рис. 4.1.3. Мочевой пузырь. Оптимизация по ВЛО + ТД 


\section{2. Плечевое сплетение. Клинические повреждения нервов}

Таблица 4.2.1

Плечевое сплетение. Оптимизация по ВЛО

\begin{tabular}{c|c|c|c|c|c}
\hline$P_{\text {КЛ }}$ & $D_{\text {Кл }}$ & $P_{\text {КЛ }}$ & $D_{\text {КЛ }}$ & $A_{1}$ & $A_{2}$ \\
\hline 0,05 & 60,0 & 0,50 & 75,0 & 77,393 & 11,668 \\
\hline
\end{tabular}

ВЛО В ОРГАНЕ ИЛИ В ТКАНИ В ЗАВИСИМОСТИ ОТ СУММАРНОЙ ДОЗЫ

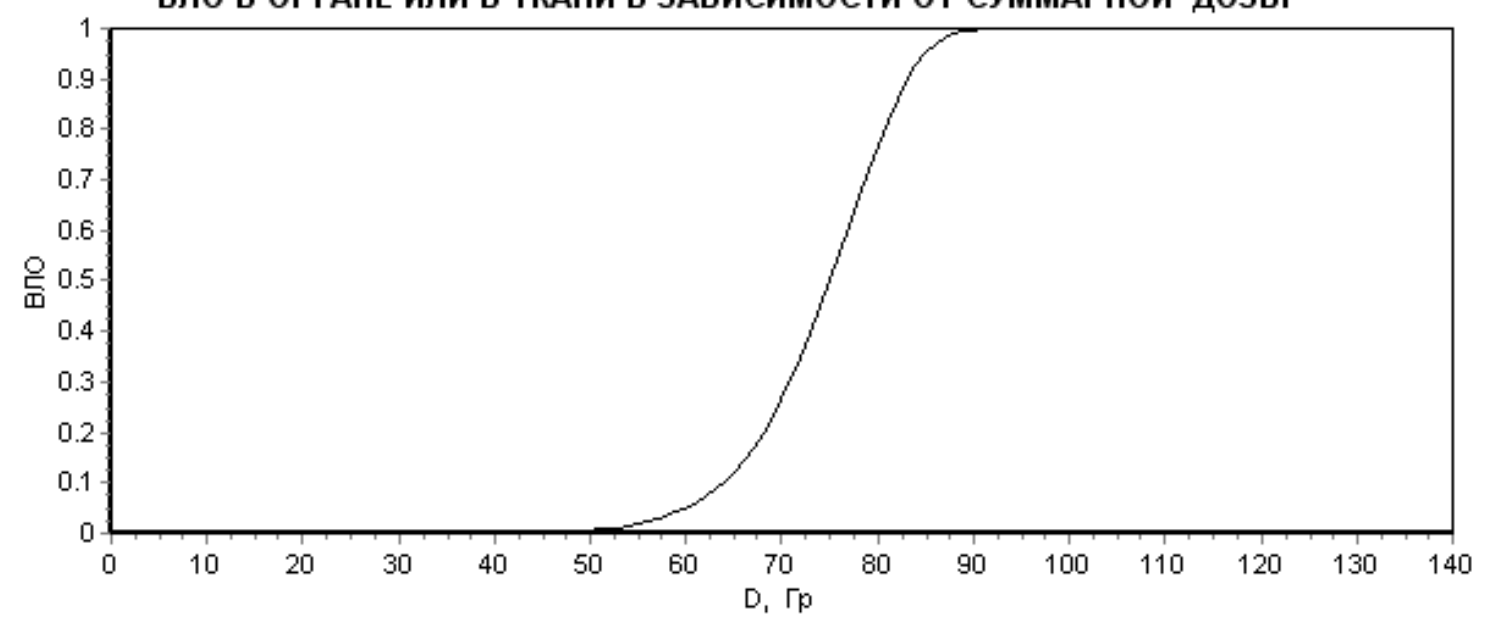

Рис. 4.2. Плечевое сплетение. Зависимость ВЛО от дозы

\section{3. Мозг. Некроз или инфаркт}

Таблица 4.3.1

Мозг. Оптимизация по ВЛО

\begin{tabular}{|c|c|c|c|c|c|c|c|c|c|}
\hline$P_{\text {Кл }}$ & $P_{\mathrm{TEOP}}$ & $\varepsilon, \%$ & $V$ & $D_{\text {Кл }}$ & $D_{\text {TEOP }}$ & $\varepsilon, \%$ & $A_{1}$ & $A_{2}$ & $b$ \\
\hline 0,05 & 0,056 & 12,01 & $1 / 3$ & 60 & 59,31 & $-1,15$ & \multirow{6}{*}{60,938} & \multirow{6}{*}{10,102} & \multirow{6}{*}{0,243} \\
\hline 0,05 & 0,049 & $-2,29$ & $2 / 3$ & 50 & 50,12 & $-2,29$ & & & \\
\hline 0,05 & 0,046 & $-8,65$ & 1 & 45 & 45,42 & 0,92 & & & \\
\hline 0,50 & 0,42 & $-15,49$ & $1 / 3$ & 75 & 76,75 & 0,42 & & & \\
\hline 0,50 & 0,51 & 1,60 & $2 / 3$ & 65 & 64,85 & 0,23 & & & \\
\hline 0,50 & 0,57 & 14,94 & 1 & 60 & 58,77 & $-2,06$ & & & \\
\hline
\end{tabular}




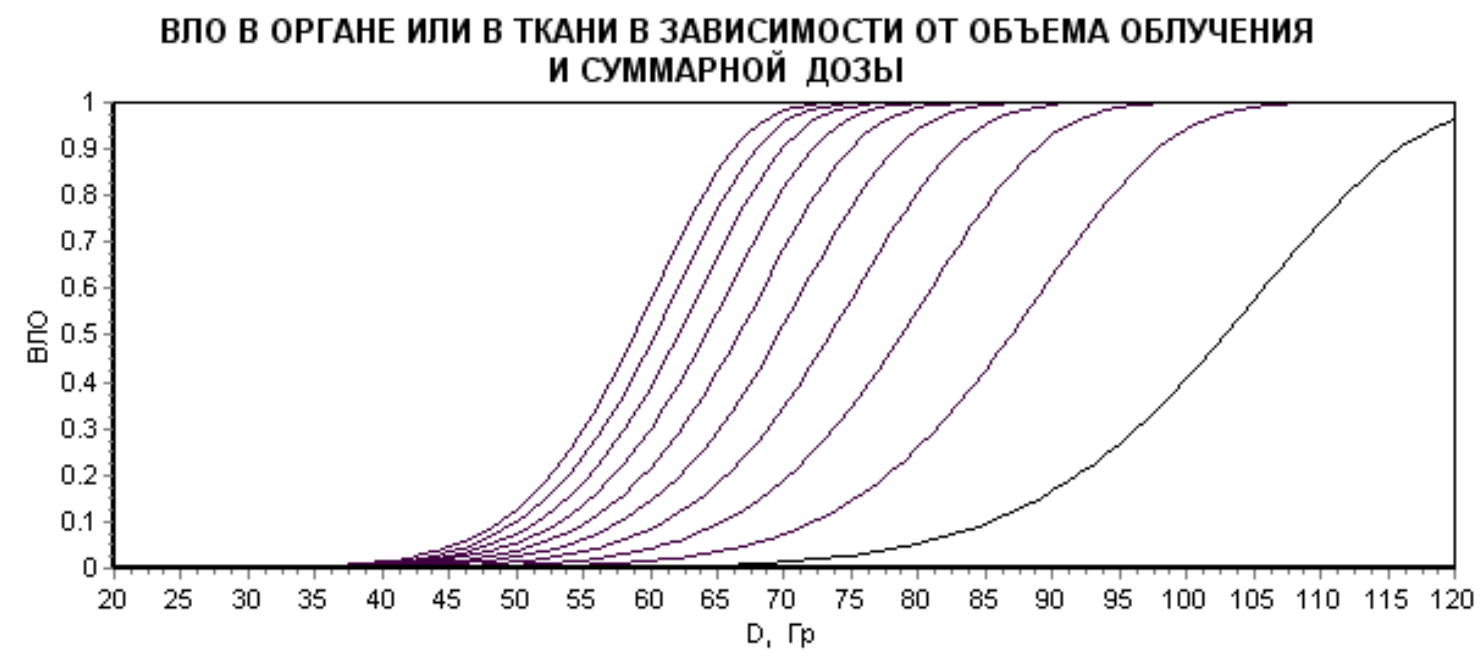

Рис. 4.3.1. Мозг. Оптимизация по ВЛО

Таблица 4.3.2

Мозг. Оптимизация по ТД

\begin{tabular}{|c|c|c|c|c|c|c|c|c|c|}
\hline$P_{\text {Кл }}$ & $P_{\mathrm{TEOP}}$ & $\varepsilon, \%$ & $V$ & $D_{\text {Кл }}$ & $D_{\mathrm{TEOP}}$ & $\varepsilon, \%$ & $A_{1}$ & $A_{2}$ & $b$ \\
\hline 0,05 & 0,059 & 18,76 & $1 / 3$ & 60 & 58,96 & $-1,74$ & \multirow{6}{*}{61,244} & \multirow{6}{*}{10,102} & \multirow{6}{*}{0,233} \\
\hline 0,05 & 0,048 & $-3,21$ & $2 / 3$ & 50 & 50,17 & 0,33 & & & \\
\hline 0,05 & 0,043 & $-13,07$ & 1 & 45 & 45,64 & 1,43 & & & \\
\hline 0,50 & 0,442 & $-11,61$ & $1 / 3$ & 75 & 76,25 & 1,72 & & & \\
\hline 0,50 & 0,505 & 0,93 & $2 / 3$ & 65 & 64,91 & $-0,13$ & & & \\
\hline 0,50 & 0,556 & 11,27 & 1 & 60 & 59,06 & $-1,56$ & & & \\
\hline
\end{tabular}

ВЛО В ОРГАНЕ ИЛИ В ТКАНИ В ЗАВИСИМОСТИ ОТ ОБЪЕМА ОБЛУЧЕНИЯ И СУММАРНОЙ ДОЗЫ

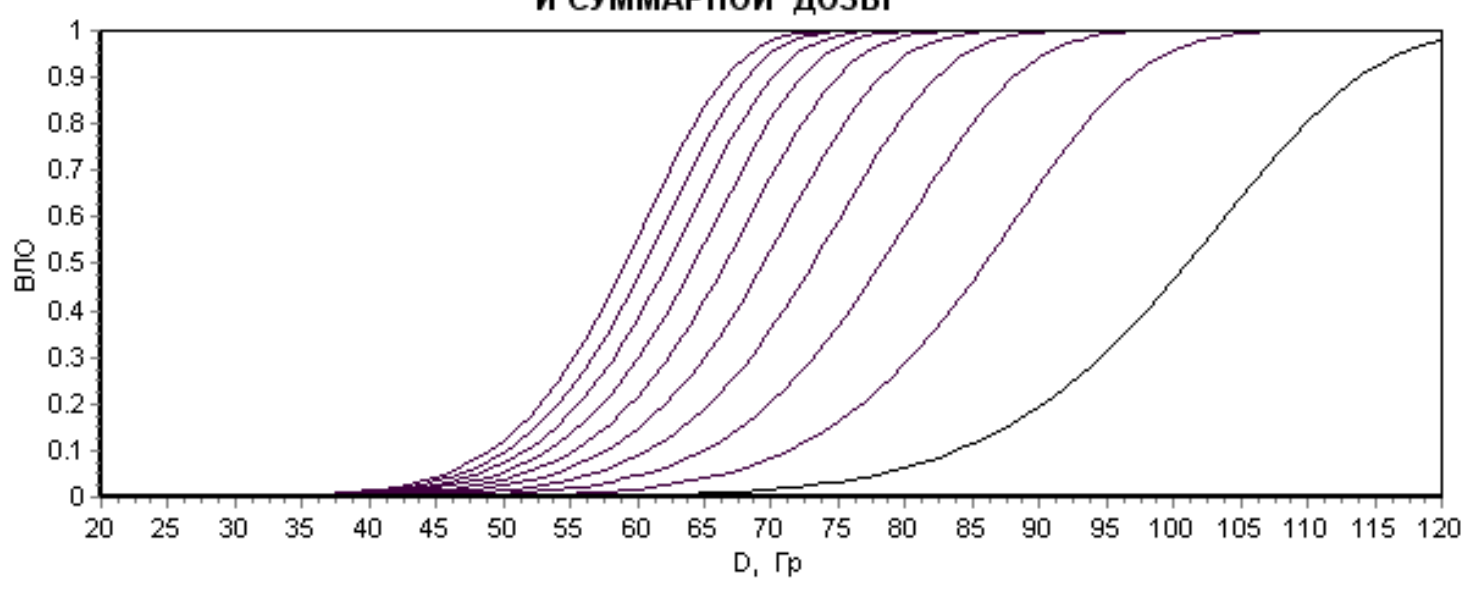

Pис. 4.3.2. Мозг. Оптимизация по ТД 


\section{Мозг. Оптимизация по ВЛО + ТД}

\begin{tabular}{|c|c|c|c|c|c|c|c|c|c|}
\hline$P_{\text {Кл }}$ & $P_{\mathrm{TEOP}}$ & $\varepsilon, \%$ & $V$ & $D_{\text {КЛ }}$ & $D_{\mathrm{TEOP}}$ & $\varepsilon, \%$ & $A_{1}$ & $A_{2}$ & $b$ \\
\hline 0,05 & 0,560 & 12,01 & $1 / 3$ & 60 & 59,31 & $-1,15$ & \multirow{6}{*}{60,938} & \multirow{6}{*}{10,102} & \multirow{6}{*}{0,243} \\
\hline 0,05 & 0,049 & $-2,29$ & $2 / 3$ & 50 & 50,12 & 0,24 & & & \\
\hline 0,05 & 0,046 & $-8,65$ & 1 & 45 & 45,42 & 0,92 & & & \\
\hline 0,50 & 0,423 & $-15,49$ & $1 / 3$ & 75 & 76,75 & 2,33 & & & \\
\hline 0,50 & 0,508 & 1,60 & $2 / 3$ & 65 & 64,85 & $-0,23$ & & & \\
\hline 0,50 & 0,575 & 14,94 & 1 & 60 & 58,77 & $-2,06$ & & & \\
\hline
\end{tabular}

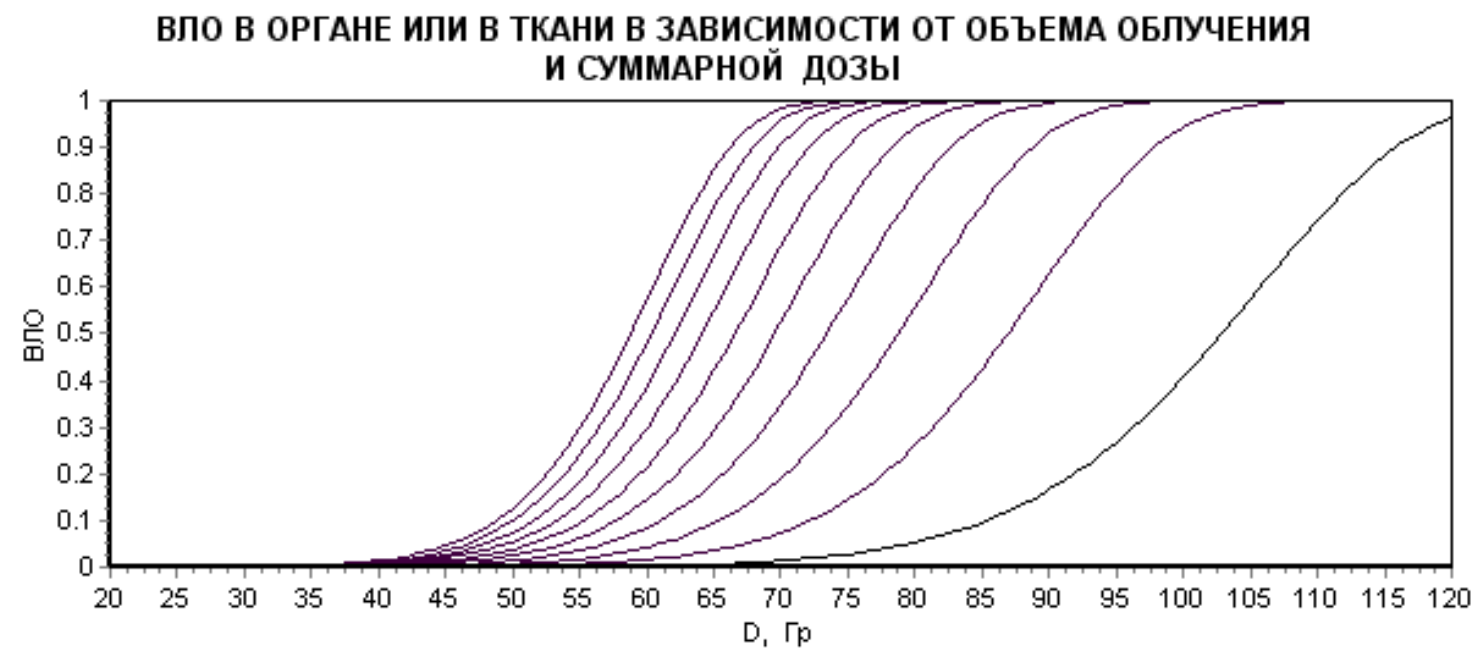

Рис. 4.3.3. Мозг. Оптимизация по ВЛО+ТД

В табл. 4.3.4 приводятся резултаты проверки согласованности систематизированной клинической информации путем раздельного расчета оптимальных значений параметров модели МРВ для критерия ВЛО $=0,05$ и ВЛО $=0,50$. Если они не совпадают, клиническую информацию можно считать плохо согласованной. Правда, как это станет ясно в дальнейшем, плохая согласованность клинической информации не исключает получение разумных оптимальных значений параметров модели МРВ при обработке всей клинической информации.

Таблица 4.3 .4

Мозг. Оптимизация по ТД, отдельно для ВЛО = 0,05 и ВЛО = 0,50

\begin{tabular}{|c|c|c|c|c|c|c|c|c|c|}
\hline$P_{\text {КЛ }}$ & $P_{\mathrm{TEOP}}$ & $\varepsilon, \%$ & $V$ & $D_{\text {Кл }}$ & $D_{\mathrm{TEOP}}$ & $\varepsilon, \%$ & $A_{1}$ & $A_{2}$ & $b$ \\
\hline 0,05 & 0,050 & 0,02 & $1 / 3$ & 60 & 59,98 & $-0,02$ & \multirow{3}{*}{509,883} & \multirow{3}{*}{1,223} & \multirow{3}{*}{0,262} \\
\hline 0,05 & 0,053 & $-0,06$ & $2 / 3$ & 50 & 50,03 & 0,05 & & & \\
\hline 0,05 & 0,050 & 0,04 & 1 & 45 & 44,98 & $-0,03$ & & & \\
\hline 0,50 & 0,50 & 0,01 & $1 / 3$ & 75 & 74,09 & $-0,08$ & \multirow{3}{*}{2164,341} & \multirow{3}{*}{0,102} & \multirow{3}{*}{0,203} \\
\hline 0,50 & 0,50 & $-0,01$ & $2 / 3$ & 65 & 65,10 & 0,16 & & & \\
\hline 0,50 & 0,50 & 0,01 & 1 & 60 & 59,96 & 0,01 & & & \\
\hline
\end{tabular}




\section{4. Ствол мозга. Некроз или инфаркт}

Таблица 4.4.1

Ствол мозга. Оптимизация по ВЛО

\begin{tabular}{|c|c|c|c|c|c|c|c|c|c|}
\hline$P_{\text {КЛ }}$ & $P_{\text {TEOP }}$ & $\varepsilon, \%$ & $V$ & $D_{\text {КЛ }}$ & $D_{\mathrm{TEOP}}$ & $\varepsilon, \%$ & $A_{1}$ & $A_{2}$ & $b$ \\
\hline 0,05 & 0,051 & 2,30 & $1 / 3$ & 60 & 59,86 & $-0,24$ & \multirow{6}{*}{67,479} & \multirow{6}{*}{9,792} & \multirow{6}{*}{0,167} \\
\hline 0,05 & 0,047 & $-9,49$ & $2 / 3$ & 53 & 53,31 & 0,59 & & & \\
\hline 0,05 & 0,052 & 3,42 & 1 & 50 & 49,82 & $-0,35$ & & & \\
\hline- & - & - & - & - & - & - & & & \\
\hline- & - & - & - & - & - & - & & & \\
\hline 0,50 & 0,50 & 0,0 & 1 & 65 & 65,0 & 0,0 & & & \\
\hline
\end{tabular}

ВЛО В ОРГАНЕ ИЛИ В ТКАНИ В ЗАВИСИМОСТИ ОТ ОБЪЕМА ОБЛУЧЕНИЯ И СУММАРНОЙ ДОЗЫ

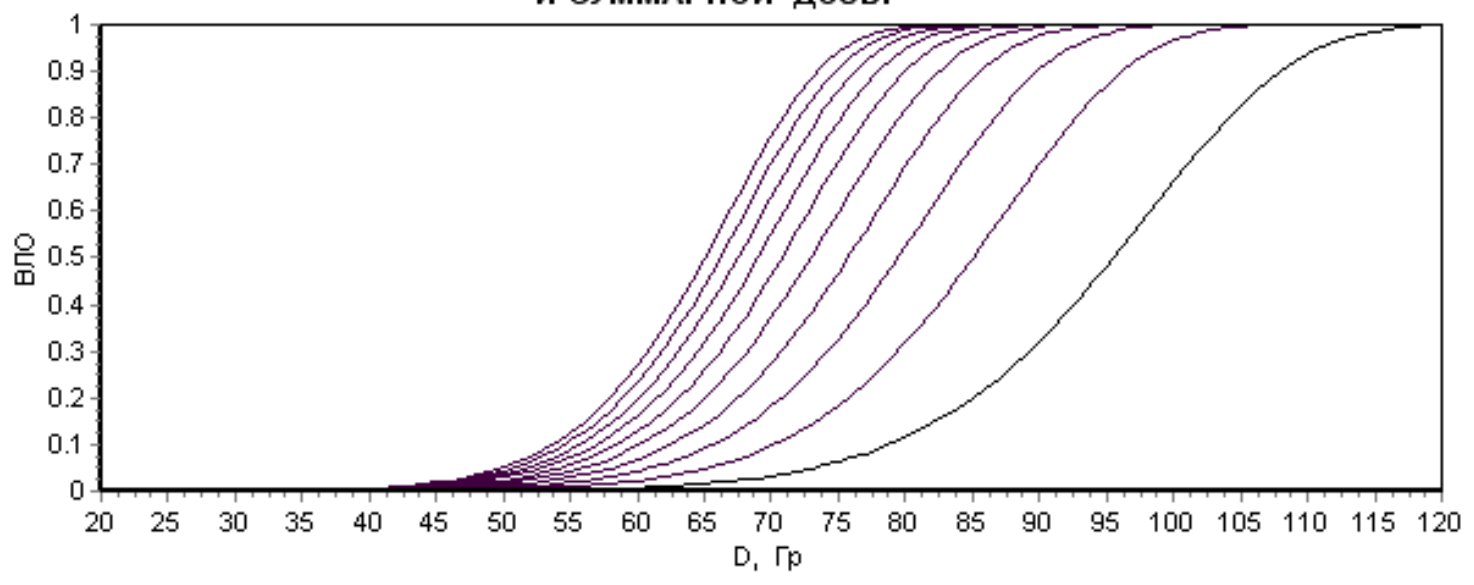

Рис. 4.4.1. Ствол мозга. Оптимизация по ВЛО

Таблица 4.4 .2

Ствол мозга. Оптимизация по ТД

\begin{tabular}{|c|c|c|c|c|c|c|c|c|c|}
\hline$P_{\text {КЛ }}$ & $P_{\mathrm{TEOP}}$ & $\varepsilon, \%$ & $V$ & $D_{\text {Кл }}$ & $D_{\text {TEOP }}$ & $\varepsilon, \%$ & $A_{1}$ & $A_{2}$ & $b$ \\
\hline 0,05 & 0,051 & 2,30 & $1 / 3$ & 60 & 59,86 & $-0,24$ & \multirow{6}{*}{67,48} & \multirow{6}{*}{9,792} & \multirow{6}{*}{0,167} \\
\hline 0,05 & 0,047 & $-5,49$ & $2 / 3$ & 53 & 53,31 & 0,59 & & & \\
\hline 0,05 & 0,052 & 3,43 & 1 & 50 & 49,82 & $-0,35$ & & & \\
\hline- & - & - & - & - & - & - & & & \\
\hline- & - & - & - & - & - & - & & & \\
\hline 0,50 & 0,50 & 0,00 & 1 & 65 & 65,00 & 0,00 & & & \\
\hline
\end{tabular}




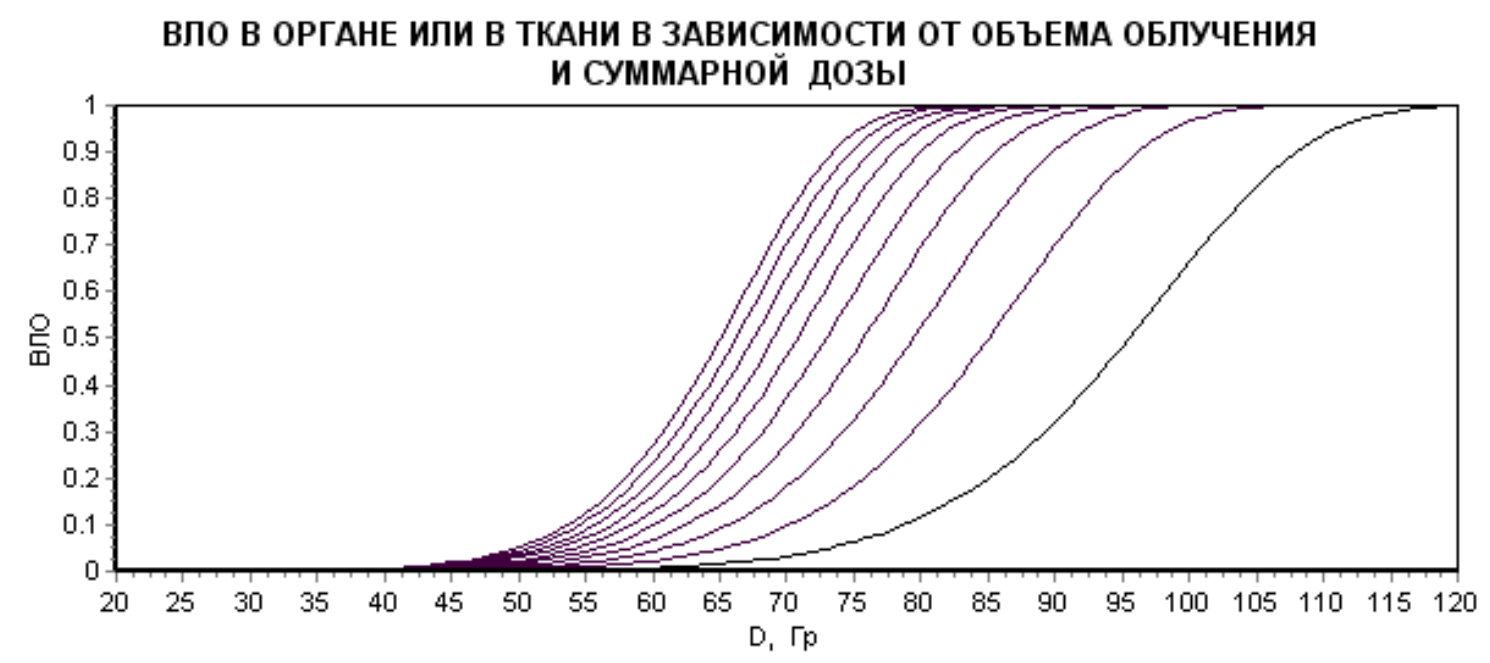

Рис. 4.4.2. Ствол мозга. Оптимизация по ТД

Таблица 4.4.3

Ствол мозга. Оптимизация по ВЛО + ТД

\begin{tabular}{|c|c|c|c|c|c|c|c|c|c|}
\hline$P_{\text {КЛ }}$ & $P_{\mathrm{TEOP}}$ & $\varepsilon, \%$ & $V$ & $D_{\text {Кл }}$ & $D_{\text {TEOP }}$ & $\varepsilon, \%$ & $A_{1}$ & $A_{2}$ & $b$ \\
\hline 0,05 & 0,051 & 2,30 & $1 / 3$ & 60 & 59,86 & $-0,24$ & \multirow{6}{*}{67,48} & \multirow{6}{*}{9,792} & \multirow{6}{*}{0,167} \\
\hline 0,05 & 0,047 & $-5,49$ & $2 / 3$ & 53 & 53,31 & 0,59 & & & \\
\hline 0,05 & 0,052 & 3,43 & 1 & 50 & 49,82 & $-0,35$ & & & \\
\hline- & - & - & - & - & - & - & & & \\
\hline- & - & - & - & - & - & - & & & \\
\hline 0,50 & 0,50 & 0,00 & 1 & 65 & 65,00 & 0,00 & & & \\
\hline
\end{tabular}

ВЛО В ОРГАНЕ ИЛИ В ТКАНИ В ЗАВИСИМОСТИ ОТ ОБЪЕМА ОБЛУЧЕНИЯ И СУММАРНОЙ ДОЗЫ

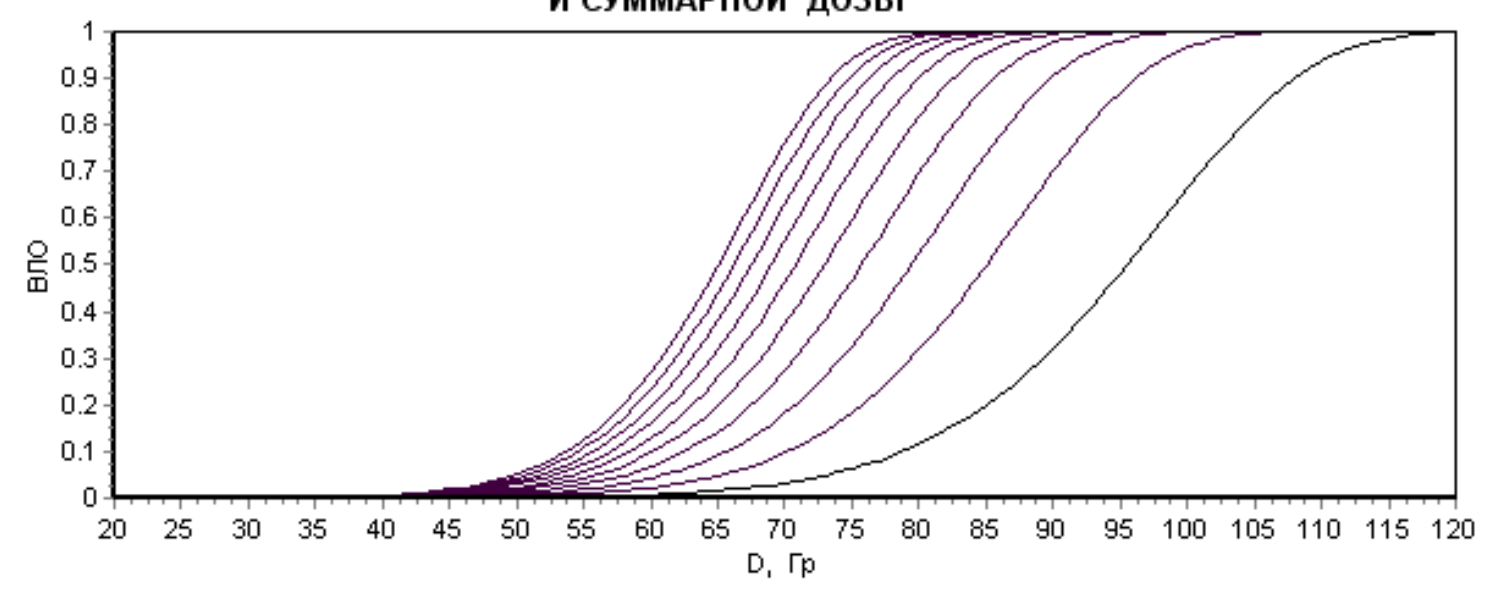

Рис. 4.4.3. Ствол мозга. Оптимизация по ВЛО + ТД 


\section{5. Конский хвост.}

Осложнение: клиническое проявление повреждения нервов

Таблица 4.5

Конский хвост

\begin{tabular}{c|c|c|c|c|c}
\hline$P_{\text {КЛ }}$ & $D_{\text {КЛ }}$ & $P_{\text {КЛ }}$ & $D_{\text {КЛ }}$ & $A_{1}$ & $A_{2}$ \\
\hline 0,05 & 60,0 & 0,50 & 75,0 & 77,393 & 11,668 \\
\hline
\end{tabular}

ВЛО В ОРГАНЕ ИЛИ В ТКАНИ В ЗАВИСИМОСТИ ОТ СУММАРНОЙ ДОЗЫ

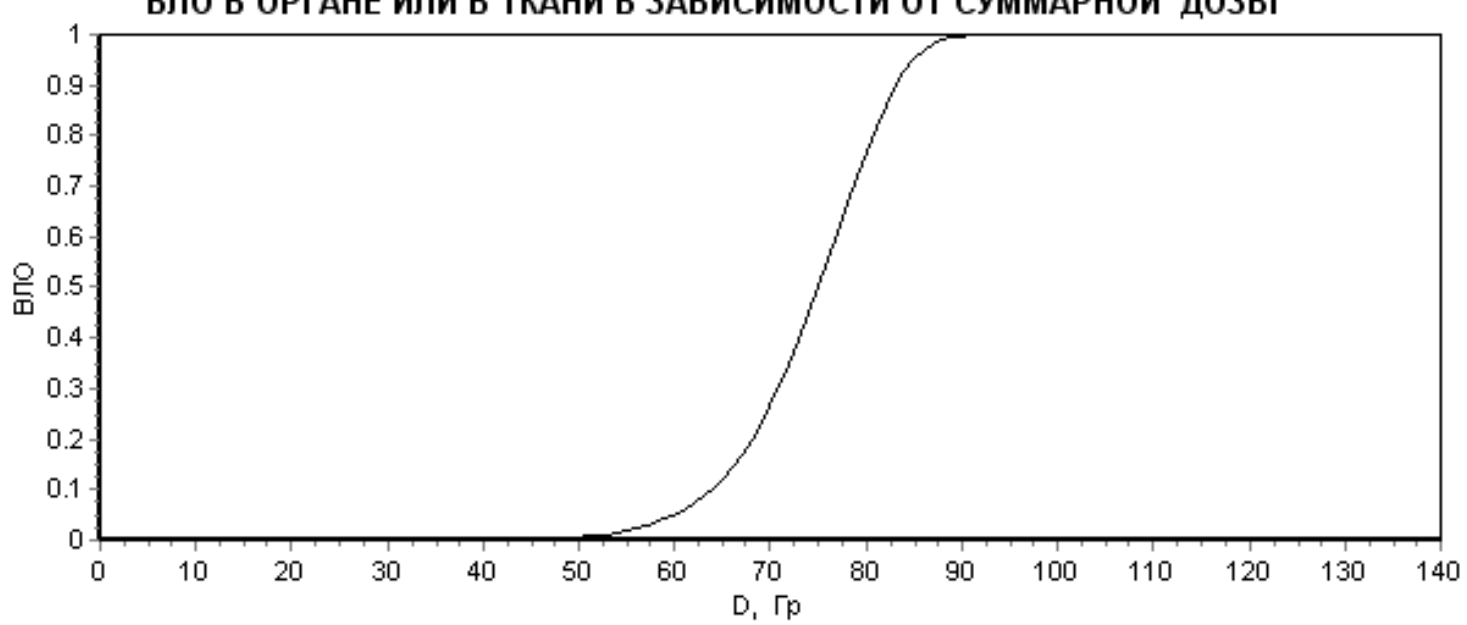

Рис. 4.5. Конский хвост. Зависимость ВЛО от дозы

\section{6. Кишка.}

Осложнение: обструкция, перфорация, изъязвление, фистула

Таблица 4.6.1

Кищка. Оптимизация по ВЛО

\begin{tabular}{|c|c|c|c|c|c|c|c|c|c|}
\hline$P_{\text {Кл }}$ & $P_{\mathrm{TEOP}}$ & $\varepsilon, \%$ & $V$ & $D_{\text {Кл }}$ & $D_{\text {TEOP }}$ & $\varepsilon, \%$ & $A_{1}$ & $A_{2}$ & $b$ \\
\hline 0,05 & 0,056 & 12,58 & $1 / 3$ & 55 & 54,53 & $-0,86$ & \multirow{6}{*}{55,981} & \multirow{6}{*}{14,161} & \multirow{6}{*}{0.167} \\
\hline- & - & - & - & - & - & - & & & \\
\hline 0,05 & 0,044 & $-11,21$ & 1 & 45 & 45,39 & 0,86 & & & \\
\hline 0,50 & 0,480 & -7.90 & $1 / 3$ & 65 & 65,54 & 0.82 & & & \\
\hline- & - & - & - & - & - & - & & & \\
\hline 0,50 & 0,541 & 8,19 & 1 & 55 & 54,55 & $-0,82$ & & & \\
\hline
\end{tabular}




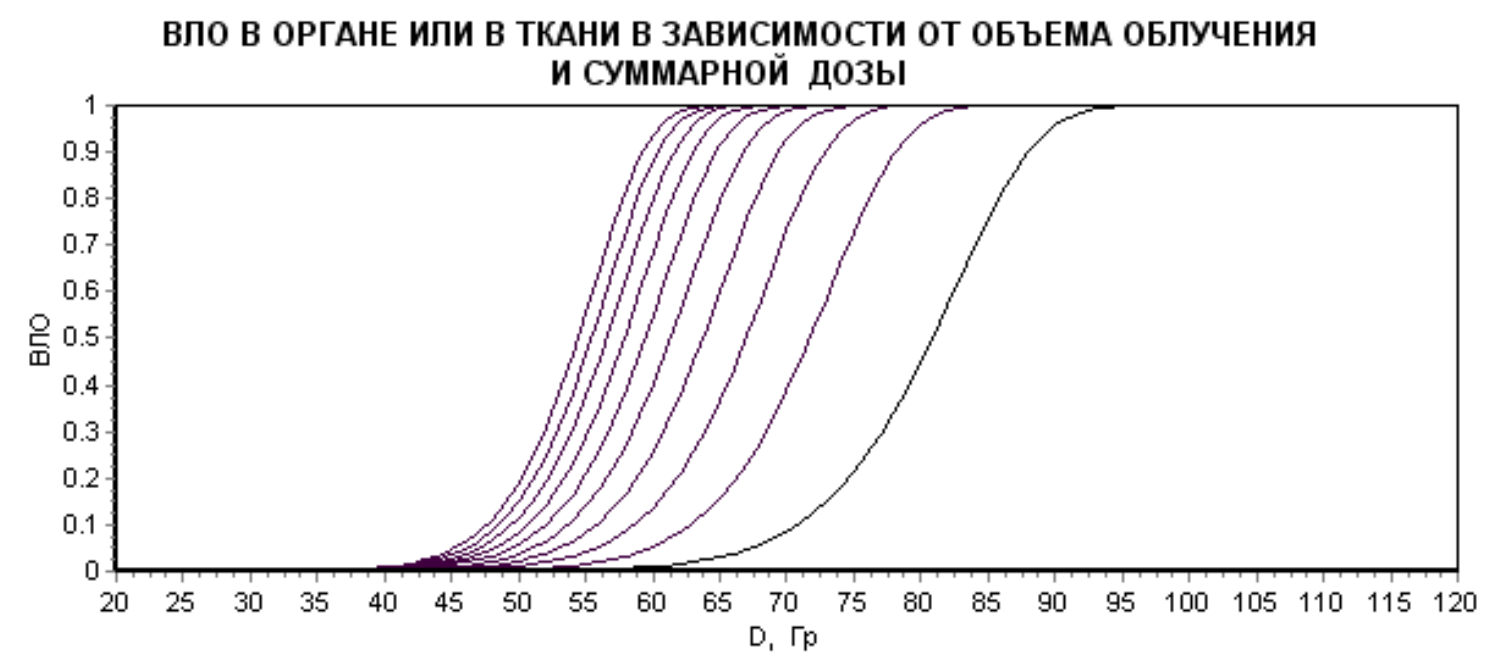

Рис. 4.6.1. Кишка. Оптимизация по ВЛО

Таблица 4.6.2

Кичка. Оптимизация по ТД

\begin{tabular}{|c|c|c|c|c|c|c|c|c|c|}
\hline$P_{\text {Кл }}$ & $P_{\mathrm{TEOP}}$ & $\varepsilon, \%$ & $V$ & $D_{\text {Кл }}$ & $D_{\mathrm{TEOP}}$ & $\varepsilon, \%$ & $A_{1}$ & $A_{2}$ & $b$ \\
\hline 0,05 & 0,056 & 12,58 & $1 / 3$ & 55 & 54,53 & $-0,86$ & \multirow{6}{*}{55,981} & \multirow{6}{*}{14,161} & \multirow{6}{*}{0.167} \\
\hline- & - & - & - & - & - & - & & & \\
\hline 0,05 & 0,044 & $-11,21$ & 1 & 45 & 65,54 & 0,82 & & & \\
\hline 0,50 & 0,480 & $-7,90$ & $1 / 3$ & 65 & 65,72 & 1,10 & & & \\
\hline- & - & - & - & - & - & - & & & \\
\hline 0,50 & 0,541 & 8,19 & 1 & 55 & 54,55 & $-0,82$ & & & \\
\hline
\end{tabular}

ВЛО В ОРГАНЕ ИЛИ В ТКАНИ В ЗАВИСИМОСТИ ОТ ОБЪЕМА ОБЛУЧЕНИЯ И СУММАРНОЙ ДОЗЫ

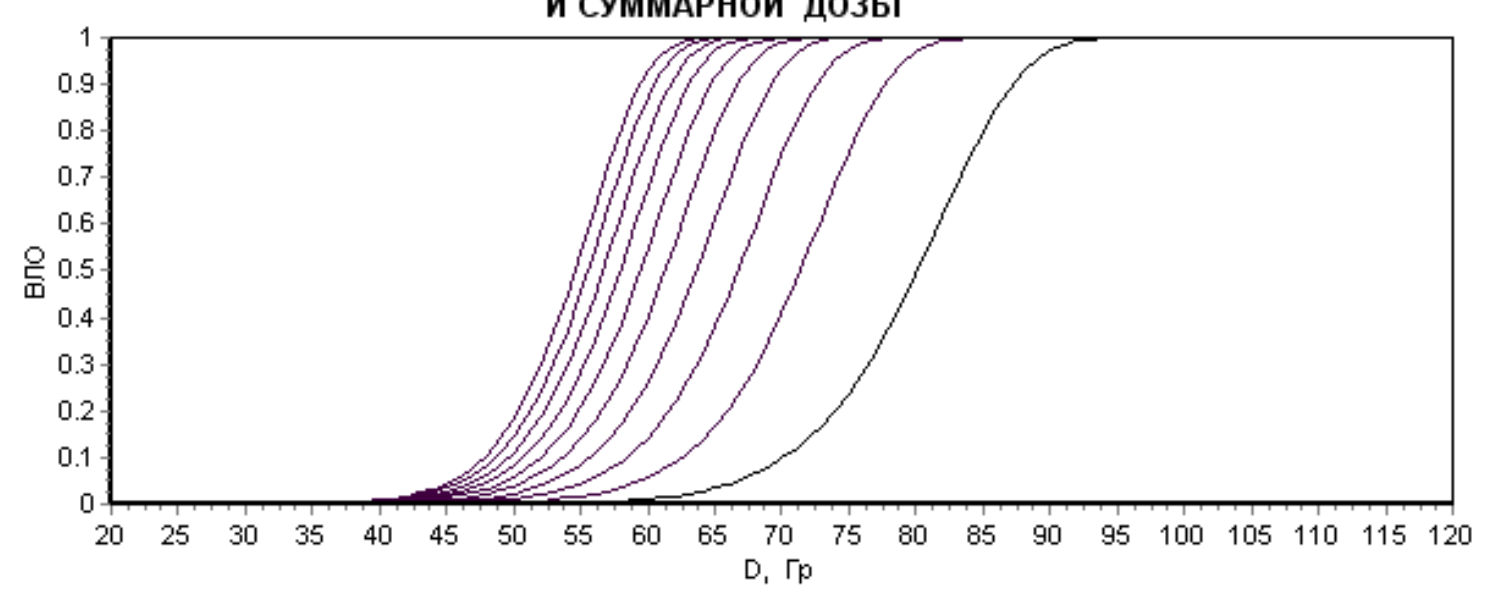

Рис. 4.6.2. Кишка. Оптимизация по ТД 
Таблица 4.6.3

Кишка. Оптимизация по ВЛО + ТД

\begin{tabular}{|c|c|c|c|c|c|c|c|c|c|}
\hline$P_{\text {Кл }}$ & $P_{\mathrm{TEOP}}$ & $\varepsilon, \%$ & V & $D_{\text {Кл }}$ & $D_{\mathrm{TEOP}}$ & $\varepsilon, \%$ & $A_{1}$ & $A_{2}$ & $b$ \\
\hline 0,05 & 0,054 & 8,41 & $1 / 3$ & 55 & 54,68 & $-0,58$ & \multirow{6}{*}{55,827} & \multirow{6}{*}{14,161} & \multirow{6}{*}{0,172} \\
\hline- & - & - & - & - & - & - & & & \\
\hline 0,05 & 0,046 & $\begin{array}{l}-7,77 \\
\end{array}$ & 1 & 45 & 45,26 & 0,59 & & & \\
\hline 0,50 & 0,448 & $-10,47$ & $1 / 3$ & 65 & 65,72 & 1,10 & & & \\
\hline- & - & - & - & - & - & - & & & \\
\hline 0,50 & 0,560 & 10,97 & 1 & 55 & 54,00 & $-1,09$ & & & \\
\hline
\end{tabular}

ВЛО В ОРГАНЕ ИЛИ В ТКАНИ В ЗАВИСИМОСТИ ОТ ОБЪЕМА ОБЛУЧЕНИЯ И СУММАРНОЙ ДОЗЫ

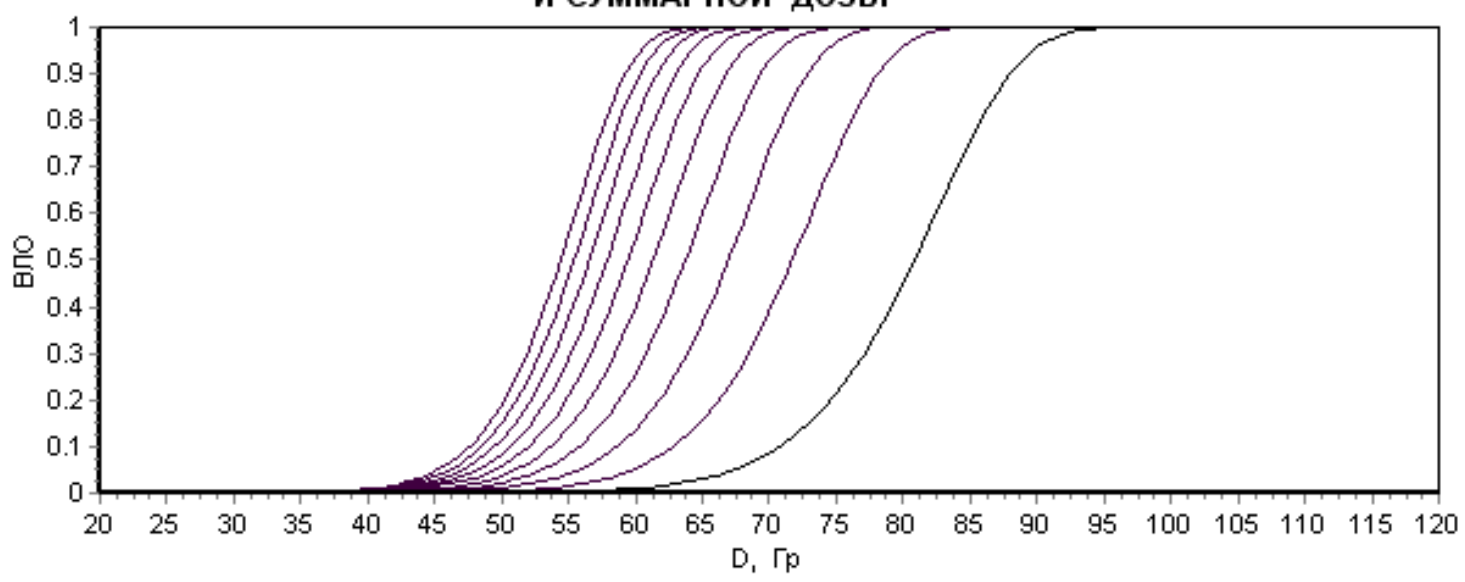

Рис. 6.3. Кишка. Оптимизация по ВЛО + ТД

4.7. Среднее ухо 1. Осложнение: Острый серозный отит

Таблица 4.7

Среднее ухо 1

\begin{tabular}{c|c|c|c|c|c}
\hline$P_{\text {КЛ }}$ & $D_{\text {КЛ }}$ & $P_{\text {КЛ }}$ & $D_{\text {КЛ }}$ & $A_{1}$ & $A_{2}$ \\
\hline 0,05 & 30,0 & 0,50 & 40,0 & 41,653 & 9,051 \\
\hline
\end{tabular}

ВЛО В ОРГАНЕ ИЛИ В ТКАНИ В ЗАВИСИМОСТИ ОТ СУММАРНОЙ ДОЗЫ

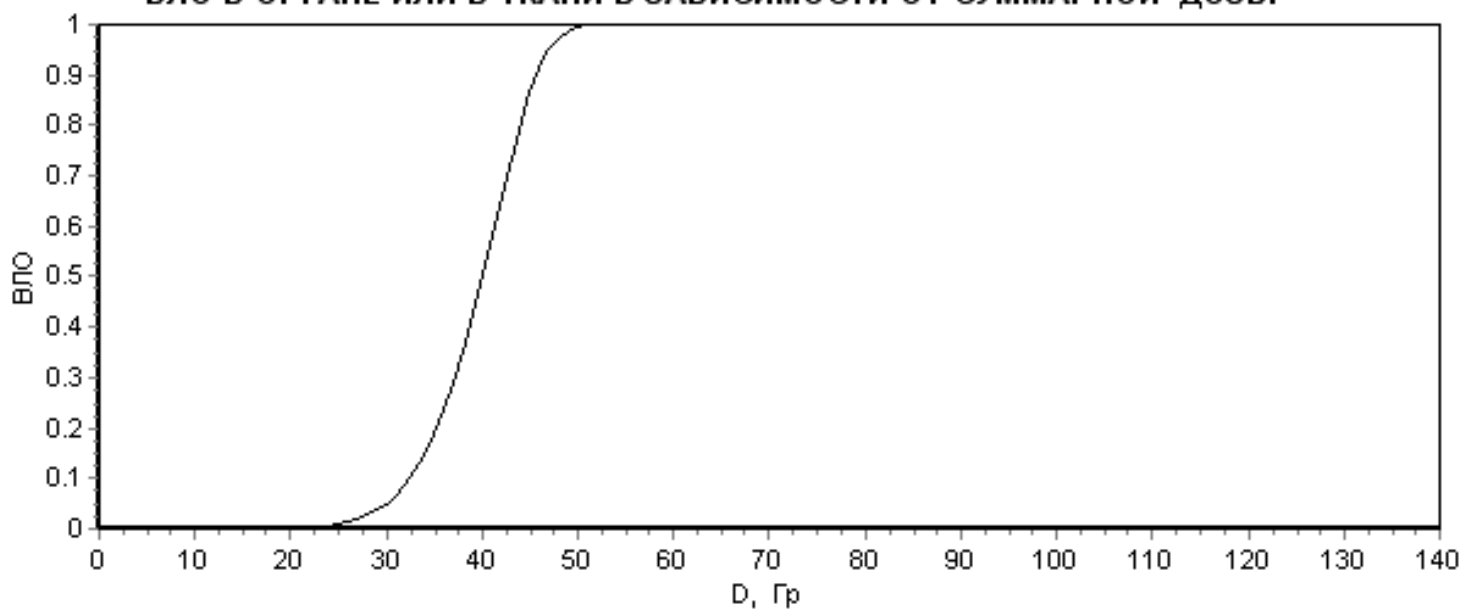

Рис. 4.7. Среднее ухо 1. Зависимость ВЛО от дозы 
4.8. Среднее ухо 2. Осложснение: хронический отит

Таблица 4.8

Среднее ухо 2

\begin{tabular}{c|c|c|c|c|c}
\hline$P_{\text {КЛ }}$ & $D_{\text {Кл }}$ & $P_{\text {КЛ }}$ & $D_{\text {КЛ }}$ & $A_{1}$ & $A_{2}$ \\
\hline 0,05 & 55,0 & 0,50 & 65,0 & 66,547 & 15,586 \\
\hline
\end{tabular}

ВЛО В ОРГАНЕ ИЛИ В ТКАНИ В ЗАВИСИМОСТИ ОТ СУММАРНОЙ ДОЗЫ

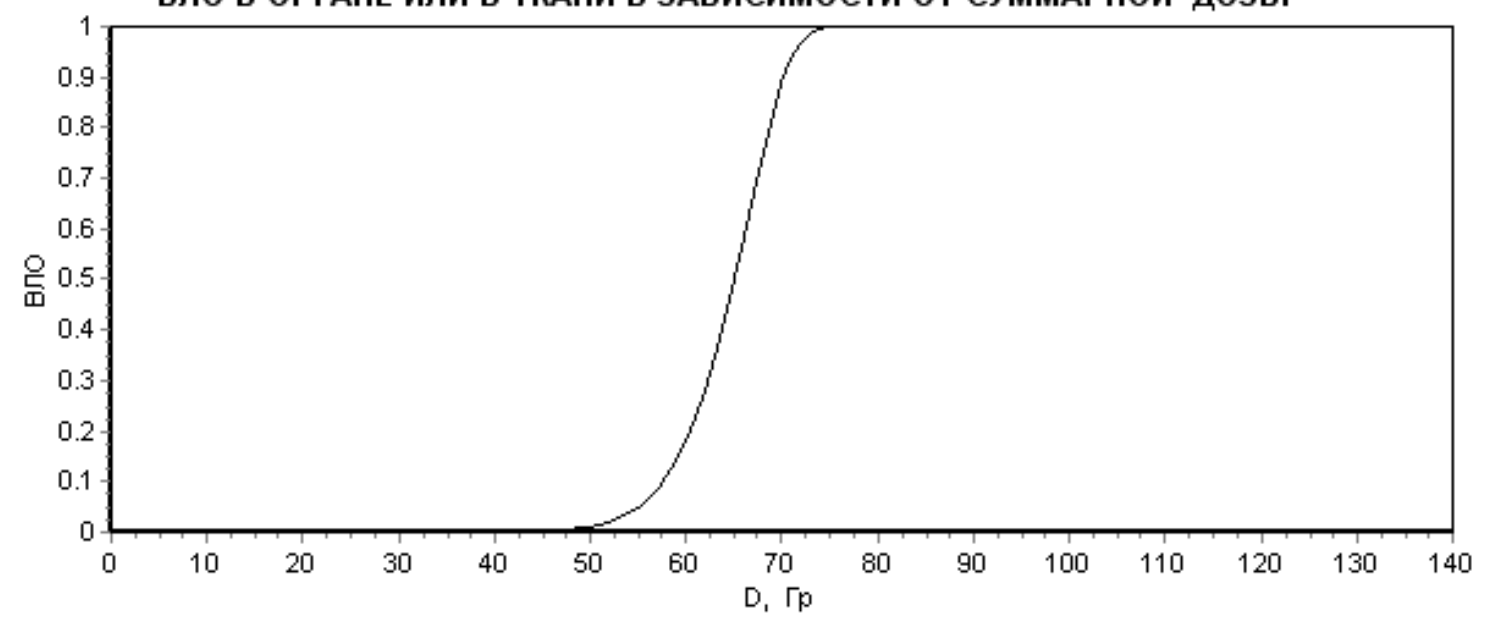

Рис. 4.8. Среднее ухо 2. Зависимость ВЛО от дозы

4.9. Пищевод. Осложнение: стриктура, перфорация

Таблица 4.9

Пищевод. Оптимизация по ВЛО

\begin{tabular}{|c|c|c|c|c|c|c|c|c|c|}
\hline$P_{\text {Кл }}$ & $P_{\mathrm{TEOP}}$ & $\varepsilon, \%$ & $V$ & $D_{\text {Кл }}$ & $D_{\text {TEOP }}$ & $\varepsilon, \%$ & $A_{1}$ & $A_{2}$ & $b$ \\
\hline 0,05 & 0,051 & 1,16 & $1 / 3$ & 60 & 59,95 & $-0,09$ & \multirow{6}{*}{69,579} & \multirow{6}{*}{13,408} & \multirow{6}{*}{0,066} \\
\hline 0,05 & 0,059 & 18,06 & $2 / 3$ & 58 & 57,27 & $-1,27$ & & & \\
\hline 0,05 & 0,041 & $-16,33$ & 1 & 55 & 55,75 & 1,37 & & & \\
\hline 0,50 & 0,450 & $-9,95$ & $1 / 3$ & 72 & 72,79 & 1,10 & & & \\
\hline 0,50 & 0,531 & 6,21 & $2 / 3$ & 70 & 69,54 & $-0,66$ & & & \\
\hline 0,50 & 0,520 & 4,10 & 1 & 68 & 67,70 & $-0,44$ & & & \\
\hline
\end{tabular}


ВЛО В ОРГАНЕ ИЛИ В ТКАНИ В ЗАВИСИМОСТИ ОТ ОБЪЕМА ОБЛУЧЕНИЯ И СУММАРНОЙ ДОЗЫ

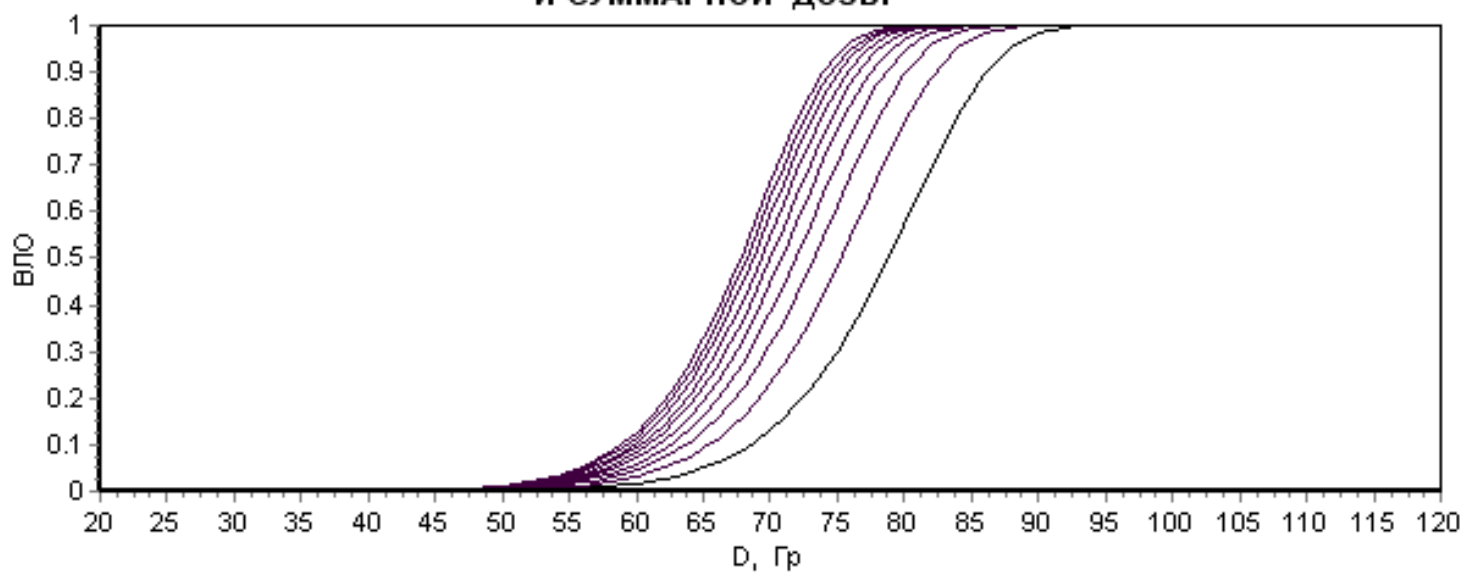

Рис. 4.9.1. Пищевод. Оптимизация по ВЛО

Таблица 4.9.2

Пищевод. Оптимизация по ТД

\begin{tabular}{|c|c|c|c|c|c|c|c|c|c|}
\hline$P_{\text {Кл }}$ & $P_{\mathrm{TEOP}}$ & $\varepsilon, \%$ & $V$ & $D_{\text {Кл }}$ & $D_{\text {TEOP }}$ & $\varepsilon, \%$ & $A_{1}$ & $A_{2}$ & $b$ \\
\hline 0,05 & 0,052 & 3,56 & $1 / 3$ & 60 & 59,84 & $-0,27$ & \multirow{6}{*}{69,684} & \multirow{6}{*}{13,408} & \multirow{6}{*}{0,063} \\
\hline 0,05 & 0,059 & 17,62 & $2 / 3$ & 58 & 57,28 & $-1,24$ & & & \\
\hline 0,05 & 0,041 & $-17,97$ & 1 & 55 & 55,84 & 1,52 & & & \\
\hline 0,50 & 0,458 & $-0,836$ & $1 / 3$ & 72 & 72,66 & 0,92 & & & \\
\hline 0,50 & 0,530 & 5,94 & $2 / 3$ & 70 & 69,56 & $-0,62$ & & & \\
\hline 0,50 & 0,513 & 2,68 & 1 & 68 & 67,81 & $-0,29$ & & & \\
\hline
\end{tabular}

ВЛО В ОРГАНЕ ИЛИ В ТКАНИ В ЗАВИСИМОСТИ ОТ ОБЪЕМА ОБЛУЧЕНИЯ И СУММАРНОЙ ДОЗЫ

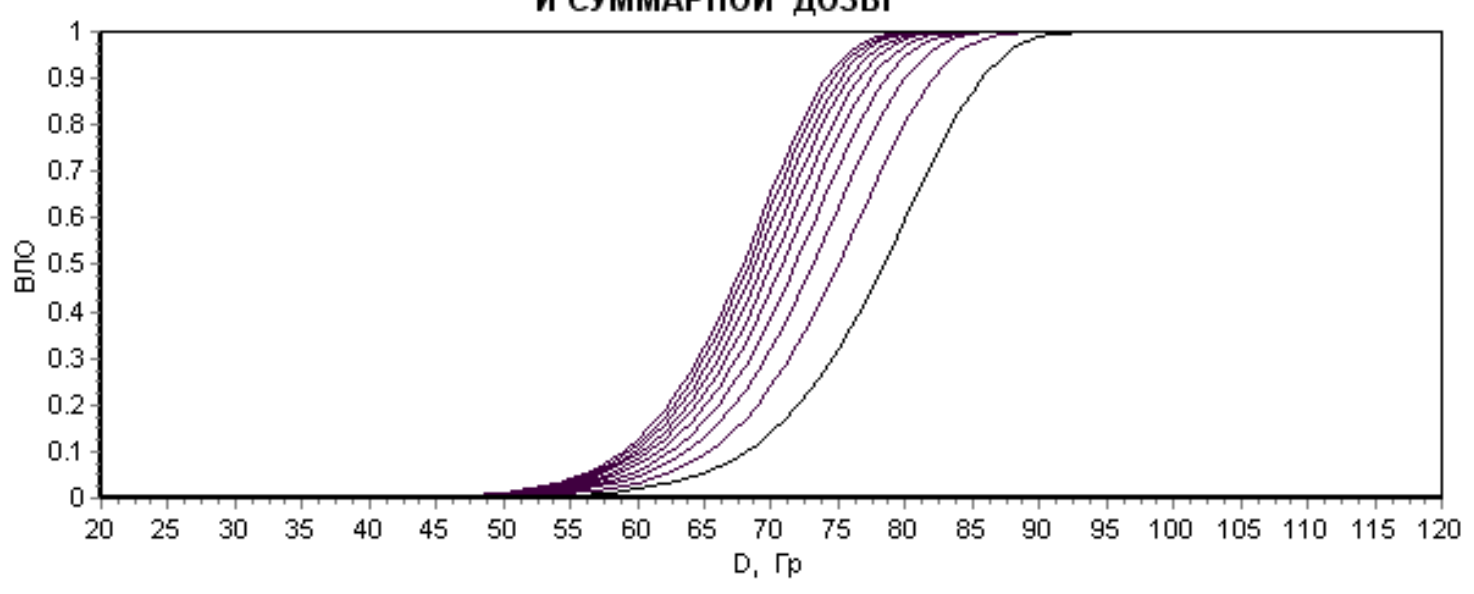

Рис. 4.9.2. Пищевод. Оптимизация по ТД 
Таблица 4.9 .3

\section{Пищевод. Оптимизация по ВЛО + ТД}

\begin{tabular}{|c|c|c|c|c|c|c|c|c|c|}
\hline$P_{\text {Кл }}$ & $P_{\text {TEOP }}$ & $\varepsilon, \%$ & $V$ & $D_{\text {КЛ }}$ & $D_{\mathrm{TEOP}}$ & $\varepsilon, \%$ & $A_{1}$ & $A_{2}$ & $b$ \\
\hline 0,05 & 0,051 & 1,16 & $1 / 3$ & 60 & 59,95 & $-0,09$ & \multirow{6}{*}{69,580} & \multirow{6}{*}{13,408} & \multirow{6}{*}{0,070} \\
\hline 0,05 & 0,059 & 18,06 & $2 / 3$ & 58 & 57,26 & $-1,26$ & & & \\
\hline 0,05 & 0,042 & $-16,33$ & 1 & 55 & 55,75 & 1,37 & & & \\
\hline 0,50 & 0,480 & $-9,95$ & $1 / 3$ & 72 & 72,79 & 1,10 & & & \\
\hline 0,50 & 0,531 & 6,21 & $2 / 3$ & 70 & 69,54 & $-0,66$ & & & \\
\hline 0,50 & 0,521 & 4,10 & 1 & 68 & 67,70 & $-0,44$ & & & \\
\hline
\end{tabular}

ВЛО В ОРГАНЕ ИЛИ В ТКАНИ В ЗАВИСИМОСТИ ОТ ОБЪЕМА ОБЛУЧЕНИЯ И СУММАРНОЙ ДОЗЫ

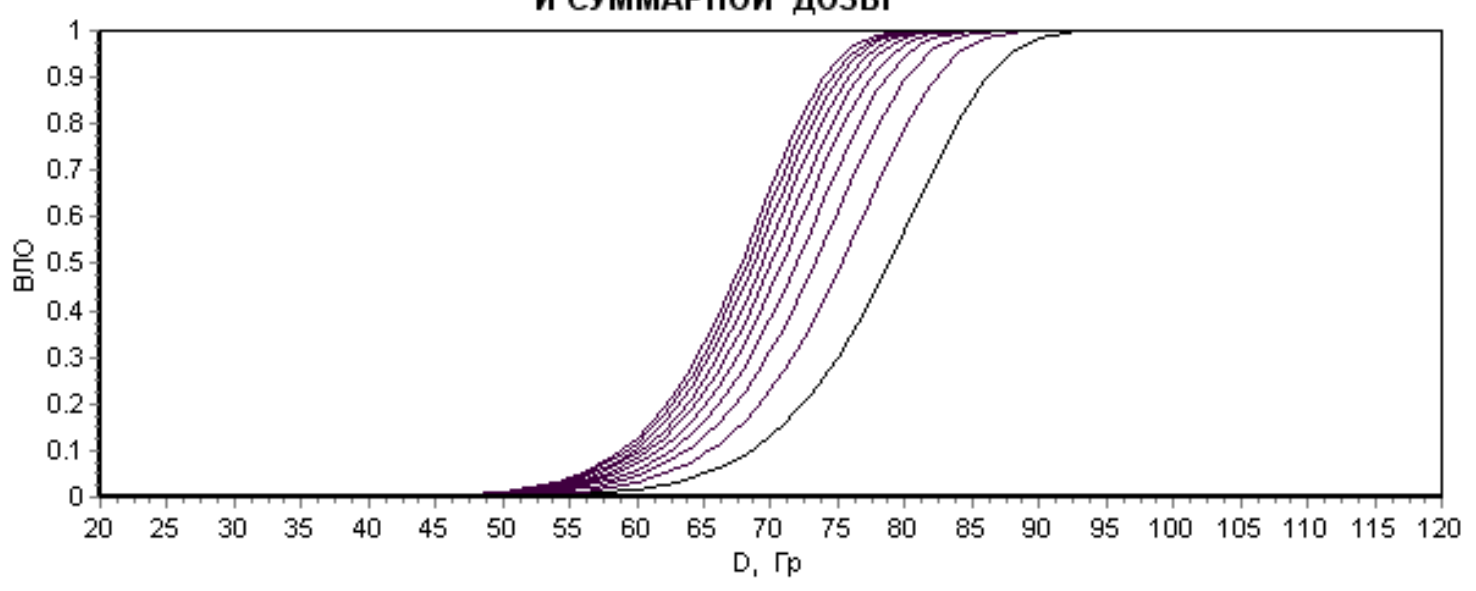

Рис. 4.9.3. Пищевод. Оптимизация по ВЛО + ТД

Таблица 4.9.4, в которой приводятся оптимальные значения параметров модели МРВ, свидетельствует о плохой согласованности систематизированной клинической информации, (оптимальные значения параметров модели для ВЛО $=0,05$ и ВЛО = 0,50 серьезно отличаются друг от друга).

Таблица 4.9 .4

Пищеевод. Оптимизация по ТД, отдельно для ВЛО = 0,05 и ВЛО = 0,50

\begin{tabular}{|c|c|c|c|c|c|c|c|c|c|}
\hline$P_{\text {Кл }}$ & $P_{\mathrm{TEOP}}$ & $\varepsilon, \%$ & $V$ & $D_{\text {Кл }}$ & $D_{\text {TEOP }}$ & $\varepsilon, \%$ & $A_{1}$ & $A_{2}$ & $b$ \\
\hline 0,05 & 0,048 & $-4,99$ & $1 / 3$ & 60 & 60,31 & 0,51 & \multirow{3}{*}{74,000} & \multirow{3}{*}{10,309} & \multirow{3}{*}{0,076} \\
\hline 0,05 & 0,057 & 14,72 & $2 / 3$ & 58 & 57,21 & $-1,36$ & & & \\
\hline 0,05 & 0,046 & $-8,29$ & 1 & 55 & 55,48 & 0,86 & & & \\
\hline 0,50 & 0,500 & $-0,01$ & $1 / 3$ & 72 & 72,10 & 0,14 & \multirow{3}{*}{4278,500} & \multirow{3}{*}{0,089} & \multirow{3}{*}{0,050} \\
\hline 0,50 & 0,500 & 0,03 & $2 / 3$ & 70 & 69,65 & $-0,51$ & & & \\
\hline 0,50 & 0,500 & $-0,02$ & 1 & 68 & 68,25 & 0,37 & & & \\
\hline
\end{tabular}


4.10. Головка и шейка бедра. Осложнение: Некроз

Таблица 4.10

Головка и шейка бедра

\begin{tabular}{c|c|c|c|c|c}
\hline$P_{\text {КЛ }}$ & $D_{\text {КЛ }}$ & $P_{\text {КЛ }}$ & $D_{\text {КЛ }}$ & $A_{1}$ & $A_{2}$ \\
\hline 0,05 & 52,0 & 0,50 & 65,0 & 67,074 & 11,668 \\
\hline
\end{tabular}

ВЛО В ОРГАНЕ ИЛИ В ТКАНИ В ЗАВИСИМОСТИ ОТ СУММАРНОЙ ДОЗЫ

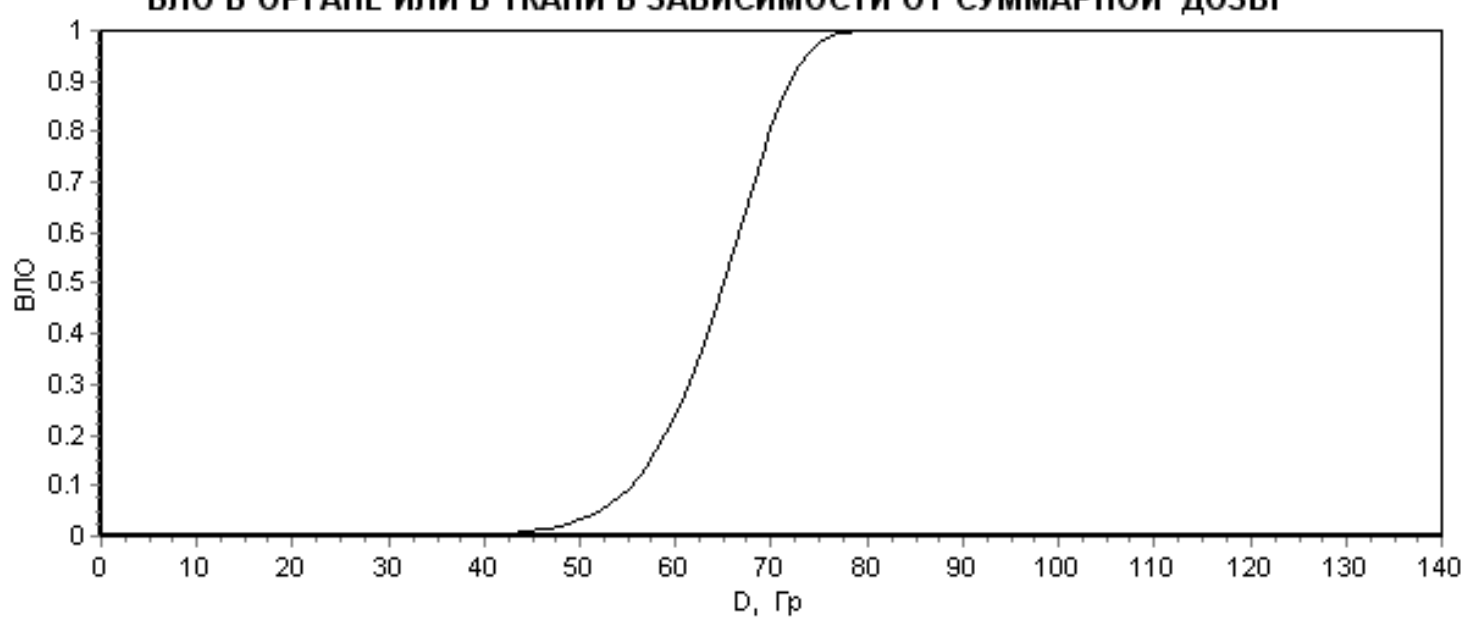

Рис. 4.10. Головка и шейка бедра. Зависимость ВЛО от дозы

\subsection{1. Сердце. Осложнение: Перикардиты}

Таблица 4.11.1

Сердце. Оптимизация по ВЛО

\begin{tabular}{|c|c|c|c|c|c|c|c|c|c|}
\hline$P_{\text {КЛ }}$ & $P_{\mathrm{TEOP}}$ & $\varepsilon, \%$ & $V$ & $D_{\text {КЛ }}$ & $D_{\text {TEOP }}$ & $\varepsilon, \%$ & $A_{1}$ & $A_{2}$ & $b$ \\
\hline 0,05 & 0,063 & 26,37 & $1 / 3$ & 60 & 58,94 & $-1,77$ & \multirow{6}{*}{49,605} & \multirow{6}{*}{13,514} & \multirow{6}{*}{0,357} \\
\hline 0,05 & 0,037 & $-25,62$ & $2 / 3$ & 45 & 46,02 & 0,037 & & & \\
\hline 0,05 & 0,058 & 6,20 & 1 & 40 & 39,82 & $-0,46$ & & & \\
\hline 0,50 & 0,408 & $-18,41$ & $1 / 3$ & 70 & 71,46 & 2,09 & & & \\
\hline 0,50 & 0,435 & $-13,03$ & $2 / 3$ & 55 & 55,80 & 1,45 & & & \\
\hline 0,50 & 0,672 & 34,30 & 1 & 50 & 48,28 & $-3,44$ & & & \\
\hline
\end{tabular}




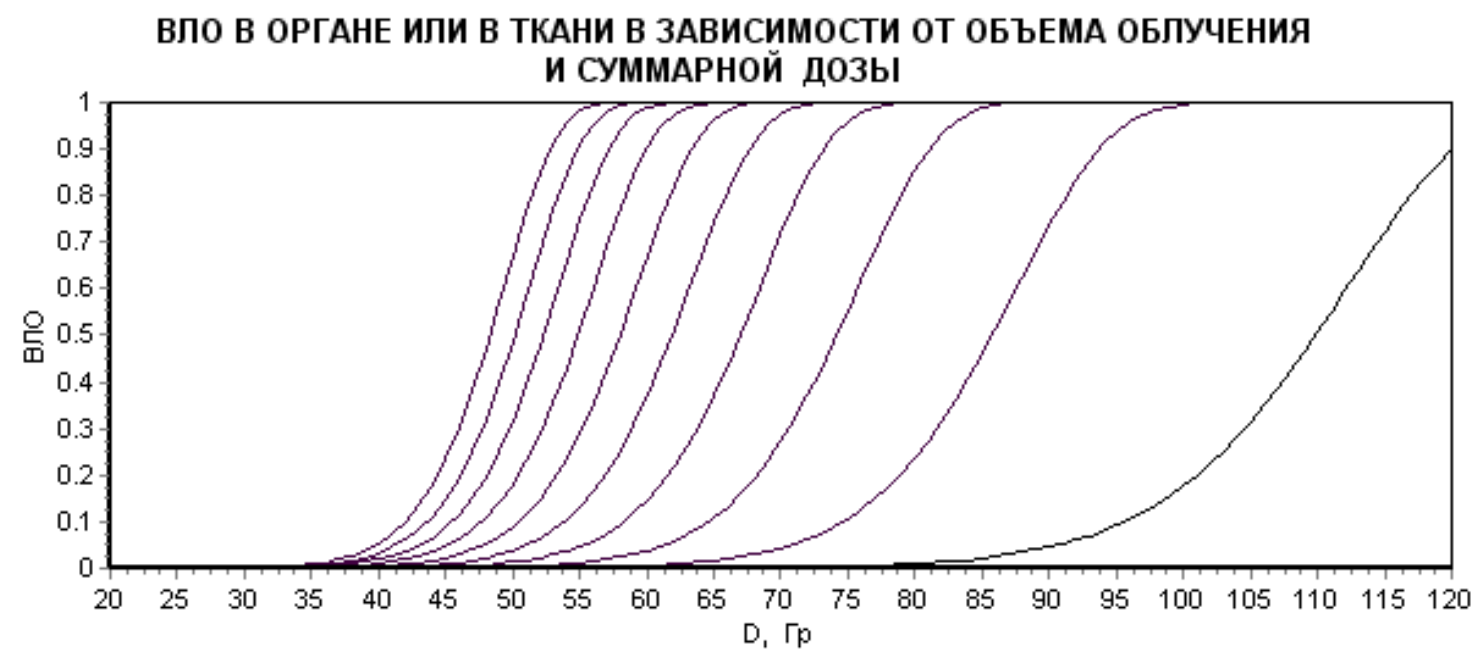

Рис. 4.11.1. Сердце. Оптимизация по ВЛО

Таблица 4.11.2

Сердце. Оптимизация по ТД

\begin{tabular}{|c|c|c|c|c|c|c|c|c|c|}
\hline$P_{\text {Кл }}$ & $P_{\text {TEOP }}$ & $\varepsilon, \%$ & $V$ & $D_{\text {Кл }}$ & $D_{\text {TEOP }}$ & $\varepsilon, \%$ & $A_{1}$ & $A_{2}$ & $b$ \\
\hline 0,05 & 0,063 & 26,37 & $1 / 3$ & 60 & 58,94 & $-1,77$ & \multirow{6}{*}{49,605} & \multirow{6}{*}{13,514} & \multirow{6}{*}{0,357} \\
\hline 0,05 & 0,037 & $-25,62$ & $2 / 3$ & 45 & 46,02 & 2,27 & & & \\
\hline 0,05 & 0,053 & 6,20 & 1 & 40 & 39,82 & $-0,46$ & & & \\
\hline 0,50 & 0,408 & $-18,41$ & $1 / 3$ & 70 & 71,46 & 2,09 & & & \\
\hline 0,50 & 0,435 & $-13,03$ & $2 / 3$ & 55 & 55,80 & 1,45 & & & \\
\hline 0,50 & 0,672 & 34,30 & 1 & 50 & 48,28 & $-3,44$ & & & \\
\hline
\end{tabular}

ВЛО В ОРГАНЕ ИЛИ В ТКАНИ В ЗАВИСИМОСТИ ОТ ОБЪЕМА ОБЛУЧЕНИЯ И СУММАРНОЙ ДОЗЫ

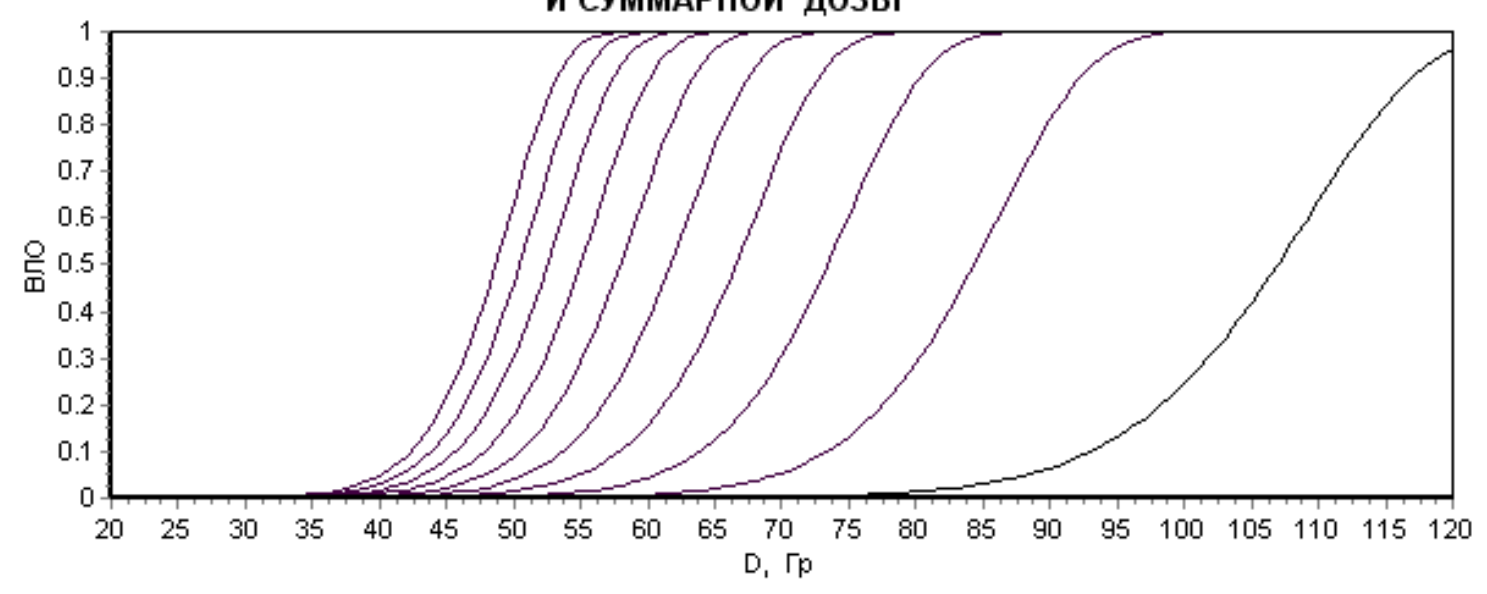

Рис. 4.11.2. Сердце. Оптимизация по ТД 
Таблица 4.11 .3

Сердце. Оптимизация по ВЛО + ТД

\begin{tabular}{|c|c|c|c|c|c|c|c|c|c|}
\hline$P_{\text {Кл }}$ & $P_{\mathrm{TEOP}}$ & $\varepsilon, \%$ & $V$ & $D_{\text {КЛ }}$ & $D_{\mathrm{TEOP}}$ & $\varepsilon, \%$ & $A_{1}$ & $A_{2}$ & $b$ \\
\hline 0,05 & 0,064 & 27,36 & $1 / 3$ & 60 & 58,90 & $-1,83$ & \multirow{6}{*}{49,630} & \multirow{6}{*}{13,515} & \multirow{6}{*}{0,356} \\
\hline 0,05 & 0,037 & $-25,72$ & $2 / 3$ & 45 & 46,02 & 2,27 & & & \\
\hline 0,05 & 0,053 & 5,50 & 1 & 40 & 39,84 & $-0,41$ & & & \\
\hline 0,50 & 0,410 & $-17,91$ & $1 / 3$ & 70 & 71,42 & 2,03 & & & \\
\hline 0,50 & 0,434 & $-13,11$ & $2 / 3$ & 55 & 55,80 & 1,46 & & & \\
\hline 0,50 & 0,669 & 33,81 & 1 & 50 & 48,30 & $-3,40$ & & & \\
\hline
\end{tabular}

ВЛО В ОРГАНЕ ИЛИ В ТКАНИ В ЗАВИСИМОСТИ ОТ ОБЪЕМА ОБЛУЧЕНИЯ И СУММАРНОЙ ДОЗЫ

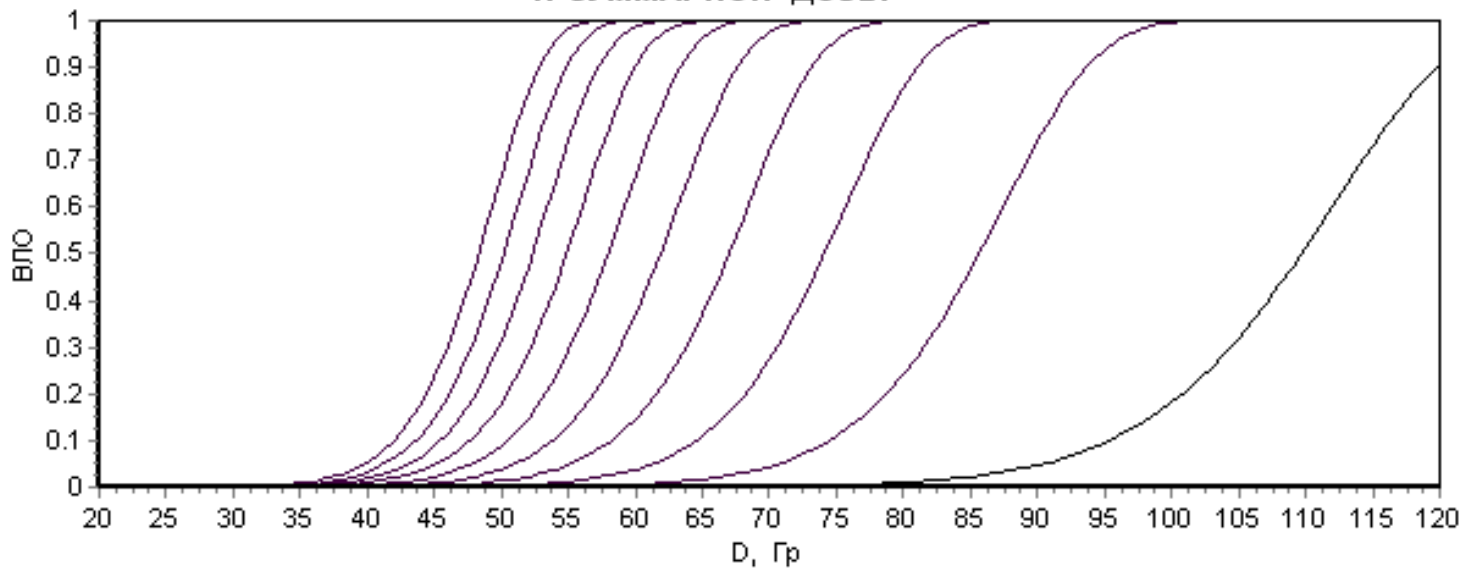

Рис 4.11.3. Сердце. Оптимизация по ВЛО + ТД

Таблица 4.11 .4

Сердце. Оптимизация по ТД, отдельно для ВЛО = 0,05 и ВЛО = 0,50

\begin{tabular}{|c|c|c|c|c|c|c|c|c|c|}
\hline$P_{\text {Кл }}$ & $P_{\mathrm{TEOP}}$ & $\varepsilon, \%$ & $V$ & $D_{\text {КЛ }}$ & $D_{\text {TEOP }}$ & $\varepsilon, \%$ & $A_{1}$ & $A_{2}$ & $b$ \\
\hline 0,05 & 0,050 & 0,74 & $1 / 3$ & 60 & 59,54 & $-0,77$ & \multirow{3}{*}{817,848} & \multirow{3}{*}{0,080} & \multirow{3}{*}{0,374} \\
\hline 0,05 & 0,049 & $-1,96$ & $2 / 3$ & 45 & 45,94 & 2,10 & & & \\
\hline 0,05 & 0,051 & 1,26 & 1 & 40 & 39,48 & $-1,30$ & & & \\
\hline 0,50 & 0,500 & 0,05 & $1 / 3$ & 70 & 69,69 & $-0,44$ & \multirow{3}{*}{439,404} & \multirow{3}{*}{0,168} & \multirow{3}{*}{0,315} \\
\hline 0,50 & 0,500 & $-0,21$ & $2 / 3$ & 55 & 56,02 & 1,86 & & & \\
\hline 0,50 & 0,500 & 0,16 & 1 & 50 & 49,30 & $-1,39$ & & & \\
\hline
\end{tabular}

Из табл. 4.11.4 следует, что клиническая информация плохо согласована. Параметры модели МРВ, рассчитанные для ВЛО = 0,05 и ВЛО = 0,50 значительно отличаются друг от друга. 


\subsection{2. Почки. Осложнения: клинические нефриты}

Таблица 4.12.1

Почки. Оптимизация по ВЛО

\begin{tabular}{|c|c|c|c|c|c|c|c|c|c|}
\hline$P_{\text {Кл }}$ & $P_{\mathrm{TEOP}}$ & $\varepsilon, \%$ & V & $D_{\text {КЛ }}$ & $D_{\text {TEOP }}$ & $\varepsilon, \%$ & $A_{1}$ & $A_{2}$ & $b$ \\
\hline 0,05 & 0,056 & 11,82 & $1 / 3$ & 50 & 49,48 & $\begin{array}{l}-1,04 \\
\end{array}$ & \multirow{6}{*}{30,018} & \multirow{6}{*}{10,987} & \multirow{6}{*}{0,701} \\
\hline 0,05 & 0,043 & $-14,39$ & $2 / 3$ & 30 & 30,44 & 1,46 & & & \\
\hline 0,05 & 0,052 & 4,42 & 1 & 23 & 22,91 & $-0,40$ & & & \\
\hline- & - & - & - & - & - & - & & & \\
\hline 0,50 & 0,643 & 28,74 & $2 / 3$ & 40 & 38,58 & $-3,56$ & & & \\
\hline 0,50 & 0,342 & $-25,56$ & 1 & 28 & 29,03 & 3,69 & & & \\
\hline
\end{tabular}

ВЛО В ОРГАНЕ ИЛИ В ТКАНИ В ЗАВИСИМОСТИ ОТ ОБЪЕМА ОБЛУЧЕНИЯ И СУММАРНОЙ ДОЗЫ

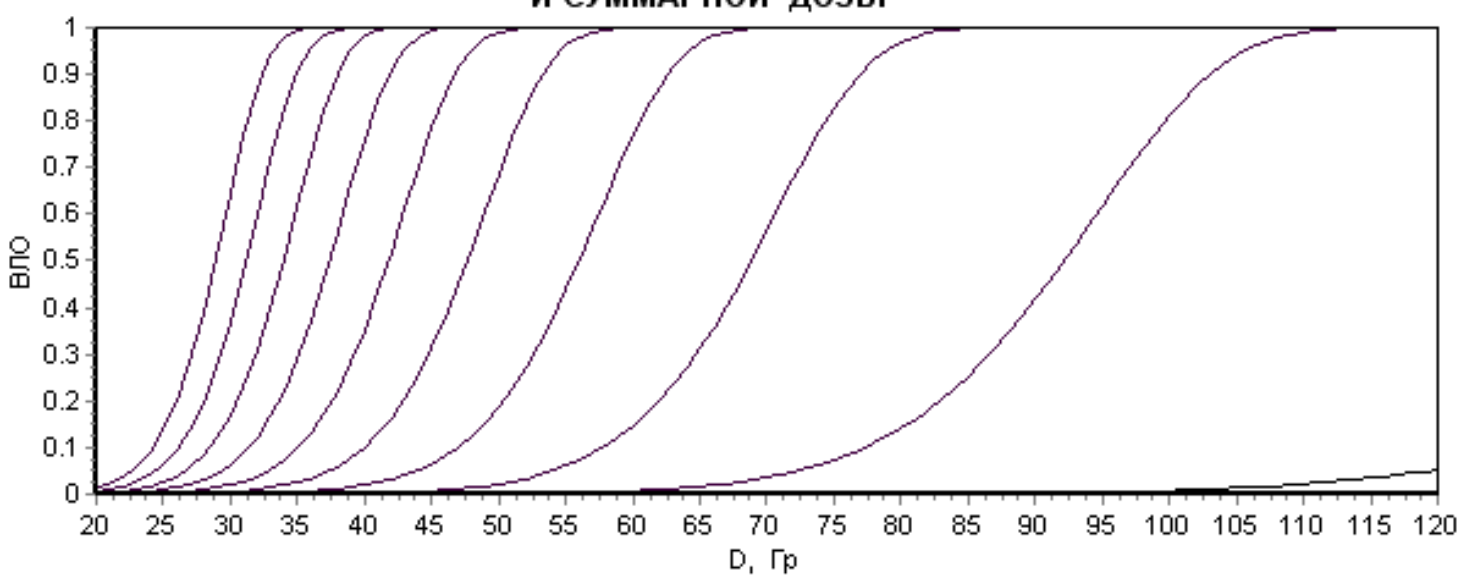

Рис. 4.12.1. Почки. Оптимизация по ВЛО

Таблица 4.12.2

Почки. Оптимизация по ТД

\begin{tabular}{|c|c|c|c|c|c|c|c|c|c|}
\hline$P_{\text {Кл }}$ & $P_{\text {TEOP }}$ & $\varepsilon, \%$ & $V$ & $D_{\text {КЛ }}$ & $D_{\text {TEOP }}$ & $\varepsilon, \%$ & $A_{1}$ & $A_{2}$ & $b$ \\
\hline 0,05 & 0,047 & $-6,86$ & $1 / 3$ & 50 & 50,34 & 0,69 & \multirow{6}{*}{29,878} & \multirow{6}{*}{10,600} & \multirow{6}{*}{0,730} \\
\hline 0,05 & 0,044 & $-11,41$ & $2 / 3$ & 30 & 30,35 & 1,18 & & & \\
\hline 0,05 & 0,061 & 21,09 & 1 & 23 & 22,58 & $-1,84$ & & & \\
\hline- & $\overline{-}$ & - & - & - & $\overline{-}$ & - & & & \\
\hline 0,50 & 0,616 & 23,12 & $2 / 3$ & 40 & 38,80 & $-2,99$ & & & \\
\hline 0,50 & 0,395 & $-21,00$ & 1 & 28 & 28,86 & 3,08 & & & \\
\hline
\end{tabular}




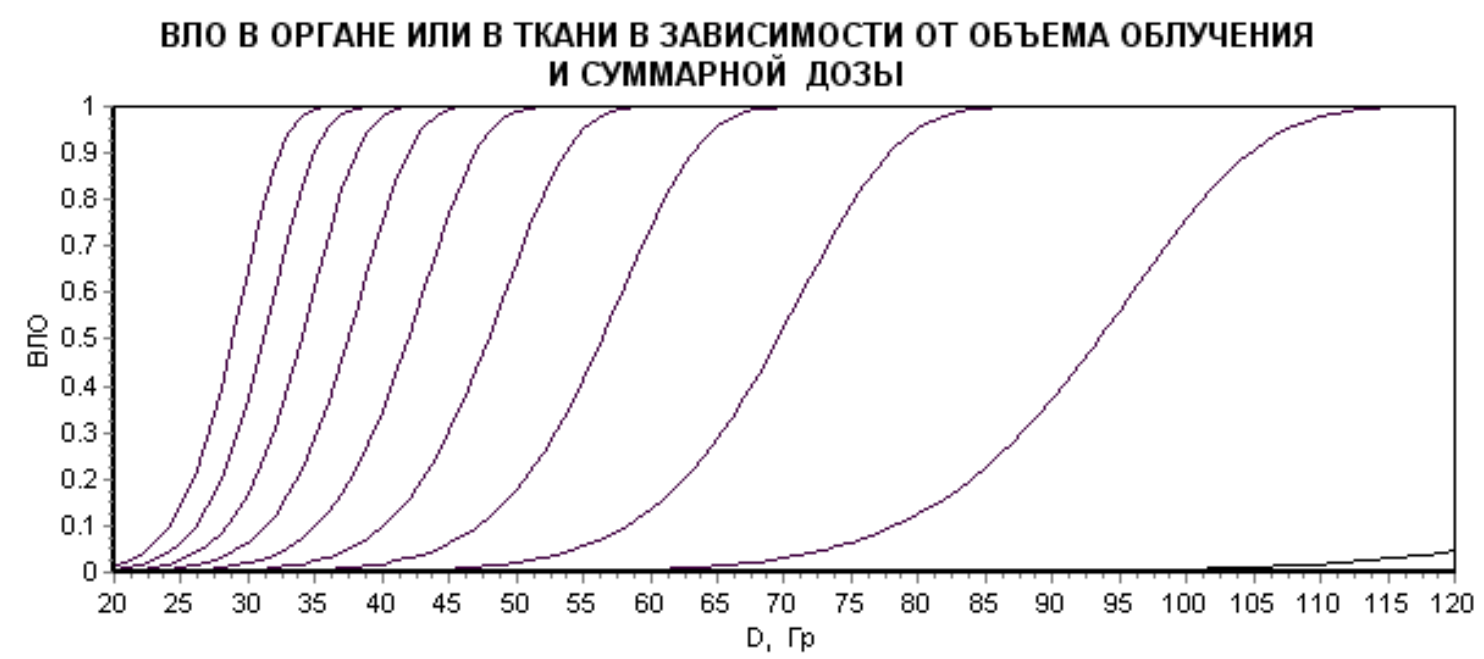

Рис. 4.12.2. Почки. Оптимизация по ТД

Таблица 4.12.3

Почки. Оптимизация по ВЛО + ТД

\begin{tabular}{|c|c|c|c|c|c|c|c|c|c|}
\hline$P_{\text {Кл }}$ & $P_{\mathrm{TEOP}}$ & $\varepsilon, \%$ & $V$ & $D_{\text {КЛ }}$ & $D_{\mathrm{TEOP}}$ & $\varepsilon, \%$ & $A_{1}$ & $A_{2}$ & $b$ \\
\hline 0,05 & 0,050 & $-0,99$ & $1 / 3$ & 50 & 50,05 & 0,09 & \multirow{6}{*}{29,916} & \multirow{6}{*}{10,730} & \multirow{6}{*}{0,720} \\
\hline 0,05 & 0,044 & $-12,42$ & $2 / 3$ & 30 & 30,38 & 1,27 & & & \\
\hline 0,05 & 0,058 & 15,21 & 1 & 23 & 22,69 & $-1,35$ & & & \\
\hline- & - & - & - & - & - & - & & & \\
\hline 0,50 & 0,625 & 25,02 & $2 / 3$ & 40 & 38,73 & $-3,18$ & & & \\
\hline 0,50 & 0,387 & $-22,56$ & 1 & 28 & 28,92 & 3,29 & & & \\
\hline
\end{tabular}

ВЛО В ОРГАНЕ ИЛИ В ТКАНИ В ЗАВИСИМОСТИ ОТ ОБЪЕМА ОБЛУЧЕНИЯ И СУММАРНОЙ ДОЗЫ

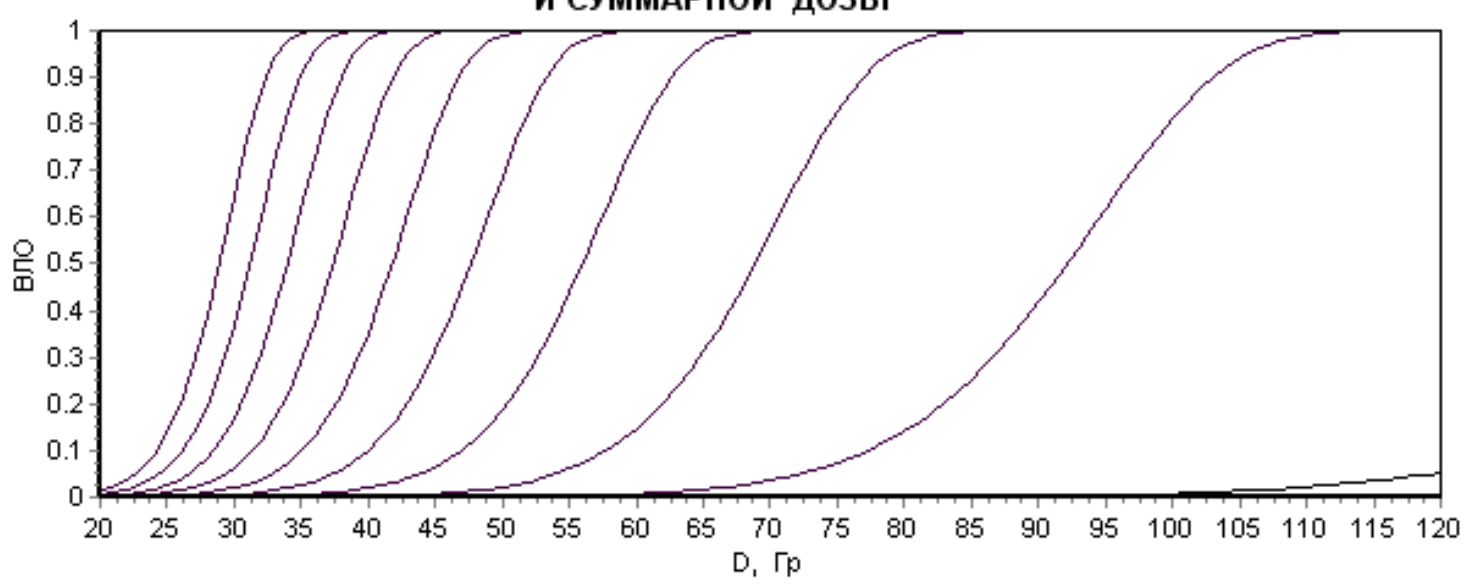

Рис. 4.12.3. Почки. Оптимизация по ВЛО+ТД 
4.13. Гортань 1. Осложнение: Некроз хряща

Таблица 4.13.1

Гортань 1. Оптимизация по ВЛО

\begin{tabular}{|c|c|c|c|c|c|c|c|c|c|}
\hline$P_{\text {Кл }}$ & $P_{\mathrm{TEOP}}$ & $\varepsilon, \%$ & $V$ & $D_{\text {Кл }}$ & $D_{\text {TEOP }}$ & $\varepsilon, \%$ & $A_{1}$ & $A_{2}$ & $b$ \\
\hline 0,05 & 0,054 & 7,01 & $1 / 3$ & 79 & 78,6 & $-0,48$ & \multirow{6}{*}{83,874} & \multirow{6}{*}{14,547} & \multirow{6}{*}{0,127} \\
\hline 0,05 & 0,034 & $-33,02$ & $2 / 3$ & 70 & 72,0 & 2,85 & & & \\
\hline 0,05 & 0,070 & 39,03 & 1 & 70 & 68,38 & $-2,31$ & & & \\
\hline 0,50 & 0,732 & 46,48 & $1 / 3$ & 90 & 86,11 & $-4,32$ & & & \\
\hline 0,50 & 0,395 & $-20,99$ & $2 / 3$ & 80 & 81,79 & 2,23 & & & \\
\hline 0,50 & 0,395 & $-20,99$ & 1 & 80 & 81,79 & 2,23 & & & \\
\hline
\end{tabular}

ВЛО В ОРГАНЕ ИЛИ В ТКАНИ В ЗАВИСИМОСТИ ОТ СУММАРНОЙ ДОЗЫ

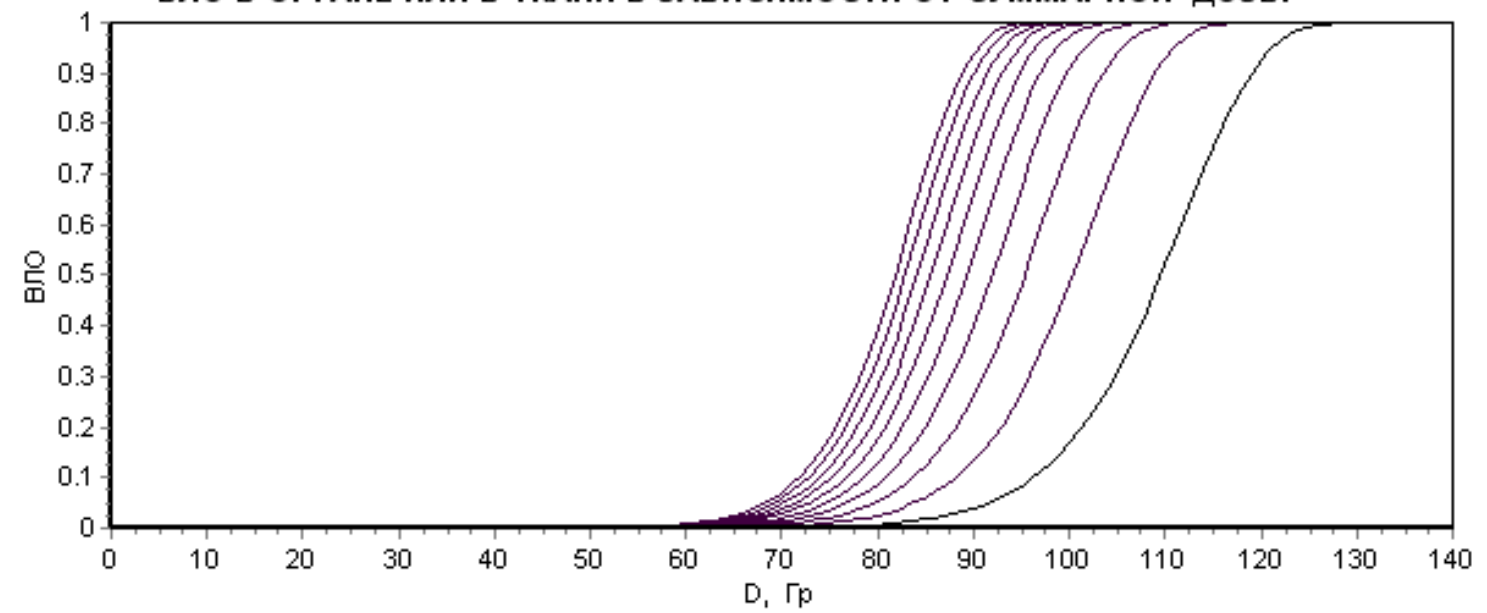

Рис. 4.13.1. Гортань 1. Оптимизация по ВЛО

Таблица 4.13.2

Гортань 1. Оптимизация по ТД

\begin{tabular}{|c|c|c|c|c|c|c|c|c|c|}
\hline$P_{\text {Кл }}$ & $P_{\mathrm{TEOP}}$ & $\varepsilon, \%$ & $V$ & $D_{\text {Кл }}$ & $D_{\text {TEOP }}$ & $\varepsilon, \%$ & $A_{1}$ & $A_{2}$ & $b$ \\
\hline 0,05 & 0,047 & $-7,10$ & $1 / 3$ & 79 & 79,43 & 0,54 & \multirow{6}{*}{83,755} & \multirow{6}{*}{14,058} & \multirow{6}{*}{0,144} \\
\hline 0,05 & 0,035 & $-30,56$ & $2 / 3$ & 70 & 71,88 & 2,69 & & & \\
\hline 0,05 & 0,077 & 54,31 & 1 & 70 & 67,80 & $-3,14$ & & & \\
\hline 0,50 & 0,701 & 40,33 & $1 / 3$ & 90 & 86,51 & $-3,88$ & & & \\
\hline 0,50 & 0,408 & $-18,34$ & $2 / 3$ & 80 & 81,60 & 2,0 & & & \\
\hline 0,50 & 0,408 & $-18,34$ & 1 & 80 & 81,60 & 2,0 & & & \\
\hline
\end{tabular}


ВЛО В ОРГАНЕ ИЛИ В ТКАНИ В ЗАВИСИМОСТИ ОТ СУММАРНОЙ ДОЗЫ

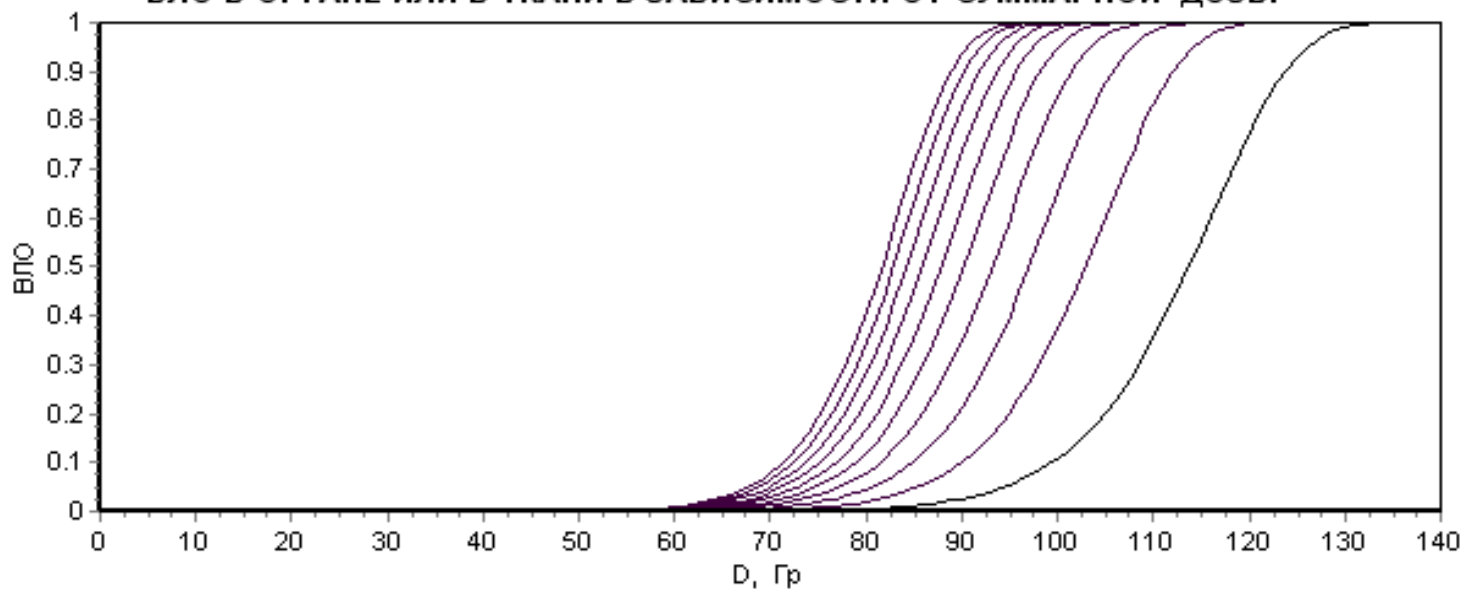

Рис. 4.13.2. Гортань 1. Оптимизация по ТД

Дальнейшие расчеты показали, что параметры модели МРВ для критерия ВЛО + ТД совпадают с параметрами, приведенными в табл. 13.1.1 (критерий ВЛО). Кроме того, параметры модели, определенные с критериями ВЛО, очень близки к параметрам модели, определенными с критерием ТД.

\subsection{4. Гортань 2. Отек гортани}

Таблица 4.14.1

Гортань 2. Оптимизация по ВЛО

\begin{tabular}{|c|c|c|c|c|c|c|c|c|c|}
\hline$P_{\text {Кл }}$ & $P_{\mathrm{TEOP}}$ & $\varepsilon, \%$ & $V$ & $D_{\text {Кл }}$ & $D_{\text {TEOP }}$ & $\varepsilon, \%$ & $A_{1}$ & $A_{2}$ & $b$ \\
\hline 0,05 & 0,046 & $-7,26$ & $1 / 3$ & 79 & 55,49 & 0,89 & \multirow{6}{*}{72,329} & \multirow{6}{*}{8,742} & \multirow{6}{*}{0,068} \\
\hline 0,05 & 0,069 & 38,35 & $2 / 3$ & 70 & 52,93 & $-3,76$ & & & \\
\hline 0,05 & 0,039 & $-22,25$ & 1 & 70 & 51,49 & 2,99 & & & \\
\hline 0,50 & 0,446 & $-10,84$ & $1 / 3$ & 90 & 71,30 & 1,86 & & & \\
\hline 0,50 & 0,528 & 5,63 & $2 / 3$ & 80 & 69,36 & $-0,91$ & & & \\
\hline 0,50 & 0,528 & 5,63 & 1 & 80 & 69,36 & $-0,91$ & & & \\
\hline
\end{tabular}

ВЛО В ОРГАНЕ ИЛИ В ТКАНИ В ЗАВИСИМОСТИ ОТ СУММАРНОЙ ДОЗЫ

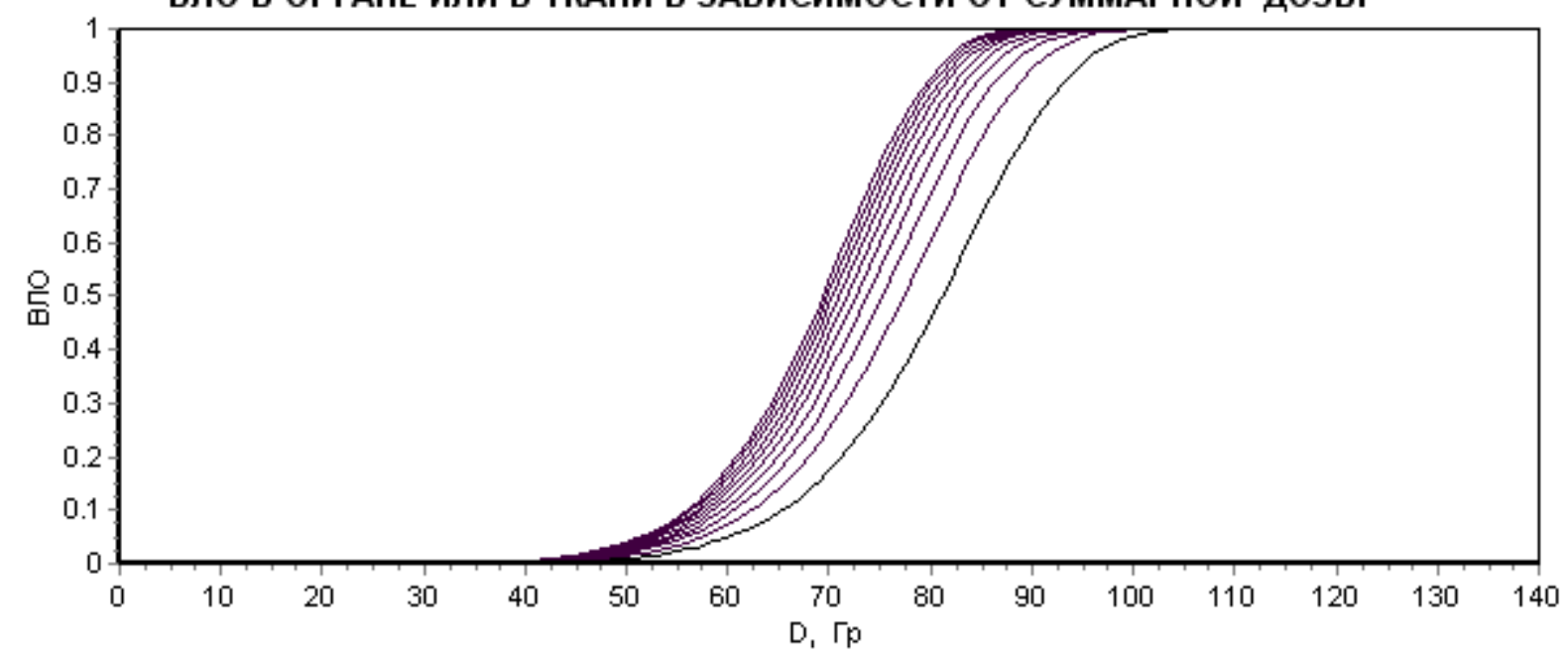

Рис. 4.14.1. Гортань 2. Оптимизация по ВЛО 
Таблица 4.14.2

Гортань 2. Оптимизация по ТД

\begin{tabular}{|c|c|c|c|c|c|c|c|c|c|}
\hline$P_{\text {Кл }}$ & $P_{\mathrm{TEOP}}$ & $\varepsilon, \%$ & $V$ & $D_{\text {КЛ }}$ & $D_{\text {TEOP }}$ & $\varepsilon, \%$ & $A_{1}$ & $A_{2}$ & $b$ \\
\hline 0,05 & 0,047 & $-6,80$ & $1 / 3$ & 79 & 55,46 & 0,83 & \multirow{6}{*}{72,335} & \multirow{6}{*}{8,752} & \multirow{6}{*}{0,067} \\
\hline 0,05 & 0,069 & 38,30 & $2 / 3$ & 70 & 52,94 & $-3,75$ & & & \\
\hline 0,05 & 0,039 & $-22,61$ & 1 & 70 & 51,52 & 3,04 & & & \\
\hline 0,50 & 0,447 & $-10,70$ & $1 / 3$ & 90 & 71,28 & 1,83 & & & \\
\hline 0,50 & 0,528 & 5,56 & $2 / 3$ & 80 & 69,37 & $-0,90$ & & & \\
\hline 0,50 & 0,528 & 5,56 & 1 & 80 & 69,37 & $-0,90$ & & & \\
\hline
\end{tabular}

ВЛО В ОРГАНЕ ИЛИ В ТКАНИ В ЗАВИСИМОСТИ ОТ СУММАРНОЙ ДОЗЫ

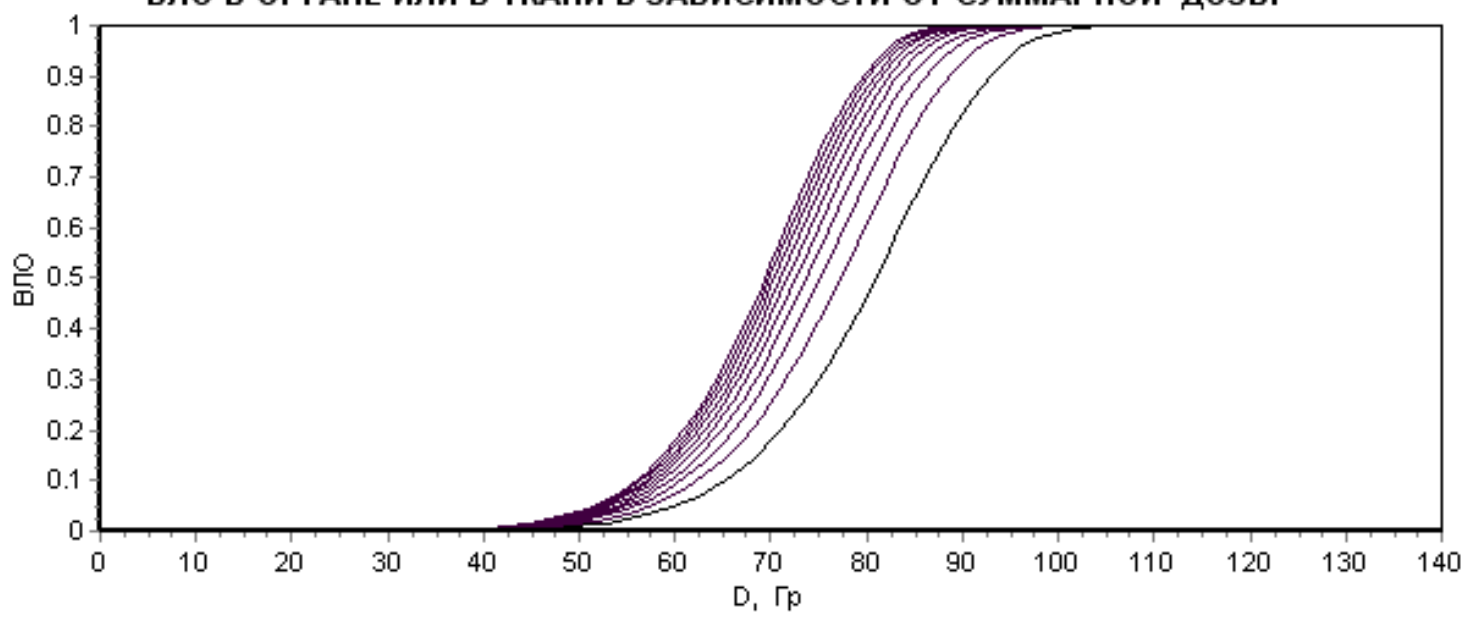

Рис. 4.14.2. Гортань 2. Оптимизация по ТД

Дальнейшие расчеты показали, что параметры модели МРВ для критерия оптимальности ВЛО + ТД совпадают с параметрами, приведенными в табл. 4.14.1 (критерий ВЛО). Кроме того, параметры модели, определенные с критерием ВЛО очень близки к параметрам модели, определенным с критерием ТД

\subsection{5. Хрусталик.}

Осложнение: катаракта, требующая вмешательства

Таблица 4.15

Хрусталик

\begin{tabular}{c|c|c|c|c|c}
\hline$P_{\text {Кл }}$ & $D_{\text {КЛ }}$ & $P_{\text {КЛ }}$ & $D_{\text {Кл }}$ & $A_{1}$ & $A_{2}$ \\
\hline 0,05 & 10,0 & 0,50 & 18,0 & 19,553 & 4,430 \\
\hline
\end{tabular}




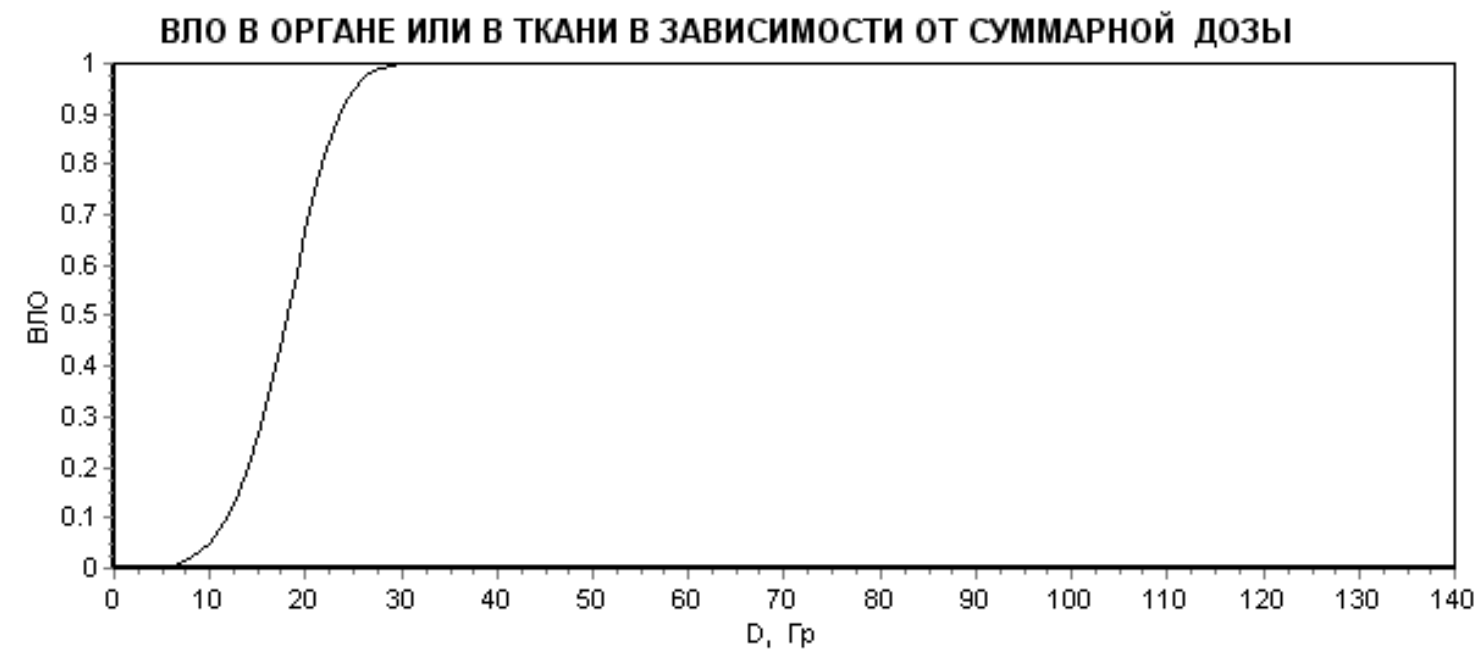

Рис. 4.15. Хрусталик. Зависимость ВЛО от дозы

\subsection{6. Печень. Осложнение: печеночная недостаточность}

Таблица 4.16.1

Печень. Оптимизация по ВЛО

\begin{tabular}{|c|c|c|c|c|c|c|c|c|c|}
\hline$P_{\text {Кл }}$ & $P_{\mathrm{TEOP}}$ & $\varepsilon, \%$ & $V$ & $D_{\text {КЛ }}$ & $D_{\mathrm{TEOP}}$ & $\varepsilon, \%$ & $A_{1}$ & $A_{2}$ & $b$ \\
\hline 0,05 & 0,069 & 38,02 & $1 / 3$ & 50 & 48,67 & $-2,66$ & \multirow{6}{*}{38,243} & \multirow{6}{*}{12,314} & \multirow{6}{*}{0,439} \\
\hline 0,05 & 0,037 & $-26,37$ & $2 / 3$ & 35 & 35,00 & 2,67 & & & \\
\hline 0,05 & 0,049 & $-1,86$ & 1 & 30 & 30,05 & 0,16 & & & \\
\hline 0,50 & 0,206 & $-58,71$ & $1 / 3$ & 55 & 60,13 & 9,32 & & & \\
\hline 0,50 & 0,563 & 12,64 & $2 / 3$ & 45 & 44,35 & $-1,44$ & & & \\
\hline 0,50 & 0,824 & 64,85 & 1 & 40 & 37,12 & $-7,20$ & & & \\
\hline
\end{tabular}

ВЛО В ОРГАНЕ ИЛИ В ТКАНИ В ЗАВИСИМОСТИ ОТ ОБЪЕМА ОБЛУЧЕНИЯ И СУММАРНОЙ ДОЗЫ

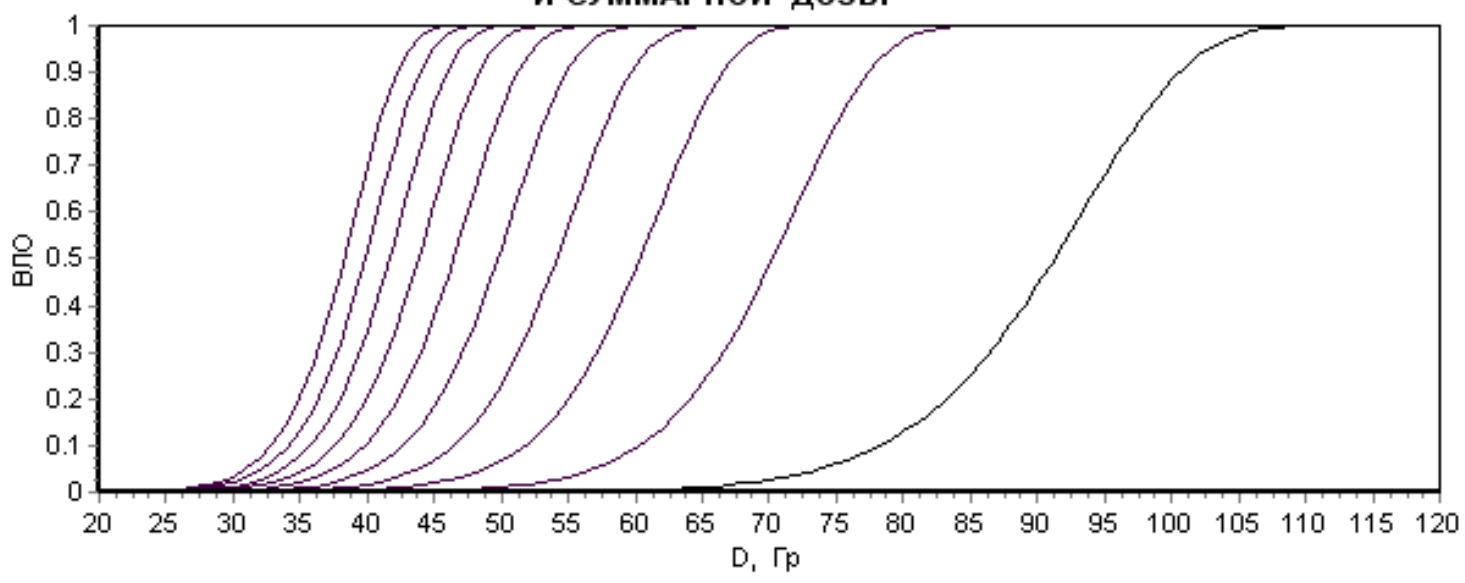

Рис. 4.16.1. Печень. Оптимизация по ВЛО 
Таблица 4.16 .2

Печень. Оптимизация по ТД

\begin{tabular}{|c|c|c|c|c|c|c|c|c|c|}
\hline$P_{\text {КЛ }}$ & $P_{\mathrm{TEOP}}$ & $\varepsilon, \%$ & $V$ & $D_{\text {КЛ }}$ & $D_{\mathrm{TEOP}}$ & $\varepsilon, \%$ & $A_{1}$ & $A_{2}$ & $b$ \\
\hline 0,05 & 0,107 & 113,36 & $1 / 3$ & 50 & 46,90 & $-6,20$ & \multirow{6}{*}{39,450} & \multirow{6}{*}{12,314} & \multirow{6}{*}{0,377} \\
\hline 0,05 & 0,034 & $-31,47$ & $2 / 3$ & 35 & 36,12 & 3,19 & & & \\
\hline 0,05 & 0,034 & $-32,54$ & 1 & 30 & 31,00 & 3,32 & & & \\
\hline 0,50 & 0,306 & $-38,86$ & $1 / 3$ & 55 & 57,94 & 5,35 & & & \\
\hline 0,50 & 0,537 & $\begin{array}{l}7,38 \\
\end{array}$ & $2 / 3$ & 45 & 44,62 & $-0,85$ & & & \\
\hline 0,50 & 0,694 & 38,90 & 1 & 40 & 0,694 & 38,90 & & & \\
\hline
\end{tabular}

ВЛО В ОРГАНЕ ИЛИ В ТКАНИ В ЗАВИСИМОСТИ ОТ ОБЪЕМА ОБЛУЧЕНИЯ И СУММАРНОЙ ДОЗЫ

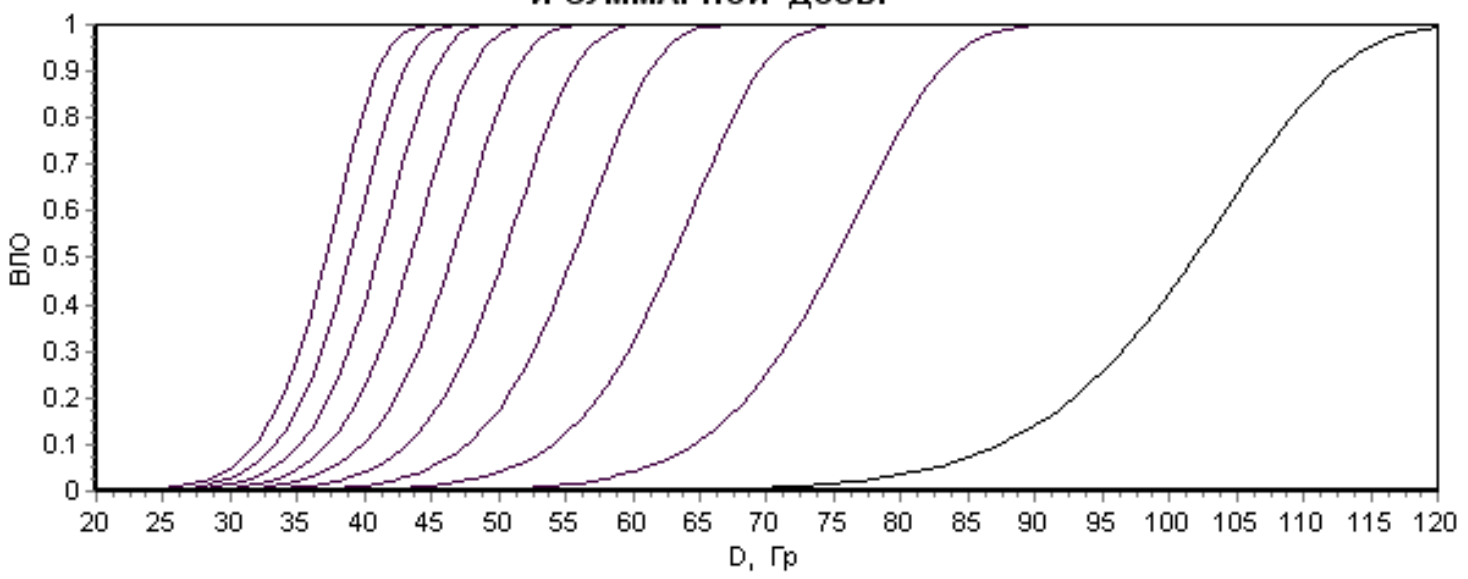

Рис. 4.16.2. Печень. Оптимизация по ТД

Таблица 4.16 .3

Печень. Оптимизация по ВЛО + ТД

\begin{tabular}{|c|c|c|c|c|c|c|c|c|c|}
\hline$P_{\text {Кл }}$ & $P_{\mathrm{TEOP}}$ & $\varepsilon, \%$ & $V$ & $D_{\text {Кл }}$ & $D_{\mathrm{TEOP}}$ & $\varepsilon, \%$ & $A_{1}$ & $A_{2}$ & $b$ \\
\hline 0,05 & 0,069 & 38,99 & $1 / 3$ & 50 & 48,64 & $-2,72$ & \multirow{6}{*}{38,262} & \multirow{6}{*}{12,314} & \multirow{6}{*}{0,438} \\
\hline 0,05 & 0,037 & $-26,45$ & $2 / 3$ & 35 & 35,90 & 2,58 & & & \\
\hline 0,05 & 0,049 & $-2,45$ & 1 & 30 & 30,06 & 0,21 & & & \\
\hline 0,50 & 0,208 & $-58,44$ & $1 / 3$ & 55 & 60,09 & 9,26 & & & \\
\hline 0,50 & 0,0563 & 12,56 & $2 / 3$ & 45 & 44,36 & $-1,43$ & & & \\
\hline 0,50 & 0,822 & 64,47 & 1 & 40 & 37,14 & $-7,15$ & & & \\
\hline
\end{tabular}




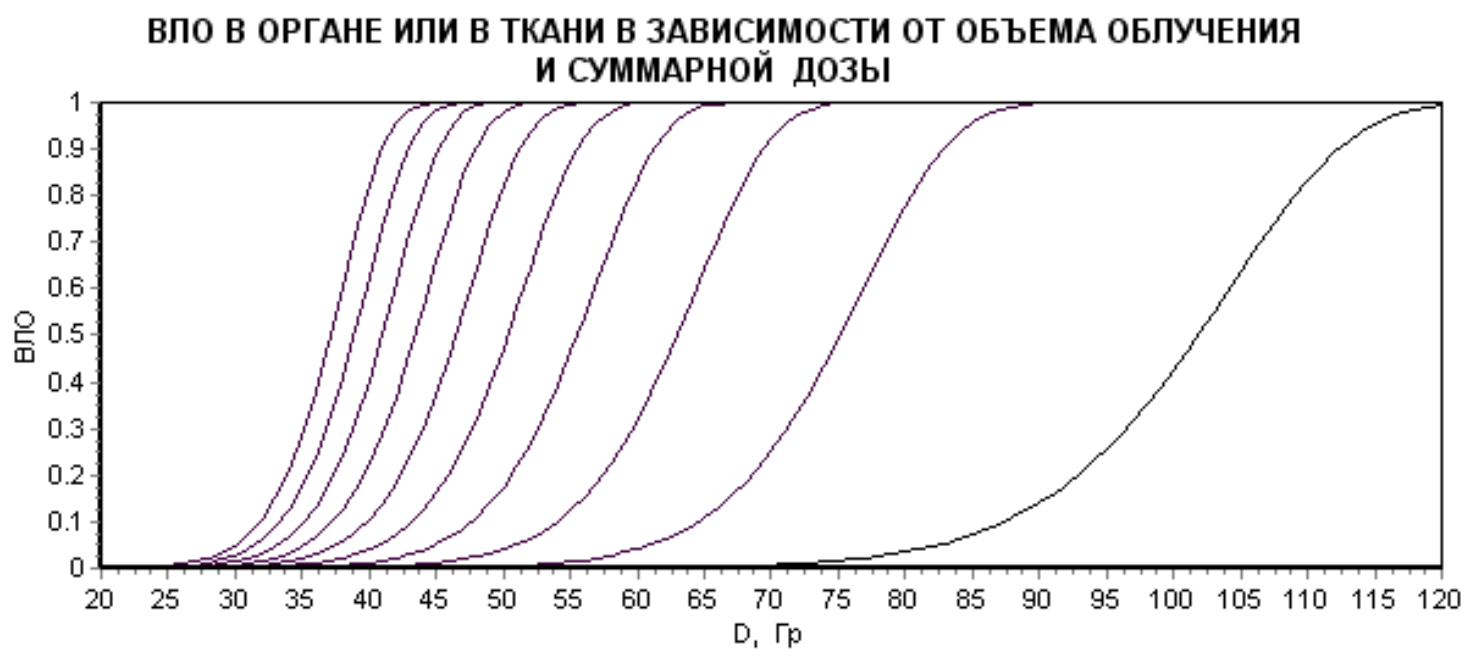

Рис. 4.16.3. Печень. Оптимизация по ВЛО + ТД

Параметры модели МРВ в табл. 4.16.4, определенные для ВЛО = 0,05 и ВЛО $=0,50$, отличаются друг от друга. Клиническая информация плохо согласована.

Таблица 4.16.4

Печень. Оптимизация по ТД, отдельно для ВЛО = 0,05 и ВЛО = 0,50

\begin{tabular}{|c|c|c|c|c|c|c|c|c|c|}
\hline$P_{\text {Кл }}$ & $P_{\mathrm{TEOP}}$ & $\varepsilon, \%$ & $V$ & $D_{\text {Кл }}$ & $D_{\text {TEOP }}$ & $\varepsilon, \%$ & $A_{1}$ & $A_{2}$ & $b$ \\
\hline 0,05 & 0,051 & 2,12 & $1 / 3$ & 50 & 49,64 & $-0,72$ & \multirow{3}{*}{80,554} & \multirow{3}{*}{2,962} & \multirow{3}{*}{0,472} \\
\hline 0,05 & 0,047 & $-2,63$ & $2 / 3$ & 35 & 35,79 & 2,25 & & & \\
\hline 0,05 & 0,052 & 4,42 & 1 & 30 & 29,55 & $-1,49$ & & & \\
\hline 0,50 & 0,500 & $-0,01$ & $1 / 3$ & 55 & 55,08 & 0,14 & \multirow{3}{*}{447,363} & \multirow{3}{*}{0,152} & \multirow{3}{*}{0,292} \\
\hline 0,50 & 0,500 & 0,01 & $2 / 3$ & 45 & 44,98 & $-0,04$ & & & \\
\hline 0,50 & 0,500 & 0,01 & 1 & 40 & 39,96 & $-0,10$ & & & \\
\hline
\end{tabular}

\subsection{7. Легкие. Осложнения: пневмониты}

Таблица 4.17.1

Легкие. Оптимизация по ВЛО

\begin{tabular}{|c|c|c|c|c|c|c|c|c|c|}
\hline$P_{\text {КЛ }}$ & $P_{\mathrm{TEOP}}$ & $\varepsilon, \%$ & $V$ & $D_{\text {Кл }}$ & $D_{\text {TEOP }}$ & $\varepsilon, \%$ & $A_{1}$ & $A_{2}$ & $b$ \\
\hline 0,05 & 0,051 & 1,58 & $1 / 3$ & 45 & 44,91 & $-0,20$ & \multirow{6}{*}{28,727} & \multirow{6}{*}{7,875} & \multirow{6}{*}{0,750} \\
\hline 0,05 & 0,120 & 140,84 & $2 / 3$ & 30 & 26,70 & $-10,99$ & & & \\
\hline 0,05 & 0,020 & $-60,05$ & 1 & 17,5 & 19,70 & $-60,05$ & & & \\
\hline 0,50 & 0,611 & 22,14 & $1 / 3$ & 65 & 62,51 & $-3,84$ & & & \\
\hline 0,50 & 0,710 & 41,91 & $2 / 3$ & 40 & 37,17 & $-7,09$ & & & \\
\hline 0,50 & 0,248 & $-50,36$ & 1 & 24,5 & 27,41 & 11,92 & & & \\
\hline
\end{tabular}




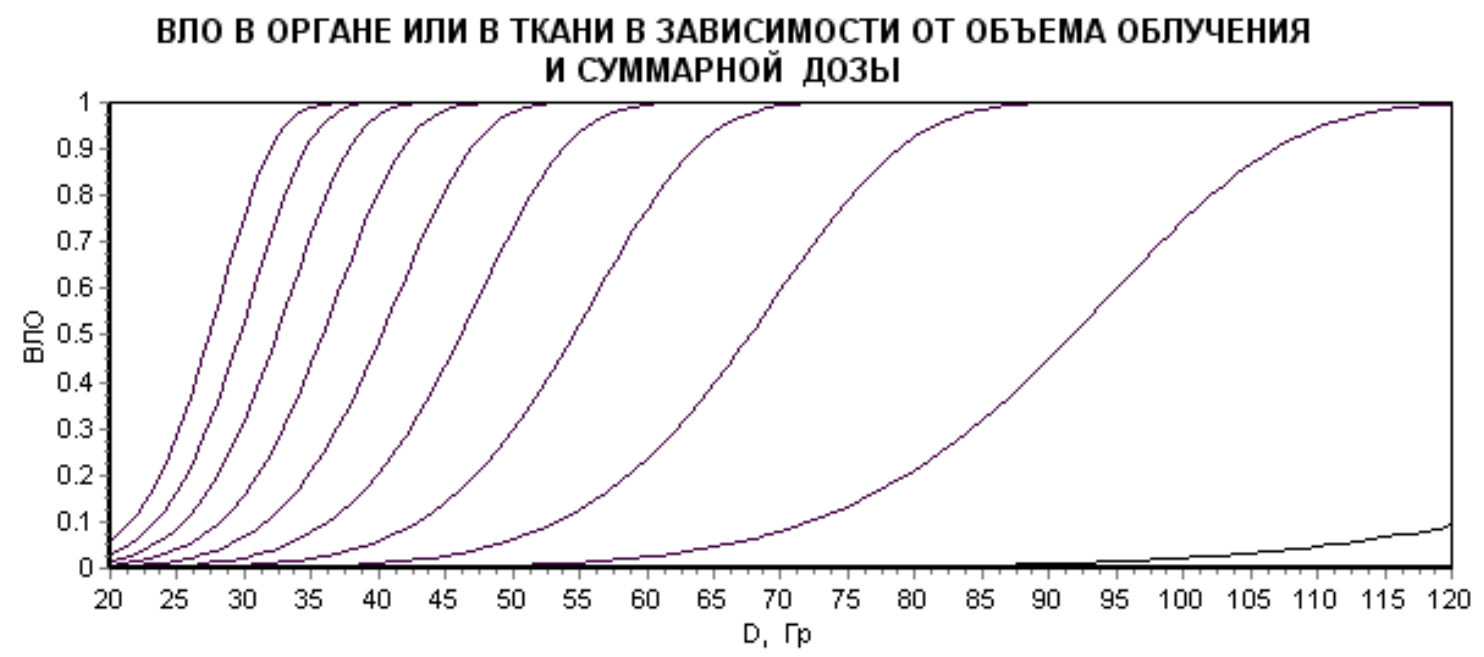

Рис. 4.17.1. Легкие. Оптимизация по ВЛО

Таблица 4.17.2

Легкие. Оптимизация по ТД

\begin{tabular}{|c|c|c|c|c|c|c|c|c|c|}
\hline$P_{\text {Кл }}$ & $P_{\mathrm{TEOP}}$ & $\varepsilon, \%$ & $V$ & $D_{\text {Кл }}$ & $D_{\text {TEOP }}$ & $\varepsilon, \%$ & $A_{1}$ & $A_{2}$ & $b$ \\
\hline 0,05 & 0,031 & $-37,09$ & $1 / 3$ & 45 & 47,49 & 6,19 & \multirow{6}{*}{27,268} & \multirow{6}{*}{7,875} & \multirow{6}{*}{0,854} \\
\hline 0,05 & 0,129 & 159,18 & $2 / 3$ & 30 & 26,44 & $-11,87$ & & & \\
\hline 0,05 & 0,030 & $-40,07$ & 1 & 17,5 & 18,70 & 6,86 & & & \\
\hline 0,50 & 0,439 & $-12,16$ & $1 / 3$ & 65 & 66,51 & 2,32 & & & \\
\hline 0,50 & 0,737 & 47,49 & $2 / 3$ & 40 & 36,80 & $-8,01$ & & & \\
\hline 0,50 & 0,359 & $-30,04$ & 1 & 24,5 & 26,03 & 6,25 & & & \\
\hline
\end{tabular}

ВЛО В ОРГАНЕ ИЛИ В ТКАНИ В ЗАВИСИМОСТИ ОТ ОБЪЕМА ОБЛУЧЕНИЯ И СУММАРНОЙ ДОЗЫ

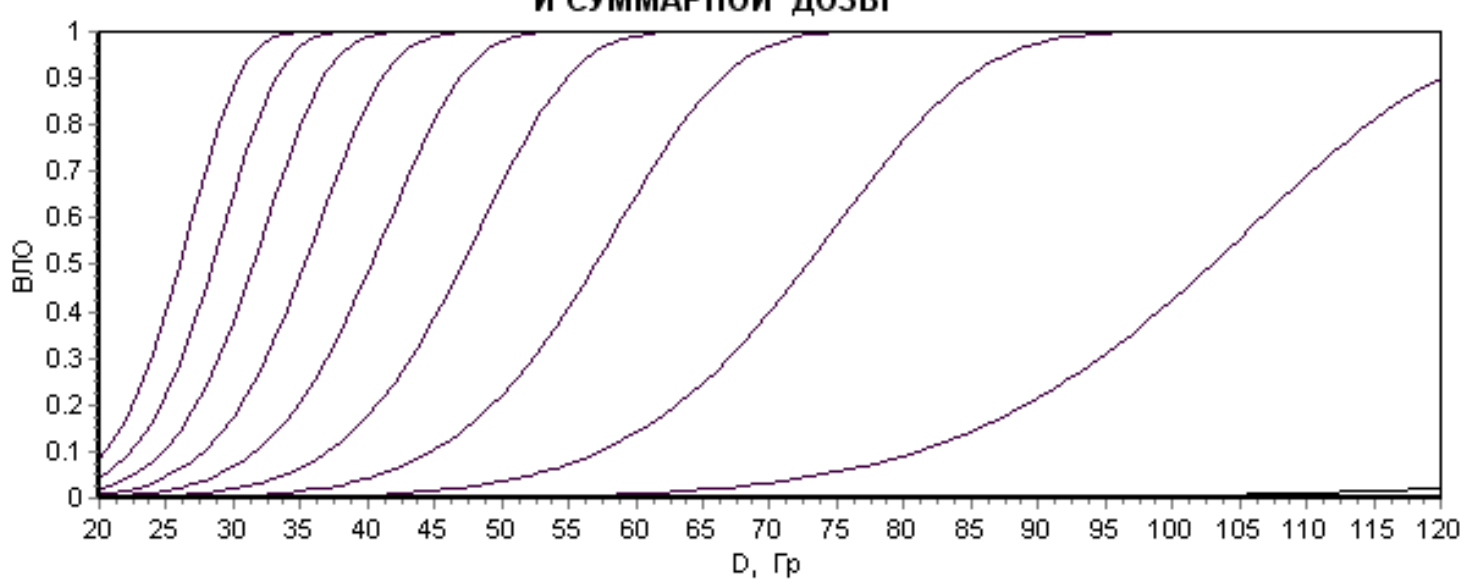

Рис. 4.17.2. Легкие. Оптимизация по ТД 
Таблица 4.17 .3

Легкие. Оптимизация по ВЛО + ТД

\begin{tabular}{|c|c|c|c|c|c|c|c|c|c|}
\hline$P_{\text {Кл }}$ & $P_{\mathrm{TEOP}}$ & $\varepsilon, \%$ & $V$ & $\overline{D_{\text {Кл }}}$ & $D_{\mathrm{TEOP}}$ & $\varepsilon, \%$ & $A_{1}$ & $\overline{A_{2}}$ & $b$ \\
\hline 0,05 & 0,050 & $-0,27$ & $1 / 3$ & 45 & 45,02 & 0,03 & \multirow{6}{*}{28,670} & \multirow{6}{*}{7,875} & \multirow{6}{*}{0,754} \\
\hline 0,05 & 0,121 & 141,52 & $2 / 3$ & 30 & 26,69 & $-11,02$ & & & \\
\hline 0,05 & 0,020 & $-59,42$ & 1 & 17,5 & 19,66 & 12,35 & & & \\
\hline 0,50 & 0,604 & 20,76 & $1 / 3$ & 65 & 62,65 & $-3,61$ & & & \\
\hline 0,50 & 0,711 & 42,13 & $2 / 3$ & 40 & 37,15 & $-7,12$ & & & \\
\hline 0,50 & 0,251 & $-49,64$ & 1 & 24,5 & 27,37 & 11,70 & & & \\
\hline
\end{tabular}

ВЛО В ОРГАНЕ ИЛИ В ТКАНИ В ЗАВИСИМОСТИ ОТ ОБЪЕМА ОБЛУЧЕНИЯ И СУММАРНОЙ ДОЗЫ

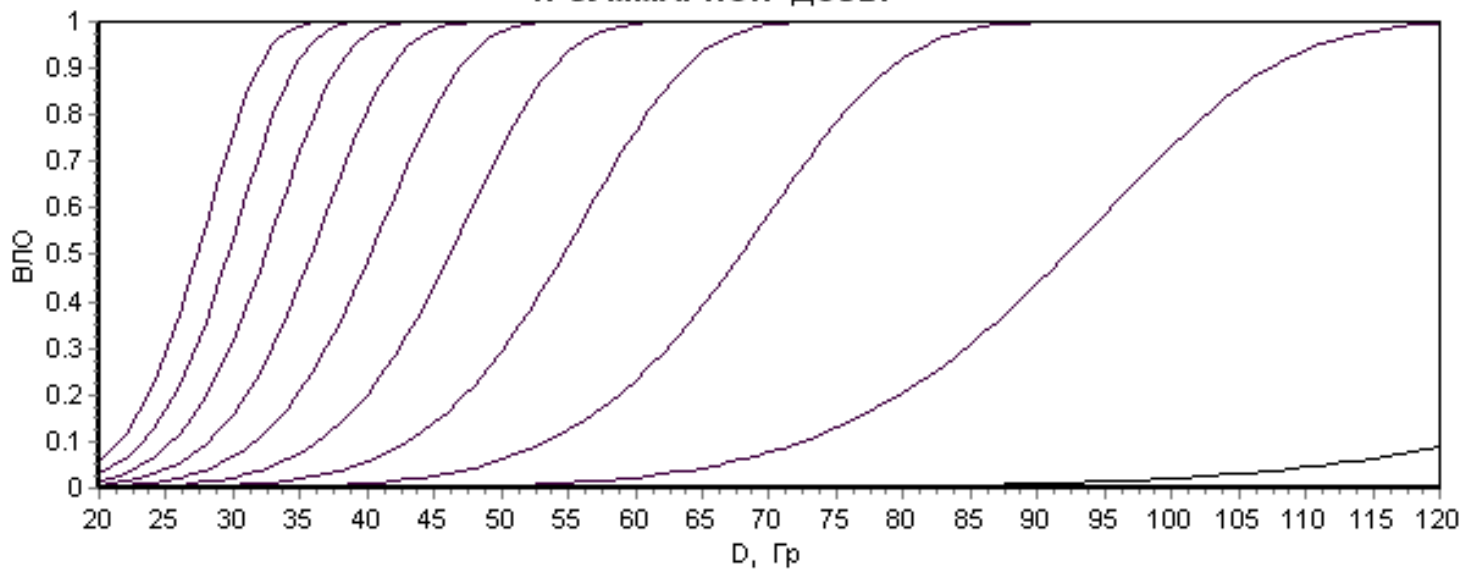

Рис. 4.17.3. Легкие. Оптимизация по ВЛО + ТД

Параметры модели МРВ в табл. 4.17.4 были определены для ВЛО = 0,05 и ВЛО $=0,50$. Они серьезно отличаются друг от друга. Клиническая информация плохо согласована.

Таблица 4.17.4

Легкие. Оптимизация по ТД, отдельно для ВЛО = 0,05 и ВЛО = 0,50

\begin{tabular}{|c|c|c|c|c|c|c|c|c|c|}
\hline$P_{\text {Кл }}$ & $P_{\mathrm{TEOP}}$ & $\varepsilon, \%$ & $V$ & $D_{\text {Кл }}$ & $D_{\text {TEOP }}$ & $\varepsilon, \%$ & $A_{1}$ & $A_{2}$ & $b$ \\
\hline 0,05 & 0,029 & $-41,34$ & $1 / 3$ & 45 & 47,27 & 5,06 & \multirow{3}{*}{24,701} & \multirow{3}{*}{11,030} & \multirow{3}{*}{0,836} \\
\hline 0,05 & 0,184 & 267,29 & $2 / 3$ & 30 & 26,48 & $-11,72$ & & & \\
\hline 0,05 & 0,022 & $-55,81$ & 1 & 17,5 & 18,87 & 7,83 & & & \\
\hline 0,50 & 0,497 & $-0,71$ & $1 / 3$ & 65 & 66,59 & 2,45 & \multirow{3}{*}{62,045} & \multirow{3}{*}{0,421} & \multirow{3}{*}{0,856} \\
\hline 0,50 & 0,512 & 2,46 & $2 / 3$ & 40 & 36,79 & $-8,03$ & & & \\
\hline 0,50 & 0,491 & $-1,73$ & 1 & 24,5 & 26,00 & 6,13 & & & \\
\hline
\end{tabular}


4.18. Зрительный нерв. Осложннение: слепота

Таблица 4.18.1

Зрительный нерв

\begin{tabular}{c|c|c|c|c|c}
\hline$P_{\text {КЛ }}$ & $D_{\text {Кл }}$ & $P_{\text {КЛ }}$ & $D_{\text {КЛ }}$ & $A_{1}$ & $A_{2}$ \\
\hline 0,05 & 50,0 & 0,50 & 65,0 & 67,446 & 9,924 \\
\hline
\end{tabular}

ВЛО В ОРГАНЕ ИЛИ В ТКАНИ В ЗАВИСИМОСТИ ОТ СУММАРНОЙ ДОЗЫ

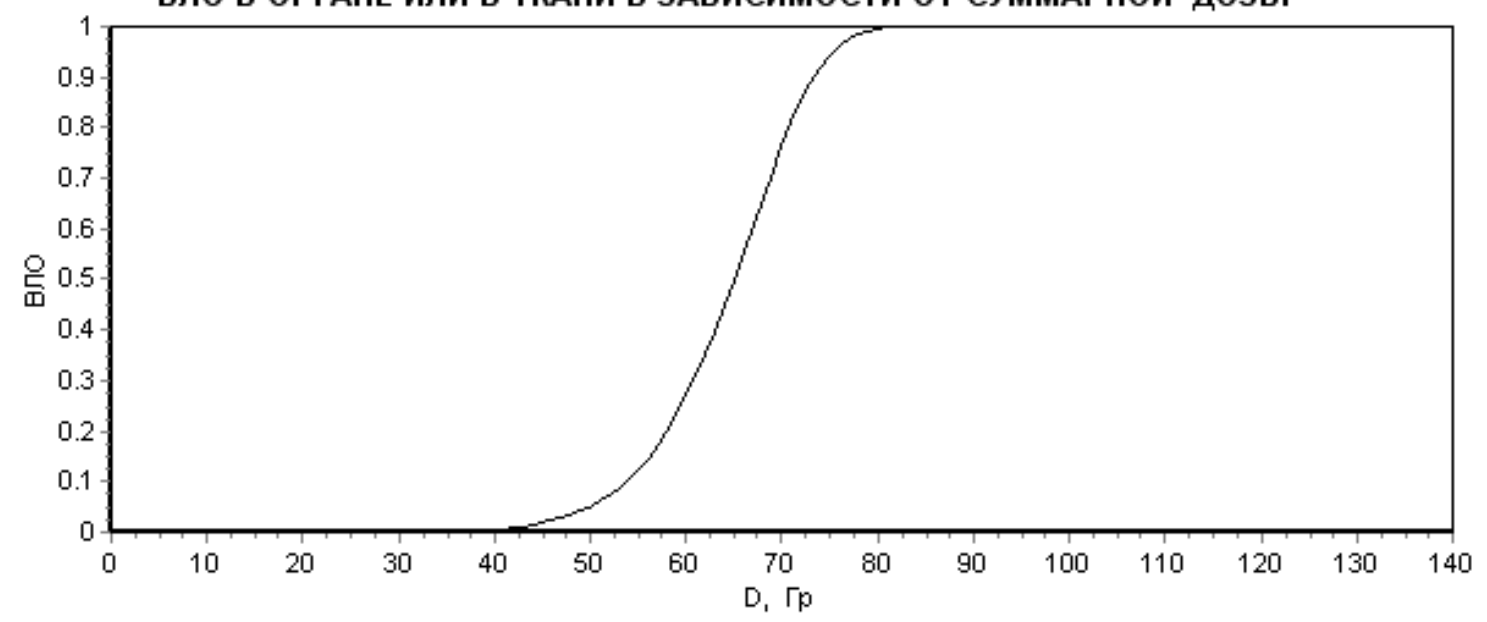

Рис. 4.18.1. Зрительный нерв. Зависимость ВЛО от дозы

\subsection{9. Зрительный нерв. Осложнение: хиазма зрительного нерва}

Параметры модели МРВ оказались одинаковыми для разных критериев оптимальности, см. табл. 4.18.1 и рис. 4.18.1.

4.20. Околоушная слюнная железа. Осложнения: ксеростомия

Таблица 4.20

Околоушная слюнная железа

\begin{tabular}{c|c|c|c|c|c}
\hline$P_{\text {КЛ }}$ & $D_{\text {КЛ }}$ & $P_{\text {КЛ }}$ & $D_{\text {КЛ }}$ & $A_{1}$ & $A_{2}$ \\
\hline 0,05 & 32,0 & 0,50 & 46,0 & 48,411 & 7,175 \\
\hline
\end{tabular}




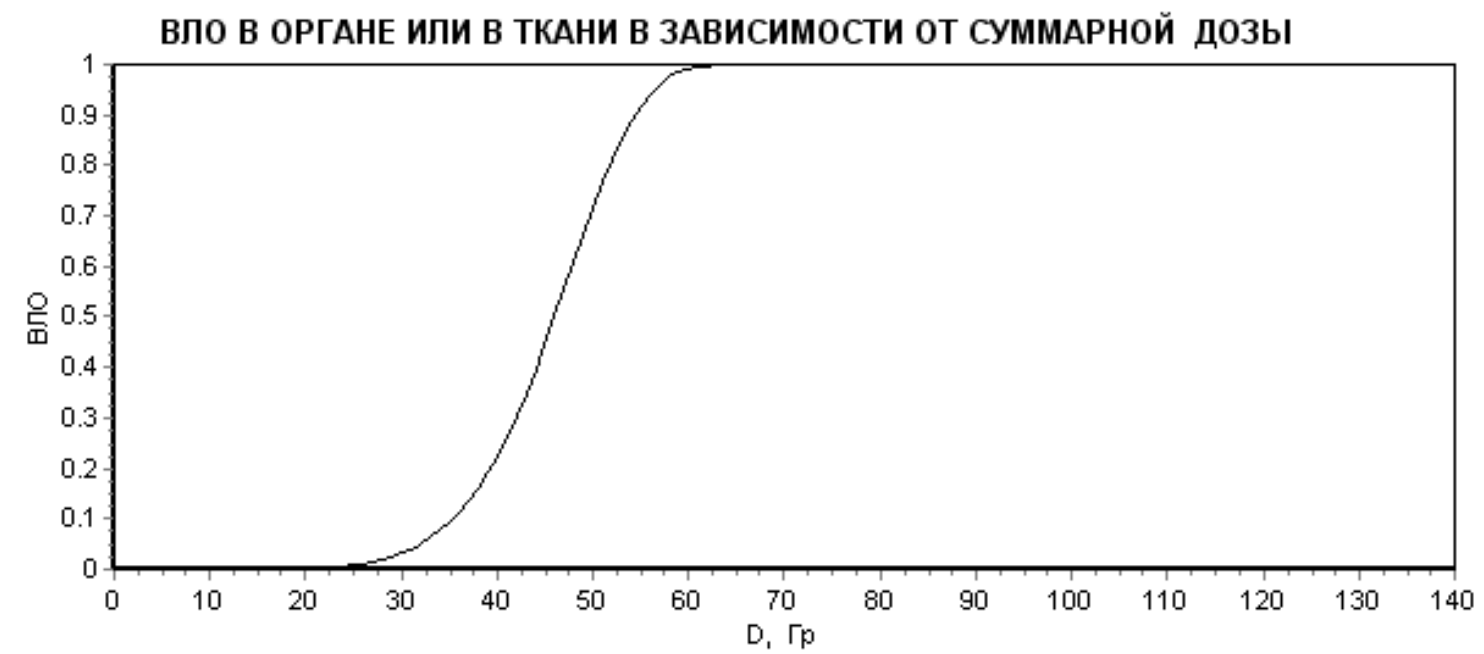

Рис. 4.20. Околоушная слюнная жселеза. Зависимость ВЛО от дозы

\subsection{1. Прямая кишка.}

Осложнения: тяжелый практит, некроз, стеноз, свищ

Таблица 4.21

\section{Прямая кишка}

\begin{tabular}{c|c|c|c|c|c}
\hline$P_{\text {КЛ }}$ & $D_{\text {КЛ }}$ & $P_{\text {КЛ }}$ & $D_{\text {КЛ }}$ & $A_{1}$ & $A_{2}$ \\
\hline 0,05 & 60,0 & 0,50 & 80,0 & 83,306 & 9,051 \\
\hline
\end{tabular}

ВЛО В ОРГАНЕ ИЛИ В ТКАНИ В ЗАВИСИМОСТИ ОТ СУММАРНОЙ ДОЗЫ

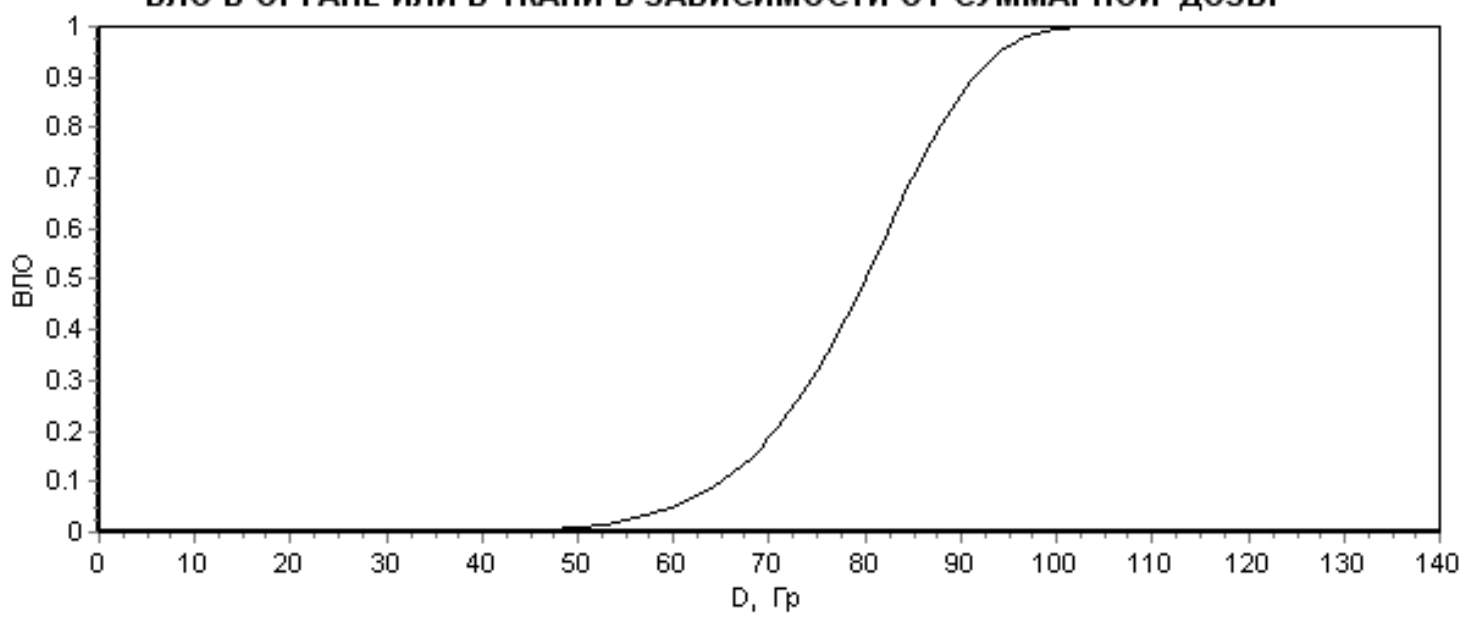

Рис. 4.21. Прямая кишка. Зависимость ВЛО от дозы 


\section{Сетчатка}

\begin{tabular}{c|c|c|c|c|c}
\hline$P_{\text {КЛ }}$ & $D_{\text {КЛ }}$ & $P_{\text {КЛ }}$ & $D_{\text {КЛ }}$ & $A_{1}$ & $A_{2}$ \\
\hline 0,05 & 45,0 & 0,50 & 65,0 & 68,453 & 7,081 \\
\hline
\end{tabular}

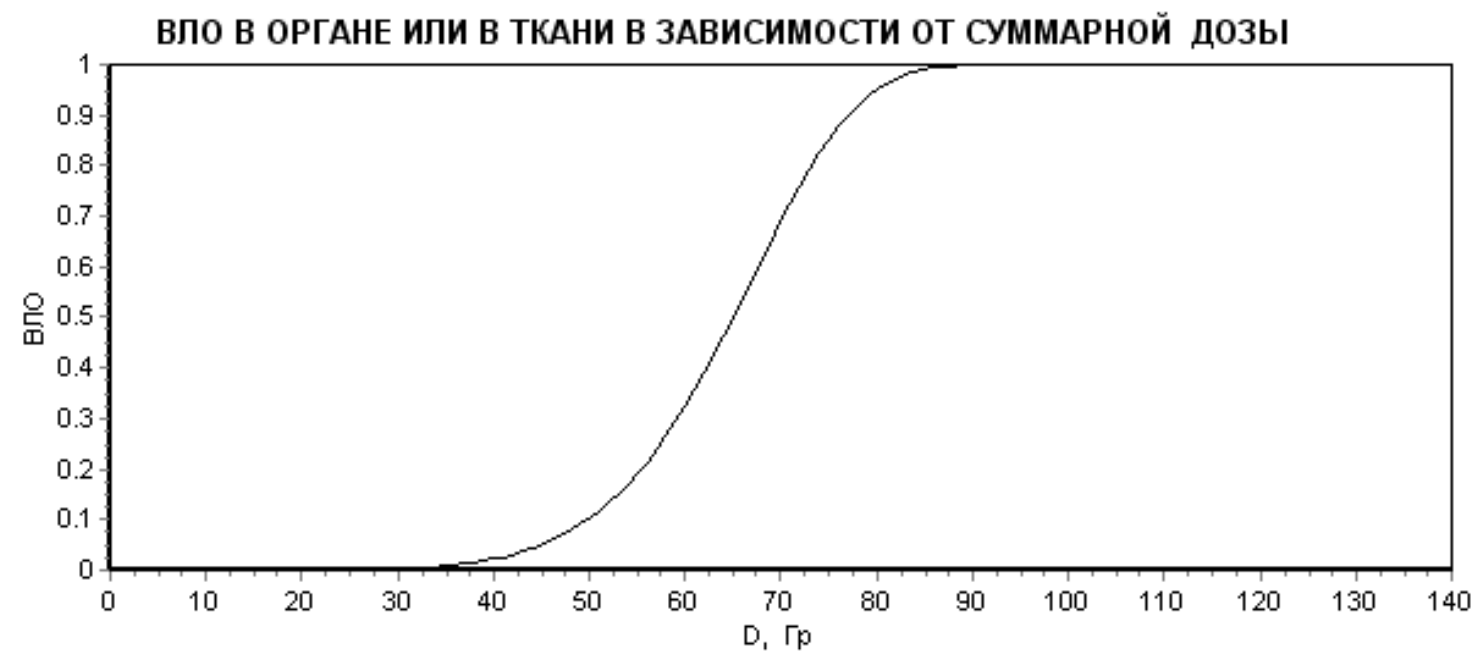

Рис. 4.22. Сетчатка. Зависимость ВЛО от дозы

\subsection{3. Ребро грудной клетки. Осложнение: патологический перелом}

Таблица 4.23

Ребро грудной клетки

\begin{tabular}{c|c|c|c|c|c}
\hline$P_{\text {КЛ }}$ & $D_{\text {КЛ }}$ & $P_{\text {КЛ }}$ & $D_{\text {КЛ }}$ & $A_{1}$ & $A_{2}$ \\
\hline 0,05 & 50,0 & 0,50 & 65,0 & 67,446 & 9,924 \\
\hline
\end{tabular}

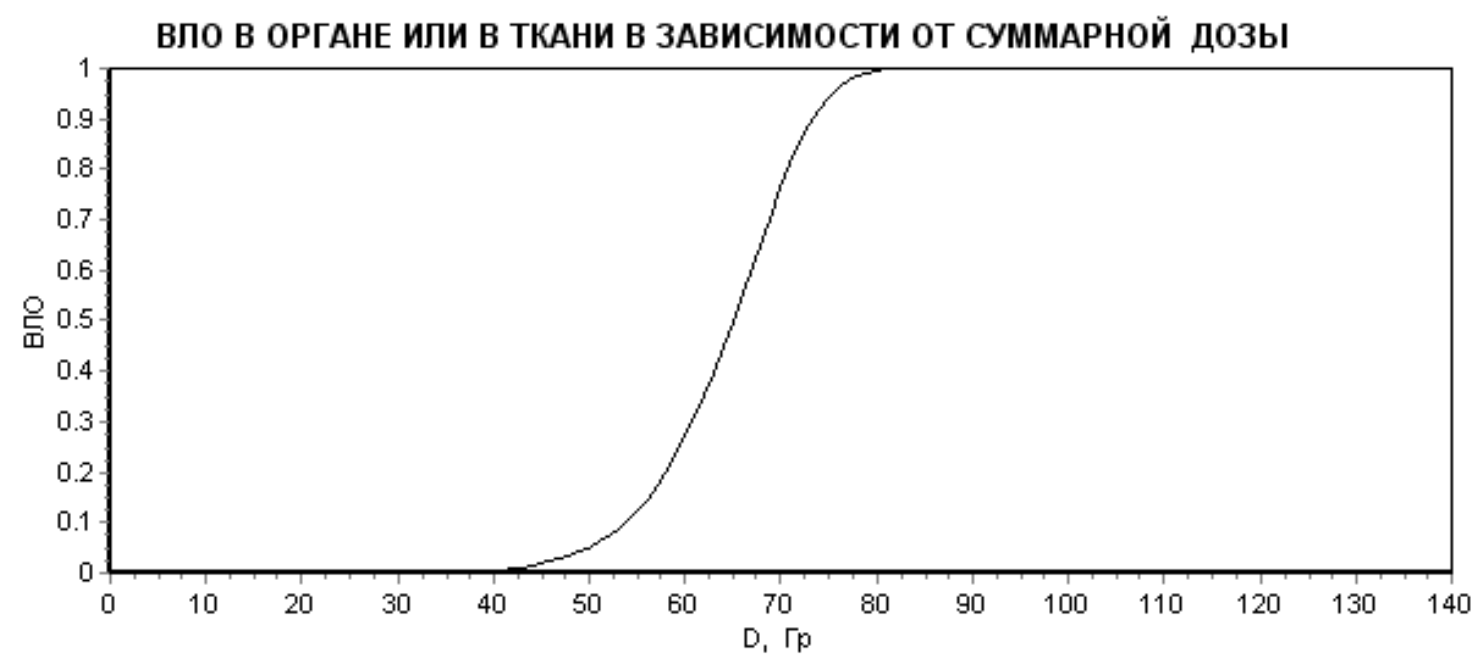

Рис. 4.23. Ребро грудной клетки. Зависимость ВЛО от дозы 
Таблица 4.24.1

Кожа. Оптимизация по ВЛО

\begin{tabular}{|c|c|c|c|c|c|c|c|c|c|}
\hline$P_{\text {Кл }}$ & $P_{\mathrm{TEOP}}$ & $\varepsilon, \%$ & $V$ & $D_{\text {КЛ }}$ & $D_{\mathrm{TEOP}}$ & $\varepsilon, \%$ & $A_{1}$ & $A_{2}$ & $b$ \\
\hline 0,05 & 0,043 & $-14,14$ & $1 / 3$ & 70 & 70,60 & 0,86 & \multirow{6}{*}{63,303} & \multirow{6}{*}{18,304} & \multirow{6}{*}{0,247} \\
\hline 0,05 & 0,058 & 16,39 & $2 / 3$ & 60 & 59,49 & $-0,85$ & & & \\
\hline 0,05 & 0,073 & $-85,32$ & 1 & 55 & 62,05 & 12,82 & & & \\
\hline- & - & - & - & - & - & - & & & \\
\hline- & - & - & - & - & - & - & & & \\
\hline 0,50 & 0,998 & 99,63 & 1 & 70 & 62,05 & $-11,35$ & & & \\
\hline
\end{tabular}

ВЛО В ОРГАНЕ ИЛИ В ТКАНИ В ЗАВИСИМОСТИ ОТ ОБЪЕМА ОБЛУЧЕНИЯ И СУММАРНОЙ ДОЗЫ

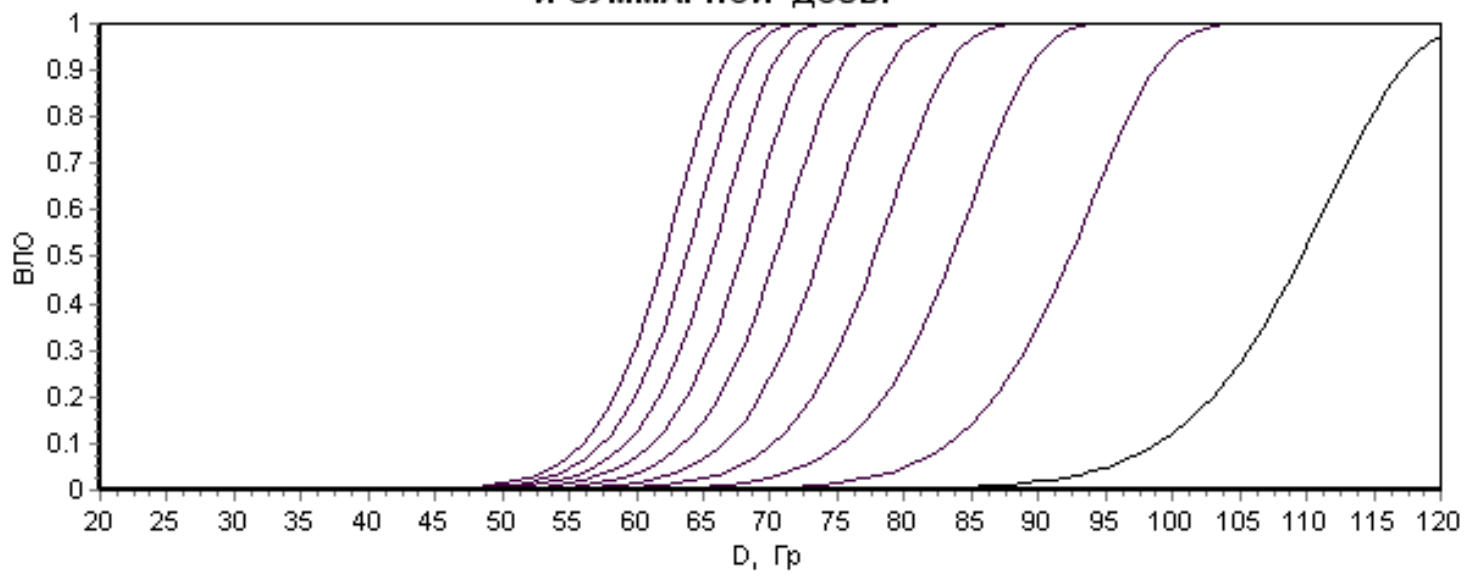

Рис. 4.24.1. Кожа. Оптимизация по ВЛО

Таблица 4.24 .2

Кожса. Оптимизация по ТД

\begin{tabular}{|c|c|c|c|c|c|c|c|c|c|}
\hline$P_{\text {Кл }}$ & $P_{\mathrm{TEOP}}$ & $\varepsilon, \%$ & $V$ & $D_{\text {КЛ }}$ & $D_{\text {TEOP }}$ & $\varepsilon, \%$ & $A_{1}$ & $A_{2}$ & $b$ \\
\hline 0,05 & 0,050 & 0,28 & $1 / 3$ & 70 & 69,99 & $-0,01$ & \multirow{6}{*}{63,136} & \multirow{6}{*}{21,092} & \multirow{6}{*}{0,222} \\
\hline 0,05 & 0,050 & $-0,28$ & $2 / 3$ & 60 & 60,01 & 0,01 & & & \\
\hline 0,05 & 0,053 & $-89,59$ & 1 & 55 & 62,05 & 12,82 & & & \\
\hline- & - & - & - & - & - & - & & & \\
\hline- & - & - & - & - & - & - & & & \\
\hline 0,50 & 0,999 & 99,97 & 1 & 70 & 62,05 & $-11,36$ & & & \\
\hline
\end{tabular}




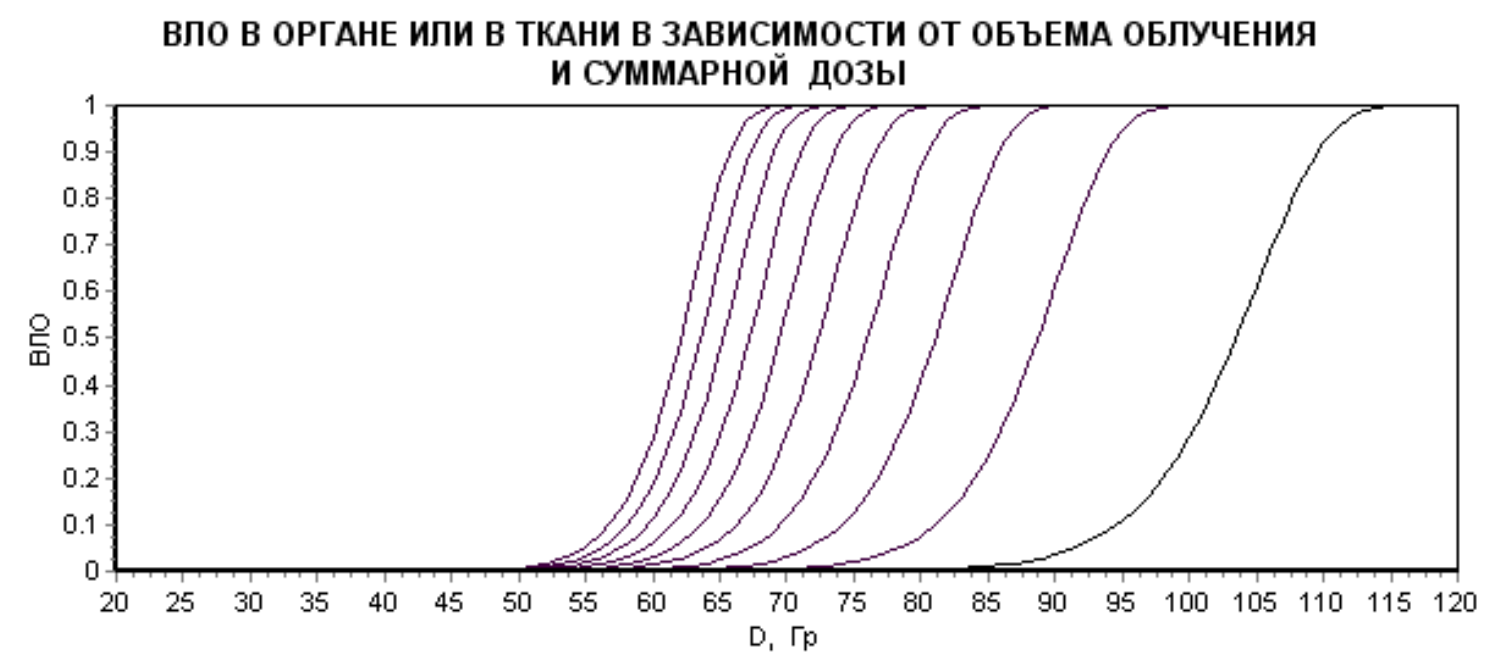

Рис. 4.24.2. Кожа. Оптимизация по ТД

Таблица 4.24.3

Кожа. Оптимизация по ВЛО + ТД

\begin{tabular}{|c|c|c|c|c|c|c|c|c|c|}
\hline$P_{\text {Кл }}$ & $P_{\text {TEOP }}$ & $\varepsilon, \%$ & V & $D_{\text {Кл }}$ & $D_{\text {TEOP }}$ & $\varepsilon, \%$ & $A_{1}$ & $A_{2}$ & $b$ \\
\hline 0,05 & 0,043 & $-14,14$ & $1 / 3$ & 70 & 70,60 & 0,86 & \multirow{6}{*}{63,303} & \multirow{6}{*}{18,304} & \multirow{6}{*}{0,247} \\
\hline 0,05 & 0,058 & 16,39 & $2 / 3$ & 60 & 59,49 & $-0,85$ & & & \\
\hline 0,05 & 0,073 & $-85,32$ & 1 & 55 & 62,05 & 12,82 & & & \\
\hline- & - & - & - & - & - & - & & & \\
\hline- & - & - & - & - & - & - & & & \\
\hline 0,50 & 0,998 & 99,63 & 1 & 70 & 62,05 & $-11,35$ & & & \\
\hline
\end{tabular}

ВЛО В ОРГАНЕ ИЛИ В ТКАНИ В ЗАВИСИМОСТИ ОТ ОБЪЕМА ОБЛУЧЕНИЯ И СУММАРНОЙ ДОЗЫ

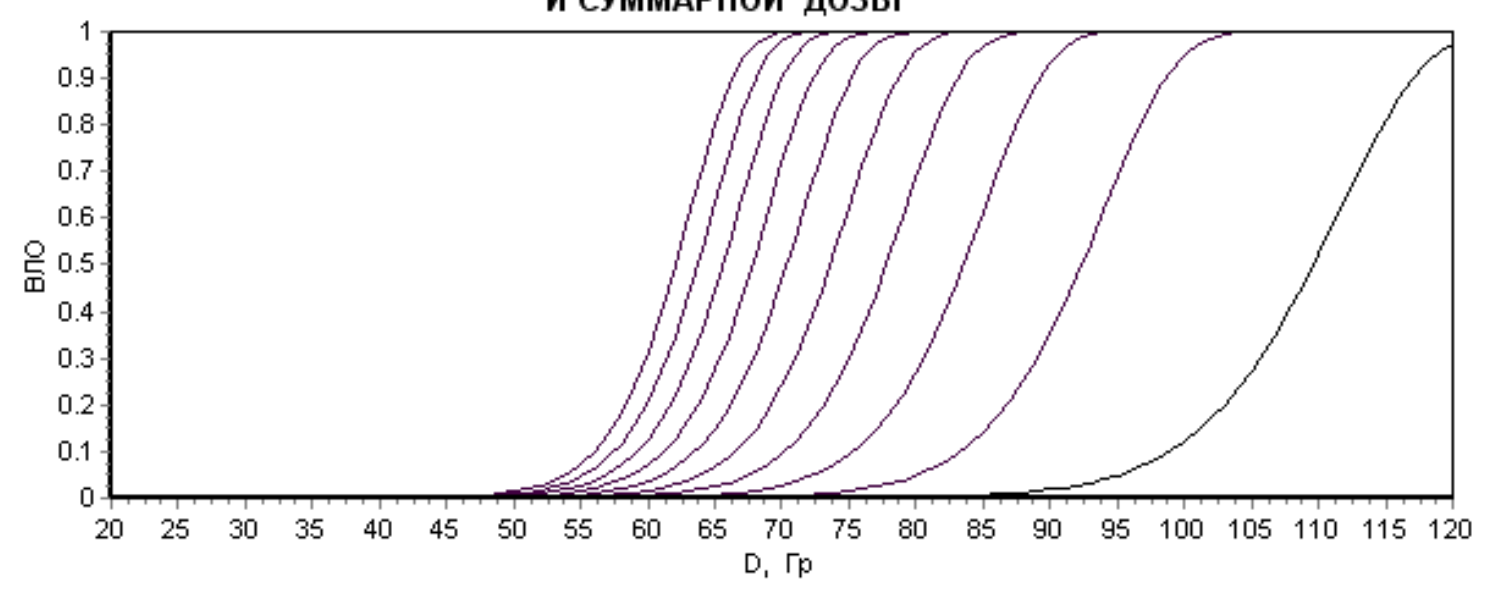

Рис. 4.24.3. Кожса. Оптимизация по ВЛО + ТД 
4.25. Тонкий кищечник. Осложнения: обструкция, перфорация

Таблица 4.25.1

Тонкий кищечник. Оптимизация по ВЛО

\begin{tabular}{|c|c|c|c|c|c|c|c|c|c|}
\hline$P_{\text {КЛ }}$ & $P_{\mathrm{TEOP}}$ & $\varepsilon, \%$ & $V$ & $D_{\text {Кл }}$ & $D_{\text {TEOP }}$ & $\varepsilon, \%$ & $A_{1}$ & $A_{2}$ & $b$ \\
\hline 0,05 & 0,062 & 29,56 & $1 / 3$ & 50 & 48,96 & $-2,07$ & \multirow{6}{*}{54,350} & \multirow{6}{*}{10,398} & \multirow{6}{*}{0,165} \\
\hline- & - & - & - & - & - & - & & & \\
\hline 0,05 & 0,040 & $-19,16$ & 1 & 40 & 40,85 & 2,12 & & & \\
\hline 0,50 & 0,346 & $-30,81$ & $1 / 3$ & 60 & 62,89 & 4,83 & & & \\
\hline- & - & - & - & - & - & - & & & \\
\hline 0,50 & 0,677 & 36,49 & 1 & 55 & 52,47 & $-4,60$ & & & \\
\hline
\end{tabular}

ВЛО В ОРГАНЕ ИЛИ В ТКАНИ В ЗАВИСИМОСТИ ОТ ОБЪЕМА ОБЛУЧЕНИЯ И СУММАРНОЙ ДОЗЫ

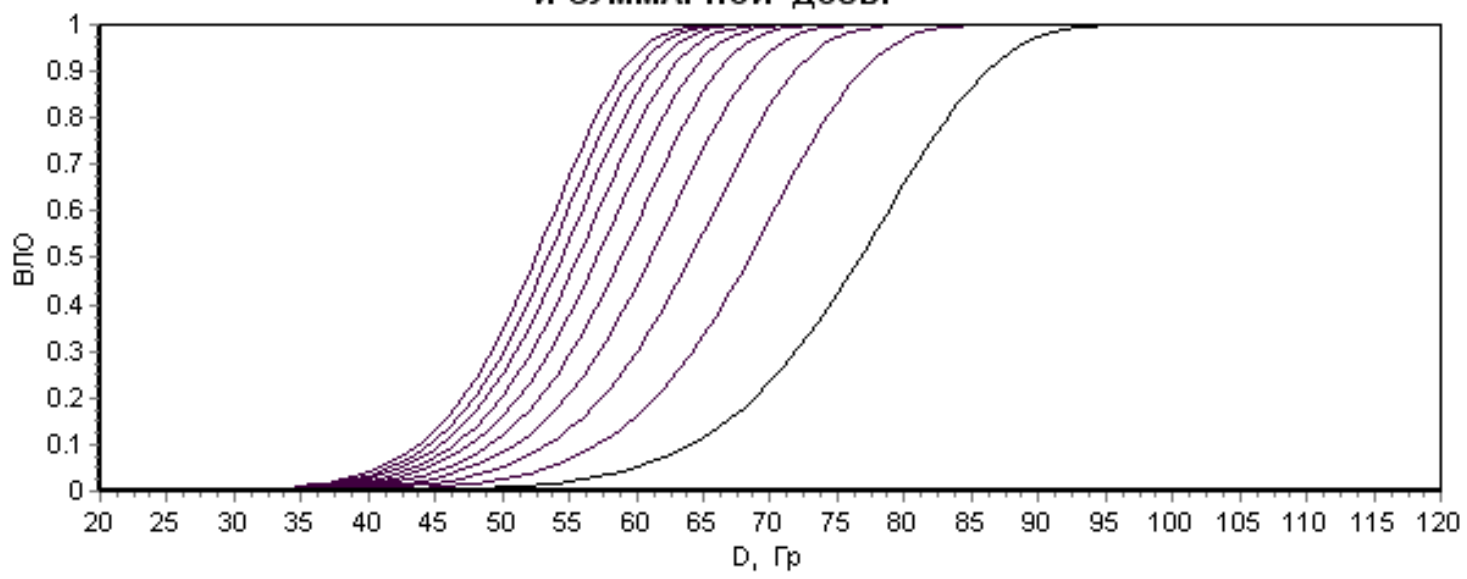

Рис. 4.25.1. Тонкий кищечник. Оптимизация по ВЛО

Таблица 4.25 .2

Тонкий кищечник. Оптимизация по ТД

\begin{tabular}{|c|c|c|c|c|c|c|c|c|c|}
\hline$P_{\text {Кл }}$ & $P_{\mathrm{TEOP}}$ & $\varepsilon, \%$ & $V$ & $D_{\text {Кл }}$ & $D_{\text {TEOP }}$ & $\varepsilon, \%$ & $A_{1}$ & $A_{2}$ & $b$ \\
\hline 0,05 & 0,062 & 23,56 & $1 / 3$ & 50 & 48,96 & $-2,07$ & \multirow{6}{*}{54,350} & \multirow{6}{*}{10,398} & \multirow{6}{*}{0,165} \\
\hline- & - & - & - & - & - & - & & & \\
\hline 0,05 & 0,040 & $-19,16$ & 1 & 40 & 40,85 & 2,12 & & & \\
\hline 0,50 & 0,346 & $-30,81$ & $1 / 3$ & 60 & 62,89 & 4,83 & & & \\
\hline- & - & - & - & - & - & - & & & \\
\hline 0,50 & 0,677 & 35,49 & 1 & 55 & 52,47 & $-4,60$ & & & \\
\hline
\end{tabular}




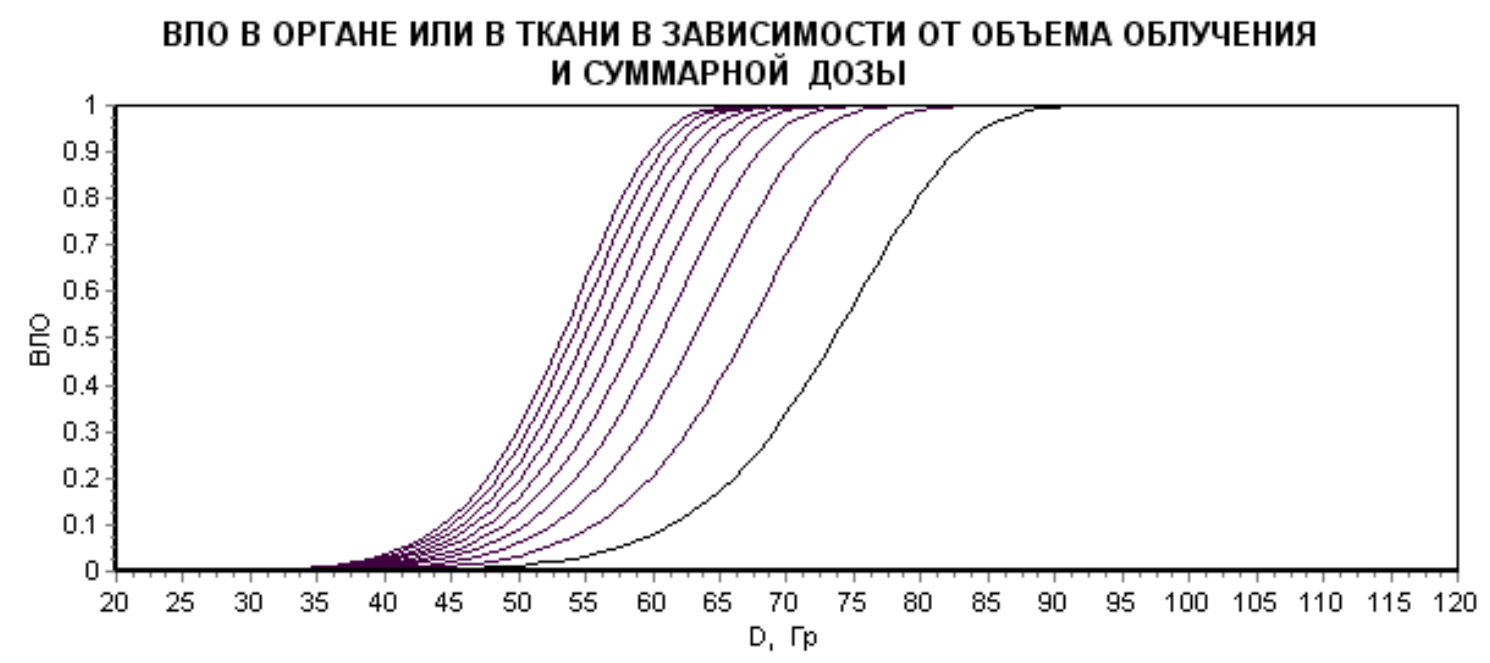

Рис. 4.25.2. Тонкий кишечник. Оптимизация по ТД

Таблица 4.25.3

Тонкий кищечник. Оптимизация по ВЛО + ТД

\begin{tabular}{|c|c|c|c|c|c|c|c|c|c|}
\hline$P_{\text {Кл }}$ & $P_{\mathrm{TEOP}}$ & $\varepsilon, \%$ & $V$ & $D_{\text {Кл }}$ & $D_{\mathrm{TEOP}}$ & $\varepsilon, \%$ & $A_{1}$ & $A_{2}$ & $b$ \\
\hline 0,05 & 0,062 & 29,56 & $1 / 3$ & 50 & 48,96 & $-2,07$ & \multirow{6}{*}{54,350} & \multirow{6}{*}{10,398} & \multirow{6}{*}{0,165} \\
\hline- & - & - & - & - & - & - & & & \\
\hline 0,05 & 0,040 & $-19,16$ & 1 & 40 & 40,85 & 2,12 & & & \\
\hline 0,50 & 0,346 & $-30,81$ & $1 / 3$ & 60 & 62,89 & 4,83 & & & \\
\hline- & - & - & - & - & - & - & & & \\
\hline 0,50 & 0,677 & 36,49 & 1 & 55 & 52,47 & $-4,60$ & & & \\
\hline
\end{tabular}

ВЛО В ОРГАНЕ ИЛИ В ТКАНИ В ЗАВИСИМОСТИ ОТ ОБЪЕМА ОБЛУЧЕНИЯ И СУММАРНОЙ ДОЗЫ

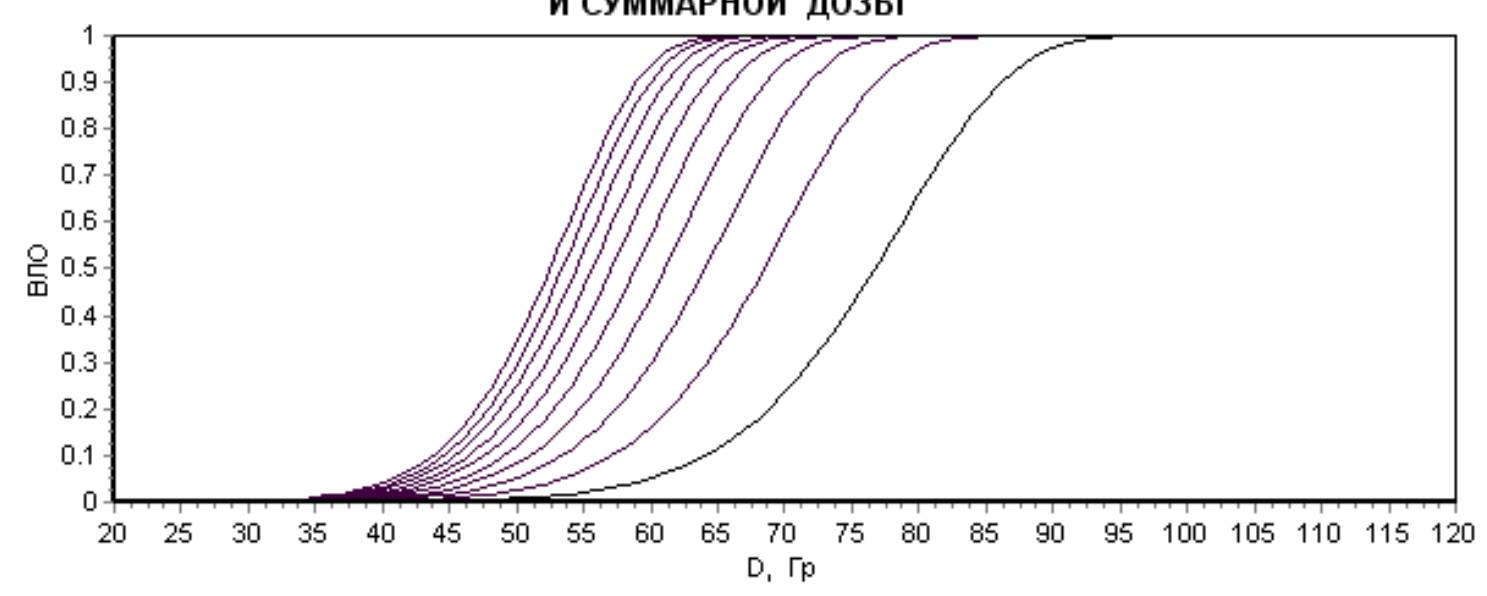

Рис. 4.25.3. Тонкий кищечник. Оптимизация по ВЛО + ТД 


\subsection{6. Спинной мозг. Осложнения: миелиты, некрозы}

Таблица 4.26.1

Спинной мозг. Оптимизация по ВЛО

\begin{tabular}{|c|c|c|c|c|c|c|c|c|c|}
\hline$P_{\text {КЛ }}$ & $P_{\text {TEOP }}$ & $\varepsilon, \%$ & $V$ & $D_{\text {Кл }}$ & $D_{\text {TEOP }}$ & $\varepsilon, \%$ & $A_{1}$ & $A_{2}$ & $b$ \\
\hline 0,05 & 0,050 & $-0,64$ & $1 / 3$ & 50 & 50,04 & 0,09 & \multirow{6}{*}{71,552} & \multirow{6}{*}{7,480} & \multirow{6}{*}{0,086} \\
\hline 0,05 & 0,060 & 19,14 & $2 / 3$ & 50 & 48,81 & $-2,38$ & & & \\
\hline 0,05 & 0,042 & $-15,59$ & 1 & 47 & 48,10 & 2,35 & & & \\
\hline 0,50 & 0,468 & $-6,37$ & $1 / 3$ & 70 & 70,88 & 1,26 & & & \\
\hline 0,50 & 0,533 & 6,56 & $2 / 3$ & 70 & 69,13 & $-1,24$ & & & \\
\hline- & - & - & - & - & - & - & & & \\
\hline
\end{tabular}

ВЛО В ОРГАНЕ ИЛИ В ТКАНИ В ЗАВИСИМОСТИ ОТ СУММАРНОЙ ДОЗЫ

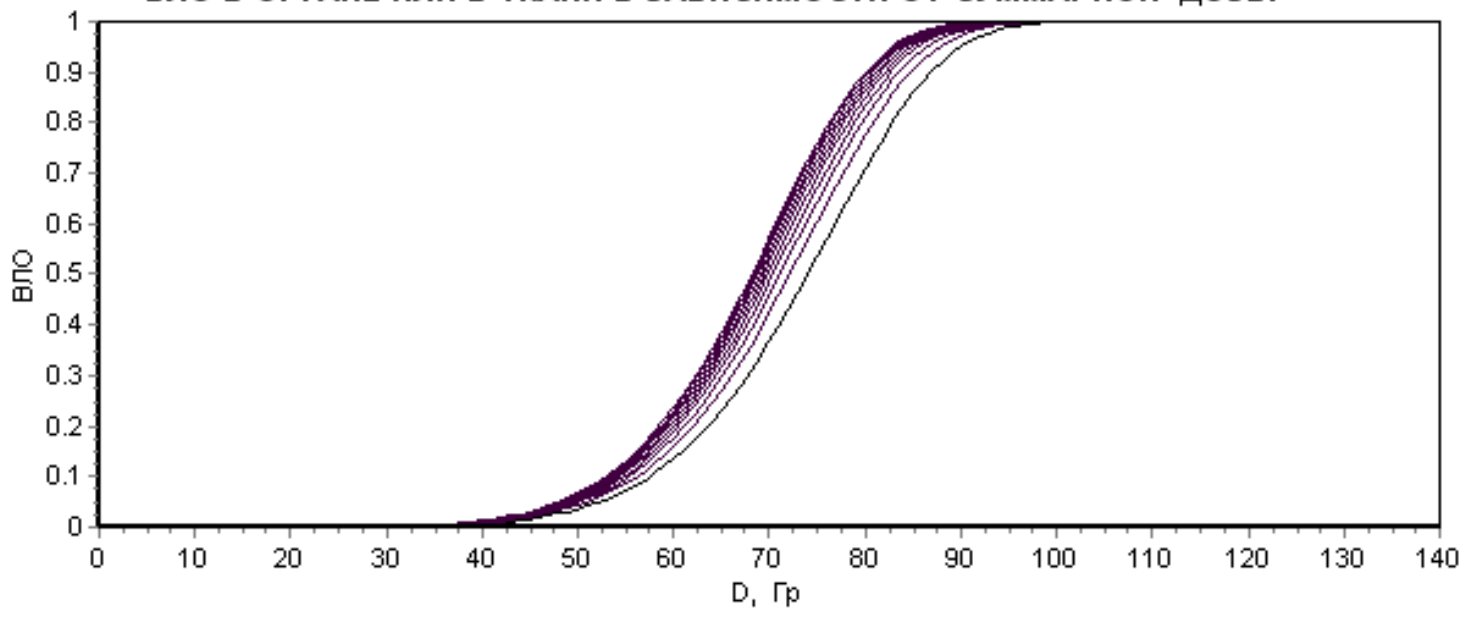

Рис. 4.26.1. Спинной мозг. Оптимизация по ВЛО

Таблица 4.26.2

Спинной мозг. Оптимизация по ТД

\begin{tabular}{|c|c|c|c|c|c|c|c|c|c|}
\hline$P_{\text {Кл }}$ & $P_{\mathrm{TEOP}}$ & $\varepsilon, \%$ & $V$ & $D_{\text {Кл }}$ & $D_{\text {TEOP }}$ & $\varepsilon, \%$ & $A_{1}$ & $A_{2}$ & $b$ \\
\hline 0,05 & 0,049 & $-1,69$ & $1 / 3$ & 50 & 50,07 & 0,15 & \multirow{6}{*}{71,495} & \multirow{6}{*}{7,486} & \multirow{6}{*}{0,037} \\
\hline 0,05 & 0,060 & 19,23 & $2 / 3$ & 50 & 48,81 & $-2,39$ & & & \\
\hline 0,05 & 0,042 & $-15,29$ & 1 & 47 & 48,08 & 2,30 & & & \\
\hline 0,50 & 0,467 & $-6,55$ & $1 / 3$ & 70 & 70,90 & 1,29 & & & \\
\hline 0,50 & 0,534 & 6,74 & $2 / 3$ & 70 & 69,11 & $-1,27$ & & & \\
\hline- & - & - & - & - & - & - & & & \\
\hline
\end{tabular}




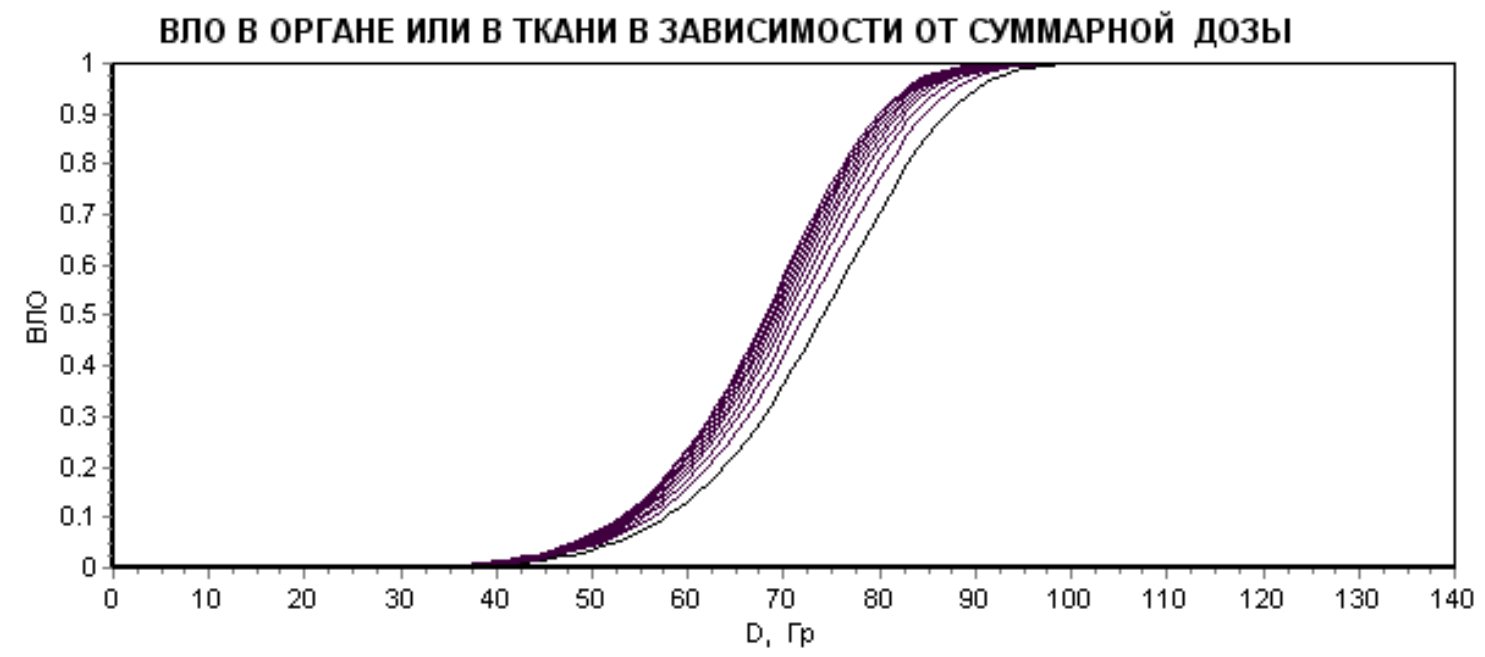

Рис. 4.26.2. Спинной мозг. Оптимизация по ТД

Результаты оптимизация с критерием ВЛО + ТД совпадают с результатом оптимизации по ВЛО.

\subsection{7. Желудок. Осложнения: изъязвление, перфорация}

Таблица 4.27.1

Желудок. Оптимизация по ВЛО

\begin{tabular}{|c|c|c|c|c|c|c|c|c|c|}
\hline$P_{\text {Кл }}$ & $P_{\mathrm{TEOP}}$ & $\varepsilon, \%$ & $V$ & $D_{\text {Кл }}$ & $D_{\text {TEOP }}$ & $\varepsilon, \%$ & $A_{1}$ & $A_{2}$ & $b$ \\
\hline 0,05 & 0,059 & 67,93 & $1 / 3$ & 60 & 59,21 & $-1,32$ & \multirow{6}{*}{64,963} & \multirow{6}{*}{12,724} & \multirow{6}{*}{0,128} \\
\hline 0,05 & 0,060 & 20,45 & $2 / 3$ & 55 & 54,18 & $-1,49$ & & & \\
\hline 0,05 & 0,035 & $-29,76$ & 1 & 50 & 51,44 & 2,88 & & & \\
\hline 0,50 & 0,351 & $-29,83$ & $1 / 3$ & 70 & 72,65 & 3,78 & & & \\
\hline 0,50 & 0,535 & 6,95 & $2 / 3$ & 67 & 66,48 & 0,77 & & & \\
\hline 0,50 & 0,635 & 26,95 & 1 & 65 & 63,11 & $-2,89$ & & & \\
\hline
\end{tabular}

ВЛО В ОРГАНЕ ИЛИ В ТКАНИ В ЗАВИСИМОСТИ ОТ ОБЪЕМА ОБЛУЧЕНИЯ И СУММАРНОЙ ДОЗЫ

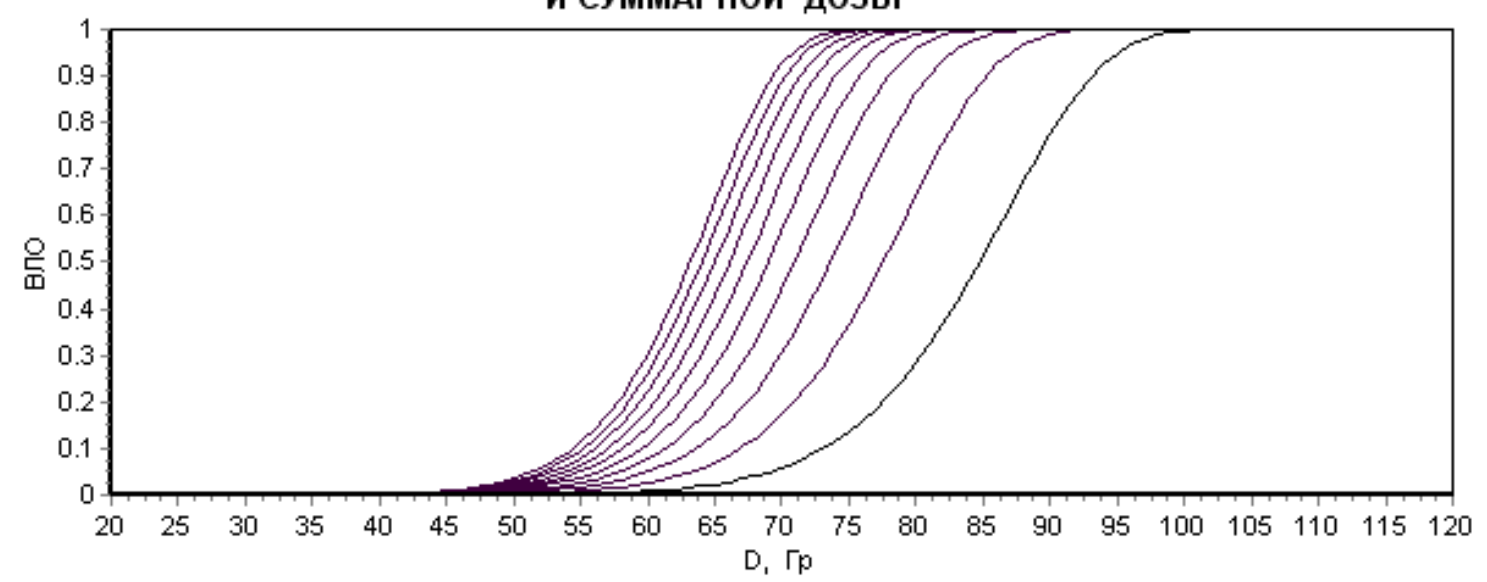

Рис. 27.1. Желудок. Оптимизация по ВЛО 
Таблица 4.27.2

Желудок. Оптимизация по ТД

\begin{tabular}{|c|c|c|c|c|c|c|c|c|c|}
\hline$P_{\text {Кл }}$ & $P_{\mathrm{TEOP}}$ & $\varepsilon, \%$ & $V$ & $D_{\text {КЛ }}$ & $D_{\text {TEOP }}$ & $\varepsilon, \%$ & $A_{1}$ & $A_{2}$ & $b$ \\
\hline 0,05 & 0,065 & 30,73 & $1 / 3$ & 60 & 58,71 & $-2,15$ & \multirow{6}{*}{65,421} & \multirow{6}{*}{12,724} & \multirow{6}{*}{0,114} \\
\hline 0,05 & 0,059 & 18,49 & $2 / 3$ & 55 & 54,25 & $-1,36$ & & & \\
\hline 0,05 & 0,032 & $-35,36$ & 1 & 50 & 51,80 & 3,60 & & & \\
\hline 0,50 & 0,382 & $-23,68$ & $1 / 3$ & 70 & 72,04 & 2,92 & & & \\
\hline 0,50 & 0,529 & 5,74 & $2 / 3$ & 67 & 66,57 & $-0,64$ & & & \\
\hline 0,50 & 0,602 & 20,39 & 1 & 65 & 63,56 & $-2,21$ & & & \\
\hline
\end{tabular}

ВЛО В ОРГАНЕ ИЛИ В ТКАНИ В ЗАВИСИМОСТИ ОТ ОБЪЕМА ОБЛУЧЕНИЯ И СУММАРНОЙ ДОЗЫ

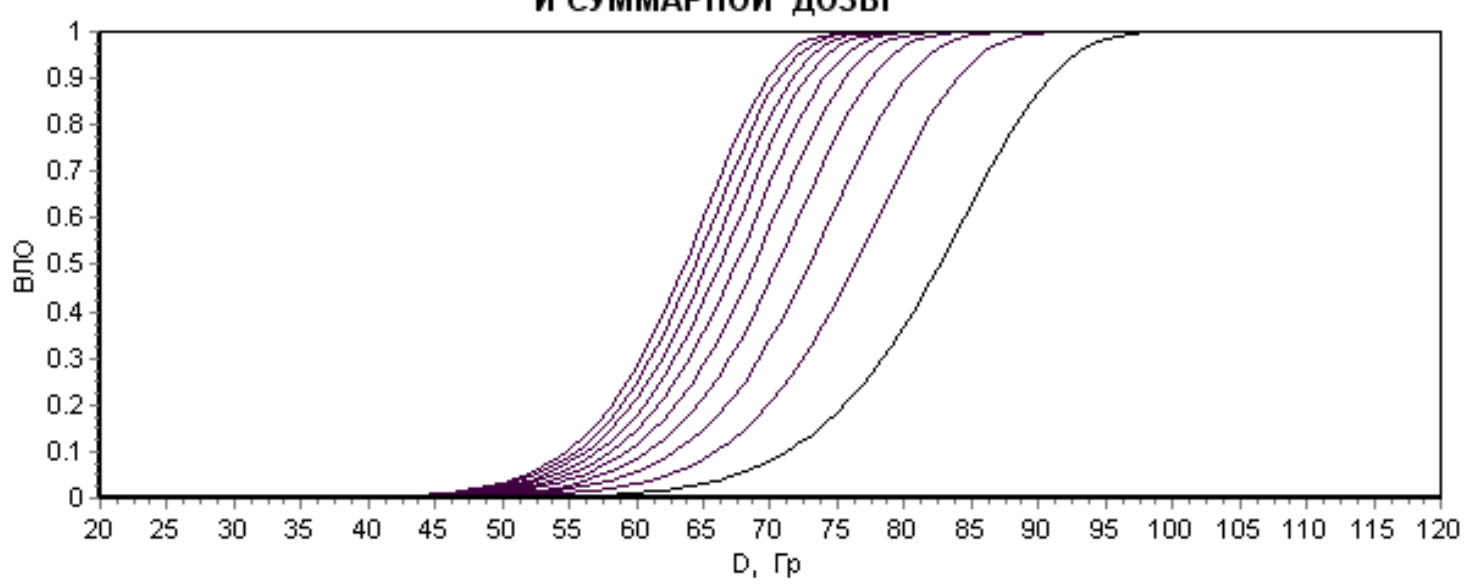

Рис. 4.27.2. Желудок. Оптимизация по ТД

Таблица 4.27.3

Желудок. Оптимизация по ВЛО + ТД

\begin{tabular}{|c|c|c|c|c|c|c|c|c|c|}
\hline$P_{\text {Кл }}$ & $P_{\text {TEOP }}$ & $\varepsilon, \%$ & $V$ & $D_{\text {Кл }}$ & $D_{\text {TEOP }}$ & $\varepsilon, \%$ & $A_{1}$ & $A_{2}$ & $b$ \\
\hline 0,05 & 0,059 & 67,93 & $1 / 3$ & 60 & 59,21 & $-1,32$ & \multirow{6}{*}{64,963} & \multirow{6}{*}{12,724} & \multirow{6}{*}{0,128} \\
\hline 0,05 & 0,060 & 20,45 & $2 / 3$ & 55 & 54,18 & $-1,49$ & & & \\
\hline 0,05 & 0,035 & $-29,76$ & 1 & 50 & 51,44 & 2,88 & & & \\
\hline 0,50 & 0,351 & $-29,83$ & $1 / 3$ & 70 & 72,65 & 3,78 & & & \\
\hline 0,50 & 0,535 & 6,95 & $2 / 3$ & 67 & 66,48 & 0,77 & & & \\
\hline 0,50 & 0,635 & 26,95 & 1 & 65 & 63,11 & $-2,89$ & & & \\
\hline
\end{tabular}




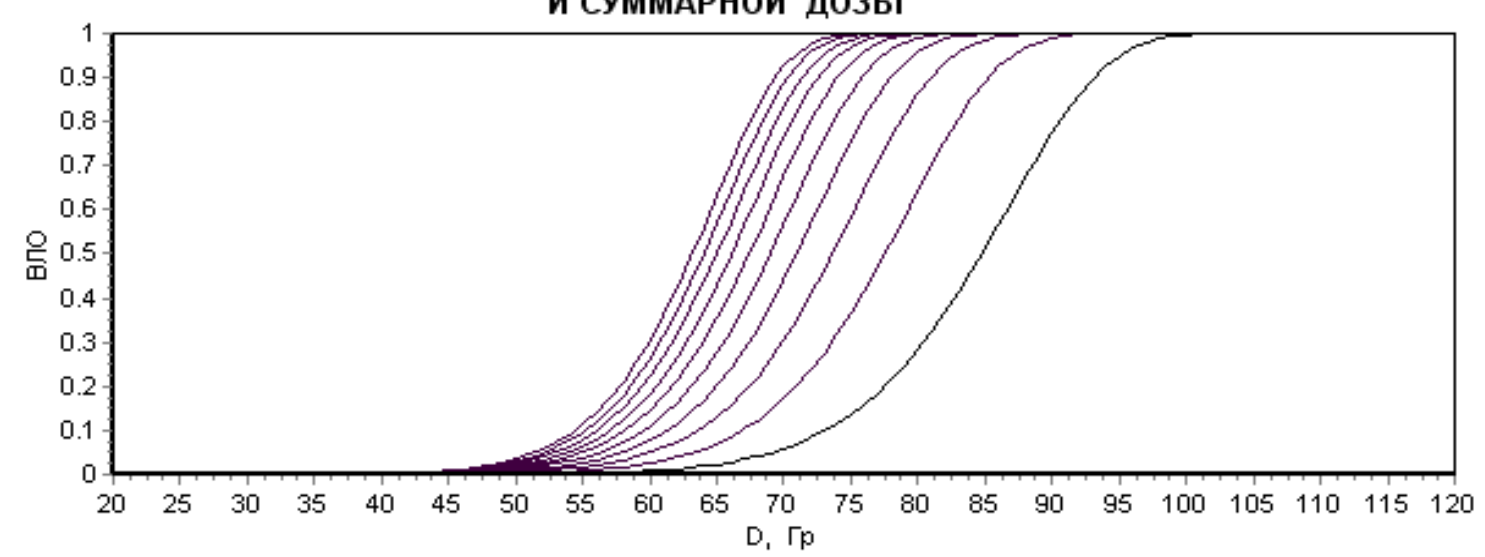

Рис. 4.27.3. Желудок. Оптимизация по ВЛО + ТД

Параметры модели МРВ в табл. 4.27.4 для ВЛО = 0,05 и ВЛО = 0,50 отличаются друг от друга. Клиническая информация плохо согласована.

Таблица 4.27 .4

Желудок. Оптимизация по ТД, отдельно для ВЛО = 0,05 и ВЛО = 0,50

\begin{tabular}{|c|c|c|c|c|c|c|c|c|c|}
\hline$P_{\text {КЛ }}$ & $P_{\mathrm{TEOP}}$ & $\varepsilon, \%$ & $V$ & $D_{\text {КЛ }}$ & $D_{\text {TEOP }}$ & $\varepsilon, \%$ & $A_{1}$ & $A_{2}$ & $b$ \\
\hline 0,05 & 0,050 & $-0,71$ & $1 / 3$ & 60 & 60,38 & 0,64 & \multirow{3}{*}{669,175} & \multirow{3}{*}{1,150} & \multirow{3}{*}{0,161} \\
\hline 0,05 & 0,051 & 2,06 & $2 / 3$ & 55 & 54,01 & $-1,80$ & & & \\
\hline 0,05 & 0,049 & $-1,32$ & 1 & 50 & 50,60 & 1,19 & & & \\
\hline 0,50 & 0,500 & $-0,05$ & $1 / 3$ & 70 & 70,09 & 0,13 & \multirow{3}{*}{124,448} & \multirow{3}{*}{0,565} & \multirow{3}{*}{0,068} \\
\hline 0,50 & 0,500 & 0,08 & $2 / 3$ & 67 & 66,86 & $-0,20$ & & & \\
\hline 0,50 & 0,500 & $-0,03$ & 1 & 65 & 65,05 & 0,07 & & & \\
\hline
\end{tabular}

\subsection{8. Щитовидная железа. Осложнения: тиреодит}

Таблица 4.28

\section{Щитовидная железа}

\begin{tabular}{c|c|c|c|c|c}
\hline$P_{\text {КЛ }}$ & $D_{\text {КЛ }}$ & $P_{\text {КЛ }}$ & $D_{\text {КЛ }}$ & $A_{1}$ & $A_{2}$ \\
\hline 0,05 & 45,0 & 0,50 & 80,0 & 86,749 & 4,525 \\
\hline
\end{tabular}

ВЛО В ОРГАНЕ ИЛИ В ТКАНИ В ЗАВИСИМОСТИ ОТ СУММАРНОЙ ДОЗЫ

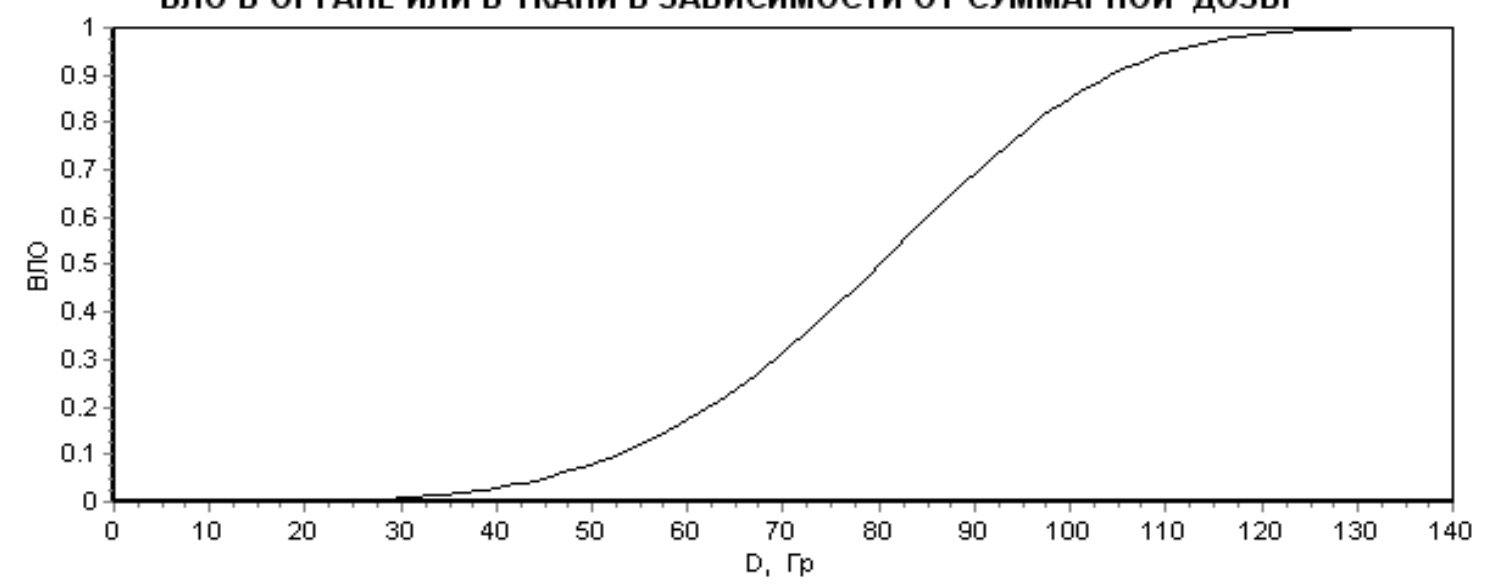

Рис. 4.28. Щитовидная железа. Зависимость ВЛО от дозы 
4.29. Кость, сустав нижней челюсти (КСНЧ).

Осложнение: выраженное ограничение функции сустава

Таблица 4.29.1

КСНЧ. Оптимизация по ВЛО

\begin{tabular}{|c|c|c|c|c|c|c|c|c|c|}
\hline$P_{\text {КЛ }}$ & $P_{\mathrm{TEOP}}$ & $\varepsilon, \%$ & $V$ & $D_{\text {Кл }}$ & $D_{\text {TEOP }}$ & $\varepsilon, \%$ & $A_{1}$ & $A_{2}$ & $b$ \\
\hline 0,05 & 0.057 & 14,90 & $1 / 3$ & 65 & 64,37 & $-0,97$ & \multirow{6}{*}{72,785} & \multirow{6}{*}{14,626} & \multirow{6}{*}{0,073} \\
\hline 0,05 & 0,038 & $-24,53$ & $2 / 3$ & 60 & 61,19 & 1,99 & & & \\
\hline 0,05 & 0,058 & 15,15 & 1 & 60 & 59,41 & $-0,99$ & & & \\
\hline 0,50 & 0,506 & 1,18 & $1 / 3$ & 77 & 76,91 & $-0,12$ & & & \\
\hline 0,50 & 0,425 & $\begin{array}{l}-14,98 \\
\end{array}$ & $2 / 3$ & 72 & 73,12 & 1,55 & & & \\
\hline 0,50 & 0,574 & 14,81 & 1 & 72 & 70,98 & $-1,41$ & & & \\
\hline
\end{tabular}

ВЛО В ОРГАНЕ ИЛИ В ТКАНИ В ЗАВИСИМОСТИ ОТ СУММАРНОЙ ДОЗЫ

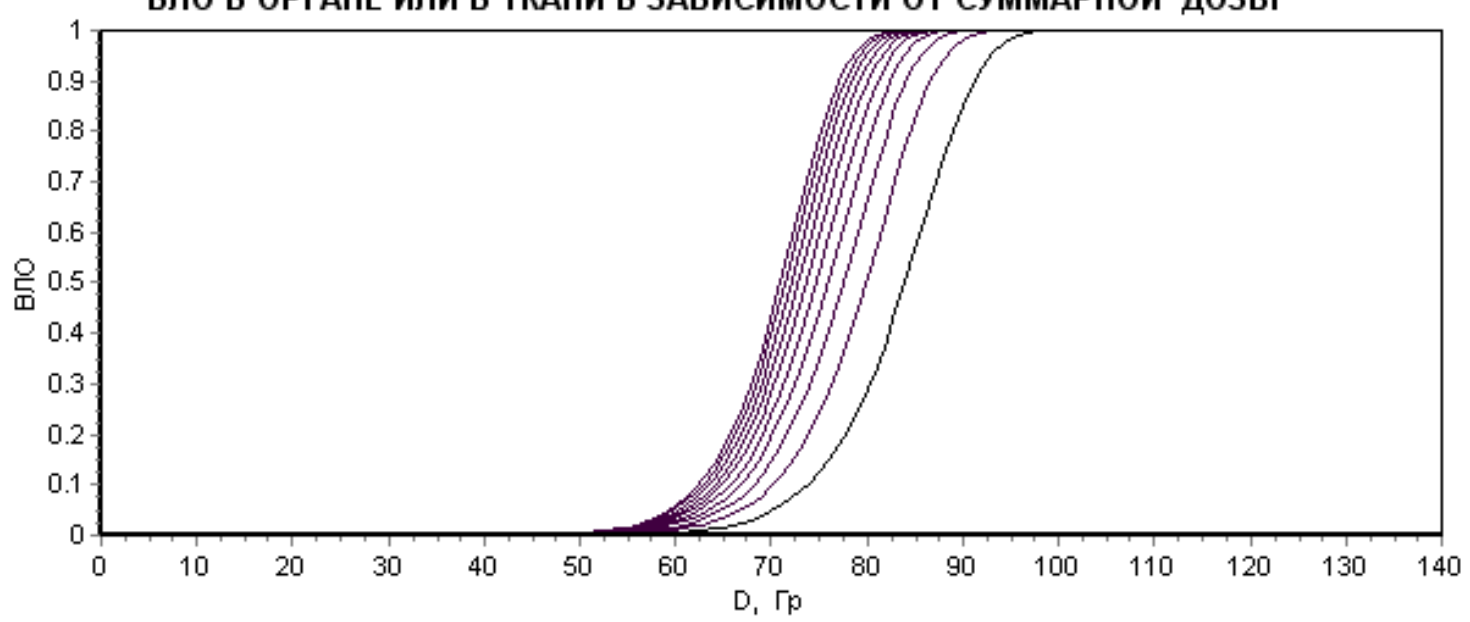

Рис. 4.29.1. КСНЧ. Оптимизация по ВЛО

Таблица 4.29.2

КСНЧ. Оптимизация по ТД

\begin{tabular}{|c|c|c|c|c|c|c|c|c|c|}
\hline$P_{\text {Кл }}$ & $P_{\mathrm{TEOP}}$ & $\varepsilon, \%$ & $V$ & $D_{\text {Кл }}$ & $D_{\mathrm{TEOP}}$ & $\varepsilon, \%$ & $A_{1}$ & $A_{2}$ & $b$ \\
\hline 0,05 & 0,058 & 16,86 & $1 / 3$ & 65 & 64,29 & $-1,09$ & \multirow{6}{*}{72,858} & \multirow{6}{*}{14,626} & \multirow{6}{*}{0,071} \\
\hline 0,05 & 0,038 & $-24,74$ & $2 / 3$ & 60 & 61,20 & 2,01 & & & \\
\hline 0,05 & 0,057 & 13,52 & 1 & 60 & 59,47 & $-0,89$ & & & \\
\hline 0,50 & 0,512 & 2,40 & $1 / 3$ & 77 & 76,82 & $-0,24$ & & & \\
\hline 0,50 & 0,424 & $-15,16$ & $2 / 3$ & 72 & 73,13 & 1,57 & & & \\
\hline 0,50 & 0,569 & 13,74 & 1 & 72 & 71,06 & $-1,31$ & & & \\
\hline
\end{tabular}




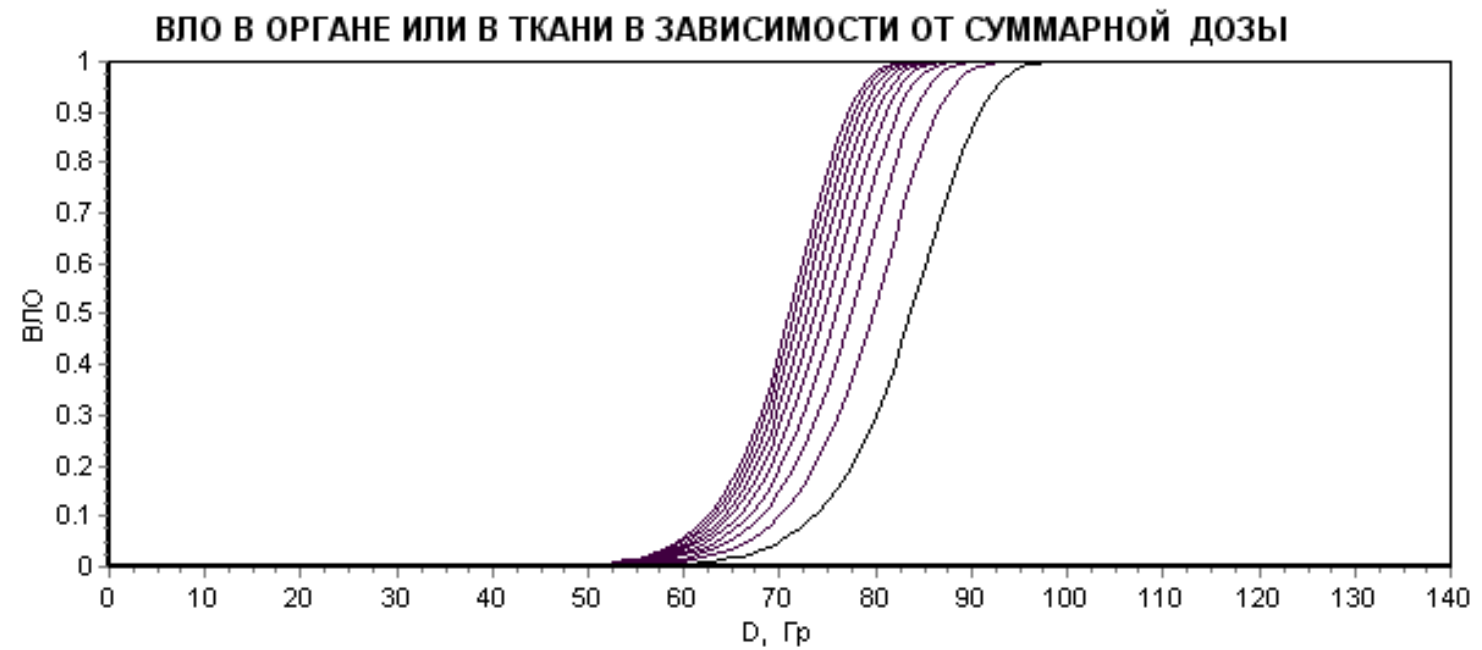

Рис. 4.29.2. КСНЧ. Оптимизация по ТД

Результаты определения параметров модели МРВ с критерием ВЛО + ТД совпадают с результатами определения параметров МРВ с критерием ВЛО.

\subsection{0. Кость, головка бедренной кости. Осложнение: некроз}

Кость, головка бедренной кости

\begin{tabular}{c|c|c|c|c|c}
\hline$P_{\text {КЛ }}$ & $D_{\text {КЛ }}$ & $P_{\text {КЛ }}$ & $D_{\text {КЛ }}$ & $A_{1}$ & $A_{2}$ \\
\hline 0,05 & 52,0 & 0,50 & 65,0 & 67,074 & 11,668 \\
\hline
\end{tabular}

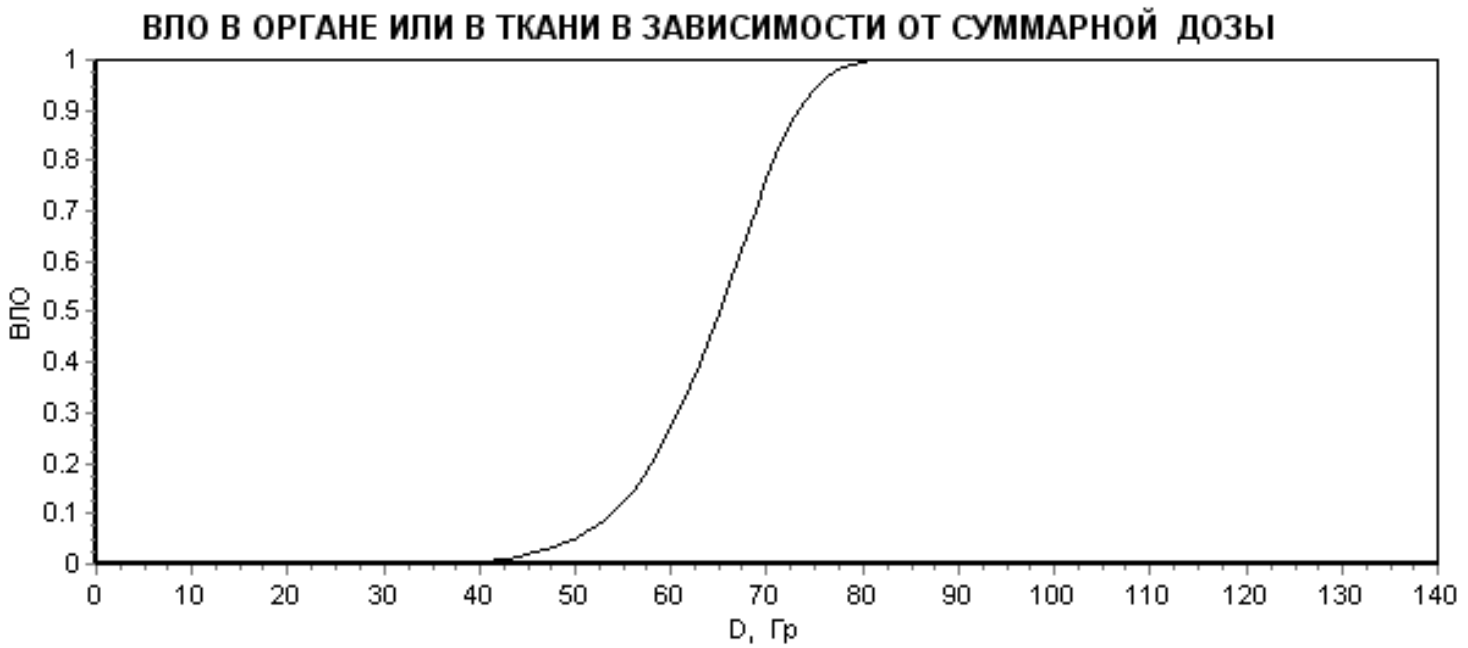

Рис. 4.30. Кость, головка бедренной кости. Зависимость ВЛО от дозы

Мы надеемся, что приведенная информация окажется полезной лучевым терапевтам, медицинским физикам и другим специалистам, которые принимают участие в планировании ЛТ опухолевых заболеваний. 


\section{Глава 5. \\ РАСЧЕТ ОПТИМАЛЬНЫХ ЗНАЧЕНИЙ ПАРАМЕТРОВ МОДЕЛИ МРВ ДЛЯ ОРГАНОВ И ТКАНЕЙ НА ОСНОВЕ СИСТЕМАТИЗИРОВАННОЙ КЛИНИЧЕСКОЙ ИНФОРМАЦИИ KEHWAR et al.}

Ниже приводятся результаты определения параметров модели МРВ на основе клинических данных Kehwar et al. для трех критериев оптимальнсти, таких же, как и в главе 4.

Номера органов и тканей, для которых определяются параметры модели MPB, связаны с порядковым номером органа или ткани в табл. 1.2. Также как и в главе 4, при определении оптимальных значений параметров модели МРВ исползовались три критерия оптимальности, ВЛО, ТД и ВЛО + ТД. На графиках, которые описывают зависимости ВЛО от дозы, рассчитанные при помощи модели MPВ, относительные объемы облученных органов и тканей, также как и в главе 4 , изменяются от 0,1 до 1,0 с шагом 0,1 .

Поскольку в табл. 1.2 (Kehwar et al.) не приводятся порядковые номера opганов и тканей, мы их расставили сами. Так будет удобней пользоваться таблицей.

При построении графиков зависимости ВЛО от дозы, относительные объемы облучения органов и тканей меняются от 0,1 до 1,0 с шагом 0,1 . Крайний левый график соответствует максимальному относительному объему облучения, равному $1,0 \%$. Крайний правый график соответствует наименьшему относительному объему облученного органа или ткани, равному $0,1 \%$.

При помощи рассчитанных оптимальных значений параметров модели МРВ, вычисляются теоретические (модельные) значения ВЛО и ТД и приводятся нормированные отклонения исходных клинических данных ВЛО и ТД от их теоретических (модельных) значений в процентах. Они облегчают анализ приведенных результатов расчетов.

Результаты расчета оптимальных значений параметров модели МРВ для каждого органа или ткани, в зависимости от использованного критерия оптимальности, приводятся в таблицах. В столбце 1 таблицы приводятся клинические значения ВЛО, в столбце 2 - значения относительных объемов, (или площадей), облученных органов или тканей, в столбцах 3 и 4 - клинические и теоретические (модельные) значения ТД, в столбце 5 - отклонения клинических значений ТД от соответствующих теоретических значений в \%, в столбцах 6 и 7 приводятся рассчитанные теоретические (модельные) значения ВЛО и отклонения клинических зна- 
чений ВЛО от их теоретических значений в \%. В последних трех столбцах приводятся оптимальные значения параметров модели МРB, $\left(A_{1}, A_{2}, b\right)$. Структуры таблиц, которые приводятся в главе 4 и 5, отличаются друг от друга. Это было сделано для того, чтобы можно было бы легко отличать клинические данные Emami et al. oт клинических данных Kehwar et al.

Мы уже отмечали, что структуры таблиц, которые приводятся в главе 4 и 5 , отличаются друг от друга. Это было сделано для того, чтобы было легко отличать клинические данные Emami et al. от клинических данных Kehwar et al.

Мы надеемся, что приведенные оптимальные значения параметров модели МРВ облегчат лучевым терапевтам и медицинским физикам практическое применение модели МРВ в планировании ЛТ опухолевых заболеваний.

\section{1. Почка}

Таблица 5.1.1

\section{Почка. Оптимизация по ВЛО}

\begin{tabular}{|c|c|c|c|c|c|c|c|c|c|}
\hline$P_{\text {Кл }}$ & $V$ & $D_{\text {Кл }}$ & $D_{\mathrm{TEOP}}$ & $\varepsilon, \%$ & $P_{\mathrm{TEOP}}$ & $\varepsilon, \%$ & $A_{1}$ & $A_{2}$ & $b$ \\
\hline 0,05 & $1 / 3$ & 43,22 & 42,15 & $-4,03$ & 0,063 & 25,40 & \multirow{6}{*}{32,020} & \multirow{6}{*}{5,660} & \multirow{6}{*}{0,728} \\
\hline 0,05 & $2 / 3$ & 27,02 & 25,45 & $-5,82$ & 0,069 & 38,99 & & & \\
\hline 0,05 & 1 & 17,12 & 18,94 & 10,65 & 0,025 & $-42,96$ & & & \\
\hline 0,50 & $1 / 3$ & 59,14 & 66,77 & 12,90 & 0,294 & $-41,11$ & & & \\
\hline 0,50 & $2 / 3$ & 42,23 & 40,31 & $-4,54$ & 0,594 & 18,83 & & & \\
\hline 0,50 & 1 & 32,34 & 30,01 & $-7,21$ & 0,653 & 30,62 & & & \\
\hline
\end{tabular}

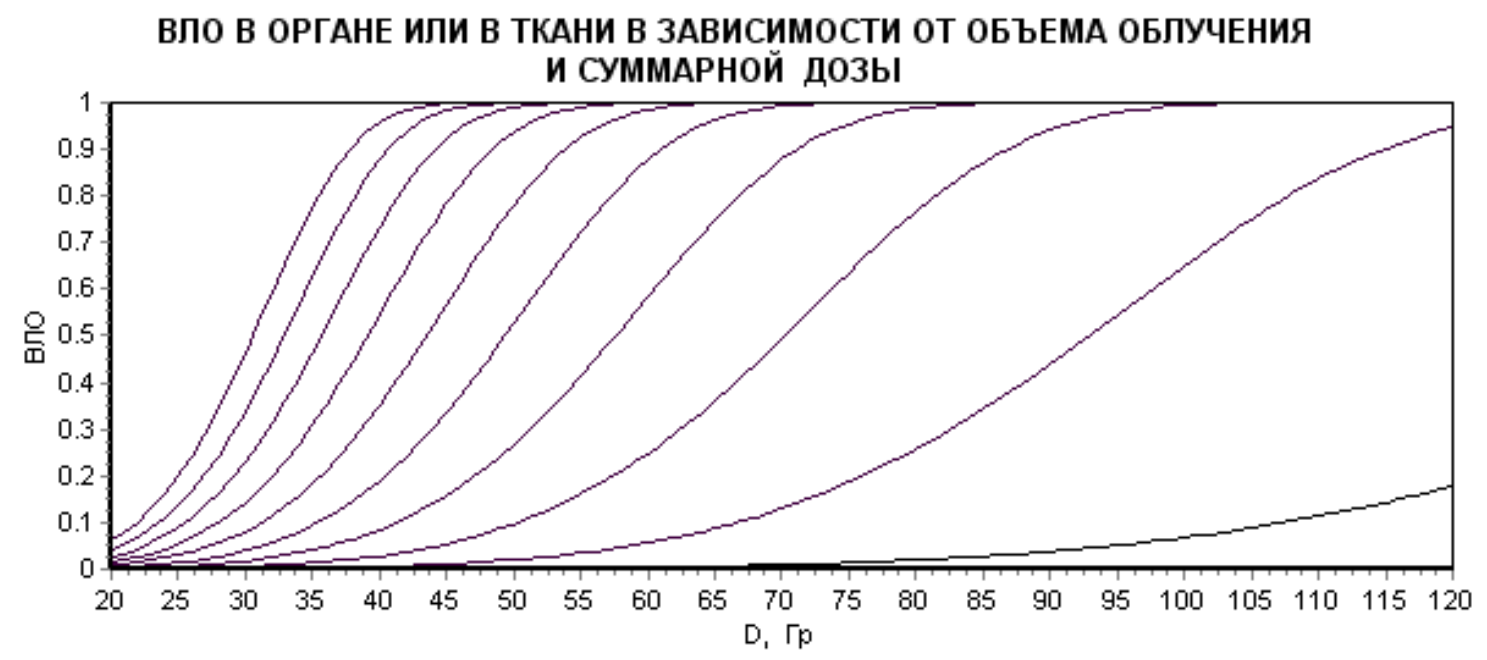

Рис. 5.1.1. Почка. Оптимизация по ВЛО 
Таблица 5.1.2

Почка. Оптимизация по ТД

\begin{tabular}{|c|c|c|c|c|c|c|c|c|c|}
\hline$P_{\text {КЛ }}$ & $V$ & $D_{\text {Кл }}$ & $D_{\text {TEOP }}$ & $\varepsilon, \%$ & $P_{\text {TEOP }}$ & $\varepsilon, \%$ & $A_{1}$ & $A_{2}$ & $b$ \\
\hline 0,05 & $1 / 3$ & 43,92 & 41,19 & $-6,21$ & 0,071 & 42,13 & \multirow{6}{*}{32,632} & \multirow{6}{*}{5,857} & \multirow{6}{*}{0,690} \\
\hline 0,05 & $2 / 3$ & 27,00 & 25,53 & $-5,43$ & 0,068 & 35,87 & & & \\
\hline 0,05 & 1 & 17,12 & 19,30 & 12,75 & 0,026 & $-48,63$ & & & \\
\hline 0,50 & $1 / 3$ & 59,14 & 65,27 & 10,37 & 0,328 & $-34,50$ & & & \\
\hline 0,50 & $2 / 3$ & 42,23 & 40,46 & $-4,19$ & 0,587 & 17,31 & & & \\
\hline 0,50 & 1 & 32,34 & 30,59 & 22,68 & 0,613 & 22,68 & & & \\
\hline
\end{tabular}

ВЛО В ОРГАНЕ ИЛИ В ТКАНИ В ЗАВИСИМОСТИ ОТ ОБЪЕМА ОБЛУЧЕНИЯ И СУММАРНОЙ ДОЗЫ

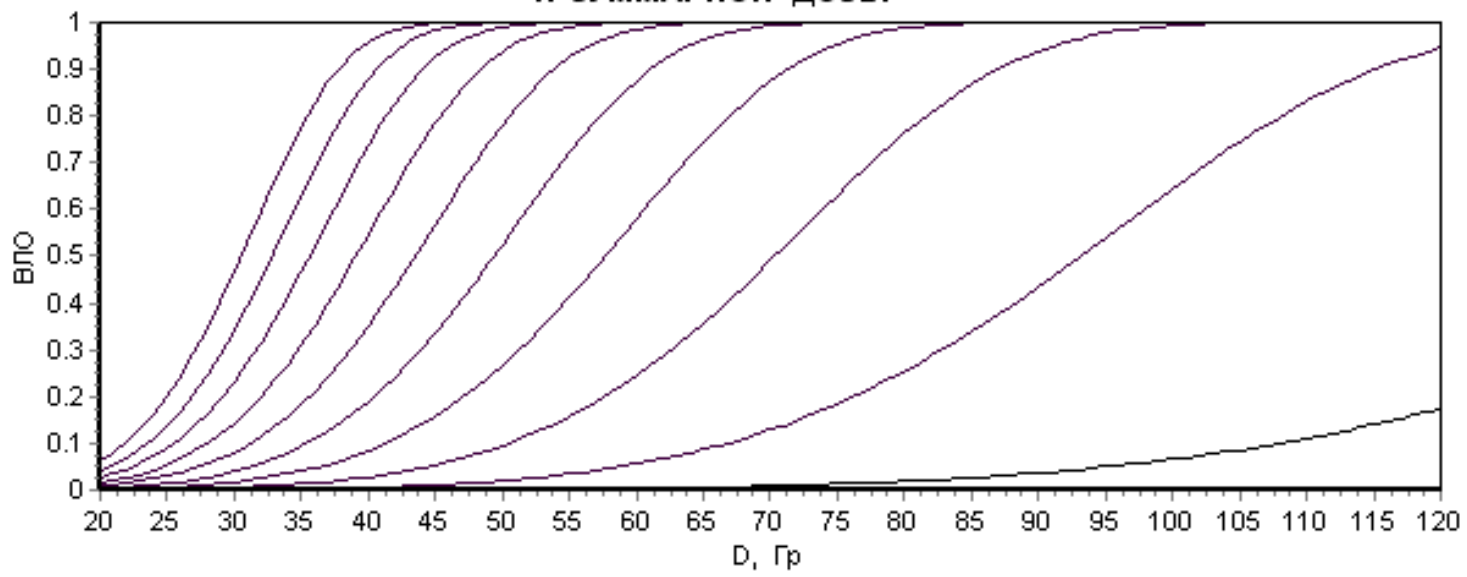

Рис. 5.1.2. Почка. Оптимизация по ТД

Таблица 5.1.3

Почка. Оптимизация по ВЛО + ТД

\begin{tabular}{|c|c|c|c|c|c|c|c|c|c|}
\hline$P_{\text {КЛ }}$ & V & $D_{\text {Кл }}$ & $D_{\mathrm{TEOP}}$ & $\varepsilon, \%$ & $P_{\mathrm{TEOP}}$ & $\varepsilon, \%$ & $A_{1}$ & $A_{2}$ & $b$ \\
\hline 0,05 & $1 / 3$ & 43,22 & 42,15 & $-4,03$ & 0,063 & 25,40 & \multirow{6}{*}{32,020} & \multirow{6}{*}{5,660} & \multirow{6}{*}{0,728} \\
\hline 0,05 & $2 / 3$ & 27,02 & 25,45 & $-5,82$ & 0,069 & 38,99 & & & \\
\hline 0,05 & 1 & 17,12 & 18,94 & 10,65 & 0,025 & $-42,96$ & & & \\
\hline 0,50 & $1 / 3$ & 59,14 & 66,77 & 12,90 & 0,294 & $-41,11$ & & & \\
\hline 0,50 & $2 / 3$ & 42,23 & 40,31 & $-4,54$ & 0,594 & 18,83 & & & \\
\hline 0,50 & 1 & 32,34 & 30,01 & $-7,21$ & 0,653 & 30,62 & & & \\
\hline
\end{tabular}


ВЛО В ОРГАНЕ ИЛИ В ТКАНИ В ЗАВИСИМОСТИ ОТ ОБЪЕМА ОБЛУЧЕНИЯ И СУММАРНОЙ ДОЗЫ

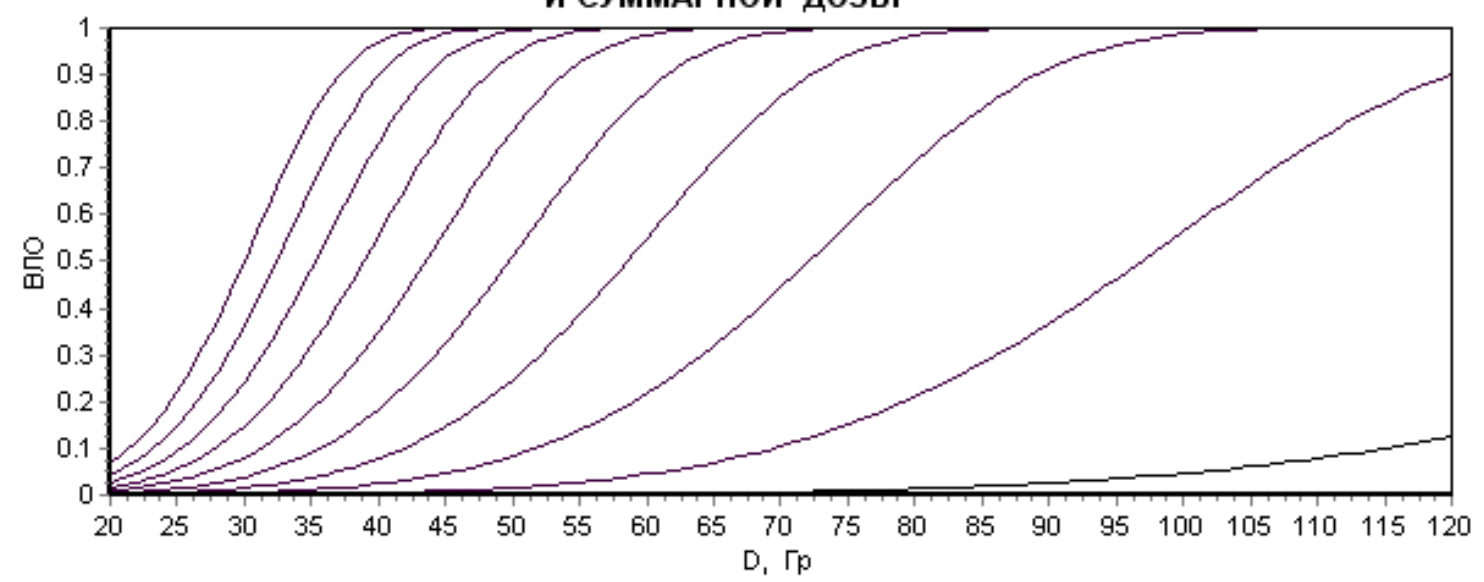

Рис. 5.1.3. Почка. Оптимизация по ВЛО + ТД

\section{2. Головной мозг}

Таблица 5.2.1

Головной мозг. Оптимизация по ВЛО

\begin{tabular}{|c|c|c|c|c|c|c|c|c|c|}
\hline$P_{\text {Кл }}$ & $V$ & $D_{\text {Кл }}$ & $D_{\text {TEOP }}$ & $\varepsilon, \%$ & $P_{\mathrm{TEOP}}$ & $\varepsilon, \%$ & $A_{1}$ & $A_{2}$ & $b$ \\
\hline 0,05 & $1 / 3$ & 58,56 & 57,78 & $-1,33$ & 0,055 & 10,39 & \multirow{6}{*}{70,808} & \multirow{6}{*}{7,615} & \multirow{6}{*}{0,170} \\
\hline 0,05 & $2 / 3$ & 51,42 & 51,36 & $-0,12$ & 0,050 & 0,86 & & & \\
\hline 0,05 & 1 & 47,25 & 47,94 & 1,46 & 0,045 & $-10,21$ & & & \\
\hline 0,50 & $1 / 3$ & 80,00 & 81,34 & 1,67 & 0,457 & $-8,57$ & & & \\
\hline 0,50 & $2 / 3$ & 72,86 & 72,30 & 0,521 & 0,521 & 4,13 & & & \\
\hline 0,50 & 1 & 68,68 & 67,48 & $-0,88$ & 0,524 & 4,71 & & & \\
\hline
\end{tabular}

ВЛО В ОРГАНЕ ИЛИ В ТКАНИ В ЗАВИСИМОСТИ ОТ ОБЪЕМА ОБЛУЧЕНИЯ И СУММАРНОЙ ДОЗЫ

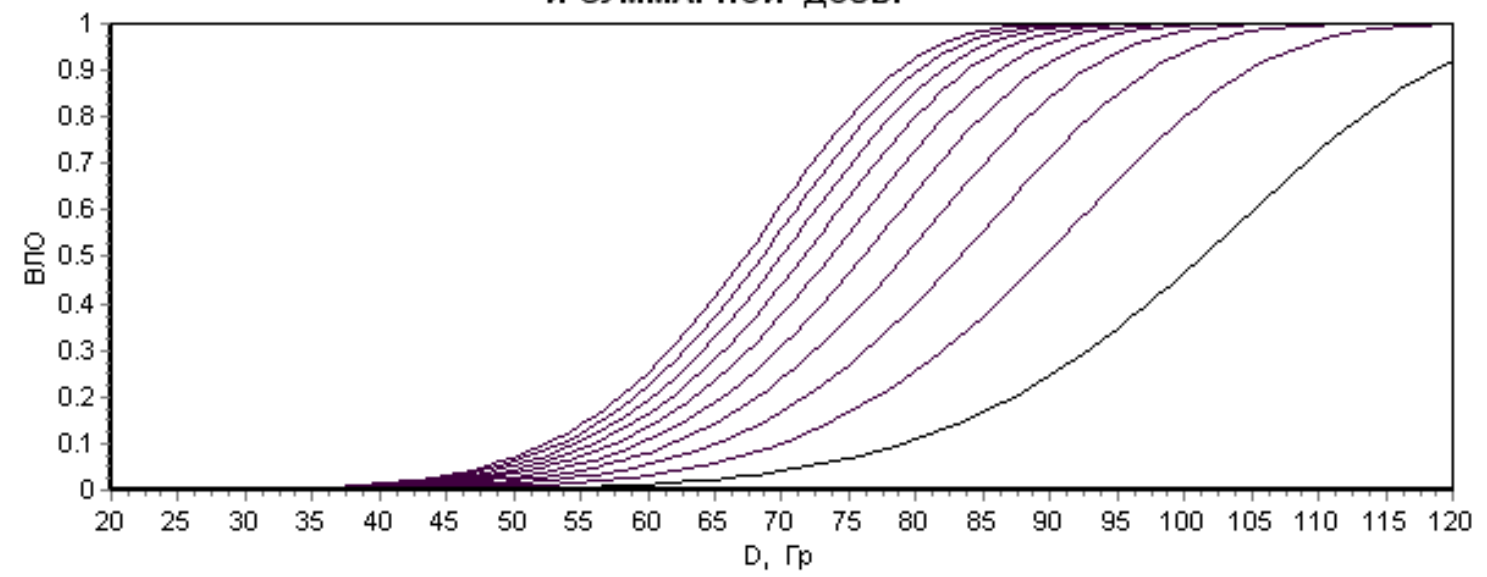

Рис. 5.2.1. Головной мозг. Оптимизация по ВЛО

Оптимальные значения параметров модели МРВ, полученные при помощи критерия ТД совпадают со значениями параметров, определенных при помощи 
критерия ВЛО. В табл. 5.2.2 приводятся параметры модели МРВ, рассчитанные раздельно, для ВЛО =0,05 и ВЛО =0,50. Полученные оптимальные значения параметров модели значительно отличаются друг от друга. Это означает, что либо использованные клинические данные являются плохо согласованными, либо модель МРВ не является адекватной клинической информации.

Таблица 5.2 .4

Головной мозг. Оптимизация по ТД, отдельно для ВЛО = 0,05 и ВЛО = 0,50

\begin{tabular}{|c|c|c|c|c|c|c|c|c|c|}
\hline$P_{\text {Кл }}$ & $V$ & $D_{\text {Кл }}$ & $D_{\mathrm{TEOP}}$ & $\varepsilon, \%$ & $P_{\mathrm{TEOP}}$ & $\varepsilon, \%$ & $\overline{A_{1}}$ & $\overline{A_{2}}$ & $b$ \\
\hline 0,05 & $1 / 3$ & 58,56 & 58,62 & 0,10 & 0,050 & $-1,00$ & \multirow{3}{*}{63,286} & \multirow{3}{*}{10,251} & \multirow{3}{*}{0,194} \\
\hline 0,05 & $2 / 3$ & 51,42 & 51,24 & $-0,35$ & 0,052 & 3,51 & & & \\
\hline 0,05 & 1 & 47,25 & 47,37 & 0,25 & 0,049 & $-2,30$ & & & \\
\hline 0,50 & $1 / 3$ & 80,00 & 80,61 & 0,77 & 0,500 & $-0,24$ & \multirow{3}{*}{151,795} & \multirow{3}{*}{0.456} & \multirow{3}{*}{0,155} \\
\hline 0,50 & $2 / 3$ & 72,86 & 72,40 & $-0,69$ & 0,501 & 0,20 & & & \\
\hline 0,50 & 1 & 68,68 & 67,99 & $-0,13$ & 0,500 & 0,04 & & & \\
\hline
\end{tabular}

\section{3. Ствол головного мозга}

Таблица 5.3.1

Ствол головного мозга. Оптимизация по ВЛО

\begin{tabular}{|c|c|c|c|c|c|c|c|c|c|}
\hline$P_{\text {КЛ }}$ & $V$ & $D_{\text {Кл }}$ & $D_{\mathrm{TEOP}}$ & $\varepsilon, \%$ & $P_{\mathrm{TEOP}}$ & $\varepsilon, \%$ & $A_{1}$ & $A_{2}$ & $b$ \\
\hline 0,05 & $1 / 3$ & 59,20 & 58,97 & $-0,39$ & 0,052 & 4,63 & \multirow{6}{*}{68,241} & \multirow{6}{*}{11,756} & \multirow{6}{*}{0,097} \\
\hline 0,05 & $2 / 3$ & 55,15 & 55,13 & $-0,03$ & 0,050 & 0,37 & & & \\
\hline 0,05 & 1 & 52,78 & 53,01 & 0,43 & 0,048 & $-4,78$ & & & \\
\hline 0,50 & $1 / 3$ & 72,99 & 73,59 & 0,82 & 0,467 & $-6,51$ & & & \\
\hline 0,50 & $2 / 3$ & 68,93 & 68,80 & $-0,19$ & 0,508 & 1,59 & & & \\
\hline 0,50 & 1 & 66,56 & 66,15 & $-0,62$ & 0,526 & 5,13 & & & \\
\hline
\end{tabular}

ВЛО В ОРГАНЕ ИЛИ В ТКАНИ В ЗАВИСИМОСТИ ОТ ОБЪЕМА ОБЛУЧЕНИЯ И СУММАРНОЙ ДОЗЫ

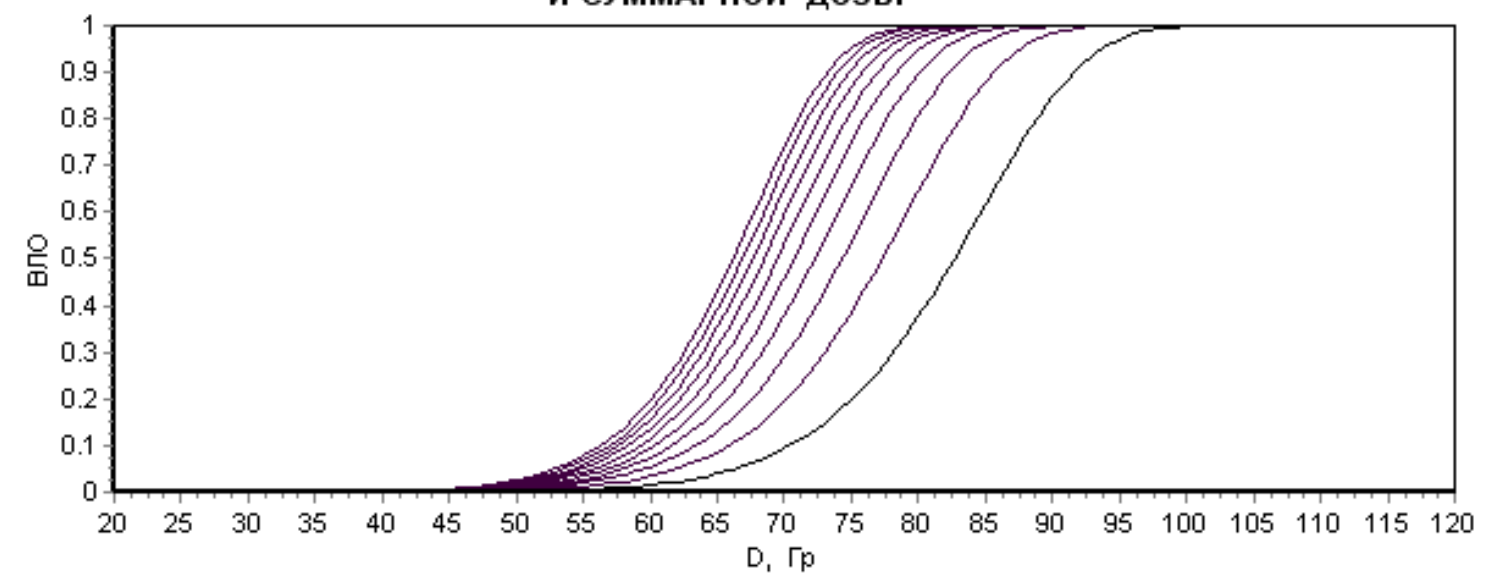

Рис. 5.3.1. Ствол головного мозга. Оптимизация по ВЛО 
Таблица 5.3.2

Ствол головного мозга. Оптимизация по ТД

\begin{tabular}{|c|c|c|c|c|c|c|c|c|c|}
\hline$P_{\text {Кл }}$ & $V$ & $D_{\text {Кл }}$ & $D_{\mathrm{TEOP}}$ & $\varepsilon, \%$ & $P_{\mathrm{TEOP}}$ & $\varepsilon, \%$ & $A_{1}$ & $A_{2}$ & $b$ \\
\hline 0,05 & $1 / 3$ & 59,20 & 58,86 & $-0,57$ & 0,053 & 6,79 & \multirow{6}{*}{68,344} & \multirow{6}{*}{11,756} & \multirow{6}{*}{0,094} \\
\hline 0,05 & $2 / 3$ & 55,15 & 55,15 & $-0,04$ & 9,050 & 0,04 & & & \\
\hline 0,05 & 1 & 52,78 & 53,09 & 0,58 & 0,047 & $-6,41$ & & & \\
\hline 0,50 & $1 / 3$ & 72,99 & 73,45 & 0,63 & 0,474 & $-5,09$ & & & \\
\hline 0,50 & $2 / 3$ & 68,93 & 68,82 & $-0,16$ & 0,507 & 1,31 & & & \\
\hline 0,50 & 1 & 66,56 & 66,25 & $-0,47$ & 0,519 & 3,88 & & & \\
\hline
\end{tabular}

ВЛО В ОРГАНЕ ИЛИ В ТКАНИ В ЗАВИСИМОСТИ ОТ ОБЪЕМА ОБЛУЧЕНИЯ И СУММАРНОЙ ДОЗЫ

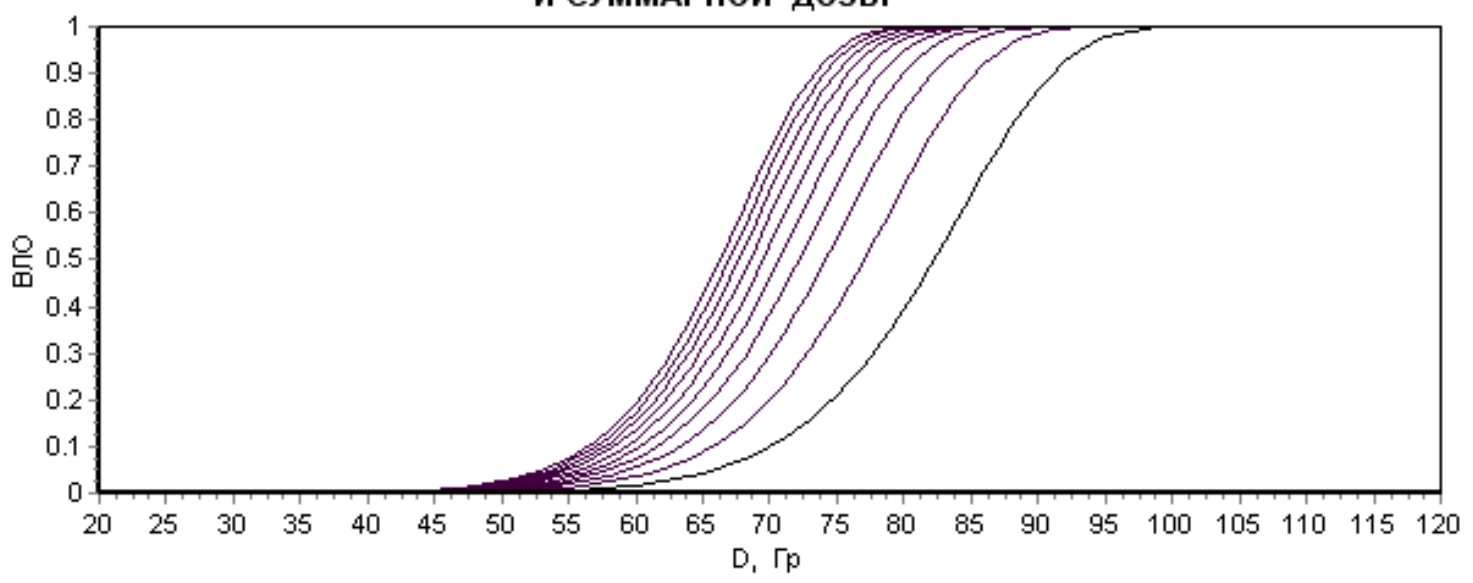

Рис. 5.3.2. Ствол головного мозга. Оптимизация по ТД

Таблица 5.3.3

Ствол головного мозга. Оптимизация по ВЛО + ТД

\begin{tabular}{|c|c|c|c|c|c|c|c|c|c|}
\hline$P_{\text {Кл }}$ & $V$ & $D_{\text {КЛ }}$ & $D_{\text {TEOP }}$ & $\varepsilon, \%$ & $P_{\mathrm{TEOP}}$ & $\varepsilon, \%$ & $A_{1}$ & $A_{2}$ & $b$ \\
\hline 0,05 & $1 / 3$ & 59,20 & 58,86 & $-0,57$ & 0,053 & 6,79 & \multirow{6}{*}{68,241} & \multirow{6}{*}{11,756} & \multirow{6}{*}{0,097} \\
\hline 0,05 & $2 / 3$ & 55,15 & 55,15 & $-0,04$ & 9,050 & 0,04 & & & \\
\hline 0,05 & 1 & 52,78 & 53,09 & 0,58 & 0,047 & $-6,41$ & & & \\
\hline 0,50 & $1 / 3$ & 72,99 & 73,45 & 0,63 & 0,474 & $-5,09$ & & & \\
\hline 0,50 & $2 / 3$ & 68,93 & 68,82 & $-0,16$ & 0,507 & 1,31 & & & \\
\hline 0,50 & 1 & 66,56 & 66,25 & $-0,47$ & 0,519 & 3,88 & & & \\
\hline
\end{tabular}


ВЛО В ОРГАНЕ ИЛИ В ТКАНИ В ЗАВИСИМОСТИ ОТ ОБЪЕМА ОБЛУЧЕНИЯ И СУММАРНОЙ ДОЗЫ

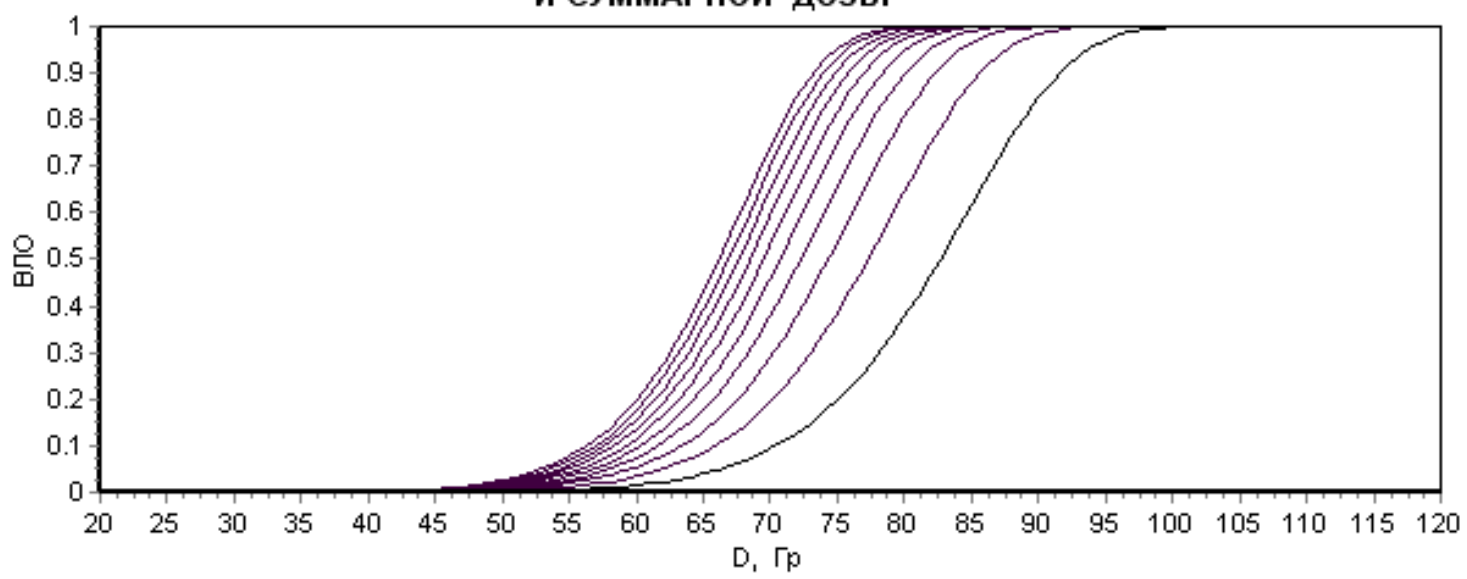

Рис. 5.3.3. Ствол головного мозга. Оптимизация по ВЛО + ТД

5.4. Ухо (среднее/внешнее). Первый вариант

Таблица 5.4

Ухо (среднее/внешнее)

\begin{tabular}{c|c|c|c|c|c}
\hline$P_{\text {КЛ }}$ & $D_{\text {КЛ }}$ & $P_{\text {КЛ }}$ & $D_{\text {КЛ }}$ & $A_{1}$ & $A_{2}$ \\
\hline 0,05 & 29,99 & 0,50 & 39,99 & 41,643 & 9,048 \\
\hline
\end{tabular}

ВЛО В ОРГАНЕ ИЛИ В ТКАНИ В ЗАВИСИМОСТИ ОТ СУММАРНОЙ ДОЗЫ

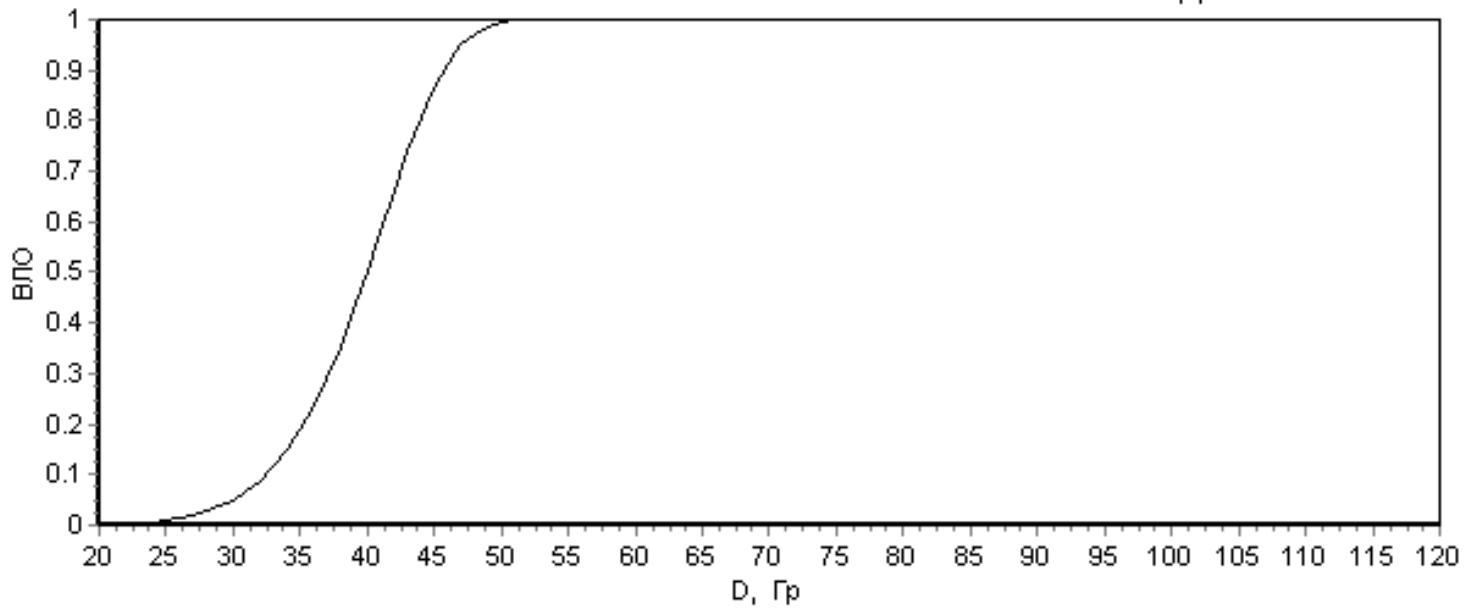

Рис. 5.4.1. Ухо (среднее/внешнее). Зависимость ВЛО от дозы

Расчеты показали, что оптимальных значений параметров для трех используемых критерив оптимальности совпадают! 
5.5. Ухо (среднее/внешнее). Второй вариант

Таблица 5.5.1

Ухо (среднее/внешнее). Оптимизация по ВЛО

\begin{tabular}{|c|c|c|c|c|c|c|c|c|c|}
\hline$P_{\text {Кл }}$ & $V$ & $D_{\text {КЛ }}$ & $D_{\text {TEOP }}$ & $\varepsilon, \%$ & $P_{\text {TEOP }}$ & $\varepsilon, \%$ & $A_{1}$ & $A_{2}$ & $b$ \\
\hline 0,05 & $1 / 3$ & 57,30 & 57,25 & $-0,09$ & 0,051 & 1,29 & \multirow{6}{*}{68,934} & \multirow{6}{*}{14,223} & \multirow{6}{*}{0,021} \\
\hline 0,05 & $2 / 3$ & 56,41 & 56,42 & 0,02 & 0,050 & $-0,24$ & & & \\
\hline 0,05 & 1 & 55,59 & 55,94 & 0,07 & 0,050 & 0,13 & & & \\
\hline 0,50 & $1 / 3$ & 68,66 & 68,75 & 0,13 & 0,494 & $-1,26$ & & & \\
\hline 0,50 & $2 / 3$ & 67,77 & 67,75 & $-0,02$ & 0,501 & 0,23 & & & \\
\hline 0,50 & 1 & 67,25 & 67,18 & $-0,10$ & 0,505 & 1,03 & & & \\
\hline
\end{tabular}

ВЛО В ОРГАНЕ ИЛИ В ТКАНИ В ЗАВИСИМОСТИ ОТ ОБЪЕМА ОБЛУЧЕНИЯ И СУММАРНОЙ ДОЗЫ

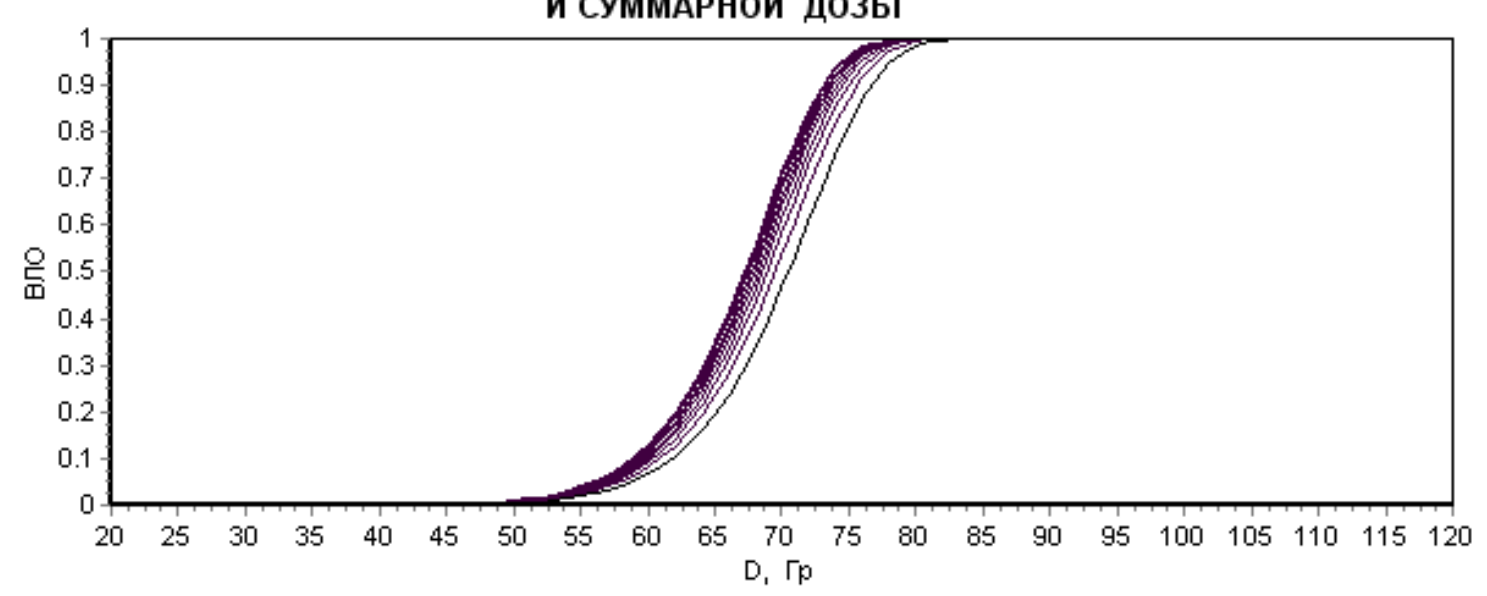

Рис. 5.5.1. Ухо (среднее/внешнее). Оптимизация по ВЛО

Таблица 5.5.2

Ухо (среднее/внешнее). Оптимизация по ТД

\begin{tabular}{|c|c|c|c|c|c|c|c|c|c|}
\hline$P_{\text {Кл }}$ & $V$ & $D_{\text {КЛ }}$ & $D_{\mathrm{TEOP}}$ & $\varepsilon, \%$ & $P_{\mathrm{TEOP}}$ & $\varepsilon, \%$ & $A_{1}$ & $A_{2}$ & $b$ \\
\hline 0,05 & $1 / 3$ & 57,30 & 57,25 & $-0,09$ & 0,051 & 1,29 & \multirow{6}{*}{68,934} & \multirow{6}{*}{14,223} & \multirow{6}{*}{0,021} \\
\hline 0,05 & $2 / 3$ & 56,41 & 56,42 & 0,02 & 0,050 & $-0,24$ & & & \\
\hline 0,05 & 1 & 55,59 & 55,94 & 0,07 & 0,050 & 0,13 & & & \\
\hline 0,50 & $1 / 3$ & 68,66 & 68,75 & 0,13 & 0,494 & $-1,26$ & & & \\
\hline 0,50 & $2 / 3$ & 67,77 & 67,75 & $-0,02$ & 0,501 & 0,23 & & & \\
\hline 0,50 & 1 & 67,25 & 67,18 & $-0,10$ & 0,505 & 1,03 & & & \\
\hline
\end{tabular}


ВЛО В ОРГАНЕ ИЛИ В ТКАНИ В ЗАВИСИМОСТИ ОТ ОБЪЕМА ОБЛУЧЕНИЯ И СУММАРНОЙ ДОЗЫ

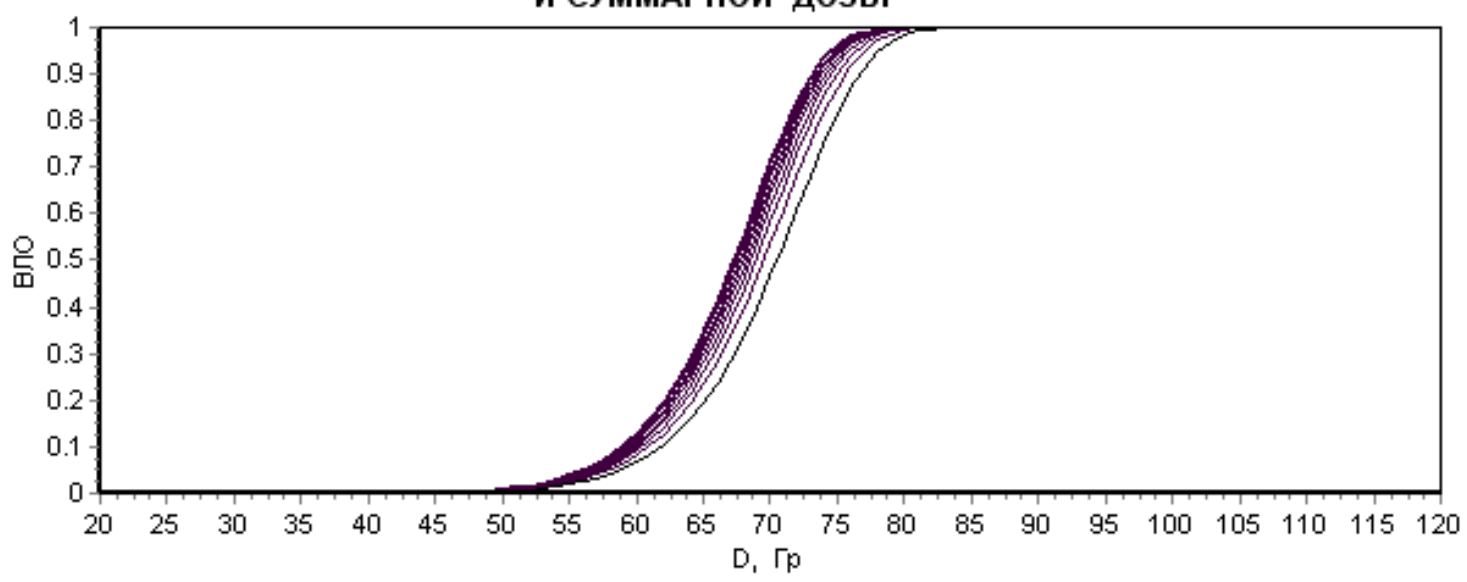

Рис. 5.5.2. Ухо (среднее/внешнее). Оптимизация по ТД

Таблица 5.3

Ухо (среднее/внешнее). Оптимизация по ВЛО + ТД

\begin{tabular}{|c|c|c|c|c|c|c|c|c|c|}
\hline$P_{\text {КЛ }}$ & $V$ & $D_{\text {КЛ }}$ & $D_{\mathrm{TEOP}}$ & $\varepsilon, \%$ & $P_{\mathrm{TEOP}}$ & $\varepsilon, \%$ & $A_{1}$ & $A_{2}$ & $b$ \\
\hline 0,05 & $1 / 3$ & 57,30 & 57,25 & $-0,09$ & 0,051 & 1,29 & \multirow{6}{*}{68,934} & \multirow{6}{*}{14,223} & \multirow{6}{*}{0,021} \\
\hline 0,05 & $2 / 3$ & 56,41 & 56,42 & 0,02 & 0,050 & $-0,24$ & & & \\
\hline 0,05 & 1 & 55,59 & 55,94 & 0,07 & 0,050 & 0,13 & & & \\
\hline 0,50 & $1 / 3$ & 68,66 & 68,75 & 0,13 & 0,494 & $-1,26$ & & & \\
\hline 0,50 & $2 / 3$ & 67,77 & 67,75 & $-0,02$ & 0,501 & 0,23 & & & \\
\hline 0,50 & 1 & 67,25 & 67,18 & $-0,10$ & 0,505 & 1,03 & & & \\
\hline
\end{tabular}

ВЛО В ОРГАНЕ ИЛИ В ТКАНИ В ЗАВИСИМОСТИ ОТ ОБЪЕМА ОБЛУЧЕНИЯ И СУММАРНОЙ ДОЗЫ

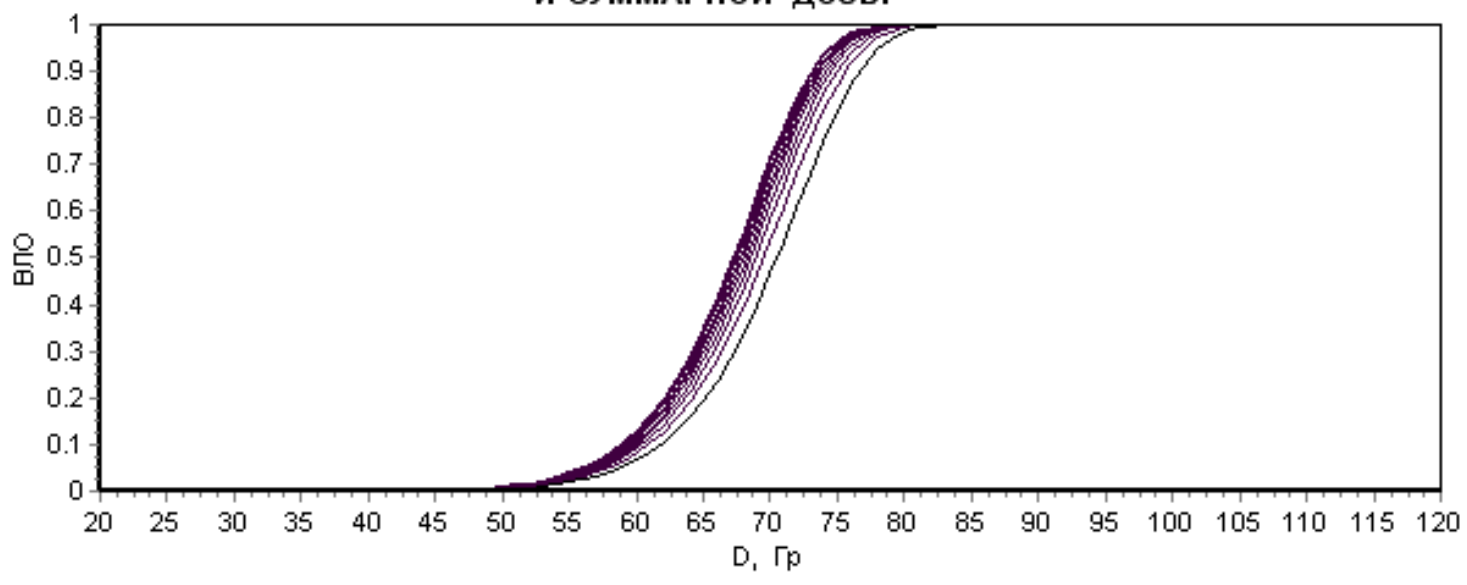

Рис. 5.3. Оптимизация по ВЛО + ТД 


\section{6. Пищевод}

Таблица 5.6.1

Пищевод. Оптимизация по ВЛО

\begin{tabular}{|c|c|c|c|c|c|c|c|c|c|}
\hline$P_{\text {Кл }}$ & $V$ & $D_{\text {КЛ }}$ & $\overline{D_{\text {TEOP }}}$ & $\varepsilon, \%$ & $P_{\mathrm{TEOP}}$ & $\varepsilon, \%$ & $A_{1}$ & $A_{2}$ & $b$ \\
\hline 0,05 & $1 / 3$ & 59,10 & 59,04 & $-0,10$ & 0,051 & 1,15 & \multirow{6}{*}{74,269} & \multirow{6}{*}{11,317} & \multirow{6}{*}{0,030} \\
\hline 0,05 & $2 / 3$ & 57,82 & 57,82 & 0,07 & 0,050 & $-0,08$ & & & \\
\hline 0,05 & 1 & 57,07 & 57,13 & 0,10 & 0,049 & $-1,06$ & & & \\
\hline 0,50 & $1 / 3$ & 74,10 & 74,31 & 0,29 & 0,490 & $-2,22$ & & & \\
\hline 0,50 & $2 / 3$ & 72,82 & 72,78 & $-0,05$ & 0,502 & 0,41 & & & \\
\hline 0,50 & 1 & 72,07 & 71,30 & -0.23 & 0,509 & 1,84 & & & \\
\hline
\end{tabular}

ВЛО В ОРГАНЕ ИЛИ В ТКАНИ В ЗАВИСИМОСТИ ОТ ОБЪЕМА ОБЛУЧЕНИЯ И СУММАРНОЙ ДОЗЫ

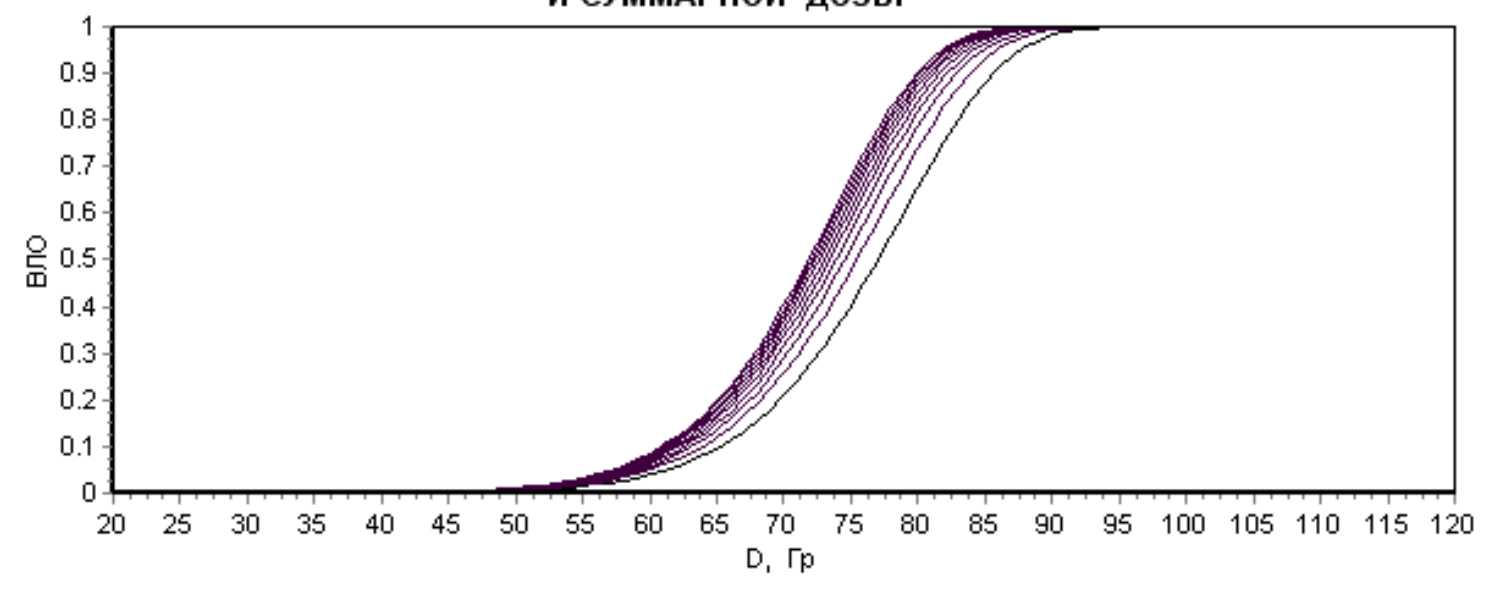

Рис. 5.6.1. Пищевод. Оптимизация по ВЛО

Результаты оптимизации по критерию ТД совпадают с результатами оптимизацией по ВЛО и с результатами оптимизациии по ВЛО + ТД. Расчет параметров модели МРВ раздельно для ВЛО =0,05 и ВЛО = 0,50 показал, что клинические данные по отношению к модели МРВ являются плохо согласованными!

Таблица 5.6.4

Пищевод. Оптимизация по ТД, отдельно для ВЛО = 0,05 и ВЛО = 0,50

\begin{tabular}{|c|c|c|c|c|c|c|c|c|c|}
\hline$P_{\text {Кл }}$ & $P_{\mathrm{TEOP}}$ & $\varepsilon, \%$ & $V$ & $D_{\text {КЛ }}$ & $D_{\text {TEOP }}$ & $\varepsilon, \%$ & $A_{1}$ & $A_{2}$ & $b$ \\
\hline 0,05 & 0,050 & 0,57 & $1 / 3$ & 59,10 & 59,00 & $-0,16$ & \multirow{3}{*}{130,847} & \multirow{3}{*}{3,586} & \multirow{3}{*}{0,029} \\
\hline 0,05 & 0,050 & $-0,06$ & $2 / 3$ & 57,82 & 57,83 & 0,02 & & & \\
\hline 0,05 & 0,050 & $-0,51$ & 1 & 57,07 & 57,15 & 0,15 & & & \\
\hline 0,50 & 0,500 & 0,00 & $1 / 3$ & 74,10 & 74,13 & 0,05 & \multirow{3}{*}{44671,344} & \multirow{3}{*}{0,057} & \multirow{3}{*}{0,026} \\
\hline 0,50 & 0,500 & 0,00 & $2 / 3$ & 72,82 & 72,81 & $-0,01$ & & & \\
\hline 0,50 & 0,500 & 0,00 & 1 & 72,07 & 72,05 & $-0,03$ & & & \\
\hline
\end{tabular}




\section{7. Сердце}

Таблица 5.7.1

Сердце. Оптимизация по ВЛО

\begin{tabular}{|c|c|c|c|c|c|c|c|c|c|}
\hline$P_{\text {Кл }}$ & $V$ & $D_{\text {Кл }}$ & $D_{\text {TEOP }}$ & $\varepsilon, \%$ & $P_{\text {TEOP }}$ & $\varepsilon, \%$ & $A_{1}$ & $A_{2}$ & $b$ \\
\hline 0,05 & $1 / 3$ & 59,91 & 59,09 & $-1,37$ & 0,058 & 15,04 & \multirow{6}{*}{48,704} & \multirow{6}{*}{10,478} & \multirow{6}{*}{0,434} \\
\hline 0,05 & $2 / 3$ & 44,53 & 43,74 & $-1,77$ & 0,060 & 19,99 & & & \\
\hline 0,05 & 1 & 35,54 & 36,68 & $-27,70$ & 0,036 & $-27,70$ & & & \\
\hline 0,50 & $1 / 3$ & 72,54 & 75,76 & 4,44 & 0,356 & $-28,85$ & & & \\
\hline 0,50 & $2 / 3$ & 57,17 & 56,08 & $-1,91$ & 0,572 & 14,37 & & & \\
\hline 0,50 & 1 & 48,18 & 47,03 & $-2,39$ & 0,591 & 18,10 & & & \\
\hline
\end{tabular}

ВЛО В ОРГАНЕ ИЛИ В ТКАНИ В ЗАВИСИМОСТИ ОТ ОБЪЕМА ОБЛУЧЕНИЯ И СУММАРНОЙ ДОЗЫ

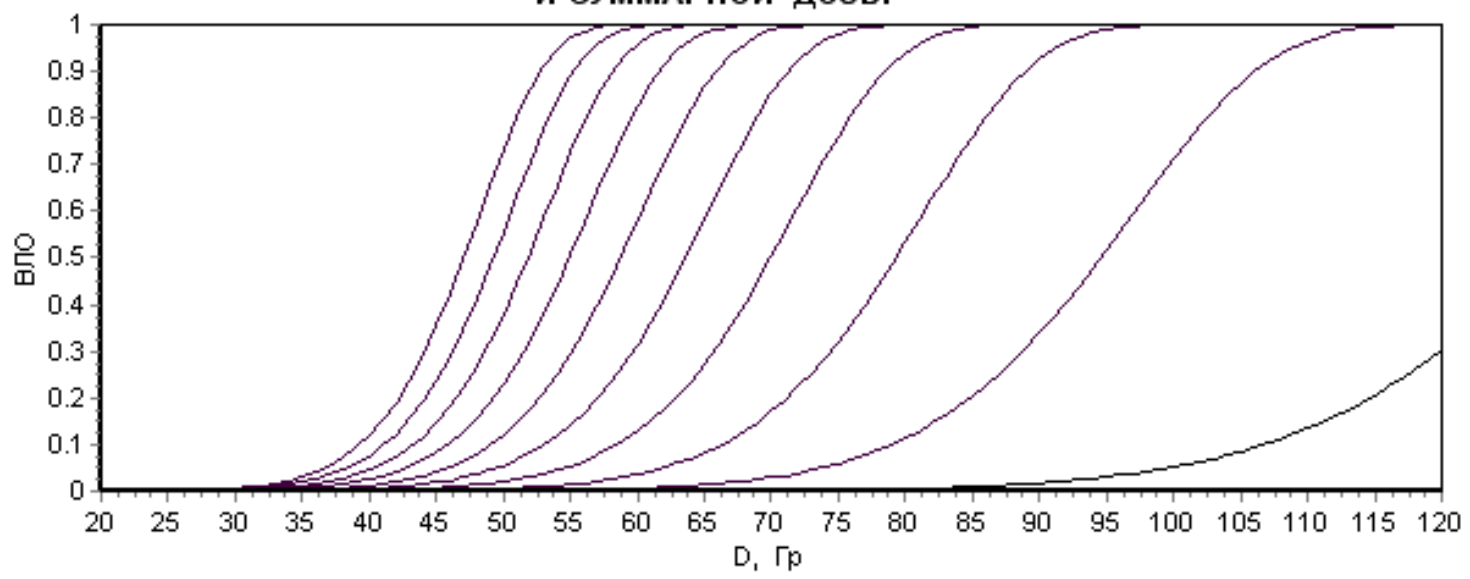

Рис 5.7.1. Сердце. Оптимизация по ВЛО

Таблица 5.7.2

Сердце. Оптимзация по ТД

\begin{tabular}{|c|c|c|c|c|c|c|c|c|c|}
\hline$P_{\text {Кл }}$ & $V$ & $D_{\text {Кл }}$ & $D_{\text {TEOP }}$ & $\varepsilon, \%$ & $P_{\text {TEOP }}$ & $\varepsilon, \%$ & $A_{1}$ & $A_{2}$ & $b$ \\
\hline 0,05 & $1 / 3$ & 59,91 & 58,60 & $-2,19$ & 0,063 & 25,24 & \multirow{6}{*}{49,047} & \multirow{6}{*}{10,478} & \multirow{6}{*}{0,420} \\
\hline 0,05 & $2 / 3$ & 44,53 & 43,80 & $-1,64$ & 0,059 & 18,37 & & & \\
\hline 0,05 & 1 & 35,54 & 36,94 & 3,94 & 0,034 & $-32,74$ & & & \\
\hline 0,50 & $1 / 3$ & 72,54 & 75,13 & 3,57 & 0,381 & $-23,76$ & & & \\
\hline 0,50 & $2 / 3$ & 57,17 & 56,15 & $-1,78$ & 0,567 & 13,35 & & & \\
\hline 0,50 & 1 & 48,18 & 47,36 & $-1,70$ & 0,564 & 12,75 & & & \\
\hline
\end{tabular}




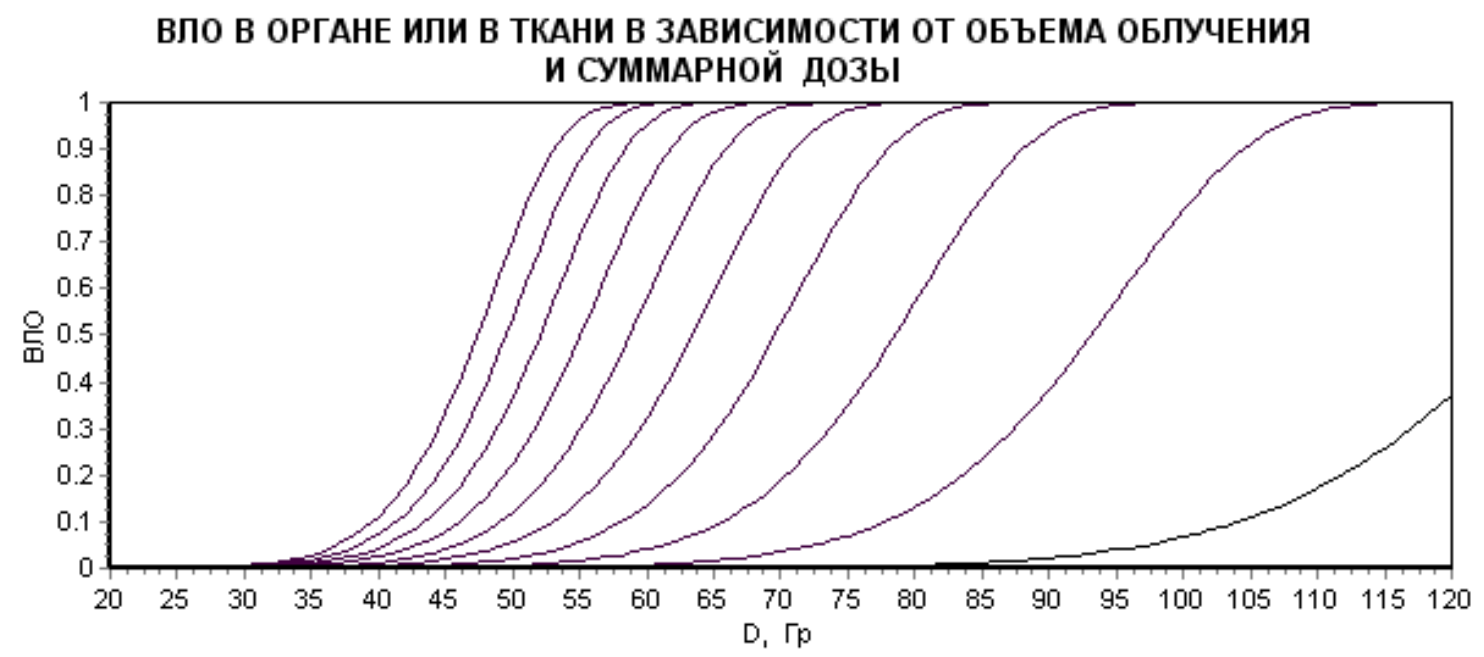

Рис. 5.7.2. Сердце. Оптимизация по ТД

Таблица 5.7.3

Сердце. Оптимизация по ВЛО

\begin{tabular}{|c|c|c|c|c|c|c|c|c|c|}
\hline$P_{\text {Кл }}$ & $V$ & $D_{\text {Кл }}$ & $D_{\text {TEOP }}$ & $\varepsilon, \%$ & $P_{\mathrm{TEOP}}$ & $\varepsilon, \%$ & $A_{1}$ & $A_{2}$ & $b$ \\
\hline 0,05 & $1 / 3$ & 59,91 & 59,09 & $-1,37$ & 0,058 & 15,04 & \multirow{6}{*}{48,704} & \multirow{6}{*}{10,478} & \multirow{6}{*}{0,434} \\
\hline 0,05 & $2 / 3$ & 44,53 & 43,74 & $-1,77$ & 0,060 & $\begin{array}{l}19,99 \\
\end{array}$ & & & \\
\hline 0,05 & 1 & 35,54 & 36,68 & $-27,70$ & 0,036 & $-27,70$ & & & \\
\hline 0,50 & $1 / 3$ & 72,54 & 75,76 & 4,44 & 0,356 & $-28,85$ & & & \\
\hline 0,50 & $2 / 3$ & 57,17 & 56,08 & $\begin{array}{l}-1,91 \\
\end{array}$ & 0,572 & 14,37 & & & \\
\hline 0,50 & 1 & 48,18 & 47,03 & $-2,39$ & 0,591 & 18,10 & & & \\
\hline
\end{tabular}

ВЛО В ОРГАНЕ ИЛИ В ТКАНИ В ЗАВИСИМОСТИ ОТ ОБЪЕМА ОБЛУЧЕНИЯ И СУММАРНОЙ ДОЗЫ

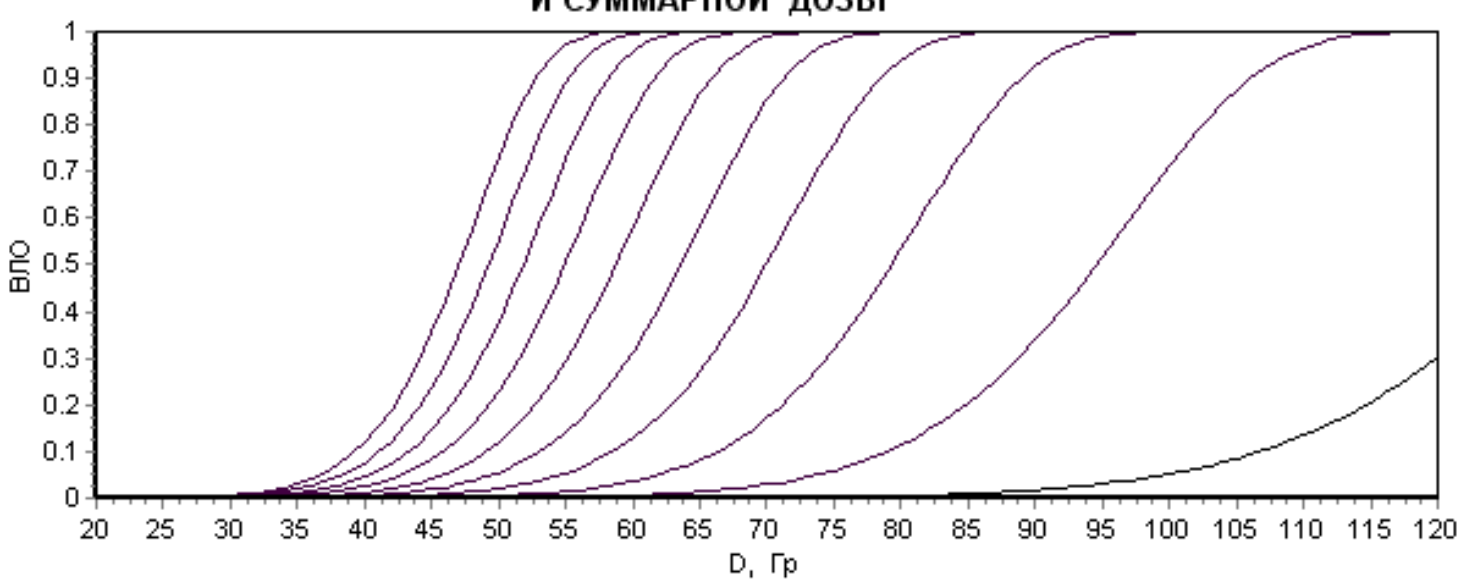

Рис. 5.7.3. Сердце. Оптимизация по ВЛО + ТД 
Таблица 5.7.4

Сердце. Оптимизация по ТД, отдельно для ВЛО =0,05 и ВЛО =0,50

\begin{tabular}{|c|c|c|c|c|c|c|c|c|c|}
\hline$P_{\text {Кл }}$ & $P_{\mathrm{TEOP}}$ & $\varepsilon, \%$ & $V$ & $D_{\text {Кл }}$ & $D_{\mathrm{TEOP}}$ & $\varepsilon, \%$ & $A_{1}$ & $A_{2}$ & $b$ \\
\hline 0,05 & 0,045 & $-10,41$ & $1 / 3$ & 59,91 & 60,41 & 0,84 & \multirow{3}{*}{44,878} & \multirow{3}{*}{13,488} & \multirow{3}{*}{0,471} \\
\hline 0,05 & 0,066 & 32,40 & $2 / 3$ & 44,53 & 43,58 & $-2,12$ & & & \\
\hline 0,05 & 0,042 & $-15,84$ & 1 & 35,54 & 36,01 & 1,32 & & & \\
\hline 0,50 & 0,500 & $-0,04$ & $1 / 3$ & 72,54 & 72,88 & 0,46 & \multirow{3}{*}{860,743} & \multirow{3}{*}{0,128} & \multirow{3}{*}{0,369} \\
\hline 0,50 & 0,501 & 0,12 & $2 / 3$ & 57,17 & $\begin{array}{l}56,43 \\
\end{array}$ & $\begin{array}{l}-1,30 \\
\end{array}$ & & & \\
\hline 0,50 & 0,500 & $-0,07$ & 1 & 48,18 & 48,59 & 0,85 & & & \\
\hline
\end{tabular}

Анализ табл. 5.7.4 свидетельствует о том, что клиническая информация по значениям ВЛО либо является плохо согласованной, либо модель МРВ является не адекватно разделенной по ВЛО клинической информацией. Вместе с тем, оптимизция по ТД показывает, что максимальное отклонение теоретических (модельных) значений ТД от клинических не превышает 3,94\%!

\section{8. Мочевой пузырь}

Таблица 5.8.1

Мочевой пузырь. Оптимизация по ВЛО

\begin{tabular}{|c|c|c|c|c|c|c|c|c|c|}
\hline$P_{\text {КЛ }}$ & $V$ & $D_{\text {КЛ }}$ & $D_{\text {TEOP }}$ & $\varepsilon, \%$ & $P_{\mathrm{TEOP}}$ & $\varepsilon, \%$ & $A_{1}$ & $A_{2}$ & $b$ \\
\hline 0,05 & $1 / 3$ & 59,40 & 59,17 & $-0,38$ & 0,051 & 2,30 & \multirow{6}{*}{91,267} & \multirow{6}{*}{6,069} & \multirow{6}{*}{0,051} \\
\hline 0,05 & $2 / 3$ & 57,10 & 57,12 & 0,03 & 0,050 & $-0,18$ & & & \\
\hline 0,05 & 1 & 55,75 & 55,95 & 0,36 & 0,049 & $-2,08$ & & & \\
\hline 0,50 & $1 / 3$ & 90,14 & $\begin{array}{l}90,87 \\
\end{array}$ & 0.81 & 0,483 & $-3,37$ & & & \\
\hline 0,50 & $2 / 3$ & 87,84 & 87,71 & $-0,14$ & 0,593 & 0,61 & & & \\
\hline 0,50 & 1 & 86,49 & 85,92 & $-0,66$ & 0,514 & 2,81 & & & \\
\hline
\end{tabular}

ВЛО В ОРГАНЕ ИЛИ В ТКАНИ В ЗАВИСИМОСТИ ОТ ОБЪЕМА ОБЛУЧЕНИЯ И СУММАРНОЙ ДОЗЫ

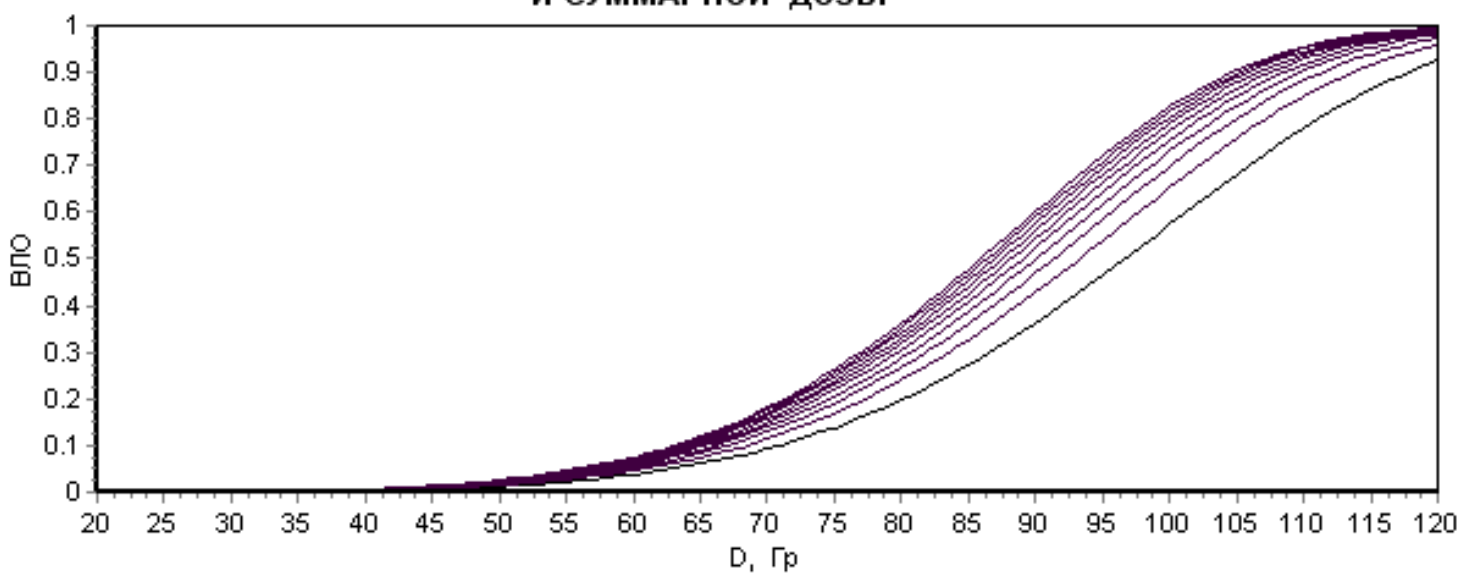

Рис. 5.8.1. Мочевой пузырь. Оптимизация по ВЛО 
Таблица 5.8.2

Мочевой пузырь. Оптимизация по ТД

\begin{tabular}{|c|c|c|c|c|c|c|c|c|c|}
\hline$P_{\text {Кл }}$ & $V$ & $D_{\text {КЛ }}$ & $D_{\mathrm{TEOP}}$ & $\varepsilon, \%$ & $P_{\mathrm{TEOP}}$ & $\varepsilon, \%$ & $A_{1}$ & $A_{2}$ & $b$ \\
\hline 0,05 & $1 / 3$ & 59,40 & 59,07 & $-0,56$ & 0,052 & 3,39 & \multirow{6}{*}{91,404} & \multirow{6}{*}{6,069} & \multirow{6}{*}{0,048} \\
\hline 0,05 & $2 / 3$ & 57,10 & 57,13 & 0,06 & 0,050 & 0,51 & & & \\
\hline 0,05 & 1 & 55,75 & 56,03 & 0,51 & 0,049 & $-2,94$ & & & \\
\hline 0,50 & $1 / 3$ & 90,14 & 90,71 & $-2,62$ & 0,487 & $-2,62$ & & & \\
\hline 0,50 & $2 / 3$ & 87,84 & 87,84 & $-0,11$ & 0,502 & 0,48 & & & \\
\hline 0,50 & 1 & 86,49 & 86,05 & $-0,51$ & 0,511 & 2,17 & & & \\
\hline
\end{tabular}

ВЛО В ОРГАНЕ ИЛИ В ТКАНИ В ЗАВИСИМОСТИ ОТ ОБЪЕМА ОБЛУЧЕНИЯ И СУММАРНОЙ ДОЗЫ

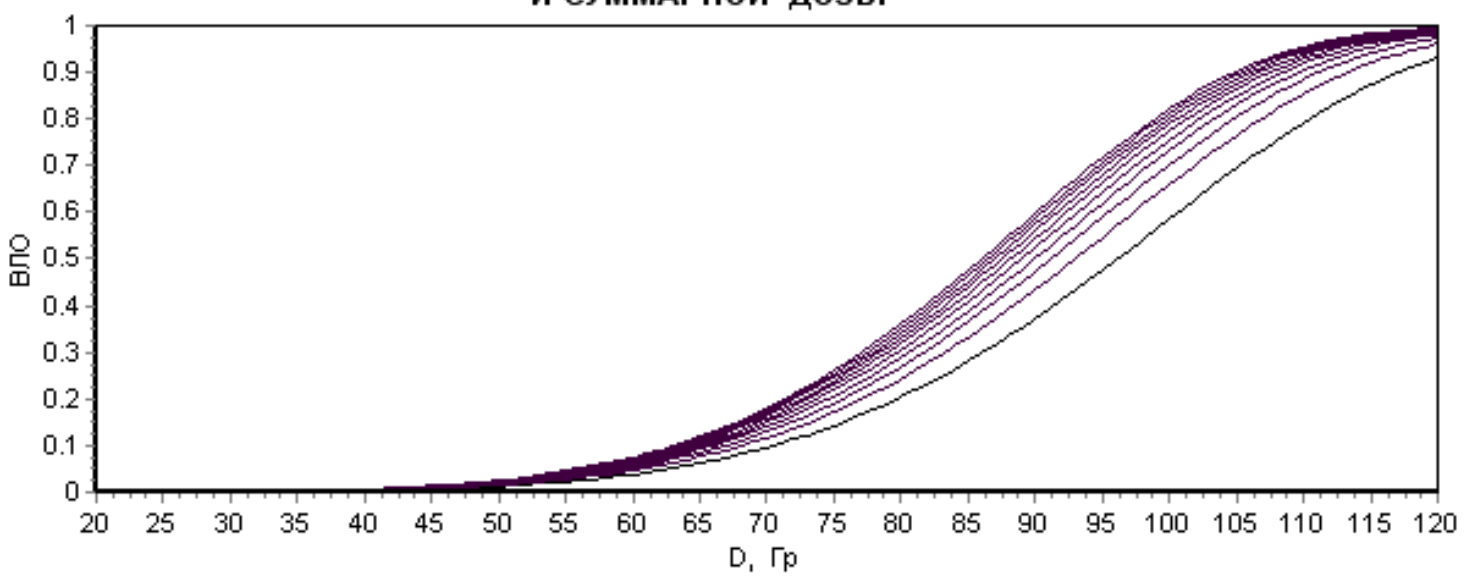

Рис. 5.8.2. Мочевой пузырь. Оптимизация по ТД

Таблица 5.8.3

Мочевой пузырь. Оптимизация по ВЛО + ТД

\begin{tabular}{|c|c|c|c|c|c|c|c|c|c|}
\hline$P_{\text {Кл }}$ & $V$ & $D_{\text {Кл }}$ & $D_{\text {TEOP }}$ & $\varepsilon, \%$ & $P_{\text {TEOP }}$ & $\varepsilon, \%$ & $A_{1}$ & $A_{2}$ & $b$ \\
\hline 0,05 & $1 / 3$ & $\begin{array}{l}59,40 \\
\end{array}$ & 59,17 & $-0,38$ & 0,051 & 2,30 & \multirow{6}{*}{91,267} & \multirow{6}{*}{6,069} & \multirow{6}{*}{0,051} \\
\hline 0,05 & $2 / 3$ & 57,10 & 57,12 & 0,03 & 0,050 & $-0,18$ & & & \\
\hline 0,05 & 1 & 55,75 & 55,95 & 0,36 & 0,049 & $-2,08$ & & & \\
\hline 0,50 & $1 / 3$ & 90,14 & 90,87 & 0.81 & 0,483 & $-3,37$ & & & \\
\hline 0,50 & $2 / 3$ & 87,84 & 87,71 & $-0,14$ & 0,593 & 0,61 & & & \\
\hline 0,50 & 1 & 86,49 & 85,92 & $-0,66$ & 0,514 & 2,81 & & & \\
\hline
\end{tabular}


ВЛО В ОРГАНЕ ИЛИ В ТКАНИ В ЗАВИСИМОСТИ ОТ ОБЪЕМА ОБЛУЧЕНИЯ

И СУММАРНОЙ ДОЗЫ

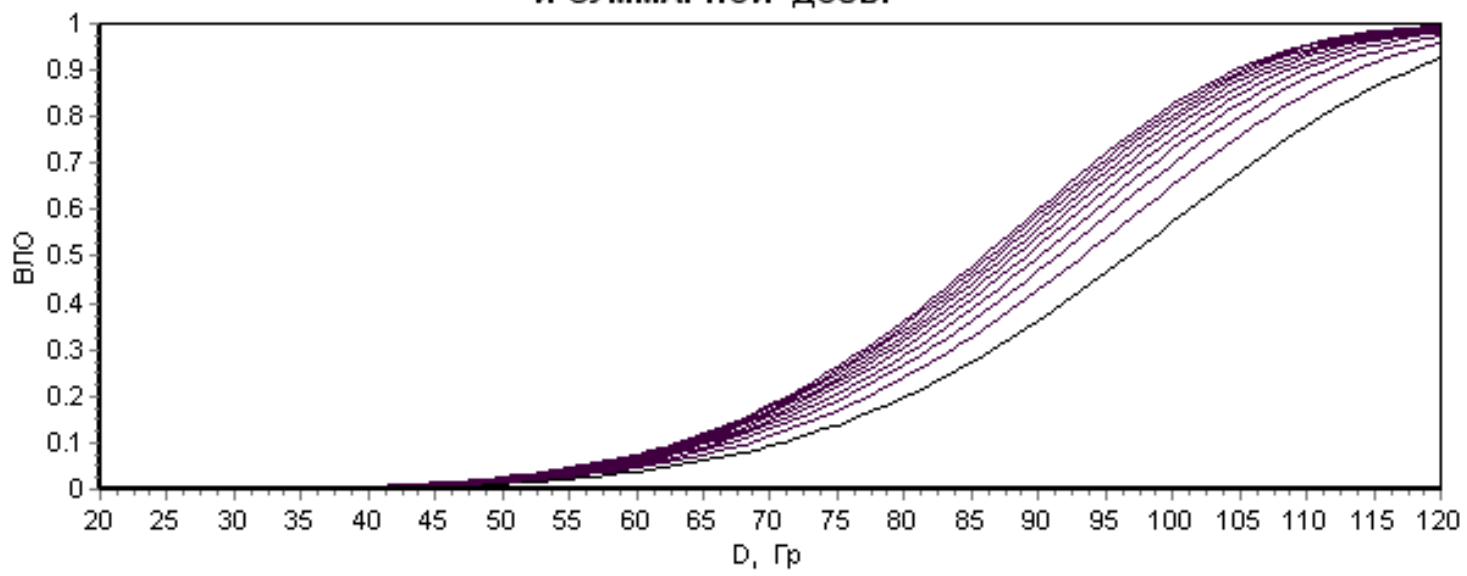

Рис. 5.8.3. Мочевой пузырь. Оптимизация по ВЛО + ТД

Анализ полученных результатов свидетельствует о согласованности клинической информации по ВЛО $=0,05$ и по ВЛО $=0,50$, а также об адекватности модели МРВ по отношению к используемой информации.

\section{9. Гортань (некроз хряща)}

Таблица 5.9.1

Гортань (некроз хряща). Оптимизация по ВЛО

\begin{tabular}{|c|c|c|c|c|c|c|c|c|c|}
\hline$P_{\text {Кл }}$ & $V$ & $D_{\text {Кл }}$ & $D_{\mathrm{TEOP}}$ & $\varepsilon, \%$ & $P_{\mathrm{TEOP}}$ & $\varepsilon, \%$ & $A_{1}$ & $A_{2}$ & $b$ \\
\hline 0,05 & $1 / 3$ & 77,90 & 77,34 & $-0,72$ & 0,057 & 14,54 & \multirow{6}{*}{79,801} & \multirow{6}{*}{19,297} & \multirow{6}{*}{0,112} \\
\hline 0,05 & $2 / 3$ & 71,57 & 71,56 & $-0,01$ & 0,050 & 0,25 & & & \\
\hline 0,05 & 1 & 67,88 & 68,38 & 0,74 & 0,044 & $-12,95$ & & & \\
\hline 0,50 & $1 / 3$ & 89,24 & 88,55 & 6,80 & 0,178 & $-64,46$ & & & \\
\hline 0,50 & $2 / 3$ & 82,91 & 81,93 & 3,44 & 0,304 & $-39,27$ & & & \\
\hline 0,50 & 1 & 79,21 & 78,29 & $-9,48$ & 0,991 & 98,19 & & & \\
\hline
\end{tabular}

ВЛО В ОРГАНЕ ИЛИ В ТКАНИ В ЗАВИСИМОСТИ ОТ ОБЪЕМА ОБЛУЧЕНИЯ И СУММАРНОЙ ДОЗЫ

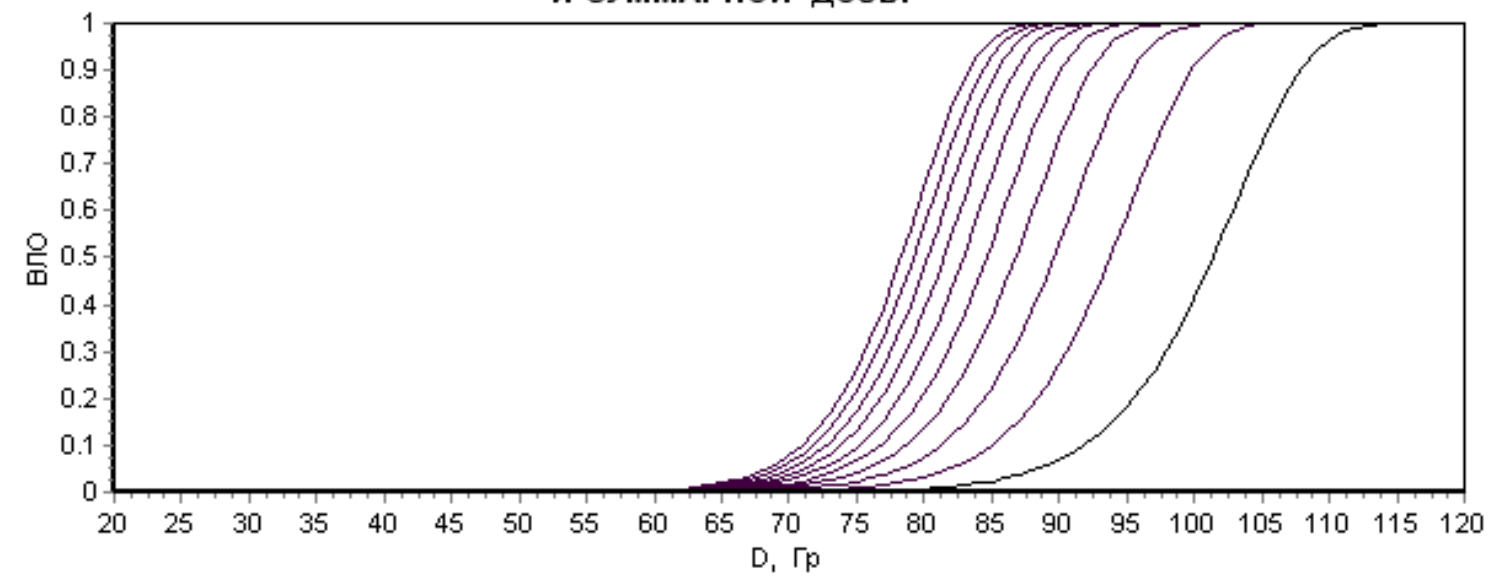

Рис. 5.9.1. Гортань (некроз хряща). Оптимизация по ВЛО 
Таблица 5.9.2

Гортань (некроз хряща). Оптимизация по ТД

\begin{tabular}{|c|c|c|c|c|c|c|c|c|c|}
\hline$P_{\text {Кл }}$ & $V$ & $D_{\text {Кл }}$ & $D_{\text {TEOP }}$ & $\varepsilon, \%$ & $P_{\mathrm{TEOP}}$ & $\varepsilon, \%$ & $A_{1}$ & $A_{2}$ & $b$ \\
\hline 0,05 & $1 / 3$ & 77,90 & 74,57 & $-4,27$ & 0,112 & 124,13 & \multirow{6}{*}{82,279} & \multirow{6}{*}{19,237} & \multirow{6}{*}{0,051} \\
\hline 0,05 & $2 / 3$ & 71,57 & 71,98 & 0,57 & 0,045 & $-10,17$ & & & \\
\hline 0,05 & 1 & 67,88 & 70,51 & 3,87 & 0,024 & $-51,19$ & & & \\
\hline 0,50 & $1 / 3$ & 89,24 & 85,38 & 2,98 & 0,326 & $-34,84$ & & & \\
\hline 0,50 & $2 / 3$ & 82,91 & 82,41 & 4,04 & 0,276 & $-44,74$ & & & \\
\hline 0,50 & 1 & 79,21 & 80,73 & $-6,66$ & 0,927 & 85,33 & & & \\
\hline
\end{tabular}

ВЛО В ОРГАНЕ ИЛИ В ТКАНИ В ЗАВИСИМОСТИ ОТ ОБЪЕМА ОБЛУЧЕНИЯ И СУММАРНОЙ ДОЗЫ

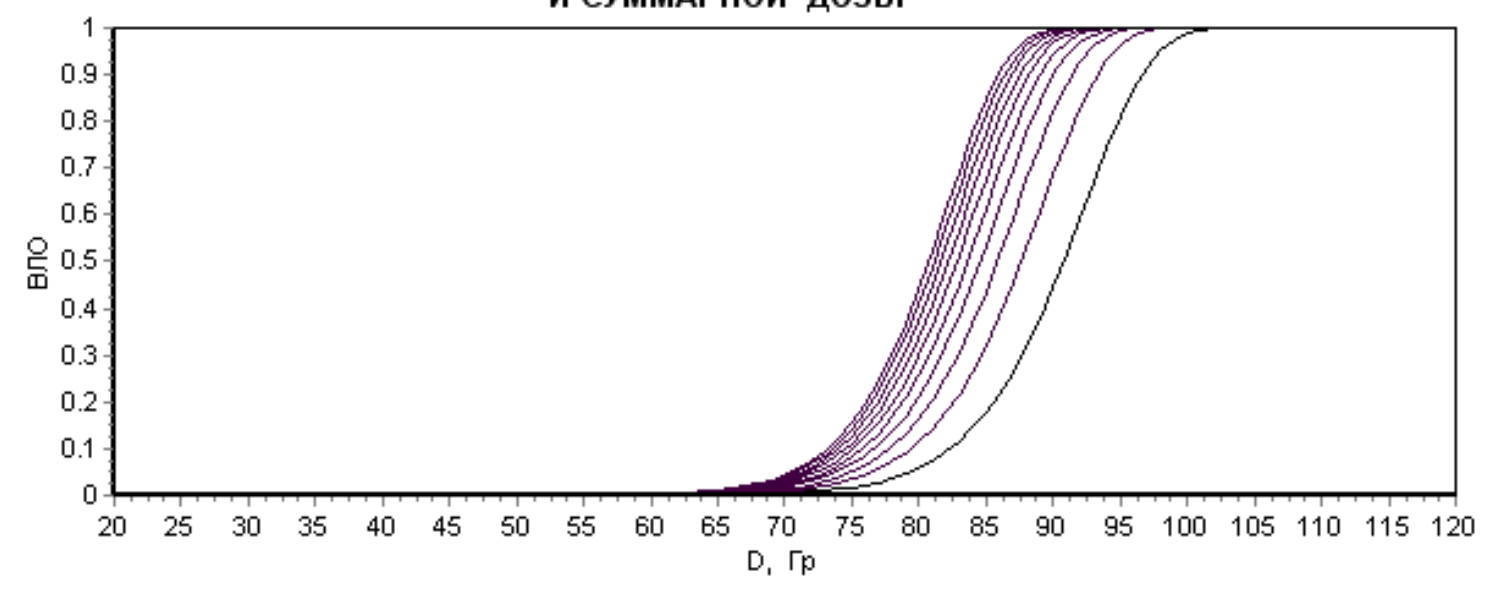

Рис. 5.9.2. Гортань (некроз хряща). Оптимизация по ТД

Таблица 5.9.3

Гортань (некроз хряща). Оптимизация по ВЛО + ТД

\begin{tabular}{|c|c|c|c|c|c|c|c|c|c|}
\hline$P_{\text {КЛ }}$ & $V$ & $D_{\text {КЛ }}$ & $D_{\text {TEOP }}$ & $\varepsilon, \%$ & $P_{\text {TEOP }}$ & $\varepsilon, \%$ & $A_{1}$ & $A_{2}$ & $b$ \\
\hline 0,05 & $1 / 3$ & 77,90 & 77,34 & $-0,72$ & 0,057 & 14,54 & \multirow{6}{*}{79,801} & \multirow{6}{*}{19,297} & \multirow{6}{*}{0,112} \\
\hline 0,05 & $2 / 3$ & 71,57 & 71,56 & $-0,01$ & 0,050 & 0,25 & & & \\
\hline 0,05 & 1 & 67,88 & 68,38 & 0,74 & 0,044 & $-12,95$ & & & \\
\hline 0,50 & $1 / 3$ & 89,24 & 88,55 & 6,80 & 0,178 & $-64,46$ & & & \\
\hline 0,50 & $2 / 3$ & 82,91 & 81,93 & 3,44 & 0,304 & $-39,27$ & & & \\
\hline 0,50 & 1 & 79,21 & 78,29 & $-9,48$ & 0,991 & 98,19 & & & \\
\hline
\end{tabular}




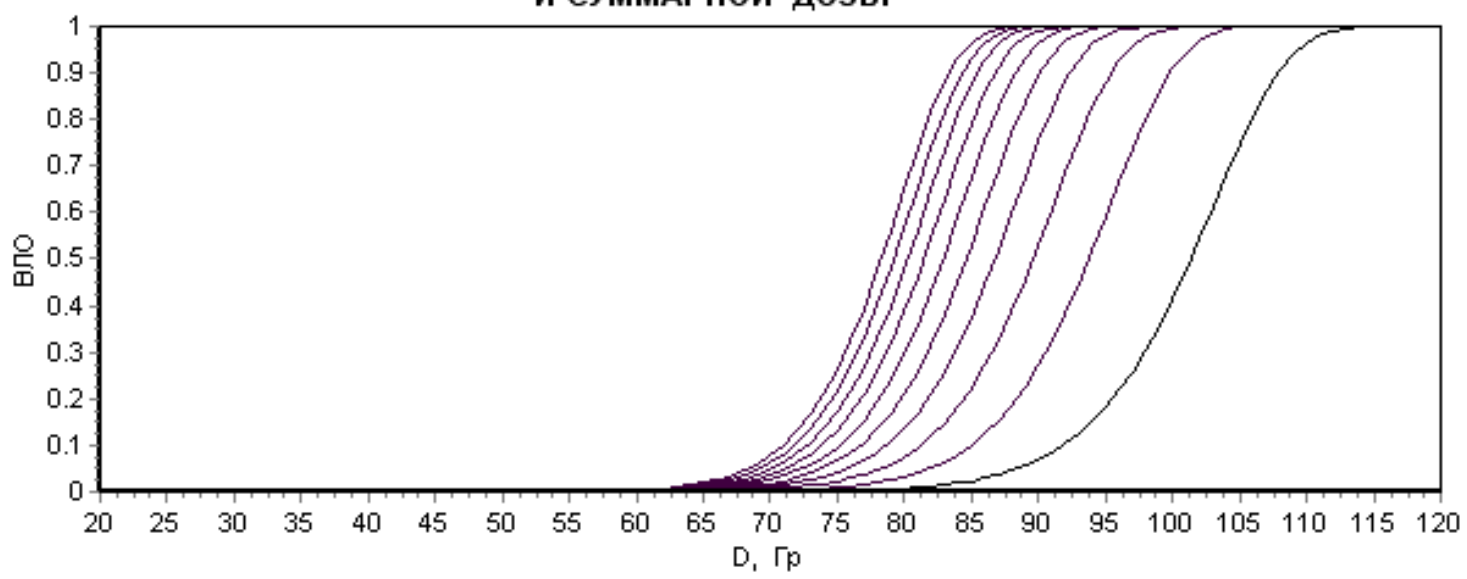

Рис. 5.9.3. Гортань (некроз хряща). Оптимизация по ВЛО + ТД

Анализ оптимальных значений параметров модели МРВ свидетельствуют о том, что критерий по ТД приводит к минимальному отклонению оптимальных теоретических значений ТД от их клинических значений. Оптимальные значения параметров модели МРВ полученные для критериев ВЛО и ВЛО+ТД - совпадают.

\subsection{0. Гортань (отек)}

Таблица 5.10.1

Гортань (отек). Оптимизация по ВЛО

\begin{tabular}{|c|c|c|c|c|c|c|c|c|c|}
\hline$P_{\text {КЛ }}$ & $V$ & $D_{\text {КЛ }}$ & $D_{\mathrm{TEOP}}$ & $\varepsilon, \%$ & $P_{\mathrm{TEOP}}$ & $\varepsilon, \%$ & $A_{1}$ & $A_{2}$ & $b$ \\
\hline 0,05 & $1 / 3$ & 41,05 & 54,81 & 33,53 & 0,006 & $-88,47$ & \multirow{6}{*}{80,347} & \multirow{6}{*}{7,550} & \multirow{6}{*}{0,010} \\
\hline 0,05 & $2 / 3$ & 55,69 & 54,44 & $-2,25$ & 0,059 & 18,20 & & & \\
\hline 0,05 & 1 & 64,25 & 52,01 & $-23,39$ & 0,318 & 536,86 & & & \\
\hline 0,50 & $1 / 3$ & 64,92 & 77,39 & 19,20 & 0,168 & $-66,38$ & & & \\
\hline 0,50 & $2 / 3$ & 79,56 & 76,85 & $-3,40$ & 0,594 & 18,72 & & & \\
\hline 0,50 & 1 & 88,13 & 76,54 & $-13,15$ & 0,866 & 73,20 & & & \\
\hline
\end{tabular}


ВЛО В ОРГАНЕ ИЛИ В ТКАНИ В ЗАВИСИМОСТИ ОТ ОБЪЕМА ОБЛУЧЕНИЯ И СУММАРНОЙ ДОЗЫ

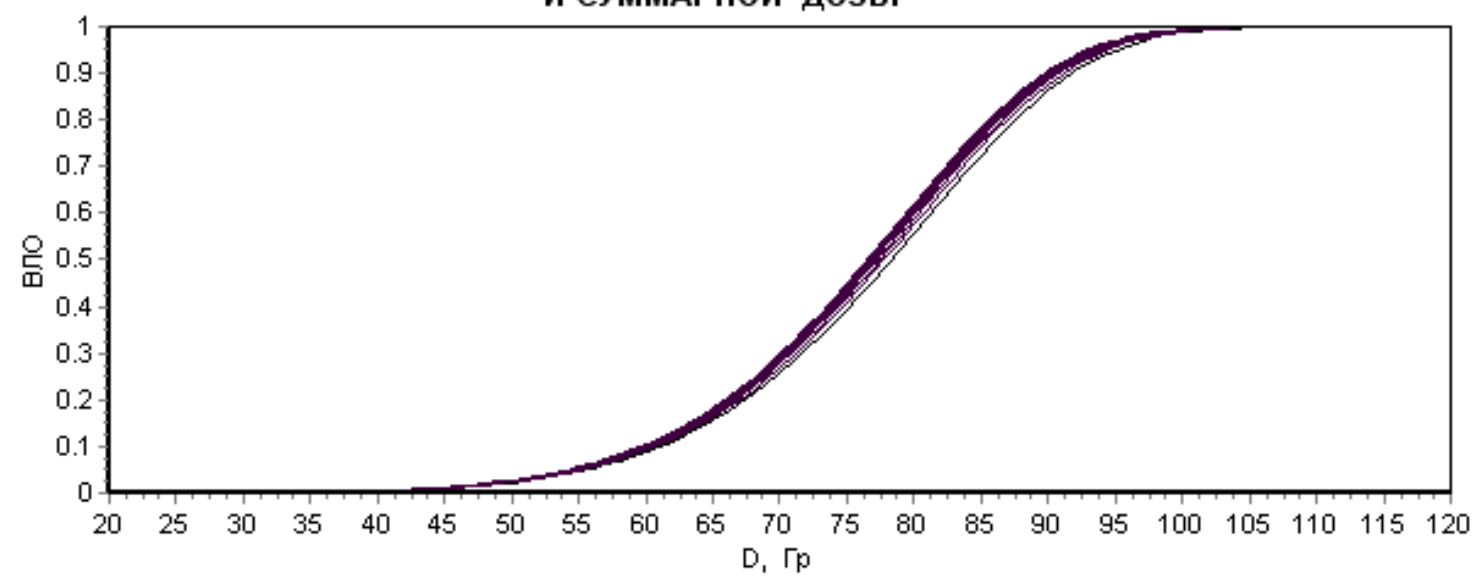

Рис. 5.10.1. Гортань (отек). Оптимизация по ВЛО

Таблица 5.10.2

Гортань (отек). Оптимизация по ТД

\begin{tabular}{|c|c|c|c|c|c|c|c|c|c|}
\hline$P_{\text {КЛ }}$ & $V$ & $D_{\text {Кл }}$ & $D_{\text {TEOP }}$ & $\varepsilon, \%$ & $P_{\mathrm{TEOP}}$ & $\varepsilon, \%$ & $A_{1}$ & $A_{2}$ & $b$ \\
\hline 0,05 & $1 / 3$ & 41,05 & 54,81 & 33,53 & 0,006 & $-88,47$ & \multirow{6}{*}{80,347} & \multirow{6}{*}{7,550} & \multirow{6}{*}{0,010} \\
\hline 0,05 & $2 / 3$ & 55,69 & 54,44 & $-2,25$ & 0,059 & 18,20 & & & \\
\hline 0,05 & 1 & 64,25 & 52,01 & $-23,39$ & 0,318 & 536,86 & & & \\
\hline 0,50 & $1 / 3$ & 64,92 & 77,39 & 19,20 & 0,168 & $-66,38$ & & & \\
\hline 0,50 & $2 / 3$ & 79,56 & 76,85 & $-3,40$ & 0,594 & 18,72 & & & \\
\hline 0,50 & 1 & 88,13 & 76,54 & $-13,15$ & 0,866 & 73,20 & & & \\
\hline
\end{tabular}

ВЛО В ОРГАНЕ ИЛИ В ТКАНИ В ЗАВИСИМОСТИ ОТ ОБЪЕМА ОБЛУЧЕНИЯ И СУММАРНОЙ ДОЗЫ

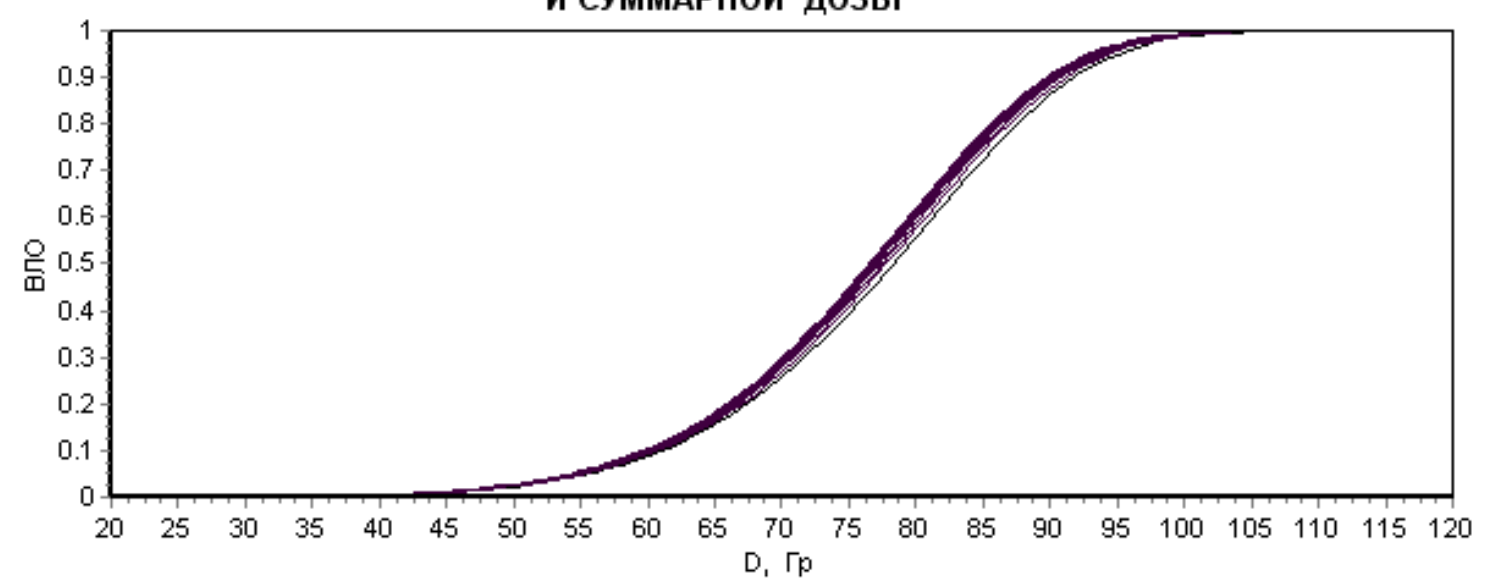

Рис. 5.10.2. Гортань (отек). Оптимизация по ТД 
Таблица 5.10.3

Гортань (отек). Оптимизация по ВЛО + ТД

\begin{tabular}{|c|c|c|c|c|c|c|c|c|c|}
\hline$P_{\text {Кл }}$ & $V$ & $D_{\text {Кл }}$ & $D_{\mathrm{TEOP}}$ & $\varepsilon, \%$ & $P_{\mathrm{TEOP}}$ & $\varepsilon, \%$ & $A_{1}$ & $A_{2}$ & $b$ \\
\hline 0,05 & $1 / 3$ & 41,05 & 54,81 & 33,53 & 0,006 & $-88,47$ & \multirow{6}{*}{80,347} & \multirow{6}{*}{7,550} & \multirow{6}{*}{0,010} \\
\hline 0,05 & $2 / 3$ & 55,69 & 54,44 & $-2,25$ & 0,059 & 18,20 & & & \\
\hline 0,05 & 1 & 64,25 & 52,01 & $-23,39$ & 0,318 & 536,86 & & & \\
\hline 0,50 & $1 / 3$ & 64,92 & 77,39 & 19,20 & 0,168 & $-66,38$ & & & \\
\hline 0,50 & $2 / 3$ & 79,56 & 76,85 & $-3,40$ & 0,594 & 18,72 & & & \\
\hline 0,50 & 1 & 88,13 & 76,54 & $-13,15$ & 0,866 & 73,20 & & & \\
\hline
\end{tabular}

ВЛО В ОРГАНЕ ИЛИ В ТКАНИ В ЗАВИСИМОСТИ ОТ ОБЪЕМА ОБЛУЧЕНИЯ И СУММАРНОЙ ДОЗЫ

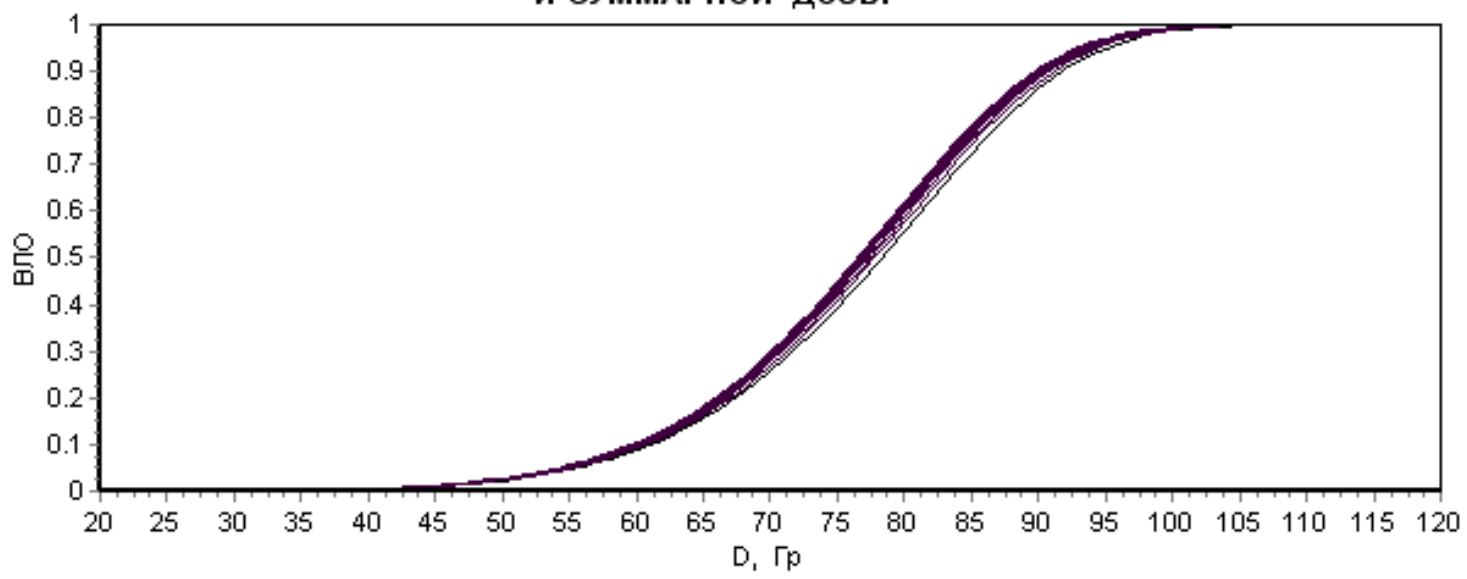

Рис. 5.10.3. Гортань (отек). Оптимизация по ВЛО + ТД

Оптимальные значения параметров модели МРВ для всех трех используемых критериев оптимальности совпадают.

\subsection{1. Печень}

Таблица 5.11.1

Печень. Оптимизация по ВЛО

\begin{tabular}{|c|c|c|c|c|c|c|c|c|c|}
\hline$P_{\text {КЛ }}$ & $V$ & $D_{\text {Кл }}$ & $D_{\text {TEOP }}$ & $\varepsilon, \%$ & $P_{\mathrm{TEOP}}$ & $\varepsilon, \%$ & $A_{1}$ & $A_{2}$ & $b$ \\
\hline 0,05 & $1 / 3$ & 44,73 & 41,22 & $-7,86$ & 0,124 & 148,58 & \multirow{6}{*}{48,585} & \multirow{6}{*}{11,616} & \multirow{6}{*}{0,083} \\
\hline 0,05 & $2 / 3$ & 34,15 & 38,91 & 13,94 & 0,011 & $-77,60$ & & & \\
\hline 0,05 & 1 & 27,96 & 26,63 & $-4,75$ & 0,086 & 72,65 & & & \\
\hline 0,50 & $1 / 3$ & 58,66 & 51,57 & $-12,09$ & 0,955 & 90,95 & & & \\
\hline 0,50 & $2 / 3$ & 48,09 & 48,69 & 1,24 & 0,452 & $-9,70$ & & & \\
\hline 0,50 & 1 & 41,90 & 47,08 & 12,35 & 0,164 & $-67,20$ & & & \\
\hline
\end{tabular}




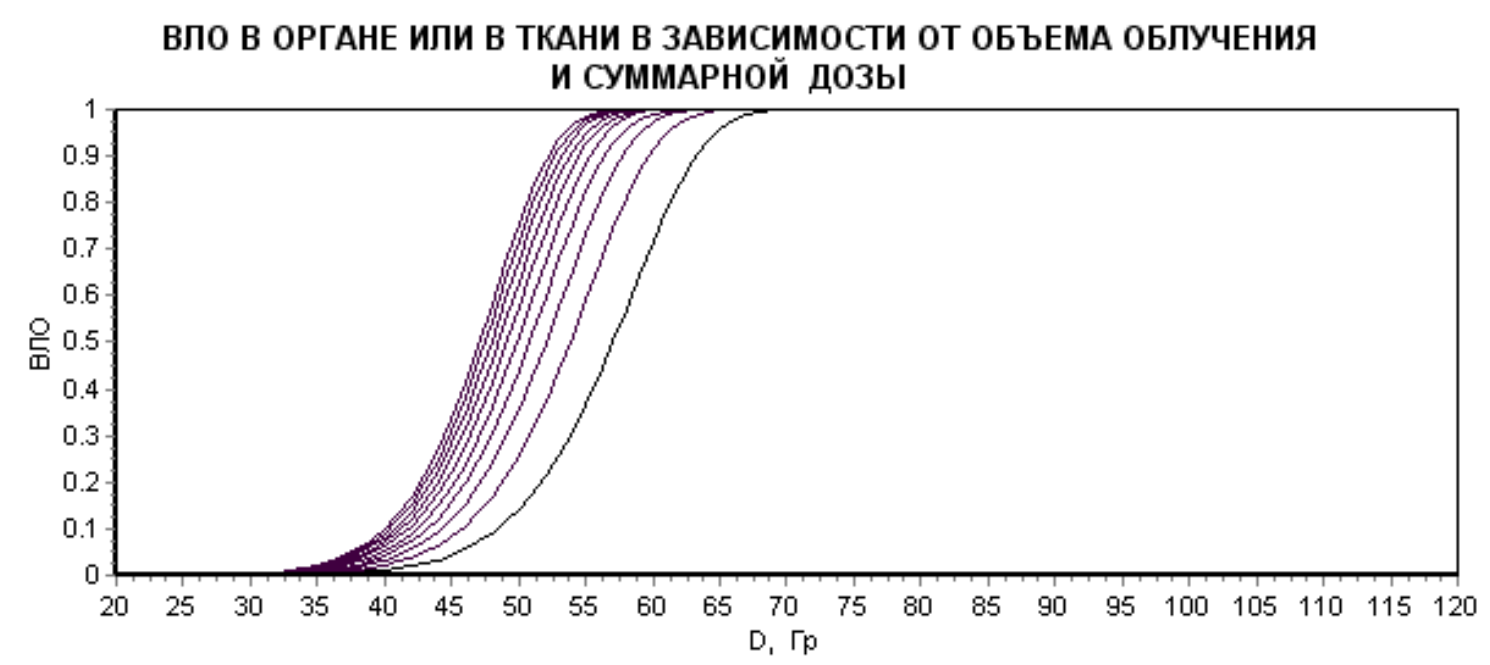

Рис. 5.11.1. Печень. Оптимизация по ВЛО

Таблица 5.11.2

\section{Печень. Оптимизация по ТД}

\begin{tabular}{|c|c|c|c|c|c|c|c|c|c|}
\hline$P_{\text {Кл }}$ & V & $D_{\text {Кл }}$ & $D_{\text {TEOP }}$ & $\varepsilon, \%$ & $P_{\text {TEOP }}$ & $\varepsilon, \%$ & $A_{1}$ & $A_{2}$ & $b$ \\
\hline 0,05 & $1 / 3$ & 44,73 & 40,81 & $-8,77$ & 0,134 & 168,65 & \multirow{6}{*}{48,750} & \multirow{6}{*}{11,267} & \multirow{6}{*}{0,078} \\
\hline 0,05 & $2 / 3$ & 34,15 & 38,66 & 13,21 & 0,013 & $-74,80$ & & & \\
\hline 0,05 & 1 & 27,96 & 27,07 & $-3,18$ & 0,071 & 42,28 & & & \\
\hline 0,50 & $1 / 3$ & 58,66 & 51,42 & $-12,35$ & 0,953 & 90,63 & & & \\
\hline 0,50 & $2 / 3$ & 48,09 & 48,71 & 1,29 & 0,451 & $-9,77$ & & & \\
\hline 0.50 & 1 & 41.90 & 47.19 & 12.63 & 0.166 & -66.82 & & & \\
\hline
\end{tabular}

ВЛО В ОРГАНЕ ИЛИ В ТКАНИ В ЗАВИСИМОСТИ ОТ ОБЪЕМА ОБЛУЧЕНИЯ И СУММАРНОЙ ДОЗЫ

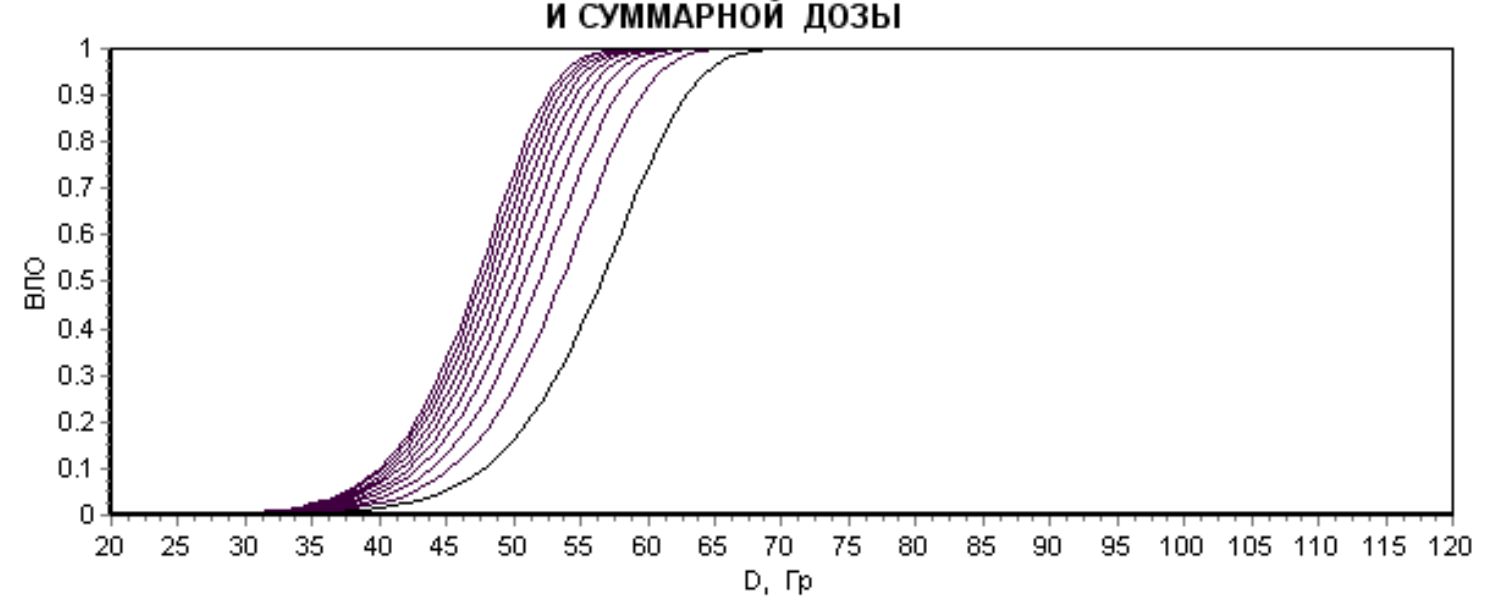

Рис. 5.11.2. Печень. Оптимизация по ТД 
Таблица 5.11.3

Печень. Оптимизация по ВЛО + ТД

\begin{tabular}{|c|c|c|c|c|c|c|c|c|c|}
\hline$P_{\text {Кл }}$ & $V$ & $D_{\text {Кл }}$ & $D_{\mathrm{TEOP}}$ & $\varepsilon, \%$ & $P_{\mathrm{TEOP}}$ & $\varepsilon, \%$ & $A_{1}$ & $A_{2}$ & $b$ \\
\hline 0,05 & $1 / 3$ & 44,73 & 40,81 & $-8,77$ & 0,134 & 168,65 & \multirow{6}{*}{48,750} & \multirow{6}{*}{11,267} & \multirow{6}{*}{0,078} \\
\hline 0,05 & $2 / 3$ & 34,15 & 38,66 & 13,21 & 0,013 & $-74,80$ & & & \\
\hline 0,05 & 1 & 27,96 & 27,07 & $-3,18$ & 0,071 & 42,28 & & & \\
\hline 0,50 & $1 / 3$ & 58,66 & 51,42 & $-12,35$ & 0,953 & 90,63 & & & \\
\hline 0,50 & $2 / 3$ & 48,09 & 48,71 & 1,29 & 0,451 & $-9,77$ & & & \\
\hline 0,50 & 1 & 41,90 & 47,19 & 12,63 & 0,166 & $-66,82$ & & & \\
\hline
\end{tabular}

ВЛО В ОРГАНЕ ИЛИ В ТКАНИ В ЗАВИСИМОСТИ ОТ ОБЪЕМА ОБЛУЧЕНИЯ И СУММАРНОЙ ДОЗЫ

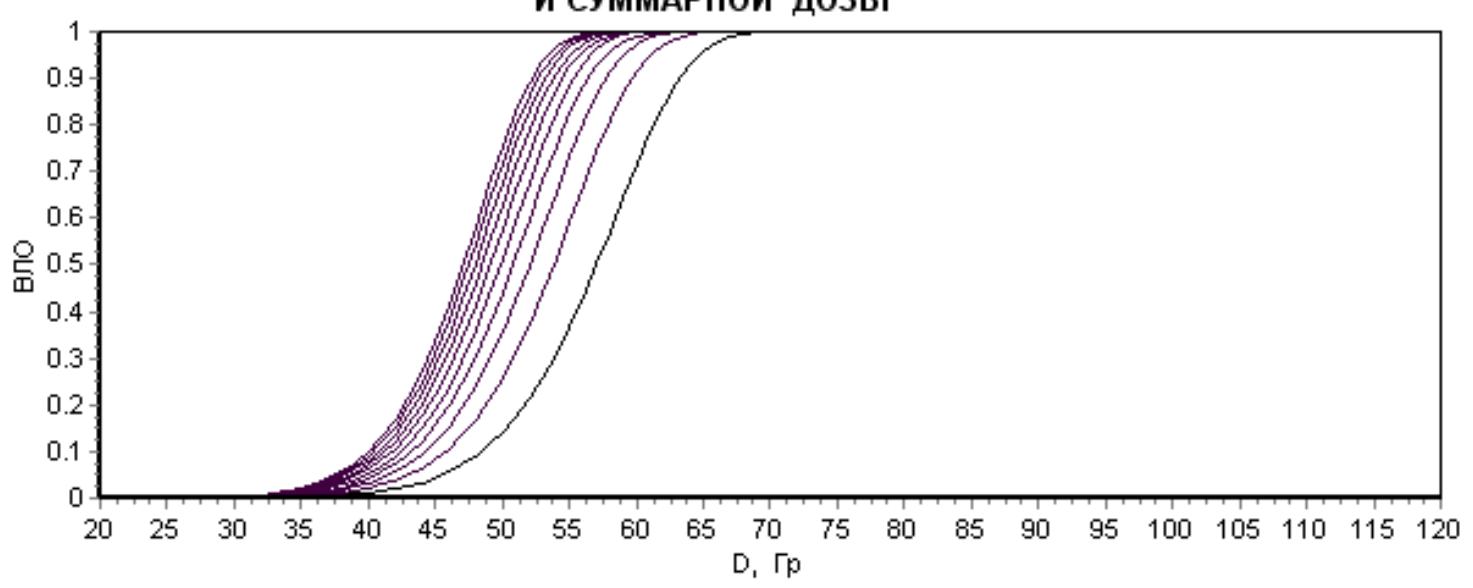

Рис. 5.11.3. Печень. Оптимизация по ВЛО + ТД

Таблица 5.11.4

Печень. Оптимизация по ТД, отдельно для ВЛО = 0,05 и ВЛО =0,50

\begin{tabular}{|c|c|c|c|c|c|c|c|c|c|}
\hline$P_{\text {КЛ }}$ & $P_{\mathrm{TEOP}}$ & $\varepsilon, \%$ & $V$ & $D_{\text {Кл }}$ & $D_{\text {TEOP }}$ & $\varepsilon, \%$ & $A_{1}$ & $A_{2}$ & $b$ \\
\hline 0,05 & 0,050 & $-0,88$ & $1 / 3$ & 44,73 & 45,06 & 0,73 & \multirow{3}{*}{302,154} & \multirow{3}{*}{1,253} & \multirow{3}{*}{0,425} \\
\hline 0,05 & 0,051 & 2,15 & $2 / 3$ & 34,15 & 33,56 & $-1,73$ & & & \\
\hline 0,05 & 0,049 & $-1,24$ & 1 & 27,96 & 28,25 & 1,03 & & & \\
\hline 0,50 & 0,500 & $-0,04$ & $1 / 3$ & 58,66 & $\begin{array}{l}58,95 \\
\end{array}$ & 0,50 & \multirow{3}{*}{1269,282} & \multirow{3}{*}{0,108} & \multirow{3}{*}{0,307} \\
\hline 0,50 & 0,500 & 0,07 & $2 / 3$ & 48,09 & 47,65 & $-0,91$ & & & \\
\hline 0,50 & 0,500 & $-0,03$ & 1 & 41,90 & 42,08 & 0,42 & & & \\
\hline
\end{tabular}

Клиническая информация по ВЛО плохо согласована. 
Таблица 5.12.1

Легкие. Оптимизация по ВЛО

\begin{tabular}{|c|c|c|c|c|c|c|c|c|c|}
\hline$P_{\text {Кл }}$ & $V$ & $D_{\text {КЛ }}$ & $D_{\text {TEOP }}$ & $\varepsilon, \%$ & $P_{\mathrm{TEOP}}$ & $\varepsilon, \%$ & $A_{1}$ & $A_{2}$ & $b$ \\
\hline 0,05 & $1 / 3$ & 29,93 & 24,64 & $-17,67$ & 0,090 & 80,88 & \multirow{6}{*}{45,992} & \multirow{6}{*}{3,158} & \multirow{6}{*}{0,298} \\
\hline 0,05 & $2 / 3$ & 14,69 & 20,04 & 36,43 & 0,019 & $-61,91$ & & & \\
\hline 0,05 & 1 & 5,77 & 5,14 & $-10,97$ & 0,071 & 42,71 & & & \\
\hline 0,50 & $1 / 3$ & 61,18 & 56,20 & $-8,15$ & 0,596 & 19,21 & & & \\
\hline 0,50 & $2 / 3$ & 45,94 & 45,71 & 0,50 & 0,506 & 1,11 & & & \\
\hline 0,50 & 1 & 37,02 & 40,51 & 9,42 & 0,406 & $-18,71$ & & & \\
\hline
\end{tabular}

ВЛО В ОРГАНЕ ИЛИ В ТКАНИ В ЗАВИСИМОСТИ ОТ ОБЪЕМА ОБЛУЧЕНИЯ И СУММАРНОЙ ДОЗЫ

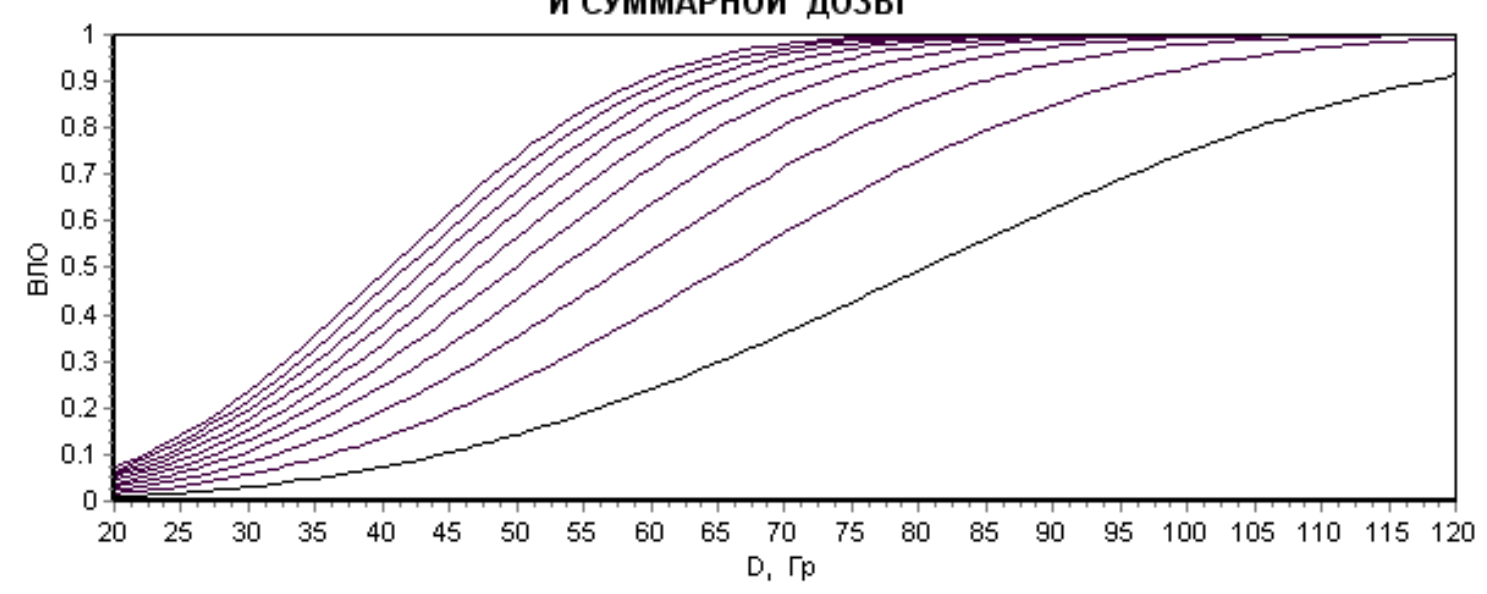

Рис. 5.12.1. Легкие. Оптимизация по ВЛО

Таблица 5.12.2

Легкие. Оптимизация по ТД

\begin{tabular}{|c|c|c|c|c|c|c|c|c|c|}
\hline$P_{\text {КЛ }}$ & $V$ & $D_{\text {Кл }}$ & $D_{\text {TEOP }}$ & $\varepsilon, \%$ & $P_{\mathrm{TEOP}}$ & $\varepsilon, \%$ & $A_{1}$ & $A_{2}$ & $b$ \\
\hline 0,05 & $1 / 3$ & 29,93 & 22,85 & $-23,65$ & 0,108 & 115,99 & \multirow{6}{*}{46,710} & \multirow{6}{*}{2,970} & \multirow{6}{*}{0,260} \\
\hline 0,05 & $2 / 3$ & 14,69 & 19,08 & 29,90 & 0,023 & $-53,36$ & & & \\
\hline 0,05 & 1 & 5,77 & 5,82 & 0,84 & 0,049 & $-2,38$ & & & \\
\hline 0,50 & $1 / 3$ & 61,18 & 54,94 & $-10,21$ & 0,615 & 22,97 & & & \\
\hline 0,50 & $2 / 3$ & 45,94 & 45,88 & $-0,14$ & 0,501 & 0,290 & & & \\
\hline 0,50 & 1 & 37,02 & 41,29 & 11,52 & 0,3995 & $-21,13$ & & & \\
\hline
\end{tabular}


ВЛО В ОРГАНЕ ИЛИ В ТКАНИ В ЗАВИСИМОСТИ ОТ ОБЪЕМА ОБЛУЧЕНИЯ И СУММАРНОЙ ДОЗЫ

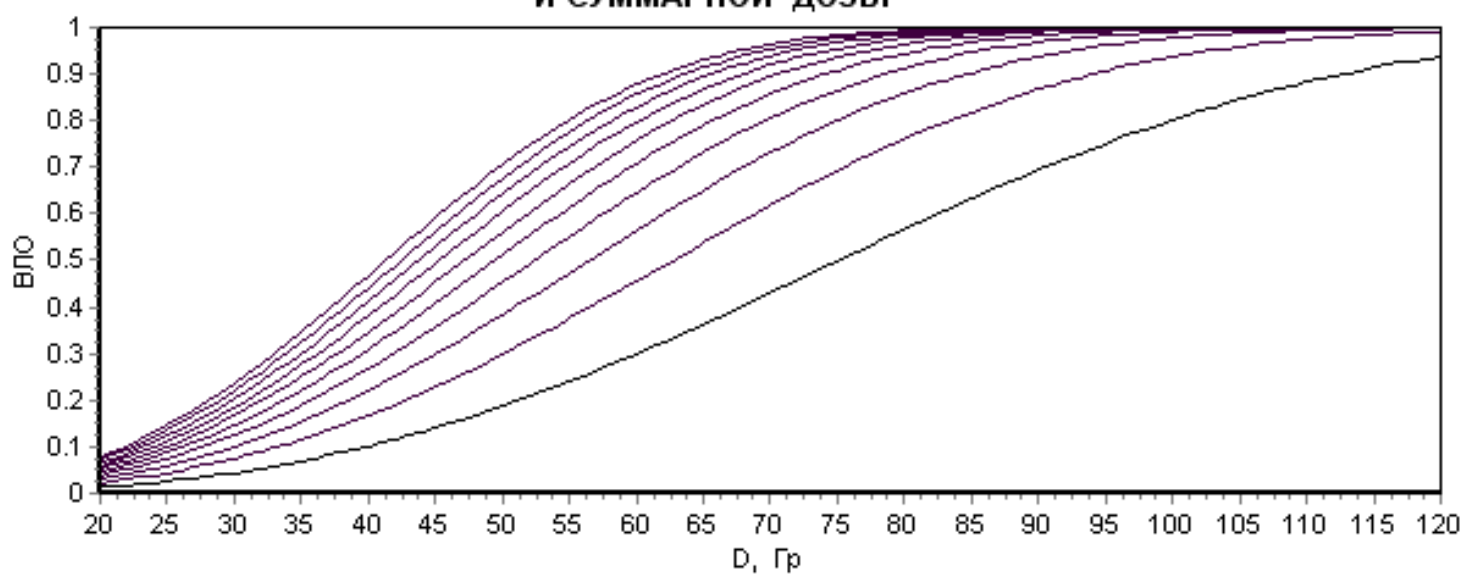

Рис. 5.12.2. Легкие. Оптимизация по ТД

Таблица 5.12.3

Легкие. Оптимизация по ВЛО + ТД

\begin{tabular}{|c|c|c|c|c|c|c|c|c|c|}
\hline$P_{\text {Кл }}$ & $V$ & $D_{\text {Кл }}$ & $D_{\text {TEOP }}$ & $\varepsilon, \%$ & $P_{\mathrm{TEOP}}$ & $\varepsilon, \%$ & $A_{1}$ & $A_{2}$ & $b$ \\
\hline 0,05 & $1 / 3$ & 29,93 & 24,54 & $-18,0$ & 0,091 & 82,69 & \multirow{6}{*}{45,555} & \multirow{6}{*}{3,147} & \multirow{6}{*}{0,296} \\
\hline 0,05 & $2 / 3$ & 14,69 & 19,99 & 36,08 & 0,019 & $-61,47$ & & & \\
\hline 0,05 & 1 & $\begin{array}{l}5,77 \\
\end{array}$ & 5,17 & $-10,38$ & 0,070 & 39,74 & & & \\
\hline 0,50 & $1 / 3$ & 61,18 & 56,13 & $-8,26$ & 0,597 & $\begin{array}{l}19,42 \\
\end{array}$ & & & \\
\hline 0,50 & $2 / 3$ & 45,94 & 45,72 & $-0,48$ & 0,505 & 1,06 & & & \\
\hline 0,50 & 1 & 37,02 & 40,55 & 9,53 & 0,406 & $\begin{array}{l}-18,84 \\
\end{array}$ & & & \\
\hline
\end{tabular}

ВЛО В ОРГАНЕ ИЛИ В ТКАНИ В ЗАВИСИМОСТИ ОТ ОБЪЕМА ОБЛУЧЕНИЯ И СУММАРНОЙ ДОЗЫ

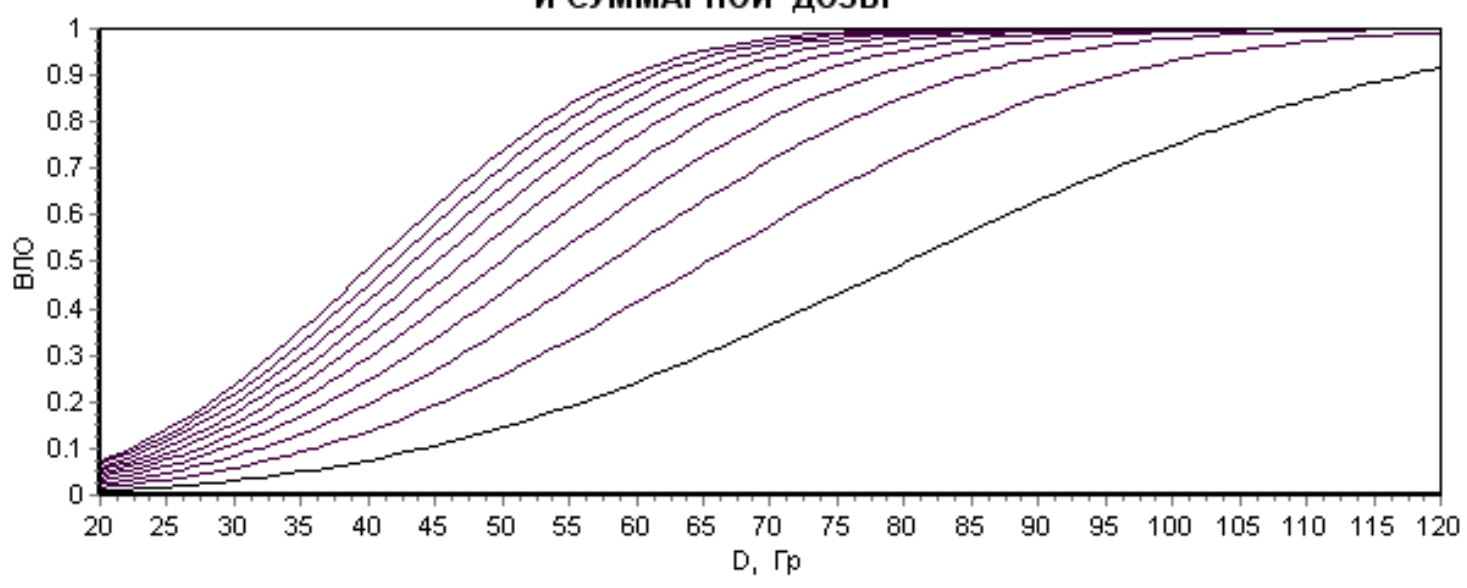

Рис. 5.12.3. Легкое. Оптимизация по ВЛО+ТД 
Таблица 5.12 .4

Легкое. Оптимизация по ТД, отдельно для ВЛО =0,05 и ВЛО = 0,50

\begin{tabular}{|c|c|c|c|c|c|c|c|c|c|}
\hline$P_{\text {КЛ }}$ & $D_{\text {Кл }}$ & $D_{\text {TEOP }}$ & $\varepsilon, \%$ & $V$ & $P_{\mathrm{TEOP}}$ & $\varepsilon, \%$ & $A_{1}$ & $A_{2}$ & $b$ \\
\hline 0,05 & 29,93 & 22,62 & $-24,41$ & $1 / 3$ & 0,135 & 170,97 & \multirow{3}{*}{37,924} & \multirow{3}{*}{3,728} & \multirow{3}{*}{0,255} \\
\hline 0,05 & 14,69 & 18,96 & 29,06 & $2 / 3$ & 0,020 & $-60,76$ & & & \\
\hline 0,05 & 5,77 & 5,91 & 2,50 & 1 & 0,046 & $-8,60$ & & & \\
\hline 0,50 & 61,18 & 61,76 & 0,94 & $1 / 3$ & 0,500 & $-0,11$ & \multirow{3}{*}{356,140} & \multirow{3}{*}{0,163} & \multirow{3}{*}{0,456} \\
\hline 0,50 & 45,94 & 45,02 & $-2,0$ & $2 / 3$ & 0,501 & 0,23 & & & \\
\hline 0,50 & 37,02 & 37,42 & 1,09 & 1 & 0,499 & $-0,12$ & & & \\
\hline
\end{tabular}

Анализ табл. 5.12.4 свидетельствует о том, что клиническая информация для значений $\mathrm{BЛО}=0,05$ и ВЛО $=0.50$ является плохо согласованной.

\subsection{3. Кожа. Некроз}

Таблица 5.13.1

Кожа. Оптимизация по ВЛО

\begin{tabular}{|c|c|c|c|c|c|c|c|c|c|}
\hline$P_{\text {Кл }}$ & $V$ & $D_{\text {Кл }}$ & $D_{\mathrm{TEOP}}$ & $\varepsilon, \%$ & $P_{\mathrm{TEOP}}$ & $\varepsilon, \%$ & $A_{1}$ & $A_{2}$ & $b$ \\
\hline 0,05 & $1 / 3$ & $\begin{array}{l}60,84 \\
\end{array}$ & 59,20 & $\begin{array}{l}-2,70 \\
\end{array}$ & 0,067 & 34,69 & \multirow{6}{*}{75,238} & \multirow{6}{*}{11,205} & \multirow{6}{*}{0,023} \\
\hline 0,05 & $2 / 3$ & 55,97 & 58,26 & 4,09 & 0,032 & $-35,60$ & & & \\
\hline 0,05 & 1 & 53,12 & 52,45 & $-1,26$ & 0,057 & 14,86 & & & \\
\hline 0,50 & $1 / 3$ & 77,92 & 74,68 & $-4,16$ & 0,672 & 34,45 & & & \\
\hline 0,50 & $2 / 3$ & 73,06 & 73,50 & 0,60 & 0,477 & $-4,61$ & & & \\
\hline 0,50 & 1 & 70,21 & 72,82 & 3,71 & 0,369 & $-26,27$ & & & \\
\hline
\end{tabular}

ВЛО В ОРГАНЕ ИЛИ В ТКАНИ В ЗАВИСИМОСТИ ОТ ОБЪЕМА ОБЛУЧЕНИЯ И СУММАРНОЙ ДОЗЫ

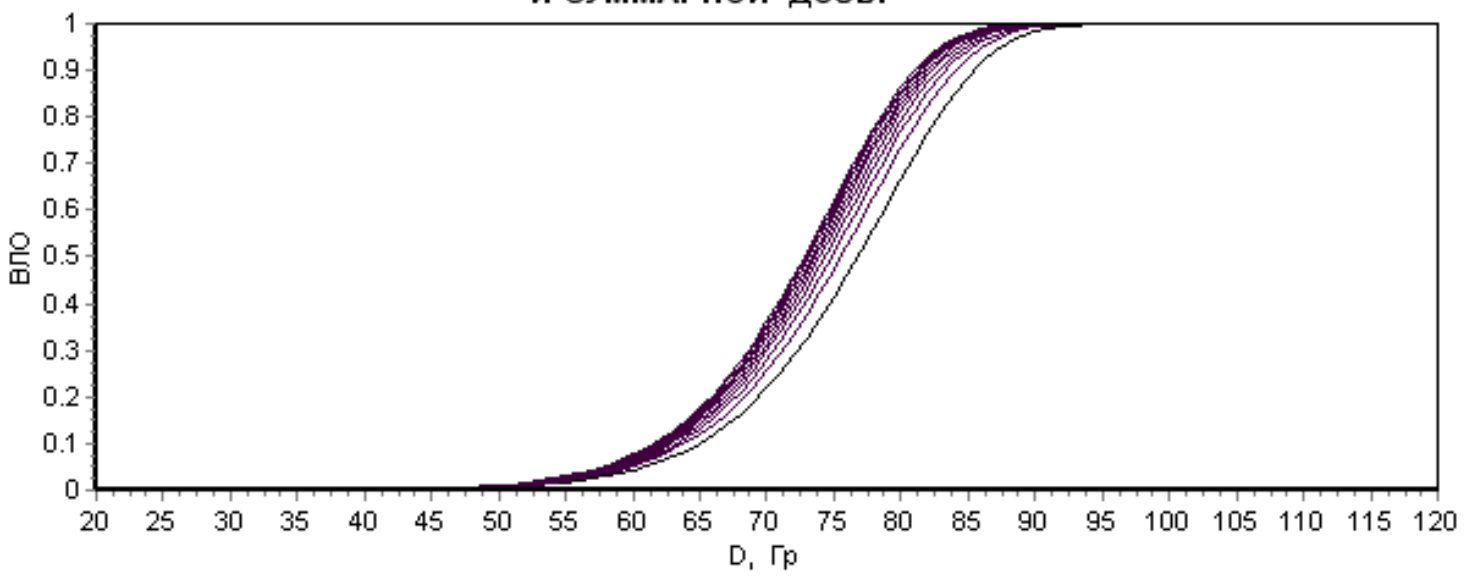

Рис. 5.13.1. Кожса. Оптимизация по ВЛО 
Таблица 5.13.2

Кожа (некроз). Оптимизация по ТД

\begin{tabular}{|c|c|c|c|c|c|c|c|c|c|}
\hline$P_{\text {Кл }}$ & $V$ & $D_{\text {КЛ }}$ & $D_{\text {TEOP }}$ & $\varepsilon, \%$ & $P_{\mathrm{TEOP}}$ & $\varepsilon, \%$ & $A_{1}$ & $A_{2}$ & $b$ \\
\hline 0,05 & $1 / 3$ & 60,84 & 59,20 & $-2,70$ & 0,067 & 34,69 & \multirow{6}{*}{75,238} & \multirow{6}{*}{11,205} & \multirow{6}{*}{0,023} \\
\hline 0,05 & $2 / 3$ & 55,97 & 58,26 & 4,09 & 0,032 & $-35,60$ & & & \\
\hline 0,05 & 1 & 53,12 & 52,45 & $-1,26$ & 0,057 & 14,86 & & & \\
\hline 0,50 & $1 / 3$ & 77,92 & 74,68 & $-4,16$ & 0,672 & 34,45 & & & \\
\hline 0,50 & $2 / 3$ & 73,06 & 73,50 & 0,60 & 0,477 & $-4,61$ & & & \\
\hline 0,50 & 1 & 70,21 & 72,82 & 3,71 & 0,369 & $-26,27$ & & & \\
\hline
\end{tabular}

ВЛО В ОРГАНЕ ИЛИ В ТКАНИ В ЗАВИСИМОСТИ ОТ ОБЪЕМА ОБЛУЧЕНИЯ И СУММАРНОЙ ДОЗЫ

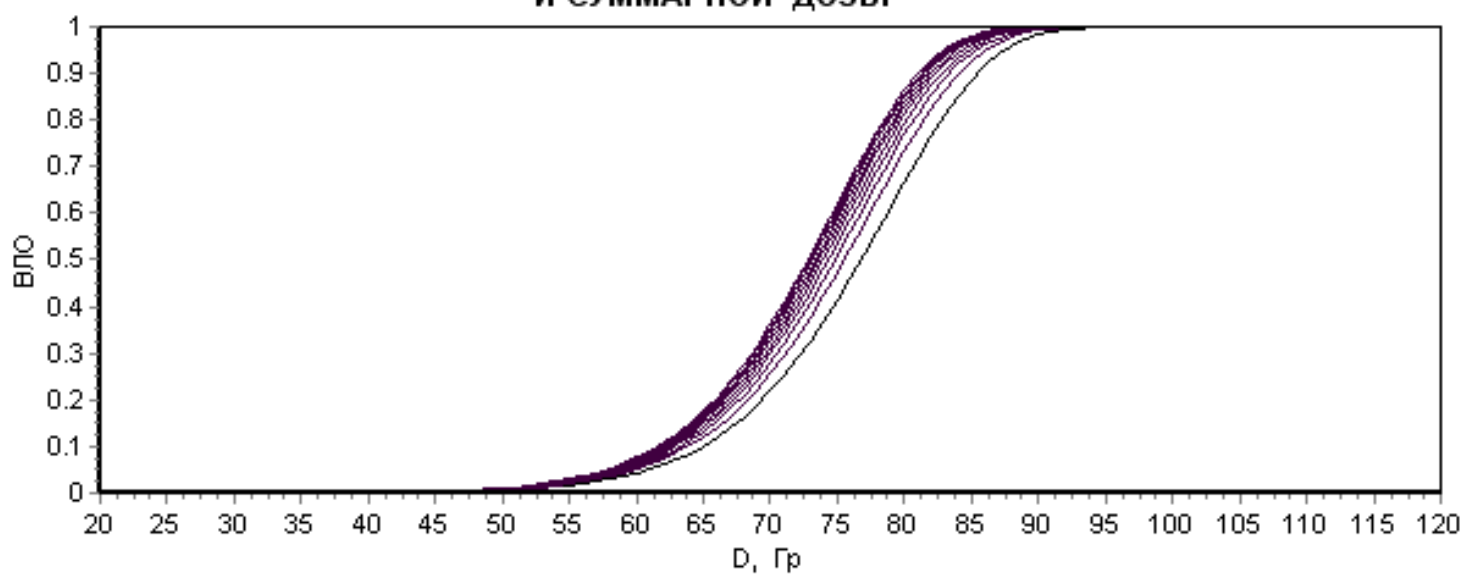

Рис. 5.13.2. Кожа. Оптимизация по ТД

Таблица 5.13.3

Кожа. Оптимизация по ВЛО + ТД

\begin{tabular}{|c|c|c|c|c|c|c|c|c|c|}
\hline$P_{\text {КЛ }}$ & $V$ & $D_{\text {Кл }}$ & $D_{\text {TEOP }}$ & $\varepsilon, \%$ & $P_{\mathrm{TEOP}}$ & $\varepsilon, \%$ & $A_{1}$ & $A_{2}$ & $b$ \\
\hline 0,05 & $1 / 3$ & 60,84 & 59,20 & $-2,70$ & 0,067 & 34,69 & \multirow{6}{*}{75,238} & \multirow{6}{*}{11,205} & \multirow{6}{*}{0,023} \\
\hline 0,05 & $2 / 3$ & 55,97 & 58,26 & 4,09 & 0,032 & $-35,60$ & & & \\
\hline 0,05 & 1 & 53,12 & 52,45 & $-1,26$ & 0,057 & 14,86 & & & \\
\hline 0,50 & $1 / 3$ & 77,92 & 74,68 & $-4,16$ & 0,672 & 34,45 & & & \\
\hline 0,50 & $2 / 3$ & 73,06 & 73,50 & 0,60 & 0,477 & $-4,61$ & & & \\
\hline 0,50 & 1 & 70,21 & 72,82 & 3,71 & 0,369 & $-26,27$ & & & \\
\hline
\end{tabular}


ВЛО В ОРГАНЕ ИЛИ В ТКАНИ В ЗАВИСИМОСТИ ОТ ОБЪЕМА ОБЛУЧЕНИЯ И СУММАРНОЙ ДОЗЫ

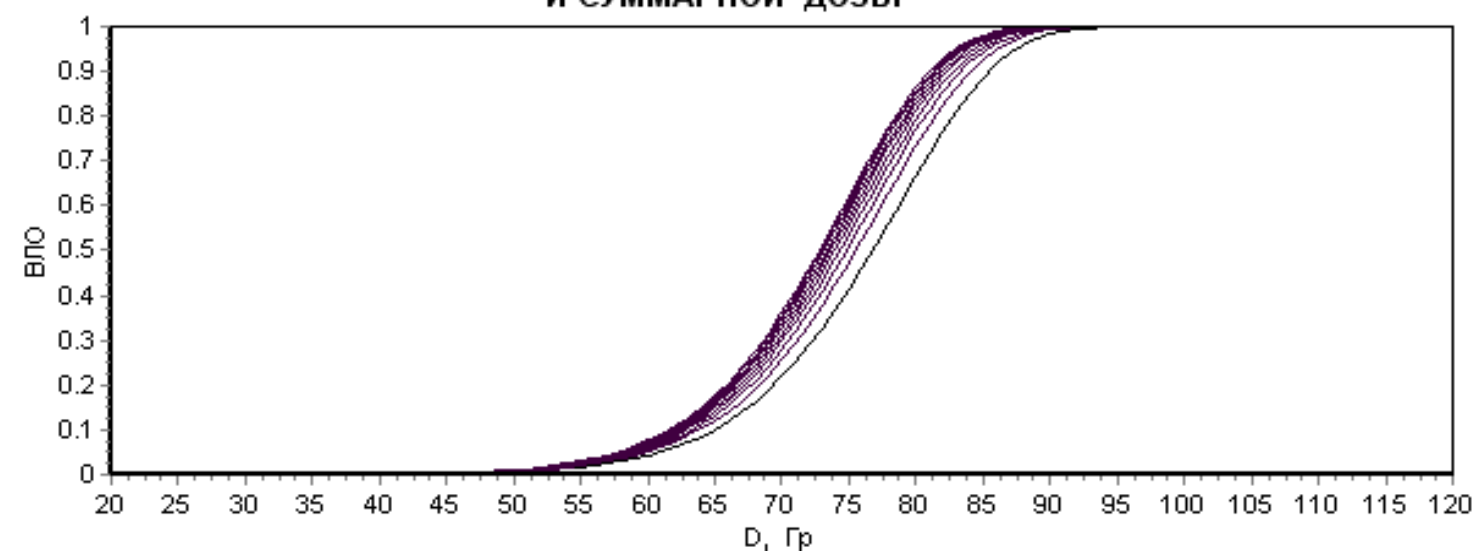

Рис. 13.3. Кожа (некроз). Оптимизация по ВЛО + ТД

Таблица 5.13.4

Кожа. Оптимзация по ТД, отдельно для ВЛО = 0,05 и ВЛО = 0,50

\begin{tabular}{|c|c|c|c|c|c|c|c|c|c|}
\hline$P_{\text {Кл }}$ & $V$ & $D_{\text {Кл }}$ & $D_{\text {TEOP }}$ & $\varepsilon, \%$ & $P_{\text {TEOP }}$ & $\varepsilon, \%$ & $A_{1}$ & $A_{2}$ & $b$ \\
\hline 0,05 & $1 / 3$ & 60,84 & 60,40 & $-0,73$ & 0,059 & 18,53 & \multirow{3}{*}{60,667} & \multirow{3}{*}{23,885} & \multirow{3}{*}{0,11} \\
\hline 0,05 & $2 / 3$ & 55,97 & 55,96 & $-0,02$ & 0,050 & 0,37 & & & \\
\hline 0,05 & 1,0 & 53,12 & 53,52 & 0,75 & 0,042 & $-16,06$ & & & \\
\hline 0,50 & $1 / 3$ & 77,91 & 79,13 & 1,56 & 0,496 & $-0,75$ & \multirow{3}{*}{117,103} & \multirow{3}{*}{0,700} & \multirow{3}{*}{0,12} \\
\hline 0,50 & $2 / 3$ & 73,06 & 72,82 & $-0,33$ & 0,501 & 0,16 & & & \\
\hline 0,50 & $\overline{1,0}$ & 70,21 & 69,36 & $-1,22$ & 0,503 & 0,59 & & & \\
\hline
\end{tabular}

\subsection{4. Кожа (телеангиктазия)}

Таблица 5.14

Кожа (телеангиэктазия)

\begin{tabular}{c|c|c|c|c|c}
\hline$P_{\text {КЛ }}$ & $D_{\text {КЛ }}$ & $P_{\text {КЛ }}$ & $D_{\text {КЛ }}$ & $A_{1}$ & $A_{2}$ \\
\hline 0,05 & 48,54 & 0,50 & 65,09 & 67,834 & 8,875 \\
\hline
\end{tabular}

ВЛО В ОРГАНЕ ИЛИ В ТКАНИ В ЗАВИСИМОСТИ ОТ СУММАРНОЙ ДОЗЫ

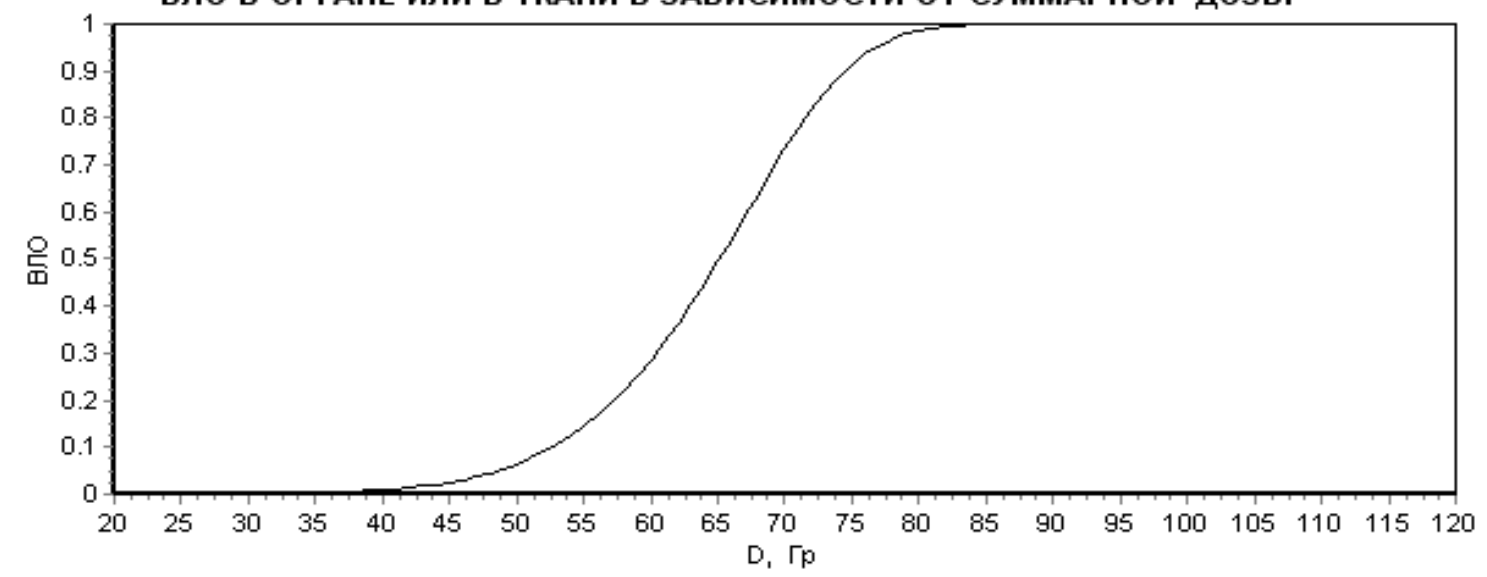

Рис. 5.14. Кожа. Зависимость ВЛО от дозы 


\subsection{5. Тонкая кишка}

Таблица 15.1

Тонкая кищка. Оптимизация по ВЛО

\begin{tabular}{|c|c|c|c|c|c|c|c|c|c|}
\hline$P_{\text {Кл }}$ & $V$ & $D_{\text {КЛ }}$ & $D_{\text {TEOP }}$ & $\varepsilon, \%$ & $P_{\mathrm{TEOP}}$ & $\varepsilon, \%$ & $A_{1}$ & $A_{2}$ & $b$ \\
\hline 0,05 & $1 / 3$ & 48,17 & 48,02 & $-0,31$ & 0,052 & 3,04 & \multirow{6}{*}{59,815} & \multirow{6}{*}{10,018} & \multirow{6}{*}{0,070} \\
\hline 0,05 & $2 / 3$ & 45,75 & 45,75 & $-0,01$ & 0,050 & 0,04 & & & \\
\hline 0,05 & 1 & 44,33 & 44,87 & 0,31 & 0,049 & $-2,99$ & & & \\
\hline 0,50 & $1 / 3$ & 61,83 & 62,28 & 0,72 & 0,475 & $-4,93$ & & & \\
\hline 0,50 & $2 / 3$ & 59,41 & 59,33 & $-0,14$ & 0,510 & 0,98 & & & \\
\hline 0,50 & 1 & 58,00 & 57,67 & $-0,58$ & 0,520 & 4,03 & & & \\
\hline
\end{tabular}

ВЛО В ОРГАНЕ ИЛИ В ТКАНИ В ЗАВИСИМОСТИ ОТ ОБЪЕМА ОБЛУЧЕНИЯ И СУММАРНОЙ ДОЗЫ

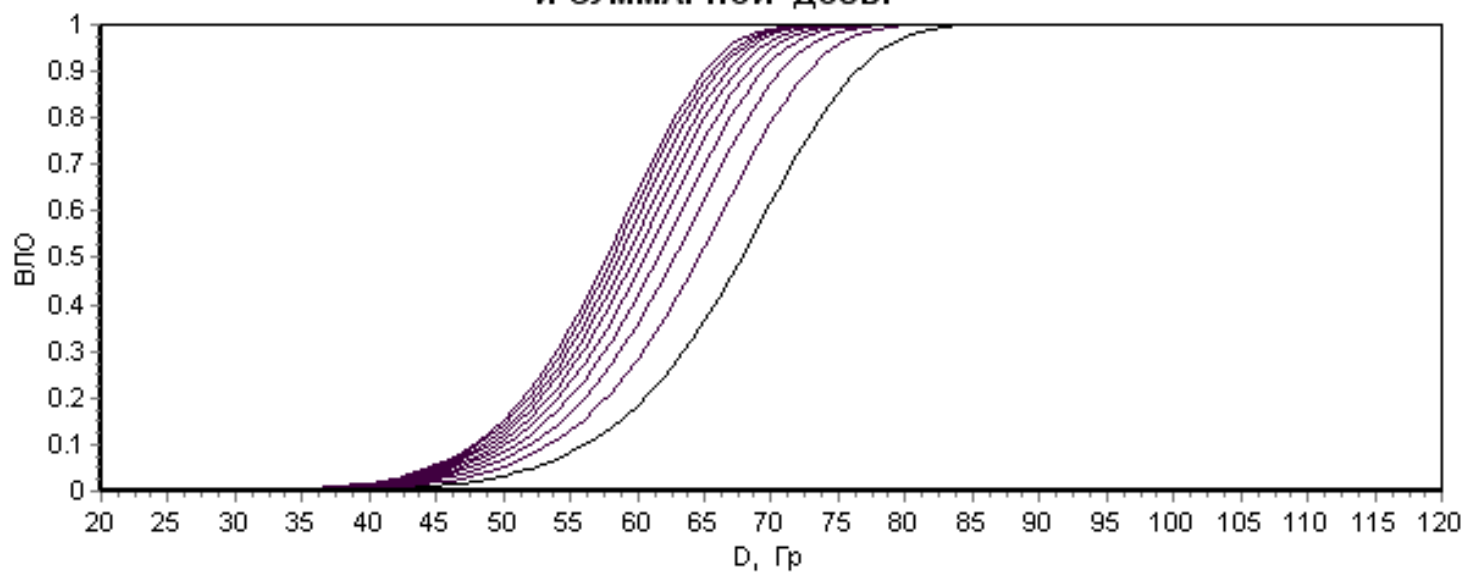

Рис. 5.15.1. Тонкая кишка. Оптимизация по ВЛО

Таблица 5.15.2

Тонкая кищка. Оптимизация по ТД

\begin{tabular}{|c|c|c|c|c|c|c|c|c|c|}
\hline$P_{\text {КЛ }}$ & $V$ & $D_{\text {КЛ }}$ & $D_{\text {TEOP }}$ & $\varepsilon, \%$ & $P_{\text {TEOP }}$ & $\varepsilon, \%$ & $A_{1}$ & $A_{2}$ & $b$ \\
\hline 0,05 & $1 / 3$ & 48,17 & 47,94 & $-0,48$ & 0,052 & 4,85 & \multirow{6}{*}{59,910} & \multirow{6}{*}{10,020} & \multirow{6}{*}{0,069} \\
\hline 0,05 & $2 / 3$ & 45,75 & 45,76 & 0,025 & 0,050 & 0,24 & & & \\
\hline 0,05 & 1 & 44,33 & 44,54 & 0,46 & 0,048 & $-4,40$ & & & \\
\hline 0,50 & $1 / 3$ & 61,83 & 62,16 & 0,54 & 0,481 & $-3,71$ & & & \\
\hline 0,50 & $2 / 3$ & 59,41 & 59,34 & $-0,11$ & 0,504 & 0,78 & & & \\
\hline 0,50 & 1 & 58,00 & 57,75 & -0.43 & 0,515 & 2,98 & & & \\
\hline
\end{tabular}




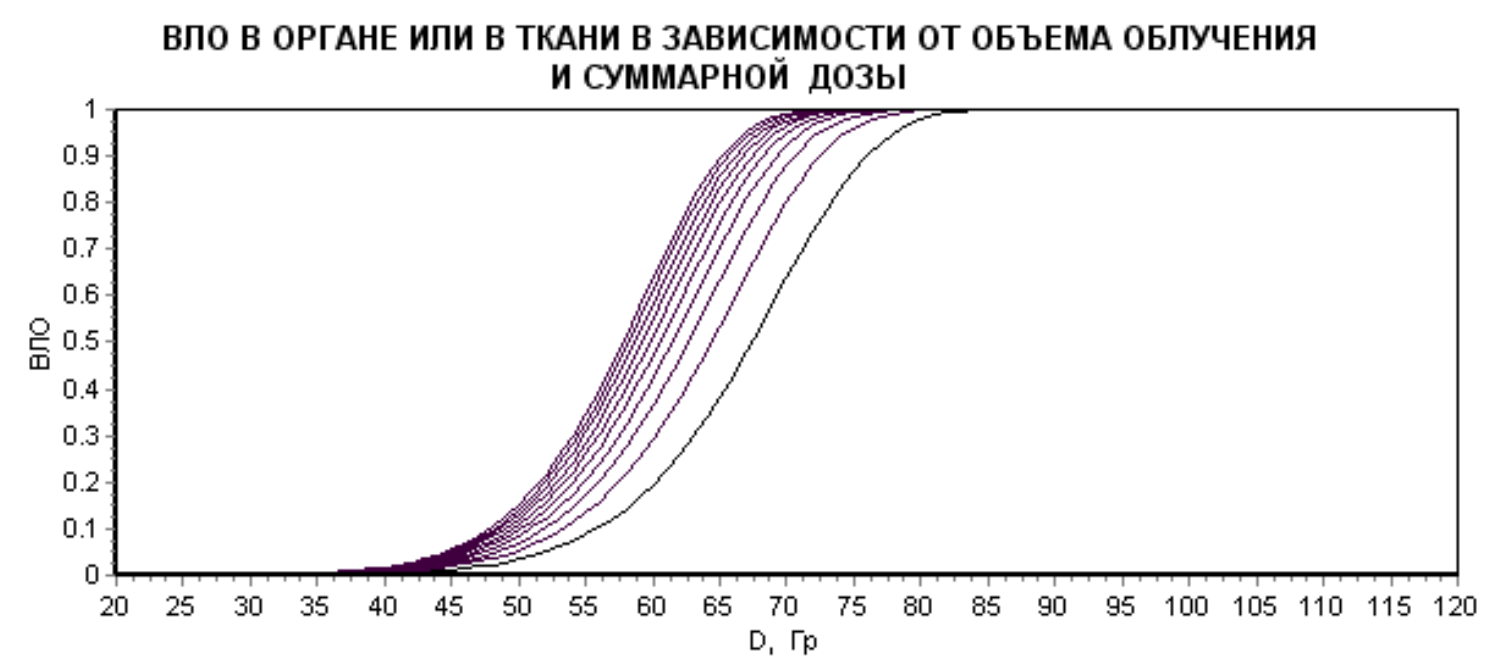

Рис. 5.15.2. Тонкая кишка. Оптимизация по ТД

Таблица 5.15.3

Тонкая кишка. Оптимизация по ВЛО + ТД

\begin{tabular}{|c|c|c|c|c|c|c|c|c|c|}
\hline$P_{\text {Кл }}$ & $V$ & $D_{\text {Кл }}$ & $D_{\mathrm{TEOP}}$ & $\varepsilon, \%$ & $P_{\mathrm{TEOP}}$ & $\varepsilon, \%$ & $A_{1}$ & $A_{2}$ & $b$ \\
\hline 0,05 & $1 / 3$ & 48,17 & 48,02 & $-0,31$ & 0,052 & 3,04 & \multirow{6}{*}{59,815} & \multirow{6}{*}{10,018} & \multirow{6}{*}{0,070} \\
\hline 0,05 & $2 / 3$ & 45,75 & 45,75 & $-0,01$ & 0,050 & 0,04 & & & \\
\hline 0,05 & 1 & 44,33 & 44,87 & 0,31 & 0,049 & $-2,99$ & & & \\
\hline 0,50 & $1 / 3$ & 61,83 & 62,28 & 0,72 & 0,475 & $-4,93$ & & & \\
\hline 0,50 & $2 / 3$ & 59,41 & 59,33 & $-0,14$ & 0,510 & 0,98 & & & \\
\hline 0,50 & 1 & 58,00 & 57,67 & $-0,58$ & 0,520 & 4,03 & & & \\
\hline
\end{tabular}

ВЛО В ОРГАНЕ ИЛИ В ТКАНИ В ЗАВИСИМОСТИ ОТ ОБЪЕМА ОБЛУЧЕНИЯ И СУММАРНОЙ ДОЗЫ

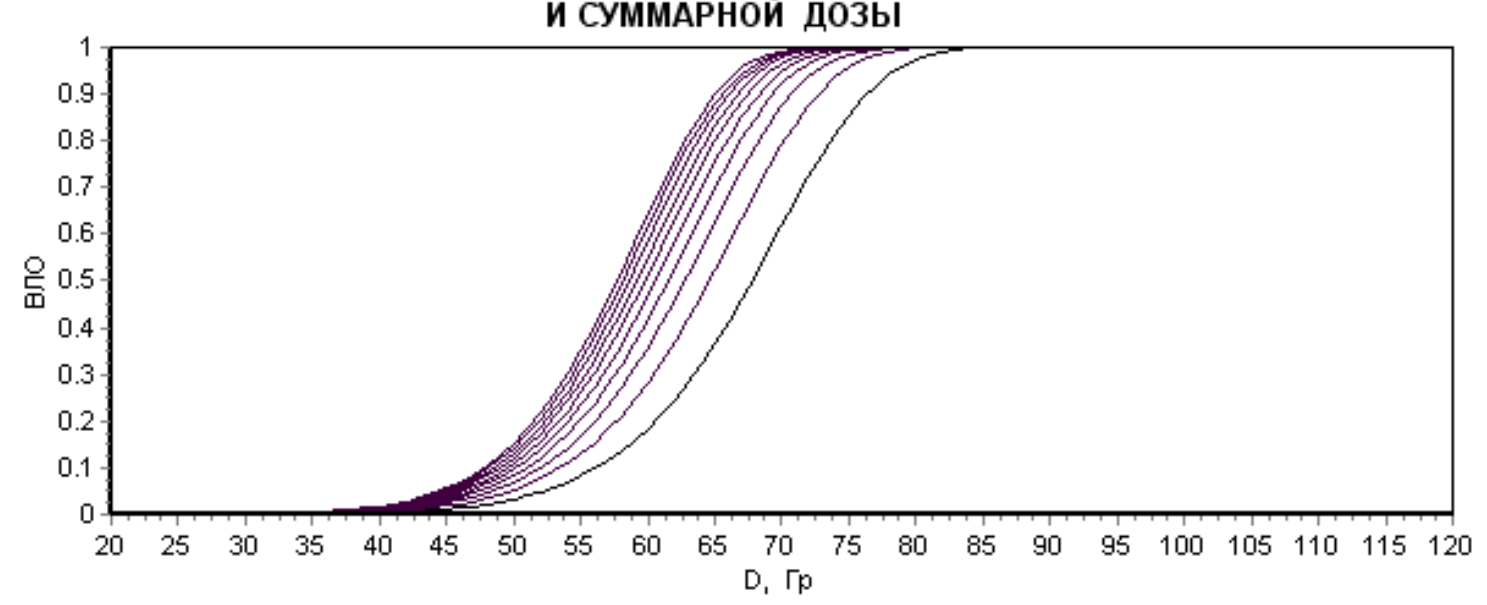

Рис. 5.15.3. Тонкая кишка. Оптимизация по ВЛО+ТД

Оптимальные значения параметров модели МРВ для всех трех критериев оптимальности принимают близкие значения 
Таблица 5.16.1

Толстая кишка. Оптимизация по ВЛО

\begin{tabular}{|c|c|c|c|c|c|c|c|c|c|}
\hline$P_{\text {КЛ }}$ & $V$ & $D_{\text {КЛ }}$ & $D_{\mathrm{TEOP}}$ & $\varepsilon, \%$ & $P_{\mathrm{TEOP}}$ & $\varepsilon, \%$ & $A_{1}$ & $A_{2}$ & $b$ \\
\hline 0,05 & $1 / 3$ & 55,00 & $\begin{array}{l}59,20 \\
\end{array}$ & $-2,70$ & 0,067 & 34,69 & \multirow{6}{*}{75,329} & \multirow{6}{*}{11,205} & \multirow{6}{*}{0,023} \\
\hline 0,05 & $2 / 3$ & 48,69 & 58,26 & 4,09 & 0,032 & $-35,60$ & & & \\
\hline 0,05 & 1 & 45,00 & 51,45 & $-1,26$ & 0,057 & 14,86 & & & \\
\hline 0,50 & $1 / 3$ & 65,00 & 74,69 & $-4,16$ & 0,672 & 34,45 & & & \\
\hline 0,50 & $2 / 3$ & 58,69 & 73,50 & 0,60 & 0,477 & $-4,61$ & & & \\
\hline 0,50 & 1 & 55,00 & 72,82 & 3,71 & 0,369 & $-26,17$ & & & \\
\hline
\end{tabular}

ВЛО В ОРГАНЕ ИЛИ В ТКАНИ В ЗАВИСИМОСТИ ОТ ОБЪЕМА ОБЛУЧЕНИЯ И СУММАРНОЙ ДОЗЫ

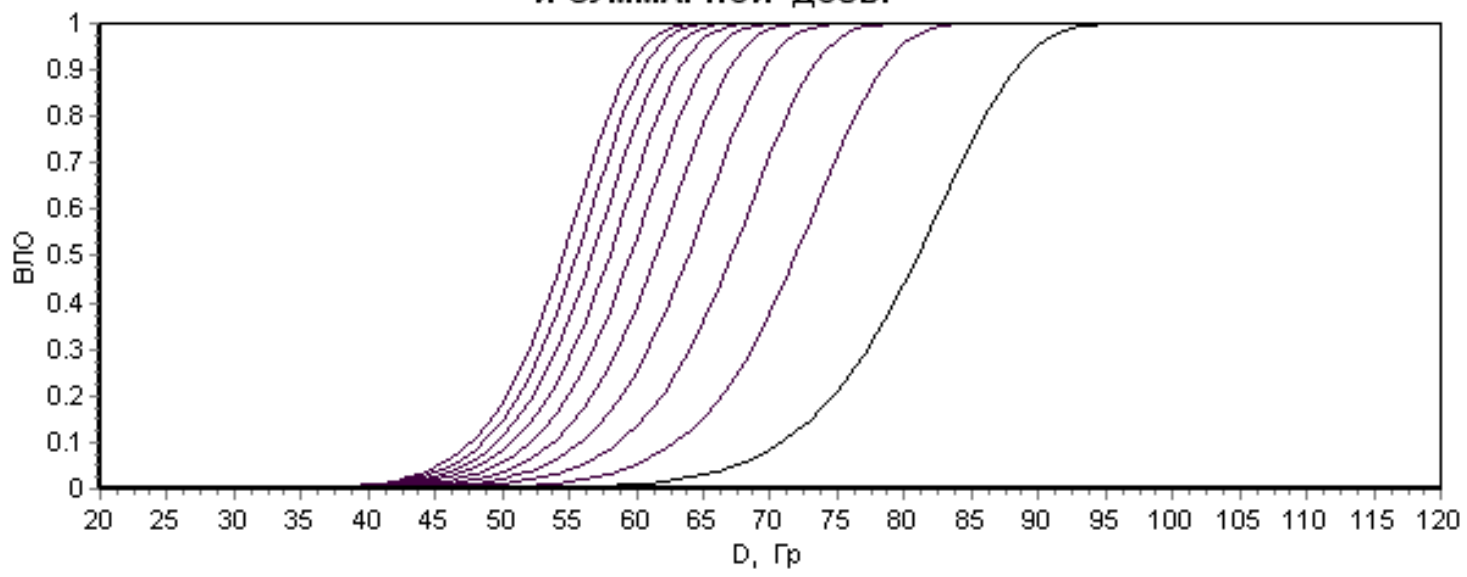

Рис. 5.16.1. Оптимизация по ВЛО

Таблица 5.16.2

Толстая кищка. Оптимизация по ТД

\begin{tabular}{|c|c|c|c|c|c|c|c|c|c|}
\hline$P_{\mathrm{K} Л}$ & $V$ & $D_{\text {КЛ }}$ & $D_{\text {TEOP }}$ & $\varepsilon, \%$ & $P_{\text {TEOP }}$ & $\varepsilon, \%$ & $A_{1}$ & $A_{2}$ & $b$ \\
\hline 0,05 & $1 / 3$ & 55,00 & 59,20 & $-2,70$ & 0,067 & 34,69 & \multirow{6}{*}{75,329} & \multirow{6}{*}{11,205} & \multirow{6}{*}{0,023} \\
\hline 0,05 & $2 / 3$ & 48,69 & 58,26 & 4,09 & 0,032 & $-35,60$ & & & \\
\hline 0,05 & 1 & 45,00 & 51,45 & $-1,26$ & 0,057 & 14,86 & & & \\
\hline 0,50 & $1 / 3$ & 65,00 & 74,69 & $-4,16$ & 0,672 & 34,45 & & & \\
\hline 0,50 & $2 / 3$ & 58,69 & 73,50 & 0,60 & 0,477 & $-4,61$ & & & \\
\hline 0,50 & 1 & 55,00 & 72,82 & 3,71 & 0,369 & $-26,17$ & & & \\
\hline
\end{tabular}




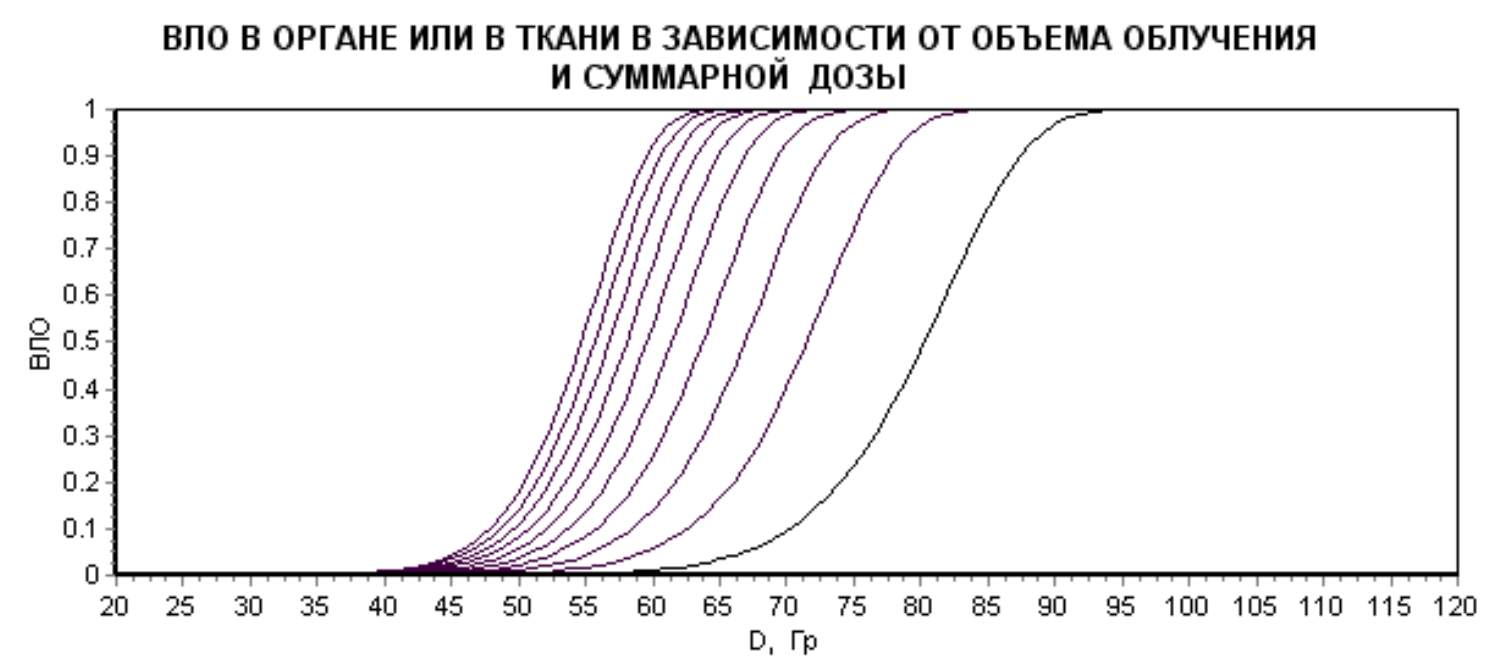

Рис. 5.16.2. Толстая кишка. Оптимизация по ТД

Таблица 5.16 .3

Толстая кищка. Оптимизация по ВЛО + ТД

\begin{tabular}{|c|c|c|c|c|c|c|c|c|c|}
\hline$P_{\text {Кл }}$ & $V$ & $D_{\text {Кл }}$ & $D_{\text {TEOP }}$ & $\varepsilon, \%$ & $P_{\text {TEOP }}$ & $\varepsilon, \%$ & $A_{1}$ & $A_{2}$ & $b$ \\
\hline 0,05 & $1 / 3$ & 55,00 & 59,20 & $-2,70$ & 0,067 & 34,69 & \multirow{6}{*}{75,329} & \multirow{6}{*}{11,205} & \multirow{6}{*}{0,023} \\
\hline 0,05 & $2 / 3$ & 48,69 & 58,26 & 4,09 & 0,032 & $-35,60$ & & & \\
\hline 0,05 & 1 & 45,00 & 51,45 & $-1,26$ & 0,057 & 14,86 & & & \\
\hline 0,50 & $1 / 3$ & 65,00 & 74,69 & $-4,16$ & 0,672 & 34,45 & & & \\
\hline 0,50 & $2 / 3$ & 58,69 & 73,50 & 0,60 & 0,477 & $-4,61$ & & & \\
\hline 0,50 & 1 & 55,00 & 72,82 & 3,71 & 0,369 & $-26,17$ & & & \\
\hline
\end{tabular}

ВЛО В ОРГАНЕ ИЛИ В ТКАНИ В ЗАВИСИМОСТИ ОТ ОБЪЕМА ОБЛУЧЕНИЯ И СУММАРНОЙ ДОЗЫ

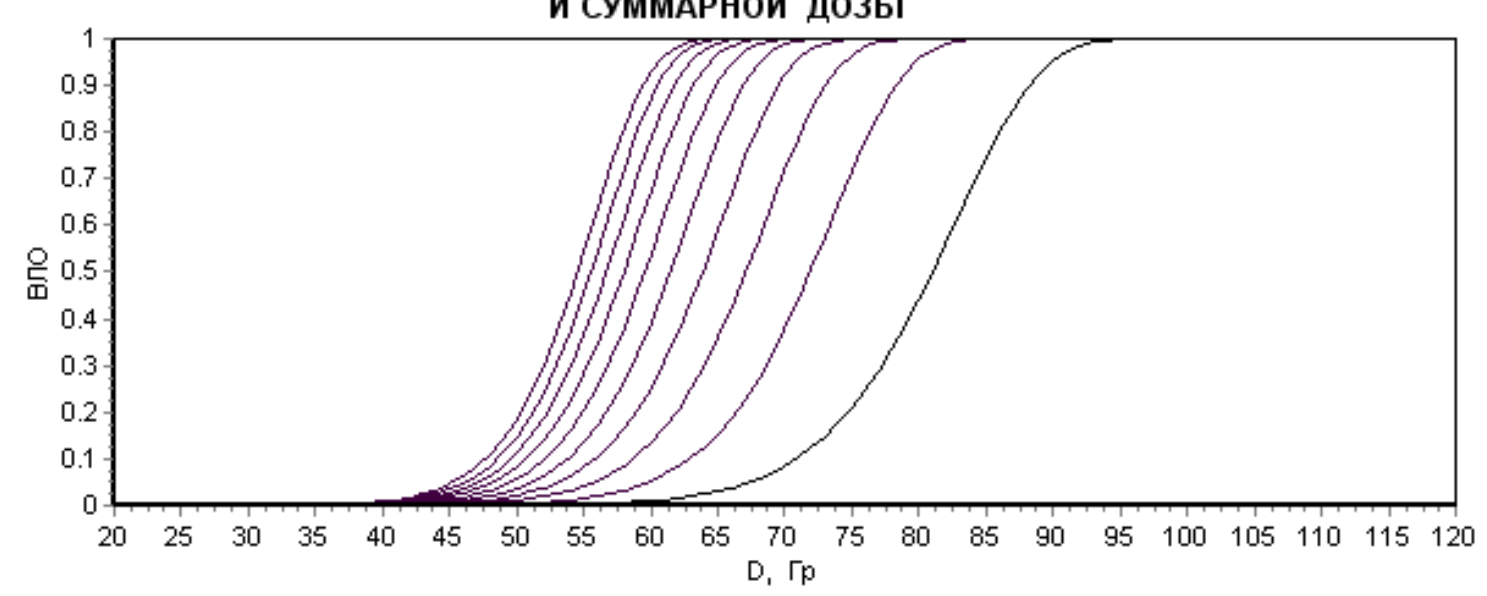

Рис. 5.16.3. Толстая кишка. Оптимизация по ВЛО + ТД

Оптимальные значения параметров модели МРВ, рассчитанные при помощи трех критериев оптимальности - совпадают. 
Таблица 5.17.1

Спинной мозг. Оптимизация по ВЛО

\begin{tabular}{|c|c|c|c|c|c|c|c|c|c|}
\hline$P_{\text {КЛ }}$ & V & $D_{\text {КЛ }}$ & $D_{\text {TEOP }}$ & $\varepsilon, \%$ & $P_{\mathrm{TEOP}}$ & $\varepsilon, \%$ & $A_{1}$ & $A_{2}$ & $b$ \\
\hline 0,05 & $1 / 3$ & 46,89 & 47,65 & 1,61 & 0,045 & $-9,48$ & \multirow{6}{*}{75,051} & \multirow{6}{*}{6,382} & \multirow{6}{*}{0,010} \\
\hline 0,05 & $2 / 3$ & 47,44 & 47,32 & $-0,26$ & 0,051 & 1,63 & & & \\
\hline 0,05 & 1 & 47,76 & 47,13 & $-1,33$ & 0,054 & 8,67 & & & \\
\hline 0,50 & $1 / 3$ & 70,74 & 71,65 & 1,28 & 0,472 & $-5,55$ & & & \\
\hline 0,50 & $2 / 3$ & 71,30 & 71,15 & $-0,21$ & 0,505 & 0,93 & & & \\
\hline 0,50 & 1 & 71,62 & 70,86 & $-1,06$ & 0,524 & 4,75 & & & \\
\hline
\end{tabular}

ВЛО В ОРГАНЕ ИЛИ В ТКАНИ В ЗАВИСИМОСТИ ОТ ОБЪЕМА ОБЛУЧЕНИЯ И СУММАРНОЙ ДОЗЫ

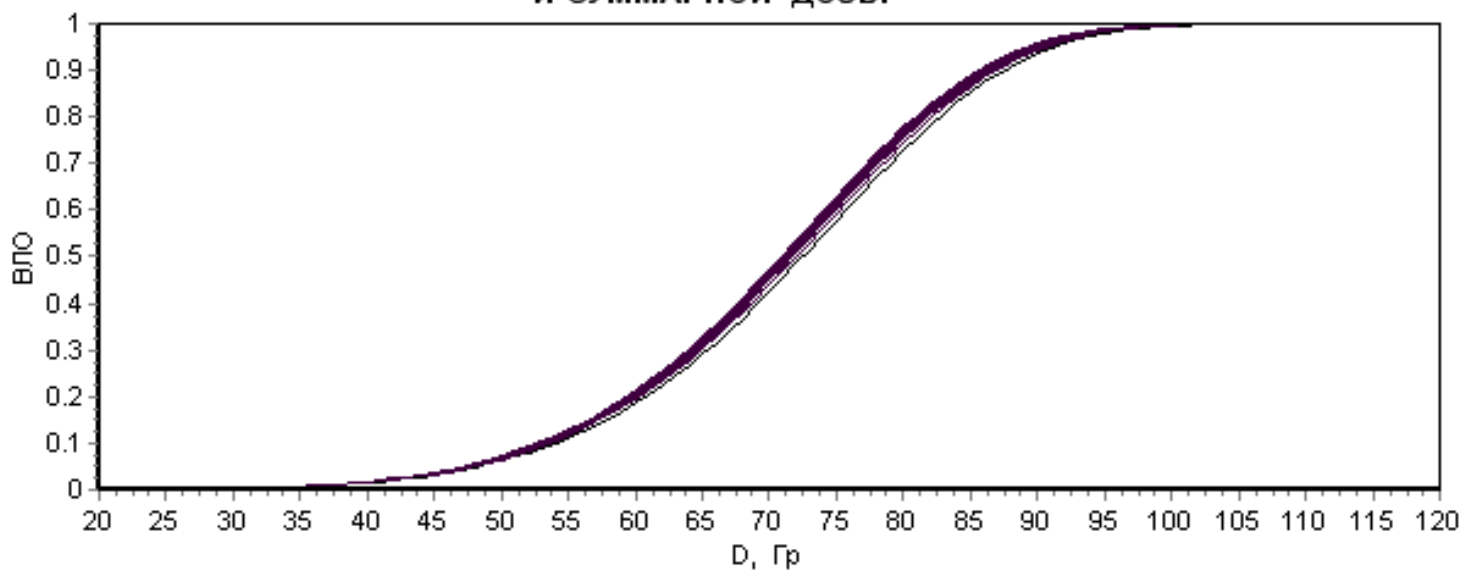

Рис. 5.17.1. Спинной мозг. Оптимизация по ВЛО

Таблица 17.2

Спинной мозг. Оптимизация по ТД

\begin{tabular}{|c|c|c|c|c|c|c|c|c|c|}
\hline$P_{\text {Кл }}$ & $V$ & $D_{\text {Кл }}$ & $D_{\text {TEOP }}$ & $\varepsilon, \%$ & $P_{\mathrm{TEOP}}$ & $\varepsilon, \%$ & $A_{1}$ & $A_{2}$ & $b$ \\
\hline 0,05 & $1 / 3$ & 46,89 & 47,65 & 1,61 & 0,045 & $-9,48$ & \multirow{6}{*}{75,051} & \multirow{6}{*}{6,382} & \multirow{6}{*}{0,010} \\
\hline 0,05 & $2 / 3$ & 47,44 & 47,32 & $-0,26$ & 0,051 & 1,63 & & & \\
\hline 0,05 & 1 & 47,76 & 47,13 & $-1,33$ & 0,054 & 8,67 & & & \\
\hline 0,50 & $1 / 3$ & 70,74 & 71,65 & 1,28 & 0,472 & $-5,55$ & & & \\
\hline 0,50 & $2 / 3$ & 71,30 & 71,15 & $-0,21$ & 0,505 & 0,93 & & & \\
\hline 0,50 & 1 & 71,62 & 70,86 & $-1,06$ & 0,524 & 4,75 & & & \\
\hline
\end{tabular}




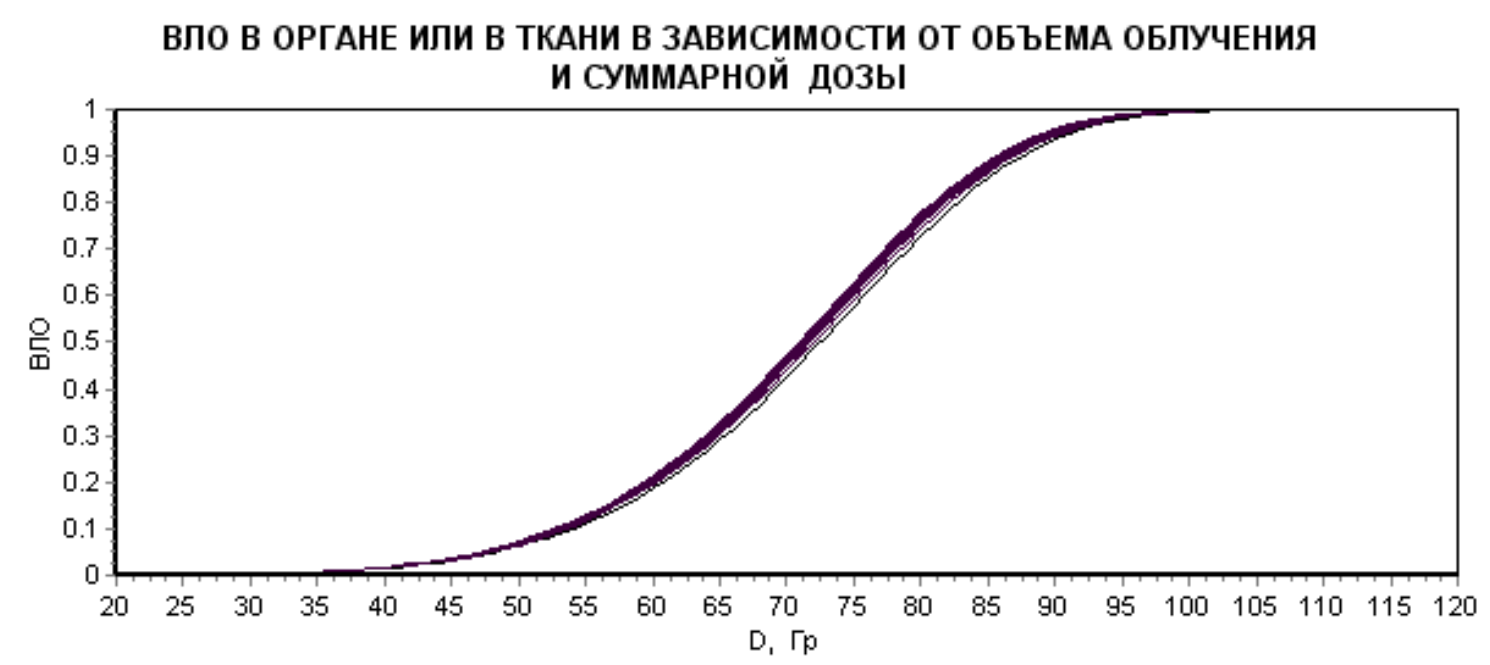

Рис. 5.17.2. Спинной мозг. Оптимизация по ТД

Таблица 5.17.3

Спинной мозг. Оптимизация по ВЛО + ТД

\begin{tabular}{|c|c|c|c|c|c|c|c|c|c|}
\hline$P_{\text {КЛ }}$ & $V$ & $D_{\text {Кл }}$ & $D_{\text {TEOP }}$ & $\varepsilon, \%$ & $P_{\mathrm{TEOP}}$ & $\varepsilon, \%$ & $A_{1}$ & $A_{2}$ & $b$ \\
\hline 0,05 & $1 / 3$ & 46,89 & 47,65 & 1,61 & 0,045 & $-9,48$ & \multirow{6}{*}{75,051} & \multirow{6}{*}{6,382} & \multirow{6}{*}{0,010} \\
\hline 0,05 & $2 / 3$ & 47,44 & 47,32 & $-0,26$ & 0,051 & 1,63 & & & \\
\hline 0,05 & 1 & 47,76 & 47,13 & $-1,33$ & 0,054 & 8,67 & & & \\
\hline 0,50 & $1 / 3$ & 70,74 & 71,65 & 1,28 & 0,472 & $-5,55$ & & & \\
\hline 0,50 & $2 / 3$ & 71,30 & 71,15 & $-0,21$ & 0,505 & 0,93 & & & \\
\hline 0,50 & 1 & 71,62 & 70,86 & $-1,06$ & 0,524 & 4,75 & & & \\
\hline
\end{tabular}

ВЛО В ОРГАНЕ ИЛИ В ТКАНИ В ЗАВИСИМОСТИ ОТ ОБЪЕМА ОБЛУЧЕНИЯ И СУММАРНОЙ ДОЗЫ

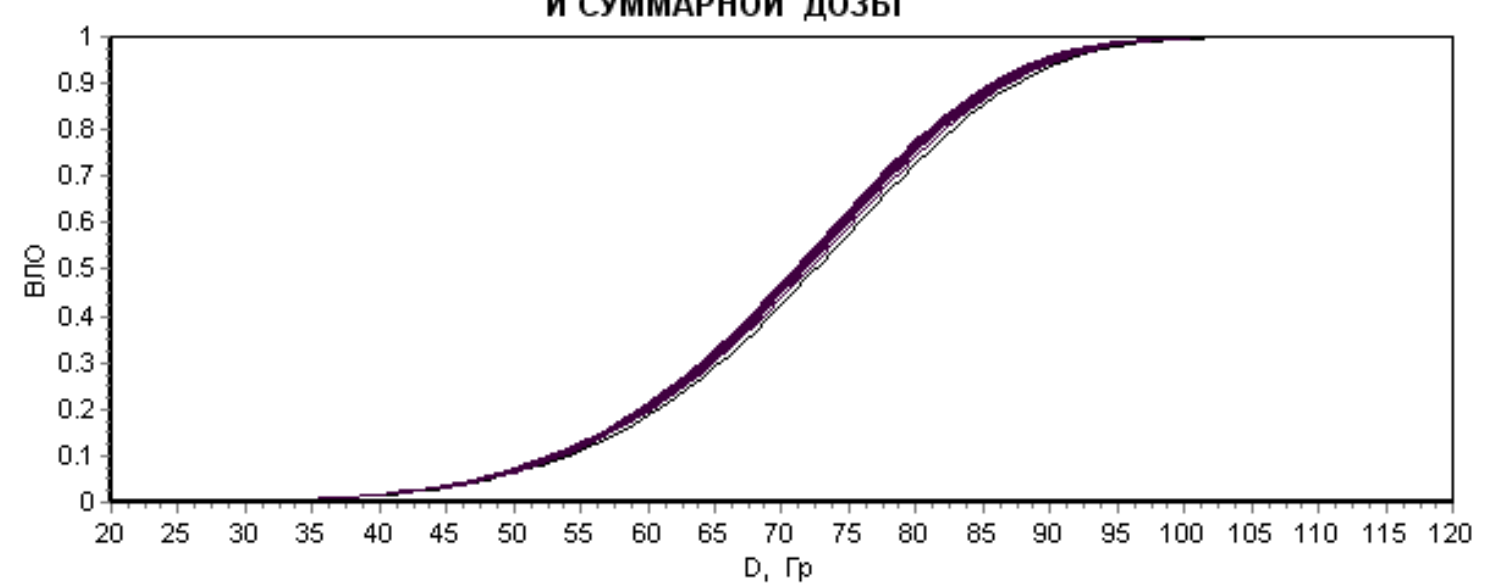

Рис. 5.17.3. Спинной мозг. Оптимизация по ВЛО + ТД

Оптимальные значения параметров модели МРВ, рассчитанные при помощи трех критериев оптимальности, совпадают. 


\subsection{8. Желудок}

Таблица 5.18.1

Желудок. Оптимизация по ВЛО

\begin{tabular}{|c|c|c|c|c|c|c|c|c|c|}
\hline$P_{\text {КЛ }}$ & $V$ & $D_{\text {КЛ }}$ & $D_{\text {TEOP }}$ & $\varepsilon, \%$ & $P_{\mathrm{TEOP}}$ & $\varepsilon, \%$ & $A_{1}$ & $A_{2}$ & $b$ \\
\hline 0,05 & $1 / 3$ & 58,33 & 57,89 & 0,76 & 0,054 & 7,83 & \multirow{6}{*}{63,315} & \multirow{6}{*}{10,141} & \multirow{6}{*}{0,185} \\
\hline 0,05 & $2 / 3$ & 51,04 & 50,92 & $-0,24$ & 0,051 & 2,36 & & & \\
\hline 0,05 & 1 & 46,77 & 47,24 & 1,01 & 0,045 & $-9,42$ & & & \\
\hline 0,50 & $1 / 3$ & 73,45 & 74,83 & 1,88 & 0,437 & $-12,67$ & & & \\
\hline 0,50 & $2 / 3$ & 66,16 & 65,83 & $-0,51$ & 0,518 & 3,60 & & & \\
\hline 0,50 & 1 & 61,90 & 61,68 & $-1,35$ & 0,549 & 9,70 & & & \\
\hline
\end{tabular}

ВЛО В ОРГАНЕ ИЛИ В ТКАНИ В ЗАВИСИМОСТИ ОТ ОБЪЕМА ОБЛУЧЕНИЯ И СУММАРНОЙ ДОЗЫ

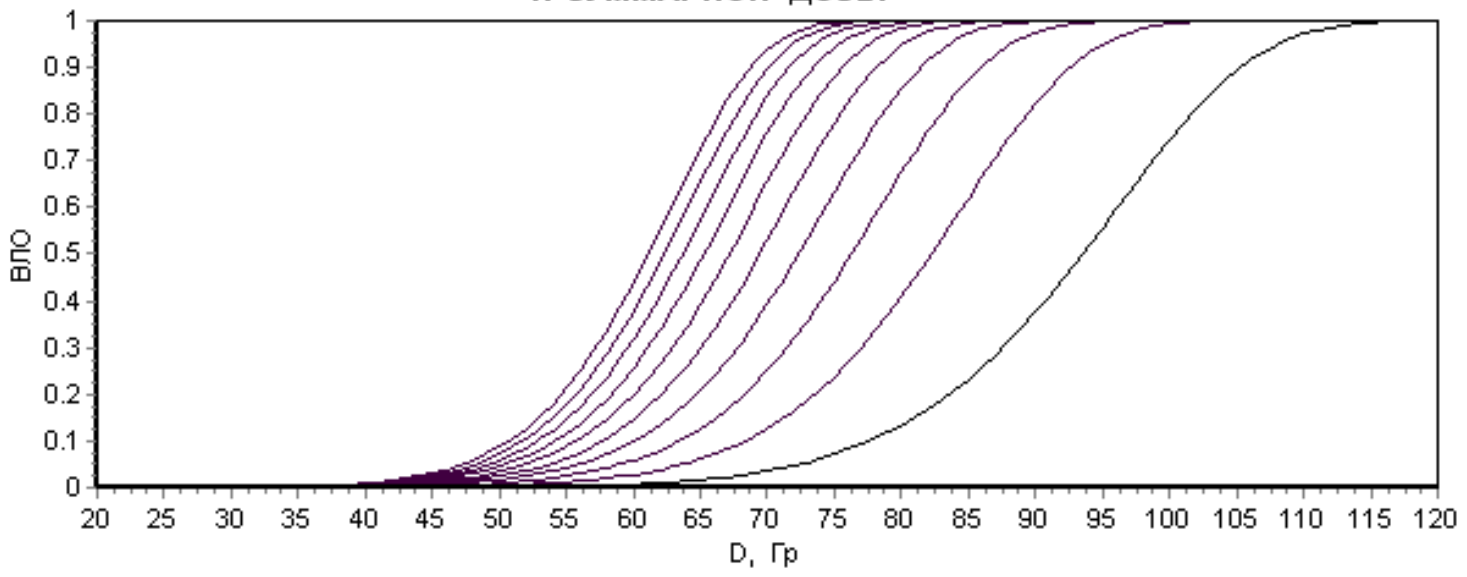

Рис. 5.18.1. Желудок. Оптимизация по ВЛО

Таблица 5.18.2

Желудок. Оптимизация по ТД

\begin{tabular}{|c|c|c|c|c|c|c|c|c|c|}
\hline$P_{\text {КЛ }}$ & $V$ & $D_{\text {КЛ }}$ & $\overline{D_{\mathrm{TEOP}}}$ & $\varepsilon, \%$ & $P_{\mathrm{TEOP}}$ & $\varepsilon, \%$ & $A_{1}$ & $A_{2}$ & $b$ \\
\hline 0,05 & $1 / 3$ & $\begin{array}{l}88,33 \\
\end{array}$ & 57,65 & $-1,18$ & 0,056 & 12,37 & \multirow{6}{*}{63,538} & \multirow{6}{*}{10,141} & \multirow{6}{*}{0,178} \\
\hline 0,05 & $2 / 3$ & 51,04 & 50,95 & $-0,17$ & 0,051 & 1,68 & & & \\
\hline 0,05 & 1 & 46,77 & 47,41 & 1,36 & 0,044 & 12,52 & & & \\
\hline 0,50 & $1 / 3$ & 73,45 & 74,52 & 1,46 & 0,451 & $-9,90$ & & & \\
\hline 0,50 & $2 / 3$ & 66,16 & 65,89 & $-0,44$ & 0,516 & 3,12 & & & \\
\hline 0,50 & 1 & 61,90 & 61,28 & $-1,0$ & 0,535 & 7,15 & & & \\
\hline
\end{tabular}




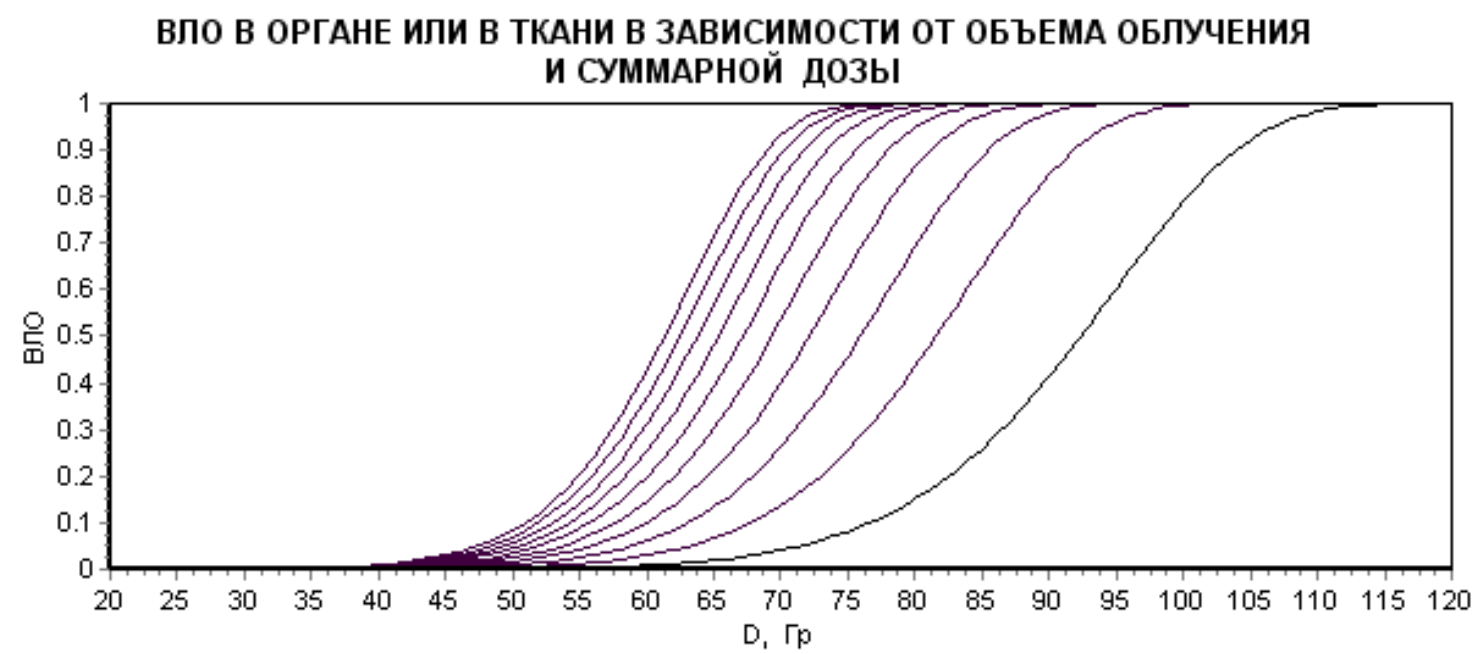

Рис. 5.18.2. Желудок. Оптимизация по ТД

Таблица 5.18.3

Желудок. Оптимизация по ВЛО + ТД

\begin{tabular}{|c|c|c|c|c|c|c|c|c|c|}
\hline$P_{\text {Кл }}$ & $V$ & $D_{\text {Кл }}$ & $D_{\text {TEOP }}$ & $\varepsilon, \%$ & $P_{\mathrm{TEOP}}$ & $\varepsilon, \%$ & $A_{1}$ & $A_{2}$ & $b$ \\
\hline 0,05 & $1 / 3$ & 58,33 & 57,65 & $-1,18$ & 0,056 & 12,37 & \multirow{6}{*}{63,538} & \multirow{6}{*}{10,141} & \multirow{6}{*}{0,178} \\
\hline 0,05 & $2 / 3$ & 51,04 & 50,95 & $-0,17$ & 0,051 & 1,68 & & & \\
\hline 0,05 & 1 & 46,77 & 47,41 & 1,36 & 0,044 & 12,52 & & & \\
\hline 0,50 & $1 / 3$ & 73,45 & 74,52 & 1,46 & 0,451 & $-9,90$ & & & \\
\hline 0,50 & $2 / 3$ & 66,16 & 65,89 & $-0,44$ & 0,516 & 3,12 & & & \\
\hline 0,50 & 1 & 61,90 & 61,28 & $-1,0$ & 0,535 & 7,15 & & & \\
\hline
\end{tabular}

ВЛО В ОРГАНЕ ИЛИ В ТКАНИ В ЗАВИСИМОСТИ ОТ ОБЪЕМА ОБЛУЧЕНИЯ И СУММАРНОЙ ДОЗЫ

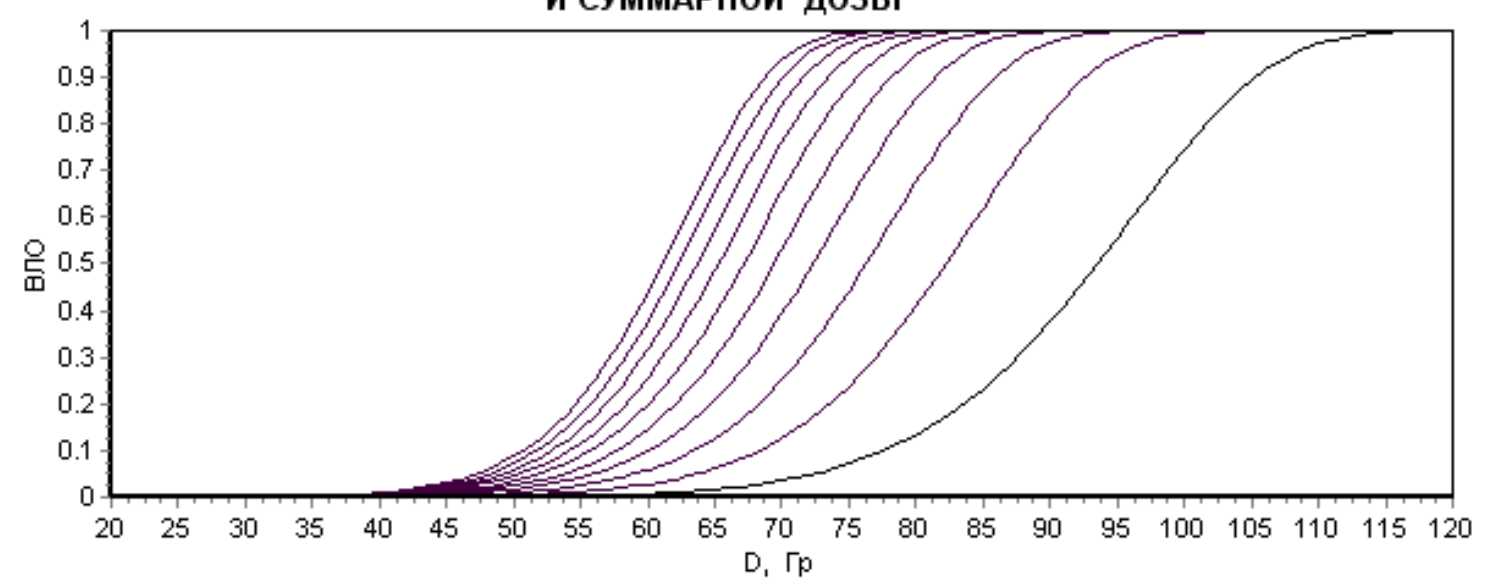

Рис. 5.18.3. Желудок. Оптимизация по ВЛО + ТД

Таблица 5.18.4

Желудок. Оптимизация по ТД, отдельно для ВЛО = 0,05 и ВЛО =0,50

\begin{tabular}{c|c|c|c|c|c|c|c|c|c}
\hline$P_{\text {КЛ }}$ & $V$ & $D_{\text {КЛ }}$ & $D_{\text {ТЕОР }}$ & $\varepsilon, \%$ & $P_{\text {ТЕОР }}$ & $\varepsilon, \%$ & $A_{1}$ & $A_{2}$ & $b$ \\
\hline 0,05 & $1 / 3$ & 58,33 & 58,41 & 0,13 & 0,050 & $-0,14$ & \multirow{2}{*}{745,883} & \multirow{2}{*}{1,073} & \multirow{2}{*}{0,200} \\
\hline 0,05 & $2 / 3$ & 51,04 & 50,85 & $-0,38$ & 0,050 & 0,40 & & \\
\hline
\end{tabular}




\begin{tabular}{c|c|c|c|c|c|c|c|c|c}
\hline 0,05 & 1 & 46,77 & 46,89 & 0,25 & 0,050 & $-0,26$ & & & \\
\hline 0,50 & $1 / 3$ & 73,45 & 73,55 & 0,13 & 0,500 & $-0,02$ & \multirow{3}{*}{350,784} & \multirow{2}{*}{0,211} & \multirow{2}{*}{0,156} \\
\hline 0,50 & $2 / 3$ & 66,16 & 66,01 & $-0,23$ & 0,500 & 0,03 & \\
\cline { 1 - 7 } 0,50 & 1 & 61,90 & 61,96 & 0,10 & 0,500 & $-0,02$ & & & \\
\hline
\end{tabular}

Анализ табл. 5.18.4 свидетелствует о том, что клиническая информация плохо согласована.

\subsection{9. Височно-нижнечелюстной сустав и нижняя челюсть (ВНС и НЧ)}

Таблица 5.19.1

ВНС и НЧ. Оптимизация по ВЛО

\begin{tabular}{|c|c|c|c|c|c|c|c|c|c|}
\hline$P_{\text {КЛ }}$ & $V$ & $D_{\text {Кл }}$ & $D_{\text {TEOP }}$ & $\varepsilon, \%$ & $P_{\mathrm{TEOP}}$ & $\varepsilon, \%$ & $A_{1}$ & $A_{2}$ & $b$ \\
\hline 0,05 & $1 / 3$ & 60,51 & 60,71 & 0,32 & 0,049 & $-3,02$ & \multirow{6}{*}{81,321} & \multirow{6}{*}{9,791} & \multirow{6}{*}{0,010} \\
\hline 0,05 & $2 / 3$ & 60,32 & 60,29 & $-0,06$ & 0,050 & 0,55 & & & \\
\hline 0,05 & 1 & 60,20 & 60,04 & $-0,26$ & 0,051 & 2,55 & & & \\
\hline 0,50 & $1 / 3$ & 78,90 & 79,20 & 0,38 & 0,487 & $-2,55$ & & & \\
\hline 0,50 & $2 / 3$ & 78,70 & 78,65 & $-0,06$ & 0,502 & 0,42 & & & \\
\hline 0,50 & 1 & 78,58 & 78,33 & $-0,31$ & 0,511 & 2,15 & & & \\
\hline
\end{tabular}

ВЛО В ОРГАНЕ ИЛИ В ТКАНИ В ЗАВИСИМОСТИ ОТ ОБЪЕМА ОБЛУЧЕНИЯ И СУММАРНОЙ ДОЗЫ

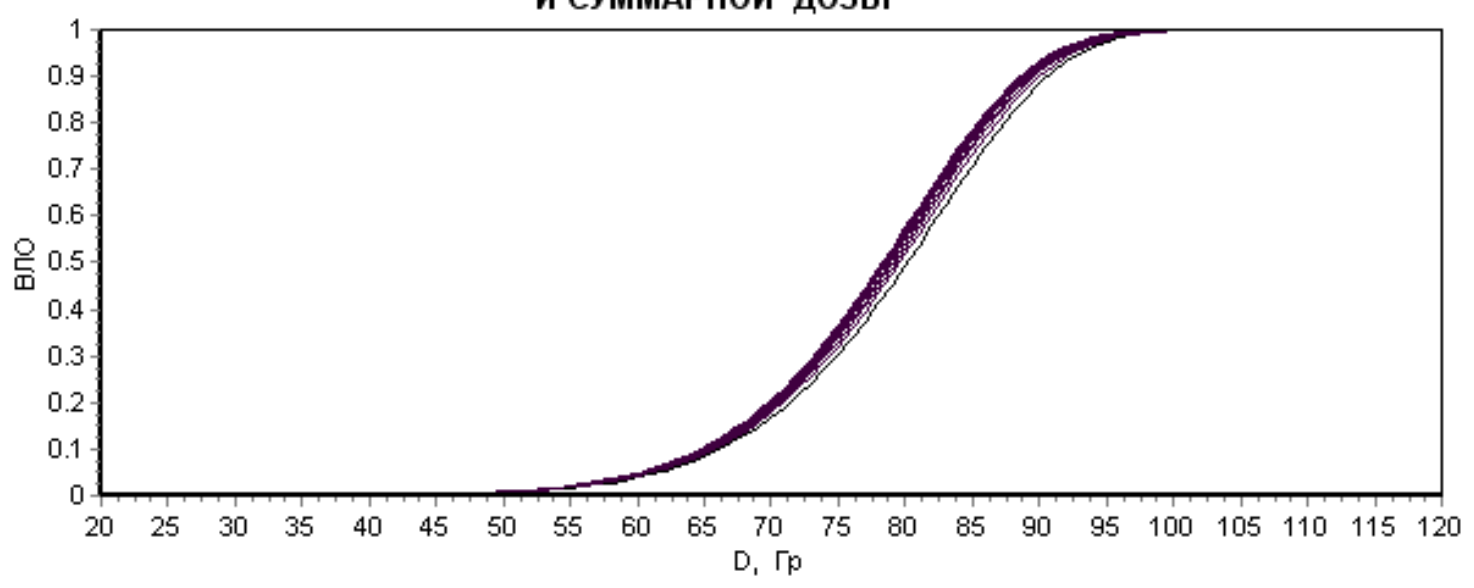

Рис. 5.19.1. ВНС и НЧ. Оптимизация по ВЛО

Таблица 5.19.2

ВНС и НЧ. Оптимизация по ТД

\begin{tabular}{|c|c|c|c|c|c|c|c|c|c|}
\hline$P_{\text {КЛ }}$ & $V$ & $D_{\text {Кл }}$ & $D_{\text {TEOP }}$ & $\varepsilon, \%$ & $P_{\mathrm{TEOP}}$ & $\varepsilon, \%$ & $A_{1}$ & $A_{2}$ & $b$ \\
\hline 0,05 & $1 / 3$ & 60,51 & 60,71 & 0,32 & 0,049 & $-3,02$ & \multirow{6}{*}{81,321} & \multirow{6}{*}{9,791} & \multirow{6}{*}{0,010} \\
\hline 0,05 & $2 / 3$ & 60,32 & 60,29 & $-0,06$ & 0,050 & 0,55 & & & \\
\hline 0,05 & 1 & 60,20 & 60,04 & $-0,26$ & 0,051 & 2,55 & & & \\
\hline 0,50 & $1 / 3$ & 78,90 & 79,20 & 0,38 & 0,487 & $-2,55$ & & & \\
\hline 0,50 & $2 / 3$ & 78,70 & 78,65 & $-0,06$ & 0,502 & 0,42 & & & \\
\hline 0,50 & 1 & 78,58 & 78,33 & $-0,31$ & 0,511 & 2,15 & & & \\
\hline
\end{tabular}




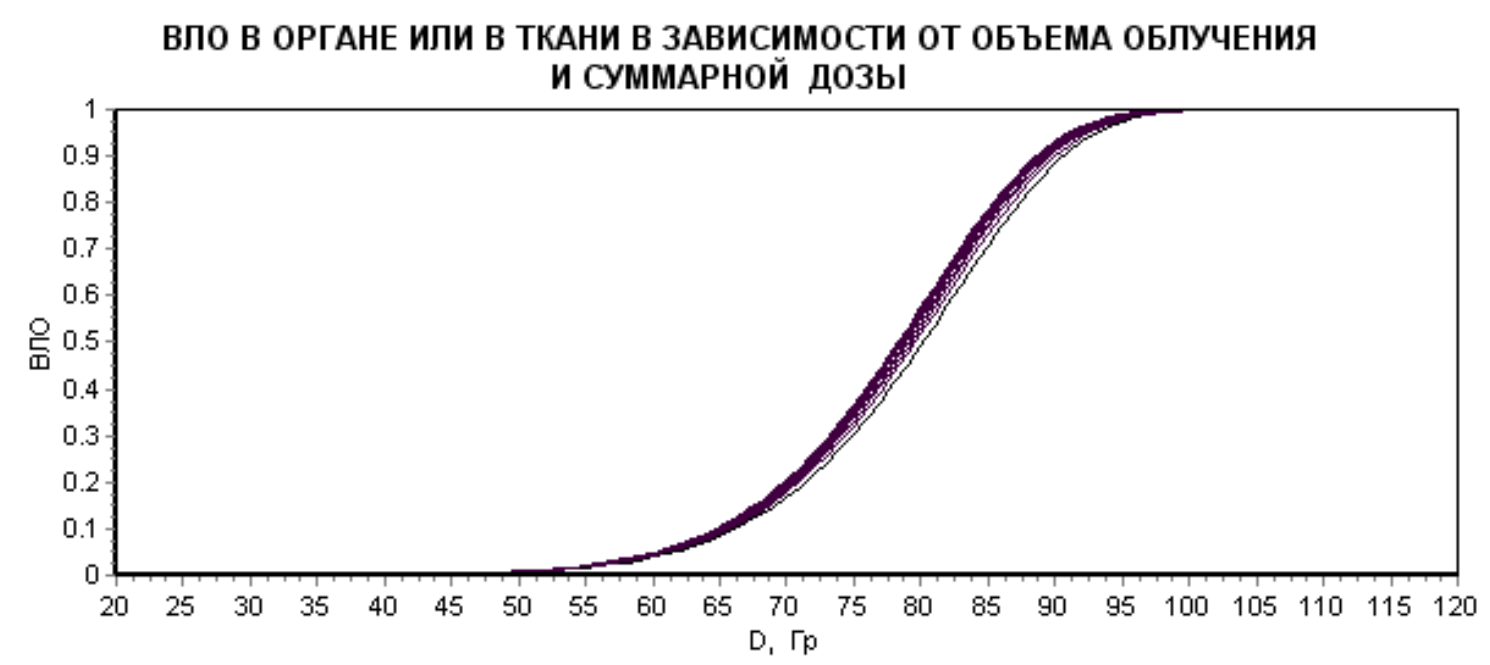

Рис. 5.19.2. ВНС и НЧ. Оптимизация по ТД

Таблица 5.19.3

ВНС и НЧ. Оптимизация по ВЛО + ТД

\begin{tabular}{|c|c|c|c|c|c|c|c|c|c|}
\hline$P_{\text {КЛ }}$ & $V$ & $D_{\text {Кл }}$ & $D_{\text {TEOP }}$ & $\varepsilon, \%$ & $P_{\mathrm{TEOP}}$ & $\varepsilon, \%$ & $A_{1}$ & $A_{2}$ & $b$ \\
\hline 0,05 & $1 / 3$ & 60,51 & 60,71 & 0,32 & 0,049 & $-3,02$ & \multirow{6}{*}{81,321} & \multirow{6}{*}{9,791} & \multirow{6}{*}{0,010} \\
\hline 0,05 & $2 / 3$ & 60,32 & 60,29 & $-0,06$ & 0,050 & 0,55 & & & \\
\hline 0,05 & 1 & 60,20 & 60,04 & $-0,26$ & 0,051 & 2,55 & & & \\
\hline 0,50 & $1 / 3$ & 78,90 & 79,20 & 0,38 & 0,487 & $-2,55$ & & & \\
\hline 0,50 & $2 / 3$ & 78,70 & 78,65 & $-0,06$ & 0,502 & 0,42 & & & \\
\hline 0,50 & 1 & 78,58 & 78,33 & $-0,31$ & 0,511 & 2,15 & & & \\
\hline
\end{tabular}

ВЛО В ОРГАНЕ ИЛИ В ТКАНИ В ЗАВИСИМОСТИ ОТ ОБЪЕМА ОБЛУЧЕНИЯ И СУММАРНОЙ ДОЗЫ

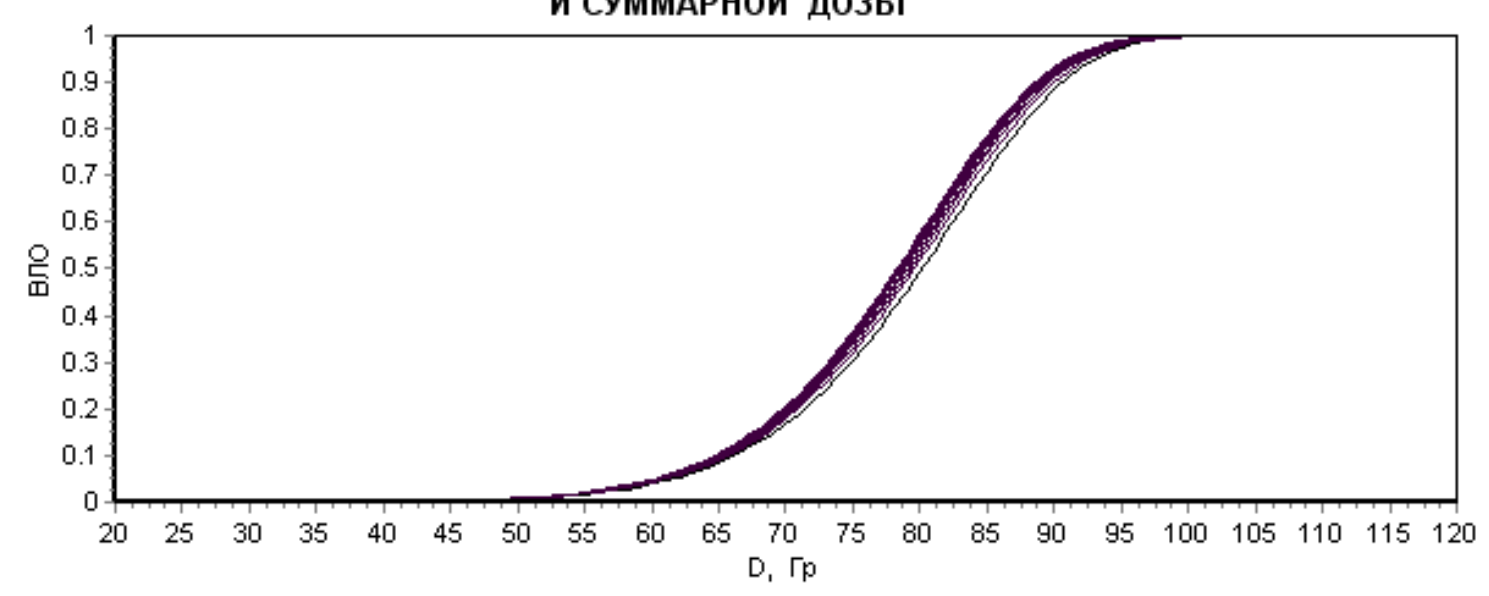

Рис. 19.3. ВНС и НЧ. Оптимизация по ВЛО+ТД

Оптимальные значения параметров модели МРВ, рассчитанные при помощи трех критериев оптимальности совпадают. 


\subsection{0. Конский хвост}

Таблица 5.20

Конский хвост

\begin{tabular}{c|c|c|c|c|c}
\hline$P_{\text {КЛ }}$ & $D_{\text {КЛ }}$ & $P_{\text {КЛ }}$ & $D_{\text {КЛ }}$ & $A_{1}$ & $A_{2}$ \\
\hline 0,05 & 58,65 & 0,50 & 75,19 & 77,866 & 10,481 \\
\hline
\end{tabular}

ВЛО В ОРГАНЕ ИЛИ В ТКАНИ В ЗАВИСИМОСТИ ОТ СУММАРНОЙ ДОЗЫ

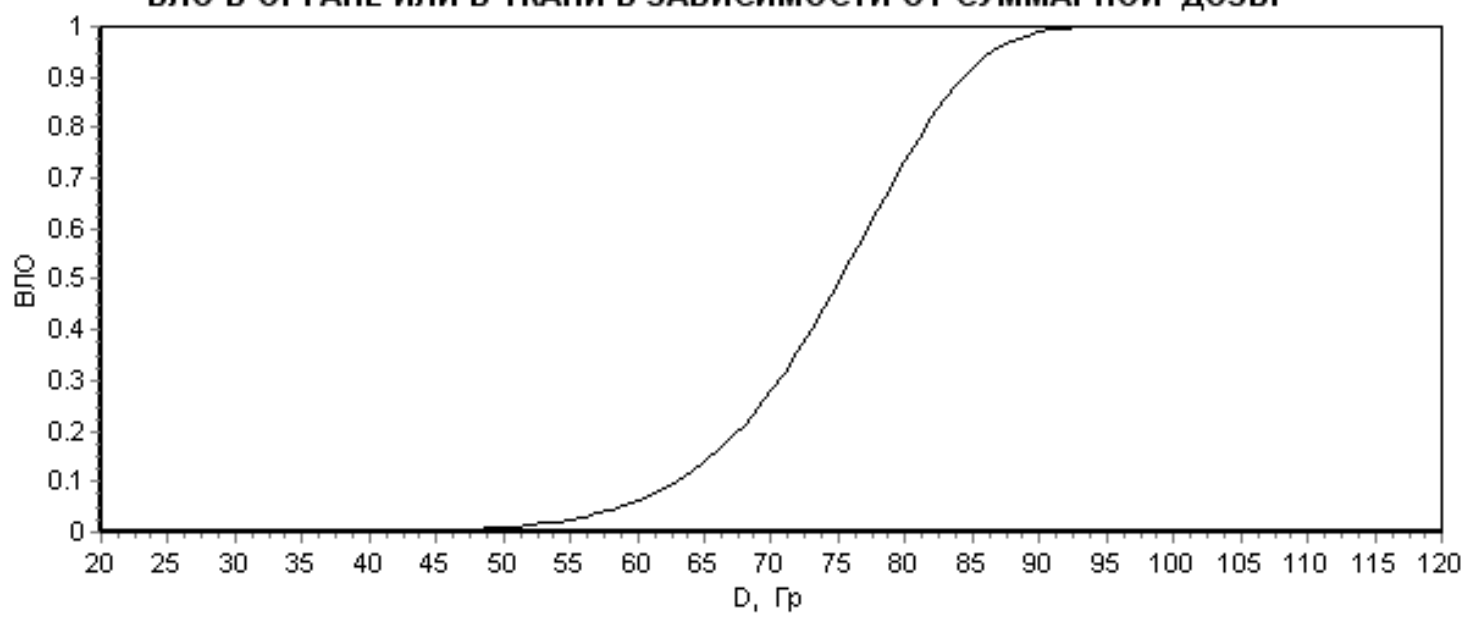

Рис. 5.20. Конский хвост. Зависимость ВЛО от дозы

\subsection{1. Плечевое сплетение}

Таблица 5.21.1

Плечевое сплетение. Оптимизация по ВЛО

\begin{tabular}{|c|c|c|c|c|c|c|c|c|c|}
\hline$P_{\text {Кл }}$ & $V$ & $D_{\text {Кл }}$ & $D_{\text {TEOP }}$ & $\varepsilon, \%$ & $P_{\mathrm{TEOP}}$ & $\varepsilon, \%$ & $\overline{A_{1}}$ & $A_{2}$ & $b$ \\
\hline 0,05 & $1 / 3$ & 61,09 & 60,95 & $-0,23$ & 0,051 & 2,23 & \multirow{6}{*}{77,271} & \multirow{6}{*}{9,978} & \multirow{6}{*}{0,055} \\
\hline 0,05 & $2 / 3$ & 58,67 & 58,67 & 0,01 & 0,050 & $-0,03$ & & & \\
\hline 0,05 & 1 & 57,25 & 57,38 & 0,22 & 0,949 & $-2,15$ & & & \\
\hline 0,50 & $1 / 3$ & 78,67 & 79,12 & 0,58 & 0,480 & $-3,94$ & & & \\
\hline 0,50 & $2 / 3$ & 76,25 & 76,16 & $-0,11$ & 0,504 & 0,78 & & & \\
\hline 0,50 & 1 & 74,83 & 74,48 & $-0,46$ & 0,516 & 3,22 & & & \\
\hline
\end{tabular}


ВЛО В ОРГАНЕ ИЛИ В ТКАНИ В ЗАВИСИМОСТИ ОТ ОБЪЕМА ОБЛУЧЕНИЯ И СУММАРНОЙ ДОЗЫ

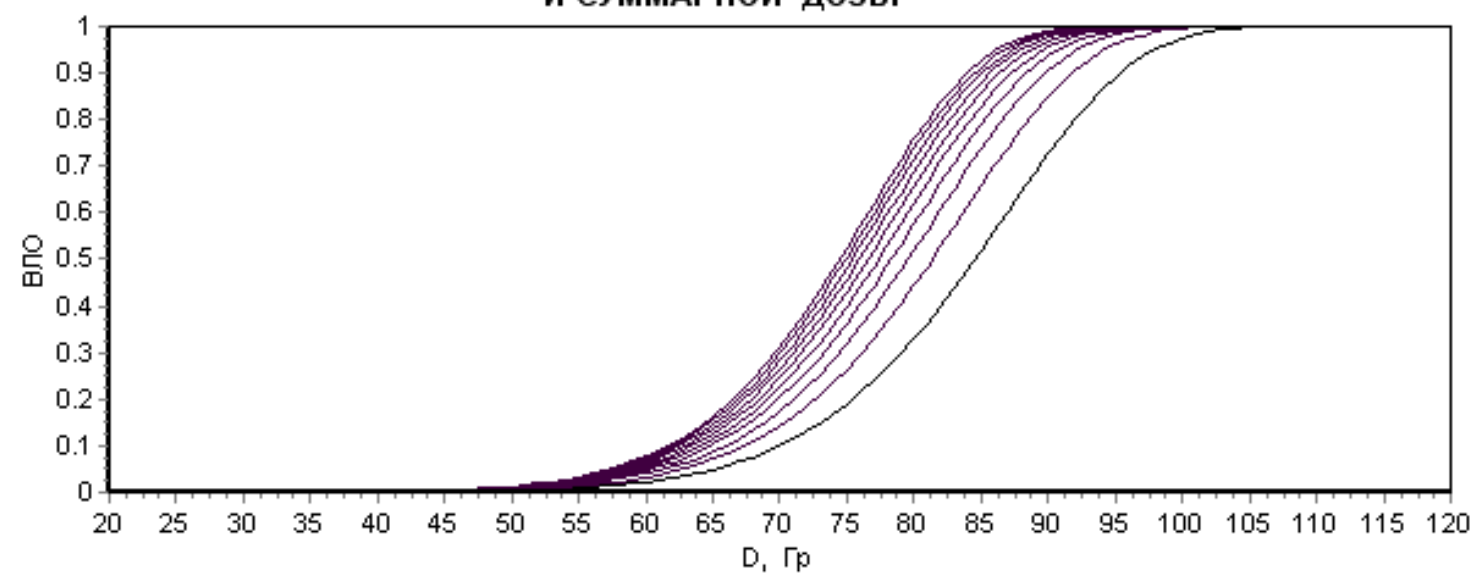

Рис. 5.21.1. Плечевое сплетение. Оптимизация по ВЛО

Таблица 5.21.2

Плечевое сплетение. Оптимизация по ТД

\begin{tabular}{|c|c|c|c|c|c|c|c|c|c|}
\hline$P_{\text {Кл }}$ & $V$ & $D_{\text {Кл }}$ & $D_{\text {TEOP }}$ & $\varepsilon, \%$ & $P_{\mathrm{TEOP}}$ & $\varepsilon, \%$ & $A_{1}$ & $A_{2}$ & $b$ \\
\hline 0,05 & $1 / 3$ & 61,09 & 60,84 & $-0,41$ & 0,052 & 4,02 & \multirow{6}{*}{77,388} & \multirow{6}{*}{9,978} & \multirow{6}{*}{0,052} \\
\hline 0,05 & $2 / 3$ & 58,67 & 58,69 & 0,03 & 0,050 & $-0,31$ & & & \\
\hline 0,05 & 1 & 57,25 & 57,46 & 0,37 & 0,048 & $-3,57$ & & & \\
\hline 0,50 & $1 / 3$ & 78,67 & 78,98 & 0,40 & 0,486 & $-2,72$ & & & \\
\hline 0,50 & $2 / 3$ & 76,25 & 76,19 & $-0,08$ & 0,503 & 0,58 & & & \\
\hline 0,50 & 1 & 74,83 & 74,60 & $-0,31$ & 0,511 & 2,17 & & & \\
\hline
\end{tabular}

ВЛО В ОРГАНЕ ИЛИ В ТКАНИ В ЗАВИСИМОСТИ ОТ ОБЪЕМА ОБЛУЧЕНИЯ И СУММАРНОЙ ДОЗЫ

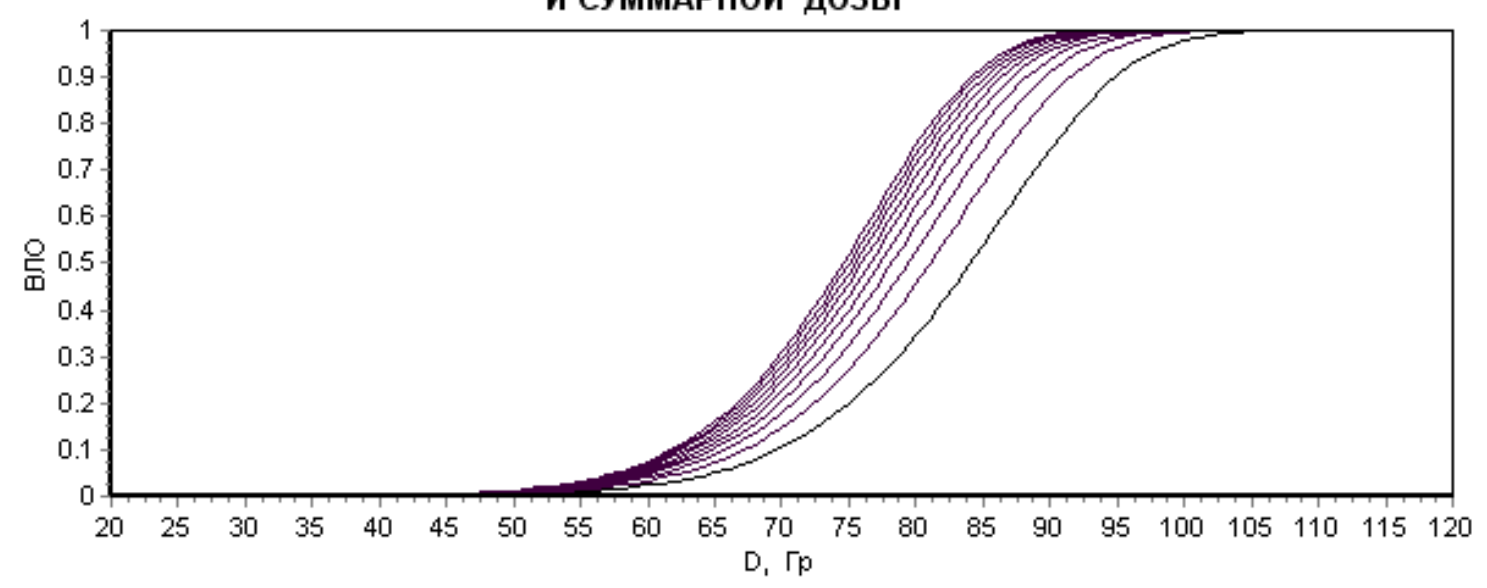

Рис. 5.21.2. Плечевое сплетение. Оптимизация по ТД 
Таблица 5.21.3

Плечевое сплетение. Оптимизация по ВДО + ТД

\begin{tabular}{|c|c|c|c|c|c|c|c|c|c|}
\hline$P_{\text {Кл }}$ & $V$ & $D_{\text {КЛ }}$ & $D_{\text {TEOP }}$ & $\varepsilon, \%$ & $P_{\mathrm{TEOP}}$ & $\varepsilon, \%$ & $A_{1}$ & $A_{2}$ & $b$ \\
\hline 0,05 & $1 / 3$ & 61,09 & 60,91 & $-0,29$ & 0,051 & 2,82 & \multirow{6}{*}{77,310} & \multirow{6}{*}{9,978} & \multirow{6}{*}{0,054} \\
\hline 0,05 & $2 / 3$ & 58,67 & 58,68 & 0,01 & 0,050 & $-0,12$ & & & \\
\hline 0,05 & 1 & 57,25 & 57,41 & 0,27 & 0,049 & $-2,62$ & & & \\
\hline 0,50 & $1 / 3$ & 78,67 & 79,08 & 0,52 & 0,482 & $-3,54$ & & & \\
\hline 0,50 & $2 / 3$ & 76,25 & 76,17 & $-0,10$ & 0,504 & 0,71 & & & \\
\hline 0,50 & 1 & 74,83 & 74,52 & $-0,41$ & 0,514 & 2,87 & & & \\
\hline
\end{tabular}

ВЛО В ОРГАНЕ ИЛИ В ТКАНИ В ЗАВИСИМОСТИ ОТ ОБЪЕМА ОБЛУЧЕНИЯ И СУММАРНОЙ ДОЗЫ

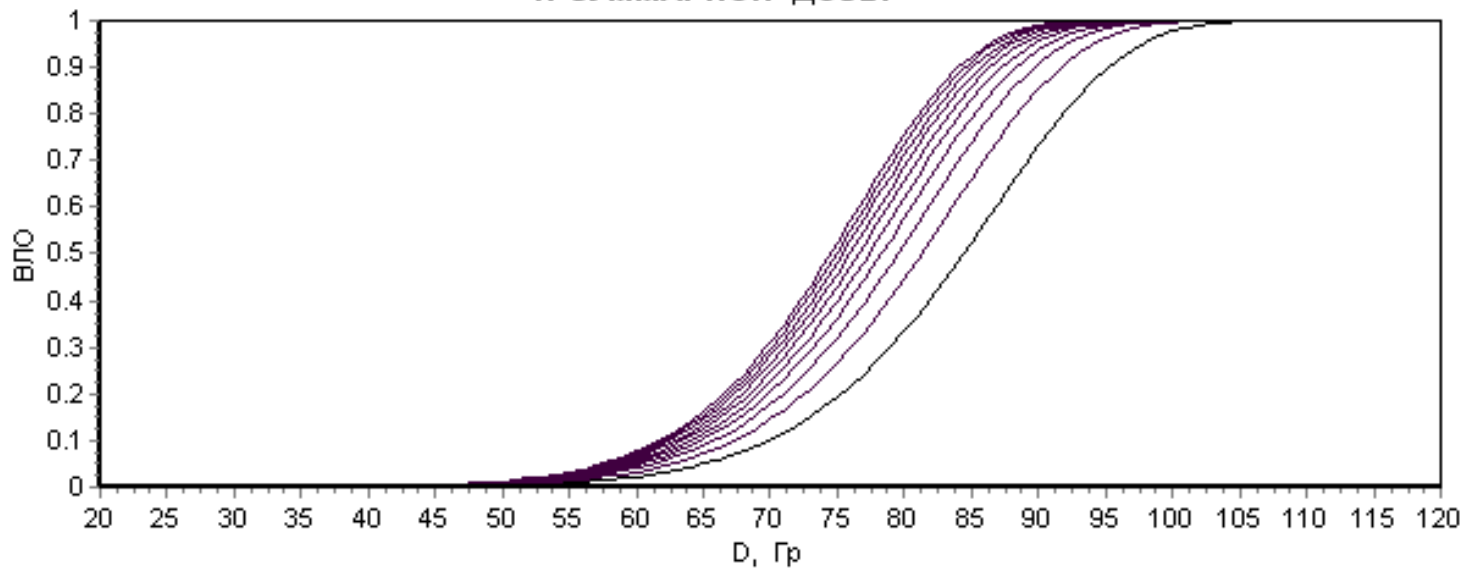

Рис. 5.21.3. Плечевое сплетение. Оптимизация по ВЛО + ТД

5.22. Головка и шейка бедренной кости (ГШБК)

Таблица 5.22

\section{ГШБК}

\begin{tabular}{c|c|c|c|c|c}
\hline$P_{\text {КЛ }}$ & $D_{\text {КЛ }}$ & $P_{\text {КЛ }}$ & $D_{\text {КЛ }}$ & $A_{1}$ & $A_{2}$ \\
\hline 0,05 & 51,61 & 0,50 & 63,70 & 65,616 & 12,371 \\
\hline
\end{tabular}




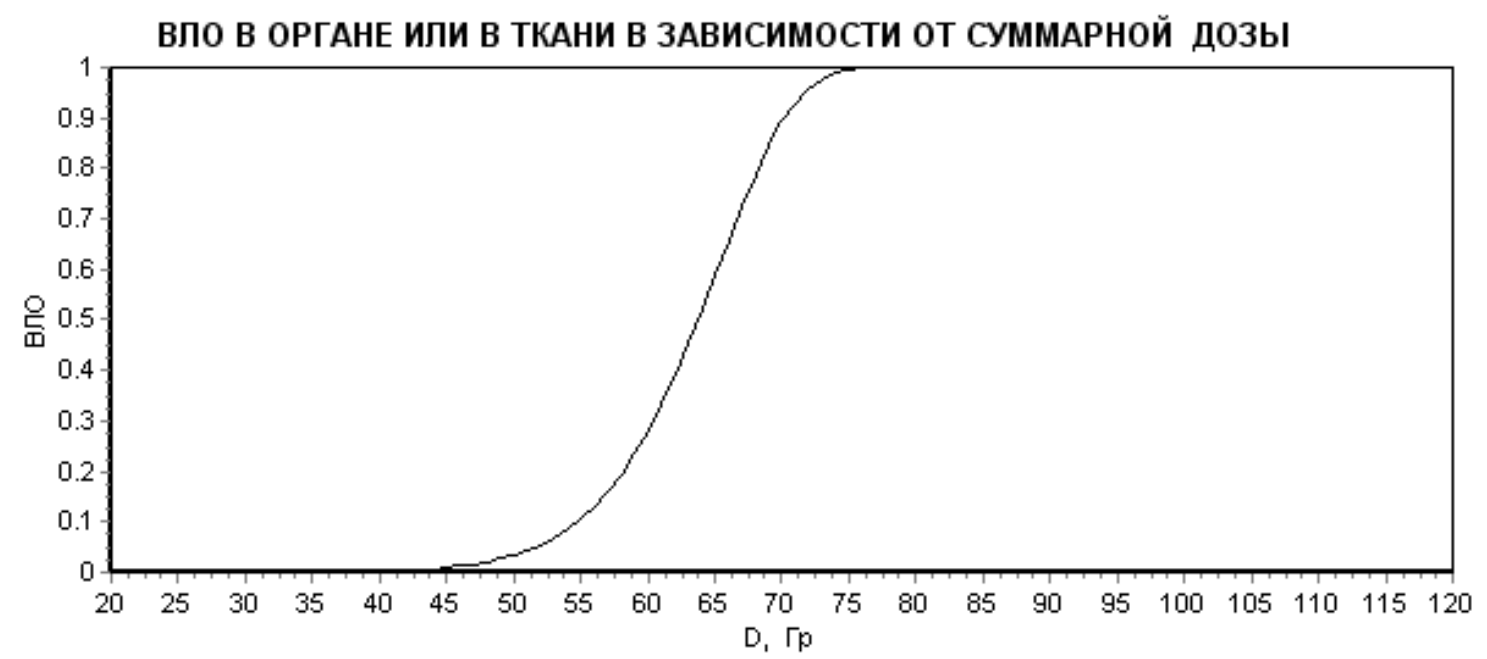

Рис. 5.22. ГШБК. Зависимость ВЛО от дозы

\subsection{3. Хрусталик глаза}

Таблица 5.23

Хрусталик глаза

\begin{tabular}{c|c|c|c|c|c}
\hline$P_{\text {КЛ }}$ & $D_{\text {КЛ }}$ & $P_{\text {КЛ }}$ & $D_{\text {КЛ }}$ & $A_{1}$ & $A_{2}$ \\
\hline 0,05 & 6,762 & 0,50 & 16,86 & 19,174 & 2,850 \\
\hline
\end{tabular}

ВЛО В ОРГАНЕ ИЛИ В ТКАНИ В ЗАВИСИМОСТИ ОТ СУММАРНОЙ ДОЗЫ

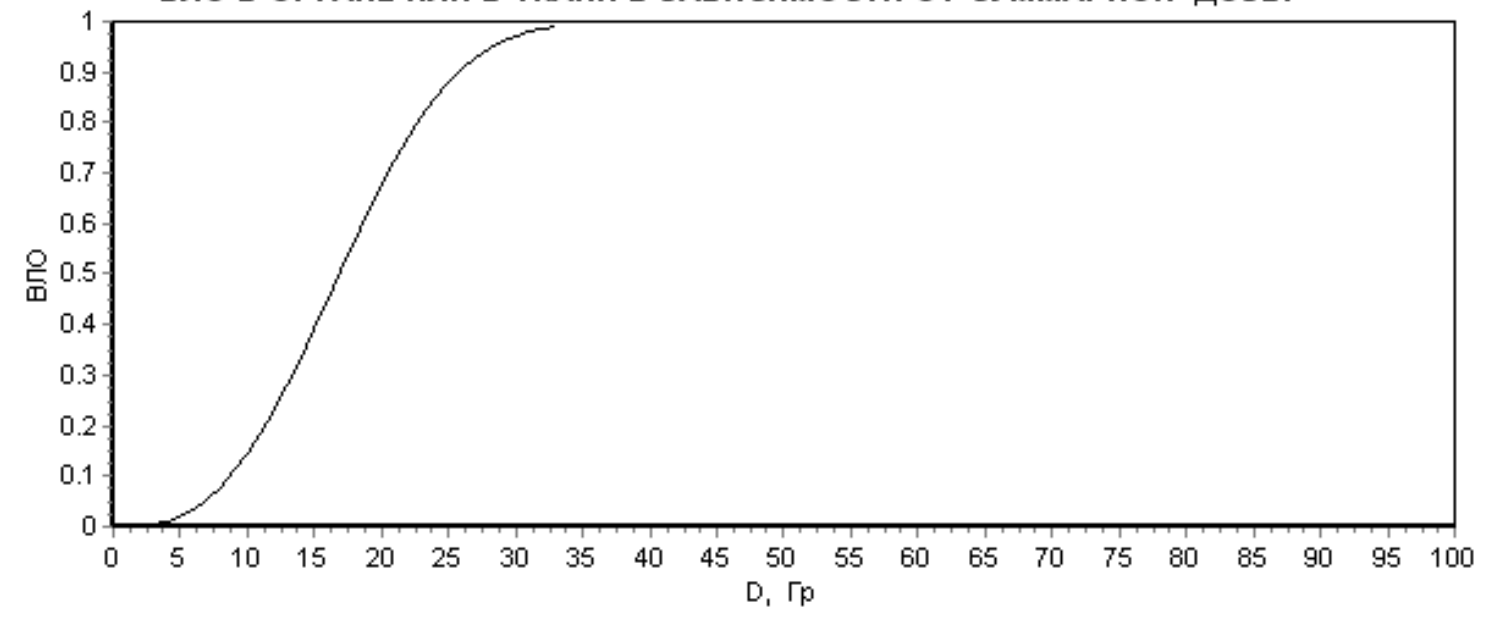

Рис. 5.23. Хрусталие глаза. Зависимость ВЛО от дозы 


\subsection{4. Зрительный нерв}

Таблица 5.24

Зрительный нерв

\begin{tabular}{c|c|c|c|c|c}
\hline$P_{\text {КЛ }}$ & $D_{\text {КЛ }}$ & $P_{\text {КЛ }}$ & $D_{\text {КЛ }}$ & $A_{1}$ & $A_{2}$ \\
\hline 0,05 & 49,34 & 0,50 & 67,02 & 69,972 & 8,502 \\
\hline
\end{tabular}

ВЛО В ОРГАНЕ ИЛИ В ТКАНИ В ЗАВИСИМОСТИ ОТ СУММАРНОЙ ДОЗЫ

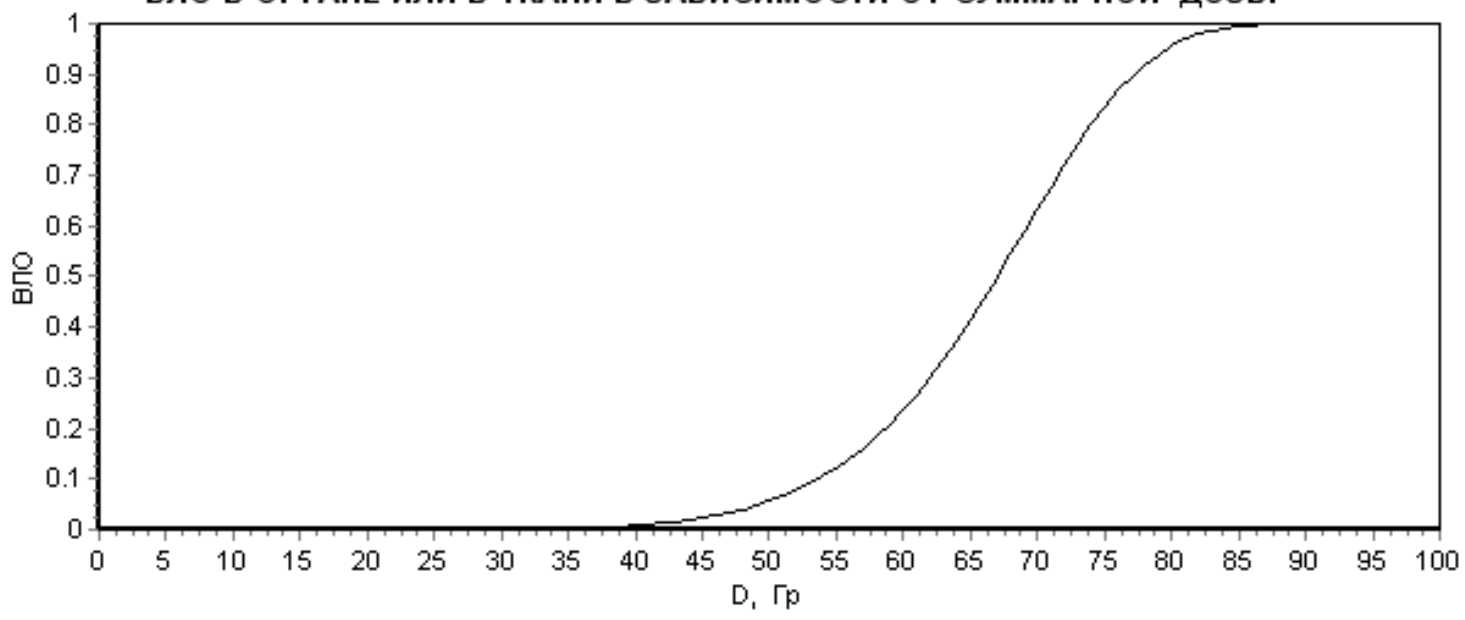

Рис. 5.24. Зрительный нерв. Зависимость ВЛО от дозы

\subsection{5. Хиазма}

Таблица 5.25

Хиазма

\begin{tabular}{c|c|c|c|c|c}
\hline$P_{\text {КЛ }}$ & $D_{\text {КЛ }}$ & $P_{\text {КЛ }}$ & $D_{\text {КЛ }}$ & $A_{1}$ & $A_{2}$ \\
\hline 0,05 & 49,54 & 0,50 & 84,57 & 91,182 & 4,869 \\
\hline
\end{tabular}

ВЛО В ОРГАНЕ ИЛИ В ТКАНИ В ЗАВИСИМОСТИ ОТ СУММАРНОЙ ДОЗЫ

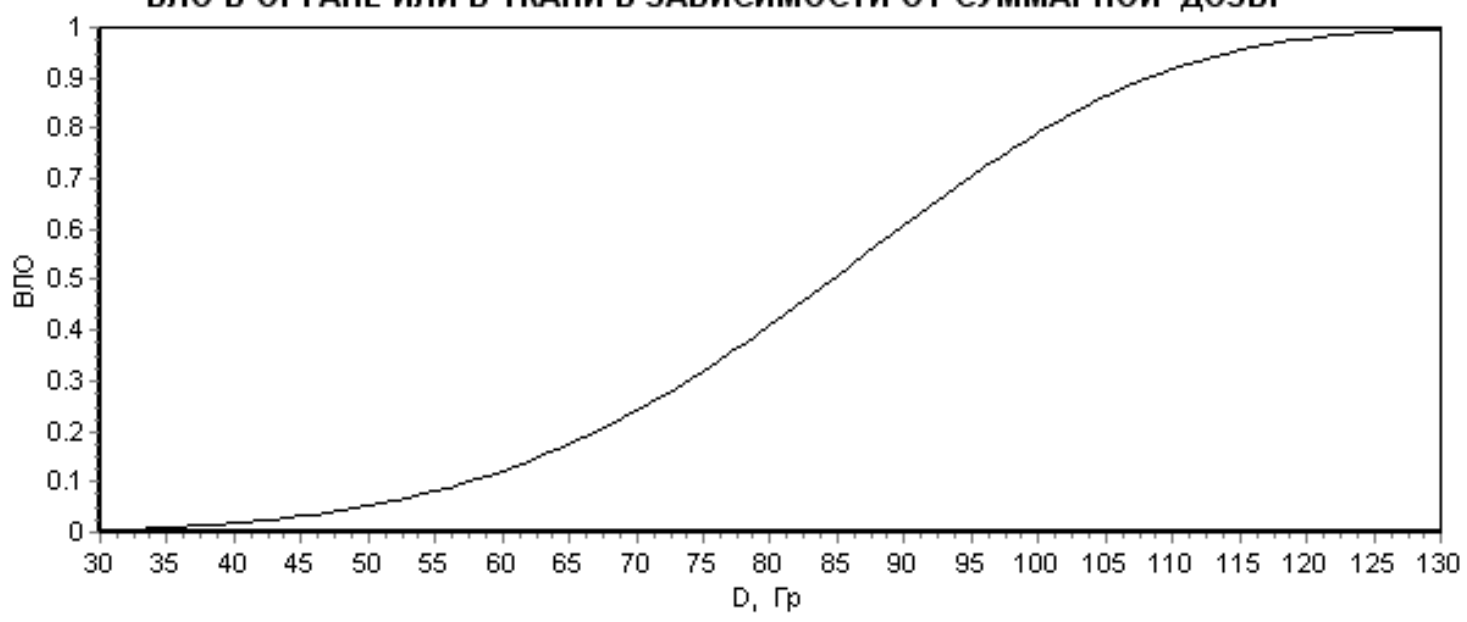

Рис. 5.25. Хиазма. Зависимость ВЛО от дозы 


\subsection{6. Сетчатка}

Таблица 5.26

\section{Сетчатка}

\begin{tabular}{c|c|c|c|c|c}
\hline$P_{\text {КЛ }}$ & $D_{\text {КЛ }}$ & $P_{\text {КЛ }}$ & $D_{\text {КЛ }}$ & $A_{1}$ & $A_{2}$ \\
\hline 0,05 & 44,67 & 0,50 & 61,58 & 64,427 & 8,110 \\
\hline
\end{tabular}

\section{ВЛО В ОРГАНЕ ИЛИ В ТКАНИ В ЗАВИСИМОСТИ ОТ СУММАРНОЙ ДОЗЫ}

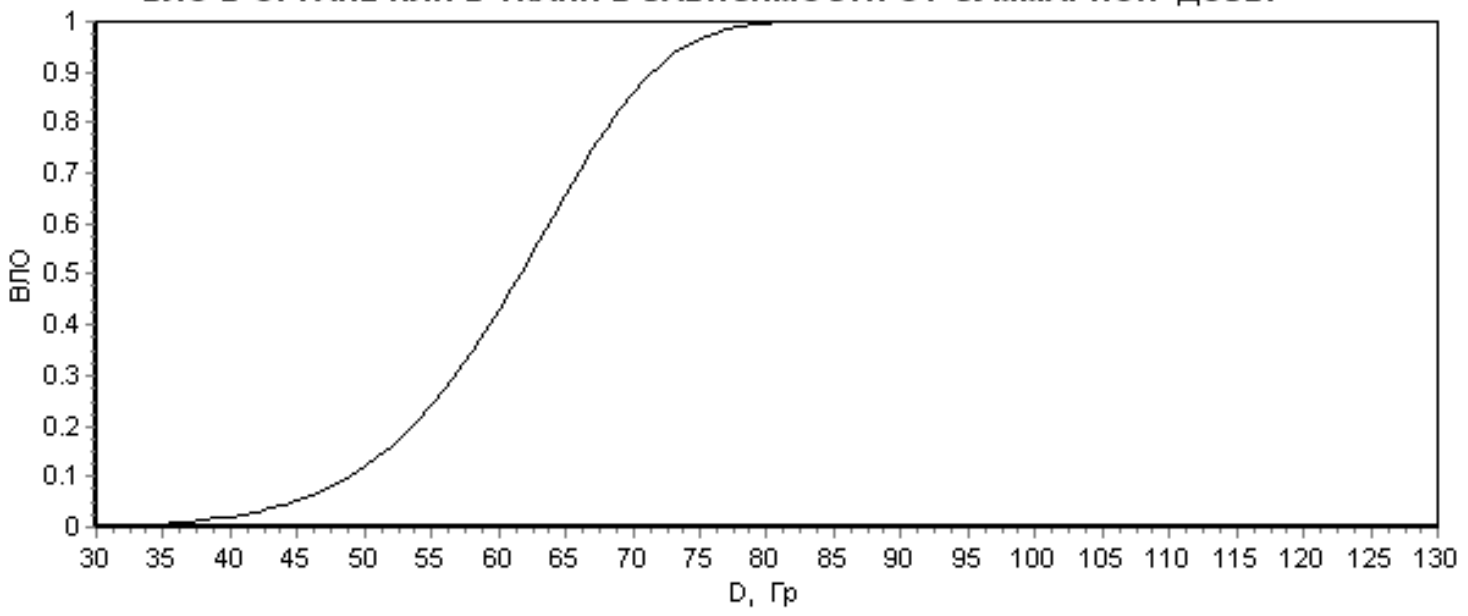

Рис. 26. Сетчатка. Зависимость ВЛО от дозы

\subsection{7. Прямая кишка}

Таблица 5.27.1

Прямая кишка. Оптимизация по ВЛО

\begin{tabular}{|c|c|c|c|c|c|c|c|c|c|}
\hline$P_{\text {Кл }}$ & $V$ & $D_{\text {Кл }}$ & $D_{\mathrm{TEOP}}$ & $\varepsilon, \%$ & $P_{\mathrm{TEOP}}$ & $\varepsilon, \%$ & $A_{1}$ & $A_{2}$ & $b$ \\
\hline 0,05 & $1 / 3$ & 58,56 & 58,29 & $-0,48$ & 0,051 & 2,90 & \multirow{6}{*}{88,401} & \multirow{6}{*}{6,099} & \multirow{6}{*}{0,064} \\
\hline 0,05 & $2 / 3$ & 55,73 & 55,75 & 0,04 & 0,050 & $-0,21$ & & & \\
\hline 0,05 & 1 & 54,08 & 54,72 & 0,45 & 0,049 & $-2,62$ & & & \\
\hline 0,50 & $1 / 3$ & 88,42 & 89,31 & 1,01 & 0,479 & $-4,19$ & & & \\
\hline 0,50 & $2 / 3$ & 85,59 & 85,43 & $-0,18$ & 0,504 & 0,77 & & & \\
\hline 0,50 & 1 & 83,93 & 83,25 & $-0,82$ & 0,517 & 3,49 & & & \\
\hline
\end{tabular}




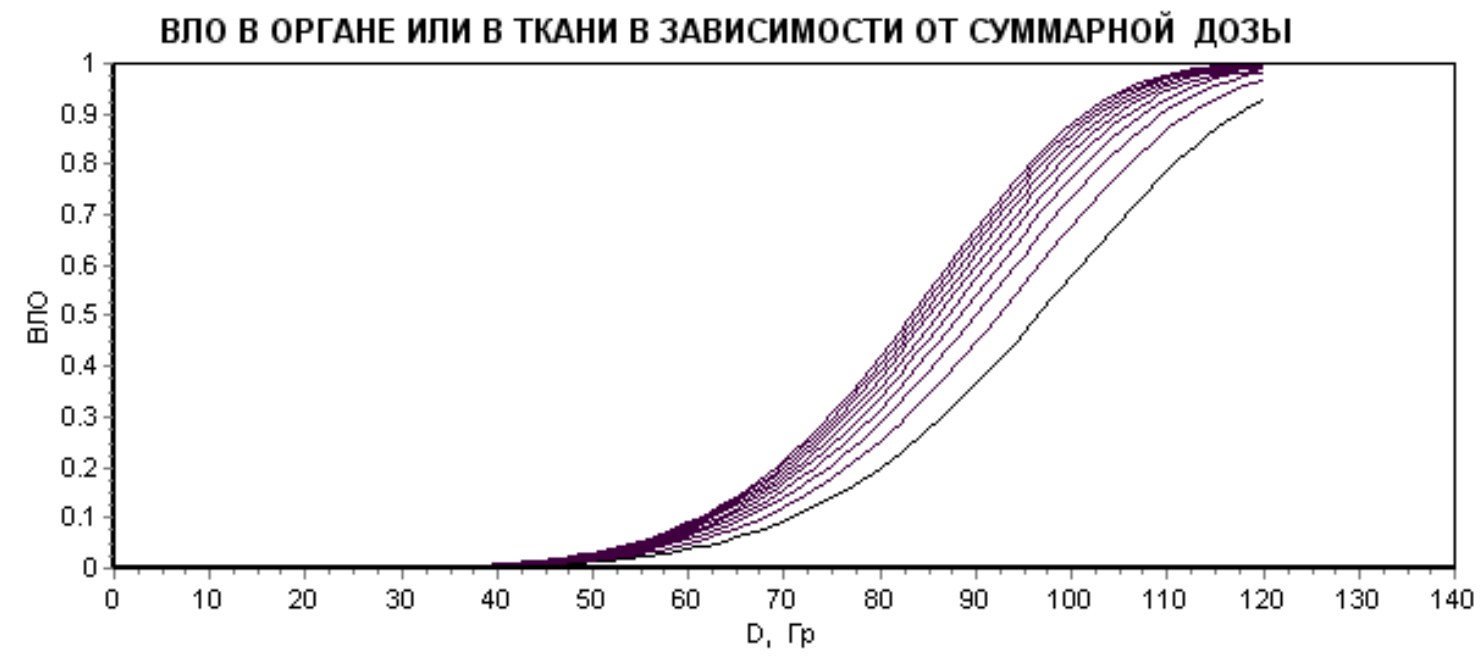

Рис. 5.27.1. Прямая кишка. Оптимизация по ВЛО

Таблица 5.27.2

\section{Прямая кишка. Оптимизация по ТД}

\begin{tabular}{|c|c|c|c|c|c|c|c|c|c|}
\hline$P_{\text {Кл }}$ & $V$ & $D_{\text {Кл }}$ & $D_{\text {TEOP }}$ & $\varepsilon, \%$ & $P_{\mathrm{TEOP}}$ & $\varepsilon, \%$ & $A_{1}$ & $A_{2}$ & $b$ \\
\hline 0,05 & $1 / 3$ & 58,56 & \begin{tabular}{|c|}
58,14 \\
\end{tabular} & $-0,72$ & 0,052 & 4,37 & \multirow{6}{*}{88,579} & \multirow{6}{*}{6,099} & \multirow{6}{*}{0,060} \\
\hline 0,05 & $2 / 3$ & 55,73 & 55,77 & 0,07 & 0,050 & $-0,44$ & & & \\
\hline 0,05 & 1 & 54,08 & 54,43 & 0,65 & 0,048 & $-3,77$ & & & \\
\hline 0,50 & $1 / 3$ & 88,42 & 89,10 & 0,76 & 0,484 & $-3,20$ & & & \\
\hline 0,50 & $2 / 3$ & 85,59 & 85,47 & $-0,14$ & 0,503 & 0,61 & & & \\
\hline 0,50 & 1 & 83,93 & 83,41 & $-0,62$ & 0,513 & 2,65 & & & \\
\hline
\end{tabular}

ВЛО В ОРГАНЕ ИЛИ В ТКАНИ В ЗАВИСИМОСТИ ОТ СУММАРНОЙ ДОЗЫ

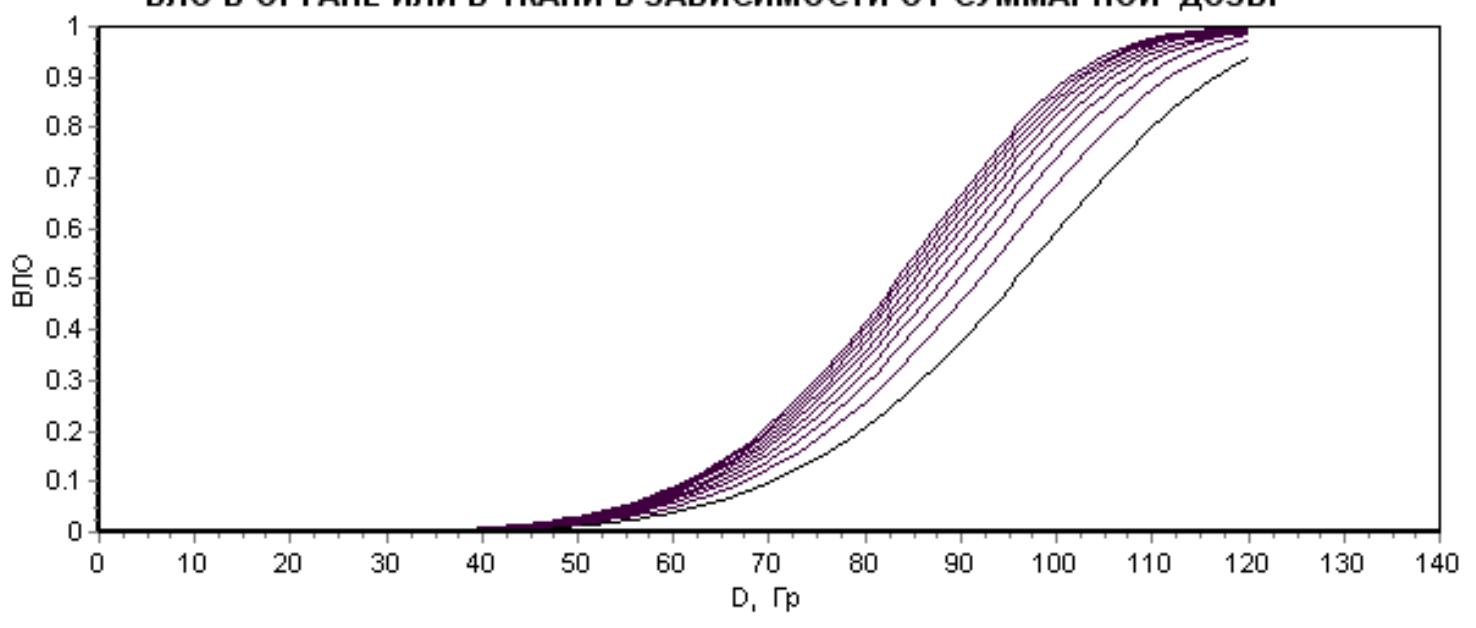

Рис. 5.27.2. Прямая кишка. Оптимизация по ТД 
Таблица 5.27.3

Прямая кишка. Оптимизация по ВДО + ТД

\begin{tabular}{|c|c|c|c|c|c|c|c|c|c|}
\hline$P_{\text {Кл }}$ & $V$ & $D_{\text {Кл }}$ & $D_{\text {TEOP }}$ & $\varepsilon, \%$ & $P_{\mathrm{TEOP}}$ & $\varepsilon, \%$ & $A_{1}$ & $A_{2}$ & $b$ \\
\hline 0,05 & $1 / 3$ & 58,56 & 58,14 & $-0,72$ & 0,052 & 4,37 & \multirow{6}{*}{88,401} & \multirow{6}{*}{6,099} & \multirow{6}{*}{0,064} \\
\hline 0,05 & $2 / 3$ & 55,73 & 55,77 & 0,07 & 0,050 & $-0,44$ & & & \\
\hline 0,05 & 1 & 54,08 & 54,43 & 0,65 & 0,048 & $-3,77$ & & & \\
\hline 0,50 & $1 / 3$ & 88,42 & 89,10 & 0,76 & 0,484 & $-3,20$ & & & \\
\hline 0,50 & $2 / 3$ & 85,59 & 85,47 & $-0,14$ & 0,503 & 0,61 & & & \\
\hline 0,50 & 1 & 83,93 & 83,41 & $-0,62$ & 0,513 & 2,65 & & & \\
\hline
\end{tabular}

ВЛО В ОРГАНЕ ИЛИ В ТКАНИ В ЗАВИСИМОСТИ ОТ СУММАРНОЙ ДОЗЫ

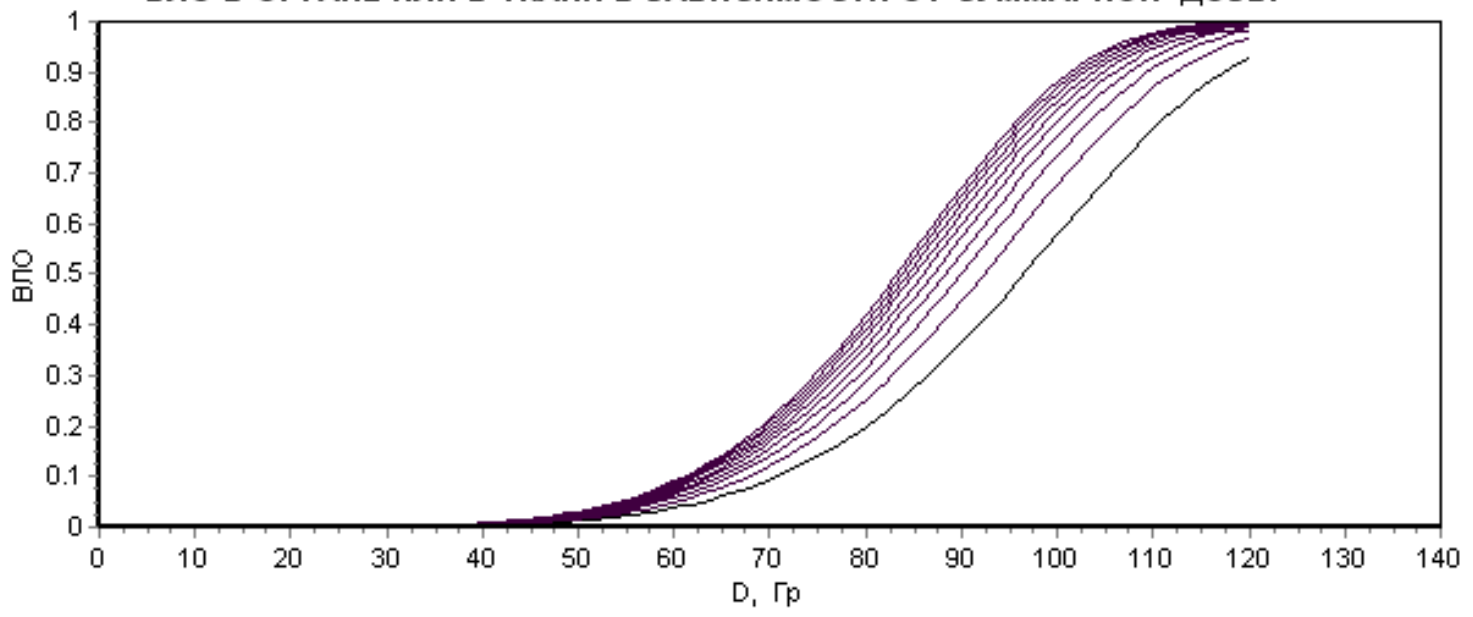

Рис. 5.27.3. Прямая кишка. Оптимизация по ВЛО + ТД

Оптимальные значения параметров МРВ модели для критерия ВЛО и ВЛО + ТД совпадают

\subsection{8. Грудная клетка}

Таблица 5.28

Грудная клетка

\begin{tabular}{c|c|c|c|c|c}
\hline$P_{\text {КЛ }}$ & $D_{\text {КЛ }}$ & $P_{\text {КЛ }}$ & $D_{\text {КЛ }}$ & $A_{1}$ & $A_{2}$ \\
\hline 0,05 & 52,23 & 0,50 & 67,74 & 70,265 & 10,013 \\
\hline
\end{tabular}




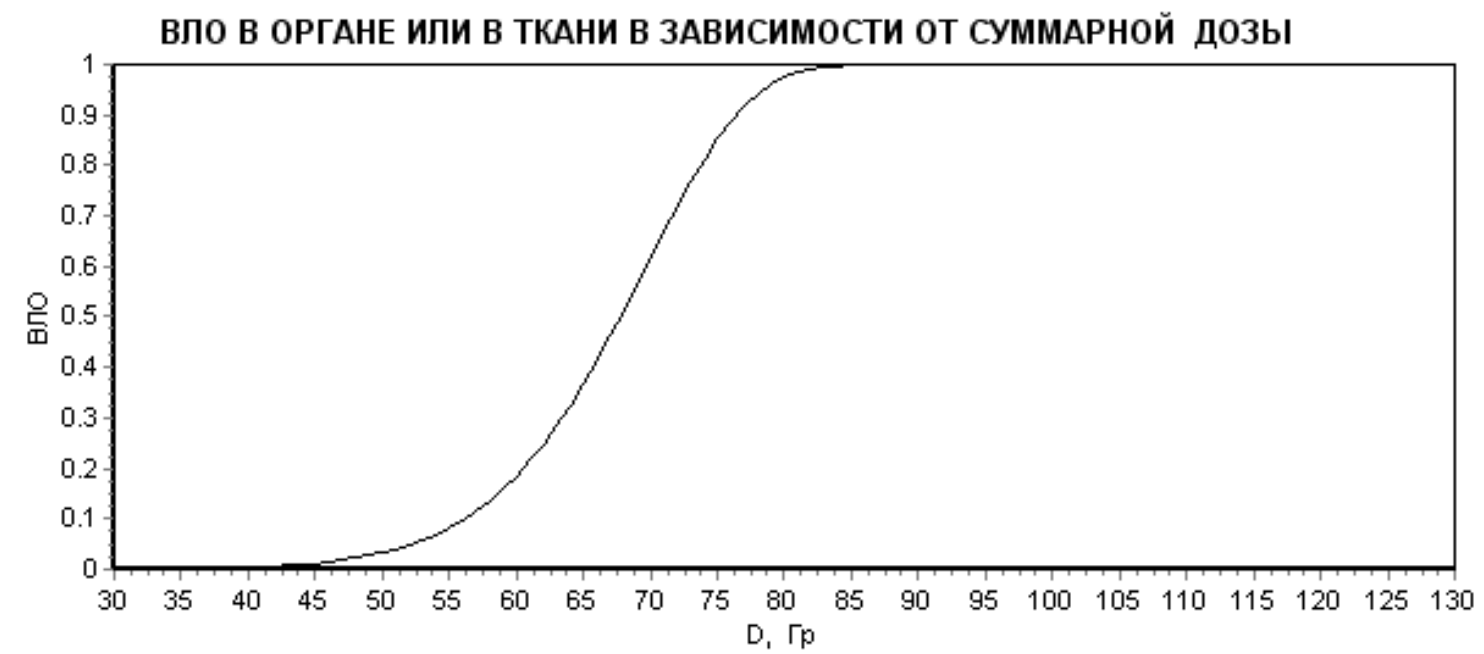

Рис. 5.28. Грудная клетка. Зависимость ВЛО от дозы

\subsection{9. Слюнные жселезы}

Таблица 5.29.1

Слюнные жселезы. Оптимизация по ВЛО

\begin{tabular}{|c|c|c|c|c|c|c|c|c|c|}
\hline$P_{\text {Кл }}$ & $V$ & $D_{\text {Кл }}$ & $D_{\mathrm{TEOP}}$ & $\varepsilon, \%$ & $P_{\mathrm{TEOP}}$ & $\varepsilon, \%$ & $A_{1}$ & $A_{2}$ & $b$ \\
\hline 0,05 & $1 / 3$ & 26,38 & 26,35 & $-0,13$ & 0,050 & 0,49 & \multirow{6}{*}{56,823} & \multirow{6}{*}{3,804} & \multirow{6}{*}{0,011} \\
\hline 0,05 & $2 / 3$ & 26,14 & 26,15 & 0,02 & 0,050 & $-0,07$ & & & \\
\hline 0,05 & 1 & 26,00 & 26,03 & 0,11 & 0,050 & $-0,41$ & & & \\
\hline 0,50 & $1 / 3$ & 52,09 & 52,23 & 0,27 & 0,496 & $-0,72$ & & & \\
\hline 0,50 & $2 / 3$ & 51,86 & 51,84 & $-0,05$ & 0,501 & 0,13 & & & \\
\hline 0,50 & 1 & 51,72 & 51,60 & $-0,022$ & 0,503 & 0,59 & & & \\
\hline
\end{tabular}

ВЛО В ОРГАНЕ ИЛИ В ТКАНИ В ЗАВИСИМОСТИ ОТ ОБЪЕМА ОБЛУЧЕНИЯ И СУММАРНОЙ ДОЗЫ

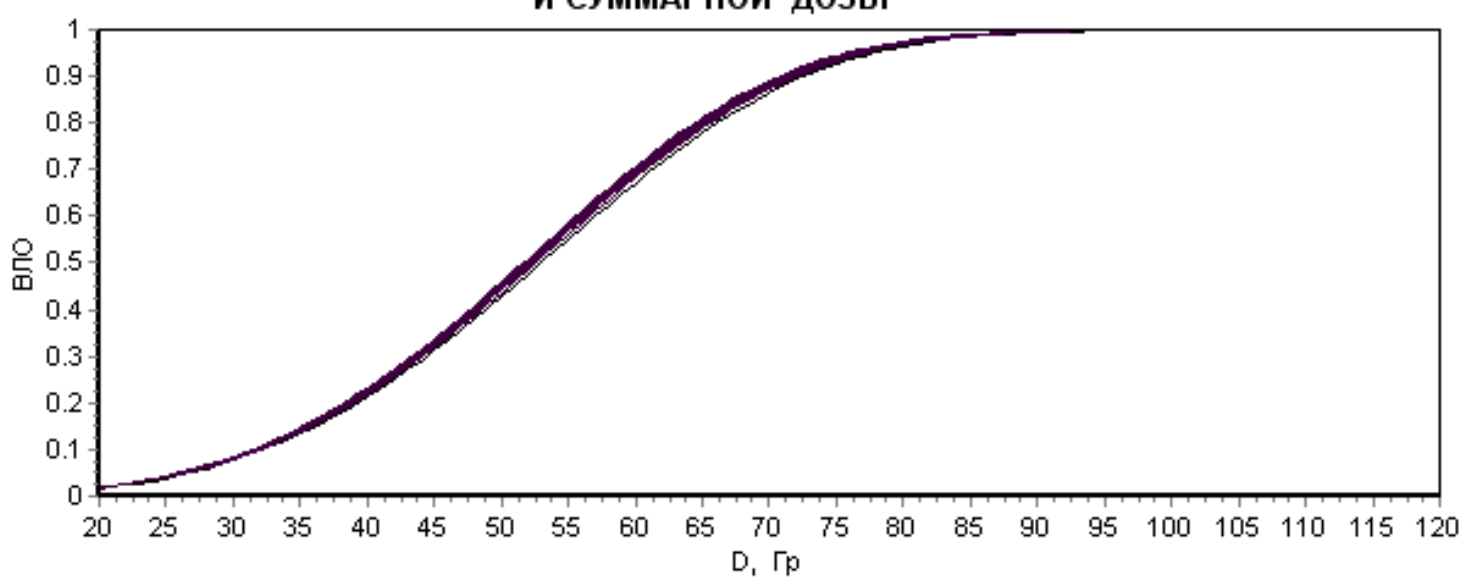

Рис. 5.29.1. Слюнные жселезы. Оптимизация по ВЛО 
Таблица 5.29.2

Слюнные жеелезы. Оптимизация по ТД

\begin{tabular}{|c|c|c|c|c|c|c|c|c|c|}
\hline$P_{\text {КЛ }}$ & $V$ & $D_{\text {Кл }}$ & $D_{\mathrm{TEOP}}$ & $\varepsilon, \%$ & $P_{\mathrm{TEOP}}$ & $\varepsilon, \%$ & $A_{1}$ & $A_{2}$ & $b$ \\
\hline 0,05 & $1 / 3$ & 26,38 & 26,33 & $-0,19$ & 0,050 & 0,71 & \multirow{6}{*}{56,852} & \multirow{6}{*}{3,804} & \multirow{6}{*}{0,010} \\
\hline 0,05 & $2 / 3$ & 26,14 & 26,15 & 0,03 & 0,050 & $-0,11$ & & & \\
\hline 0,05 & 1 & 26,00 & 26,04 & 0,16 & 0,050 & $-0,60$ & & & \\
\hline 0,50 & $1 / 3$ & $\begin{array}{l}52,09 \\
\end{array}$ & 52,20 & 0,21 & 0,497 & $-0,56$ & & & \\
\hline 0,50 & $2 / 3$ & 51,86 & 51,84 & $-0,04$ & 0,501 & 0,10 & & & \\
\hline 0,50 & 1 & 51,72 & 51,63 & $-0,17$ & 0,502 & 0,46 & & & \\
\hline
\end{tabular}

ВЛО В ОРГАНЕ ИЛИ В ТКАНИ В ЗАВИСИМОСТИ ОТ ОБЪЕМА ОБЛУЧЕНИЯ

И СУММАРНОЙ ДОЗЫ

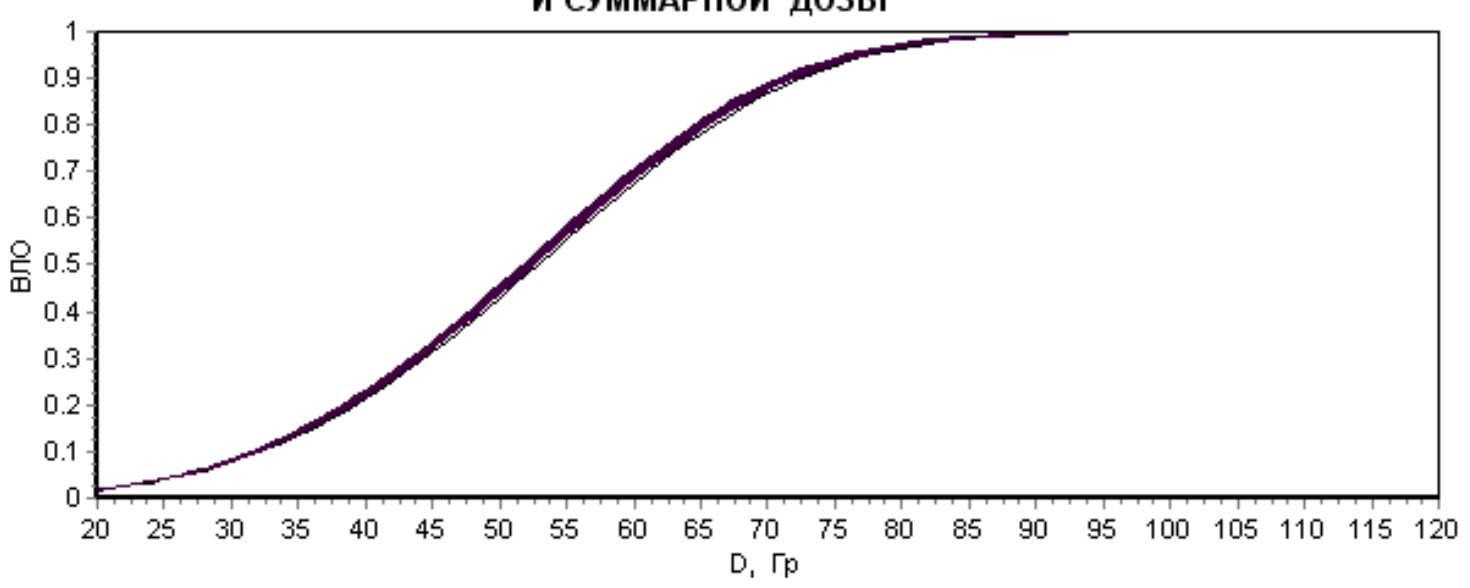

Рис. 5.29.2. Слюнные железы. Оптимизация по ТД

Таблица 5.29.3

Слюнные железы. Оптимизация по ВЛО + ТД

\begin{tabular}{|c|c|c|c|c|c|c|c|c|c|}
\hline$P_{\text {Кл }}$ & $V$ & $D_{\text {Кл }}$ & $D_{\text {TEOP }}$ & $\varepsilon, \%$ & $P_{\mathrm{TEOP}}$ & $\varepsilon, \%$ & $A_{1}$ & $A_{2}$ & $b$ \\
\hline 0,05 & $1 / 3$ & 26,38 & 26,35 & $-0,13$ & 0,050 & 0,49 & \multirow{6}{*}{56,823} & \multirow{6}{*}{3,804} & \multirow{6}{*}{0,011} \\
\hline 0,05 & $2 / 3$ & 26,14 & 26,15 & 0,02 & 0,050 & $-0,07$ & & & \\
\hline 0,05 & 1 & 26,00 & 26,03 & 0,11 & 0,050 & $-0,41$ & & & \\
\hline 0,50 & $1 / 3$ & 52,09 & 52,23 & 0,27 & 0,496 & $-0,72$ & & & \\
\hline 0,50 & $2 / 3$ & 51,86 & 51,84 & $-0,05$ & 0,501 & 0,13 & & & \\
\hline 0,50 & 1 & 51,72 & 51,60 & $-0,022$ & 0,503 & 0,59 & & & \\
\hline
\end{tabular}

Оптимальные значения параметров для критериев ВЛО и ВЛО + ТД совпадают. 
Щитовидная железа

\begin{tabular}{c|c|c|c|c|c}
\hline$P_{\text {КЛ }}$ & $D_{\text {КЛ }}$ & $P_{\text {КЛ }}$ & $D_{\text {КЛ }}$ & $A_{1}$ & $A_{2}$ \\
\hline 0,05 & 27,50 & 0,50 & 132,5 & 165,303 & 1,656 \\
\hline
\end{tabular}

ВЛО В ОРГАНЕ ИЛИ В ТКАНИ В ЗАВИСИМОСТИ ОТ СУММАРНОЙ ДОЗЫ

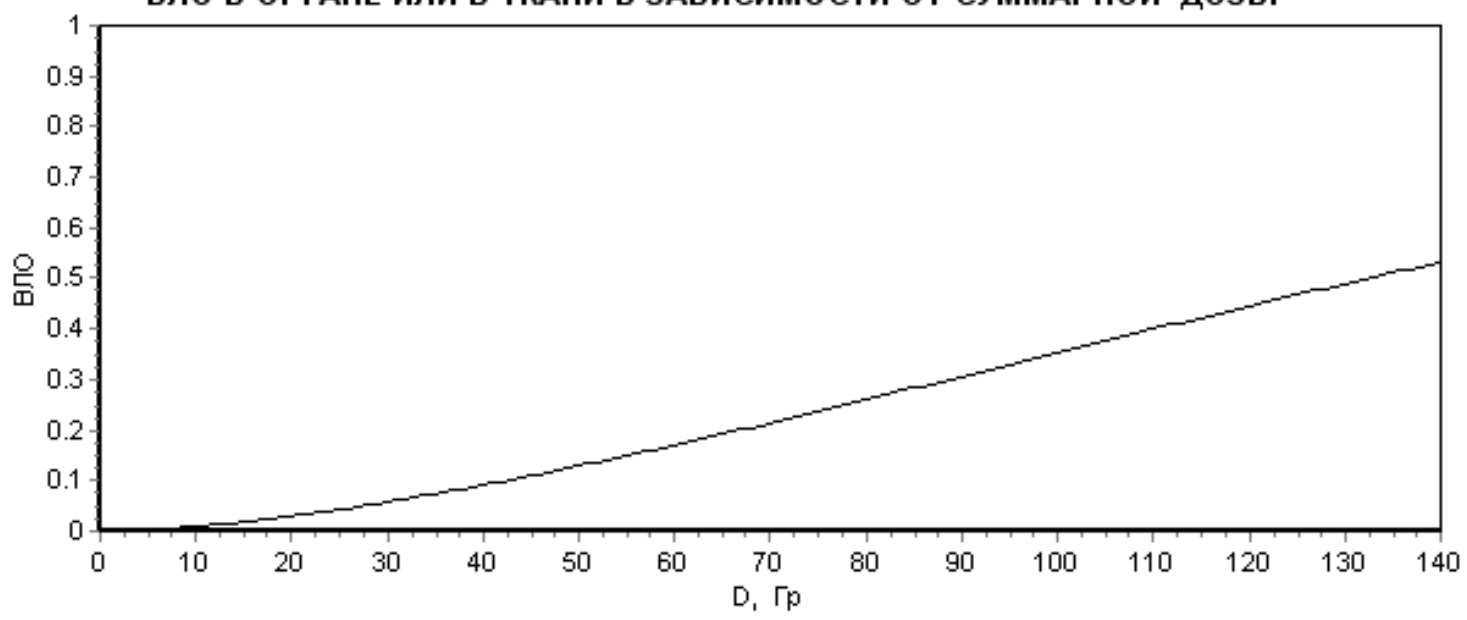

Рис. 5.30. Щитовидная железа. Зависимость ВЛО от дозы

Хочется выразить надежду, что модель МРВ и рассчитанные оптимальные значения параметров модели, окажутся полезными лучевым терапевтом для эффективного планирования ЛТ злокачественных опухолей. Анализ результатов, полученных в главах 4 и 5 свидетельствует о том, что модель МРВ удовлетворительно описывает клинические значения ТД.

Исходная систематизированная клиническая информация, которая используется для настройки модели МРВ, состоит из значений доз однородного облучения органа или ткани (значения ТД), относительного значения объема облучения и значений ВЛО, которые возникают при их однородном облучении. В этом легко убедиться, рассмотрев табл. 1.1 или 1.2. Но, в действительности, распределение дозы в органе или в ткани может быть неоднородным. Тогда не адекватными могут оказаться как оптимальные значения параметров модели МРВ, так и рассчитанные с ними значения ТД и ВЛО. Выйти из этого положения можно по-разному. Можно поступить так, как это сделали Kehwar et al., чьи систематизированные клинические данные о значениях ТД однородного облучения органов и тканей приводятся с доверительными интервалами. Приводится наиболее вероятная средняя доза однородного облучения органа или ткани и значения доз, которые образуют 95\% доверительный интервал, в котором может заключаться средняя доза однородного 
облучения ткани. Эта информация чрезвычайно важна и, как показали расчеты, точность настройки модели МРВ и точность воспроизведения ею клинических данных ТД гораздо выше, чем клинических данных Emami et al. Правда, при этом остается в стороне вопрос о повышении точности расчета значений ВЛО в здоровых органах и тканях, и расчета ВЛИ в опухолевой ткани.

Мы уже отмечали, что значения ВЛО в органах и тканях и значение ВЛИ в опухолевой ткани являются очень важными прогностическими величинами, которые характеризуют эффективность плана ЛТ. При помощи ВЛИ и ВЛО лучевой терапевт может для каждого альтернативного плана ЛТ соразмерить тяжесть онкозаболевания с риском вызвать тяжелые необратимые лучевые повреждения в здоровых органах и тканях, а также в ложе опухолевого заболевания. Можно ли улучшить ситуацию с расчетом значений ВЛИ и ВЛО? Если основной причиной плохой согласованности теоретических и клинических значений ВЛО является неоднородность дозовых распределений в органах и тканях, тогда в этом можно убедиться, если при систематизации (упорядоченности) клинической информации воспользоваться методами редукции неоднородных дозовых распределений, которые приводят к расчету эквивалентных по значению ВЛО редуцированных однородных дозовых распределений. 


\section{Глава 6. \\ ОПТИМАЛЬНЫЕ ЗНАЧЕНИЯ ПАРАМЕТРОВ МОДЕЛИ МРВ \\ ДЛЯ НЕКОТОРЫХ ЗЛОКАЧЕСТВЕННЫХ \\ НОВООБРАЗОВАНИЙ}

Ниже приводятся оптимальные значения параметров модели МРВ для расчета ВЛИ для некоторых опухолевых образований.

\section{1. ВЛИ эпидермоидного рака кожи (ЭРК)}

В работе [2] было показано, что, основываясь на клинических данных по излечению эпидермоидного рака кожи (ЭРК), приведенных в [34], можно успешно настроить и использовать модель МРВ для эффективного практического планирования ЛТ ЭРК. Для описания ВЛИ ЭРК в зависимости от условий его облучения мы получили следующее выражение:

$$
P(D, S)=1-\exp \left[-\frac{D S^{-0,076}}{9,63}\right],
$$

где $D$ - доза в Гр; $S$ - площадь облученной мишени. Исходные клинические наблюдения соответствовали ВЛИ эпидермоидного рака в интервале ВЛИ $\in(0,85 ; 0,95)$. Модель (6.1) нуждается в проверке. Необходимо установить, будет ли модель хорошо описывать ВЛИ эпидермоидного рака для всего диапазона изменения $D$. Из (6.1) получаем зависимость ТД уровня ВОЛО $=Q$ для относительной площади ЭРК, равной $S$

$$
D(Q, S)=9,3|\ln (Q)| S^{0,076} \text {. }
$$

С помощью формул (6.1) и (6.2) можно получить различные полезные соотношения.

1. Для $P_{1}=P, D_{1} \neq D, S_{1} \neq S$, получаем

$$
\frac{D}{D_{1}}=\left(\frac{S}{S_{1}}\right)^{0,076} \text {. }
$$

2. Для $S_{1}=S, D_{1} \neq D, Q_{1} \neq Q$, получаем

$$
\frac{D}{D_{1}}=\frac{\ln (Q)}{\ln \left(Q_{1}\right)} \text {. }
$$

3. Для $D_{1}=D, S_{1} \neq S, Q_{1} \neq Q$, получаем

$$
\frac{\ln (Q)}{\ln \left(Q_{1}\right)}=\left(\frac{S}{S_{1}}\right)^{0,076} \text {. }
$$


Эти формулы могут быть полезны при определении адекватности структуры модели клиническим наблюдениям.

\section{2. ВЛИ плоскоклеточного рака глотки (ПРГ)}

Для описания ВЛИ плоскоклеточного рака глотки (ПРГ) мы воспользовались данными, которые приводятся в работе [35] для стадий заболевания $T_{2}$ и $T_{3}$ и при стандартной схеме ФД (пять облучений в неделю с разовой дозой 2 Гр/сеанс). Модель МРВ для описания ВЛИ ПРГ имеет следующий вид:

$$
P(D)=1-\exp \left[-\left(\frac{D}{67,0}\right)^{6,83}\right] \text {. }
$$

\section{3. Лимфогранулематоз (болезнь Ходжкина)}

Клинические данные, соответствующие ВЛИ болезни Ходжкина в зависимости от дозы, были взяты из работы [36]. Параметры модели МРВ были рассчитаны в результате решения экстремальной задачи. Модель МРВ для описания вероятности излечения лимфогранулематоза $P(D)$ в зависимости от дозы облучения имеет следующий вид:

$$
P(D)=1-\exp \left[-\left(\frac{D}{18,85}\right)^{1,059}\right] .
$$

\section{4. Рак гортани}

Клинические данные для построения модели МРВ были взяты из работы [37]. Модель МРВ имеет следующий вид:

$$
P(D)=1-\exp \left[-\left(\frac{D}{56,70}\right)^{13,377}\right] \text {. }
$$

В описанных математических моделях, предназначенных для расчета ВЛИ опухолевого заболевания, при наличии соответствующей клинической информации, можно ввести в рассмотрение объемы (или площади) облученных опухолей, как это было сделано нами для ЭРК (модель 6.1). 
Глава 7.

\section{РЕДУКЦИЯ НЕОДНОРОДНЫХ ДОЗОВЫХ РАСПРЕДЕЛЕНИЙ В ОРГАНАХ И ТКАНЯХ В ОДНОРОДНЫЕ РАСПРЕДЕЛЕНИЯ ДОЗЫ (В АД). РАСЧЕТ СООТВЕТСТВУЮЩИХ РЕДУЦИРОВАННЫХ ЗНАЧЕНИЙ ВОЛОР (ВЛОР)}

\section{1. Проблема оценки неоднородного распределения дозы и оценка соответствующего значения ВЛО в органе или ткани при планировании ЛТ злокачественных опухолей}

Актуальной проблемой современной ЛТ злокачественных опухолей, является проблема перехода от неоднородного распределения дозы в органе или ткани к редуцированному однородному распределению дозы, и проблема расчета соответствующего однородному распределению дозы редуцированного значения ВОЛОр (ВЛОр).

Дело заключается в том, что в настоящее время все разработанные математические модели для расчета ВЛО в органах и тканях, а также для расчета вероятности локального излечения (ВЛИ) опухолевого заболевания, в основном созданы для однородных дозовых распределений в органах и тканях, для которых дозовое поле описывается одним числом, значением дозы. Для того, чтобы корректно настраивать и использовать эти модели при планирования ЛТ, необходимо разработать методы перехода от неоднородных распределений дозы в органах и тканях, с которыми лучевой терапевт реально имеет дело при планировании ЛТ, к редуцированным однородным распределениям дозы и к соответствующим им редуцированным значениям ВЛОр (или ВОЛОр). Естественно, что если клиническая информация настроена корректно, тогда редуцированное значение ВЛОр должно быть близким к клиническому значению ВЛО.

\section{2. Математические модели для расчета редуцированного} значения дозы однородного облучения органа или ткани, и для расчета соответствующего ей редуцированного значения ВОЛОр (ВЛОр)

Будем считать, что в нашем распоряжении имеется корректно настроенная математическая модель МРВ 


$$
\begin{aligned}
& \text { ВЛО }=P(D, V)=1-\exp \left[-\left(\frac{D V^{b}}{A_{1}}\right)^{A_{2}}\right], \\
& \text { ВОЛО }=Q(D, V)=\exp \left[-\left(\frac{D V^{b}}{A_{1}}\right)^{A_{2}}\right] .
\end{aligned}
$$

Она позволяет рассчитывать значения ВЛО (ВОЛО) при однородном облучении органа или ткани объема $V$ дозой $D$. Значения $\left(b, A_{1}, A_{2}\right)$ - параметры модели MPB.

Наша задача заключается в том, чтобы разработать метод редукции неоднородного дозового распределения в эквивалентное по ВЛО однородное распределение дозы, и разработать метод расчета значения ВЛОр, соответствующего редуцированному распределению дозы. Очевидно, что это значение должно совпадать или быть близким к клиническому значению ВЛО.

Рассмотрим неоднородное распределение дозы в ткани с относительным объемом $V$. Разделим объем $V$ ткани на $m$ одинаковых относительных элементарных объема $g=V / m$. Пусть распределение доз в элементарных объемах описывается вектором $D=\left(D_{1}, \ldots, D_{m}\right)$. Здесь и далее будем считать, что $V=1$ соответствует тотальному облучению выделенного органа или ткани (или выделенной части органа или ткани). Необходимо разработать математическую модель для редукции (свертки) неоднородного распределения дозы в ткани, рассчитать адекватную дозу (АД), соответствующую однородному облучению ткани, а также рассчитать редуцированное значение ВЛОр, (или ВОЛОр), соответствующее полученному однородному распределению дозы.

Для решения поставленной задачи наше внимание привлекла хорошо известная в настоящее время математическая модель, которая предлагается для редукции неоднородных дозовых распределений в ткани в адекватную дозу (АД) ее однородного облучения [9]. Она известна давно. В дальнейшем мы будем называть ее «традиционной моделью». К сожалению, мы не смогли выяснить кем, когда и как была получена эта модель (см., например, описание этой модели в лучшем, по словам рекламы, зарубежном учебнике Джойнера и Ван дер Когель «Основы клинической радиобиологии», 2013 г. [9]). В ней приводится описание традиционной модели, но не описывается метод построения этой модели. Традиционная модель имеет следующий вид:

$$
\begin{aligned}
& D_{\text {АД }}(D, V)=\left[\frac{g}{V} \sum_{i=1}^{m} D_{i}^{A}\right]^{1 / A}, V=m g, \\
& D=\left(D_{1}, \ldots, D_{m}\right),
\end{aligned}
$$

где (7.3) - заданное неоднородное распределение дозы; $g / V$ - элементарный относительный объем облученной ткани. Объем $V=1$ соответсвует тотальному относи- 
тельному объему облучаемой ткани; $A$ - параметр модели. Далее мы покажем, что этот параметр совпадает с одним из параметров модели МРВ (точнее, с параметром $A_{2}$ модели (7.1)). Неоднородное распределение доз (7.3) может быть описано в виде дифференциальной гистограммы доза - объем, ДГДО [26-30]:

$$
G^{*}(D, g)=\left\{\left(D_{1}, g\right), \ldots,\left(D_{m}, g\right)\right\} \text { или } G(D, V)=\left\{\left(D_{1}, V_{1}\right), \ldots,\left(D_{K}, V_{K}\right)\right\},
$$

где $V_{i}=n_{i} g, n_{i}$ - число элементарных объемов $g$ ткани с одинаковыми значениями дозы облучения $D_{i}$. В этом случае, как это нетрудно видеть, редуцированная доза однородного облучения ткани будет описываться следующей моделью:

$$
D_{\text {Ад }}(D, V)=\left[\sum_{i=1}^{K}\left(\frac{V_{i}}{V}\right) D_{i}^{A}\right]^{1 / A}, V=\sum_{i=1}^{K} V_{i}, D=\left(D_{1}, \ldots, D_{K}\right) .
$$

В 1986 г., в работе [6] мы показали, каким образом можно получить модель (7.2) при помощи созданной нами модифицированной модели МРВ (7.1). Процедура ее получения была основана на следующих предположениях:

Предположение 7.1. ВОЛО в элементарном объеме $g$ ткани зависит от его объема, суммарной дозы $D$ его однородного облучения, относительного объема всей облученной ткани $V$, частью которого он является, и не зависит от распределения доз в других элементарных объемах.

Предположение 7.2. ВОЛО в $i$-м элементарном объеме $g$ при его однородном облучении суммарной дозой $D_{i}$, с учетом относительного объема всей облученной ткани $V>g$, можно приближенно рассчитать при помощи модифицированной модели MPB:

$$
Q\left(D_{i}, V, g\right)=\exp \left[-\frac{g}{V}\left(\frac{D_{i} V^{b}}{A_{1}}\right)^{A_{2}}\right]=\left\{\exp \left[-\left(\frac{D_{i} V^{b}}{A_{1}}\right)^{A_{2}}\right]\right\}^{g / V},
$$

где $g / V$ - относительное значение элементарного объема ткани. ВОЛО в элементарном объеме $g / V$ основано на вычислении ВОЛО при тотальном объеме облучения ткани $V$ заданной дозой $D_{i}$, и в ее коррекции с учетом рассматриваемого элементарного объема ткани $g / V$.

На рис. 7.1 приводятся графики зависимости ВЛО от дозы при различных условиях облучения кожи:

1) зависимость ВЛО от дозы при тотальном облучении кожи $S=1$, рассчитанная при помощи модели МРВ (левый график);

2) зависимость ВЛО от дозы при облучении элементарной площади кожи $g / S=0,1$, описанная модифицированной моделью МРВ (7.6) с учетом всей площади облученной кожи $S=1$ (средний график); 
3) зависимость ВЛО от дозы при облучении изолированной элементарной относительной площади кожи $g / S=0,1$ (правый график).

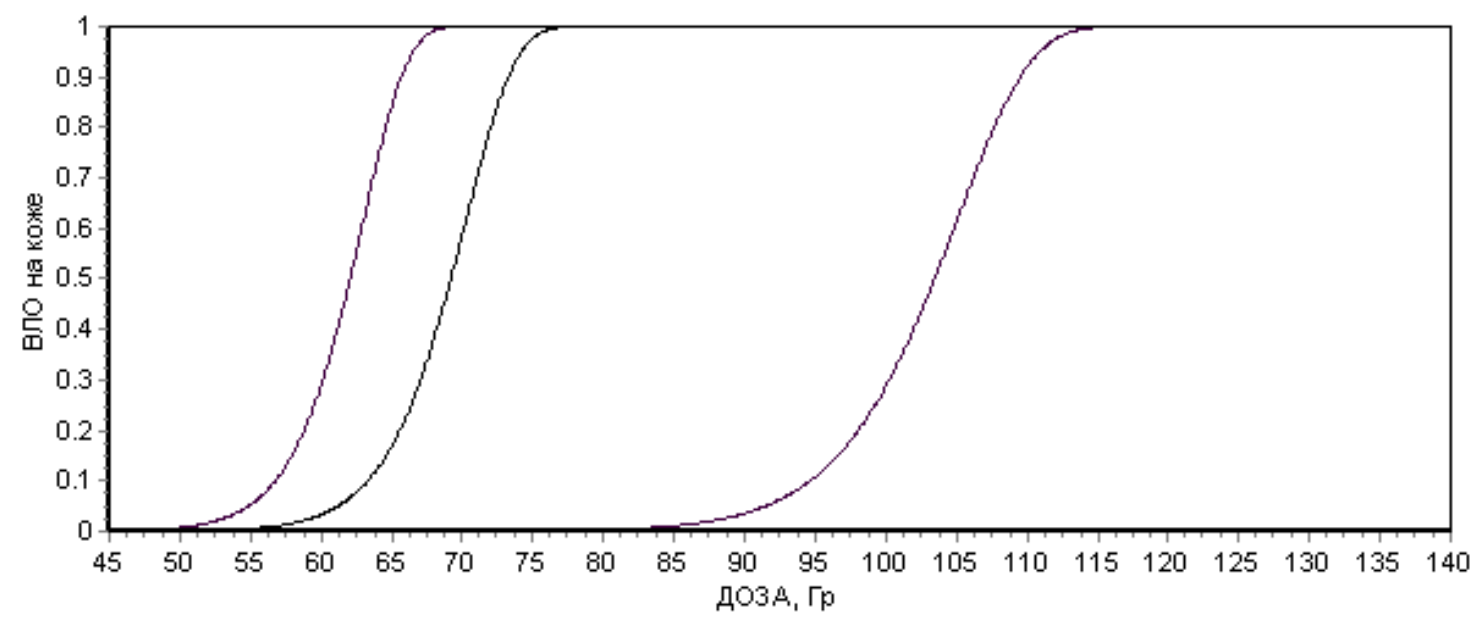

Рис. 7.1. Зависимости ВЛО на коже от дозы при различных условиях ее облучения

Следствие 7.1. Если сделанные предположения справедливы, то редуцированное значение ВОЛОр $=Q(D, V)$ можно получить следующим образом:

$$
Q(D, V)=\prod_{T=1}^{m} Q\left(D_{T}, V, g\right)=\prod_{T=1}^{K} Q\left(D_{T}, V, V_{T}\right)=\prod_{T=1}^{K} Q\left(D_{T}, V\right)^{V_{T} / V},
$$

где $V_{T}=\sum_{j=1}^{R_{T}} \frac{g}{V}$ - суммарный относительный объем ткани, соответствующий $R_{T}$ элементарным относительным объемам $g$, которые облучаются одинаковыми дозами $D_{T} ; V=1$ - относительный тотальный объем облученной ткани.

Утверждение 7.1. Если предположения 7.1 и 7.2 справедливы и неоднородное распределение дозы в элементарных объемах ткани описываются в виде множества ДГДО $=\left\{\left(D_{1}, g\right), \ldots,\left(D_{m}, g\right)\right\}$, ВОЛО в элементарных объемах ткани описывается в виде $Q\left(D_{i}, V, g\right), i=1, \ldots, m$, (модель 7.6), тогда значение ВОЛОр в ткани объема $V$ есть среднее геометрическое от ВОЛО в элементарных объемах $g$, которые описываются моделью (7.7).

Доказательство. Доказательство тривиально! ВОЛО $=Q(D, V)$ в ткани объема $V$ при неоднородном облучении $m$ составляющих ее одинаковых элементарных объемов $g$ дозами $D_{i}, i=1, \ldots, m$, можно описать следующим образом:

$$
Q(D, V, g)=\prod_{i=1}^{m} Q\left(D_{i}, V, g\right)=\prod_{i=1}^{m} Q\left(D_{i}, V\right)^{g / V}=\left[\prod_{i=1}^{m} Q\left(D_{i}, V\right)\right]^{g / V} .
$$

\section{Утверждение доказано.}


Утверждение 6.2. Если предположения 7.1 и 7.2 верны, то при помощи модели (7.8) можно получить математическую модель (7.2) или (7.5).

Доказательство. Пусть неоднородное дозовое распределение представлено в виде множества ДГДО $=\left\{\left(D_{1}, g\right), \ldots,\left(D_{m}, g\right)\right\}$, где $m$ - число рассматриваемых элементарных объемов ткани, $V=m g=1-$ относительный тотальный объем всей облученной ткани. Модель (7.2) для неоднородного распределения дозы, которая представлена в форме ДГДО, может быть вычленена из модели (7.8) следующим образом:

$$
\begin{aligned}
& Q(D, V)=\prod_{i=1}^{m} Q\left(D_{i}, V, g\right)=\prod_{i=1}^{m} \exp \left[-\frac{g}{V}\left(\frac{D_{i} V^{b}}{A_{1}}\right)^{A_{2}}\right]= \\
& =\exp \left\{-\left(\frac{V^{b}}{A_{1}}\right)^{A_{2}}\left[\left(\frac{g}{V} \sum_{i=1}^{m} D_{i}^{A_{2}}\right)^{1 / A_{2}}\right]^{A_{2}}\right\}=\exp \left\{-\left(\frac{V^{b}}{A_{1}}\right)^{A_{2}}\left[D_{\text {АД }}(D, V)\right]^{A_{2}}\right\}= \\
& =\exp \left[-\left(\frac{D_{\mathrm{AД}}(D, V) \cdot V^{b}}{A_{1}}\right)^{A_{2}}\right],
\end{aligned}
$$

где $A_{2}$ - параметр модели МРВ. В модели (7.9) выделена редуцированная адекватная доза, $D_{\mathrm{Aд}}(D, V)$, однородного облучения ткани, которая сохраняет структуру модели МРВ и эквивалентна по ВОЛОр (или по ВЛОр) ее неоднородному облучению в форме ДГДО. Формула, которая описывает адекватную дозу, $D_{\text {Ад}}(D, V)$, имеет следующий вид:

$$
D_{\text {АД }}(D, V)=\left(\frac{g}{V} \sum_{i=1}^{m} D_{i}^{A_{2}}\right)^{1 / A_{2}} .
$$

\section{Утверждение доказано.}

Нетрудно видеть, что в том случае, когда неоднородное дозовое поле описывается в виде ДГДО (не с элементарными объемами $g / V$ ), получаем:

$$
D_{\text {АД }}(D, V)=\left[\sum_{i=1}^{K}\left(\frac{V_{i}}{V}\right) D_{i}^{A_{2}}\right]^{1 / A_{2}}, V=\left(V_{1}, \ldots, V_{K}\right) .
$$

Таким образом, мы получили две математические модели (7.7) и (7.10) (а также (7.11)), предназначенные для свертки (редукции) ВОЛО (модель (7.7)), и редукции неоднородных дозовых распределений в форме ДГДО (формулы (7.10) и (7.11)).

К сожалению, реально оценить точность редукции неоднородных распределений дозы при помощи модели (7.7) и (7.10), (или (7.11), можно при наличии соответствующим образом подготовленной специальной клинической информа- 
ции, которой мы в настоящее время не располагаем. Но, кое-что все же удалось выяснить.

\section{3. Свойства математических моделей (7.7) и (7.11)}

Модели (7.7) и (7.11) являются приближенными! Рассмотрим свойства модели (7.7), которая может быть использована для расчета значения ВОЛО в элементарном объеме с учетом всего облученного объема ткани. Эти свойства могут быть полезными при анализе редуцированных значений ВОЛОр или редуцированных значений доз, АД.

1. $Q(D, V, g)$ является убывающей функцией от значения объема $g$.

2. Если в (7.7) облучаемый объем ткани $V$ уменьшить до размеров элементарного объема, т.е. $V=g$, то из (7.7) получаем значение ВОЛО в элементарном объеме $g$

$$
Q(D, g, g)=\exp \left[-\frac{g}{g}\left(\frac{D g^{b}}{A_{1}}\right)^{A_{2}}\right]=\exp \left[-\left(\frac{D g^{b}}{A_{1}}\right)^{A_{2}}\right]=Q(D, g) .
$$

3. Если элементарный объем облученной ткани $g$ увеличить до объема всей облученной ткани $V$, то получаем ВОЛО в облучаемом объеме $V$, т.е.

$$
Q(D, V, V)=\exp \left[-\frac{V}{V}\left(\frac{D V^{b}}{A_{1}}\right)^{A_{2}}\right]=\exp \left[-\left(\frac{D V^{b}}{A_{1}}\right)^{A_{2}}\right]=Q(D, V) .
$$

4. Пусть рассматривается два объема $V_{1} \neq V_{2}$, которые облучаются одной и той же дозой $D$, и соответствующие значения ВОЛО, с учетом всего облучаемого объема $V$, могут быть рассчитаны при помощи модели (7.7)

$$
Q\left(D, V, V_{1}\right)=\exp \left[-\frac{V_{1}}{V}\left(\frac{D V^{b}}{A_{1}}\right)^{A_{2}}\right], Q\left(D, V, V_{2}\right)=\exp \left[-\frac{V_{2}}{V}\left(\frac{D V^{b}}{A_{1}}\right)^{A_{2}}\right] .
$$

Отношение логарифмов (7.14) будет равно

$$
\frac{\ln \left[Q\left(D, V, V_{1}\right)\right]}{\ln \left[Q\left(D, V, V_{2}\right)\right]}=\frac{V_{1}}{V_{2}} \text {. }
$$

5. При тех же условиях, при равных значениях элементарных объемов $V_{1}=V_{2}$, но не равных значениях доз $D_{1} \neq D_{2}$, получим

$$
\frac{\ln \left[Q\left(D_{1}, V, V_{1}\right)\right]}{\ln \left[Q\left(D_{2}, V, V_{1}\right)\right]}=\left(\frac{D_{1}}{D_{2}}\right)^{A_{2}} \text {. }
$$

6. Пусть предположения 7.1 и 7.2 верны. Чем тогда отличаются друг от друга результаты расчета ВЛО в элементарном объеме $g$ при помощи модели (7.6), в которой учитывается влияние тотального объема облученной ткани на дозу в 
элементарном объеме $g$, и математическая модель МРВ, при помощи которой рассчитывается ВЛО в элементарном объеме $g$, без учета тотального объема облучения ткани. Для этого достаточно рассмотреть, при каких условиях значения ВОЛО в элементарном объеме $g$, расчитанное при помощи модели МРВ и модели (7.6), будут приводить к одинаковым значениям ВОЛО, т.е. к равенству

$$
\exp \left[-\left(\frac{D_{i} g^{b}}{A_{1}}\right)^{A_{2}}\right]=\left\{\exp \left[-\left(\frac{D_{i} V^{b}}{A_{1}}\right)^{A_{2}}\right]\right\}^{g / V} .
$$

После необходимых преобразований, получаем:

$$
\Psi=b A_{2}=1 .
$$

Таким образом, только при условии (7.18) использование модели (7.6) будет приводить к точным редуцированным значениям ВОЛОр, и к редуцированным значениям АД.

В работе [7] мы показали, что при условии (7.18) лучевые повреждения элементарных объемов ткани не зависят от распределения дозы в элементарных объемах ткани и объема всей облученной ткани.

7. Если ВОЛО в элементарном объеме зависит от объема всей облученной ткани, ей должно соответствовать неравенство

$$
\text { ВЛО }(D, g)<\text { ВЛО }(D, V, g),
$$

так как чем больше объем облученной ткани, тем больше может быть значение ВЛО (но, не меньше, эмпирический факт), тем меньше должно быть значение ВОЛО $=1-$ ВЛО, и

$$
\text { ВОЛО }(D, g)>\text { ВОЛО }(D, V, g) \text {, }
$$

Отсюда следует, что

$$
\begin{aligned}
& \exp \left[-\left(\frac{D g^{b}}{A_{1}}\right)^{A_{2}}\right]>\exp \left[-\frac{g}{V}\left(\frac{D V^{b}}{A_{1}}\right)^{A_{2}}\right], \\
& \left(\frac{D g^{b}}{A_{1}}\right)^{A_{2}}>\frac{g}{V}\left(\frac{D V^{b}}{A_{1}}\right)^{A_{2}},
\end{aligned}
$$

и

$$
\Psi=b A_{2}>1 \text {. }
$$

Таким образом, при $\Psi=b A_{2}=1$, ВЛО (ВОЛО) в элементарных объемах не зависят от объема всей облученной ткани, а при $\Psi=b A_{2}>1$ - зависят. Отсюда следует, что для здоровых органов и тканей организма величина $\Psi>1$, а для опухолевых тканей может быть близка к 1 . Это в том случае, если клетки опухолевой ткани не образуют системных, связанных агломераций из опухолевых клеток (см. работу [27], главу 7), и их можно рассматривать как независимые клоногенные клетки. 
8. Из (7.19) следует, что при одной и той же дозе облучения элементарных объемов в ткани, (однородное облучение), редуцированное значение ВОЛОр будет точно равно значению ВОЛО в ткани. Чем меньше будет неоднородность в распределении дозы, тем ближе будут редуцированные значения параметров облучения ткани к их истинным значениям, ВОЛОр и АД.

Но, как мы уже отмечали, реальную точность расчетов можно будет оценить на основе обработки систематизированных клинических данных по неоднородным облучениям органов и тканей и соответствующих им значений ВЛО (ВОЛО).

\section{4. Проверка согласованности систематизированной}

клинической информации для некоторых органов и тканей, основанная на расчете редуцированных значений ВОЛОр и АД при помощи моделей (7.7) и (7.11)

Для приближенной оценки согласованности систематизированных клинических данных, которые используются для настройки модели МРВ, мы использовали метод редукции неоднородных дозовых распределений, которые приводятся в табл. 1.1 и 1.2. Для расчета редуцированных значений ВОЛО и редуцированных значений АД мы воспользуемся моделями (7.7) и (7.11). Будем рассматривать клинические данные, которые содержат максимальную клиническую информацию, т.е. такие, для которых значениям ВЛО $=0,05$ и ВЛО $=0,50$, соответствуют три относительных объема (площади) облучения, равные $1 / 3,2 / 3$ и 1.0, и соответствующие значения ТД (см. главы 4 и 5).

Рассмотрим в качестве примера кожу (данные Kehwar et al., табл. 1.2), головной мозг (данные Emami et al., (табл. 1.1), и данные Kehwar et al. (табл. 1.2). Они выделены нами и приводятся в табл. 7.1-7.3.

Максимальная разница между клиническими и теоретическими значениями суммарных ТД для кожи не превышает 0,89\%. Для ВЛО - не превышает 7,91\%!

Для головного мозга (данные Emami et al., табл. 7.2) максимальная разница в описании клинических и теоретических значений суммарных ТД не превышает $1,74 \%$. Для ВЛО она составляет $18,76 \%$ !

Для головного мозга (данные Kehwar et al., табл. 7.3) максимальная разница в описании клинических и теоретических значений суммарных ТД не превышает $1,66 \%$. Для ВЛО - она составляет $12,25 \%$ ! 
Кожса. Параметры модели МРВ, определенные

на основе клинических данных Kehwar et al.

\begin{tabular}{|c|c|c|c|c|c|c|c|c|c|}
\hline$P_{\text {Кл }}$ & $V$ & $D_{\text {Кл }}$ & $D_{\text {TEOP }}$ & $\varepsilon, \%$ & $P_{\text {TEOP }}$ & $\varepsilon, \%$ & $b$ & $A_{1}$ & $A_{2}$ \\
\hline 0,05 & $1 / 3$ & 60,84 & 60,36 & $-0,79$ & 0,054 & 7,91 & \multirow{6}{*}{0,109} & \multirow{6}{*}{72,388} & \multirow{6}{*}{9,852} \\
\hline 0,05 & $2 / 3$ & 55,97 & 55,97 & $-0,01$ & 0,050 & 0,06 & & & \\
\hline 0,05 & 1,0 & 53,12 & 53,55 & 0,80 & 0,046 & $-7,42$ & & & \\
\hline 0,50 & $1 / 3$ & 77,92 & 78,62 & 0,89 & 0,470 & $-5,93$ & & & \\
\hline 0,50 & $2 / 3$ & 73,06 & 72,90 & $-0,22$ & 0,508 & 1,54 & & & \\
\hline 0,50 & 1,0 & 70,21 & 69,74 & $-0,66$ & 0,523 & 4,59 & & & \\
\hline
\end{tabular}

Критерий оптимальности - ТД. Лучевые осложнения на коже - некрозы и изъязвления.

Таблица 7.2

Головной мозг. Параметры модели МРВ, определенные на основе клинических данных Emami et al.

\begin{tabular}{|c|c|c|c|c|c|c|c|c|c|}
\hline$P_{\text {Кл }}$ & $V$ & $D_{\text {Кл }}$ & $D_{\text {TEOP }}$ & $\varepsilon, \%$ & $P_{\text {TEOP }}$ & $\varepsilon, \%$ & $b$ & $A_{1}$ & $A_{2}$ \\
\hline 0,05 & $1 / 3$ & 60 & 58,96 & $-1,74$ & 0,059 & 18,76 & \multirow{6}{*}{0,233} & \multirow{6}{*}{61,244} & \multirow{6}{*}{10,102} \\
\hline 0,05 & $2 / 3$ & 50 & 50,17 & 0,33 & 0,048 & $-3,21$ & & & \\
\hline 0,05 & 1 & 45 & 45,64 & 1,43 & 0,043 & $-13,07$ & & & \\
\hline 0,50 & $1 / 3$ & 75 & 76,25 & 1,72 & 0,442 & $-11,61$ & & & \\
\hline 0,50 & $2 / 3$ & 65 & 64,91 & $-0,13$ & 0,505 & 0,93 & & & \\
\hline 0,50 & 1 & 60 & $\begin{array}{l}59,06 \\
\end{array}$ & $-1,56$ & 0,556 & 11,27 & & & \\
\hline
\end{tabular}

Критерий оптимальности - ТД. Лучевые осложнения - инфаркт, некроз.

Таблица 7.3

Головной мозг. Параметры модели МРВ, определенные на основе клинических данных Kehwar et al.

\begin{tabular}{|c|c|c|c|c|c|c|c|c|c|}
\hline$P_{\text {Кл }}$ & $V$ & $D_{\text {Кл }}$ & $\overline{D_{\mathrm{TEOP}}}$ & $\varepsilon, \%$ & $P_{\mathrm{TEOP}}$ & $\varepsilon, \%$ & $b$ & $A_{1}$ & $A_{2}$ \\
\hline 0,05 & $1 / 3$ & 58,56 & 57,65 & $-1,58$ & 0,056 & 12,25 & \multirow{6}{*}{0,166} & \multirow{6}{*}{71,188} & \multirow{6}{*}{7,551} \\
\hline 0,05 & $2 / 3$ & 51,42 & 51,38 & $-0,08$ & 0,050 & 0,57 & & & \\
\hline 0,05 & 1 & 47,25 & 48,04 & 1,66 & 0,044 & $-11,45$ & & & \\
\hline 0,50 & $1 / 3$ & 80,00 & 81,38 & 1,73 & 0,456 & $-8,77$ & & & \\
\hline 0,50 & $2 / 3$ & 72,86 & 72,54 & $-0,44$ & 0,511 & 2,34 & & & \\
\hline 0,50 & 1 & 68,68 & 67,81 & $-1,26$ & 0,534 & 6,72 & & & \\
\hline
\end{tabular}

Критерий оптимальности - ТД. Лучевые осложнения - инфаркт, некроз.

Клиническую информацию, приведенную в табл. 7.1-7.3 запишем следующим образом:

$$
\begin{aligned}
& \text { Для ВЛО }=0,05:\left[\left(D_{1} ; V_{1}=1 / 3\right),\left(D_{2} ; V_{2}=2 / 3\right),\left(D_{3} ; V_{3}=1,0\right)\right], \\
& \text { Для ВЛО }=0,50:\left[\left(D_{4} ; V_{4}=1 / 3\right),\left(D_{5} ; V_{5}=2 / 3\right),\left(D_{6} ; V_{6}=1,0\right)\right] .
\end{aligned}
$$

Для ВЛО = 0,05 первые два члена в (7.24) и (7.25) можно рассматривать как неоднородное облучения ткани объема $1 / 3$ дозой $D_{1}$ и облучения ткани объема $2 / 3$ дозой $D_{2}$. Такое облучение мы можем рассматривать, как тотальное неоднородное облучение ткани. Клинический результат такого тотального облучения ткани 
нам известен, он соответствует $\left(D_{3} ; V_{3}=1,0\right)$. Аналогичное рассуждение можно провести для $\mathrm{B} Л О=0,50$. Таким образом, у нас появляется возможость оценить согласованность клинической информации для ВЛО $=0,05$ и для ВЛО $=0,50$.

В разд. 7.5 приводятся результаты редукции неоднородных распределений значений ВЛО (расчеты ВЛОр), соответствующих каждому органу или ткани, и их сравнению с заданным клиническим значением ВЛО $=0,05$ и ВЛО $=0,50$, а также результаты редукции неоднородных распределений доз для ВЛО $=0,05$ и $\mathrm{BЛО}=0,50$, и их сравнение с клиническими значениями доз тотального облучения ткани.

\section{5. Редукция неоднородного распределения ВОЛО при помощи модели (7.7)}

Для кожи и головного мозга (табл. 7.1-7.3) мы имеем информацию всего для двух значений ВЛО, равных 0,05 и 0,50 .

Кожса (Kehwar et al.). Исходя из клинических данных и используя модель (7.7), получаем следующую формулу для вычисления редуцированного значения ВОЛОр для кожи:

$$
\text { ВОЛОр }=Q(D, V)=\prod_{i=1}^{2} Q\left(D_{i}, V, V_{i}\right)=\prod_{i=1}^{2} Q\left(D_{i}, V\right)^{V_{i} / V} .
$$

Клиническую информацию, представленную в табл. 7.1 для кожи можно проверить, воспользовавшись моделью (7.26). Для ВЛО $=0,05$ (ВОЛО $=0,95)$ должно выполняться равенство (стрелкой показано ожидаемое значение ВОЛО $=Q(D, V)$ :

$$
\begin{aligned}
& Q(D, V)=\prod_{i=1}^{2} Q\left(D_{i}, V, V_{i}\right)=Q(60,84 ; 1 ; 1 / 3) \cdot Q(55,97 ; 1 ; 2 / 3)= \\
& =Q(60,84 ; 1)^{1 / 3} \cdot Q(55,97 ; 1)^{2 / 3}=0,95^{1 / 3} \cdot 0,95^{2 / 3} \rightarrow 0,95 ;
\end{aligned}
$$

для $\mathrm{BЛО}=0,50($ ВОЛО $=0,50)$ равенство:

$$
\begin{aligned}
& Q(D, V)=\prod_{i=1}^{2} Q\left(D_{i}, V, V_{i}\right)=Q(77,92 ; 1 ; 1 / 3) \cdot Q(73,06 ; 1 ; 2 / 3)= \\
& =Q(77,92 ; 1)^{1 / 3} \cdot Q(73,06 ; 1)^{2 / 3}=0,50^{1 / 3} \cdot 0,50^{2 / 3} \rightarrow 0,50 .
\end{aligned}
$$

Но, в действительности мы получаем:

$$
\begin{aligned}
& Q(D, V)=Q(60,84 ; 1)^{1 / 3} \cdot Q(55,97 ; 1)^{2 / 3}=0,893 \neq 0,95 ! \\
& Q(D, V)=Q(77,92 ; 1)^{1 / 3} \cdot Q(73,06 ; 1)^{2 / 3}=0,242 \neq 0,50 !
\end{aligned}
$$


Для ВЛО $=0,05$ отклонение расчитанного значения ВОЛОр от клинического значения составляет величину: $100 \frac{0,95-0,893}{0,95}=6 \%$. При ВЛО =0,50, отклонение составляет: $100 \frac{0,50-0,242}{0,50}=51,6 \%$.

Такая же картина наблюдается для головного мозга (данные Emami et al., табл. 7.2). Используя клинические данные для ВЛО =0,05 (ВОЛО $=0,95)$ и ВЛО $=0,50$ (ВОЛО $=0,50)$, получаем:

$$
\begin{aligned}
& Q(60 ; 1)^{1 / 3} \cdot Q(55 ; 1)^{2 / 3}=0,700 \rightarrow 0,95, \\
& Q(75 ; 1)^{1 / 3} \cdot Q(65 ; 1)^{2 / 3}=0,495 \rightarrow 0,50 .
\end{aligned}
$$

Отклонения составляют 26,32 и $1,0 \%$.

Аналогично обстоит дело и для головного мозга (данные Kehwar et al), табл. 6.3:

$$
\begin{aligned}
& Q(58,56 ; 1)^{1 / 3} \cdot Q(51,42 ; 1)^{2 / 3}=0,875 \rightarrow 0,95, \\
& Q(80 ; 1)^{1 / 3} \cdot Q(72,86 ; 1)^{2 / 3}=0,202 \rightarrow 0,50 .
\end{aligned}
$$

Отклонения составляют 7,9 и 59,6\%.

Таким образом, мы получили возможность оценить рассогласованность клинических и теоретических (модельных) значений ВОЛО. Плохая согласованность значений ВОЛО не должна удивлять нас, поскольку в настоящее время планирование ЛТ опухолевых заболеваний происходит при помощи заданных опухолевых доз и предельных значений ТД в здоровых органах и тканях.

\section{6. Редукция неоднородного распределения ТД в АД при помощи модели (7.11)}

Клиническая информация для кожи и головного мозга соответствует значениям ВЛО $=0,05$ и ВЛО = 0,50 при относительных объемах (площадях) облучения $V=1 / 3 ; 2 / 3 ; 1,0$, и заданных значениях ТД (см. табл. 7.1-7.3). Нам предстоит выяснить, насколько близко к клиническим значениям ТД находятся теоретические (модельные) значения АД, рассчитанные при помощи модели (7.11). Ниже приводятся значения ДГдО, выделенные из трех приведенных таблиц:

1) значения ДГДО, выделенные из табл. 7.1 для кожи (Kehwar et al.) имеют следующий вид:

$$
\begin{aligned}
& \text { ДГдО }=\{(60,84 ; 1 / 3),(55,97 ; 2 / 3)\} \rightarrow 0,05 \rightarrow(53,12 ; 1), \\
& \text { ДГдО }=\{(77,92 ; 1 / 3),(73,06 ; 2 / 3)\} \rightarrow 0,50 \rightarrow(70,21 ; 1) .
\end{aligned}
$$


Стрелки указывают на значения ВЛО и значения клинических доз, которым должны соответствовать редуцированные значения АД, соответствующие тотальному облучению кожи;

2) значения ДГДО, выделеные из табл. 7.2 для головного мозга, (Emami et $\mathrm{al})$, для ВЛО=0,05 и ВЛО=0,50 имеют следующий вид:

$$
\begin{aligned}
& \text { ДГДО }=\{(60 ; 1 / 3),(50 ; 2 / 3)\} \rightarrow 0,05 \rightarrow(45 ; 1), \\
& \text { ДГДО }=\{(75 ; 1 / 3),(65 ; 2 / 3)\} \rightarrow 0,50 \rightarrow(60 ; 1) ;
\end{aligned}
$$

3) ДГДО, выделенное из табл. 7.3 для головного мозга, (данные Kehwar et al.), будет:

$$
\begin{aligned}
& \text { ДГдО }=\{(58,56 ; 1 / 3),(51,42 ; 2 / 3)\} \rightarrow 0,05 \rightarrow(47,25 ; 1), \\
& \text { ДГдО }=\{(80 ; 1 / 3),(72,86 ; 2 / 3)\} \rightarrow 0,50 \rightarrow(68,68 ; 1) .
\end{aligned}
$$

Из (7.33) для кожи следует, что при ВЛО = 0,05 неоднородность дозового поля можно описать в виде дозы 60,84 Гр для относительной площади кожи $V=1 / 3$, и дозы 55,97 Гр для относительной площади облученной кожи $V=2 / 3$. Эта информациия приводится в виде ДГДО в (7.33) для ВЛО $=0,05$ и в (7.34) для ВЛО $=0,50$.

Два плана облучения, приведенные в ДГДО, эквивалентны тотальному неоднородному облучению кожи $(1 / 3+2 / 3=1)$. При тотальном облучении кожи, как это следует из табл. 7.1, доза 53,12 Гр должна приводить к ВЛО = 0,05. Редукция неоднородного распределения дозы в форме ДГДО (7.33) при помощи модели (7.11) приводит к следующему значению АД

$$
\text { АД }=\left[(1 / 3) \cdot 60,84^{9,852}+(2 / 3) \cdot 55,97^{9,852}\right]^{1 / 9,852}=58,02 \Gamma \text { р. }
$$

Но, из табл. 7.1 следует, что АД должна быть равно клиническому значению $D_{3}=55,12$ Гр. Отклонение АД от клинического значения дозы составляет $-9,22 \%$. При ВЛО = 0,50 редукция неоднородного дозового распределения, рассчитанная при помощи модели (7.11) приводит к АД, равной

$$
\text { АД }=\left[(1 / 3) \cdot 77,92^{9,852}+(2 / 3) \cdot 73,06^{9,852}\right]^{1 / 9,852}=75 \Gamma \mathrm{p} .
$$

Отклонение редуцированной дозы АД $=75$ Гр от клинической дозы 70,21 Гр составляет $-6,82 \%$.

Результаты расчетов АД для кожи и головного мозга приводятся в табл. 7.4. В ней первые четыре строки (1-4) относятся к коже (Kehwar et al.), строки (5-8) - к головному мозгу (Emami et al.), и строки (9-12) - к головному мозгу (Kehwar et al.). О результатах расчетов, которые приводятся в строках 3 и 4, 7 и 8, 11 и 12, мы поговорим в главе 8 . 
В 9 и 10 столбцах табл. 7.4 приводятся значения АД, полученные в результате обработки ДГДО, и расчеты отклонения редуцированных значений АД от заданных клинических значений тотальных доз облучения $D_{\text {тот }}$ Отклонения рассчитывалось по формуле $\varepsilon, \%=100 \frac{D_{\text {Tот }}-\text { АД }}{D_{\text {ТОт }}}$. Все отклонения отрицательные, так как рассчитанные значения АД превышают соответствующие значения $D_{\text {тот}}$.

Анализ полученных результатов по облучению кожи, приведенных в первых двух строках табл. 7.4, соответствуют традиционному методу редукции неоднородного распределения дозы и свидетельствует о том, что отклонение АД от

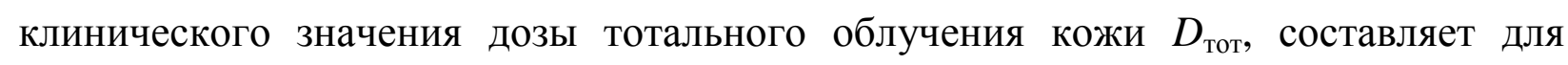
ВЛО $=0,05$ и для ВЛО $=0,50:-9,22 \%$ и $-6,82 \%$. Результаты аналогичных расчетов для головного мозга приводятся в строках 5 и 6 и в строках 9 и 10 табл. 7.4.

Результаты расчетов, которые приводятся в строках 3 и 4, 7 и 8, и 11 и 12, табл. 7.4, (они выделены жирным шрифтом), относятся к использованию разработанного нами нового метода редукции неоднородного распределения дозы, основанного на приведении неоднородного распределения дозы к тотальным объемам облучения и к последующей редукции неоднородного дозового распределения, который оказался более точным, чем традиционный метод редукции неоднородных дозовых распределений, и о котором речь пойдет в главе 8.

Таблица 7.4

Редукция неоднородных дозовых распределений при помощи модели (7.11) для кожи (Kehwar et al.), головного мозга (Emami et al.)

и головного мозга (Kehwar et al.)

\begin{tabular}{c|c|c|c|c|c|c|c|c|c}
\hline № & $P$ & $V_{1}$ & $D_{1}$ & $V_{2}$ & $D_{2}$ & $V_{3}$ & $D_{\text {ТОТ }}$ & АД & $\varepsilon, \%$ \\
\hline \multicolumn{8}{|c}{ Кожа, Kehwar et al. } \\
\hline 1 & 0,05 & $1 / 3$ & 60,84 & $2 / 3$ & 55,97 & 1,0 & 53,12 & 58,02 & $-9,22$ \\
2 & 0,50 & $1 / 3$ & 77,92 & $2 / 3$ & 73,06 & 1,0 & 70,21 & 75,00 & $-6,82$ \\
3 & 0,05 & $1 / 3$ & 60,84 & $2 / 3$ & 55,97 & 1,0 & 53,12 & 57,69 & $-\mathbf{8 , 6 0}$ \\
4 & 0,50 & $1 / 3$ & 77,92 & $2 / 3$ & 73,06 & 1,0 & 70,21 & 74,59 & $-\mathbf{6 , 2 4}$ \\
\hline \multicolumn{8}{|c}{ Головной мозг, Emami et al. } \\
\hline 5 & 0,05 & $1 / 3$ & 60 & $2 / 3$ & 50 & 1,0 & 45 & 55,31 & $-22,91$ \\
6 & 0,50 & $1 / 3$ & 75 & $2 / 3$ & 65 & 1,0 & 60 & 69,89 & $-16,48$ \\
7 & 0,05 & $1 / 3$ & 60 & $2 / 3$ & 50 & 1,0 & 45 & 49,26 & $-\mathbf{9 , 4 7}$ \\
8 & 0,50 & $1 / 3$ & 75 & $2 / 3$ & 65 & 1,0 & 60 & 62,79 & $-\mathbf{4 , 6 5}$ \\
\hline \multicolumn{8}{|c|}{ Головной мозг, Кehwar et al. } \\
\hline 9 & 0,05 & $1 / 3$ & 58,56 & $2 / 3$ & 51,42 & 1,0 & 47,25 & 54,52 & $-15,39$ \\
10 & 0,50 & $1 / 3$ & 80,0 & $2 / 3$ & 72,86 & 1,0 & 68,68 & 75,75 & $-10,29$ \\
11 & 0,05 & $1 / 3$ & 58,56 & $2 / 3$ & 51,42 & 1,0 & 47,25 & 53,10 & $-\mathbf{1 2 , 3 8}$ \\
12 & 0,50 & $1 / 3$ & 80,0 & $2 / 3$ & 72,86 & 1,0 & 68,68 & 73,90 & $-7,60$ \\
\hline
\end{tabular}

Расчеты велись для ВЛО $=P=0,05$ и ВЛО $=P=0,50$. 
Мы установили (главы 4 и 5), что модель МРВ достаточно хорошо описывает ТД и хуже описывает значения ВЛО. Поэтому, как и следовало ожидать, модель (7.11) будет точнее описывать редуцированные значения ТД, чем модель (7.7) редуцированные значения ВОЛОр.

Для кожи, при ВЛО $=0,05$ величина АД $=58,02$ Гр. и приводит к ВЛО $=0,107$ (не равна 0,05). При ВЛО $=0,50$ величина АД $=75,0$ приводит $к$ ВЛО $=0,758$ (не равна 0,50 ). 


\section{ГЛАВА 8.}

\section{ПОВЫШЕНИЕ ТОЧНОСТИ РЕДУКЦИИ НЕОДНОРОДНОГО} ДОЗОВОГО РАСПРЕДЕЛЕНИЯ В РЕЗУЛЬТАТЕ

\section{«ПРИВЕДЕНИЯ НЕОДНОРОДНОГО ДОЗОВОГО РАСПРЕДЕЛЕНИЯ К ТОТАЛЬНЫМ ОБЬЕМАМ ОБЛУЧЕНИЯ» ПРИ ПОМОЩИ МОДЕЛИ МРВ}

Рассмотрим приведенный нами в главе 7 метод расчета значения редуцированного неоднородного распределения ВОЛО, ВОЛОр, и метод расчета АД редуцированного неоднородного распределения дозы. Они позволили нам получить традиционную математическую модель (7.2) и (7.5) для расчета АД, основанную на приближенной модели (7.6) для расчета ВЛО в элементарном объеме ткани с учетом всего ее облучаемого объема. Создание двух математических моделей были основаны на следующих предположениях (приведем их):

Предположение 8.1. ВОЛО в элементарном объеме $g$ ткани зависит от его объема, суммарной дозы $D$ его однородного облучения, относительного объема всей облученной ткани $V$, частью которого он является, и не зависит от распределения доз в других элементарных объемах.

Предположение 8.2. ВОЛО в $i$-м элементарном объеме $g$ при его однородном облучении суммарной дозой $D_{i}$, с учетом относительного тотального объема всей облученной ткани $V>g$, можно представить в виде

$$
Q\left(D_{i}, V, g\right)=\exp \left[-\frac{g}{V}\left(\frac{D_{i} V^{b}}{A_{1}}\right)^{A_{2}}\right]=\left\{\exp \left[-\left(\frac{D_{i} V^{b}}{A_{1}}\right)^{A_{2}}\right]\right\}^{g / V},
$$

где $g / V$ - значение относительного элементарного объема. Мы указали на то, что модель (8.1) - это модификация модели МРB, предназначенная для приближенного расчета ВОЛО в элементарном объеме ткани с учетом ВОЛО, которое соответствует тотальному облучению ткани, $V=1$. Заметим, что в (8.1) видно, что значение ВОЛО $=Q\left(D_{i}, V, g\right)$ в элементарном объеме $g$, основано на вычислении ВОЛО при тотальном объеме облучения ткани $V$ заданной дозой $D_{i}$, и в ее коррекции с учетом рассматриваемого элементарного объема ткани $g / V$. Мы показали, что:

$1)$ если в (8.1) облучаемый объем ткани $V$ уменьшить до размеров элементарного объема, т.е. $V=g$, то из (7.7) получаем значение ВОЛО в элементарном объеме $g$, равное его значению, вычисленному при помощи модели МРВ, т.е.

$$
Q(D, g, g)=\exp \left[-\frac{g}{g}\left(\frac{D g^{b}}{A_{1}}\right)^{A_{2}}\right]=\exp \left[-\left(\frac{D g^{b}}{A_{1}}\right)^{A_{2}}\right]=Q(D, g)
$$


2) если элементарный объем облученной ткани $g$ увеличить до объема всей облученной ткани $V$, то получаем ВОЛО в облучаемом объеме $V$, вычисленный при помощи модели МРВ

$$
Q(D, V, V)=\exp \left[-\frac{V}{V}\left(\frac{D V^{b}}{A_{1}}\right)^{A_{2}}\right]=\exp \left[-\left(\frac{D V^{b}}{A_{1}}\right)^{A_{2}}\right]=Q(D, V) .
$$

Таким образом, если объем облученной ткани увеличить до тотального объема $V$, тогда (8.1) будет рассчитывать точное значение ВОЛО $=Q(D, V)$, которое совпадет с результатом ее расчета при помощи модели (8.1) и модели МРВ! Следовательно для того, чтобы рассчитать значения $Q\left(D_{i}, V, V_{i}\right)$ с большей точностью необходмо при помощи модели МРВ перейти к эквивалентному по ВЛО $_{i}=Q\left(D_{i}, V_{i}\right)$ значению ВЛО $_{i}=Q\left(D_{i}^{*}, 1\right), i=1, \ldots, K$, т.е. к таким новым значениям доз тотального облучения ткани $D_{i}^{*}$, которые оставляют неизменнвми значения ВЛО $i, i=1, \ldots, K$.

Пересчет значений доз при переходе к тотальным облучениям ткани осуществляется следующим образом. Полагаем, что редуцированное значение ВОЛОр может быть получено при помощи модели:

$$
\text { ВОЛОр }\left(D^{*}, 1\right)=\prod_{i=1}^{K} Q\left(D_{i}^{*}, 1\right),
$$

Значение $D_{i}^{*}, i=1, \ldots, K$, получим из условий,

$$
Q\left(D_{i}, V_{i}\right)=\exp \left[-\left(\frac{D_{i} V_{i}^{b}}{A_{1}}\right)^{A_{2}}\right]=\exp \left[-\left(\frac{D_{i}^{*} \cdot 1}{A_{1}}\right)^{A_{2}}\right]=Q\left(D_{i}^{*}, 1\right), i=1, \ldots, K,
$$

откуда следует, что:

$$
D_{i}^{*}=D_{i} V_{i}^{b}, i=1, \ldots, K .
$$

В известной степени (8.6) позволяет проверить и степенную зависимость ТД от объема облученной ткани. Редуцированное значение ВОЛОр, можно рассчитать при помощи модели:

$$
\text { ВОЛОр }=\prod_{i=1}^{K} Q\left(D_{i}, V_{i}\right)=\prod_{i=1}^{K} Q\left(D_{i}^{*}, 1\right) .
$$

Модель для расчета АД, при $V_{i}=1, i=1, \ldots, K$, принимает следующий вид:

$$
D_{\text {Ад }}(D, 1)=\left[\sum_{i=1}^{K}\left(D_{i}^{*}\right)^{A_{2}}\right]^{1 / A_{2}}, D=\left(D_{1}^{*}, \ldots, D_{K}^{*}\right) \text {. }
$$

Таким образом, связь между величинами ВОЛОр $D_{\text {Ад }}$ и $V=1$, может быть описана при помощи модели МРВ 
ВОЛОр $\left(D_{\text {АД }}, 1\right)=\exp \left[-\left(\frac{D_{\text {АД }} \cdot 1^{b}}{A_{1}}\right)^{A_{2}}\right]$.

Для того чтобы изменить АД $=D_{\text {Ад }} f$ раз, необходимо изменить значение дозы $D_{i}^{*}$ для каждого $i=1, \ldots, K$ в $f$ раз,

$$
f D_{\text {АД }}=f\left[\sum_{i=1}^{K}\left(D_{i}^{*}\right)^{A_{2}}\right]^{1 / A_{2}}=\left[f^{A_{2}} \sum_{i=1}^{K}\left(D_{i}^{*}\right)^{A_{2}}\right]^{1 / A_{2}}=\sum_{i=1}^{K}\left(f D_{i}^{*}\right)^{A_{2}} .
$$

Для того чтобы привести редуцированное значение дозы к некоторому объему $V_{*}$, необходимо

$$
\begin{aligned}
& D_{\mathrm{Aд}} V_{*}^{-b}=\left(V_{*}^{-b}\right) \cdot\left[\sum_{i=1}^{K}\left(D_{i}^{*}\right)^{A_{2}}\right]^{1 / A_{2}}=\left[\left(V_{*}^{-b}\right)^{A_{2}} \sum_{i=1}^{K}\left(D_{i}^{*}\right)^{A_{2}}\right]^{1 / A_{2}}= \\
& =\left[\sum_{i=1}^{K}\left(D_{i}^{*} V_{*}^{-b}\right)^{A_{2}}\right]^{1 / A_{2}} \cdot
\end{aligned}
$$

Когда надо изменить редуцированную дозу в $f$ раз и объем облученной ткани свести к $V_{*}$, необходимо воспользоваться следующей формулой

$$
\begin{aligned}
& f D_{\mathrm{AД}} V_{*}^{-b}=f\left[\sum_{i=1}^{K}\left(D_{i}^{*}\right)^{A_{2}}\right]^{1 / A_{2}} V_{*}^{-b}=\left[\left(f V_{*}^{-b}\right)^{A_{2}} \sum_{i=1}^{K}\left(D_{i}^{*}\right)^{A_{2}}\right]^{1 / A_{2}}= \\
& =\left[\sum_{i=1}^{K}\left(f D_{i}^{*} V_{*}^{-b}\right)^{A_{2}}\right]^{1 / A_{2}} .
\end{aligned}
$$

Результаты расчетов АД для кожи и головного мозга приводятся в табл. 7.4. В ней первые четыре строки относятся к коже (Kehwar et al.), строки (5-8) и (9-12) - к головному мозгу (данные Emami et al. и Kehwar et al.). Для каждого органа или ткани первые две строки из четырех относятся к расчету редуцированных доз традиционным методом, последующие две строки относятся к расчету редуцированных значений доз методом приведения неоднородных распределений дозы к тотальным объемам облучения. Они выделены жирным шифтом. В девятом и десятом столбцах табл. 8.1 приводятся значения АД, полученные в результате обработки неоднородного распределения дозы, представленного в виде ДГДО, и отклонения клинических значений $D_{\text {Тот }}$ (значения доз тотального облучения ткани для ВЛО $=0,05$ и ВЛО $=0,50)$ от рассчитанных значений АД в виде $\varepsilon, \%=100 \frac{\mathrm{AД}-D_{3}}{D_{3}}$.

Анализ полученных результатов по облучению кожи, и головного мозга, приведенные в табл. 7.4 свидетельствуют о следующем. 
1. Во всех рассмотренных случаях, АД тотального облучения органа или ткани превышает соответствующее клиническое значение тотального облучения органа или ткани $D_{\text {тот }}$.

2. Разработанный метод приведения неоднородных распределений доз к тотальным облучениям органов или тканей для последующей редукции неоднородных дозовых распределений, оказался точнее традиционного метода редукции неоднородных дозовых распределений. В этом легко убедиться, проанализировав значения отклонений $D_{\text {Тот }}$ от соответствующих значений АД, приведенных в последнем столбце табл. 7.4.

Например, для кожи традиционный метод редукции неоднородных дозовых распределений для ВЛО $=0,05$ приводит к отклонению АД от $D_{\text {Тот }}-9,22 \%$, а для ВЛО $=0,50-$ к $6,82 \%$.

Использование метода приведения неоднородных доз к тотальному облучению приводит к меньшим отклонениям: для ВЛО $=0,05-$ к 8,60\%, для ВЛО $=0,50-$ к 6,24\%. Отклонение теоретических (модельных) значений АД от клинических значений $D_{\text {Tот }}$, оказываются меньше, чем при традиционном методе редукции неоднородных дозовых распределений.

Для головного мозга и данных Emami et al., как легко в этом убедиться, значения отклонений больше, чем для кожи. Для головного мозга и клинических дан-

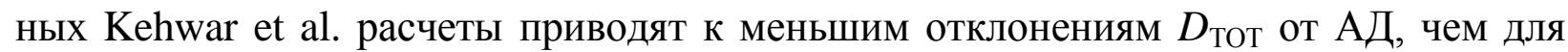
клинических данных Emami et al. Это, скорее всего, связано с тем, что Kehwar et al. более тщательно провели статистическую обработку клинической информации.

Предложенный нами новый метод редукции неоднородных дозовых распределений основан на приведении доз неоднородного распределения к тотальным объемам облученного органа или ткани. Но конечно, аналогичным образом можно построить алгоритм редукции неоднородных дозовых распределений путем приведения дозовых распределений к некоторому элементарному (малому) объему облучения. При этом точность расчетов будет мало отличаться от точности, полученной при приведении неоднородных дозовых распределений к тотальному объему облучения, но объем вычислений при этом увеличится из-за дробления объема облучения на множество элементарных объемов.

В целом мы видим, что значения АД, рассчитанные методом приведения неоднородного распределения доз к тотальному облучению для рассмотренных органов и тканей, оказываются гораздо ближе к клиническим значениям, к $D_{\text {тот }}$. Но, в то же время, следует признать, что отклонения при традиционном методе расчета АД не всегда являются катастрофическими. 
Хочется надеяться, что развитие этого научного направления позволит решить вопрос об оценке точности расчета редуцированных дозовых распределений и повышении эффективности ЛТ злокачественных опухолей неоднородными дозовыми полями. Оно позволит также решить старый вопрос о том, в каких случаях неоднородное облучение опухоли через решетчатые диафрагмы (РД) может быть эффективней, чем облучение опухоли однородными дозовыми распределениями, (открытыми полями).

В заключение этой главы считаю необходимым отметить тот вклад, который внес в проблему использования неоднородного дозового распределения для ЛТ опухолевых заболеваний Б.М. Алиев и его сотрудники [77-80]. Алиев писал: «Нам кажется, что пришло время пересмотреть существующие представления относительно характера распределения доз в месте расположения злокачественных новообразований и на этой основе разработать рациональные подходы к их лечению». Далее он отмечал, «что сформулированное положение о необходимости равномерного облучения злокачественных новообразований независимо от клинической стадии развития опухолевого процесса, без достаточного радиобиологического обоснования, укоренилось в клинической практике и до настоящего времени применяется для сравнительной оценки различных программ и методов лучевой терапии». Он отмечал, что «неравномерное облучение опухолей открывает широкие перспективы для дальнейших физико-дозиметрических, радиобиологических и клинических исследований. Применение неравномерного облучения опухолей в сочетании с другими методами позволит разработать более рациональные схемы комплексного лечения злокачественных новообразований и на этой основе существенно улучшить эффективность лечения онкологических больных».

Мы полагаем, что в настоящее время стремление к повышению эффективности ЛТ опухолевых заболеваний, делают актуальной проблему исследования и применения неоднородных дозовых распределений. В России пионерами этого нового направления в ЛТ безусловно были Б.М. Алиев и его сотрудники [77-80]. 


\section{ЗАКЛЮЧЕНИЕ}

Основные проблемы, которые были затронуты в настоящей работе и которые, на наш взгляд, требуют проведения следующих дальнейших исследований.

1. Создание математических моделей для учета влияния объема облученного органа или ткани на толерантные дозы (ТД) для произвольного уровня ВЛО.

2. Создание новых математических моделей для описания ВЛО в здоровых органах и тканях и ВЛИ опухолевых заболеваний в зависимости от планов ЛТ. Увеличение множества таких моделей расширит возможности выбора эффективных моделей для описания лучевого воздействия на различные нормальные органы и ткани организма, а также на опухолевые ткани.

3. Разработка СМ моделей для расчета ВЛО в здоровых органах и тканях и ВЛИ опухолевых заболеваний, предназначенных для планирования ЛТ с учетом суммарной и разовой дозы для произвольных объемов облученных органов или тканей.

4. Исследование проблемы облучения Системы «опухоль + ложе опухоли» и Системы «опухоль + здоровые органы и ткани организма». Разработан метод определения оптимальных условий облучения Системы, основанный на радиобиологических параметрах $\gamma$ для опухоли и ложа опухоли и суммарных и разовых дозах. Необходимо клинически обосновать, что значения параметров радиочувствительности опухоли $\gamma_{0}$ и ложа опухоли $\gamma_{L O}$ играют главную роль в выборе оптимальных планов ЛТ Систем «опухоль + ложе опухоли» и в определении метода ЛТ (метода ФД): стандартного, ГипоФ или ГиперФ.

5. Использование теории «надежности систем» для описания вероятностей возникновения лучевых осложнений в органах и тканях, (отказах систем) как функции от гибели составляющих их клеток. Мы показали, что она расширяет возможности адекватного описания вероятностей лучевых осложнений в органах и тканях путем их математического моделирования.

6. Разработан метод «Трех графиков» (ТГ), который может быть использован для расчета радиобиологических параметров опухоли на основе феноменологического описания зависимости ВЛИ от суммарной опухолевой дозы (СОД). Необходимо исследовать возможности применения метода ТГ для различных опухолевых заболеваний, оценить эффективность использования различных феноменологических моделей для описания ВЛИ от СОД и установления точности, с которой могут быть рассчитаны радиобиологические параметры для моделей опухолевых образований.

Использование метода ТГ для сканирования структуры опухолевой ткани в зависимости от дозы облучения $[27,30]$, показало, что предположение о том, что 
опухолевая ткань может состоять не из множества независимых клоногенных клеток, а из тканевых единиц, (связанных систем опухолевых клеток), не лишено оснований. Необходимо исследовать это явление и выяснить, могут ли новые знания о структуре опухолевой ткани в зависимости от дозы облучения внести положительный вклад в задачу повышения эффективности планирования ЛТ опухолевых заболеваний.

7. В настоящее время предполагается, что эффективный путь перехода от неоднородного дозового распределения к эквивалентному по ВЛО (или по ВЛИ) однородному дозовому распределению заключается в его преобразовании в гистограммы, ДГДО, с ее последующей редукцией. Необходимо доказать, теоретически и клинически, что реальные неоднородные дозовые распределения действительно эквиваленты по ВЛО, (или по ВЛИ опухолевых заболеваний), дозовым распределениям в форме гистограмм, ДГДО, с последующей их редукцией в однородные дозовые распределения.

8. Разработаны математические модели для свертки ДГДО в Адекватные Дозы однородного облучения органов и тканей. Каждая модель содержит в качестве параметра один из параметров модели МРВ $A_{2}$. Редуцированная доза, АД, однородного облучения ткани является возрастающей функцией от параметра $A_{2}$. При одном и том же неоднородном распределении дозы, как это может наблюдаться в Системе «опухоль + ложе опухоли», большая редуцированная доза будет в той ткани, которой соответствует большее значение параметра $A_{2}$.

Разработка математических моделей для редукции неоднородных дозовых распределений в органах и тканях в эквивалентные по ВЛОр (или по ВЛИр) однородные дозовые распределения, является актуальной проблемой современной ЛТ опухолевых заболеваний. Без создания таких моделей проблема широкого применения математических методов для оптимального планирования ЛТ опухолевых заболеваний становится неполной и проблематичной.

9. Анализ созданных математических моделей предназначенных для редукции ДГДО показал, что минимальное значение ВЛО в здоровой ткани (например, ложе опухоли), будет иметь место при минимальном значении интегральной поглощенной дозы и при однородном распределении средней дозы в ткани. Если опухоль рассматривать как ткань, то при фиксированной интегральной дозе максимальное значение ВЛИ опухолевого заболевания будет наблюдаться при неоднородном распределении дозы. Таким образом, если описанные модели редукции неоднородных дозовых распределений адекватны реальной действительности, то необходимо исследовать проблему выбора не однородного, а эффективного неоднородного компромиссного дозового распределения в Системе «опухоль + ложе опухоли». 
10. Из анализа созданных математических моделей для редукции неоднородных дозовых распределений следует, что величину $\Psi=A_{2} b$, где $A_{2}$ и $b$ параметры модели MPB, можно рассматривать как меру связанности клеток в ткани (в тканевую организацию клеток). Необходимо установить, в какой степени этот параметр может действительно использоваться для характеристики меры связанности клеток в ткани и действительно ли, что при $\Psi=A_{2} b \approx 1$ множество клеток ткани можно рассматривать как независимые.

11. Следует отметить, что если описанные в работе математические модели редукции ДГДО адекватны реальной действительности, то полученные в настоящей работе результаты моделирования неоднородного лучевого воздействия на опухолевые и здоровые ткани организма свидетельствуют о том, что дальнейшее совершенствование методов ЛТ злокачественных опухолей может быть расширено и связано с разумным и обоснованным использованием как однородных, так и неоднородных дозовых распределений.

Полученные результаты свидетельствуют о том, что оптимальным дозовым распределением в Системе «опухоль + ложе опухоли» может быть не только однородное дозовое поле, как это принято считать в настоящее время, а некоторое компромиссное неоднородное дозовое распределение. В работах $[27,28,30]$ мы показали, что требование однородности дозового поля в Системе «опухоль + ложе опухоли» приводит к минимальному лучевому воздействию на ложе опухоли и отнюдь не к оптимальному лучевому воздействию на опухоль.

Для того чтобы обоснованно и целенаправленно выбирать оптимальные однородные и неоднородные терапевтические дозовые распределения для лучевого лечения злокачественных опухолей необходимо всесторонне изучить проблему воздействия неоднородных дозовых распределений на различные типы опухолей и на различные здоровые органы и ткани организма.

Мы полагаем, что дальнейший прогресс ЛТ опухолевых заболеваний может быть связан с направленным формированием как однородных, так и неоднородных дозовых распределений. Они могут существенно расширить возможности ЛТ, но для их эффективного использования необходимо изучить воздействие неоднородных дозовых распределений на опухолевые и здоровые ткани организма, создать адекватные математические модели для их целенаправленного генерирования, для формирования оптимальных неоднородных (или однородных), терапевтических дозовых полей.

12. Следует подчеркнуть, что изучение и направленное формирование однородных и неоднородных дозовых распределений имеет большое значение в различных областях деятельности человека, как на нашей планете, так и в космосе. 


\section{СПИСОК ЛИТЕРАТУРЫ}

1. Клеппер Л.Я. О выборе оптимальных условий облучения злокачественных опухолей. М.: ЦЭМИ АН СССР, 1964.

2. Клеппер Л.Я. Применение методов линейного программирования и ЭВМ для выбора оптимальных условий облучения в дистанционной лучевой терапии злокачественных опухолей. М.: ЦЭМИ АН СССР, 1965.

3. Гольштейн Е.Г., Юдин Д.Б. Новые направления в линейном программировании. М.: Наука, 1979.

4. Павлов А.С. Внутритканевая гамма-, бета-терапия злокачественных опухолей. М.: Медицина, 1967.

5. Клеппер Л.Я. Формирование дозовых полей радиоактивными препаратами и аппликаторами. М.: Энергоатомиздат, 1983.

6. Клеппер Л.Я. Формирование дозовых полей дистанционными источниками излучения. М.: Энергоатомиздат, 1986.

7. Клеппер Л.Я. Формирование дозовых полей радиоактивными источниками излучения. М.: Энергоатомиздат, 1993.

8. Х Хуг О., Келлерер А. Стохастическая радиобиология. М.: Атомиздат, 1969.

9. Основы клинической радиобиологии / под ред. М.С. Джойнера и О. Дж. ван дер Когель. М.: Бином. Лаборатория знаний, 2013.

10. Клеппер Л.Я. Методы математического моделирования и оптимизации лучевой терапии рака молочной железы. М.: ЦЭМИ РАН, 2015.

11. Костылев В.A. Медицинская физика. Краткая история (прошлое, настоящее и будущеe). M.: 2010. URL: https://youtu.be/VvEknQmb96I.

12. Клеппер Л.Я. Математические модели редукции неоднородных распределений дозы в тканях в адекватные дозы их однородного облучения // Медицинская радиология и радиационная безопасность. 2010. Т. 55. № 6. С. 58-65.

13. Кейрим-Маркус И.Б. Эквидозиметрия. М.: Атомиздат, 1980.

14. Ellis F. Dose, time and fractionation: A clinical hypothesis // Clin. Radiol. 1969. Vol. 2. P. 1-7.

15. Kirk J., Gray W., Watson E. Cumulative radiation effect. Part 1. Fractionated treatment regiones // Clin. Radiol. 1971. № 22. P. 145-155.

16. Orton C., Ellis F. A simplification in the use of the NSD concept in practical radiotherapy // Brit. J. Radiol. 1973. Vol. 46. № 457. P. 529-537.

17. Barendsen G.W. Dose fractionation, dose rate and isoeffect relationships for normal tissue response // Int. J. Rradiat. Oncol. Biol. Phys. 1982. № 8. P. 1981-1997.

18. Клеппер Л.Я. Синтез радиологических моделей и радиологические инварианты. Часть первая // Медицинская техника. 2005. № 3. С. 27-32.

19. Клеппер Л.Я. Синтез радиологических моделей и радиологические константы. Часть вторая // Медицинская техника. 2006. № 3. С. 23-27. 
20. Клеппер Л.Я. Синтез радиологических моделей и радиологические константы. Часть третья. Синтез популяционно-феноменологических моделей и ММ Клеппера // Meдицинская техника. 2006. № 4. С. 22-26.

21. Клеппер Л.Я. Синтез радиологических моделей и радиологические константы. Часть четвертая. Синтез популяционно-феноменологических моделей и математической модели Lyman // Медицинская техника. 2006. № 5. С. 36-40.

22. Клеппер Л.Я. Синтез модифицированной LQ модели и функции нормального распределения вероятностей для прогнозирования лучевых осложнений в органах и тканях // Медицинская радиология и радиационная безопасность. 2011. № 1 (56). C. $43-50$.

23. Lyman J.T., Wolbarst A.B. Optimisation of radiation therapy, 111: A method of assessing complication probabilities from dose-volume histograms // Int. J. Radiat. Oncol. Biol. Phys. 1987. Vol. 13. P. 103-109.

24. Клеппер Л.Я. LKB модель для расчета вероятности возникновения лучевых осложнений в органах и тканях и ее анализ // Медицинская физика. 2015. № 1 (65). С. 32-38.

25. Munro N.R., Gilbert C.W. The relation between tumour lethal doses end the radiosensitivity of tumour cells // Brit. J. Radiol. 1961. № 34. P. 246-251.

26. Клеппер Л.Я. Об определении оптимальных условий облучения Системы «опухольевая ткань+нормальная ткань (ложе опухоли)» (теоретические аспекты проблемы) // Медицинская физика. 2015. № 2 (66). С. 23-33.

27. Клеппер Л.Я. Методы математического моделирования и оптимального планирования лучевой терапии злокачественных опухолей. М.: ЦЭМИ РАН, 2017.

28. Клеппер Л.Я. Неоднородные дозовые распределения и планирование лучевой терапии злокачественных опухолей. М.: ЦЭМИ РАН, 2018.

29. Клеппер Л.Я. Обоснование метода гипофракционирования при ЛТ ранних стадий рака молочной железы на основе математического моделирования // Медицинская физика. 2016. № 1 (68). С. 8-14.

30. Клеппер Л.Я. Проблемы стохастической радиологии. М.: ЦЭМИ РАН, 2019.

31. Emami B., Lyman J., Brown A., Coia L., Goiten M., Munzenride J.E., Shank B., Solin L.J., Wesson M. Tolerance of normal tissue to therapeutic radiation // Int. J. Radiat. Oncol. Biol. Phys. 1991. Vol. 21. № 1. P. 109-122.

32. Kehwar T.S., Sharma S.C. Use of normal tissue tolerance doses into linear quadratic equation to estimate normal tissue complication probability // Radiation Oncology Online Journal. 2003. URL: www.rooj.com.

33. Kehwar T.S., Sharma S.C. Use of normal tissue tolerance doses into linear quadratic equation to estimate normal tissue complication probability // J. Cancer Res. Ther. 2005. Vol. 1. Iss. 3. P. 1-27.

34. Клеппер Л.Я. Математическое моделирование оптимальных дозовых полей в лучевой терапии злокачественных опухолей. Часть 2 (контактные методы лучевой терапии) // Медицинская техника. 2004. № 4. С. 27-32. 
35. Evaluation of treatment planning for particle beam radiotherapy / Publiched by the radiotherapy development branch radiation research program division of cancer treatment national cancer institute, 1987.

36. Kaplan N.E. Evidence for a tumoricidal dose level in the radiotherapy of Hodgkin's disease // Cancer Research. 1966. № 26. Pt. 1. P. 1221-1224.

37. Shukovsky L.J. Dose, time, volume radiftionshipson squamous cell carcinoma of the supraglotic larynx // Amer. J. Roentgenol. 1971. Vol. 108. № 1. P. 27-29.

38. Феллер Б. Введение в теорию вероятностей и ее приложения. М.: Мир, 1967.

39. Моисеев Н.Н. Математические задачи системного анализа. М.: Наука, 1981.

40. Подиновский В.В., Ногин В.Д. Парето оптимальные решения многокритериальных задач. М.: Наука,1982.

41. Radiation pneumonits: A new approach to the derivation of time-dose factors / Wara W.M., Phillips T.L., Margolis L.W. et al. // Cancer. 1973. № 32. P. 547-552.

42. Холин B.B. Радиобиологические основы лучевой терапии злокачественных опухолей. Л.: Медицина, 1979.

43. Qi X.S., White J., Li X.A. Is $\alpha / \beta$ for breast cancer really low? // Radiotherapy and Oncology. 2011. № 100. Р. 282-288.

44. Олейник Ю.А., Клеппер Л.Я. О выборе оптимальных динамических условий облучения злокачественных опухолей // Кибернетика. 1968. № 6. С. 93-98.

45. Клеппер Л.Я., Олейник Ю.А. Задачи выбора оптимальных физических и динамических условий облучения злокачественных опухолей // Мед. радиология. 1969. № 3. С. 64-73.

46. Олейник Ю.А., Клеппер Л.Я. Определение оптимальных динамических условий облучения злокачественных новообразований и адаптивное лучевое лечение // Мед. радиология. 1973. № 2. С. 49-54.

47. Bentzen S.M., Thames H.D., Overgaard J. Dose variation in the in vitro cellular radiosensitivity explain the chalow clinical dose-cjntrol curve for malignant melanoma? // Int. J. Radiat. Biol. Phys. 1990. № 57. P. 117-126.

48. ICRU (1999). Prescribing, recording and reporing photon beam therapy/ ICRU, Rtport 62, Oxford University Press, 1999.

49. Клеппер Л.Я., Олейник Ю.А. Определение оптимальных условий облучения методом последовательного ввода ограничений // Мед. радиология. 1978. № 8. С. 64-72.

50. Клеппер Л.Я. Программа решения задачи нелинейного программирования с нелинейными ограничениями. Ротапринт. М.: ЦЭМИ АН СССР, 1979.

51. Клеппер Л.Я. Решение задачи линейного программирования специального вида с выделением структурных переменных. М.: ЦЭМИ АН СССР, 1980.

52. Клеппер Л.Я. Определение оптимальных планов облучения с ограниченным числом направлений облучения // Мед. радиология. 1981. № 2. С. 51-55. 
53. Клеппер Л.Я. Об устойчивости оптимального решения задачи определения оптимальных физико-технических условий облучения // Мед. радиология. 1983. № 3. C. 33-38.

54. Клеппер Л.Я. Актуальные проблемы дозиметрического планирования лучевой терапии злокачественных опухолей // Медицинская техника. 1992. № 6. С. 21.

55. Клеппер Л.Я. Проблема выбора оптимальных точек центрации пучков излучения при планировании лучевой терапии злокачественных опухолей // Медицинская техника. 1999. № 3. С. 26-30.

56. Клеппер Л.Я. Нелинейная экстремальная задача определения оптимального плана облучения с выбором оптимальной точки пересечения центральных осей пучков излучения // Медицинская радиология и радиационная безопасность. 1999. Т. 44. № 2. C. 61-66.

57. Клеппер Л.Я. Математическое моделирование оптимальных дозовых полей в лучевой терапии злокачественных опухолей. Часть 1 (дистанционная лучевая терапия) // Медицинская техника. 2004. № 1. С. 30-37.

58. Клеппер Л.Я. Математическое моделирование оптимальных дозовых полей в лучевой терапии злокачественных опухолей. Часть 2 (контактные методы лучевой терапии) // Медицинская техника. 2004. № 4. С. 27-32.

59. Клеппер Л.Я., Антипина Н.А. Определение вероятности излечения опухолевого заболевания при внутритканевой лучевой терапии злокачественных новообразований // Медицинская физика. 2006. № 4 (32). С. 30-41.

60. Клеппер Л.Я. Синтезированная математическая модель для планирования мультифракционированной лучевой терапии опухолевых заболеваний. Сообщение 1 . Неравномерный метод облучения // Медицинская радиология и радиационная безопасность. 2007. № 5. С. 61-70.

61. Клеппер Л.Я. Синтезированная математическая модель для планирования мультифракционированной лучевой терапии опухолевых заболеваний. Сообщение 2. Анализ математических моделей мультифракционированного облучения // Медицинская радиология и радиационная безопасность. 2007. Т. 52. № 6. С. 56-62.

62. Strandqvist $M$. Studien uber die kumulative wirkung der rontgenstrahlen bei fraktionierung // Acta Radiol. 1944. Suppl. P. 55-57.

63. Fowler J.F., Denekamp J. Radiation effects on normal tissues // Cancer. A comprehensive treatise. Ney-Iork, Lodon, 1977, Vol. 6.

64. Рудерман А.И., Вайнберг М.Ш., Жолкивер К.И. Дистанционная гамма-терапия злокачественных опухолей. М.: Медицина, 1977.

65. Schultheiss T.E., Orton C.G., Peck R.A. Model in radiotherapy: Volume effects // Med. Phys. 1983. № 10 (4). P. 410.

66. Ulmer $W$. Aspects of the volume effect in the linear-quadratic and cubic model // Strahlentherapie und Onkologie. 1987. Vol. 163. № 2. P. 123-129. 
67. Жолкивер К.И., Зевриева И.Ф., Досаханов А.Х. Количественная оценка биологического эффекта радиации в нормальных тканях при лучевой терапии злокачественных новообразований: методические рекомендации. Алма-Ата, МЗ Каз. ССР, 1983.

68. Факторы время-доза-фракционирование и их использование в лучевой терапии злокачественных опухолей (методические рекомендации) / Фадеева М.А., Костромина К.Н., Даценко В.С. и др. М.: МЗ СССР, ЦОЛИУ врачей, 1987.

69. Chadwick K.H., Leenhouts H.P. A molecular theory of cell survival // Phis. Med. Biol. 1973. № 18. P. 78-87.

70. Kellerer A.M., Rossi H.H. RBE and the primary mechanism of radiation action // Radiat. Res. 1971. № 47. P. 14-34.

71. Cohen L. Dose-time relationship: computation of cell lethality following fractionated radiation therapy // Int. J. Radiat. Oncol. Biol. Phys. 1978. № 3. P. 267-271.

72. Orton C.G., Cohen L. A unified approach to dose effect relationships in radiotherapy. 1: Modified TDF and Linear quadratic equations // Int. J. Rradiat.Oncol.Biol.Phys.,1988, № 3, P. 601.

73. Videroe R. // Acta Radiol. 1966. № 4. P. 257.

74. Pitkanen M., Viitanen J., Ojava A. Application of linear quadratic model for clinical radiotherapy. Teshnical Research Centr of Finland, 1988.

75. Weibull W. A statistical distribution function of wide applicability // J. Appl. Mechanics. 1951. № 18. P. 293.

76. Ярмоненко С.П., Вайнсон А.А., Календо Г.С., Рампан Ю.И. Биологические основы лучевой терапии опухолей. М.: Медицина, 1976.

77. Алиев Б.М. Неравномерное облучение злокачественных опухолей (физикодозиметрические, экспериментальные и клинические исследования). Дисертация ... д-ра мед. наук. М., 1973.

78. Алиев Б.М. Лучевая терапия запущенных форм злокачественных новообразований. М.: Медицина, 1978.

79. Харитонова Н.T. Возможности неравномерной дистанционной гамма-терапии при распространённом раке пищевода. Автореф. дис. ... канд. мед. наук. М.: 1980.

80. Юрьева T.B. Возможности дистанционно гамма-терапии местно-распространённых сарком мягких тканей в плане комбинированного лечения. Автореф. дис. ... канд. мед. наук. М.: 1982. 
Монография

Л.Я. Клеппер

МРВ МОДЕЛИ ДЛЯ ПЛАНИРОВАНИЯ ЛТ ЗЛОКАЧЕСТВЕННЫХ ОПУХОЛЕЙ

(справочное пособие

для лучевых терапевтов

и медицинских физиков)

Подписано в печать 12.01.2021 г.

Формат 60×90/16. Печ. л. 12,2. Тираж 80 экз. Заказ № 2

ФГБУН Центральный экономико-математический институт РАН

117418, Москва, Нахимовский пр., 47

Тел. 8 (499) 724-21-39

E-mail: ecr@cemi.rssi.ru

http://www.cemi.rssi.ru/

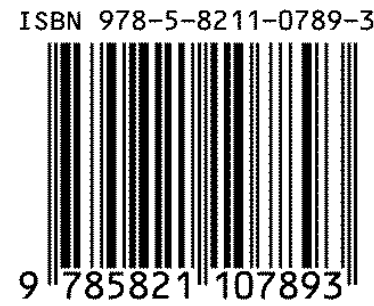

